\title{
Improving health of broiler chickens by early life modulation of immune responsiveness and intestinal microbiota
}

Recruiting Natural Killer cells for increased resistance

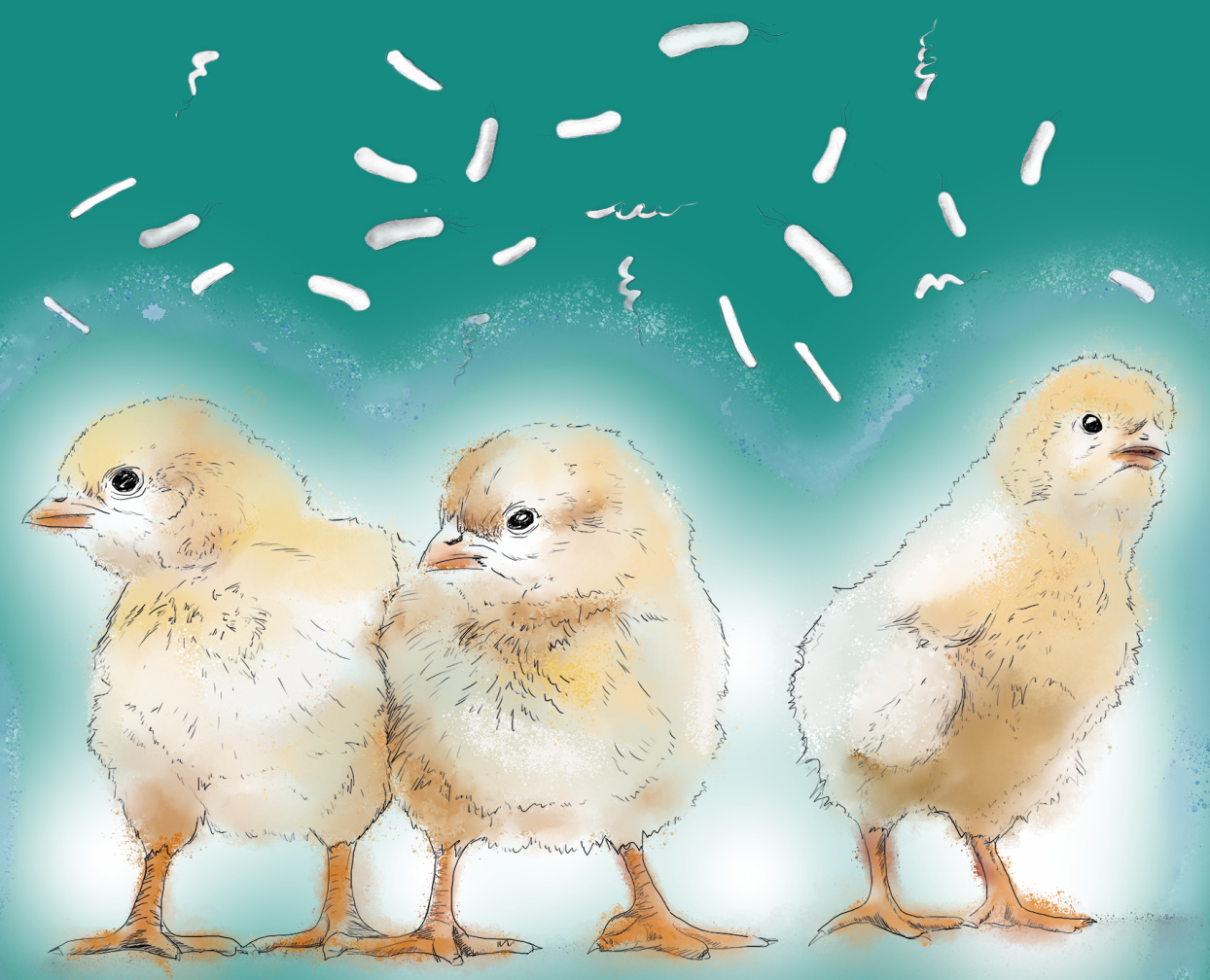

Nathalie Meijerink 


\section{Improving health of broiler chickens by early life modulation of immune responsiveness and intestinal microbiota}

Recruiting Natural Killer cells for increased resistance

Nathalie Meijerink 


\section{Colofon}

(C) 2021 Nathalie Meijerink

ISBN: 978-90-393-7407-8

(Cover) illustrations: Rike van de Grift

Cover design and layout: Jasmijn de Boer

Thesis printing: proefschrift-aio.nl

All rights reserved. No part of this thesis may be reproduced, stored or transmitted in any form or by any means without prior permission of the author.

The research described in this thesis was carried out at the Division of Infectious Diseases \& Immunology, Department of Biomolecular Health Sciences, Faculty of Veterinary Medicine, Utrecht University, the Netherlands and was funded by the Dutch Research Council (NWO) under the NWO Earth and Life Sciences (ALW) research programme.

Printing of this thesis was financially supported by Infection \& Immunity Utrecht, by the Division Infectious Diseases \& Immunology, Faculty of Veterinary Medicine, Utrecht University, the Netherlands and by Cargill Inc. 
Improving health of broiler chickens

by early life modulation of immune responsiveness and intestinal microbiota

Recruiting Natural Killer cells for increased resistance

Het verbeteren van de gezondheid van vleeskuikens door modulatie van immuunresponsiviteit en darmmicrobiota op jonge leeftijd

Rekruteren van Natural Killer cellen voor een verhoogde weerstand

(met een samenvatting in het Nederlands)

Proefschrift

ter verkrijging van de graad van doctor aan de Universiteit Utrecht op gezag van de rector magnificus, prof.dr. H.R.B.M. Kummeling, ingevolge het besluit van het college voor promoties in het openbaar te verdedigen op woensdag 8 december 2021 des middags te 2.15 uur

door

Nathalie Meijerink

geboren op 26 mei 1991

te IJsselmuiden 
Promotoren: Prof.dr. V.P.M.G. Rutten

Prof.dr. J.A. Stegeman

Copromotor: Dr. C.A. Jansen 


\section{Contents}

$\begin{array}{lll}\text { Chapter } 1 & \text { General introduction } & 7\end{array}$

Chapter 2 Analysis of chicken intestinal natural killer cells, a major IEL subset 25 during embryonic and early life

Chapter 3 Early life inoculation with adult-derived microbiota accelerates maturation of intestinal microbiota and enhances NK cell activation in broiler chickens

Chapter 4 Glucose oligosaccharide and long-chain glucomannan feed additives induce enhanced activation of intraepithelial NK cells and relative abundance of commensal lactic acid bacteria in broiler chickens

Chapter 5 A detailed analysis of innate and adaptive immune responsiveness upon infection with Salmonella enterica serotype Enteritidis in young broiler chickens

Chapter 6 Long-chain glucomannan supplementation modulates immune responsiveness and intestinal microbiota resulting in improved resistance to Salmonella enterica serotype Enteritidis in broiler chickens

Chapter 7

Summarizing discussion

Dankwoord / Acknowledgements

Curriculum Vitae

List of Publications 

CHAPTER 1

General introduction 
The poultry sector is a major contributor to human food industry and poultry meat is considered a key sustainable source of high protein and low fat, due to the high growth rate and efficient feed conversion by broiler chickens and consequently low production costs ${ }^{1,2}$. Global consumption of poultry meat is expected to increase to $145 \mathrm{Mt}$ (million metric tons, 145 billion $\mathrm{kg}$ ) over the next ten years, an increase of $\sim 10 \%$ of its current consumption, that accounts for $50 \%$ of the total increase in global meat consumption. Consequently, poultry becomes the most widely consumed meat as compared to pork, beef and goat/sheep ${ }^{1}$. Hence, the health and production efficiency of broiler chickens are of great importance. However, the ban on preventive use of antimicrobials in animal production by EU legislations ${ }^{3}$ have led to an increase in intestinal health problems in chickens and therefore, alternative strategies to improve their health are required ${ }^{4,5}$. Infections with intestinal pathogens including Clostridium perfringens and Salmonella serovars Enteritidis or Typhimurium may not only induce severe disease in chickens, but also increase the risk of foodborne disease in humans $s^{6,7}$. Furthermore, infections with other Salmonella serovars and Campylobacter are mainly asymptomatic in chickens but still have an high public health impact ${ }^{8}$.

Both the immune system and intestinal microbiota are important entities contributing to the health of broiler chickens. Potentiation of protection by immune cells, by increased numbers as well as activity, and modulation of intestinal microbiota composition may increase the resistance of broiler chickens to infectious agents. In the next sections of this introduction an overview is provided of the chicken systemic and intestinal immune systems, the intestinal microbiota, the interaction between these as well as important intestinal pathogens in chickens are described. Furthermore, possible strategies to stimulate number and function of immune cells, and modulate intestinal microbiota that may improve the health of chickens are introduced.

\section{The avian immune system}

The immune system constitutes of a complex interplay between many immune cells and immune proteins aimed to protect the host against diseases caused by invading pathogens. In general, the structure and function of the chicken immune system is similar to that in mammals, although chickens lack structured peripheral or mesenteric lymph nodes ${ }^{9,10}$. The immune system can be divided into innate and adaptive components. The innate immune system is the first to respond, rapid and non-specific, and may directly reduce replication or spread of bacteria and viruses by cytotoxic activity, phagocytosis or production of antimicrobial products. Innate immune cells are activated by pathogen-associated molecular patterns (PAMPs), which are recognized through pattern recognition receptors (PRRs) ${ }^{11}$. Amongst the cells of the innate immune system, natural killer (NK) cells are important players. NK cells kill virus-infected cells, tumor cells and bacteria and recruit or activate other immune cells by the secretion of chemokines and cytokines. Furthermore, macrophages, heterophils (equivalent to mammalian neutrophils) and dendritic cells (DCs) are part of the innate immune system. Macrophages and heterophils are involved in phagocytosis and destruction of bacteria. DCs are also involved in phagocytosis similar to macrophages and both of them secrete cytokines that activate other immune cells and act as professional antigen-presenting cells (APCs). Professional APCs present peptides from phagocytosed pathogenic material or intracellular pathogens on both major histocompatibility complexes (MHC) class I and II to T cells of the adaptive immune system. The activation and differentiation of $\mathrm{T}$ cells by APCs requires recognition of antigens presented on $\mathrm{MHCl}$ (differentiation to $\mathrm{CD}^{+}$ $\mathrm{T}$ cells) or $\mathrm{MHCll}$ (differentiation to $\mathrm{CD}^{+} \mathrm{T}$ cells), binding of co-stimulatory receptors and stimulation by cytokines. 
The adaptive immune system is thus activated by specific antigens and innate immune reactivity that results in proliferation of effector cells. Therefore, it takes longer, days or weeks, to develop an effective adaptive immune response. Adaptive immune cells include $T$ and $B$ cells and their responses are highly specific for particular pathogens. Following initial activation of $\mathrm{T}$ and $\mathrm{B}$ cells, some differentiate to effector cells, others to memory cells that respond faster and stronger upon subsequent encounters with the same pathogen ${ }^{12,13}$. The majority of T cells acquire the $\alpha \beta$ T cell receptor (TCR) while other $T$ cells express the $\gamma \delta T C R$, alongside expression of the TCR co-receptors CD4 and CD8. CD4 ${ }^{+}$and $\mathrm{CD}^{+} \mathrm{T}$ cells are selected based on strong affinity engagement with respectively either $\mathrm{MHCll}$ or $\mathrm{MHCl}$ molecules (positive selection) and weak interactions with MHC-presented self-antigens on APCs (negative selection) ${ }^{14,15}$. The CD8 co-receptor is composed of two chains and the most common isoform is $C D 8 \alpha \beta$, which is involved in the specific antigen-driven cytotoxicity ${ }^{16,17}$. In contrast, the other isoform CD8 $\alpha \alpha$ does not follow conventional positive selection and is not expressed on naïve T cells, but can be readily induced on activated T cells independent of the TCR-MHC restriction including CD4+, $\mathrm{CD} 8 \alpha \beta^{+} \mathrm{CD} 3^{+} \mathrm{TCR} \alpha \beta^{+} \mathrm{T}$ cells and $\mathrm{CD} 3^{+} \mathrm{TCR} \gamma \delta^{+} \mathrm{T}$ cells ${ }^{18}$. In addition, the positive selection of intestinal CD $8 \alpha \alpha^{+}$precursors results in double negative TCR $\alpha \beta^{+}$or TCR $\gamma \delta^{+}$T cells that directly migrate to the intestinal epithelium where they acquire CD8 $\alpha \alpha$ expression. Intraepithelial $\gamma \delta T$ cells and $\alpha \beta T$ cells therefore mostly express CD8 $\alpha \alpha$ alone ${ }^{14,17}$. The antigen specificity of intestinal CD8 $\alpha \alpha^{+} T$ cells is unclear, however, their reactivity is restricted by classical or nonclassical $\mathrm{MHCl}$ molecules ${ }^{16,19}$. With regard to function, activated CD4+ helper T cells such as Th1, Th2 and Th17 secrete cytokines to optimize immune responses, whereas $C D 4^{+}$regulatory $T$ cells secrete cytokines to balance immune responses. CD8 $\alpha \beta^{+}$cytotoxic T cells kill infected or dysfunctional cells. The role of CD8 $\alpha \alpha^{+} T$ cells is not completely understood since these cells show rapid non-specific cytotoxicity as well as anti-inflammatory cytokine production to promote integrity of the intestinal barrier ${ }^{16,18}$. The $\gamma \delta T$ cells rapidly respond to foreign agents and thereby strengthen the innate immune response ${ }^{20-23}$. High numbers of $\gamma \delta T$ cells are found in the intestine of both mammals and birds, whereas circulating $\gamma \delta$ T cells are abundantly present in chickens $^{24}$, ruminants ${ }^{25}$ and pigs ${ }^{26}$ in contrast to humans ${ }^{27}$. Functions of $\gamma \delta T$ cells comprise cytotoxicity $\left(\mathrm{CD} 8 \alpha \alpha^{-/+}\right.$and $\left.\mathrm{CD} 8 \alpha \beta^{+}\right)$and production of cytokines such as TNF $\alpha$ and interleukin-10 (IL-10), and antimicrobial proteins such as Regllly $\left(\mathrm{CD} 8 \alpha \alpha^{-/+}\right)^{16,19}$. In addition, $\mathrm{CD} 8 \alpha \alpha^{-/+} \gamma \delta$ T cells maintain epithelial barrier functions by production of factors such as TGF $\beta$ that stimulate wound healing ${ }^{16,19}$.

Activated B cells differentiate into plasma cells that secrete antibodies. Other functions of B cells include secretion of cytokines and antigen presentation to T cells. Antibodies can inactivate foreign agents such as pathogens, neutralize toxins, form antigen-antibody complexes that activate the complement system and its bactericidal activity, facilitate phagocytosis (opsonization) and bind to target cells to facilitate antibody-dependent cellular cytotoxicity by NK cells (ADCC) ${ }^{28}$. Ultimately, innate and adaptive immune cells work closely together to induce effective immune responses that control infections.

The development of the immune system in chickens starts early in the embryonic phase. Cells of the innate and adaptive immune system arise from haematopoietic stem cells that populate the developing bone marrow in the embryo. The differentiation of innate immune cells starts around embryonic day (ED) 6. Innate immune cells subsequently migrate from the bone marrow to peripheral tissues such as the spleen and intestine, and are functional at hatch ${ }^{29-31}$. In contrast, $\mathrm{T}$ and B cell-precursors migrate from the bone marrow to respectively the thymus and bursa of Fabricius. The differentiation to mature but as yet naïve T and B cells starts at approximately ED12, followed by a gradual migration of these $T$ and $B$ cells to the periphery. Hence at hatch, the adaptive immune system is not fully developed yet and only from two to three weeks of life on, shows effective responses upon exposure to pathogens $\mathrm{s}^{29,30}$. As a consequence immediately post-hatch, the defense against pathogens of young chickens relies on maternal antibodies, obtained from the yolk in the egg, and on innate immune responses in which NK 
cells are key players. This is especially applicable to the intestine, the site of entry of the majority of environmental infections early in life.

\section{Intestinal immune system}

The intestinal tract includes the largest compartment of the immune system, as compared to other tissues, that is continuously exposed to dietary components and commensal microbes. An important feature of the immune system in the intestine is the requirement not to respond to the majority of foreign agents derived from food or commensal microbes. Such immune responses would otherwise result in gut inflammation and consequently impair health and production efficiency ${ }^{32}$.

The first line of defense in the intestine is a physical barrier including the mucus layer and the epithelium layer directly underneath ${ }^{33}$. The small intestine of chickens consists of the duodenum, jejunum and ileum, and the large intestine consists of two caeca and the colon. In terms of immune responsiveness, the ileum is one of the sites of immune activation with many lymphoid structures ${ }^{34,35}$. In mammals, gut-associated lymphoid tissues (GALT) consist of organized series of lymphoid structures throughout the intestine. In chickens, however, GALT is more scattered along the intestinal tract in specialized lymphoid structures located at strategic sites. Furthermore, lymphoid cells reside in the epithelial lining of the gut and are distributed in the underlying lamina propria in both mammals and chickens ${ }^{32}$. Lymphocytes scattered between the epithelial cells are referred to as intraepithelial lymphocytes (IELs). Chicken IELs include high numbers of NK cells, the majority of which express the IL-2 receptor alpha chain (IL-2R $\alpha$, designated CD25) ${ }^{36,37}$. In addition, the IEL population consists of a large number of T cells including $\gamma \delta T$ cells and $C D 8^{+} \alpha \beta$ T cells $^{36,37}$. In the lamina propria, mainly macrophages, heterophils, $D C s, B$ cells and $C D 4^{+} T$ cells are located as well as the specialized lymphoid structures ${ }^{38}$. These specialized structures include lymphoid aggregates located within the lamina propria such as Meckel's diverticulum (remnant of the yolk stalk located at the onset of the ileum), mammalian-like Peyer's patches (PPs) and caecl tonsils ${ }^{35}$. Lymphoid aggregates consist of specialized epithelium containing microfold (M) cells. These M cells continuously sample gut lumen content, which is transferred to underlying macrophages and DCs. In addition, lymphoid follicles rich in B and T cells are in close proximity to the $\mathrm{M}$ cells (Fig. 1).

IELs are the first immune cells to respond to invading pathogens as well as to be affected by commensal microbes and feed constituents. The IEL population communicates with the underlying immune cells and substantial cellular traffic exists between the gut immune structures and systemic immune sites including the bone marrow and spleen ${ }^{32}$. Therefore, intraepithelial NK cells, $\gamma \delta T$ cells and $\mathrm{CD}^{+} \alpha \beta \mathrm{T}$ cells are important in initiating and mediating immune responsiveness. Upregulation of presence and function of IELs is likely to increase the resistance of chickens to infections. Since the resistance of young chickens mainly depends on innate immune responses, early life potentiation of intraepithelial NK cells in particular may contribute to reducing disease susceptibility. 


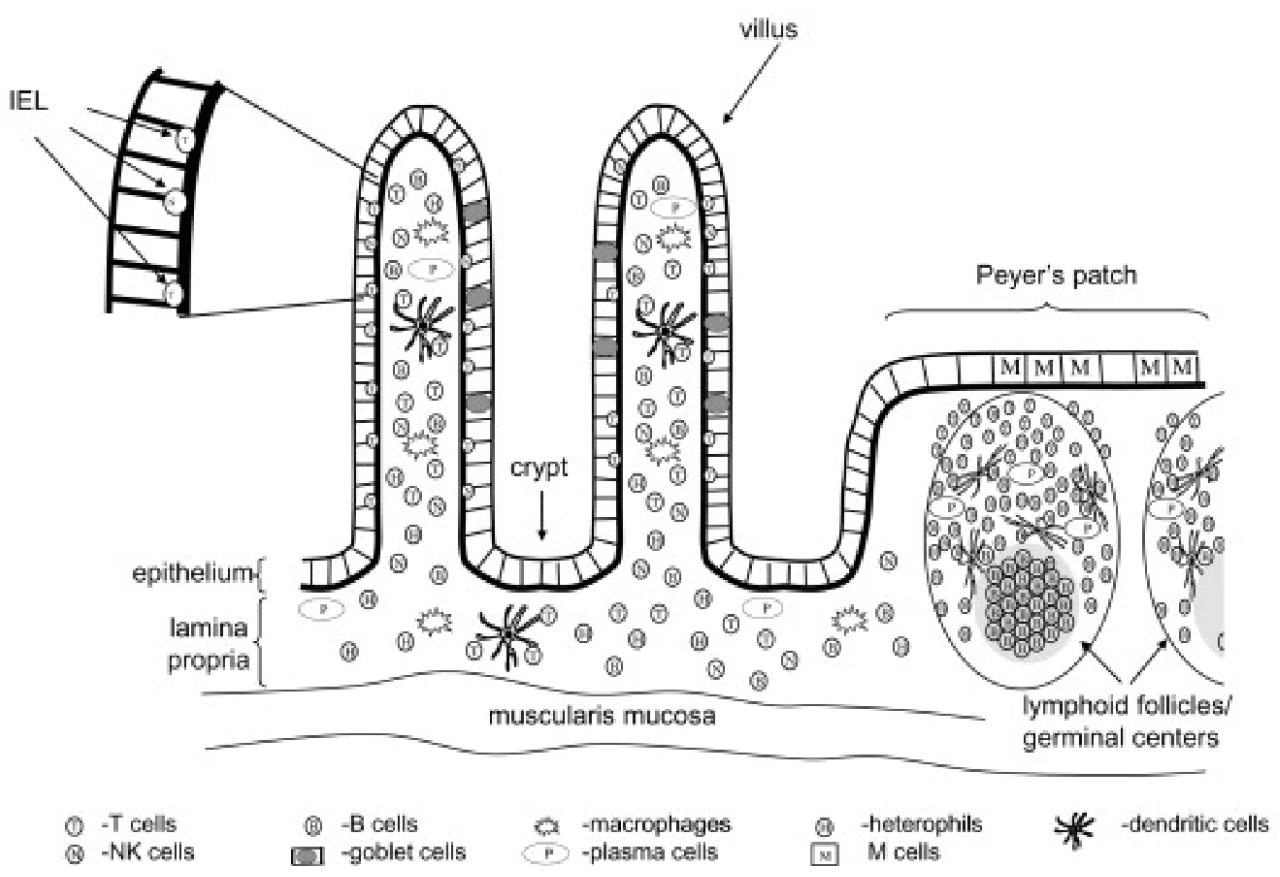

Figure 1. Schematic representation of the immune cell compartments in the small intestine of chickens. The intraepithelial lymphocytes (IELs) are scattered between epithelial cells and include natural killer (NK) cells, $\gamma \delta$ T cells and $C D 8^{+} \alpha \beta$ T cells. Adapted from Smith et al. $2013^{32}$

\section{Natural Killer cells}

The mechanisms of NK cell activation are complex and determined by the balance between pathogen-related signaling through activating and inhibitory NK receptors (NKRs) ${ }^{39-42}$. Human NKRs are constituted of three major families; the killer cell Ig-like receptor (KIR) superfamily, the C-type lectin superfamily including CD94/NKG2 receptors and natural cytotoxicity receptors (NCRs) ${ }^{39}$. Similar NKRs have been found on NK cells in cattle, pigs and chickens as well as mice and rats, that in addition express C-type lectin-like Ly49 receptors, which are absent in humans ${ }^{42-45}$. Furthermore, chickens express Ig-like receptors (CHIRs) that share features of $\mathrm{KIRs}^{42}$. Activation of NK cells results from non-engaged inhibitory receptors due to $\mathrm{MHCl}$ molecules that lack self or foreign antigens or by the down-regulation of $\mathrm{MHCl}$ on infected or tumor cells. Another way is by the engagement of activating receptors with stress-induced ligands on infected or transformed cells, or virus-encoded ligands ${ }^{46}$. Other receptors involved in activation of NK cells are $F_{c}$ receptors $\left(F_{c} \gamma R\right.$ RIII/CD16) triggering $\mathrm{ADCC}^{39}$, and Toll-like receptors (TLRs) $)^{47,48}$ and NOD-like receptors (NLRs) ${ }^{49}$ that recognize bacterial or viral PAMPs. One of the CHIRs, CHIR-AB1, was recognized as $F_{c}$ receptor with high binding affinity for $\lg Y$ (equivalent to mammalian $\lg G$ ) and may be involved in $A D C C$ in chickens ${ }^{50}$. Furthermore, NK cells express several chemokine and cytokine receptors that are involved in respectively chemotaxis and activation by binding to APC and T cell-derived cytokines such as IL-2, IL-12, IL-15, IL-18 or IFN $\gamma^{39,40,46,51}$. In other cases, NK cell activation requires both recognition of PAMPs by TLRs, NLRs and retinoic acid inducible gene I (RIG-I)-like receptors, and cytokine stimulation by APCs ${ }^{52,53}$. In response 
to viruses, human and murine NK cell activation was induced by several NKRs including NKp4654, NKp44 ${ }^{55}$, NKp30 $0^{56}$, activating receptor NKG2D ${ }^{57}, \mathrm{CD} 4-\mathrm{NKG}^{2} \mathrm{E}^{58}$ and Ly49H ${ }^{59}$, or by TLR2 and TLR3 ${ }^{51}$. In chickens, the activating receptor CHIR-A was reported to recognize avian influenza virus ${ }^{41}$. During bacterial infections, activation of human NK cells was mediated by KIR3DL2 ${ }^{47}, T L R 2^{60}, T L R 3^{61}$ and $\mathrm{TLR}^{48}$. Activation of NK cells leads to cytotoxicity for target cells; by the release of perforins and granzymes (degranulation), production of IFNY or by engagement of death ligands like TNF-related apoptosis-inducing ligand (TRAIL) and FasL on NK cells with the respective death receptors on infected or tumor cells, resulting in apoptosis ${ }^{62}$. Secondly, NK cells produce chemokines such as XCL1 and CCL to recruit other immune cells, and immunoregulatory cytokines including IL-2, IL-10, IL-12, TNF- $\alpha$ and predominantly IFN $\gamma^{46,63}$. Furthermore, human NK cells were shown to be a source of antimicrobial peptides such as $\alpha$-defensins and cathelicidins that directly kill bacteria ${ }^{64}$. Lastly, NK cells are suggested to be involved in antigen presentation, since human, murine and porcine NK cells showed expression of APC-associated markers including MHCII, CD80 and CD86, and were able to internalize antigens derived from killed target cells $s^{65-67}$. NK cells are thus important in the early defense against pathogens by both direct killing and stimulation of other immune cells that results in killing of target cells.

However, the understanding of NK cell biology is hampered by the various phenotypic definitions of NK cells across species. Since NK cells share phenotypic properties with T cells, identification of NK cells by flowcytometry requires the marker CD3 to exclude T cells. Human NK cells are identified as CD3 $\mathrm{CD}^{+} 6^{+}$lymphocytes ${ }^{39}$, whereas murine NK cells do not express a CD56 homolog and are recognized as $\mathrm{CD}^{-} \mathrm{NK}^{-1.1^{+}}$or $\mathrm{CD}^{-} \mathrm{CD}^{-}$(9b $^{+}$depending on the mouse strain ${ }^{68}$. In rats, the marker NKR-P1 is used to identify NK cells among CD3 negative lymphocytes ${ }^{69}$. In addition, expression of the NCR NKp46 is used to identify human and murine NK cells ${ }^{70}$. In veterinary species including cows, pigs, sheep and chickens, CD56 is not used as a NK cell marker. Bovine NK cells are identified as CD3- NKp46+71, ovine NK cells as CD3- NKp46 ${ }^{+72}$ and porcine NK cells were described as CD3- perforin ${ }^{+}$CD2 ${ }^{+}$CD8 $\alpha^{+}$CD $16^{+}$ with a distinct population of these cells expressing NKp4645. In chickens, expression of CD56 was only observed on subpopulations of NK cells in the lung and embryonic spleen ${ }^{73}$ and NKp46 has not been identified yet in chickens ${ }^{42}$. To date, a pan-NK marker is not available for chickens. However, several markers have been identified, such as IL-2R $\alpha, 20 \mathrm{E} 5$ and 5C7, that are expressed on CD3-lymphocytes that show NK cell functions in the intestine, spleen, blood and lung of layer chickens ${ }^{36,74,75}$. It is of note that the antigens recognized by most of these markers in chickens, i.e. $20 \mathrm{E} 5$ and $5 \mathrm{C7}$, are still unknown, which complicates comparisons with NK cell subsets in other species. Nevertheless, CD3- IL-2R $\alpha^{+} \mathrm{NK}^{-}$ cells are abundantly present in the intraepithelial layer of the chicken intestine ${ }^{36}$ and a subset of these NK cells also expresses the chicken Fc receptor CHIR-AB150.

Following their identification, NK cells in humans have been divided into two functionally different subsets based on expression of surface markers in blood. Circulating NK cells are divided in a CD56 dim CD16 bright subset that has a naturally higher cytotoxic capacity and a CD56 bright CD $16^{\text {-/dim }}$ subset with a natural higher capacity to produce immunoregulatory cytokines ${ }^{39,40,76}$. The CD56 dim subset constitutes the majority of total NK cells in blood and bone marrow, whereas the CD56 bright NK cells are the majority in secondary lymphoid organs, such as lymph nodes, spleen and GALT, and most other non-lymphoid tissues $^{77}$. It is of note that within the tissue-resident CD56 bright subset, a high phenotypical and functional diversity is observed that can be distinct from circulating CD $56^{\text {bright }} \mathrm{NK}$ cells ${ }^{77}$. In addition, the functional distinction between subsets is less clear upon stimulation of circulating NK cells and emphasizes the complexity of NK cells by their high heterogeneity and plasticity. Stimulation with cytokines such as IL-2 and IL-12 induced similar levels of cytotoxicity ${ }^{78,79}$ and resulted in cytokine production such as IFNy by both circulating NK cell subsets ${ }^{80,81}$. These observations are related to expression of the high-affinity heterotrimeric IL-2R (IL-2R $\alpha \beta \gamma)$, which is considered as an early activation marker. Circulating CD56 bright 
NK cells constitutively express IL-2R $\alpha \beta \gamma$ and show high proliferation in response to low levels of IL-2, whereas the CD56 dim NK cells express only the low-affinity heterodimeric IL-2R $\beta Y$ and show almost no proliferation ${ }^{78,79}$. LPS stimulation resulted in enhanced cell cytotoxicity and cytokine production accompanied with up-regulation of IL-2R $\alpha$ on circulating $\mathrm{CD}^{-} \mathrm{CD} 56^{+} \mathrm{NK}$ cells and expansion of this subset only, which was not observed for CD3- CD16 ${ }^{+}$NK cells ${ }^{82}$. The CD56 dim CD16 bright NK cells did show expression of IL-2R $\alpha$ upon pre-stimulation of $\mathrm{F}_{\mathrm{c}}$ receptors ${ }^{83,84}$ or combinatorial stimulation of the IL-12R, IL-15R and IL-18R ${ }^{85}$, and subsequently resulted in increased cytotoxicity and cytokine production. Despite the complexity of NK cell subsets, functionally different NK cells with specific tissue distribution are also described in other species. Murine CD3- NK1.1+ NK cells are divided in CD27 high ${ }^{86}$ and CD94 high 87 NK cells that show higher IFNy production as well as cytotoxicity in comparison to CD27 low/- and CD94 low/- NK cells. The CD27 high NK cells are predominantly found in secondary lymphoid tissues and the CD27 low/- subset preferentially resided in non-lymphoid tissues ${ }^{86,88}$, whereas CD94 ${ }^{\text {high }}$ and CD94 $4^{\text {low/ }}$ NK cells are similarly distributed between lymphoid and non-lymphoid tissues in mice ${ }^{87}$. Within the bovine CD3- NKp46+ NK cell population, the CD2 ${ }^{-}$subset showed a higher IL-2R $\alpha$ expression and IFN $\gamma$ production than the $\mathrm{CD}_{2}{ }^{+}$subset, but the level of cytotoxicity was similar between subsets ${ }^{89}$. Regarding tissue distribution, CD2- NK cells are the main subset present in lymph nodes whereas the $\mathrm{CD}_{2}{ }^{+} \mathrm{NK}$ cells are the predominant subset in blood ${ }^{90,91}$. Within the porcine CD3- perforin ${ }^{+} \mathrm{CD} 8 \alpha^{+}$ $\mathrm{CD} 16^{+} \mathrm{NK}$ cell population, the NKp46 $6^{+}$subset showed higher production of IFN $\gamma$ compared to the NKp46 subset but both subsets showed similar cell cytotoxicity ${ }^{45}$. The tissue distribution of porcine NKp46+ and NKp46- NK cells was similar in blood, spleen and liver, although in the lymph nodes the majority of NK cells was NKp46 ${ }^{+45}$. In chickens, however, functionally different NK cell subsets have not been described yet. It is currently unknown whether the CD3- IL-2R $\alpha^{+} N K$ cells correlate to the human CD56 bright subset that constitutively express IL-2R $\alpha$, or to the murine CD27 high, bovine CD2- or porcine NKp46+ subset since these NK cells are mostly present in secondary lymphoid organs. More insight in the functional capacities and tissue distribution of NK cell subsets is warranted to distinguish functionally different NK cells in chickens. This knowledge will aid in the development of strategies to stimulate number and function of NK cells at early life that may increase the resistance of chickens to infections.

\section{Intestinal microbiota}

The intestinal lumen contains numerous bacteria referred to as microbiota. In general, microbiota are ecological communities of assembled commensal, symbiotic and pathogenic living microorganisms in a defined environment in or on plants, animals and humans, and include Bacteria, Archaea, Fungi, Protists and Algae $^{92}$. The intestinal microbiota play an important role in many physiological processes of the host including nutrient digestion and absorption, cell metabolism, intestinal barrier function and the development of the immune system ${ }^{93}$. Some bacteria, referred to as beneficial, provide health benefits for the host like the production of vitamins and short chain fatty acids (SCFAs). These beneficial species for chickens and humans include amongst others lactic acid bacteria (LAB) such as Lactobacilli and Bifidobacteria ${ }^{94,95}$. Imbalance in the composition or metabolic activity of the microbiota, termed dysbiosis, has been associated with disease susceptibility in chickens and humans such as intestinal infections $s^{95,96}$.

The development of the intestinal microbial population in chickens starts during embryonic development, since from ED18 on hen-derived microbiota are observed that have passed through the eggshell ${ }^{17,98}$. Post-hatch, the bacterial community's richness increases rapidly over time due to environmental and biological factors such as litter, feed and the gut region, and stabilizes by day 14 as the intestinal microbiota matures from a simple to a more complex and diverse composition ${ }^{99,100}$. 
Under natural circumstances, eggs and young chickens are in close contact with adult hens in a nest and their intestines are colonized with microbes acquired from a rich and diverse microbial environment ${ }^{97,101}$. However, in a commercial production setting chickens hatch in a clean hatchery environment in the absence of adult hens, which results in a delayed development of a healthy intestinal microbiota as compared to natural circumstances ${ }^{101,102}$. Microbiota compositions differ between the small intestine and caeca, and greatest microbial abundance and diversity is found in the caeca ${ }^{103,104}$. The major phyla in the small intestine are Firmicutes and Proteobacteria, of which the genera Lactobacillus, Enterococcus and various genera within Clostridiaceae are dominant. In the caeca Firmicutes, Bacteroidetes, Proteobacteria and Actinobacteria are the major phyla, of which the families Bacteroidaceae, Ruminococcaceae, Lachnospiraceae and Clostridiaceae are dominant ${ }^{103-105}$. In spite of structural differences of the intestinal tract between poultry and mammals, the major phylum in gut microbiota is Firmicutes, followed by Bacteroidetes, Proteobacteria and Actinobacteria in both animal groups ${ }^{106}$.

The continuous interaction between the microbiota and the intestinal immune system occurs throughout life, which is observed both in chickens ${ }^{107,108}$ and mammals ${ }^{109,110}$. The development of the immune system requires microbial colonization. Colonization of $L A B$, amongst others, influences the maintenance and function of epithelial cells, macrophages, DCs, T and B cells to promote homeostasis and limit inflammation and disease $\mathrm{e}^{107,109,111,112}$. Furthermore, it was shown in humans and mice that $L A B$ enhanced proliferation and function of NK cells either directly through stimulation of TLRs or indirectly through cytokine stimulation by macrophages and $D C s^{113,114}$. Since disrupted microbial colonization leads to higher susceptibility to infections such as with Salmonella ${ }^{115}$, it is hypothesized that a rapid colonization of the intestine with beneficial species such as $L A B$ at the earliest possible time increases the resistance of chickens to such infections. The early and rapid colonization of $L A B$ may prevent intestinal colonization of the pathogen and stimulate immune responsiveness of chickens.

\section{Intestinal pathogens in chickens}

Several intestinal pathogens cause significant infectious diseases in chickens including Salmonella, Clostridium perfringens (causing necrotic enteritis) and Escherichia coli (causing colibacillosis) ${ }^{116}$. Salmonella infections, those with Salmonella enterica serotype Enteritidis (SE) in particular, can lead to severe disease and mortality rates to $10 \%$ in chickens up to approximately ten days of age. Symptoms include inflammation of internal organs and joints in young chickens, whereas chickens older than ten days are often subclinically infected and carry the bacteria in their intestines ${ }^{117,118 .}$ Salmonella are facultative intracellular bacteria of which Salmonella enterica infections, serotype Enteritidis or Typhimurium (ST), are one of the most common causes of foodborne diseases in humans. Salmonella-associated foodborne diseases result from consumption of contaminated poultry products that are not well prepared ${ }^{119}$. Prevention of SE infection in poultry is thus important for health and welfare of chickens and to avoid substantial economic losses in the poultry sector and food recalls ${ }^{120}$. In addition, SE prevention in poultry is relevant for the health and wellbeing of humans in terms of food safety as well as to avoid loss of productivity and medical costs ${ }^{121}$.

Salmonella infection in chickens can occur through the egg but most often occurs via oral or respiratory routes $^{122}$. Important sources of Salmonellae are humans carrying faeces or dust on clothing, vermin, feed and consumables. After entry most Salmonellae only colonize the intestinal tract, which are subsequently shed in the faeces and consequently spread within the flock. However, SE and ST are able to disseminate systemically to tissues such as the liver, spleen and ovary ${ }^{118,123}$. Preventive treatment with antibiotics is not allowed and therapeutic treatment of Salmonella-infected chickens is restricted 
due to limited effectiveness against Salmonella strains, the risk of residues in poultry products, and potential induction of antibiotic resistance ${ }^{18,124}$. Therefore, alternative strategies are warranted to prevent Salmonella infections in poultry. Stimulation of the immune responsiveness and modulation of the intestinal microbiota are potential strategies to increase the resistance of young chickens to such infections.

\section{Potentiation of the immune system and modulation of intestinal microbiota}

The resistance of chickens to pathogens such as SE may be increased by stimulation of the number and function of the immune system, modulation of the intestinal microbiota and the interaction between these. This is shown in studies in which the application of adult-derived microbiota (AM) in ovo ${ }^{97,125}$ or to hatchlings ${ }^{126-128}$ accelerated microbial colonization and increased resistance to pathogens by competitive exclusion. In addition, early life transplantation of AM resulted in decreased pro-inflammatory responses as long-term effects ${ }^{129}$. As a consequence of application of $A M$, reduced colonization and shedding of Salmonellae were observed ${ }^{127,128,130}$. Furthermore, probiotics and prebiotics are administrated in chickens as well as in humans to prevent dysbiosis and promote intestinal health ${ }^{131,132}$. Probiotics are beneficial microorganisms including $L A B$ and yeasts that confer health benefits to the host such as promoting competitive exclusion of pathogens and stimulation of the epithelial barrier and immune activity by vitamin and SCFA production. Prebiotics are non-digestible dietary fibers for the host that are metabolized by the probiotics to promote their growth and activity, and consequently health benefits to the host ${ }^{131,132}$. Commonly used probiotics are Bifidobacterium, Lactobacillus, Enterococcus and Streptococcus. Fermentable oligosaccharides are commonly used prebiotics like fructooligosaccharides, galactooligosaccharides, and mannanoligosaccharides.

Another strategy to stimulate immune responsiveness and modulate intestinal microbiota includes nutrition. Dietary components such as mushroom and plant polysaccharides, organic acids and vitamins were shown to increase numbers and activity of macrophages, NK cells, B and T cells in the intestine and systemic immune tissues of chickens and mammals ${ }^{133-135}$. Furthermore, feed additives including organic acids, exogenous enzymes and phytochemicals/essential oils promoted beneficial microbial populations such as LAB in the intestine of chickens ${ }^{136,137}$. Thus, feed may affect the immune system directly and/or indirectly through alteration of the composition and activity of the intestinal microbiota and subsequent interactions between immune cells and commensal microbes (Fig. 2), thereby promoting chicken health and productivity ${ }^{138}$. Since chickens are most susceptible to infectious diseases in the first week of life, due to an immature adaptive immune system, this is hypothesized to be the most ideal period to stimulate innate immunity and modulate intestinal microbiota composition by feed additives ${ }^{135}$. Moreover, applying feed additives that stimulate innate immune responses may provide protection against a variety of pathogens due to the non-specific nature of innate immune cells ${ }^{11}$. Several nutritional strategies supplemented directly post-hatch increased resistance to Salmonella infections in young chickens by stimulating macrophage and heterophil functions as well as increasing the relative abundance of beneficial microorganisms ${ }^{139-141}$. However, stimulation of NK cells and its potential to increase resistance of chickens to infectious agents has not been investigated yet. Therefore, early life supplementation of feed additives that potentiate intraepithelial NK cells and commensal LAB may be an effective alternative approach to increase the resistance of chickens, and hence improve the health of poultry. 


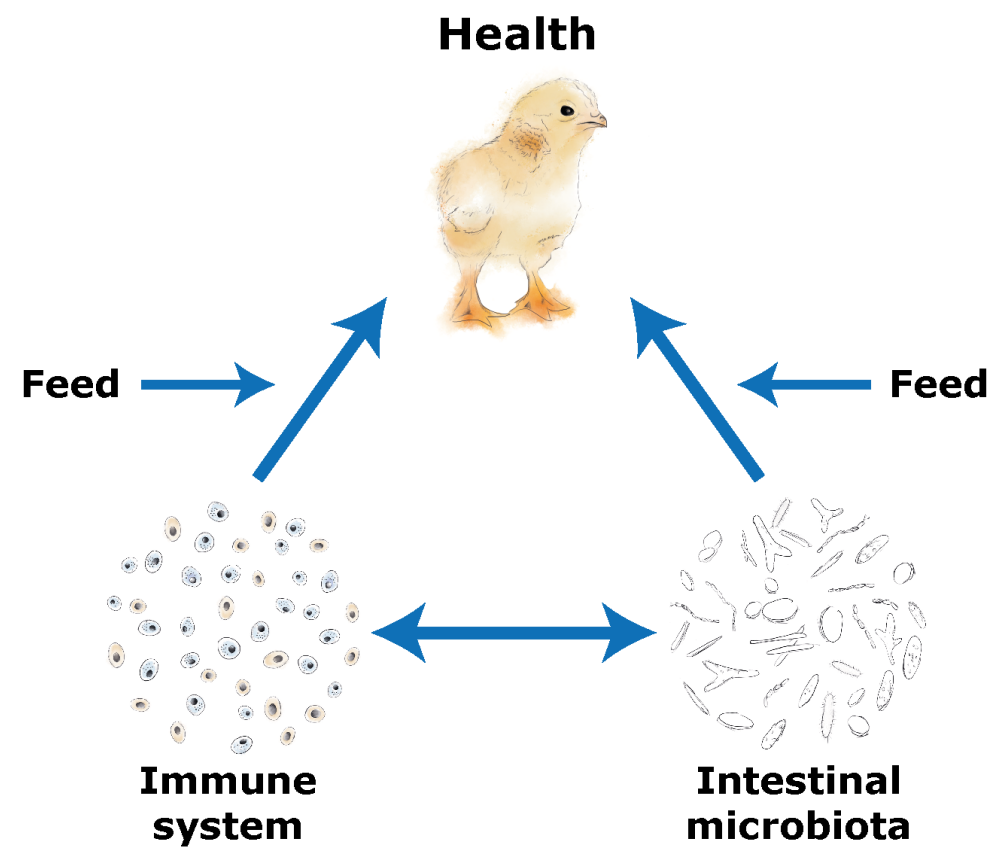

Figure 2. Simplified schematic representation of the interaction between chicken health, immune system, intestinal microbiota and feed. Important aspects in the health of chickens are the immune system and intestinal microbiota, which both can be directly modulated by feed additives. In addition, feed additives can indirectly stimulate the immune system due to the interaction between immune cells and commensal microbes. The stimulation of immune responsiveness and modulation of intestinal microbiota composition by nutritional strategies may increase the resistance of young chickens and consequently improve their health.

\section{Aim and outline of this thesis}

The research described in this thesis aimed to investigate stimulation of immune responsiveness, modulation of intestinal microbiota and the interaction between these by nutritional treatment strategies in order to increase the resistance of young broiler chickens. In young chickens, resistance to pathogens relies on the innate immune system of which NK cells are important players. NK cells are highly abundant among IELs in the intestine and the first cells to be affected by intestinal microbiota and feed additives, and thereby the main interest of the conducted studies. More insight in NK cell subsets and their function will contribute to the understanding of NK cell biology in chickens and will aid to develop strategies specifically targeting NK cells to strengthen the first line of defense. It is hypothesized that enhanced responsiveness of NK cells may increase the resistance to pathogens and consequently improve the health of chickens.

In chapter 2, NK and T cell subsets present in the IEL population of the ileum as well as in other tissues are analyzed and quantified in course of time from late embryonic development up to three weeks of life. In parallel, functional parameters of NK cells are assessed and used as a baseline for following studies pertaining modulation of NK cell number and function in broiler chickens. In chapter $\mathbf{3}$, the interaction between the immune system and the intestinal microbiota in young broiler chickens is described. To this purpose, inoculation of adult-derived microbiota (AM) immediately post-hatch was used as a model to create a different microbiota composition as compared to that of control chickens. In chapter 4, modulatory properties of nutritional compounds are investigated. Potential compounds 
were tested for their capacity to enhance activation of NK cells and macrophages (in vitro screening), and evaluated for safety during embryonic development (in ovo screening) and impact on performance traits (in vivo screening). Based on these results, two compounds, glucose oligosaccharide and long-chain glucomannan, were selected as feed additives. Both nutritional strategies were subsequently assessed for their ability to stimulate the immune system and/or modulate the intestinal microbiota composition as well as for the interaction between these when applied directly post-hatch.

Next, more insight was required in the role of NK cells in response to a Salmonella enterica serotype Enteritidis (SE) infection. In chapter 5, a detailed analysis of innate and adaptive immune responses upon SE infection in young broiler chickens is described. Then in chapter $\mathbf{6}$, the potency of long-chain glucomannan supplementation to increase the resistance to SE infection in young broiler chickens is assessed.

The results reported in this thesis are summarized and discussed in chapter $\mathbf{7}$ in a broader context and implications as well as future perspectives on NK cell stimulation are given. 


\section{References}

1. OECD, Food and Agriculture Organization of the United Nations. OECD-FAO Agricultural Outlook 2020-2029. 2020 doi:10.1787/1112c23b-en.

2. Zuidhof MJ, Schneider BL, Carney VL, Korver DR, Robinson FE. Growth, efficiency, and yield of commercial broilers from 1957, 1978, and 20051. Poult Sci. 2014;93(12):2970-2982. doi:10.3382/ps.2014-04291.

3. More SJ. European perspectives on efforts to reduce antimicrobial usage in food animal production. Ir Vet J. 2020;73(1). doi:10.1186/ s13620-019-0154-4.

4. McEwen SA, Angulo FJ, Collignon PJ, Conly JM. Unintended consequences associated with national-level restrictions on antimicrobial use in food-producing animals. Lancet Planet Health. 2018;2(7):e279-e282. doi:10.1016/S2542-5196(18)30138-4.

5. MehdiY,Létourneau-MontminyM-,GaucherM-,etal.Useofantibioticsinbroilerproduction:Globalimpactsandalternatives.AnimNutr. 2018;4(2):170-178. doi:10.1016/j.aninu.2018.03.002.

6. VanImmerseelF,DeBuckJ, PasmansF,HuyghebaertG, HaesebrouckF, DucatelleR.Clostridiumperfringensinpoultry:Anemergingthreat for animal and public health. Avian Pathol. 2004;33(6):537-549. doi:10.1080/03079450400013162.

7. KoutsoumanisK, AllendeA, Alvarez-OrdóñezA, etal.Salmonella control in poultryflocks and its publichealthimpact. EFSAJ.2019;17(2). doi:10.2903/j.efsa.2019.5596.

8. Wagenaar JA, Jacobs-Reitsma W, Hofshagen M, Newell D. Poultry Colonization with Campylobacter and Its Control at the Primary Production Level. Poultry Colonization with Campylobacter and Its Control at the Primary Production Level. American Society of Microbiology; 2008.

9. Nochi T, Jansen CA, Toyomizu M, Eden WV. The Well-Developed Mucosal Immune Systems of Birds and Mammals Allow for Similar Approaches of Mucosal Vaccination in Both Types of Animals. Front Nutr. 2018;5. doi:10.3389/fnut.2018.00060.

10. Wigley P. Immunology of Birds. eLS. 2017:1-8. doi:10.1002/9780470015902.a0026259.

11. Juul-Madsen HR, Viertlböeck B, Härtle S, Smith AL, Göbel TW. Innate Immune Responses. Innate Immune Responses. ; 2013:121-147.

12. Ratcliffe MJH, Härtle S. B Cells, the Bursa of Fabricius and the Generation of Antibody Repertoires. B Cells, the Bursa of Fabricius and the Generation of Antibody Repertoires. ; 2013:65-89.

13. Smith AL, Göbel TW. Avian T Cells: Antigen Recognition and Lineages. Avian T Cells: Antigen Recognition and Lineages. ; 2013:91-102.

14. Cheroutre H. Starting at the beginning: New perspectives on the biology of mucosal T cells. Vol 22. ; 2004.

15. MoebiusU, KoberG,GriscelliAL, HercendT,MeuerSC. ExpressionofdifferentCD8isoformsondistincthumanlymphocytesubpopulations. Eur J Immunol. 1991;21(8):1793-1800. doi:10.1002/eji.1830210803.

16. Van Kaer L, Olivares-Villagomez D. Development, homeostasis, and functions of intestinal intraepithelial lymphocytes. J Immunol. 2018;200(7):2235-2244. doi:10.4049/jimmunol.1701704.

17. Kadivar M, Petersson J, Svensson L, Marsal J.CD8 $\alpha \beta+\gamma \delta$ T cells: A novel T cell subset with a potential role in inflammatory bowel disease. J Immunol. 2016;197(12):4584-4592. doi:10.4049/jimmunol.1601146.

18. GangadharanD,CheroutreH.TheCD8isoform CD8 $\alpha$ aisnotafunctional homologue oftheTCRco-receptorCD $8 \alpha \beta$.CurrOpin Immunol. 2004;16(3):264-270. doi:10.1016/j.coi.2004.03.015.

19. Cheroutre H, Lambolez F, Mucida D. The light and dark sides of intestinal intraepithelial lymphocytes. Nat Rev Immunol. 2011;11(7):445-456. doi:10.1038/nri3007.

20. Shanmugasundaram R, Selvaraj RK. Regulatory T cell properties of chicken CD4+CD25 + cells. J Immunol. 2011;186(4):1997-2002. doi:10.4049/jimmunol.1002040.

21. Wong MT, Ong DEH, Lim FSH, etal.AHigh-Dimensional Atlas of Human TCell Diversity Reveals Tissue-Specific Trafficking and Cytokine Signatures. Immunity. 2016;45(2):442-456. doi:10.1016/j.immuni.2016.07.007.

22. Degen WGJ, Van Daal N, Rothwell L, Kaiser P, Schijns, V. E.J.C. Th1/Th2 polarization by viral and helminth infection in birds. Vet Microbiol. 2005;105(3-4):163-167. doi:10.1016/j.vetmic.2004.12.001.

23. Walliserl, GöbelTW. Chicken IL-17A is expressed in $\alpha \beta$ and $\gamma \delta$ T cell subsets and binds to a receptor presenton macrophages, and T cells. Dev Comp Immunol. 2018;81:44-53. doi:10.1016/j.dci.2017.11.004.

24. Sowder JT, Chen C-H, Lanier Ager L, Chan MM, Cooper MD. Large subpopulation of avian T cells express a homologue of the

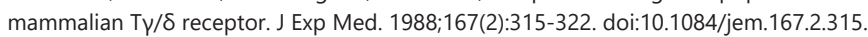

25. Mackay CR, Hein WR. Alarge proportion of bovine T cells express the $\gamma \delta$ T cell receptor and showa distinct tissue distributionand surface phenotype. Int Immunol. 1989;1(5):540-545. doi:10.1093/intimm/1.5.540.

26. Piriou-Guzylack L, Salmon H. Membrane markers of the immune cells in swine: An update. Vet Res. 2008;39(6). doi:10.1051/ vetres:2008030.

27. Shekhar S, Milling S, Yang X. Migration of $\gamma \delta$ T cells in steady-state conditions. Vet Immunol Immunopathol. 2012;147(1-2):1-5. doi:10.1016/j.vetimm.2012.03.016.

28. Forthal DN. Functions of antibodies. Microbiol Spectr. 2014;2(4). doi:10.1128/microbiolspec.AID-0019-2014.

29. Klasing KC LT. Functions, costs and benefits of the immune system during development and growth. 1999;In: Adams NJ, and Slotow R (eds)(Proc. 22. Int. Ornithol. Congr. H. Birdlife South Africa, Johannesburg):2817-2835.

30. Sharma JM, Tizard I. Avian cellular immune effector mechanisms - a review. Avian Pathol. 1984;13(3):357-376. doi:10.1080/03079458408418541. 
31. Alkie TN, Yitbarek A, Hodgins DC, Kulkarni RR, Taha-Abdelaziz K, Sharif S. Development of innate immunity in chicken embryos and newly hatched chicks: a disease control perspective. Avian Pathol. 2019;48(4):288-310. doi:10.1080/03079457.2019.1607966.

32. Smith AL, Powers C, Beal RK. The Avian Enteric Immune System in Health and Disease. The Avian Enteric Immune System in Health and Disease. ; 2013:227-250.

33. Uni Z, Smirnov A, Sklan D. Pre- and posthatch development of goblet cells in the broiler small intestine: Effect of delayed access to feed. Poult Sci. 2003;82(2):320-327. doi:10.1093/ps/82.2.320.

34. Simon K, De Vries Reilingh G, Kemp B, Lammers A. Development of lleal cytokine and immunoglobulin expression levels in response to early feeding in broilers and layers. Poult Sci. 2014;93(12):3017-3027. doi:10.3382/ps.2014-04225.

35. Befus AD, Johnston N, Leslie GA, Bienenstock J. Gut-associated lymphoid tissue in the chicken. I. Morphology, ontogeny, and some functional characteristics of Peyer's patches. J Immunol. 1980;125(6):2626-2632.

36. Göbel TWF, Kaspers B, Stangassinger M. NK and T cells constitute two major, functionally distinct intestinal epithelial lymphocyte subsets in the chicken. Int Immunol. 2001;13(6):757-762.

37. Fenzl L, Göbel TW, Neulen M-. $\gamma \delta$ T cells represent a major spontaneously cytotoxic cell population in the chicken. Dev Comp Immunol. 2017;73:175-183. doi:10.1016/j.dci.2017.03.028.

38. Lillehoj HS, Trout JM. Avian gut-associated lymphoid tissues and intestinal immune responses to Eimeria parasites. Clin Microbiol Rev. 1996;9(3):349-360. doi:10.1128/cmr.9.3.349.

39. Cooper MA, Fehniger TA, Caligiuri MA. The biology of human natural killer-cell subsets. Trends in Immunology. 2001;22(11): 633-640. doi:10.1016/S1471-4906(01)02060-9.

40. Lanier LL. NK cell recognition. Vol 23. ; 2005.

41. Jansen CA, Van Haarlem DA, Sperling B, et al. Identification of an activating chicken Ig-like receptor recognizing avian influenza viruses. J Immunol. 2016;197(12):4696-4703. doi:10.4049/jimmunol.1600401.

42. Straub C, Neulen M-, Sperling B, et al. Chicken NK cell receptors. Dev Comp Immunol. 2013;41(3):324-333. doi:10.1016/j. dci.2013.03.013.

43. Smith HRC, Idris AH, Yokoyama WM. Murine natural killer cell activation receptors. Immunol Rev. 2001;181:115-125. doi:10.1034/ j.1600-065X.2001.1810109.x.

44. Parham P. MHC class I molecules and KIRS in human history, health and survival. Nat Rev Immunol. 2005;5(3):201-214. doi:10.1038/ nri1570.

45. Mair KH, Essler SE, Patzl M, Storset AK, Saalmüller A, Gerner W. NKp46 expression discriminates porcine NK cells with different functional properties. Eur J Immunol. 2012;42(5):1261-1271. doi:10.1002/eji.201141989.

46. Horowitz A, Stegmann KA, Riley EM. Activation of natural killer cells during microbial infections. Front Immunol. 2012;2(JAN). doi:10.3389/fimmu.2011.00088.

47. Sivori S, Falco M, Carlomagno S, et al. A novel KIR-associated function: Evidence that CpG DNA uptake and shuttling to early endosomes is mediated by KIR3DL2. Blood. 2010;116(10):1637-1647. doi:10.1182/blood-2009-12-256586.

48. Chalifour A, Jeannin P, Gauchat J-, et al. Direct bacterial protein PAMP recognition by human NK cells involves TLRs and triggers Q-defensin production. Blood. 2004;104(6):1778-1783. doi:10.1182/blood-2003-08-2820.

49. Qiu F, Maniar A, Quevedo Diaz M, Chapoval Al, Medvedev AE. Activation of cytokine-producing and antitumor activities of natural killer cells and macrophages by engagement of Toll-like and NOD-like receptors. Innate Immun. 2011;17(4):375-387. doi:10.1177/1753425910372000.

50. Viertlboeck BC, Schweinsberg S, Hanczaruk MA, et al. The chicken leukocyte receptor complex encodes a primordial, activating, high-affinity IgY Fc receptor. Proc Natl Acad Sci U S A. 2007;104(28):11718-11723. doi:10.1073/pnas.0702011104.

51. Della Chiesa M, Marcenaro E, Sivori S, Carlomagno S, Pesce S, Moretta A. Human NK cell response to pathogens. Semin Immunol. 2014;26(2):152-160. doi:10.1016/j.smim.2014.02.001.

52. Perrot I, Deauvieau F, Massacrier C, et al. TLR3 and Rig-like receptor on myeloid dendritic cells and Rig-like receptor on human NK cells are both mandatory for production of IFN- $\gamma$ in response to double-stranded RNA. J Immunol. 2010;185(4):2080-2088. doi:10.4049/jimmunol.1000532.

53. Souza-Fonseca-Guimaraes F, Adib-Conquy M, Cavaillon JM. Natural killer (NK) cells in antibacterial innate immunity: angels or devils? Mol Med. 2012;18(1):270-285. doi:10.2119/molmed.2011.00201.

54. Mandelboim O, Lieberman N, Lev M, et al. Recognition of haemagglutinins on virus-infected cells by NKp46 activates lysis by human NK cells. Nature. 2001;409(6823):1055-1060. doi:10.1038/35059110.

55. Hershkovitz O, Rosental B, Rosenberg LA, et al. NKp44 receptor mediates interaction of the envelope glycoproteins from the West Nile and dengue viruses with NK cells. J Immunol. 2009;183(4):2610-2621. doi:10.4049/jimmunol.0802806.

56. Chisholm SE, Reyburn HT. Recognition of vaccinia virus-infected cells by human natural killer cells depends on natural cytotoxicity receptors. J Virol. 2006;80(5):2225-2233. doi:10.1128/JVI.80.5.2225-2233.2006.

57. Fang M, Lanier LL, Sigal LJ. A role for NKG2D in NK cell-mediated resistance to poxvirus disease. PLoS Pathog. 2008;4(2). doi:10.1371/journal.ppat.0040030.

58. Fang M, Orr M, Spee P, Egebjerg T, Lanier L, Sigal L. CD94 Is Essential for NK Cell-Mediated Resistance to a Lethal Viral Disease. Immunity. 2011;34(4):579-589. doi:10.1016/j.immuni.2011.02.015.

59. Smith HRC, Heusel JW, Mehta IK, et al. Recognition of a virus-encoded ligand by a natural killer cell activation receptor. Proc Natl Acad Sci U S A. 2002;99(13):8826-8831. doi:10.1073/pnas.092258599.

60. Marcenaro E, Ferranti B, Falco M, Moretta L, Moretta A. Human NK cells directly recognize Mycobacterium bovis via TLR2 and acquire the ability to kill monocyte-derived DC. Int Immunol. 2008;20(9):1155-1167. doi:10.1093/intimm/dxn073. 
61. Sivori S, Falco M, Della Chiesa M, et al. CpG and double-stranded RNA trigger human NK cells by toll-like receptors: Induction of cytokine release and cytotoxicity against tumors dendritic cells. Proc Natl Acad Sci U S A. 2004;101(27):10116-10121. doi:10.1073/pnas.0403744101.

62. Smyth MJ, Cretney E, Kelly JM, et al. Activation of NK cell cytotoxicity. Mol Immunol. 2005;42(4 SPEC. ISS.):501-510. doi:10.1016/j. molimm.2004.07.034.

63. Robertson MJ. Role of chemokines in the biology of natural killer cells. J Leukocyte Biol. 2002;71(2):173-183.

64. Agerberth B, Charo J, Werr J, et al. The human antimicrobial and chemotactic peptides LL-37 and $\alpha$-defensins are expressed by specific lymphocyte and monocyte populations. Blood. 2000;96(9):3086-3093. doi:10.1182/blood.v96.9.3086.

65. De Pelsmaeker S, Devriendt B, Leclercq G, Favoreel HW. Porcine NK cells display features associated with antigen-presenting cells. J Leukocyte Biol. 2018;103(1):129-140. doi:10.1002/JLB.4A0417-163RR.

66. Hanna J, Mandelboim O. When killers become helpers. Trends Immunol. 2007;28(5):201-206. doi:10.1016/j.it.2007.03.005.

67. Evans JH, Horowitz A, Mehrabi M, et al. A distinct subset of human NK cells expressing HLA-DR expand in response to IL-2 and can aid immune responses to BCG. Eur J Immunol. 2011;41(7):1924-1933. doi:10.1002/eji.201041180.

68. Walzer T, Bléry M, Chaix J, et al. Identification, activation, and selective in vivo ablation of mouse NK cells via NKp46. Proc Natl Acad Sci U S A. 2007;104(9):3384-3389. doi:10.1073/pnas.0609692104.

69. Giorda R, Rudert WA, Vavassori C, Chambers WH, Hiserodt JC, Trucco M. NKR-P1, a signal transduction molecule on natural killer cells. Science. 1990;249(4974):1298-1300. doi:10.1126/science.2399464.

70. Walzer T, Jaeger S, Chaix J, Vivier E. Natural killer cells: from CD3-NKp46+ to post-genomics meta-analyses. Curr Opin Immunol. 2007;19(3):365-372. doi:10.1016/j.coi.2007.04.004.

71. Storset AK, Kulberg S, Berg I, Boysen P, Hope JC, Dissen E. NKp46 defines a subset of bovine leukocytes with natural killer cell characteristics. Eur J Immunol. 2004;34(3):669-676. doi:10.1002/eji.200324504.

72. Connelley T, Storset AK, Pemberton A, et al. NKp46 defines ovine cells that have characteristics corresponding to NK cells. Vet Res. 2011;42(1). doi:10.1186/1297-9716-42-37.

73. Neulen M-, Göbel TW. Chicken CD56 defines NK cell subsets in embryonic spleen and lung. Dev Comp Immunol. 2012;38(3):410415. doi:10.1016/j.dci.2012.08.001.

74. Jansen CA, De Geus ED, Van Haarlem DA, et al. Differential lung NK cell responses in avian influenza virus infected chickens correlate with pathogenicity. Sci Rep. 2013;3. doi:10.1038/srep02478.

75. Jansen CA, van de Haar, P. M., van Haarlem D, et al. Identification of new populations of chicken natural killer (NK) cells. Dev Comp Immunol. 2010;34(7):759-767. doi:10.1016/j.dci.2010.02.009.

76. Nagler A, Lanier LL, Cwirla S, Phillips JH. Comparative studies of human FcRIII-positive and negative natural killer cells. J Immunol. 1989;143(10):3183-3191.

77. Melsen JE, Lugthart G, Lankester AC, Schilham MW. Human Circulating and Tissue-Resident CD56bright Natural Killer Cell Populations. Front Immunol. 2016;7(JUN). doi:10.3389/fimmu.2016.00262.

78. Caligiuri MA, Zmuidzinas A, Manley TJ, Levine H, Smith KA, Ritz J. Functional consequences of interleukin 2 receptor expression on resting human lymphocytes: Identification of a novel natural killer cell subset with high affinity receptors. J Exp Med. 1990;171(5):1509-1526. doi:10.1084/jem.171.5.1509.

79. Nagler A, Lanier LL, Phillips JH. Constitutive expression of high affinity interleukin 2 receptors on human CD16- natural killer cells in vivo. J Exp Med. 1990;171(5):1527-1533. doi:10.1084/jem.171.5.1527.

80. Cooper MA, Fehniger TA, Turner SC, et al. Human natural killer cells: A unique innate immunoregulatory role for the CD56bright subset. Blood. 2001;97(10):3146-3151. doi:10.1182/blood.V97.10.3146.

81. De Maria A, Bozzano F, Cantoni C, Moretta L. Revisiting human natural killer cell subset function revealed cytolytic CD56dimCD16+ NK cells as rapid producers of abundant IFN- $\gamma$ on activation. Proc Natl Acad Sci U S A. 2011;108(2):728-732. doi:10.1073/ pnas. 1012356108.

82. Rudnicka K, Matusiak A, Chmiela M. CD25 (IL-2R) expression correlates with the target cell induced cytotoxic activity and cytokine secretion in human natural killer cells. Acta Biochim Pol. 2015;62(4):885-894. doi:10.18388/abp.2015_1152.

83. Márquez M-, Millet C, Stekman H, et al. CD16 cross-linking induces increased expression of CD56 and production of IL-12 in peripheral NK cells. Cell Immunol. 2010;264(1):86-92. doi:10.1016/j.cellimm.2010.05.002.

84. Duggan MC, Campbell AR, McMichael EL, et al. Co-stimulation of the fc receptor and interleukin-12 receptor on human natural killer cells leads to increased expression of cd25. Oncolmmunology. 2018;7(2). doi:10.1080/2162402X.2017.1381813.

85. Leong JW, Chase JM, Romee R, et al. Preactivation with IL-12, IL-15, and IL-18 induces cd25 and a functional high-affinity il-2 receptor on human cytokine-induced memory-like natural killer cells. Biol Blood Marrow Transplant. 2014;20(4):463-473. doi:10.1016/j.bbmt.2014.01.006.

86. Hayakawa Y, Smyth MJ. CD27 dissects mature NK cells into two subsets with distinct responsiveness and migratory capacity. J Immunol. 2006;176(3):1517-1524. doi:10.4049/jimmunol.176.3.1517.

87. Yu J, Wei M, Mao H, et al. CD94 defines phenotypically and functionally distinct mouse NK cell subsets. J Immunol. 2009;183(8):49684974. doi:10.4049/jimmunol.0900907.

88. Hayakawa Y, Huntington ND, Nutt SL, Smyth MJ. Functional subsets of mouse natural killer cells. Immunol Rev. 2006;214(1):47-55. doi:10.1111/j.1600-065X.2006.00454.x.

89. Boysen P, Olsen I, Berg I, Kulberg S, Johansen GM, Storset AK. Bovine CD2-/NKp46+ cells are fully functional natural killer cells with a high activation status. BMC Immunol. 2006;7. doi:10.1186/1471-2172-7-10.

90. Hamilton CA, Mahan S, Bell CR, et al. Frequency and phenotype of natural killer cells and natural killer cell subsets in bovine lymphoid compartments and blood. Immunology. 2017;151(1):89-97. doi:10.1111/imm.12708. 
91. Boysen P, Storset AK. Bovine natural killer cells. Vet Immunol Immunopathol. 2009;130(3-4):163-177. doi:10.1016/j. vetimm.2009.02.017.

92. Berg G, Rybakova D, Fischer D, et al. Microbiome definition re-visited: old concepts and new challenges. Microbiome. 2020;8(1). doi:10.1186/s40168-020-00875-0.

93. Pedroso AA, Lee MD. The composition and role of the microbiota in chickens. The composition and role of the microbiota in chickens. ; 2015:21-50.

94. Novik G, Savich V. Beneficial microbiota. Probiotics and pharmaceutical products in functional nutrition and medicine. Microbes Infect. 2020;22(1):8-18. doi:10.1016/j.micinf.2019.06.004.

95. Stanley D, Hughes RJ, Moore RJ. Microbiota of the chicken gastrointestinal tract: Influence on health, productivity and disease. Appl Microbiol Biotechnol. 2014;98(10):4301-4310. doi:10.1007/s00253-014-5646-2.

96. Wang B, Yao M, Lv L, Ling Z, Li L. The Human Microbiota in Health and Disease. Engineering. 2017;3(1):71-82. doi:10.1016/J. ENG.2017.01.008.

97. Pedroso $A A$, Batal $A B$, Lee MD. Effect of in ovo administration of an adult-derived microbiota on establishment of the intestinal microbiome in chickens. Am J Vet Res. 2016;77(5):514-526. doi:10.2460/ajvr.77.5.514.

98. Lee S-, La T-, Lee H-, et al. Characterization of microbial communities in the chicken oviduct and the origin of chicken embryo gut microbiota. Scientific Reports. 2019;9(1). doi:10.1038/s41598-019-43280-w.

99. Oakley BB, Kogut MH. Spatial and Temporal Changes in the Broiler Chicken Cecal and Fecal Microbiomes and Correlations of Bacterial Taxa with Cytokine Gene Expression. Front Vet Sci. 2016;3:11. doi:10.3389/fvets.2016.00011.

100. Jurburg SD, Brouwer MSM, Ceccarelli D, van der Goot J, Jansman AJM, Bossers A. Patterns of community assembly in the developing chicken microbiome reveal rapid primary succession. MicrobiologyOpen. 2019;8(9). doi:10.1002/mbo3.821.

101. Kubasova T, Kollarcikova M, Crhanova M, et al. Contact with adult hen affects development of caecal microbiota in newly hatched chicks. PLoS ONE. 2019;14(3). doi:10.1371/journal.pone.0212446.

102. Bailey R. Intestinal microbiota and the pathogenesis of dysbacteriosis in broiler chickens. University of East Anglia; 2010.

103. Gong J, Si W, Forster RJ, et al. 16S rRNA gene-based analysis of mucosa-associated bacterial community and phylogeny in the chicken gastrointestinal tracts: From crops to ceca. FEMS Microbiol Ecol. 2007;59(1):147-157. doi:10.1111/j.1574-6941.2006.00193.x.

104. Yeoman CJ, Chia N, Jeraldo P, Sipos M, Goldenfeld ND, White BA. The microbiome of the chicken gastrointestinal tract. Anim Health Res Rev. 2012;13(1):89-99. doi:10.1017/S1466252312000138.

105. Oakley BB, Lillehoj HS, Kogut MH, et al. The chicken gastrointestinal microbiome. FEMS Microbiol Lett. 2014;360(2):100-112. doi:10.1111/1574-6968.12608.

106. Ley RE, Hamady M, Lozupone C, et al. Evolution of mammals and their gut microbes. Science. 2008;320(5883):1647-1651. doi:10.1126/science.1155725.

107. Broom LJ, Kogut MH. The role of the gut microbiome in shaping the immune system of chickens. Vet Immunol Immunopathol. 2018;204:44-51. doi:10.1016/j.vetimm.2018.10.002.

108. Schokker D, Jansman AJM, Veninga G, et al. Perturbation of microbiota in one-day old broiler chickens with antibiotic for 24 hours negatively affects intestinal immune development. BMC Genomics. 2017;18(1). doi:10.1186/s12864-017-3625-6.

109. Round JL, Mazmanian SK. The gut microbiota shapes intestinal immune responses during health and disease. Nature Reviews Immunology. 2009;9(5):313-323. doi:10.1038/nri2515.

110. Macpherson AJ, Harris NL. Interactions between commensal intestinal bacteria and the immune system. Nature Reviews Immunology. 2004;4(6):478-485. doi:10.1038/nri1373.

111. Ismail AS, Behrendt CL, Hooper LV. Reciprocal interactions between commensal bacteria and $\gamma \delta$ intraepithelial lymphocytes during mucosal injury. J Immunol. 2009;182(5):3047-3054. doi:10.4049/jimmunol.0802705.

112. Hoytema van Konijnenburg, D. P., Reis BS, Pedicord VA, Farache J, Victora GD, Mucida D. Intestinal Epithelial and Intraepithelial T Cell Crosstalk Mediates a Dynamic Response to Infection. Cell. 2017;171(4):783-794.e13. doi:10.1016/j.cell.2017.08.046.

113. Ferlazzo G, Rizzello V, Bonaccorsi I, Dongarr ML, Fink LN. Role of natural killer and dendritic cell crosstalk in immunomodulation by commensal bacteria probiotics. J Biomed Biotechnol. 2011;2011. doi:10.1155/2011/473097.

114. Makino S, Sato A, Goto A, et al. Enhanced natural killer cell activation by exopolysaccharides derived from yogurt fermented with Lactobacillus delbrueckii ssp. bulgaricus OLL1073R-1. J Dairy Sci. 2016;99(2):915-923. doi:10.3168/jds.2015-10376.

115. Varmuzova K, Kubasova T, Davidova-Gerzova L, et al. Composition of gut microbiota influences resistance of newly hatched chickens to Salmonella enteritidis infection. Front Microbiol. 2016;7(JUN). doi:10.3389/fmicb.2016.00957.

116. Gezondheidsdienst voor dieren. Dierziekten. https://www.gddiergezondheid.nl/dierziekten. Published 2020. Retrieved 2021.

117. Suzuki S. Pathogenicity of Salmonella enteritidis in poultry. Int J Food Microbiol. 1994;21(1-2):89-105. doi:10.1016/01681605(94)90203-8.

118. Gezondheidsdienst voor dieren. Salmonellose. https://www.gddiergezondheid.nl/salmonellose-pluimvee. Published 2020. Retrieved 2021.

119. Arlet G, Barrett TJ, Butaye P, Cloeckaert A, Mulvey MR, White DG. Salmonella resistant to extended-spectrum cephalosporins: prevalence and epidemiology. Microbes Infect. 2006;8(7):1945-1954. doi:10.1016/j.micinf.2005.12.029.

120. Scharff RL. Food attribution and economic cost estimates for meat- And poultry-related illnesses. J Food Protection. 2020;83(6):959967. doi:10.4315/JFP-19-548.

121. Hoffmann S, Batz MB, Morris Jr. JG. Annual cost of illness and quality-adjusted life year losses in the united states due to 14 foodborne pathogens. J Food Protection. 2012;75(7):1292-1302. doi:10.4315/0362-028X.JFP-11-417. 
122. Kallapura G, Morgan MJ, Pumford NR, et al. Evaluation of the respiratory route as a viable portal of entry for Salmonella in poultry via intratracheal challenge of Salmonella Enteritidis and Salmonella Typhimurium. Poult Sci. 2014;93(2):340-346. doi:10.3382/ps.2013-03602.

123. Kallapura G, Kogut MH, Morgan MJ, et al. Fate of Salmonella Senftenberg in broiler chickens evaluated by challenge experiments. Avian Pathol. 2014;43(4):305-309. doi:10.1080/03079457.2014.923554.

124. World Organisation for Animal Health, (OIE). Prevention, detection and control of Salmonella in poultry. OIE, ed. 28th ed. Office International des Epizooties; 2019.

125. Richards-Rios P, Leeming G, Fothergill J, Bernardeau M, Wigleya P. Topical application of adult cecal contents to eggs transplants spore-forming microbiota but not other members of the microbiota to chicks. Appl Environ Microbiol. 2020;86(5). doi:10.1128/ AEM.02387-19.

126. Yin Y, Lei F, Zhu L, et al. Exposure of different bacterial inocula to newborn chicken affects gut microbiota development and ileum gene expression. ISME Journal. 2010;4(3):367-376. doi:10.1038/ismej.2009.128.

127. Ferreira AJP, Ferreira CSA, Knobl T, et al. Comparison of three commercial competitive-exclusion products for controlling Salmonella colonization of broilers in Brazil. J Food Protection. 2003;66(3):490-492. doi:10.4315/0362-028X-66.3.490.

128. Nakamura A, Ota Y, Mizukami A, Ito T, Ngwai YB, Adachi Y. Evaluation of aviguard, a commercial competitive exclusion product for efficacy and after-effect on the antibody response of chicks to Salmonella. Poult Sci. 2002;81(11):1653-1660. doi:10.1093/ ps/81.11.1653.

129. Metzler-Zebeli BU, Siegerstetter S-, Magowan E, Lawlor PG, O'Connell NE, Zebeli Q. Fecal microbiota transplant from highly feed efficient donors affects cecal physiology and microbiota in low- And high-feed efficient chickens. Front Microbiol. 2019;10(JULY). doi:10.3389/fmicb.2019.01576.

130. Schneitz C, Koivunen E, Tuunainen P, Valaja J. The effects of a competitive exclusion product and two probiotics on Salmonella colonization and nutrient digestibility in broiler chickens. J Appl Poult Res. 2016;25(3):396-406. doi:10.3382/japr/pfw025.

131. Ducatelle R, Eeckhaut V, Haesebrouck F, Van Immerseel F. A review on prebiotics and probiotics for the control of dysbiosis: Present status and future perspectives. Animal. 2014;9(1):43-48. doi:10.1017/S1751731114002584.

132. Pourabedin M, Zhao X. Prebiotics and gut microbiota in chickens. FEMS Microbiol Lett. 2015;362(15). doi:10.1093/femsle/fnv122.

133. Guo FC, Savelkoul HFJ, Kwakkel RP, Williams BA, Verstegen MWA. Immunoactive, medicinal properties of mushroom and herb polysaccharides and their potential use in chicken diets. World's Poult Sci J. 2003;59(4):427-440. doi:10.1079/WPS20030026.

134. Klasing KC. Nutrition and the immune system. Brit Poult Sci. 2007;48(5):525-537. doi:10.1080/00071660701671336.

135. Kogut MH. Impact of nutrition on the innate immune response to infection in poultry. J Appl Poult Res. 2009;18(1):111-124. doi:10.3382/japr.2008-00081.

136. Yadav S, Jha R. Strategies to modulate the intestinal microbiota and their effects on nutrient utilization, performance, and health of poultry. J Animal Sci Biotechnol. 2019;10(1). doi:10.1186/s40104-018-0310-9.

137. Kogut MH. The effect of microbiome modulation on the intestinal health of poultry. Anim Feed Sci Technol. 2019;250:32-40. doi:10.1016/j.anifeedsci.2018.10.008.

138. Carrasco JMD, Casanova NA, Miyakawa MEF. Microbiota, gut health and chicken productivity: What is the connection? Microorg. 2019;7(10). doi:10.3390/microorganisms7100374.

139. Lowry VK, Farnell MB, Ferro PJ, Swaggerty CL, Bahl A, Kogut MH. Purified $\beta$-glucan as an abiotic feed additive up-regulates the innate immune response in immature chickens against Salmonella enterica serovar Enteritidis. Int J Food Microbiol. 2005;98(3):309-

318. doi:10.1016/j.jijfoodmicro.2004.06.008.

140. Kogut MH, He H, Genovese KJ, Jiang YW. Feeding the BT cationic peptides to chickens at hatch reduces cecal colonization by salmonella enterica serovar enteritidis and primes innate immune cell functional activity. Foodborne Pathog Dis. 2010;7(1):23-30. doi:10.1089/fpd.2009.0346.

141. Babu US, Raybourne RB. Impact of dietary components on chicken immune system and Salmonella infection. Expert Rev Anti-Infect Ther. 2008;6(1):121-135. doi:10.1586/14787210.6.1.121. 



\section{CHAPTER 2}

\section{Analysis of chicken intestinal natural killer cells, a major IEL subset during embryonic and early life}

Nathalie Meijerink1, Daphne A. van Haarlem', Francisca C. Velkers², J. Arjan Stegeman², Victor P.M.G. Rutten ${ }^{1,3}$, Christine A. Jansen ${ }^{1}$

1 Department Biomolecular Health Sciences, Division Infectious Diseases and Immunology, Faculty of Veterinary Medicine, Utrecht University, Utrecht, the Netherlands

2 Department Population Health Sciences, Division Farm Animal Health, Faculty of Veterinary Medicine, Utrecht University, Utrecht, the Netherlands

3 Department of Veterinary Tropical Diseases, Faculty of Veterinary Science, University of Pretoria, Pretoria, South Africa 


\section{Abstract}

Restrictions on antimicrobials demand alternative strategies to improve broiler health, such as supplying feed additives which stimulate innate immune cells like natural killer (NK) cells. The main objective of this study was to characterize intestinal NK cells in broiler chickens during embryonic and early life and compare these to NK cells in spleen, blood and bone marrow. Also T-cell subsets were determined. The majority of intestinal NK cells expressed IL-2R $\alpha$ rather than $20 \mathrm{E} 5$ and $5 \mathrm{C7}$, and showed low level of activation. Within intestinal NK cells the activation marker CD107 was mostly expressed on IL-2R $\alpha^{+}$cells while in spleen and blood 20E5 $5^{+}$KK cells primarily expressed CD107. High percentages of intestinal $C D 8 \alpha \alpha^{+}, C D 8 \alpha \beta^{+}$and from 2 weeks onward also gamma delta T cells were found.

Taken together, we observed several intestinal NK subsets in broiler chickens. Differences in NK subsets were mostly observed between organs, rather than differences over time. Targeting these intestinal NK subsets may be a strategy to improve immune-mediated resistance in broiler chickens.

Keywords: broiler chickens, innate immunity, NK cells, intraepithelial lymphocytes, IEL 


\section{Introduction}

Restrictions on the use of antimicrobials in poultry production have made search for other strategies to maintain or improve poultry health, such as enhanced immune responsiveness by feed interventions important ${ }^{1}$. The gut-associated immune system provides the interface between body and intestinal content and protects against invasion of pathogens. Gut-associated lymphoid tissue (GALT) is situated throughout the intestinal epithelium and consists of the Peyer's patches, the intraepithelial lymphocytes (IEL) and the lamina propria leukocytes (LPL) underneath the IEL ${ }^{2,3}$. Between epithelial cells of the villi, the abundantly present IEL preserve the integrity of the epithelium, regulate interactions with microbiota and complement the first line of defense against pathogens ${ }^{4}$. The IEL population consists of various types of immune cells including innate lymphoid cells, $\gamma \delta T$ cells, CD8 ${ }^{+} T$ cells and natural killer (NK) cells ${ }^{5-7}$.

NK cells share many characteristics with group I innate lymphoid cells; both cells express the transcription factor T-bet and secrete Th1 cell-associated cytokines like IFN $\gamma$ and TNF $\alpha$ as reviewed in Artis and Spits (2015). These cytokines are involved in killing of infected cells and especially IFNY plays a role in the induction of subsequent adaptive immune responses ${ }^{9}$. NK cells lyse target cells without prior sensitization and without restriction by major histocompatibility (MHC) antigens. In humans, NK cells exit continuously from the bone marrow into blood and tissues like spleen and intestine, and undergo progressive phenotypic and functional maturation ${ }^{10}$. A phenotypic distinction is made between NK cells with predominant cytotoxic effector functions (CD56 ${ }^{\text {dim }}$ CD16 ${ }^{\text {high }}$, IL2-R $\alpha$ ) involved in killing of

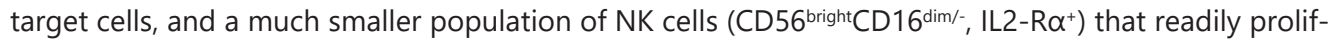
erates and mainly produces cytokines ${ }^{11-13}$. The CD56 bright $N K$ cells constitutively express the high-affinity heterotrimeric IL-2R including the $\alpha$-chain, whereas CD56 dim NK cells express intermediate-affinity IL$2 \mathrm{R}$ and upregulate expression of the $\alpha$-chain only upon activation ${ }^{14,15}$. Increased IL2-R $\alpha$ expression on human NK cells correlates with target cell induced cytotoxicity and cytokine production ${ }^{16}$. Human NK cells mainly reside in the intestine in frequencies of $40 \%$ in IEL, and in lower frequencies of $10-20 \%$ in blood and secondary lymphoid tissues like spleen ${ }^{17-20}$. The predominantly cytotoxic NK cell subset was shown in humans and mice to be circulating ${ }^{11,21}$, whereas the mainly cytokine-producing subset is observed in tissues in close contact with T cells ${ }^{12,21}$.

Although NK cell biology in chickens is less advanced compared to humans and mice, chicken NK cells have been described as a population of cells that lack surface expression of $T$ and $B$ cell-specific surface markers and are able to kill a NK-susceptible target cell line ${ }^{22}$. A high percentage of these NK cells was observed in embryonic spleen ${ }^{22-24}$ and in the IEL of the duodenum ${ }^{22}$ of layer chickens. More recent studies have shown the presence of NK cells that are able to degranulate, which is a measure for NK cell activation ${ }^{25}$, in various tissues including spleen, lung and blood of layer chickens ${ }^{24,26,27}$.

Despite the fact that a pan NK marker is still missing for chickens, expression of many markers has been reported on CD3 negative cells that show enhanced CD107 expression, indicating that these markers are expressed on cells with NK cell function. Interestingly, differential expression of these markers in various organs suggests that also in chickens different NK cell subsets exist. For instance, in the duodenum of adult SPF chickens and layer chickens a high percentage of NK cells expressed the marker $28-4^{22,28}$, identified as the chicken orthologue of the IL-2R $\alpha$ chain ${ }^{29}$. In blood, spleen and lung of layer chickens, NK cells differentially expressed $5 C 7$, which is defined as putative $C D 11 \mathrm{~b} / \mathrm{C}^{30}$ or $C D 11 \mathrm{~d}^{31}$, both molecules involved in adhesion, $20 \mathrm{E} 5$ and $7 C 1^{24,26}$. The avian orthologue of CD56, a pan NK marker in humans, was expressed on a small population of NK cells in the lung and embryonic spleen but not in other tissues ${ }^{32}$. Moreover, although CD8 $\alpha$ was expressed on chicken NK cells ${ }^{22,32}$, its expression was 
shown to be downregulated upon activation ${ }^{24}$. Since at the onset of the present study limited data on NK cells in broiler chickens was available, we set out to first investigate presence and function of the various NK cell subtypes in broiler chickens in the absence of pathogenic challenges, in multiple organs in course of time from the embryonal stage ED14 up to 21 days of age. In addition, other IEL like the $\gamma \delta \mathrm{T}$ cells and $\mathrm{CD} 8^{+} \mathrm{T}$ cell subsets have not been described in broiler chickens at multiple timepoints during embryonic and early life.

Stimulating intestinal NK cells through feed may be a strategy to improve health of chickens. Feed additives can influence, either directly or indirectly through the microbiota, the intestinal development and immune responses ${ }^{1,33,34}$. Probiotics were shown to increase intestinal mRNA levels of toll-like receptors $(T L R s)^{35}$, which play a key role in innate immunity and are also expressed on NK cells. Small cationic peptide supplementation of broiler chickens was shown to increase mRNA levels of TLRs and type I and II interferons in caecal tissue upon infection with Salmonella ${ }^{36}$, including IFN $\gamma$ which is secreted by NK cells. However, complementation of these results with phenotypic characterization including cellular assays of intestinal cells such as NK cells are needed to assess possibilities of modulating these intestinal NK cells to improve chicken health.

In this study, we set out to analyze various subsets of NK cells in the intestine and compared these with subsets in spleen, blood and bone marrow of chicken embryos and in young chickens during the first three weeks of life. We used the monoclonal antibodies specific for 28-4, $20 \mathrm{E} 5$ and $5 \mathrm{C7}$ to analyze NK cell subsets over time and we assessed activation of NK cells by determining surface expression of CD107. Analysis of CD107 expression within the IL-2R $\alpha^{+}$and $20 \mathrm{E} 5^{+} \mathrm{NK}$ cell subsets enabled us to investigate whether in chickens also NK cell subsets with varying functions can be observed. Presence of intraepithelial T cells was assessed over time in comparison to T cells in spleen, blood and bone marrow. Characterization of intestinal NK cell subsets will aid in investigating possibilities to modulate NK cells through for instance feed interventions, which may result in strengthening of the innate immune defense well as subsequent adaptive immune responses in young broiler chickens.

\section{Materials and methods}

\section{Animals and tissues}

Embryonated Ross 308 eggs were obtained from a commercial hatchery. Eggs were placed in a hatcher at the facilities of the Division Farm Animal Health, Faculty of Veterinary Medicine, Utrecht University, the Netherlands. From literature ${ }^{22}$ and earlier work of our group ${ }^{24}$, we know that spleens of fourteen day-old embryos contain a high quantity of NK cells. Subsequently, embryonic day (ED) 16 and ED18 embryos were analyzed as representative of late embryonic development. At ED 14, 16 and 18 , embryos $(n=10)$ were euthanized and the small intestines, spleens, femurs and tibiae were collected. Samples of either small intestine or spleen were pooled to obtain sufficient cells and homogenized using a $70 \mu \mathrm{M}$ cell strainer (Beckton Dickinson (BD) Biosciences, NJ, USA) to obtain single cell suspensions. Lymphocytes were isolated by Ficoll-Paque Plus (GE Healthcare, the Netherlands) density gradient centrifugation for $12 \mathrm{~min}\left(1700 \mathrm{rpm}, 20^{\circ} \mathrm{C}\right)$, washed in phosphate-buffered saline (PBS; Lonza, Switzerland) by centrifugation for $5 \mathrm{~min}(1300 \mathrm{rpm})$ and resuspended at $4.0 \times 10^{6} \mathrm{cells} / \mathrm{ml}$ in 'complete medium' (IMDM supplemented with $8 \%$ heat-inactivated FCS (Lonza); $2 \%$ heat-inactivated chicken serum, $100 \mathrm{U} / \mathrm{ml}$ penicillin/streptomycin and $2 \mathrm{mM}$ glutamax l; Gibco BRL, United Kingdom). Bone marrow was collected as previously described ${ }^{37}$ by cutting the edges of femur and tibia bones and flushing the bone marrow with $10 \mathrm{ml}$ complete medium using a $21 \mathrm{G}$ needle and a $10 \mathrm{ml}$ syringe (BD Biosciences). Subsequently, bone marrow samples of 10 embryos were pooled, homogenized 
using a $70 \mu \mathrm{M}$ cell strainer, centrifuged for $10 \mathrm{~min}\left(1200 \mathrm{rpm}, 20^{\circ} \mathrm{C}\right)$ and resuspended at $4.0 \times 10^{6}$ cells/ $\mathrm{ml}$ in complete medium.

For the analyses during three weeks post hatch, 40 chickens were housed in one floor pen lined with wood shavings $\left(2 \mathrm{~kg} / \mathrm{m}^{2}\right)$ and received a commercial broiler feed ad libitum. At day 1 , fifteen chickens were euthanized and ileum, spleen, bone marrow and blood were collected and tissue samples of three birds were pooled to obtain biological replicates $(n=5)$ with sufficient cells to perform the analyses. At day $3,5,7,14$ and 21 , chickens $(n=5)$ were euthanized and individual tissues were collected. lleum segments of approximately $10 \mathrm{~cm}$ were harvested and flushed with PBS to remove contents. Next, segments were cut in sections of $1 \mathrm{~cm}^{2}$ and washed again in PBS. Subsequently, IEL were collected by stirring the sections three times at $200 \mathrm{rpm}$ for $15 \mathrm{~min}$ at $37^{\circ} \mathrm{C}$ in EDTA-medium (HBSS $1 \times$ (Gibco BRL) supplemented with $10 \%$ heat-inactivated FCS (Lonza); 0.005M EDTA (Sigma-Aldrich, the Netherlands)). Supernatants containing the IEL were collected after each incubation in EDTA-medium and centrifuged for $5 \mathrm{~min}$ at $1200 \mathrm{rpm}$ at $20^{\circ} \mathrm{C}$. Cell pellets were then resuspended in PBS, and lymphocytes were isolated by Ficoll-Paque density gradient centrifugation (12 min, $1700 \mathrm{rpm}, 20^{\circ} \mathrm{C}$ ), washed and resuspended in complete medium at $4.0 \times 10^{6} \mathrm{cells} / \mathrm{ml}$. Lymphocytes were isolated from spleen as described in embryos, however, with different Ficoll-Paque density gradient centrifugation conditions (20 min, $2200 \mathrm{rpm}, 20^{\circ} \mathrm{C}$ ). Isolation of lymphocytes from bone marrow was done according to the procedure described above for embryos. Blood, at least $5 \mathrm{ml}$, was collected in a tube containing $200 \mu \mathrm{l}$ of heparin (5000 IE/ml, LEO Pharma A/S, Denmark), diluted with an equal volume of PBS, layered on Ficoll-Paque and centrifuged $\left(20 \mathrm{~min}, 2200 \mathrm{rpm}, 20^{\circ} \mathrm{C}\right.$ ). Subsequently, lymphocytes were harvested, washed and resuspended in complete medium at $4.0 \times 10^{6} \mathrm{cells} / \mathrm{ml}$. Unless mentioned otherwise, lymphocytes were stained for flow cytometric analyses directly after isolation.

To localize markers expressed on immune cells in situ, immunohistochemical stainings were performed on ileum tissue of chickens from the control group (PBS inoculation) of a different animal experiment (AVD1080020174425). Chickens $(n=4)$ were euthanized at day 14 and $3 \mathrm{~mm}$ cross sections of ileum were frozen in liquid nitrogen and stored at $-80^{\circ} \mathrm{C}$.

To analyze CD107 expression within the IL-2R $\alpha^{+}$and $20 \mathrm{E5} 5^{+}$NK cell subsets, viably frozen lymphocytes of IEL, spleen and blood from 21-day-old broiler chickens (AVD1080020174425) were thawed and subsequently stained. Pellets of isolated lymphocytes were resuspended in FCS (Lonza) and ice cold complete medium supplemented with 20\% DMSO (Sigma-Aldrich) was added dropwise. Subsequently, cells were stored at $-140^{\circ} \mathrm{C}$. Frozen cells were rapidly thawed in a $37^{\circ} \mathrm{C}$ water bath followed by adding cold complete medium. Next, the cell suspension was centrifuged, the supernatant was discarded and the pellet was resuspended in complete medium and washed again. Viability of the thawed cells was approximately $90 \%$.

Chickens were housed, handled and treated according to approval by the Dutch Central Authority for Scientific Procedures on Animals and the Animal Experiments Committee of Utrecht University (the Netherlands) under registration numbers AVD108002017863 and AVD1080020174425 and all procedures were performed in accordance with all relevant legislation.

\section{Immunohistochemistry on ileum cryostat sections}

Cryostat sections $(7 \mu \mathrm{m})$ of the ileum, were placed on precoated slides (Superfrost/Plus, Germany) and stored over silica gel. First, slides were fixed for $10 \mathrm{~min}$ in pure acetone and air-dried. Sections were then incubated for one hour at room temperature (RT) in a closed humidified box with previously 
optimized concentrations of mouse-anti-chicken-CD3 (CT3; IgG1), mouse-anti-chicken-CD4 (CT4; IgG1), mouse-anti-chicken-TCRy $\delta$ (TCR-1; IgG1), mouse-anti-chicken-CD25 (IL-2R $\alpha$ AV142; IgG1, Bio-Rad Laboratories, CA, USA) and mouse-anti-chicken-20E5 (IgG1, Developmental Studies Hybridoma Bank (DSHB), University of lowa, IA, USA) diluted in PBS (Lonza) containing $0.5 \%$ bovine serum albumin and $0.1 \%$ sodium azide (PBA). Unless described otherwise, antibodies were obtained from Southern Biotech, Birmingham, AL, USA. Following washing in PBS, tissue sections were stained using the VECTASTAIN ${ }^{\circledR}$ Elite ${ }^{\circledR} A B C$ HRP Peroxidase, Standard Kit according to the manufacturer's protocol (Vector Laboratories, United Kingdom). Sections were incubated with horse-anti-mouseIgG-biotin, washed in PBS followed by incubation in ABC solution. Tissue sections were washed and HRP-enzyme reactivity was revealed by adding $0.5 \mathrm{mg}$ 3,3-diaminobenzidine-tetrahydrochloride (DAB; Sigma-Aldrich) per $\mathrm{ml}$ Tris buffer $\left(0.05 \mathrm{M}, \mathrm{pH}\right.$ 7.6) containing $0.05 \% \mathrm{H}_{2} \mathrm{O}_{2^{\prime}}$ and incubation at RT for 10 min. Sections were washed, briefly counterstained with haematoxylin (Sigma-Aldrich) and rinsed with tap water. Finally, tissue sections were mounted in Kaiser's Glycerol/Gelatine (Boom, the Netherlands) and microscopically analyzed to determine types and location of immune cells in IEL and lamina propria of the ileum.

\section{Phenotypic characterization of lymphocytes by flow cytometry}

Lymphocytes of the intestine, spleen, bone marrow and blood were stained with markers that are known to be expressed on NK cells and T cell subsets. Based on prevalence in our pilot experiments, the following markers were selected to classify NK cell subsets: $28-4$, recognizing the IL-2R $\alpha$ chain 22,24 , 20E5, with unknown specificity ${ }^{22,24}$, and $5 C 7$, recognizing CD11b/c or CD11 $\mathrm{d}^{30,31}$. Lymphocytes $\left(5 \times 10^{5}\right)$ were stained with two panels of antibodies specific for the above NK cell markers and anti-CD3 to be able to exclude T cells (Table 1). In addition, lymphocytes were stained using two panels of antibodies specific for markers on $\gamma \delta T$ and various T cell subsets (Table 1). Staining with primary and secondary antibodies (Table 1) was performed using previously optimized concentrations of the various antibodies in $50 \mu \mathrm{l}$ for $20 \mathrm{~min}$ at $4^{\circ} \mathrm{C}$ in the dark. Next, cells were washed two times in PBS supplemented with $0.5 \%$ BSA and $0.005 \% \mathrm{NaN}_{3}$ (PBA). In addition, lymphocytes were stained with a live/dead marker according the instructions of the manufacturer (Zombie Aqua ${ }^{\text {TM }}$ Fixable Viability Kit, Biolegend, CA, USA) for 15 min at room temperature (RT) in the dark. After washing in PBA, lymphocytes were resuspended and fixed using 2\% paraformaldehyde in PBS (Merck, Germany) for 10 min at RT. Following fixation, cells were washed again and resuspended in $200 \mu \mathrm{l}$ PBA of which $150 \mu \mathrm{l}$ was used to assess fluorescence of cells in a FACSCANTO II Flowcytometer (BD Biosciences). Analysis was performed using FlowJo software (Tree star Inc, OR, USA). 
Table 1. Flow cytometry staining reagents.

\begin{tabular}{|c|c|c|c|c|}
\hline Cell population & Mix & $\begin{array}{l}\text { Primary antibody } \\
\text { (mouse-anti- } \\
\text { chicken) }\end{array}$ & $\begin{array}{l}\text { Clone/ } \\
\text { Isotype }\end{array}$ & Secondary antibody \\
\hline \multirow[t]{5}{*}{ NK } & a & CD3-APC 1 & CT3/lgG1 & - \\
\hline & & 28-4-UNL ${ }^{2}$ & $\lg G 3$ & Goat-anti-mouse-IgG3-FITC ${ }^{1}$ \\
\hline & & $20 \mathrm{E} 5-\mathrm{BIOT}^{2}$ & $\lg \mathrm{g} 1$ & Streptavidin-Perc $\mathrm{P}^{5}$ \\
\hline & b & $\mathrm{CD}-\mathrm{APC}^{1}$ & CT3/lgG1 & - \\
\hline & & $5 \mathrm{C} 7-\mathrm{BIOT}^{2}$ & $\lg \mathrm{G} 1$ & Streptavidin-Perc $\mathrm{P}^{5}$ \\
\hline \multirow[t]{7}{*}{$\mathrm{T}$} & a & $\mathrm{CD}^{4} 5-\mathrm{APC}^{1}$ & $\lg M$ & - \\
\hline & & $\mathrm{TCR} \gamma \delta-\mathrm{PE}^{1}$ & TCR-1/lgG1 & - \\
\hline & & BU-1-FITC ${ }^{1}$ & AV20/lgG1 & - \\
\hline & $b$ & CD3-FITC $^{1}$ & $\mathrm{CT} 3 / \operatorname{lgG} 1$ & - \\
\hline & & CD4-APC ${ }^{1}$ & $\mathrm{CT} 4 / \lg \mathrm{G} 1$ & - \\
\hline & & $C D 8 \alpha-P E^{1}$ & CT8/lgG1 & - \\
\hline & & CD8ß-BIOT ${ }^{1}$ & $\mathrm{EP} 42 / \lg \mathrm{g} 2 \mathrm{a}$ & Streptavidin-PercP ${ }^{5}$ \\
\hline \multirow[t]{8}{*}{ NK activation } & a & CD107-APC ${ }^{3}$ & $\begin{array}{l}\text { LEP-100 I 5G10/ } \\
\text { IgG1 }\end{array}$ & - \\
\hline & & $\mathrm{CD} 3-\mathrm{PE}^{1}$ & $\mathrm{CT} 3 / \operatorname{lgG} 1$ & - \\
\hline & & CD41/61-FITC ${ }^{4}$ & $11 \mathrm{C} 3 / \operatorname{lgG} 1$ & - \\
\hline & $b$ & CD107-APC 3 & $\begin{array}{l}\text { LEP-100 I 5G10/ } \\
\text { IgG1 }\end{array}$ & - \\
\hline & & $\mathrm{CD} 3-\mathrm{PE}^{1}$ & $\mathrm{CT} 3 / \lg \mathrm{g} 1$ & - \\
\hline & & CD41/61-FITC ${ }^{4}$ & $11 \mathrm{C} 3 / \operatorname{lgG} 1$ & - \\
\hline & & 28-4-UNL² & $\lg G 3$ & Goat-anti-mouse-lgG3-APC/Cy $7^{1}$ \\
\hline & & $20 \mathrm{E} 5-\mathrm{BIOT}^{2}$ & $\lg G 1$ & Streptavidin-PercP5 \\
\hline
\end{tabular}

Manufacturer: 'Southern Biotech. ${ }^{2}$ Purified supernatant of hybridoma provided by Göbel, T.W., Ludwig Maximilians University, Germany. ${ }^{3}$ Developmental Studies Hybridoma Bank (DSHB), University of lowa, IA, USA. ${ }^{4}$ Serotec, United Kingdom. ${ }^{5}$ BD Biosciences.

\section{Assessment of NK cell activation in the CD107 assay}

The CD107 assay, which determines NK cell activation by enhanced surface expression of CD107 as a result of releasing granules containing perforin and granzymes, was carried out as described previously ${ }^{24}$. Briefly, $1 \times 10^{6}$ lymphocytes were incubated in $0.5 \mathrm{ml}$ complete medium for 4 hours at $37^{\circ} \mathrm{C}, 5 \% \mathrm{CO}_{2}$ in the presence of $1 \mu \mathrm{l} / \mathrm{ml}$ Golgistop (BD Biosciences) and $0.5 \mu \mathrm{l} / \mathrm{ml}$ mouse-anti-chicken-CD107-APC. After anti-CD107 staining, lymphocytes were stained with anti-CD3 and anti-CD41/61 mAbs to exclude T cells and thrombocytes from the analyses (Table 1). A second panel was used to analyze CD107 expression within the IL-2R $\alpha^{+}$and $20 \mathrm{E5}{ }^{+} \mathrm{NK}$ cells (Table 1). After incubation, cells were washed, stained and analyzed as described in section 'Phenotypic characterization of lymphocytes by flow cytometry'.

\section{Statistical analysis}

The assumptions for normal distributed data were not met and therefore non-parametric statistical tests were used. Differences in percentages of immune cell subsets in course of time were analyzed using the Kruskal-Wallis tests accompanied with Dunn's multiple comparisons tests. Differences in percentages of immune cell subsets within a tissue and between tissues were analyzed using Friedman's tests with Dunn's multiple comparisons tests. A $p$-value of $<0.05$ was considered statistically significant. All statistical analyses were performed using the software program GraphPad Prism 7 (GraphPad Software, CA, USA). 


\section{Results}

\section{High prevalence of IL-2R $\alpha+$ NK cells in the IEL of the ileum}

Immunohistochemistry was performed on tissue sections from the intestine of two week-old broiler chickens to localize IL-2R $\alpha^{+}$and $20 \mathrm{E5}^{+}$cells, $\mathrm{CD}^{+}, \mathrm{CD}^{+}$and $\gamma \delta \mathrm{T}$ cells (Fig. 1). The IL-2R $\alpha^{+}$cells were present in high numbers among the epithelial cells of villi, crypts and glands (IEL) compared to mild to moderate numbers in the lamina propria of the ileum (Fig. 1A). Unfortunately, we were unable to detect $20 \mathrm{E} 5^{+}$cells in the ileum because the antibody did not work in our hands in immunohistochemical staining (data not shown). The presence of $\mathrm{CD}^{+} \mathrm{T}$ cells was observed more in the IEL compared to the lamina propria (Fig. 1B). CD4 ${ }^{+}$T cells were mainly present in the lamina propria compared to minimal numbers in IEL (Fig. 1C). Moderate numbers of $\gamma \delta$ T cells were observed both in IEL and lamina propria (Fig. 1D).

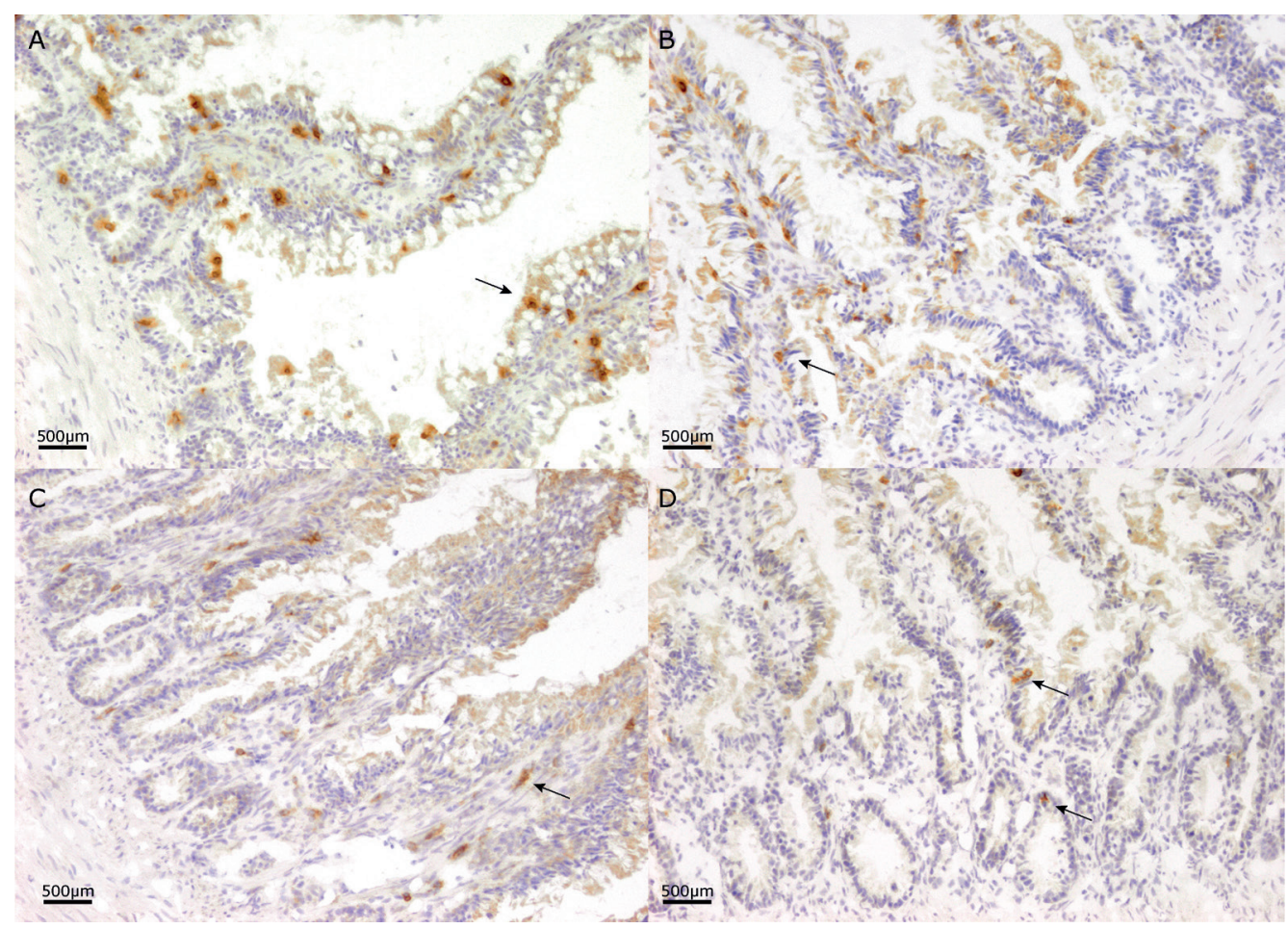

Figure 1. Localization of cell subsets in IEL using immunohistochemistry. (A) Sections of ileum were stained with IL-2R $\alpha$, (B) $C D 3$, (C) CD4 and (D) TCR $\delta \delta$ and subsequently counterstained with haematoxylin. Immune cells were microscopically analyzed for their localization in the IEL population or in the lamina propria, stained cells are depicted by arrows. Representative images of a two week-old broiler chicken in a $20 \times$ magnification, scale bar $=500 \mu \mathrm{m}$.

Based on FACS analyses, the presence of various NK cell subsets in the intestine was determined in embryos and chickens of different ages by analyzing the relative number, defined by the percentage, of $\mathrm{CD} 3$ negative cells that express $\mathrm{IL}-2 \mathrm{R} \alpha^{+}, 20 \mathrm{E} 5^{+}$and $5 \mathrm{C}^{+}$cells, and $\mathrm{CD} 107$ as marker for NK cell activation (Fig. 2A). The percentage of intestinal IL-2R $\alpha^{+} \mathrm{NK}$ cells tended to be increased at day 1 post hatch $(60.74 \pm 3.2 \%)$ compared to ED14 (15.32 $\pm 5.9 \%, p=0.06)$ and ED18 $(19.25 \pm 6.5 \%, p=0.08)$, and remained similar throughout aging (Fig. 2B). The percentage of intestinal $20 \mathrm{E} 5^{+}$NK cells was lower 
at day $1(3.9 \pm 0.76 \%)$ compared to ED14 $(14.87 \pm 1.4 \%, p=0.05)$ and subsequently increased towards day $21(11.38 \pm 1.9 \%)$ to levels similar to those in early embryonic life (Fig. $2 \mathrm{C})$. Also, the percentage of intestinal $5 \mathrm{C7}^{+} \mathrm{NK}$ cells was lower at day $1(1.4 \pm 0.27 \%)$ than at ED14 $(7.3 \pm 1.4 \%, p=0.07)$ and increased again to $7.2 \pm 2.1 \%$ at day 21 (Fig. 2D). When comparing these subsets of NK cells in the intestine post hatch, a considerably higher percentage of IL-2R $\alpha^{+} \mathrm{NK}$ cells was observed compared to $20 \mathrm{E} 5^{+}$NK cells (Fig 2B-D). The percentage of CD107 ${ }^{+}$NK cells varied between $7.66 \pm 1.7 \%$ (day 21) and $13.71 \pm 2.3 \%$ (day 5) during embryonic phase and first three weeks of age (Fig. 2E). In addition, CD107 expressing cells were predominantly observed in the intestinal IL-2R $\alpha^{+}$NK cell subset $(17.3 \pm 0.5 \%)$ and less CD107 expression was observed in 20E5+ NK cells (6.4 $\pm 0.4 \%$, Fig. 2F).

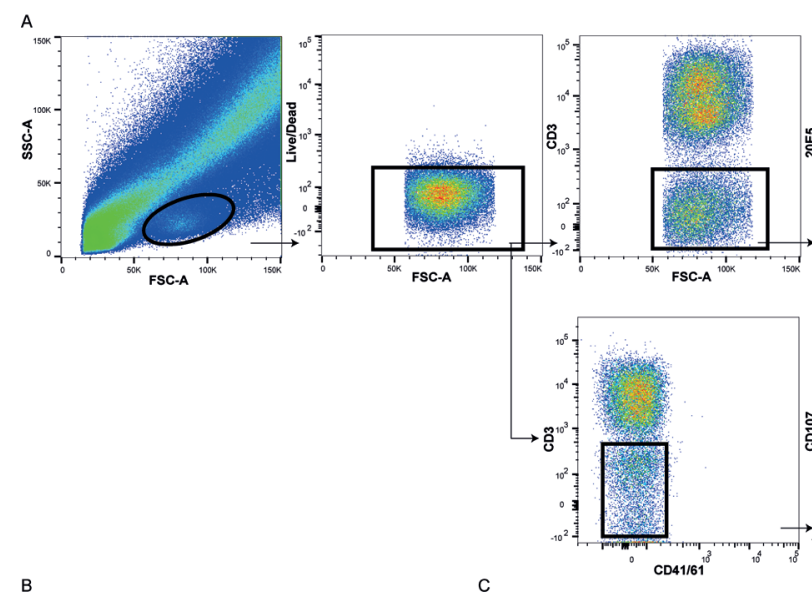

B IL-2Ra ${ }^{+}$NK cells
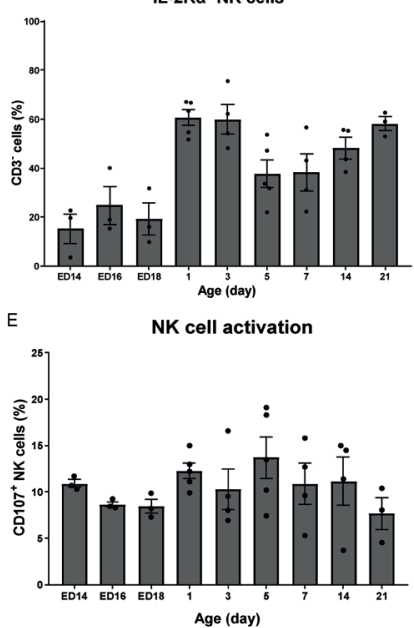

C
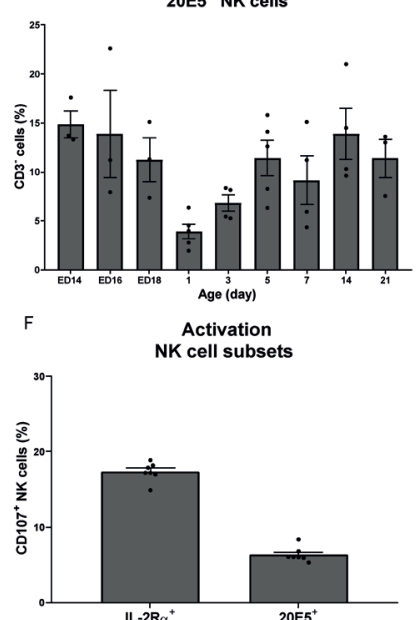

NK cell subsets

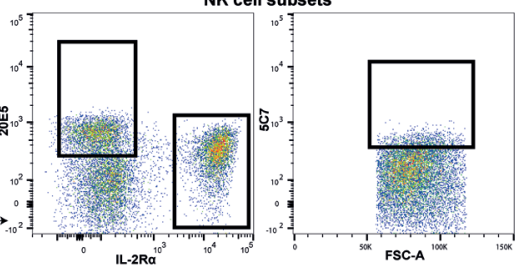

NK cell activation

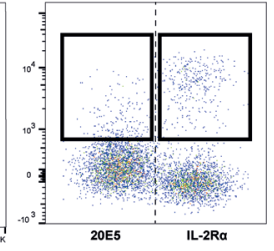

$5 \mathrm{C7} 7^{+}$NK cells

D

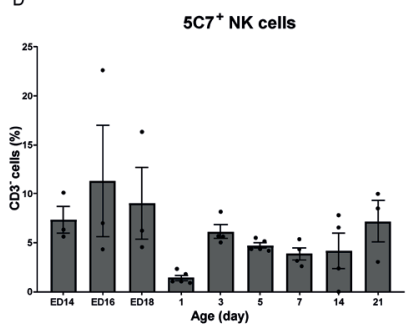

Figure 2. Phenotypic characterization of intestinal NK cells in embryos and chickens. (A) Gating strategy after isolation of lymphocytes from IEL to analyze NK cell subsets and their activation; lymphocytes were gated, within lymphocytes the live lymphocytes, therein the CD3 negative cells followed by selection of cells expressing IL-2R $\alpha, 20 E 5$ and 5C7 (NK cell subsets) or the CD3 and CD41/61 negative cells followed by selection of cells expressing CD107 in total NK cells and within the IL-2R $\alpha^{+}$and $20 \mathrm{E} 5^{+}$NK cells. (B) Percentage of CD3 negative cells (mean \pm SEM) that express surface markers IL-2R $\alpha,(C) 20 E 5$ and (D) $5 C 7$. (E) NK cell activation was assessed by measuring the surface marker CD107 (mean \pm SEM) in total NK cells and in (F) NK cell subsets. In B-E for embryos $n=3$, chickens $n=$ 5 , and in $\mathrm{F}$ two independent experiments with in total 7 replicates. 


\section{Splenic NK cells predominantly express IL-2R $\alpha$ while blood and bone marrow-derived NK cells are mostly 20E5+}

In addition to intestinal NK cells, the presence of NK cell subsets in spleen, blood and bone marrow was investigated at different ages. The percentage of splenic IL-2R $\alpha^{+}$and $20 \mathrm{E} 5^{+} \mathrm{NK}$ cells did not change significantly during embryonic and early life (Fig. 3A, B). The percentage of splenic $5 \mathrm{C7}^{+} \mathrm{NK}$ cells was lower at day 1 ( $4.2 \pm 0.2 \%)$ compared to ED14 ( $8.5 \pm 0.9 \%, p=0.02)$. At day $7(7.5 \pm 0.4 \%, p=0.03)$, the percentage of $5 \mathrm{C}^{+} \mathrm{NK}$ cells in spleen increased again to similar levels as observed in early embryonic life (Fig. 3C). Similar to intestinal NK cells, also the majority of splenic NK cells in young chickens was $\mathrm{IL}-2 \mathrm{R} \alpha^{+}$. Percentages of blood-derived IL-2R $\alpha^{+}, 20 \mathrm{E} 5^{+}$and $5 \mathrm{C} 7^{+} \mathrm{NK}$ cells were similar during the first three weeks of age (Fig. 3D-F). While the percentage of bone marrow-derived IL-2R $\alpha^{+} \mathrm{NK}$ cells did not change in time (Fig. 3G), the percentage of 20E5 $5^{+}$NK cells increased post hatch from day 1 (2.7 \pm $0.3 \%)$ to day $7(16.4 \pm 1.9 \%, p=0.01)$ and $14(17.8 \pm 1.9 \%, p<0.01)$ to levels comparable to those in embryonic life (Fig. $3 \mathrm{H})$. Bone marrow-derived $5 \mathrm{C} 7^{+} \mathrm{NK}$ cells were lower at day 1 (3.0 $\left.\pm 0.2 \%\right)$ compared to $\operatorname{ED} 18(7.1 \pm 2.1 \%, p=0.04$, Fig. 3). In contrast to findings in intestine and spleen, most blood and bone marrow-derived NK cells where 20E5+ (Fig. 3D-I).
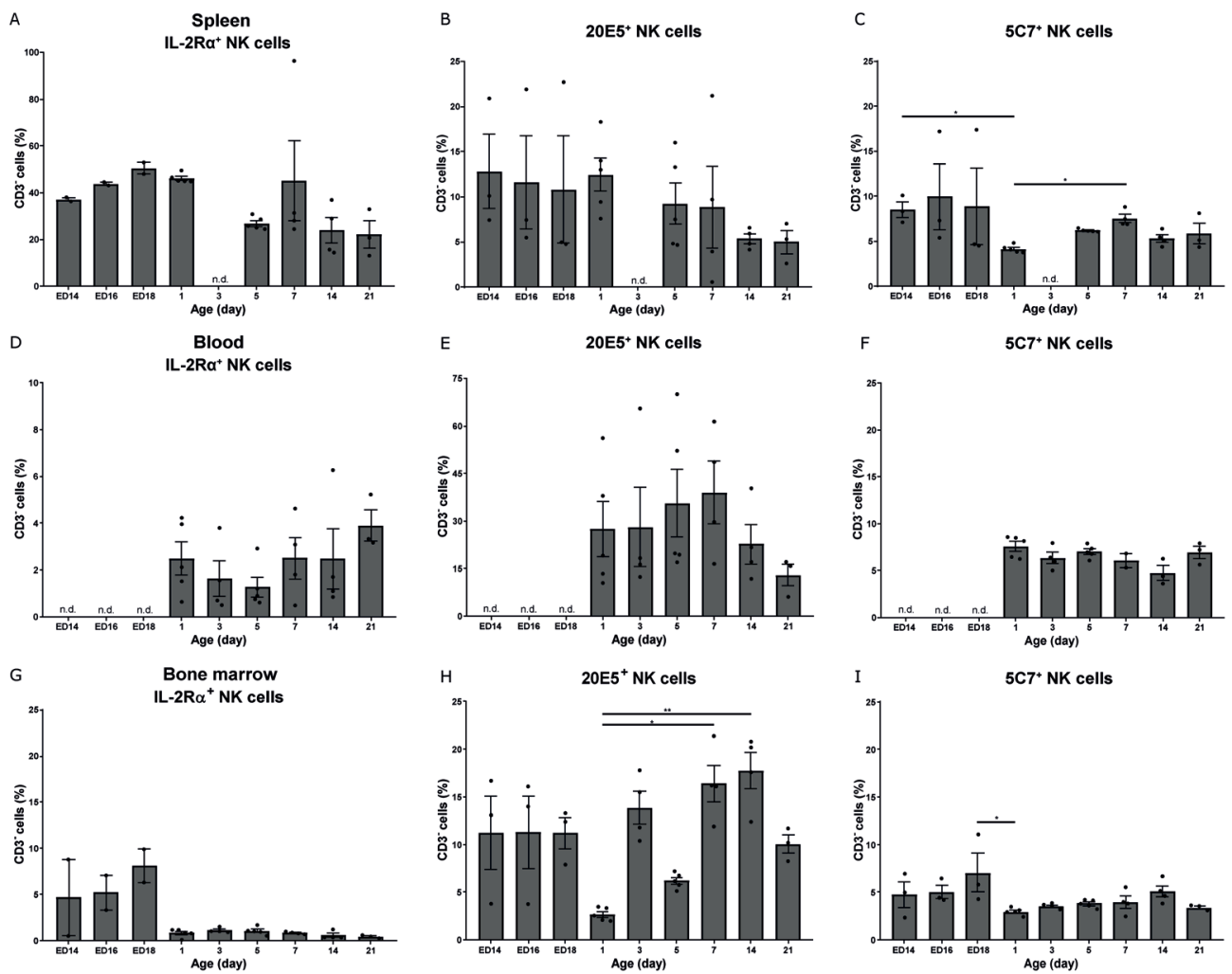

Figure 3. Phenotypic characterization of NK cells in spleen, blood and bone marrow of embryos and chickens. Gating strategy is similar to IEL. (A-C) Percentage of CD3 negative cells (mean \pm SEM) that express surface markers IL-2R $\alpha, 20 E 5$ and $5 C 7$ in spleen, (D-F) blood and (G-I) bone marrow. In A-I for embryos $n=3$, chickens $n=5$, n.d. means not determined. Statistical significance between ages is indicated as ${ }^{*} p<0.05$ and ${ }^{* *} p<0.01$. 
No significant differences in activation of splenic NK cells was observed upon hatch (Fig. 4A). The percentage of blood-derived CD107 ${ }^{+}$NK cells tended to increase between day 7 (5.3 $\pm 1.2 \%$, Fig. 4C) and $14(19.4 \pm 5.0 \%, p=0.07)$. Percentages of bone marrow-derived CD107 ${ }^{+}$NK cells tended to be lower at day $1(7.3 \pm 0.3 \%)$ compared to ED14 $(16.0 \pm 1.2 \%, p=0.09)$ and significantly decreased at day $7(5.1 \pm 0.2 \%)$ compared to $\operatorname{ED14}(p<0.01), \operatorname{ED16}(p=0.02)$ and $\operatorname{ED18}(p=0.02)$, which was followed with a tendency to increase again (Fig. 4E). A higher percentage of $\mathrm{CD} 107^{+}$cells was observed within the $20 \mathrm{E5}^{+} \mathrm{NK}$ cell subset compared to IL-2R $\alpha^{+} \mathrm{NK}$ cells in spleen and blood, the opposite of what was observed in intestine (Fig. 4B, D).

A

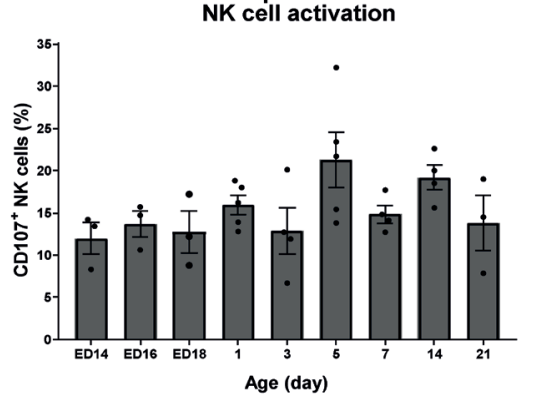

$\mathrm{C}$

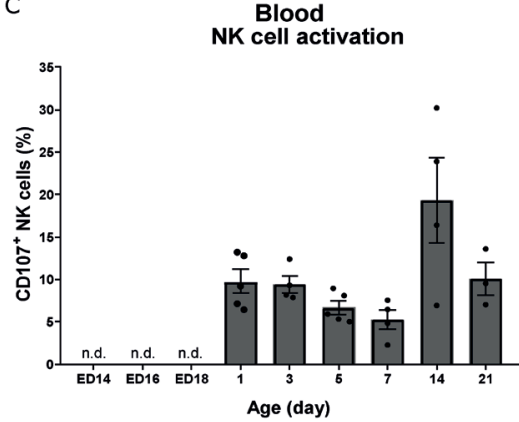

E

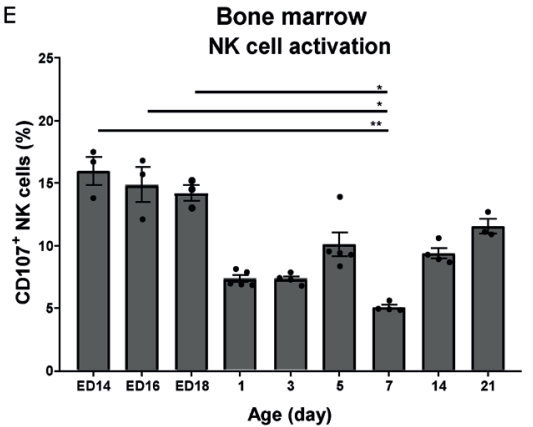

B

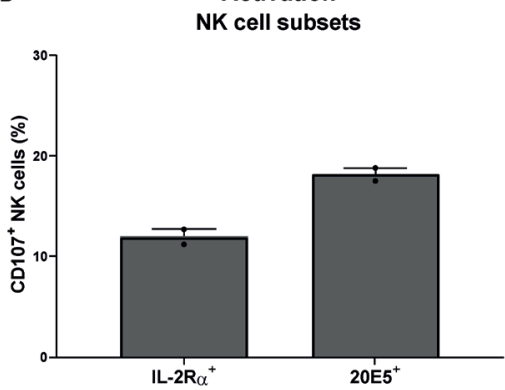

D

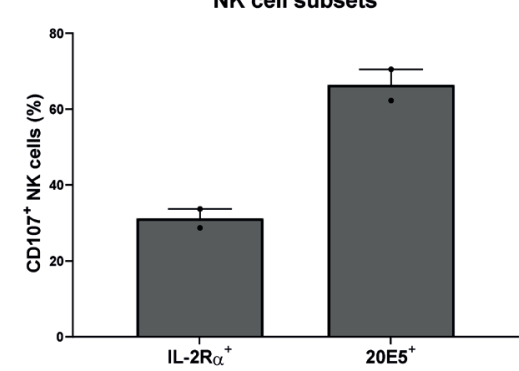

Figure 4. Phenotypic characterization of activated NK cells in spleen, blood and bone marrow of embryos and chickens. Gating strategy is similar to IEL. (A) NK cell activation was assessed by measuring the surface marker CD107 (mean \pm SEM) in total NK cells of spleen, (C) blood and (E) bone marrow and in NK cell subsets of (B) spleen and (D) blood. In $A, C, E$ for embryos $n=3$, chickens $n=5$, and in $\mathrm{B}, \mathrm{D}$ two independent experiments. Statistical significance between ages is indicated as ${ }^{*} p<0.05$ and ${ }^{* *} p<0.01$. 


\section{High prevalence of $y \delta$ T cells, CD8 $\alpha \alpha+$ and CD $8 \alpha \beta+$ T cells in IEL of the ileum in young broiler chickens}

Next, the presence of T cell subsets amongst the IEL was determined during embryonic development and in chickens during the first three weeks post hatch (Fig. 5A). The presence of total $\mathrm{CD}^{+} \mathrm{T}$ cells in IEL increased during aging to approximately $70 \%$ (data not shown). No CD4+ intestinal T cells were detected in embryos, whereas in young chickens a low presence was found up to day 21 (data not shown). Percentages of $\gamma \delta$ T cells in the IEL remained similar upon hatch, but increased from day 14 on to $37.9 \pm 5.8 \%$ at day 21 (Fig. $5 \mathrm{~B}$ ). Although not significant, a gradual increase was also observed in the percentages of intestinal CD8 $\alpha \alpha^{+}$T cells upon hatch in ED18 (18.6 $\left.\pm 8.7 \%\right)$ versus $32.4 \pm 4.5 \%$ in chickens of 21 days (Fig. $5 C$ ). The percentages of CD8 $\alpha \beta^{+}$T cells increased significantly upon hatch from $1.0 \pm$ $0.2 \%$ at ED14 to $49.2 \pm 3.7 \%$ in chickens of 14 days ( $p=0.02$, Fig. $5 C$ ). No significant differences were found between the percentage of intestinal $\gamma \delta$ T cells, CD $8 \alpha \alpha^{+}$and $C D 8 \alpha \beta^{+} T$ cells during embryonic development between ED14 and ED18 (Fig. 5B, C). In chickens younger than 7 days, more CD8 $\alpha \alpha^{+}$and CD8 $\alpha \beta^{+}$T cells were observed compared to $\gamma \delta$ T cells (Fig. 5B, C). However, presence of intestinal $\gamma \delta$ $T$ increased to levels similar to those of $C D 8 \alpha \alpha^{+}$and $C D 8 \alpha \beta^{+}$T cells from day 14 onwards (Fig. 5B, C).
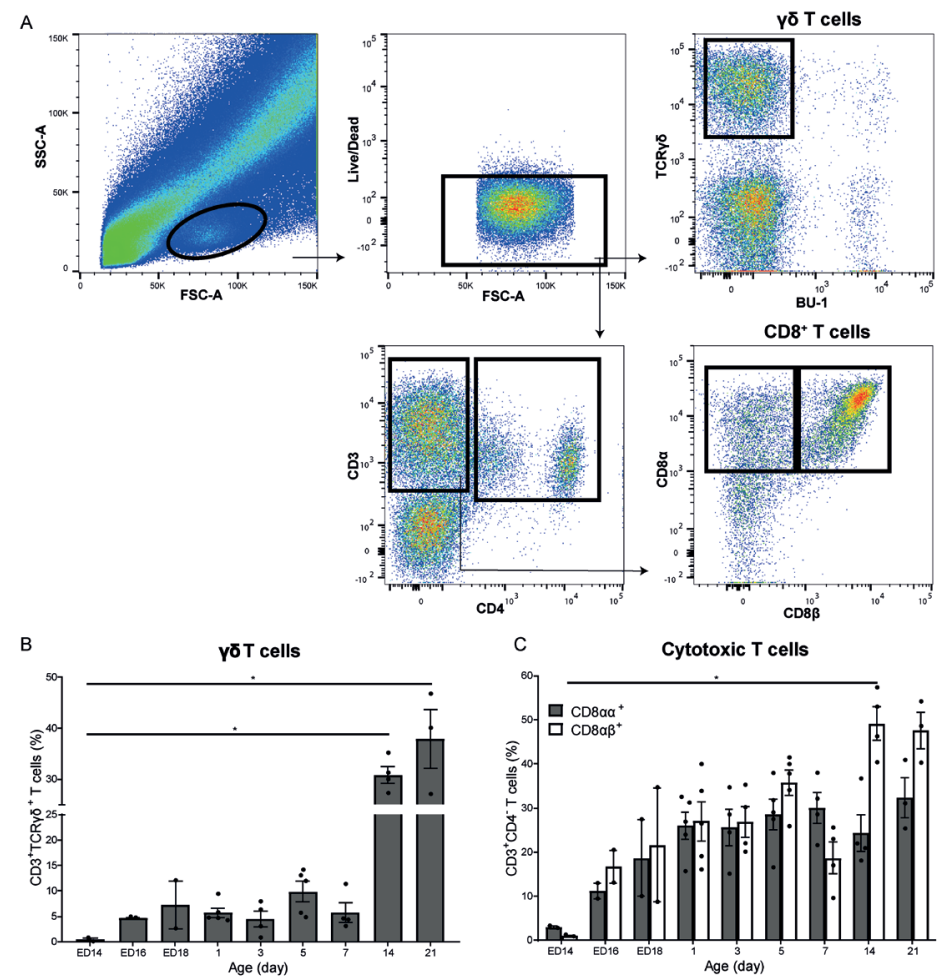

Figure 5. Phenotypic characterization of intestinal T cells in embryos and chickens. (A) Gating strategy after isolation of lymphocytes from IEL to analyze T cell subsets; lymphocytes were gated, within lymphocytes the live lymphocytes, followed by selection of TCR-1 ${ }^{+} \mathrm{BU}-1^{-} \mathrm{T}$ cells $\left(\gamma \delta \mathrm{T}\right.$ cells) or selection within the $\mathrm{CD} 3^{+} \mathrm{CD} 4$ - cells of $\mathrm{T}$ cells expressing CD8 $\alpha \alpha$ and $C D 8 \alpha \beta$ (CD8 ${ }^{+} \mathrm{T}$ cells). (B) Percentage of live cells (mean \pm SEM) that express the surface marker TCR $\gamma \delta$ to distinguish $\gamma \delta$ T cells. (C) Percentage of CD3 ${ }^{+} C D 4-T$ cells (mean \pm SEM) that express CD8 $\alpha \alpha$ and CD8 $\alpha \beta$ to distinguish cytotoxic CD8 ${ }^{+} T$ cells. In B-C for embryos $n=3$, chickens $n=5$. Statistical significance between ages is indicated as * $p<0.05$. 


\section{Highest prevalence of CD8 $\alpha \beta+T$ cells in spleen, blood and bone marrow of young broiler chickens}

Next, $\mathrm{T}$ cell subsets in spleen, blood and bone marrow were analyzed in broiler chickens of different ages. The percentage of $\mathrm{CD}^{+} \mathrm{T}$ cells in spleen (54\%) and blood $(75 \%)$ increased during aging similar to what was observed in IEL (70\%), whereas in bone marrow a low presence of approximately $2 \%$ was found throughout aging (data not shown). An increase was observed in percentages of $\gamma \delta$ T cells upon hatch in spleen (Fig. 6A), however, no differences were found in percentages of $\gamma \delta \mathrm{T}$ cells in blood (Fig. $6 \mathrm{C}$ ) and bone marrow (Fig. 6E). Furthermore, a higher percentage of CD8 $\alpha \alpha^{+} T$ cells was found in spleen (Fig. 6B) and bone marrow (Fig. 6F) of embryos compared to young chickens, whereas the percentage of CD8 $\alpha \alpha^{+} T$ cells in blood did not change upon hatch (Fig. 6D). Percentages of CD8 $\alpha \beta^{+} T$ cells were higher in spleen, blood and bone marrow of young chickens versus embryos (Fig. 6B, D, F).

In embryonic spleen and bone marrow, the majority of the T cells expressed CD8 $\alpha \alpha^{+}$or CD8 $\alpha \beta^{+}$(Fig. 6). In young chickens, the percentage splenic $C D 8 \alpha \beta^{+} T$ cells was higher compared to the percentage $\gamma \delta T$ cells at day $14(p=0.01)$ and $21(p=0.03$, Fig. $6 \mathrm{~A}, \mathrm{~B})$. In blood of young chickens, percentages of $\gamma \delta \mathrm{T}$ cells and $C D 8^{+} T$ cells were similar until two weeks of age, whereas at day 21 the $C D 8 \alpha \beta^{+} T$ cell subset showed highest presence (Fig. 6C, D). In young chickens, no $\gamma \delta$ T cells were found in bone marrow and highest percentages were found of $C D 8 \alpha \beta^{+} T$ cells from two weeks onwards (Fig. 6E, F).

\section{Discussion}

The present study was designed to get insight in phenotypical and functional characteristics of subsets of NK cells in general, and intestinal NK cells more specifically, in broiler chickens in course of time during embryonic development and the first three weeks of life.

The majority of intestinal NK cells in the ileum was located in the IEL and expressed the IL-2R $\alpha$. Their relative presence, expressed as percentage of total CD3 negative cells, increased post hatch. The high percentage of intestinal IL-2R $\alpha^{+}$NK cells is in agreement with earlier studies in layer chickens and SPF chickens that showed similar percentages at similar ages ${ }^{22,28}$. Also $20 \mathrm{E}^{+}$and $5 \mathrm{C}^{+}$intestinal NK cells were readily detected with no major changes in presence in the course of embryonic stages and the first weeks of life. It should be noted that the $5 \mathrm{C} 7$ marker could not be included in the staining panel with IL-2R $\alpha$ and 20E5, which means we cannot define $5 \mathrm{C7} 7^{+}$NK cells as a distinct subset as $5 \mathrm{C7}$ may be expressed on either IL-2R $\alpha^{+}$and $20 \mathrm{E5} 5^{+}$NK cells or both. Intestinal percentages of either $20 \mathrm{E} 5$ or $5 C 7$ as single markers were found to a lesser extent compared to IL-2R $\alpha$. In addition, $10 \%$ of intestinal NK cells expressed CD107 and this expression was mainly observed in the IL-2R $\alpha^{+} N K$ subset. One day after hatch, the percentage of NK cells expressing IL-2R $\alpha$ showed an increase, whereas the $20 \mathrm{E} 5^{+} \mathrm{NK}$ cells decreased.

In our study, the relative number of IL-2R $\alpha^{+}$NK cells in intestine of embryos was similar to bone marrow, but lower in intestine compared to spleen. However, the percentage of IL-2R $\alpha^{+} \mathrm{NK}$ cells was highest after hatch in IEL compared to the other tissues. This may indicate trafficking of IL-2R $\alpha^{+} \mathrm{NK}$ cells in the first week after hatch from spleen to the intestine, which has been shown in adult mice where spleen-derived NK cells were found in all NK-containing organs after intravenous adoptive transfer ${ }^{38}$. Alternatively, local NK cells in the intestine may have up-regulated the IL-2R $\alpha^{+}$expression due to dual stimulation of Fc receptors and IL-12R ${ }^{14}$, or combination of the IL-12R, IL15R and IL-18R ${ }^{15}$, which promotes activation as has been shown for human blood-derived NK cells ${ }^{14,15}$. Interestingly, we observed that the representation of NK cell subsets within the individual tissues varied, since in 
both intestine and spleen, the IL-2R $\alpha^{+}$NK cell subset was more abundant whereas in blood and bone marrow, the majority of the NK cells expressed $20 \mathrm{E}^{+}$. The distinction of these NK cell subsets has not been demonstrated in layer chickens during embryonic life. In four-week-old layer chickens, a higher relative presence of splenic $20 \mathrm{E} 5^{+}$and $5 C 7^{+} \mathrm{NK}$ cells was observed compared to IL-2R $\alpha^{+} \mathrm{NK}$ cells ${ }^{24}$. We hypothesized that different tissue distributions of IL-2R $\alpha^{+}$and $20 \mathrm{E} 5^{+} \mathrm{NK}$ cell subsets may be related to specific functions such as cytotoxic activity, which is measured in humans ${ }^{25}$ and chicken ${ }^{24}$ by enhanced surface expression of CD107.

A

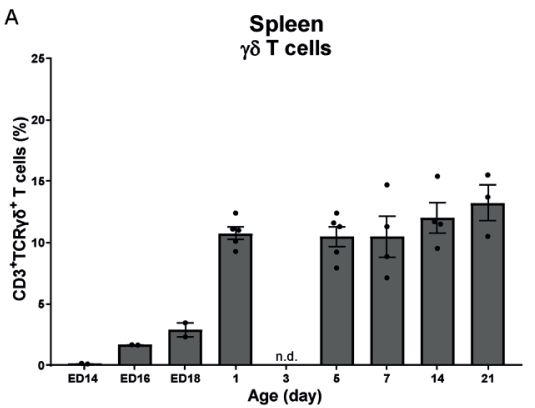

C
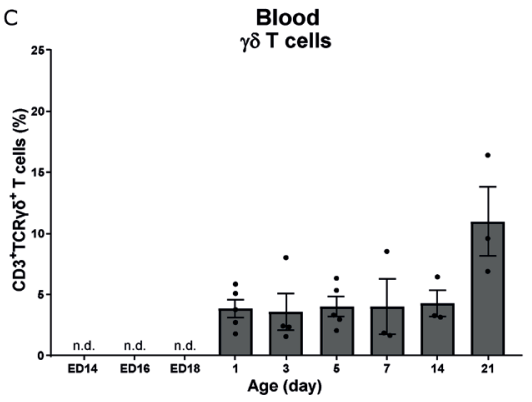

E

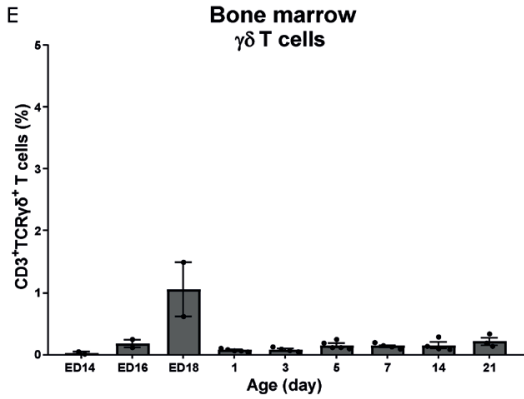

B

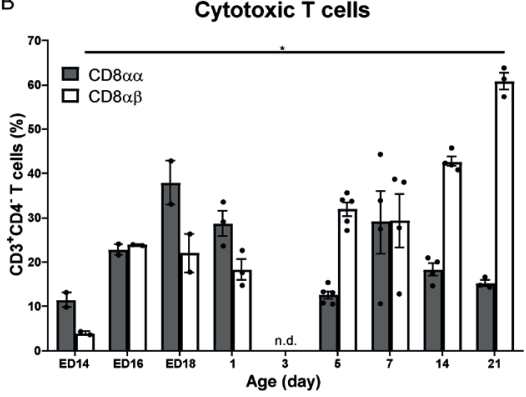

D

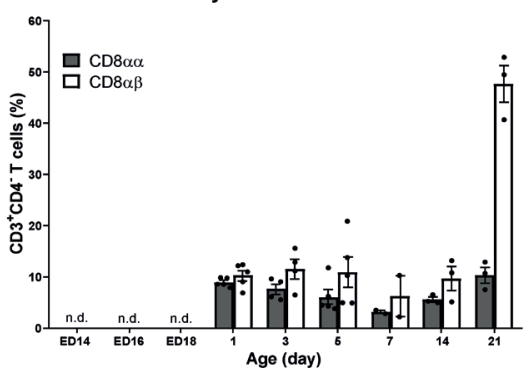

$\mathrm{F}$

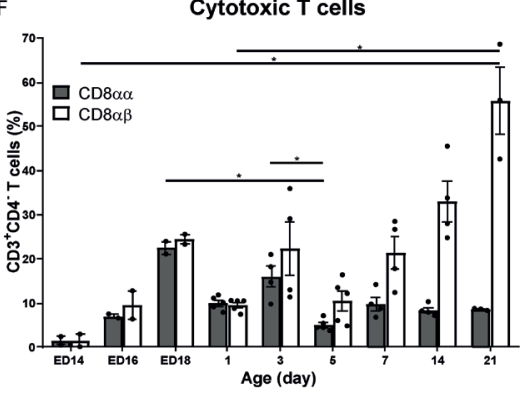

Figure 6. Phenotypic characterization of T cells in spleen, blood and bone marrow of embryos and chickens. Gating strategy is similar to IEL. (A) Percentage of live cells (mean \pm SEM) that express the surface marker TCR $\gamma \delta$ to distinguish $\gamma \delta T$ cells in spleen, (C) blood and (E) bone marrow. (B) Percentage of CD3 ${ }^{+} \mathrm{CD} 4-\mathrm{T}$ cells (mean $\pm \mathrm{SEM}$ ) that express CD8 $\alpha \alpha$ and $\mathrm{CD} 8 \alpha \beta$ to distinguish cytotoxic CD $8^{+}$ $T$ cells in spleen, (D) blood and (F) bone marrow. In $A-F$ for embryos $n=3$, chickens $n=5$. Statistical significance between ages is indicated as * $p<0.05$. 
Comparable percentages of CD107 expression were observed on NK cells in spleen, blood, bone marrow and intestine. The percentage of NK cell activation detected in this study was similar to those observed in spleen ${ }^{24}$ and blood ${ }^{27}$ of non-infected layer chickens in earlier studies, but lower compared to NK cell activation in infected layer chickens, of which relative presence may increase up to $30 \%{ }^{27}$. Interestingly, CD107 expression in spleen and blood was mainly observed on 20E5+ NK cells, which is different from what we found in the intestine. Based on these data, both NK cell subsets are implicated in cytotoxic activity, which was different from what we initially hypothesized. Studies with human NK cells have shown that increased IL-2R $\alpha$ expression was associated with an early stage of NK cell activation ${ }^{14-16}$. This may also be the case in chickens, suggesting that the higher percentage of IL-2R $\alpha^{+}$ NK cells in the intestine and spleen may reflect a population of NK cells in an early stage of activation. The finding that intestinal IL-2R $\alpha^{+}$NK cells are involved in cytotoxicity has also been shown in adult layer chickens where IL-2R $\alpha^{+}$IEL as putative NK cells showed killing of target cells ${ }^{22,29}$. The activation of intestinal IL-2R $\alpha^{+} \mathrm{NK}$ cells may be dependent on local signals, such as interactions with the microbiota either directly through TLRs or indirectly via cytokine production of exposed resident cells $s^{39,40}$. Further research into the IL-2R $\alpha^{+}$and $20 \mathrm{E} 5^{+}$NK cell subsets is necessary to clarify their functions, which will contribute to the understanding of NK cell biology in chickens. Inducing a higher prevalence of IL-2R $\alpha^{+}$ NK cells in the intestine, by for instance early life feed interventions, may infer higher protective potency and strengthen the innate immune response during post-hatch development, when adaptive immunity is still immature.

In addition to NK cells, the presence of T cells was also analyzed. In the intestine, the major T cell subsets found in the IEL were $\gamma \delta$ - and $C D 8^{+}$cytotoxic $T$ cells, whereas $C D 4^{+} T$ cells were mainly present in the lamina propria. This was confirmed with immunohistochemistry data and relative numbers were in agreement with reported levels of intestinal $\gamma \delta T^{41,42}, C D 8^{+} T^{41}$ and $C D 4+T$ cells ${ }^{2,43}$ in layer chickens. From two weeks of age onwards, $\gamma \delta T$ cells were more abundant in the IEL compared to spleen, blood and bone marrow and a similar tissue distribution of $\gamma \delta \mathrm{T}$ cells was observed in layer chickens $^{44}$, rodents and humans ${ }^{45,46}$. Percentage of intestinal $\gamma \delta$ T cells was similar to those found in ruminants ${ }^{47}$ and pigs ${ }^{48}$. Percentages $\gamma \delta \mathrm{T}$ cells in the blood of broiler chickens were higher compared to humans ${ }^{49}$. This indicates that $\gamma \delta T$ cells in chickens are important effector cells at the interface of innate and adaptive immunity in different locations ${ }^{29}$. Within the cytotoxic CD8 ${ }^{+} \mathrm{T}$ cell population, high percentages CD8 $\alpha \alpha^{+}$T cells were observed in the IEL, whereas in the other tissues high percentages of $C D 8 \alpha \beta^{+} T$ cells were found. At three weeks of age, percentages of $C D 8 \alpha \beta^{+} T$ cells were highest in all tissues. Percentages of intestinal cytotoxic $C D 8 \alpha \alpha^{+}$and $C D 8 \alpha \beta^{+}$T cell subsets were similar to that found in layer chickens ${ }^{41}$, whereas in mice higher prevalence of CD8 $\alpha \alpha^{+} T$ cells was observed ${ }^{50}$. An overview of the data generated in broiler chickens as compared to layer chickens is given in Table S1. Similar to chicken, human T cells predominantly express CD8 $\alpha \beta$, however, $C D 8 \alpha \alpha$ is only expressed on activated T cells, including $\gamma \delta T$ and cytotoxic T cells, and forms a small population which significantly expands during chronic infections ${ }^{51}$. Higher prevalence of CD8 $\alpha \alpha^{+} T$ cells was also associated with higher protective immune responses towards viral ${ }^{52}$ and bacterial ${ }^{53}$ infections in chickens. Another study reported that intestinal $C D 8 \alpha \alpha^{+} \mathrm{T}$ cells showed innate functional characteristics in mice and humans ${ }^{54}$, which might be suggested to be similar in chickens. A higher prevalence of $\gamma \delta T$ cells or $\mathrm{CD} 8 \alpha \alpha^{+} \mathrm{T}$ cells, which might be induced by feed interventions, may strengthen the innate response by collaborating with NK cells to improve health of broiler chickens.

In conclusion, we were able to isolate NK cell subsets from IEL and showed that most NK cells expressed IL-2R $\alpha$. IL-2R $\alpha^{+}$NK cells are predominantly present in intestine and spleen, while in blood and bone marrow NK cells are mostly 20E5 $5^{+}$Interestingly, the majority of intestinal $\mathrm{CD} 107^{+}$cells is detected in IL-2R $\alpha^{+}$NK cells, whereas in spleen and blood, the majority of $\mathrm{CD} 107^{+}$cells is observed within the 
$20 \mathrm{E} 5^{+}$NK cell subset. In addition, the IEL showed highest percentages of $\gamma \delta \mathrm{T}$ and cytotoxic CD8 $\alpha \alpha^{+}$ $T$ cells compared to the other tissues after hatch. The higher prevalence of IL-2R $\alpha^{+} N K$ cells in the intestine compared to the other tissues may indicate an early activation stage of intestinal NK cells, which might be caused by local interactions with the microbiota. Higher prevalence of IL-2R $\alpha^{+} \mathrm{NK}$ cells in the intestine may infer higher protective potency and future studies should investigate whether the presence of this intestinal NK cell subset could be manipulated by early life feed interventions either directly or through the microbiota. This may result in strengthening the first line of defense in broiler chickens during post-hatch development when their adaptive immunity is still immature and may open possibilities to use immune-mediated protection to raise broiler chickens resistant to infectious diseases and reduce antimicrobial use.

\section{Acknowledgements}

We thank the animal caretakers of the Department Population Health Sciences, division Farm Animal Health, Faculty of Veterinary Medicine, Utrecht University, for their help during the animal experiments. We thank R.H.G.A. van den Biggelaar for his help during the isolation of immune cells. We thank the Dutch Molecular Pathology Centre (DMPC), Department Biomolecular Health Sciences, Faculty of Veterinary Medicine, Utrecht University, for the immunohistochemistry analysis and G.J.A. Arkesteijn for maintaining optimal working conditions of the Flow Cytometry and Cell Sorting Facility, Faculty of Veterinary Medicine, Utrecht University. This work is part of the research programme NWO Earth and Life Sciences (ALW) with project number 868.15.020, which is financed by the Dutch Research Council (NWO) and by Cargill Animal Nutrition and Health. 


\section{Supplementary data}

Table S1. Characterization of immune cells generated in broiler chickens in the present study compared to data known in layer chickens.

\begin{tabular}{|c|c|c|}
\hline Immune cells & Broiler chickens & Layer chickens \\
\hline CD3-CD56 ${ }^{+}$NK cells & Not determined & Embryonic spleen ${ }^{1}$, lung ${ }^{1,2}$, blood $^{2}$ (not present in intestine ${ }^{1}$ ) \\
\hline CD3-IL-2R $\alpha^{+}$NK cells & $\begin{array}{l}\text { lleum, spleen, blood, bone } \\
\text { marrow }\end{array}$ & Duodenum ${ }^{3,4,5}$, (embryonic) spleen ${ }^{3}$, blood ${ }^{2,3,6,7}$, lung $^{2}$ \\
\hline CD3-20E5 ${ }^{+}$NK cells & $\begin{array}{l}\text { lleum, spleen, blood, bone } \\
\text { marrow }\end{array}$ & (Embryonic) spleen ${ }^{8}$, blood ${ }^{2,6,7}$, lung $^{2}$ \\
\hline CD3-CD11b/c NK cells & $\begin{array}{l}\text { lleum, spleen, blood, bone } \\
\text { marrow }\end{array}$ & (Embryonic) spleen ${ }^{8}$ \\
\hline CD107+ NK cells & $\begin{array}{l}\text { lleum, spleen, blood, bone } \\
\text { marrow }\end{array}$ & (Embryonic) spleen ${ }^{8}$, lung ${ }^{2}$, blood ${ }^{2}$ \\
\hline$\gamma \delta \mathrm{T}$ cells & $\begin{array}{l}\text { Adult spleen }{ }^{15} \text {, thymus }{ }^{15} \\
\text { ileum embryonic/ early life }\end{array}$ & $\begin{array}{l}\text { Small intestine } 9,11, \text { caeca }^{10} \text {, spleen } \\
\text { of fabricius }{ }^{13}, \text { skin }^{14}\end{array}$ \\
\hline $\mathrm{CD}^{+} \mathrm{T}$ cell & $\begin{array}{l}\text { Adult spleen }{ }^{15} \text {, thymus }{ }^{15} \text {, } \\
\text { ileum embryonic/ early life }\end{array}$ & $\begin{array}{l}\text { Small intestine }{ }^{11}, \text { duodenum }^{4}, \text { caeca }^{16} \text {, spleen }{ }^{11,16} \text {, blood }{ }^{11} \text {, thymus }{ }^{11} \text {, } \\
\text { bursa of fabricius }{ }^{13}\end{array}$ \\
\hline
\end{tabular}

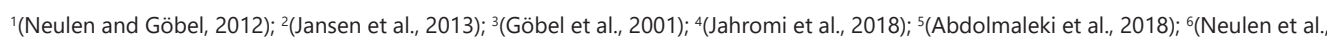
2015); ${ }^{7}\left(\right.$ van der Eijk et al., 2019); ${ }^{8}$ (Jansen et al., 2010); ${ }^{9}$ (Bucy et al., 1988); ${ }^{10}$ (Pieper et al., 2011); ${ }^{11}$ (Tregaskes et al., 1995$) ;{ }^{12}$ (Sowder et al., 1988); ${ }^{13}$ (Kim et al., 2000); ${ }^{14}$ (Sgonc et al., 1996); ${ }^{15}$ (Erf et al., 1998); ${ }^{16}$ (Perumbakkam et al., 2016). 


\section{References}

1. Taha-Abdelaziz K, Hodgins DC, Lammers A, Alkie TN, Sharif S. Effects of early feeding and dietary interventions on development of lymphoid organs and immune competence in neonatal chickens: A review. Vet Immunol Immunopathol. 2018;201:1-11. doi:10.1016/j. vetimm.2018.05.001.

2. Vervelde L, Jeurissen SHM. Postnatal development of intra-epithelial leukocytes in the chicken digestive tract: phenotypical characterization in situ. Cell Tissue Res. 1993;274(2):295-301. doi:10.1007/BF00318748.

3. Casteleyn C, Doom M, Lambrechts E, van den Broeck W, Simoens P, Cornillie P. Locations of gut-associated lymphoid tissue in the 3-month-old chicken: A review. Avian Pathol. 2010;39(3):143-150. doi:10.1080/03079451003786105.

4. Smith AL, Powers C, Beal RK. The Avian Enteric Immune System in Health and Disease. In: Avian Immunology, second ed; 2013:227-250.

5. Schat KA, Wakenell PS. Preparation and characterization of chicken intraepithelial leukocytes. Avian Pathol. 1993;22(3):509-523. doi:10.1080/03079459308418939

6. Cheroutre H, Lambolez F, Mucida D. The light and dark sides of intestinal intraepithelial lymphocytes. Nat Rev Immunol. 2011;11(7):445-456. doi:10.1038/nri3007.

7. Van Kaer L, Olivares-Villagomez D. Development, homeostasis, and functions of intestinal intraepithelial lymphocytes. J Immunol. 2018;200(7):2235-2244. doi:10.4049/jimmunol.1701704.

8. Artis D, Spits H. The biology of innate lymphoid cells. Nature. 2015;517(7534):293-301. doi:10.1038/nature14189.

9. Fuchs $A$, Vermi $W$, Lee JS, et al. Intraepithelial type 1 innate lymphoid cells are a unique subset of il-12- and il-15-responsive ifn- $\gamma$-producing cells. Immunity. 2013;38(4):769-781. doi:10.1016/j.immuni.2013.02.010.

10. Bonanni V, Sciumè G, Santoni A, Bernardini G. Bone marrow NK cells: Origin, distinctive features, and requirements for tissue localization. Front Immunol. 2019;10(JULY). doi:10.3389/fimmu.2019.01569.

11. Lanier LL, Le AM, Civin Cl, Loken MR, Phillips JH. The relationship of CD16 (Leu-11) and Leu-19 (NKH-1) antigen expression on human peripheral blood NK cells and cytotoxic T lymphocytes. J Immunol. 1986;136(12):4480-4486.

12. Cooper MA, Fehniger TA, Caligiuri MA. The biology of human natural killer-cell subsets. Trends in Immunology. 2001;22(11):633640. doi:10.1016/s1471-4906(01)02060-9.

13. Nagler A, Lanier LL, Phillips JH. Constitutive expression of high affinity interleukin 2 receptors on human CD16- natural killer cells in vivo. J Exp Med. 1990;171(5):1527-1533. doi:10.1084/jem.171.5.1527.

14. Duggan MC, Campbell AR, McMichael EL, et al. Co-stimulation of the fc receptor and interleukin-12 receptor on human natural killer cells leads to increased expression of cd25. Oncolmmunology. 2018;7(2).

15. Leong JW, Chase JM, Romee R, et al. Preactivation with IL-12, IL-15, and IL-18 induces cd25 and a functional high-affinity il-2 receptor on human cytokine-induced memory-like natural killer cells. Biol Blood Marrow Transplant. 2014;20(4):463-473. doi:10.1016/j.bbmt.2014.01.006.

16. Rudnicka K, Matusiak A, Chmiela M. CD25 (IL-2R) expression correlates with the target cell induced cytotoxic activity and cytokine secretion in human natural killer cells. Acta Biochim Pol. 2015;62(4):885-894. doi:10.18388/abp.2015_1152.

17. Caligiuri MA. Human natural killer cells. Blood. 2008;112(3):461-469. doi:10.1182/blood-2007-09-077438.

18. Leon F, Roldan E, Sanchez L, Camarero C, Bootello A, Roy G. Human small-iintestinal epithelium contains functional natural killer lymphocytes. Gastroenterology. 2003;125(2):345-356. doi:10.1016/S0016-5085(03)00886-2.

19. Lynch L, O'Donoghue D, Dean J, O'Sullivan J, O'Farrelly C, Golden-Mason L. Detection and characterization of hemopoietic stem cells in the adult human small intestine. J Immunol. 2006;176(9):5199-5204. doi:10.4049/jimmunol.176.9.5199.

20. Ferlazzo G, Thomas D, Lin S-, et al. The Abundant NK Cells in Human Secondary Lymphoid Tissues Require Activation to Express Killer Cell Ig-Like Receptors and Become Cytolytic. J Immunol. 2004;172(3):1455-1462. doi:10.4049/jimmunol.172.3.1455.

21. Farag SS, Caligiuri MA. Human natural killer cell development and biology. Blood Rev. 2006;20(3):123-137. doi:10.1016/ j.blre.2005.10.001.

22. Göbel TWF, Kaspers B, Stangassinger M. NK and T cells constitute two major, functionally distinct intestinal epithelial lymphocyte subsets in the chicken. Int Immunol. 2001;13(6):757-762. doi:10.1093/intimm/13.6.757.

23. Zhang L, Katselis GS, Moore RE, et al. MHC class I target recognition, immunophenotypes and proteomic profiles of natural killer cells within the spleens of day-14 chick embryos. Dev Comp Immunol. 2012;37(3-4):446-456. doi:10.1016/j.dci.2012.03.007.

24. Jansen CA, van de Haar PM, van Haarlem D, et al. Identification of new populations of chicken natural killer (NK) cells. Dev Comp Immunol. 2010;34(7):759-767. doi:10.1016/j.dci.2010.02.009.

25. Alter G, Malenfant JM, Altfeld M. CD107a as a functional marker for the identification of natural killer cell activity. J Immunol Methods. 2004;294(1-2):15-22. doi:10.1016/j.jim.2004.08.008.

26. Jansen CA, De Geus ED, Van Haarlem DA, et al. Differential lung NK cell responses in avian influenza virus infected chickens correlate with pathogenicity. Sci Rep. 2013;3. doi:10.1038/srep02478.

27. Vervelde L, Matthijs MGR, van Haarlem DA, de Wit JJ, Jansen CA. Rapid NK-cell activation in chicken after infection with infectious bronchitis virus M41. Vet Immunol Immunopathol. 2013;151(3-4):337-341. doi:10.1016/j.vetimm.2012.11.012.

28. Jahromi MZ, Bello MB, Abdolmaleki M, Yeap SK, Hair-Bejo M, Omar AR. Differential activation of intraepithelial lymphocytenatural killer cells in chickens infected with very virulent and vaccine strains of infectious bursal disease virus. Dev Comp Immunol. 2018;87:116-123. doi:10.1016/j.dci.2018.06.004.

29. Fenzl L, Göbel TW, Neulen M-. $\gamma \delta T$ cells represent a major spontaneously cytotoxic cell population in the chicken. Dev Comp Immunol. 2017;73:175-183. doi:10.1016/j.dci.2017.03.028. 
30. Wu Z, Rothwell L, Young JR, Kaufman J, Butter C, Kaiser P. Generation and characterization of chicken bone marrow-derived dendritic cells. Immunology. 2010;129(1):133-145. doi:10.1111/j.1365-2567.2009.03129.x.

31. Deeg CA, Degroote RL, Giese IM, et al. CD11d is a novel antigen on chicken leukocytes. J Proteomics. 2020;225. doi:10.1016/ j.jprot.2020.103876.

32. Neulen M-, Göbel TW. Chicken CD56 defines NK cell subsets in embryonic spleen and lung. Dev Comp Immunol. 2012;38(3):410415. doi:10.1016/j.dci.2012.08.001.

33. Ao Z, Kocher A, Choct M. Effects of dietary additives and early feeding on performance, gut development and immune status of broiler chickens challenged with clostridium perfringens. Asian-Australas J Anim Sci. 2012;25(4):541-551. doi:10.5713/ ajas.2011.11378.

34. Rostami F, Ghasemi HA, Taherpour K. Effect of Scrophularia striata and Ferulago angulata, as alternatives to virginiamycin, on growth performance, intestinal microbial population, immune response, and blood constituents of broiler chickens. Poult Sci. 2015;94(9):2202-2209. doi:10.3382/ps/pev198.

35. Bai $\mathrm{SP}, \mathrm{Wu} \mathrm{AM}$, Ding $\mathrm{XM}$, et al. Effects of probiotic-supplemented diets on growth performance and intestinal immune characteristics of broiler chickens. Poult Sci. 2013;92(3):663-670. doi:10.3382/ps.2012-02813.

36. Kogut MH, Genovese KJ, He H, Swaggerty CL, Jiang Y. Modulation of chicken intestinal immune gene expression by small cationic peptides as feed additives during the first week posthatch. Clin Vaccine Immunol. 2013;20(9):1440-1448. doi:10.1128/ CVI.00322-13.

37. De Geus ED, Jansen CA, Vervelde L. Uptake of particulate antigens in a nonmammalian lung: Phenotypic and functional characterization of avian respiratory phagocytes using bacterial or viral antigens. J Immunol. 2012;188(9):4516-4526. doi:10.4049/ jimmunol.1200092.

38. Grégoire C, Chasson L, Luci C, et al. The trafficking of natural killer cells. Immunol Rev. 2007;220(1):169-182. doi:10.1111/j.1600065X.2007.00563.x.

39. Poggi A, Benelli R, Venè R, et al. Human gut-associated natural killer cells in health and disease. Front Immunol. 2019;10(MAY). doi:10.3389/fimmu.2019.00961.

40. Sonnenberg G, Artis D. Innate Lymphoid Cell Interactions with Microbiota: Implications for Intestinal Health and Disease. Immunity. 2012;37(4):601-610. doi:10.1016/j.immuni.2012.10.003.

41. Tregaskes CA, Kong F-, Paramithiotis E, et al. Identification and analysis of the expression of CD8 $\alpha \beta$ and CD8 $\alpha \alpha$ isoforms in chickens reveals a major TCR- $\gamma \delta C D 8 \alpha \beta$ subset of intestinal intraepithelial lymphocytes. J Immunol. 1995;154(9):4485-4494.

42. Bucy RP, Chen C-H, Cihak J, Losch U, Cooper MD. Avian T cells expressing $\gamma \delta$ receptors localize in the splenic sinusoids and the intestinal epithelium. J Immunol. 1988;141(7):2200-2205.

43. Lillehoj HS, Chung KS. Postnatal development of T-lymphocyte subpopulations in the intestinal intraepithelium and lamina propria in chickens. Vet Immunol Immunopathol. 1992;31(3-4):347-360. doi:10.1016/0165-2427(92)90021-H.

44. Sowder JT, Chen C-H, Lanier Ager L, Chan MM, Cooper MD. Large subpopulation of avian T cells express a homologue of the mammalian T $\gamma / \delta$ receptor. J Exp Med. 1988;167(2):315-322. doi:10.1084/jem.167.2.315.

45. Carding SR, Egan PJ. $\gamma \delta$ T cells: Functional plasticity and heterogeneity. Nat Rev Immunol. 2002;2(5):336-345. doi:10.1038/nri797.

46. Haas W, Pereira P, Tonegawa S. Gamma/delta cells. Vol 11. ; 1993. Accessed 15 July 2020.

47. Mackay $C R$, Hein WR. A large proportion of bovine $T$ cells express the $\gamma \delta T$ cell receptor and show a distinct tissue distribution and surface phenotype. Int Immunol. 1989;1(5):540-545. doi:10.1093/intimm/1.5.540.

48. Piriou-Guzylack L, Salmon H. Membrane markers of the immune cells in swine: An update. Vet Res. 2008;39(6). doi:10.1051/ vetres:2008030.

49. Shekhar S, Milling S, Yang X. Migration of $\gamma \delta$ T cells in steady-state conditions. Vet Immunol Immunopathol. 2012;147(1-2):1-5. doi:10.1016/j.vetimm.2012.03.016.

50. Mayassi T, Jabri B. Human intraepithelial lymphocytes. Mucosal Immunol. 2018;11(5):1281-1289. doi:10.1038/s41385-018-0016-5.

51. Walker LJ, Marrinan E, Muenchhoff M, et al. CD8aa expression marks terminally differentiated human CD8+ T cells expanded in chronic viral infection. Front Immunol. 2013;4(AUG). doi:10.3389/fimmu.2013.00223.

52. Perumbakkam S, Hunt HD, Cheng HH. Differences in CD8aa and cecal microbiome community during proliferation and late cytolytic phases of Marek's disease virus infection are associated with genetic resistance to Marek's disease. FEMS Microbiol Ecol. 2016;92(12). doi:10.1093/femsec-fiw188.

53. Pieper J, Methner U, Berndt A. Characterization of avian $\gamma \delta$ T-cell subsets after Salmonella enterica serovar typhimurium infection of chicks. Infect Immun. 2011;79(2):822-829. doi:10.1128/IAI.00788-10.

54. VanKaer L, Algood HSC, Singh K, et al. CD8 $\alpha \alpha+$ innate-type lymphocytes in the intestinal epithelium mediate mucosal immunity. Immunity. 2014;41(3):451-464. doi:10.1016/j.immuni.2014.08.010. 

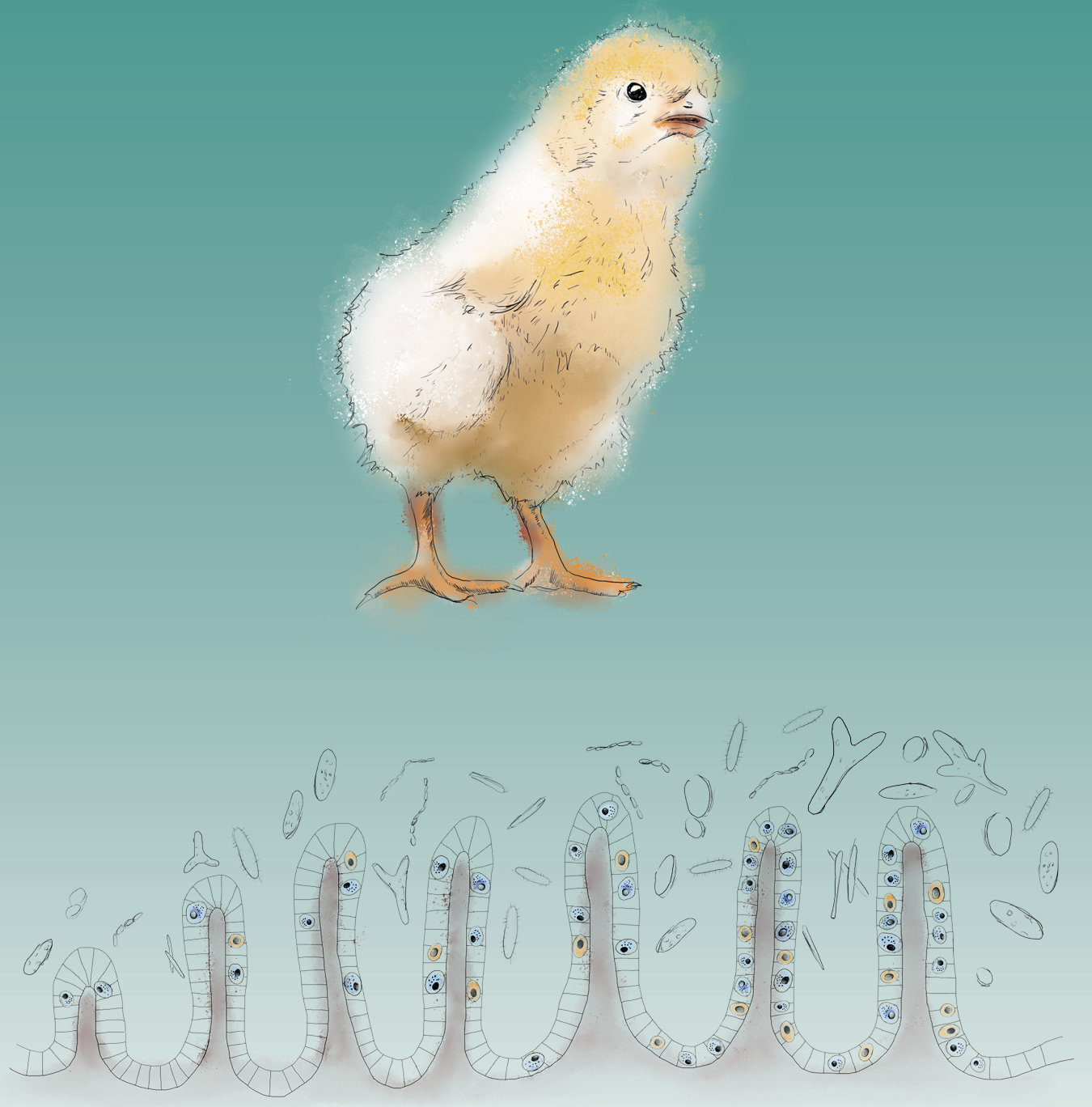


\section{Early life inoculation with adult-derived microbiota accelerates maturation of intestinal microbiota and enhances NK cell activation in broiler chickens}

Nathalie Meijerink ${ }^{1 *}$, Jannigje G. Kers ${ }^{2,3^{*}}$, Francisca C. Velkers², Daphne A. van Haarlem¹, David M. Lamot ${ }^{4}$, Jean E. de Oliveira ${ }^{5}$, Hauke Smidt ${ }^{3}$, J. Arjan Stegeman ${ }^{2}$, Victor P.M.G. Rutten ${ }^{1,6}$, Christine A. Jansen ${ }^{1}$

1 Department Biomolecular Health Sciences, Division Infectious Diseases and Immunology, Faculty of Veterinary Medicine, Utrecht University, Utrecht, the Netherlands

2 Department Population Health Sciences, Division Farm Animal Health, Faculty of Veterinary Medicine, Utrecht University, Utrecht, the Netherlands

3 Laboratory of Microbiology, Wageningen University \& Research, Wageningen, the Netherlands

${ }^{4}$ Cargill Animal Nutrition and Health Innovation Center, Velddriel, the Netherlands

5 Cargill R\&D Center Europe, Vilvoorde, Belgium

6 Department of Veterinary Tropical Diseases, Faculty of Veterinary Science, University of Pretoria, Pretoria, South Africa

* Contributed equally to this work 


\section{Abstract}

Studies in mammals, including chickens, have shown that the development of the immune system is affected by interactions with intestinal microbiota. Early life microbial colonization may affect the development of innate and adaptive immunity and may contribute to lasting effects on health and resilience of broiler chickens. We inoculated broiler chickens with adult-derived-microbiota (AM) to investigate their effects on intestinal microbiota composition and natural killer (NK) cells, amongst other immune cells. We hypothesized that AM inoculation directly upon hatch (day 0 ) would induce an alteration in microbiota composition shortly after hatch, and subsequently affect (subsets of) intestinal NK cells and their activation. Microbiota composition of caecal and ileal content of chickens of 1,3 , 7, 14, 21 and 35 days of age was assessed by sequencing of 16S ribosomal RNA gene amplicons. In parallel, subsets and activation of intestinal NK cells were analyzed by flow cytometry.

In caecal content of 1- and 3-day-old AM chickens, a higher alpha-diversity (Faith's phylogenetic diversity) was observed compared to control chickens, whereas ileal microbiota were unaffected. Regarding beta-diversity, caecal microbiota profiles could be clustered into three distinct community types. Cluster A represented caecal microbiota of 1-day-old AM chickens and 1- and 3-day-old control chickens. Cluster B included microbiota of seven of eight 3- and 7-day-old AM and 7-day-old control chickens, and cluster C comprised microbiota of all chickens of 14-days and older, independent of inoculation. In 3-day-old AM chickens an increase in the percentages of intestinal IL-2R $\alpha^{+} \mathrm{NK}$ cells and activated NK cells was observed compared to control chickens of the same age. In addition, an increase in relative numbers of intestinal cytotoxic CD8 $\alpha \alpha^{+}$T cells was observed in 14- and 21-day-old AM chickens. Taken together, these results indicate that early exposure to AM shapes and accelerates the maturation of caecal microbiota, which is paralleled by an increase in IL-2R $\alpha^{+} N K$ cells and enhanced NK cell activation. The observed association between early life development of intestinal microbiota and immune system indicates possibilities to apply microbiota-targeted strategies that can accelerate maturation of intestinal microbiota and strengthen the immune system, thereby improving the health and resilience of broiler chickens.

Keywords: poultry, avian immunology, intestinal microbiota, intraepithelial lymphocytes, innate immunity, NK cells 


\section{Introduction}

Health and production efficiency of broiler chickens are of major importance, as chicken meat is a key sustainable source of animal protein for the growing human population ${ }^{1,2}$. In poultry production, restrictions of the use of antimicrobials have made other strategies to maintain or improve poultry health, such as enhanced immune responsiveness, increasingly important.

A crucial role in chicken health and production performance is played in many physiological processes by intestinal microbiota, including nutrient digestion and absorption, metabolism, intestinal barrier function, and development of intestinal immunity ${ }^{3,4}$. The maturation of the intestinal microbiota of chickens entails rapid successional changes, developing from a simple, to a more complex and diverse composition due to gradual colonization with microbiota ${ }^{5-7}$. Early life exposure to microbiota is an important driver of this development, which can also affect health later in life. This has been shown in human infants ${ }^{8-10}$, and other mammals and hatchlings treated with antibiotics early in life or raised under extreme hygienic conditions, e.g. germ-free or SPF environments ${ }^{11-15}$. Also, in commercial chickens under normal circumstances, early transiently colonizing bacteria have been shown to have a large effect on intestinal microbiota composition later in life ${ }^{16-18}$. However, due to hatching in a hatchery environment, colonization in commercial chickens starts with microbiota from environmental, rather than parental sources. As these environmental microorganisms may include pathogenic bacteria, competitive exclusion products derived from intestinal microbiota of healthy adult chickens have been developed to compete with colonization by pathogenic bacteria and are widely used in poulty production systems to induce a healthy microbiota ${ }^{19}$. When supplied in ovo or to hatchlings, adult-derived microbiota has been shown to accelerate bacterial colonization ${ }^{20-22}$ and to decrease the occurrence of undesirable bacteria such as Salmonella and Escherichia coli19,23,24.

The intestinal immune system plays an important role in the defense against pathogens that enter a host via the gut. Underneath the mucus layer (the first protective barrier in the intestinal $\operatorname{tract}^{25}$ ), a layer of epithelial cells including immune cells such as the intraepithelial lymphocytes (IEL) is observed. The population of IEL consists of high numbers of $\gamma \delta$ T cells, adaptive CD8 ${ }^{+} T$ cells and innate natural killer (NK) cells $^{26}$. During embryonic development and early life, when resistance against pathogens relies on innate immune responses since the adaptive immune system is not yet fully developed, NK cells are important players ${ }^{27,28}$. Chicken NK cells have also been reported in multiple organs including the intestine, lung, spleen and blood ${ }^{26,29,30}$. Previously, we and others showed that a high percentage of intestinal NK cells in chickens are recognized by the marker 28-426,31, which was identified as CD25 or IL-2R ${ }^{26}$. In mammals, the IL-2R $\alpha$ chain is expressed on NK cells early upon activation ${ }^{32}$, and this is followed by enhanced NK cell mediated killing and IFN $\gamma$ production ${ }^{32}$. Another marker found to be expressed on intestinal NK cells was $20 \mathrm{E} 5^{31}$. It is also expressed on cells that show NK cell activation ${ }^{29}$. Furthermore, elsewhere in the body, increased surface expression of CD107 indicative of NK cell activation was observed on primary chicken NK cells in lung, spleen and blood upon infections with avian viruses ${ }^{30,33,34}$.

In the intestinal tract many interactions occur between the microbiota and immune cells ${ }^{35,36}$. These interactions are important for the development of the immune system, as was shown in mammals ${ }^{21,37,38}$ and chickens ${ }^{14,39}$. For example, early life transplantation of adult microbiota has resulted in increased natural antibody titers in laying chickens ${ }^{40}$ paralleled by long lasting effects on mRNA levels of pro-inflammatory cytokines ${ }^{41}$. Disturbing the early life microbiota in 1-day-old broiler chickens by antibiotics resulted in reduced numbers of macrophage-like cells in the jejunum ${ }^{14}$, whereas differences in rearing environment, e.g., a reduction in environmental microbial exposure resulted, in two phylogenetically distinct lines of broiler chickens, in lower expression levels of $\beta$-defensins ${ }^{42}$. 
Studies in rodents and humans have shown that specific probiotic microorganisms enhance intestinal NK cell activity and cytokine production ${ }^{43}$ either directly via their interaction with receptors expressed on NK cells $s^{44,45}$, or indirectly via cytokine production of resident myeloid or epithelial cell ${ }^{46}$. Also the adaptive immune system can be modulated via interactions with the microbiota ${ }^{47-50}$, or indirectly through innate immune cell activities. As other studies in rodents and humans have shown, the microbiota affects activation of $\gamma \delta T$ cells $5^{51,52}$ and $C D 8^{+} T$ cells ${ }^{53}$. Taken together, this indicates that the composition and activity of the microbiota and its effects on the immune system in early life may have long term consequences on the health of individuals.

In chickens, previous studies addressed the effect of microbiota on innate immune responses in the intestine, spleen and blood by studying mRNA levels of immune related genes ${ }^{41,42}$, by immunohistochemistry ${ }^{14}$ and by analysis of natural antibody titers ${ }^{40}$. In this study, we used tools that we developed previously for the analysis of the phenotype and the function of chicken innate immune cells ${ }^{29,54}$ to assess whether and to what extent differences in early life microbial colonization would affect the development of NK cells locally (in the intestine) and systemically (in spleen and blood).

We hypothesized that inoculation with adult-derived microbiota (AM) upon hatch would induce an alteration in microbiota development and affect the presence and activation of intestinal NK cells. To induce early colonization with a rich, complex microbiota to stimulate immune development, we used Aviguard $^{\oplus}$ (MSD Animal Health, the Netherlands), as this product derived from microbiota of healthy adult chickens has been shown to be able to colonize the intestinal tract and induce early maturation of the intestinal microbiota in previous studies with hatchlings $\mathrm{s}^{22,55}$. In this study, AM inoculation resulted in an accelerated maturation of the intestinal microbiota, an increase of IL-2R $\alpha^{+} \mathrm{NK}$ cells and enhanced activation of NK cells. The observed association between early life development of intestinal microbiota and the immune system indicates possibilities to apply microbiota-targeted strategies that can accelerate maturation of intestinal microbiota and strengthen the immune system to improve the health and resilience of broiler chickens.

\section{Materials and methods}

\section{Birds and husbandry}

Ross 308 broiler 17- and 18-day old embryonated eggs were obtained from the same parent flock of a commercial hatchery (Lagerwey, the Netherlands). ED17 (hatch group A, n = 52) and ED18 eggs (hatch group $B, n=52$ ) were disinfected with $3 \%$ hydrogen peroxide and placed in disinfected egg hatchers. All eggs hatched at ED21. Directly upon hatch, chickens (day 0 in age) were randomly divided into two treatment groups, weighed, labelled and inoculated. Next, the chickens of the two treatment groups were placed in separate floor pens of $2 \times 1.5 \mathrm{~m}$ (pens 1 and 2), with a solid wall separating the pens. Each pen was divided in two equal parts of $1 \times 1.5 \mathrm{~m}$ for chickens from hatch group $\mathrm{A}$ and $B$. The pens were lined with wood shavings $\left(2 \mathrm{~kg} / \mathrm{m}^{2}\right.$, sterilized by autoclavation). Non-sterilized standard commercial starter and grower feeds (Research Diet Services, the Netherlands) and water was provided ad libitum. No antibiotics, coccidiostatic drugs or commercial vaccines were applied during the experiment. A standard lighting, temperature scheme for Ross broiler chickens was used, and conditions were kept the same for all compartments. The chickens were observed daily for clinical signs, abnormal behavior or mortality and were also evaluated for presence of abnormalities during post-mortem. No signs of disease or impaired health were observed in both groups throughout the experiment. Feed intake and body weight were assessed in both groups at each sampling moment 
and followed the expectations based on the Ross 308 broiler performance standards in both groups. The experimental room was equipped with a mechanical negative pressure ventilation system.

The animal experiment was approved by the Dutch Central Authority for Scientific Procedures on Animals and the Animal Experiments Committee (registration number AVD1080020174425) of Utrecht University (the Netherlands) and all procedures were done in full compliance with all relevant legislation.

\section{Experimental design}

Chickens were inoculated once immediately after hatch to reduce opportunities for prior exposure to microbiota. First, the control group received an oral inoculation with $0.5 \mathrm{ml}$ PBS (Lonza, Basel, Switzerland). The other group, henceforth referred to as the AM group, was inoculated with $0.5 \mathrm{ml}$ of PBS containing $0.05 \mathrm{~g} / \mathrm{ml}$ of competitive exclusion product Aviguard ${ }^{\oplus}$ (MSD Animal Health, the Netherlands). This is a freeze-dried powder, soluble in water, consisting of fermented, undefined cultures from intestinal microbiota of healthy specific-pathogen-free birds and was used according to manufacturer's instructions. To determine the microbial composition of the AM inoculum and compare this to the microbiota in the chickens, four aliquots of $2 \mathrm{ml}$ were stored at $-80^{\circ} \mathrm{C}$ for DNA extraction. The experimental design of the study is shown in Fig. S1.

\section{Sample collection}

At day 0 (upon hatch), four non-inoculated chickens per hatch group were randomly selected and sacrificed, to collect caecal and ileal content for microbiota analyses, as has been described in ${ }^{56}$. lleal content was collected distal and close to the Meckel's diverticulum. The intestinal content was gently squeezed into a $2 \mathrm{ml}$ sterile cryotube, snap frozen on dry ice and stored at $-80^{\circ} \mathrm{C}$ for DNA extraction. The time between sacrificing and placing the intestinal samples on dry ice was between 3-5 min. To avoid cross contamination, all management and biotechnical procedures were completed first with the control group and for each compartment at the same time. At days 1 (24 hours after inoculation), 3, 7, 14, 21 and 35, eight chickens (four from the control and four from the AM group) were randomly selected per hatch group (A/B) and sacrificed to collect caecal and ileal content as described above. At day 0 and day 1 , the chickens were too small to collect sufficient cells for immunological analyses. Therefore ileum tissue, spleen and blood were collected from day 3 onwards from six of these eight chickens ( $n=3$ per hatch group). All chickens were weighed prior to post-mortem analyses.

\section{DNA extraction}

In total, 104 caecal and 104 ileal content samples, consisting of 52 samples per treatment group, and four samples of the AM inoculum were analyzed for microbiota composition. DNA was extracted from $0.25 \mathrm{~g}$ content, using $700 \mu \mathrm{l}$ of Stool Transport and Recovery (STAR) buffer (Roche Diagnostics Nederland BV, the Netherlands). All samples were transferred to sterile screw-capped $2 \mathrm{ml}$ tubes (BIOplastics BV, the Netherlands) containing $0.5 \mathrm{~g}$ of zirconium beads $(0.1 \mathrm{~mm}$; BioSpec Products, Inc., USA) and 5 glass beads ( $2.5 \mathrm{~mm}$; BioSpec Products). All samples were treated in a bead beater (Precellys 24, Bertin technologies, France) at a speed of $5.5 \mathrm{~m} / \mathrm{s}$ for $3 \times 1 \mathrm{~min}$, followed by incubation at $95^{\circ} \mathrm{C}$ with agitation (15 min and $300 \mathrm{rpm}$ ). The lysis tube was centrifuged $\left(13,000 \mathrm{~g}\right.$ for $5 \mathrm{~min}$ at $4^{\circ} \mathrm{C}$ ), and the supernatant was transferred to a $2 \mathrm{ml}$ microcentrifuge tube. Thereafter, the above-described process was repeated with $300 \mu \mathrm{I}$ STAR buffer. An aliquot $(250 \mu \mathrm{l})$ of the combined supernatants from the sample lysis was then transferred into the custom Maxwell ${ }^{\circledR} 16$ Tissue LEV Total RNA Purification Kit cartridge. The remainder of the extraction protocol was then carried out in the Maxwell ${ }^{\circledast} 16$ Instrument 
(Promega, the Netherlands) according to the manufacturer's instructions. DNA concentrations were measured with a NanoDrop ND-1000 spectrophotometer (NanoDrop ${ }^{\circledR}$ Technologies, DE, USA), and the DNA samples were stored at $-20^{\circ} \mathrm{C}$ until further use.

\section{qPCR, 16S rRNA gene amplification, sequencing and data processing}

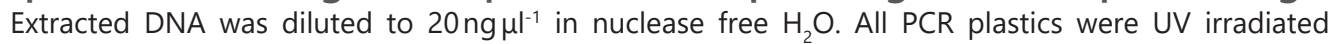
for $15 \mathrm{~min}$ before use. To validate the AM inoculation, absolute quantification of the bacterial $16 \mathrm{~S}$ ribosomal RNA (rRNA) genes by real-time PCR amplification was performed for the caecal content samples of day-old chickens. For ileal content samples the amount of DNA was too low to reliably determine gene copy numbers. All qPCR assays (CFX384 ${ }^{\mathrm{TM}}$ real-time PCR detection system, Bio-Rad, CA, USA) were performed in triplicate with $25 \mu \mathrm{l}$ reactions and was described previously ${ }^{57}$. For $16 \mathrm{~S}$ rRNA gene-based microbial composition profiling, barcoded amplicons covering the variable regions V5-V6 of the bacterial 16S rRNA gene were generated by PCR using the 784F and 1064R primers as described before ${ }^{58}$. Each sample was amplified in duplicate using Phusion hot start II high fidelity polymerase (Finnzymes, Finland), checked for correct size and concentration on a $1 \%$ agarose gel and subsequently combined and purified using CleanNA magnetic beads (CleanNA, the Netherlands). A detailed description of the PCR conditions is given elsewhere ${ }^{56}$. Positive and negative controls were added to the data set to ensure high quality sequencing data. As positive controls we used synthetic mock communities of known composition ${ }^{58}$, and as negative controls we used nuclease free water. The resulting libraries were sent to Eurofins Genomics GmbH (Germany) for sequencing on an Illumina Hiseq2500 instrument. The $16 \mathrm{~S}$ rRNA data was analyzed using NG-tax 2.059. In short, paired-end libraries were filtered to contain only read pairs with a perfect match to the primers and perfectly matching barcodes, to demultiplex reads by sample. Amplicon sequence variants (ASVs) were defined as unique sequences. The ASV picking strategy was based on a de novo reference approach. Taxonomy was assigned using the SILVA 128 16S rRNA gene reference database ${ }^{60}$. Caecal content samples of day 0 and ileal content samples of day 0 and 1 were excluded from the analysis, because these contained a large number of families associated with the negative control samples, and therefore did not pass our quality control standards. Raw sequence data were deposited into the Sequence Read Archive (SRA) at NCBI under accession number PRJNA670739.

\section{Isolation of tissues and cells}

lleum segments ( $\pm 10 \mathrm{~cm}$ distal from Meckel's diverticulum), spleens and blood (5 ml) were collected. lleum segments were washed with PBS to remove contents and cut in sections of $1 \mathrm{~cm}^{2}$ and washed again. Subsequently, IELs were collected by incubating three times in EDTA-medium [HBSS 1× (Gibco BRL, United Kingdom) supplemented with 10\% heat-inactivated FCS (Lonza) and 1\% 0.5M EDTA (Sigma-Aldrich)] at $200 \mathrm{rpm}$ for $15 \mathrm{~min}$ at $37^{\circ} \mathrm{C}$. Supernatants were collected and centrifuged for $5 \mathrm{~min}$ at $1200 \mathrm{rpm}$ at $20^{\circ} \mathrm{C}$. Cells were then resuspended in PBS, lymphocytes were isolated using Ficoll-Paque Plus (GE Healthcare, the Netherlands) density gradient centrifugation for 12 min at 1700 rpm, washed in PBS using centrifugation for $5 \mathrm{~min}$ at $1300 \mathrm{rpm}$ and resuspended at $4.0 \times 10^{6}$ cells/ $\mathrm{ml}$ in NK medium [IMDM 2 mM glutamax I (Gibco BRL) supplemented with $8 \%$ heat-inactivated FCS (Lonza), 2\% heat-inactivated chicken serum and $100 \mathrm{U} / \mathrm{ml}$ penicillin/streptomycin (Gibco BRL)]. Spleens were homogenized using a $70 \mu \mathrm{m}$ cell strainer (Beckton Dickinson (BD) Biosciences, NJ, USA) to obtain a single cell suspension. Next, lymphocytes in spleen and blood were isolated by Ficoll-Paque density gradient centrifugation (20 min at $2200 \mathrm{rpm}$ ), washed in PBS and resuspended at $4.0 \times 10^{6} \mathrm{cells} / \mathrm{ml}$ in NK medium as described for ileum. 


\section{Flow cytometry}

Presence and activation of NK and T cell subsets were determined in IEL, spleen and blood. Unless described otherwise, all antibodies were obtained from Southern Biotech (AL, USA). Markers known to be expressed on chicken NK cells (hybridomas provided by Göbel, T.W., Ludwig Maximilians University, Germany), such as mouse-anti-chicken-28-4 (IL-2R $;$; IgG3) and -20E5-BIOT (IgG1) were co-stained with mouse-anti-chicken-CD45-FITC (IgM) and -CD3-APC (CT3; IgG1) mAb to exclude T cells. The T cell panel included the following markers: mouse-anti-chicken-CD3-PE (CT3; IgG1), -CD4-APC (CT4; IgG1),

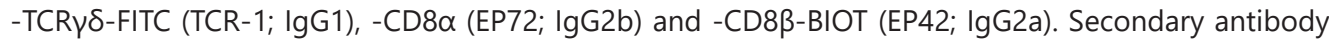
staining was performed using goat-anti-mouse-lgG3-PE and streptavidin (SA)-PercP (BD Biosciences) in the NK cell panel, and goat-anti-mouse-IgG2b-APC/Cy7 and SA-PercP in the T cell panel. To asses CD107 expression on NK cells, lymphocytes were washed in PBA and stained with mouse-anti-chicken-CD3-PE, -TCRYס-BIOT (TCR-1; IgG1), -28-4 and -CD41/61-FITC (11C3; IgG1, Serotec) to exclude thrombocytes from analysis. Secondary antibody staining was performed using SA-PercP and goat-anti-mouse-lgG3-APC/Cy7. All staining procedures were incubated for 20 min at $4^{\circ} \mathrm{C}$ in the dark, washed in PBA and subsequently stained with a live/dead marker (Zombie Aqua ${ }^{\mathrm{TM}}$ Fixable Viability Kit, Biolegend, CA, USA) for $15 \mathrm{~min}$ at room temperature (RT) in the dark to exclude dead cells. Finally, lymphocytes were fixed using 2\% paraformaldehyde (Merck, Germany) for 10 min at RT, washed and resuspended in $200 \mu \mathrm{l}$ PBA. Fluorescence of cells was assessed in $150 \mu \mathrm{l}$ or 50,000 lymphocytes in the live gate using a FACSCANTO II Flowcytometer (BD Biosciences), and data was analyzed with software program FlowJo (Tree star Inc, OR, USA).

\section{NK cell activation assay}

NK cell activation was determined using the CD107-assay, which measures increased surface expression of CD107 as a result of degranulation; the release of perforin and granzymes ${ }^{29}$. Briefly, lymphocytes isolated from IEL, spleen and blood were resuspended in NK medium, and $1 \times 10^{6}$ lymphocytes per sample were used. Lymphocytes were cultured in presence of $1 \mu \mathrm{l} / \mathrm{ml}$ Golgistop (BD Biosciences) and mouse-anti-chicken-CD107-APC mAb (5G10; IgG1, hybridomas provided by Göbel) during 4 hours at $37^{\circ} \mathrm{C}, 5 \% \mathrm{CO}_{2}$. Next, cells were washed, stained with monoclonal antibodies and analyzed as described in section 'Flow cytometry'.

\section{Data analysis}

Statistical analyses for microbiota and the relation between microbiota and the immune system were performed in R version 3 (R Foundation for Statistical Computing, Austria), using the packages Phyloseq, Microbiome, Vegan and DirichletMultinomial ${ }^{61-64}$. A Kruskal-Wallis test was used to test for difference in 16S rRNA gene counts in caecal content of day-old chickens between treatment groups. Alpha and beta diversity metrics and multivariate statistical analyses were applied to determine differences in the measured intestinal microbiota between the two treatment groups and with age. The alpha diversity (within sample) data was determined using Faith's phylogenetic diversity. Faith's phylogenetic diversity not only takes the number of different taxa (ASVs) into account, but also the phylogenetic relatedness of these taxa ${ }^{65}$. To test for differences in relative abundance of genera between treatment groups, we used a Wilcoxon rank-sum test and corrected for multiple comparisons using the Benjamini-Hochberg $(\mathrm{BH})$ procedure. The beta diversity (between samples) was determined using weighted and unweighted UniFrac metrics ${ }^{66}$. Multivariate microbiota data were visualized using principal coordinates analysis (PCoA, multidimensional scaling method), and non-parametric permutational analysis of variance (PERMANOVA) tests were used to analyze group differences within multivariate community data ${ }^{67}$. 
To assess whether the development of the microbiota proceeded through different stages of maturation in the two treatment groups, Dirichlet Multinomial Mixtures (DMM) modeling was applied, using a probabilistic model, to identify possible clusters (types) of microbial composition 16S rRNA gene sequence data ${ }^{68}$ based on the relative abundance of the microbial groups at genus level. Two separate DMM models were used to study clustering of the microbiota data of the caecal content and ileal content separately. Next, to test whether the observed differences in the microbial development between treatments were associated with differences in immune development, Wilcoxon rank-sum test, corrected for multiple comparisons using $\mathrm{BH}$, was used to test for associations between the identified DMM clusters of microbial composition and immunological parameters. As ileal microbiota clustering did not indicate differences in microbial development between treatments, only the clusters identified for the caecal microbiota profiles were used. Associations were tested for a subset of immunological parameters that showed differences between AM and control chickens of the same age. Furthermore, parameters with fewer than four observations per treatment group and day of age were omitted. The final selection of parameters included percentages and absolute numbers of intestinal IL-2R $\alpha^{+}, 20 \mathrm{E5}{ }^{+}$ and $\mathrm{CD} 107^{+} \mathrm{NK}$ cells, and CD8$\alpha \alpha^{+} \mathrm{T}$ cells.

Statistical analyses for the immunological parameters were done with GraphPad Prism 7.0 software (GraphPad Software Inc., USA), using the Mann-Whitney U-test to test differences between treatment groups at a specific day of age. A $p$-value of $<0.05$ was considered statistically significant.

\section{Results}

\section{AM treatment influences the composition and development of the intestinal microbiota in newly hatched chickens}

The total bacterial 16S rRNA gene copy numbers 24 hours after inoculation were significantly higher in caecal content samples at day 1 in AM inoculated compared to control chickens, indicating the presence of a higher quantity of bacteria after inoculation with AM (Fig. S2).

To investigate the effect of $\mathrm{AM}$ inoculation on the microbiota composition at different ages in the broiler chickens, alpha and beta diversities, as well as differences in relative abundance of individual microbial taxa, were assessed. The phylogenetic diversity metric, providing information on the number as well as phylogenetic relatedness of observed microbial taxa at the ASV level, was used as an alpha diversity measure to determine differences between $A M$ and control chickens. The phylogenetic diversity of the caecal content was higher in 1- and 3-day-old AM chickens compared to controls, but not for any of the other ages (Fig. 1A). In contrast, the phylogenetic diversity of ileal content microbiota did not differ between treatment groups at any age (Fig. 1B).

Beta diversity, i.e., the similarity in composition between samples, was determined using the weighted and unweighted UniFrac distance metrics to determine the influence of age and treatment on the composition. Two dimensional visualization of the caecal content microbiota profiles in PCoA plots placed 3- and 7-day-old AM inoculated chickens closely together, indicating high similarity in microbiota composition between these age groups (Fig. 2). PERMANOVA of caecal content microbiota showed that treatment explained $6-9 \%$ of the variation in caecal microbiota composition between samples ( $p<5$-04; unweighted UniFrac, $p<2 \mathrm{e}-04$; weighted UniFrac), whereas age explained $49-41 \%$ of the variation between samples ( $p<5$ e-04; unweighted UniFrac, $p<2 \mathrm{e}-04$; weighted UniFrac). PERMANOVA of ileal content samples showed that treatment explained $4 \%$ of the variation 
in ileal microbiota composition based on unweighted UniFrac, whereas treatment did not significantly contribute to explaining the observed variation using the weighted UniFrac distance metrics $(p=0.038$; unweighted UniFrac, $p=0.355$; weighted UniFrac, Fig. 2B), indicating that differences in microbial profiles of ileal samples between treatment groups concerned mostly the presence/absence of taxa occurring at low relative abundance. Age explained $29-24 \%$ of the variation between the ileal content samples ( $p<1 \mathrm{e}-04$; unweighted UniFrac, $p<1 \mathrm{e}-04$; weighted UniFrac).

In the AM inoculum 24 different genera were detected, for which the relative abundances in caecal and ileal samples were compared between AM and control chickens. A higher relative abundance in caecal content of AM chickens compared to controls was found for ten of these 24 genera at day 1, five on day 3, four at day 7 and two at day 14 and 21. At day 35 none of these genera differed in relative abundance between $A M$ and control chickens (Table 1). This indicates that AM inoculation had an impact on the relative abundance of genera at an early age, but did not permanently influence the relative abundance of these genera in the caecal content samples. For ileal content, no differences in the relative abundances of the 24 genera of the inoculum were observed at any of the different ages (data not shown).
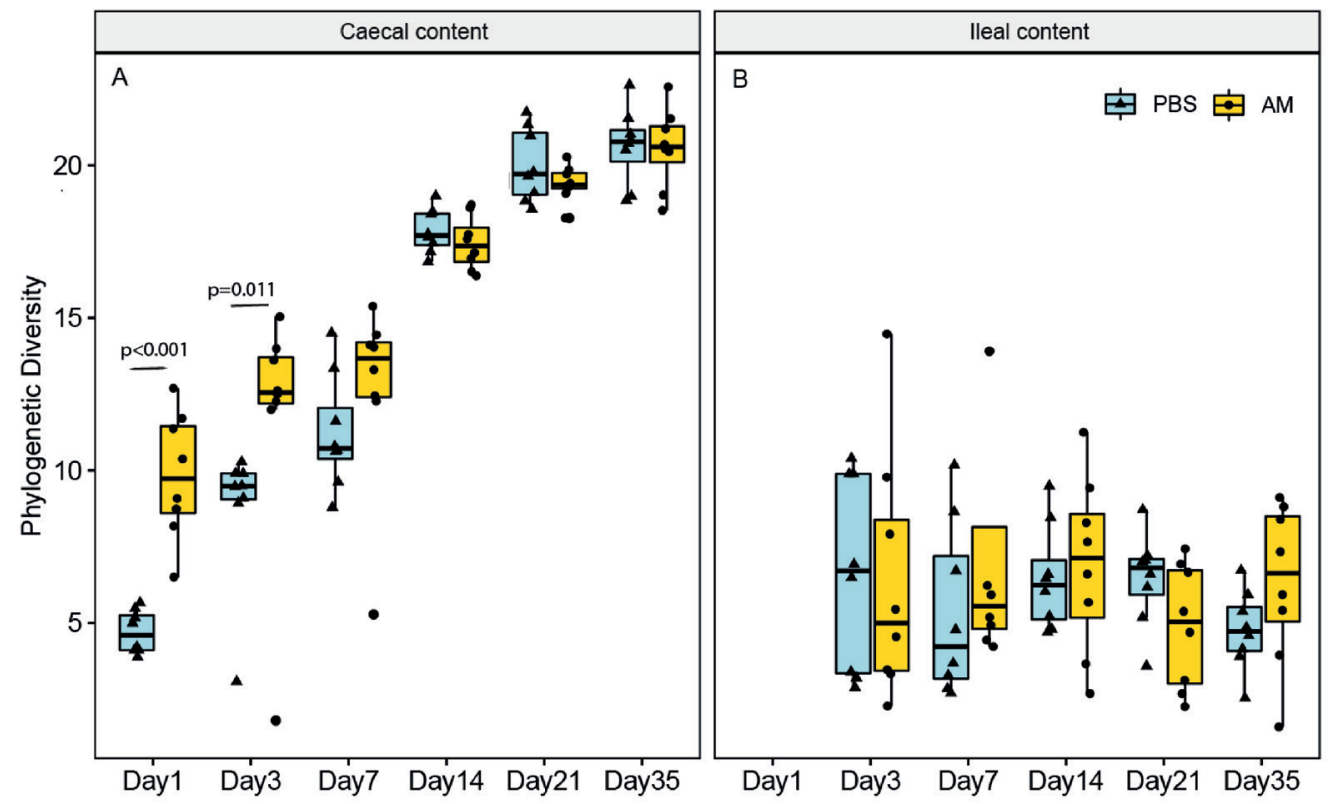

Figure 1. Phylogenetic diversity of the caecal and ileal content microbiota at different ages. (A) The phylogenetic diversity (alpha diversity, at ASV level) was only significantly higher in the caecal content of AM chickens compared to controls on day 1 and day 3 ( $p$ $<0.05$ ). (B) In the ileal content microbiota no differences were observed at any of the ages. $n=8$ chickens per treatment per day of age, whiskers show $95 \%$ interval, box $50 \%$ interval. 


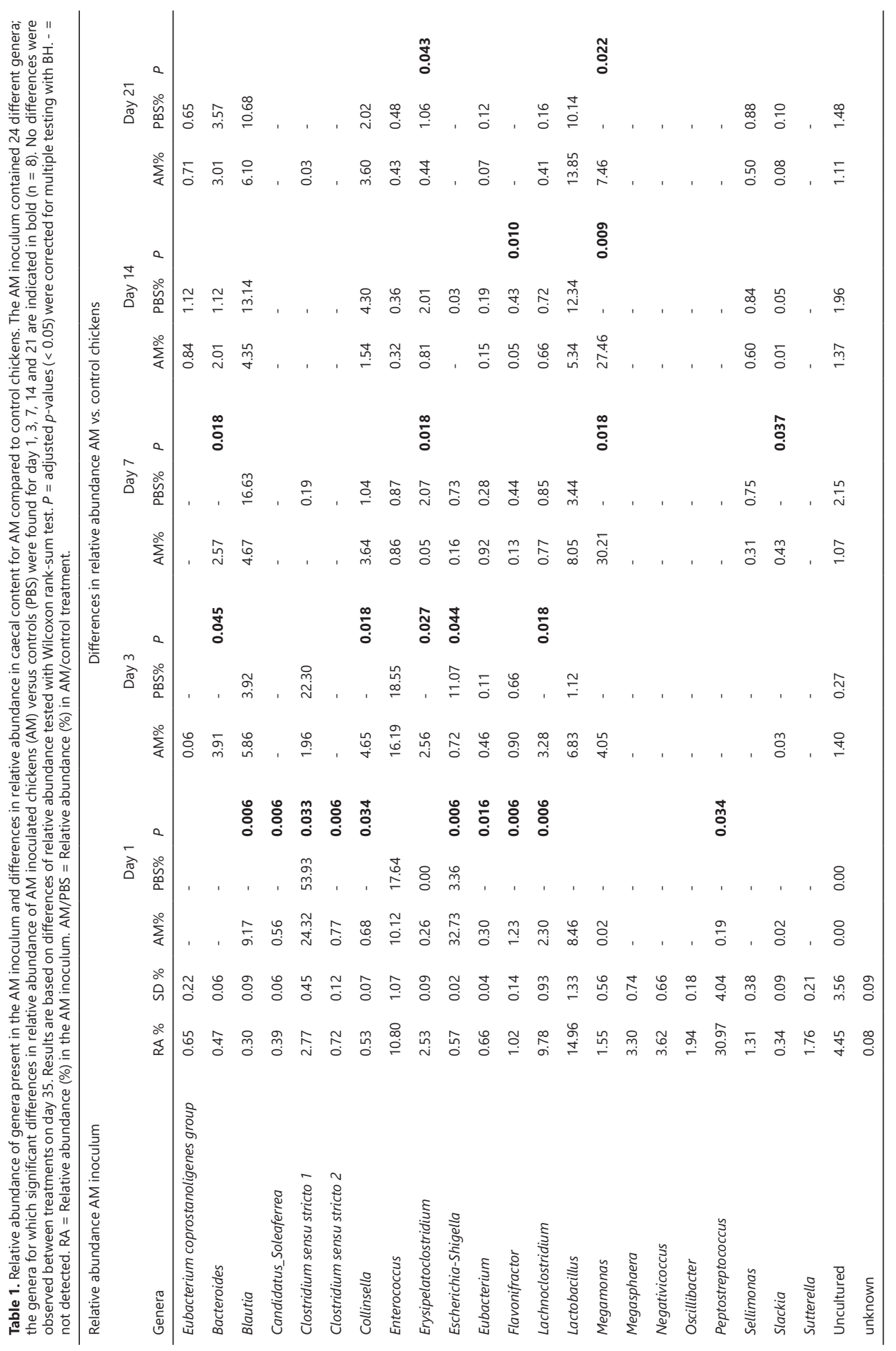




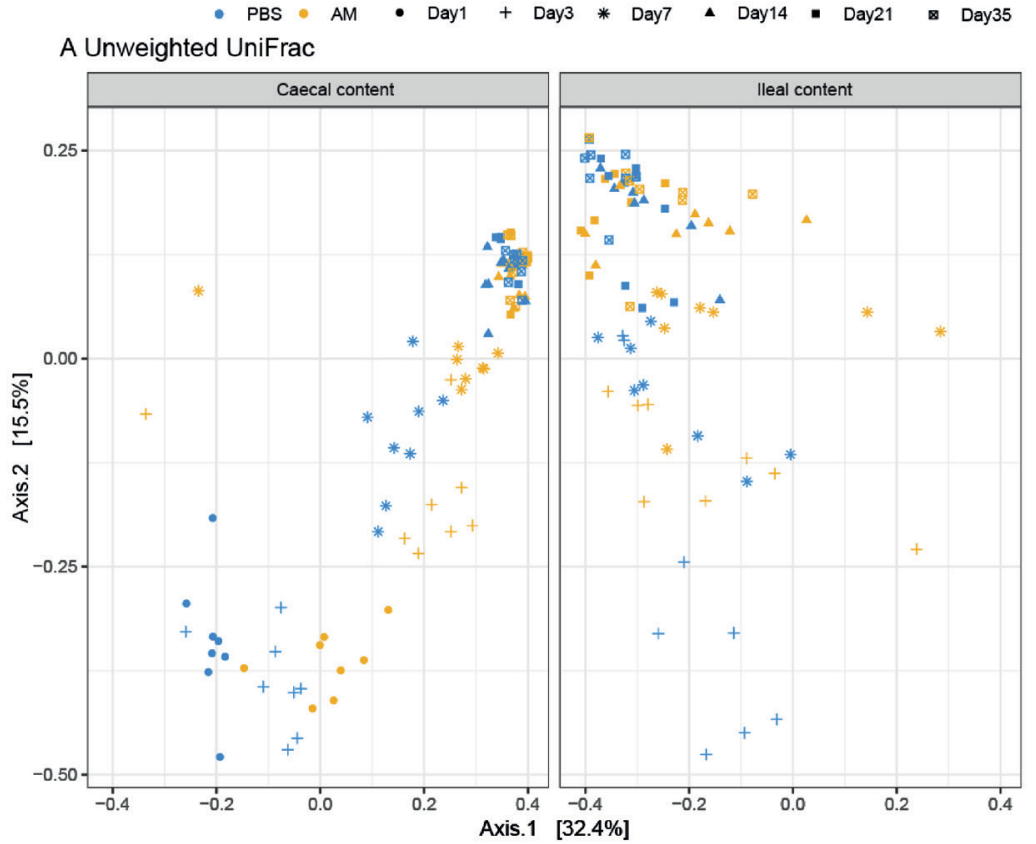

B Weighted UniFrac

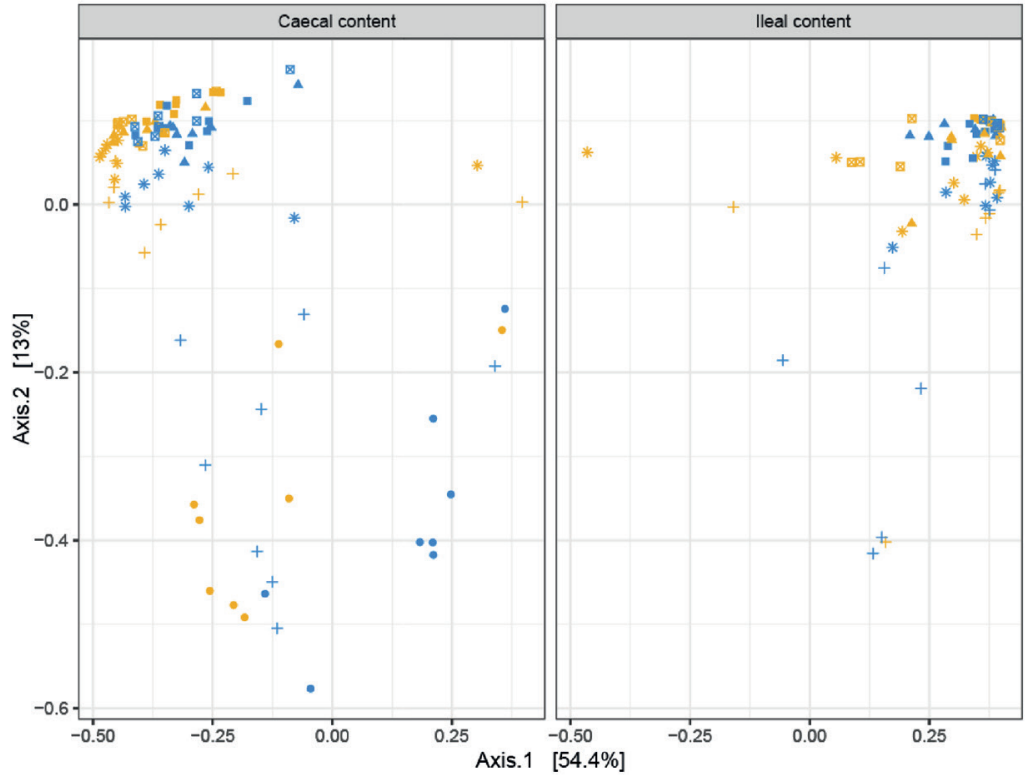

Figure 2. $\mathrm{PCoA}$ plot visualizing caecal and ileal content microbial profiles. Unweighted (A) and weighted (B) UniFrac distance based PCoA on caecal (left) and ileal (right) content samples. PERMANOVA of caecal content microbiota showed that treatment explained $6-9 \%$ of the variation in caecal microbiota composition between samples ( $p<5 e-04$; unweighted UniFrac, $p<2 e-04$; weighted UniFrac), whereas age explained $49-41 \%$ of the variation between samples ( $p<5 e-04$; unweighted UniFrac, $p<2 e-04$; weighted UniFrac). PERMANOVA of ileal content samples showed that treatment explained $4 \%$ of the variation in ileal microbiota composition based on unweighted UniFrac, whereas treatment did not significantly contribute to explaining the observed variation using the weighted UniFrac distance metrics ( $p=0.038$; unweighted UniFrac, $p=0.355$; weighted UniFrac). $\mathrm{n}=8$ chickens per treatment per day of age. 
To assess if AM inoculation affected the development of the microbial composition from hatch towards a mature microbiota, microbial profiles were subjected to DMM clustering of $16 \mathrm{~S}$ rRNA gene sequencing data based on the relative abundance of microbial taxa at genus level. The DDM method showed the best model fit, based on lowest Laplace approximation, for three clusters in the caecal content profiles (Fig. 3A). Cluster A contained 26 samples, with all 1-day-old AM and control chickens and all 3-day-old controls. Cluster B consisted of 21 samples, containing seven of the eight 3-day-old AM chickens and 7-day-old AM and control chickens. The remaining 48 samples were in cluster $C$, which contained all AM and control chickens of 14, 21 and 35 days old. This difference in distribution of AM and control chickens over cluster A and B in the first week of life indicates an accelerated maturation of caecal microbiota profiles for AM chickens. In contrast, clustering for the ileal content profiles only showed an effect of age, with cluster D dominated by 3-and 7-day-old chickens of both treatments, and cluster E by chickens of 14, 21 and 35 days old of both treatments (Fig. 3B). The relative microbial abundance of the clusters observed in the caecal content was analyzed and although PBS and AM chickens varied in their relative abundance of microbial families, PBS and AM chickens can be part of the same cluster based on relative abundance of genera (Fig. 3 C).

A caecal content

\begin{tabular}{ccccccc} 
Day 1 & 3 & 7 & 14 & 21 & 35 \\
\cline { 2 - 7 } Group & PBS AM & PBS AM & PBS AM & PBS AM & PBS AM & PBS AM
\end{tabular}
Cluster

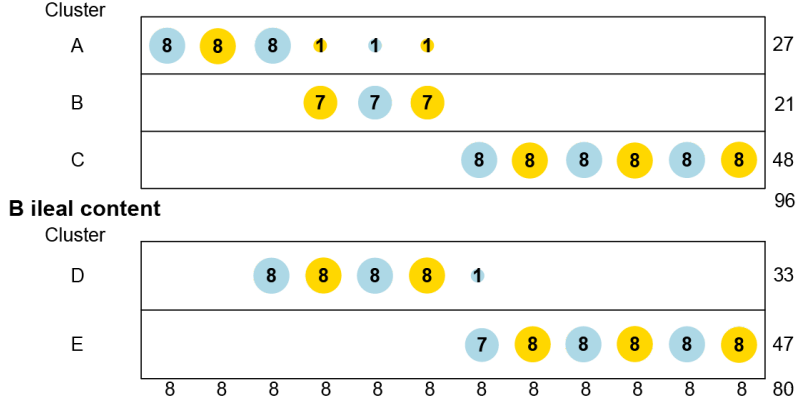

C

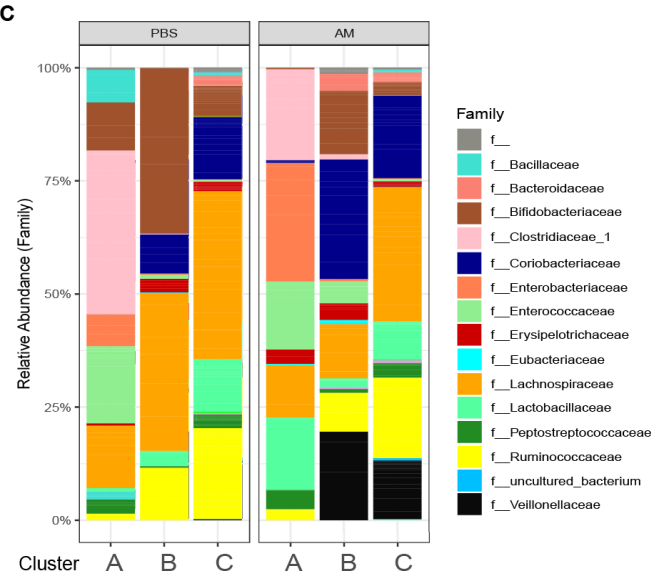

Figure 3. Dirichlet multinomial mixtures (DMM) clustering of $16 \mathrm{~S}$ rRNA gene sequencing data for caecal and ileal microbial profiles. (A) DMM clustering showed the best model fit for three clusters in the caecal content profiles (lowest Laplace approximation, $\mathrm{n}=$ 96). Cluster A contains 27 samples, Cluster B 21 samples and the remaining 48 samples are in cluster C. Cluster B contains seven of eight 3-day old AM chickens, and 7-day-old AM and control chickens, indicating acceleration of microbiota maturation in the caecal content. (B) In the ileal content samples two distinct clusters were observed, but no evidence for acceleration of the development of the microbiota $(n=80)$. Nodes are colored according to intervention (AM or PBS) and ordered according to age. (C) Relative microbial abundance of the clusters observed in the caecal content stratified by the intervention at family level.

\section{AM treatment affects presence of NK cell subsets and their activation} Possible differences in subsets and activation of intestinal NK cells from AM and control chickens were determined. Local effects of AM inoculation on intestinal NK cells were compared to systemic effects measured in spleen and blood. Within the live lymphocytes, the CD3 negative IL-2R $\alpha^{+}$or $20 \mathrm{E} 5^{+} \mathrm{NK}$ cells were quantified (Fig. 4A). In parallel, NK cell activation was determined by analysis of enhanced CD107 surface expression on CD3 negative and CD41/61 negative cells. At day 3, the percentage of intestinal IL-2R $\alpha^{+} \mathrm{NK}$ cells tended to be higher in AM chickens (5.61 $\left.\pm 0.95 \%\right)$ compared to controls ( $3.25 \pm 0.93 \%, p=0.09$, Fig. 4B). No differences between treatment groups were observed in intestinal 
20E5 ${ }^{+}$NK cells (Fig. 4C). Increased CD107 expression on intestinal NK cells was observed at day 3 in AM chickens (10.52 $\pm 0.70 \%)$, when compared to controls $(8.07 \pm 0.47 \%, p=0.06$, Fig. 4D). At day 35 , an increase in activation of intestinal NK cells was observed in AM chickens (14.86 $\pm 1.27 \%$ ) compared to the controls $(11.71 \pm 0.75 \%, p=0.04$, Fig. 4D). No differences between treatment groups were observed in CD107 expression of intestinal NK cells at other ages (Fig. 4D).

Relative numbers of IL-2R $\alpha^{+}$and $20 \mathrm{E5}^{+} \mathrm{NK}$ cells in spleen and blood were similar in both treatment groups (Fig. 4E, F and Fig. S3A, B). However, NK cell activation was significantly increased in splenic NK cells in 3-day-old AM chickens ( $20.74 \pm 1.10 \%$ ) compared to controls (15.35 $\pm 0.40 \%, p=0.004$, Fig. $4 \mathrm{G}$ ). No difference in CD107 surface expression on blood-derived NK cells was found between treatment groups

A
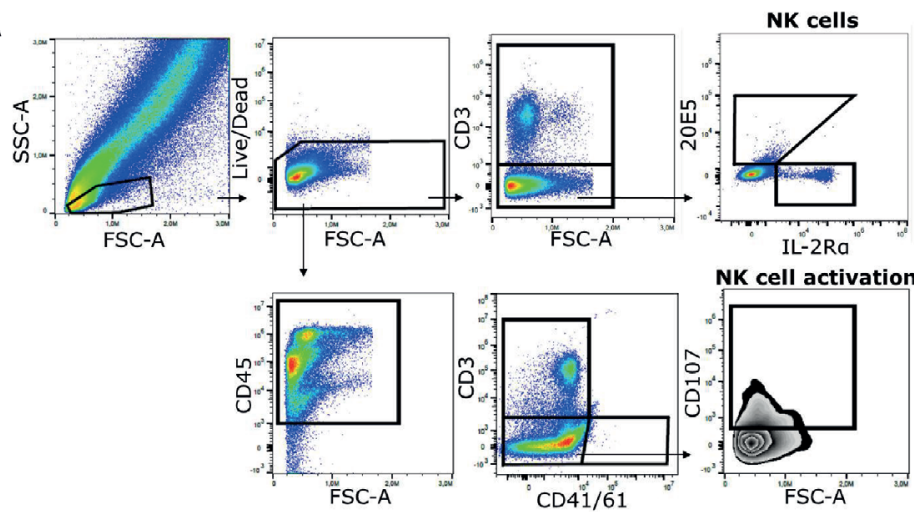

B
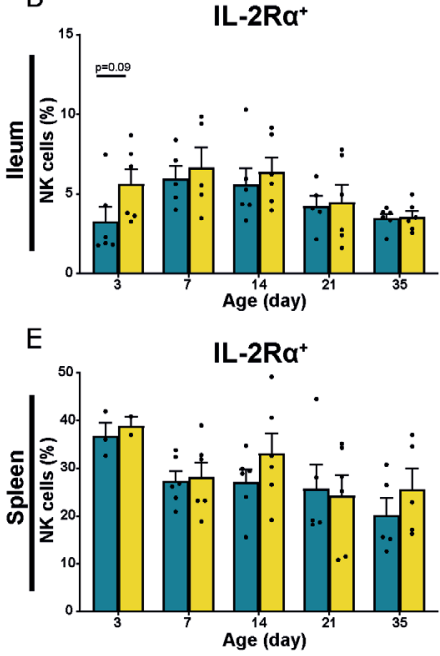

C

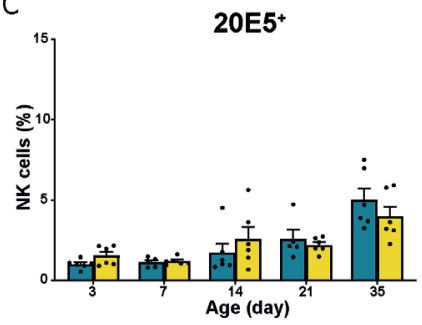

F

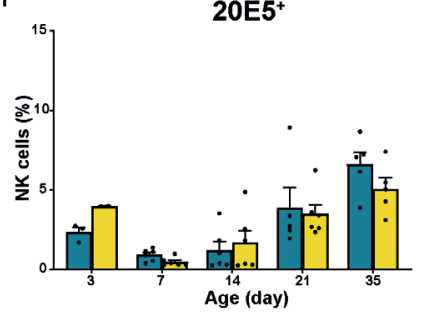

D

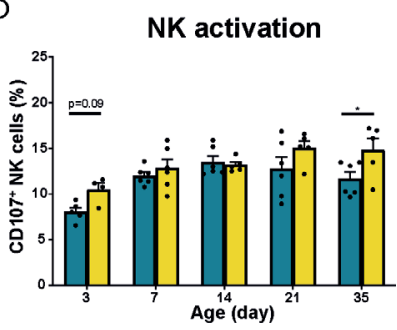

G

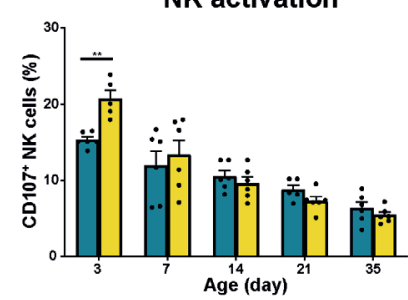

Figure 4. Effect of adult microbiota (AM) on NK cells in broiler chickens. (A) Gating strategy after isolation of lymphocytes from IEL to analyze NK cell subsets and activation. (B,E) Percentages of NK cell subsets by characterization of surface markers IL-2R $\alpha,(C, F) 20 E 5$ during aging in (B-D) IEL and (E-G) spleen. $(D, G)$ Percentages of NK cell activation during aging as assessed by measuring the surface marker CD107. Mean + SEM of chickens is shown $(n=6)$, however, chickens were excluded from analysis when numbers of events acquired in the gate of interest were $<100$. Statistical significance is indicated as ${ }^{*} p<0.05$ and ${ }^{* *} p<0.01$. 
(Fig. S3C). Furthermore, AM inoculation did not affect total lymphocyte numbers in the intestine, spleen and blood (Fig. S4A, E, I). In addition to the percentages of the different NK subsets, absolute numbers were determined. Similar trends were observed in absolute number of IL-2R $\alpha^{+}, 20 \mathrm{E} 5^{+}$and $\mathrm{CD} 107^{+} \mathrm{NK}$ cells although the differences between treatments were less pronounced (Fig. S4).

\section{AM treatment affects intestinal cytotoxic CD8 $\alpha \alpha$ T cells in 14- and 21-day-old chickens}

In addition to NK cell subsets and NK cell activation, effects of AM inoculation on presence and function of $\gamma \delta T$ cells and presence of cytotoxic CD8 ${ }^{+} T$ cells were studied. Within the $\mathrm{CD}^{+}$and $C D 4$ lymphocytes, both TCR $\gamma \delta^{+}$and TCR $\gamma \delta$ - cell populations were analyzed for CD $8 \alpha \alpha$ and CD8 $\alpha \beta$ expression (Fig. 5A). In parallel, activation of $\gamma \delta T$ cells was determined at day 7, 14 and 21 by analyzing increased surface expression of CD107 on $\mathrm{CD}^{+}{ }^{+} \mathrm{CD} 41 / 61-\mathrm{TCR} \gamma \delta^{+}$cells (Fig. 5A). No differences between $\mathrm{AM}$ and control chickens were observed in the percentage of intestinal $\gamma \delta$ T cells (Fig. 5B), CD8, CD8 $\alpha \alpha^{+}$and $\mathrm{CD} 8 \alpha \beta^{+}$gamma delta subsets (data not shown) and activation of $\gamma \delta$ T cells (Fig. $5 \mathrm{C}$ ). The percentage of intestinal CD8 $\alpha \alpha^{+}$T cells tended to be higher in 14- (25.3 $\left.\pm 1.5 \%\right)$ and 21-day-old AM chickens (33.2 \pm $4.2 \%$ ) compared to controls ( $19.5 \pm 1.7 \%, p=0.08$ and $24.0 \pm 1.3 \%, p=0.07$, respectively, Fig. 5D). No differences between groups were found at any age in the percentages of intestinal CD8 $\alpha \beta^{+}$T cells (Fig. $5 \mathrm{E})$. Furthermore, no differences between $\mathrm{AM}$ and control chickens were observed in the percentage of $\gamma \delta$ T cells (Fig. 5F and Fig. S3D), subsets (data not shown), $\gamma \delta T$ cell activation (Fig. 5G and Fig. S3E) and cytotoxic T cells in spleen and blood (Fig. 5H, I and Fig. S3F, G). Absolute numbers of these parameters were investigated and did not show any differences between AM and control chickens, although an increase in numbers of both treatments was observed with age (Fig. S5).

\section{Association between caecal microbiota clusters and immune cells}

Clustering of the caecal content profiles suggests that AM chickens showed an earlier maturation of caecal microbiota profiles compared to controls. Also, differences in IL-2R $\alpha^{+} \mathrm{NK}$ cells, NK cell activation and CD8 $\alpha \alpha^{+}$T cells were observed between AM chickens and the controls. To assess a possible relationship between the observed differences in the microbial development between treatments and the detected differences in immune parameters, we used the previously identified DMM clusters to test for correlations between the caecal microbiota profiles (i.e., stages of successive microbiota maturation) and immune parameters. Clusters $A, B$ and $C$ were based on relative abundance of genera present in the caecal microbiota of chickens and represent different stages during the early life development of caecal microbiota. Correlations to relative and absolute numbers of IL-2R $\alpha^{+}, 20 \mathrm{E} 5^{+}, \mathrm{CD} 107^{+} \mathrm{NK}$ cells and cytotoxic CD8aa+ $T$ cells in the ileum were investigated. The percentage of intestinal IL-2R $\alpha^{+} \mathrm{NK}$ cells was higher in cluster B compared to cluster A ( $p=0.026$, Table 2$)$, and compared to cluster C ( $p=$ 0.044 , Table 2) regardless of treatment (Fig. $6 A$ ). The percentage of IL-2R $\alpha^{+} N K$ cells in cluster $C$ tended to be higher compared to cluster $A$, but this was not significant ( $p=0.068$, Table 2, Fig 6A). Relative numbers of intestinal 20E5 ${ }^{+}$NK cells were similar between clusters $A$ and $B$ and highest in cluster $C$ (Table 2, Fig. 6B). Relative numbers of intestinal CD107+ NK cells were highest in cluster $\mathrm{C}$ and lowest in cluster $A$ (Table 2, Fig. 6C). Within cluster $C$, the percentage of $C D 107^{+} \mathrm{NK}$ cells tended to be higher in AM chickens (Fig. 6C). Relative numbers for intestinal cytotoxic CD8 $\alpha \alpha^{+}$T cells were higher in cluster $B$ and $C$ compared to cluster A and did not differ between cluster B and C (Table 2, Fig. 6D). Similar correlations were observed between clusters and absolute numbers of intestinal $20 \mathrm{E} 5^{+}, \mathrm{CD} 107^{+} \mathrm{NK}$ cells and cytotoxic CD8 $\alpha \alpha^{+}$T cells (Table 2). These results indicate significant associations between caecal microbiota clusters and subsets of intestinal immune cells. 


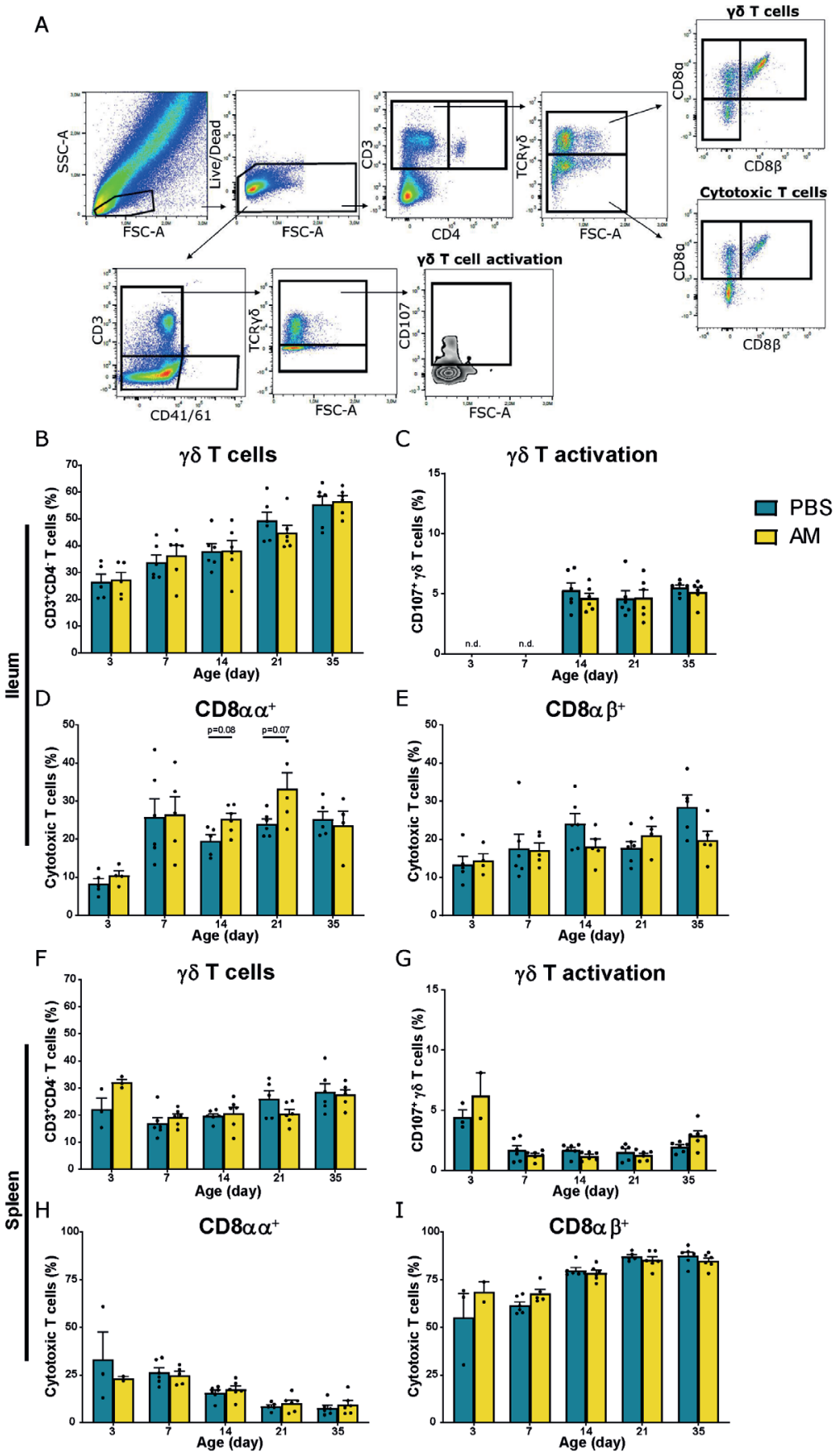

Figure 5. Effect of adult microbiota (AM) on T cells in broiler chickens. (A) Gating strategy after isolation of lymphocytes from IEL to analyze T cell subsets and $\gamma \delta T$ cell activation. $(B, F)$ Percentages of total $\gamma \delta T$ cells and $(C, G) \gamma \delta T$ cell activation by characterization of surface markers TCR $\gamma \delta$ and CD107 during aging in (B-E) IEL and (F-I) spleen. $(D, H)$ Percentages of cytotoxic T cell subsets using the surface markers CD8 $\alpha \alpha$ and $(E, l) C D 8 \alpha \beta$ during aging. Mean + SEM of chickens is shown $(n=6)$, however, chickens were excluded from analysis when numbers of events acquired in the gate of interest were $<100$. 


\section{Discussion}

In this study, we aimed to induce an alteration in the intestinal microbiota shortly after hatch by administration of adult-derived microbiota, and compared presence and function of NK cells, as representatives of developing innate immunity, to those of non-inoculated controls. We hypothesized that early exposure to adult-derived microbiota would accelerate intestinal microbiota colonization and affect subsets and activation of intestinal NK cells. Our results indicate that the inoculation with the adult-derived microbes mostly affected the early development of the caecal microbiota, and induced an earlier maturation of caecal microbiota compared to control broiler chickens. This development was paralleled by an increase in intestinal IL-2R $\alpha^{+}$NK cells and enhanced activation of NK cells early in life and CD8 $\alpha \alpha^{+} T$ cells later in life.

Table 2. Statistical differences in relative (\%) and absolute (cells/mg) numbers of intestinal immune cells between caecal microbiota clusters. Significant differences are indicated in bold.

\begin{tabular}{|c|c|c|c|}
\hline Immune cells & Cluster A vs. B & Cluster B vs. C & Cluster A vs. C \\
\hline IL-2R $\alpha^{+}$NK (\%) & 0.026 & 0.044 & 0.068 \\
\hline $20 \mathrm{E} 5^{+}$NK (\%) & 0.124 & $3.0 e^{-4}$ & 0.001 \\
\hline CD107+ NK (\%) & 0.003 & 0.020 & 0.001 \\
\hline CD8 $\alpha \alpha^{+} \mathrm{T}(\%)$ & 0.001 & 0.254 & $4.1 e^{-4}$ \\
\hline IL-2R $\alpha^{+}$NK (cells/mg) & 0.011 & 0.051 & $2.7 e^{-4}$ \\
\hline 20E5+ NK (cells/mg) & 0.039 & $5.3 e^{-7}$ & $2.1 e^{-6}$ \\
\hline CD107+ NK (cells/mg) & 0.398 & $4.0 e^{-6}$ & $1.1 \mathrm{e}^{-4}$ \\
\hline CD8 $\alpha \alpha^{+} \mathrm{T}$ (cells/mg) & 0.008 & $9.5 e^{-6}$ & $6.4 e^{-4}$ \\
\hline
\end{tabular}

The AM inoculation delivered immediately after hatch successfully altered intestinal microbiota composition, especially in the first week of life, but did not permanently influence the diversity of caecal microbiota. In addition, with respect to the genera found in the AM product, a higher relative abundance was only found shortly after inoculation. More specifically, a higher relative abundance in AM chickens was found for ten of the 24 genera in the inoculum on day 1 , but this quickly declined to two genera by the end of the first week. These findings are in line with previous studies with the same product: inoculation with Aviguard in ovo enhanced development of intestinal microbiota of broiler chickens and increased diversity and reduced the abundance of Enterobacteriaceae ${ }^{22}$. Similar to our study, not all genera present in the inoculum permanently colonized the intestine; they were assumed to have been transient colonizers facilitating the development of a complex microbiota by temporarily altering the microenvironment ${ }^{22}$. Similar observations have been reported for 1-day-old laying hens inoculated with Aviguard. Not all bacteria of the product, nor of the mother hen, were effectively transferred to the chickens' gut, but compared to controls, caecal microbiota enriched for the phyla Bacteroidetes and Actinobacteria was observed within a week in both Aviguard treated chickens and in chickens naturally exposed to a mother hen ${ }^{55}$.

Like chickens hatched in commercial hatcheries, the control chickens in our study were gradually exposed to microbiota in the hours and days after hatch from different sources, such as the housing environment, litter, feed and water. This colonization was delayed compared to the chickens inoculated with AM directly after hatch, as indicated by the clustering of caecal content profiles of 3-day-old controls with 1-day-old AM inoculated chickens, and of 7-day-old controls with 3-day-old AM chickens. This accelerated maturation of caecal microbiota composition has not only been observed in Aviguard studies $^{22,55}$, but also in a study in which topical spray treatment of eggs with adult caecal content 
significantly altered broiler chicken microbiota immediately after hatch, and accelerated the normal microbiota development ${ }^{69}$. As in our study, the effect on the caecal microbiota was highest at 3 days of age, and diminished over time ${ }^{69}$. In contrast, swabbing of the egg surface once during incubation with diluted adult caecal content did not lead to significant differences in alpha diversity nor in the pattern of bacterial colonization between treated and control broiler chickens ${ }^{70}$. This difference may be a result of the egg inoculation technique, suggesting that perhaps a lower number of spores and vegetative cells was applied to the eggshell in the latter study.

Although many of the available poultry microbiota studies have focused on broiler chickens, its relation with the innate immune system has not previously been elaborately investigated. We observed an increase in IL-2R $\alpha^{+}$NK cells and activation of NK cells within the first days of life, together with an increase in relative numbers of cytotoxic CD8 $\alpha \alpha^{+} T$ cells from day 14 onwards in chickens that were inoculated with AM.

A

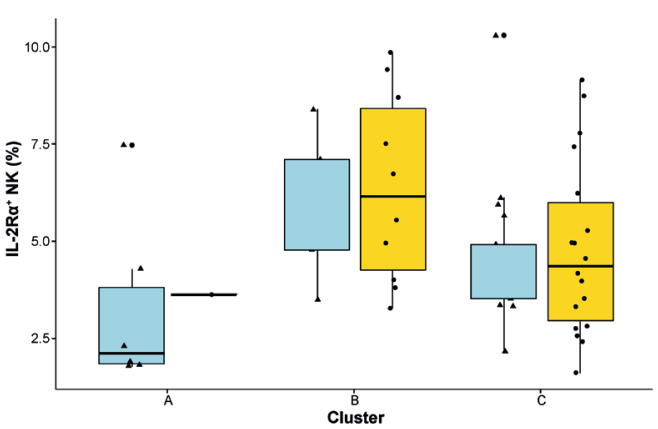

C

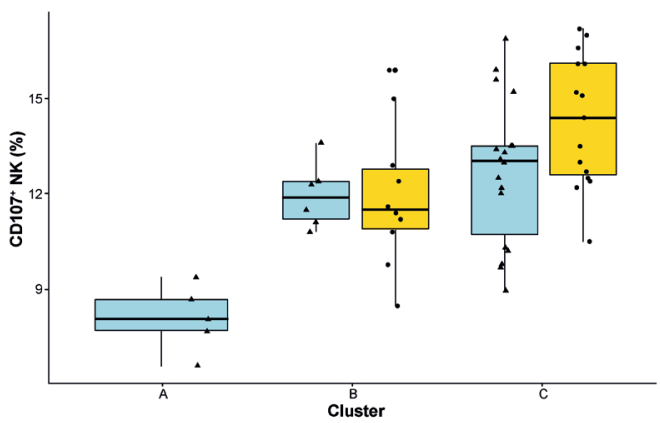

B 安 PBS 由

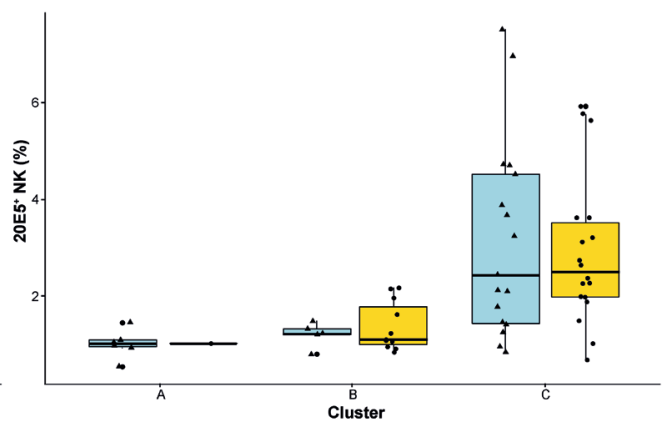

D

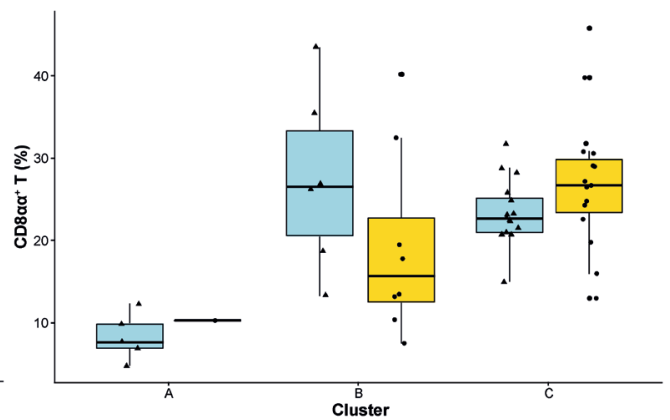

Figure 6. Associations between caecal microbiota clusters and immune cells. Associations between the identified DMM clusters of caecal microbiota composition and relative numbers of intestinal NK cell subsets (A) IL-2R $\alpha^{+},(B) 20 E 5^{+}$, (C) CD107 ${ }^{+}$and (D) cytotoxic $\mathrm{CD} 8 \alpha \alpha^{+} \mathrm{T}$ cells were analyzed using Wilcoxon rank-sum test. Adjusted $p$-values $(<0.05)$ were corrected for multiple testing with $\mathrm{BH}$.

The increased NK cell activation observed in AM chickens may suggest a mildly increased cytotoxic capacity against potential pathogens, as the CD107 expression can increase up to $30 \%$ upon viral infections ${ }^{33}$, which is more than two fold higher than the NK cell activation observed in this study. This result is in line with the observed increase in IL-2R $\alpha^{+}$NK cells in this study. Studies in humans have 
shown that increased IL-2R $\alpha$ expression is associated with an early stage of NK cell activation ${ }^{32}$, and this was also observed in chickens ${ }^{31,71,72}$. In addition to the local effect on NK cell activation, our observation of increased splenic NK cell activation in 3-day-old AM chickens also indicates there is a systemic effect. No effects of AM inoculation on immune cells in the blood were observed.

The observed differences between $\mathrm{AM}$ and control chickens with respect to immune parameters suggest an interaction between microbial and immune development. This was further substantiated by the significant associations between IL-2R $\alpha^{+} \mathrm{NK}, \mathrm{CD} 107^{+} \mathrm{NK}$ cells, and CD8 $\alpha \alpha^{+} \mathrm{T}$ cells and caecal microbiota clusters: cluster A includes chickens with a starting microbiota, cluster B chickens in the middle of the maturation process and cluster $C$ chickens with a more matured successive microbiota composition from day 14 onwards. These clusters follow the successional patterns of microbiota development as previously described for broiler chickens, with bacterial community richness increasing rapidly over time and stabilizing from day 14 onwards $^{5-7}$. Our analyses showed that cluster B was associated with an increase in IL-2R $\alpha^{+}$NK cells and an enhanced NK cell activation regardless of treatment. This suggests that the accelerated microbiota colonization due to AM inoculation affected the development of NK cells locally and systemically. Interestingly, the IL-2R $\alpha^{+}$NK cell subset was higher in relative numbers in cluster B compared to the starting microbiota cluster $A$, but subsequently decreased in relative numbers in the more mature microbiota cluster $C$. The $20 \mathrm{E} 5^{+} \mathrm{NK}$ cell subset and NK cells that express CD107 further increased in relative numbers between cluster B and cluster C. This fits with the observation in mammals that an increase in IL-2R expression is associated with an early stage of NK cell activation, which is followed by enhanced NK cell mediated killing. Cluster C was associated with an increased relative number of intestinal cytotoxic CD8 $\alpha \alpha^{+} T$ cells. As the caecal microbiota in this cluster shows a matured composition similar in AM and control chickens of the same age, this suggests that early life inoculation with $\mathrm{AM}$ also affected the adaptive immune development in the intestine.

Although these results indicate associations between early life microbiota colonization and immune system development, the data from this study cannot elucidate exactly how these processes are related. As has been shown in humans and mice, microbiota can signal to immune cells in various ways either locally or systemically ${ }^{46,73}$. Locally, microorganisms interact directly with NK cells via TLRs and NCRs resulting in cytokine production by NK cells, and indirectly via cytokine production of resident myeloid or epithelial cells that consequently affect NK cell responses ${ }^{46,74}$. Systemically, microbiota can induce instructive signals to non-mucosal antigen-presenting cells and by producing among others IL-15, TNF $\alpha$ and IFN, subsequently prime optimal splenic NK cell responses ${ }^{73}$. Since chicken NK cells have been shown to express $\operatorname{TLRs}^{75}$ and $\mathrm{NCRs}^{76,77}$, the interactions between microbiota and NK cells probably follow similar routes to those in humans and mice.

In mammals, specific commensal bacterial strains have been linked to modulation of NK cells. Several reports established that bacteria within the Lactobacillus genus can induce IFNY and cytotoxicity responses in intestinal NK cells as a result of IL-12 production by dendritic cells after TLR engagement with bacteria ${ }^{43,78,79}$. Furthermore, Bacteroides fragilis can stimulate innate and adaptive immune pathways directly through TLR signaling and indirectly by inducing cytokine productione ${ }^{80}$. Although we did observe significant differences in the relative abundance of genera between AM and control chickens at day 1 and 3, we cannot pinpoint a specific genus responsible for the observed effect on NK cells. Interestingly, the genus Bacteroides showed a significantly higher prevalence and relative abundance in 3- and 7-day-old AM chickens and the genus was absent in control chickens of similar age. This could suggest that the observed effects on NK cells in 3-day-old AM chickens may be linked to a higher presence of Bacteroides bacteria as shown previously ${ }^{80}$. We did not find differences in 
the prevalence of Lactobacillus bacteria due to AM inoculation. Other genera that showed significant differences in their prevalence and/or relative abundance between AM and control chickens at 1 and 3 days of age have not been described as specifically interacting with NK cells.

In addition, microbiota has been shown in mice and humans to interact directly with $\gamma \delta \mathrm{T}$ cells, and increased frequencies of $\mathrm{CD}^{+} \gamma \delta \mathrm{T}$ cells and $\gamma \delta \mathrm{T}$ cell activation were observed during intestinal inflammation ${ }^{51,81}$. Under non-inflammatory conditions similar to those of our study, application of adult caecal content on eggs altered and accelerated the microbiota of 3-day-old chickens but did not affect $\gamma \delta \mathrm{T}$ cells in caecal tonsils ${ }^{69}$. Furthermore, AM inoculated chickens in our study showed an increased presence of intestinal CD8 $\alpha \alpha^{+}$T cells at two and three weeks of age. Although in previous studies with mice no $C D 8 \alpha \alpha$ and $C D 8 \alpha \beta$ subsets were investigated, microbiota was shown to have direct $^{53}$ and indirect ${ }^{82}$ effects on cytotoxic T cells, as IFN $\gamma$ production was induced.

Further research including challenge models is needed to answer the question if chickens with an accelerated maturation of intestinal microbiota and enhanced NK cell responses early in life are indeed more resilient against infections.

Interestingly we observed a relation between changes in caecal microbiota and intestinal NK cell responses. It would have been highly interesting to investigate the interaction between immune system and microbiota at caecum level, but unfortunately this was not possible since only few NK cells can be obtained from the caecum in young chickens ${ }^{83,84}$. Although we set out to analyze the relation between NK cells and microbiota composition in the ileum, we did not observe differences between treatment groups at any age in the phylogenetic diversity of ileal microbiota nor in the relative abundances of genera. Not being able to show a difference at ileal level, especially considering the relatively small number of chickens at each time point, was not surprising, and exactly the reason why we also collected caecal content. Nevertheless, the shift in microbiota composition as measured in the caeca showed that the AM treatment has successfully affected microbiota development in parts of the intestinal tract. For the AM treatment to be able to alter caecal microbiota composition, the microbiota of the AM product at least must have passed, and to some extent may have colonized upstream parts of the intestinal tract as well, albeit not inducing a measurable shift in microbiota composition in ileum. Therefore, we expect that the observed effects on ileal NK cells are associated with the AM treatment. In conclusion, our study showed a relation between an accelerated maturation of intestinal microbiota and the enhanced NK cell response early in life. This interaction between microbiota and the developing innate immune system indicates possibilities in developing strategies to improve health and resilience of broiler chickens. One such possibility is through feed interventions or the use of products with adult-derived microbiota directly after hatch, both of which can affect microbiota composition and may accelerate microbiota maturation. Consequently, this can strengthen the innate immune system, conferring direct protective effects early in life as well as influencing adaptive immunity later in life. The combination of a well-developed microbiota and immune system may result in more robust broiler chickens with higher resilience against health challenges, such as disturbances in gut health and invading pathogens. Future research including challenge studies are warranted to test this hypothesis.

\section{Acknowledgements}

We thank the animal caretakers of the Department Population Health Sciences, division Farm Animal Health, Faculty of Veterinary Medicine, Utrecht University, for their help during the animal experiments. We acknowledge R.H.G.A. van den Biggelaar and I.S. Ludwig for their help during the isolation of 
immune cells. We acknowledge G.J.A. Arkesteijn for maintaining optimal working conditions of the Flow Cytometry and Cell Sorting Facility, Faculty of Veterinary Medicine, Utrecht University. We thank M. van Gaal and I. Heikamp-de Jong of the Laboratory of Microbiology, Wageningen University \& Research for their help with molecular analyses of microbial composition. This work was part of the research programme of NWO Earth and Life Sciences (ALW) with project number 868.15.020, which is financed by the Dutch Research Council (NWO) and by Cargill Animal Nutrition and Health. 


\section{Supplementary data}
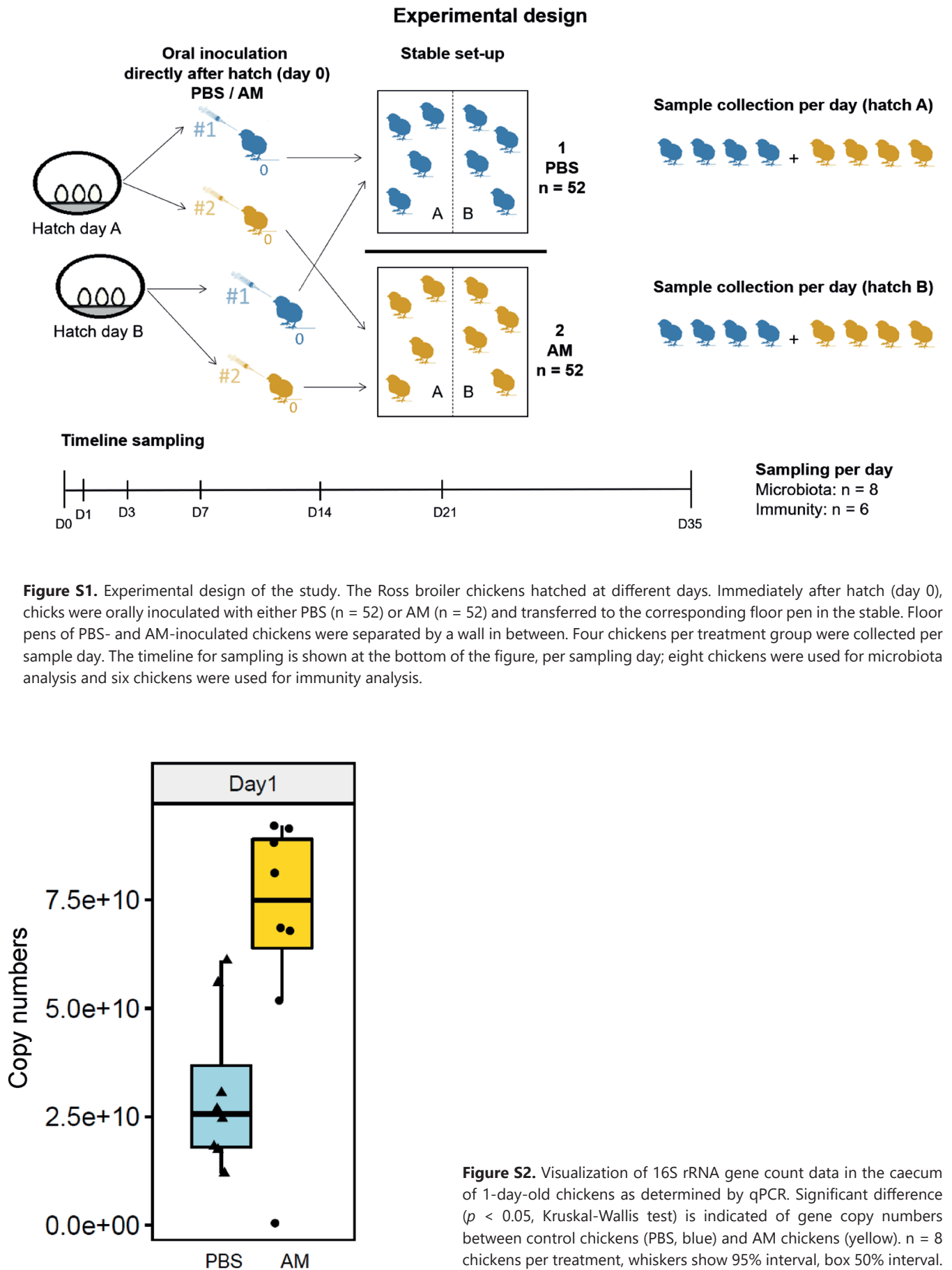

Figure S2. Visualization of $16 \mathrm{~S}$ rRNA gene count data in the caecum of 1-day-old chickens as determined by qPCR. Significant difference $(p<0.05$, Kruskal-Wallis test) is indicated of gene copy numbers between control chickens (PBS, blue) and AM chickens (yellow). $n=8$ chickens per treatment, whiskers show $95 \%$ interval, box $50 \%$ interval. 

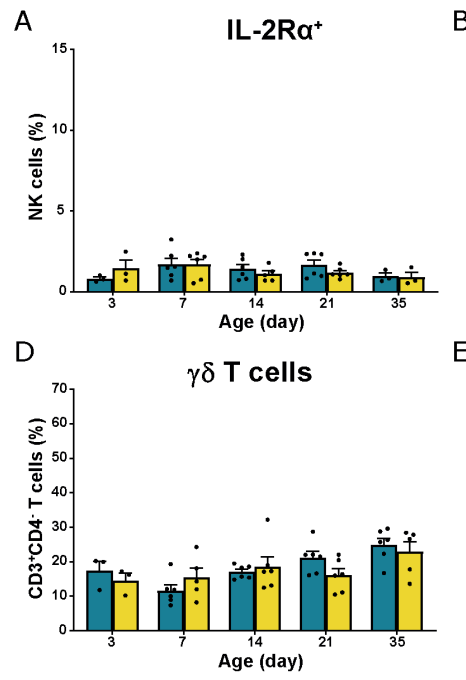

$\mathrm{F}$

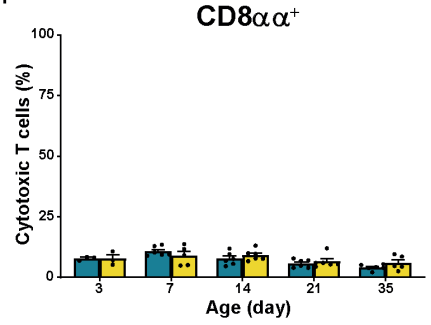

B

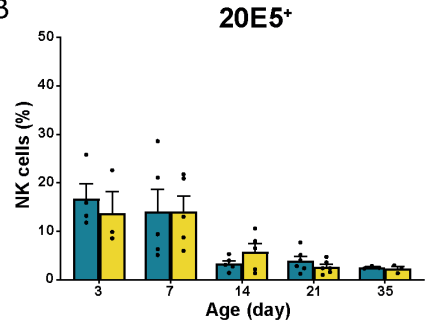

E

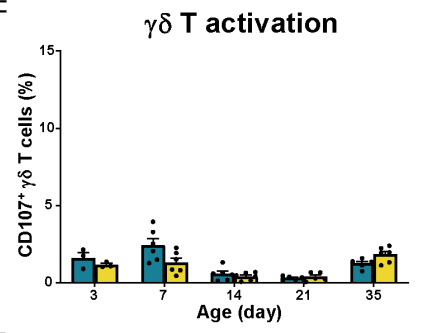

G

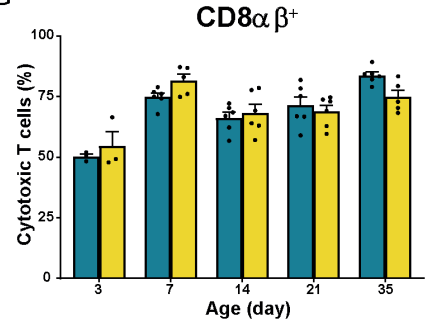

C

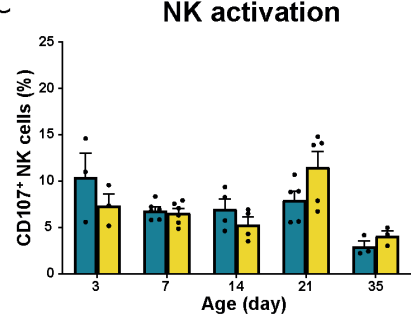

$\square$ PBS

$\square$ AM

Figure S3. Effect of adult microbiota (AM) on NK and T cells in blood of broiler chickens. (A) Percentages of NK cell subsets characterized by surface markers IL-2R $\alpha$ and (B) $20 \mathrm{E} 5$ during aging in blood. (C) Percentages of NK cell activity during aging as assessed by measuring the surface marker CD107. (D) Percentages of total $\gamma \delta T$ cells and $(E) \gamma \delta T$ cell activation by characterization of surface markers TCR $\gamma \delta$ and CD107, respectively. (F) Percentages of cytotoxic T cell subsets using the surface markers CD8 $\alpha \alpha$ and (G) CD8 $\alpha \beta$ during aging in blood. Mean + SEM of chickens is shown $(n=6)$, however, chickens were excluded from analysis when numbers of events acquired in the gate of interest were $<100$. 


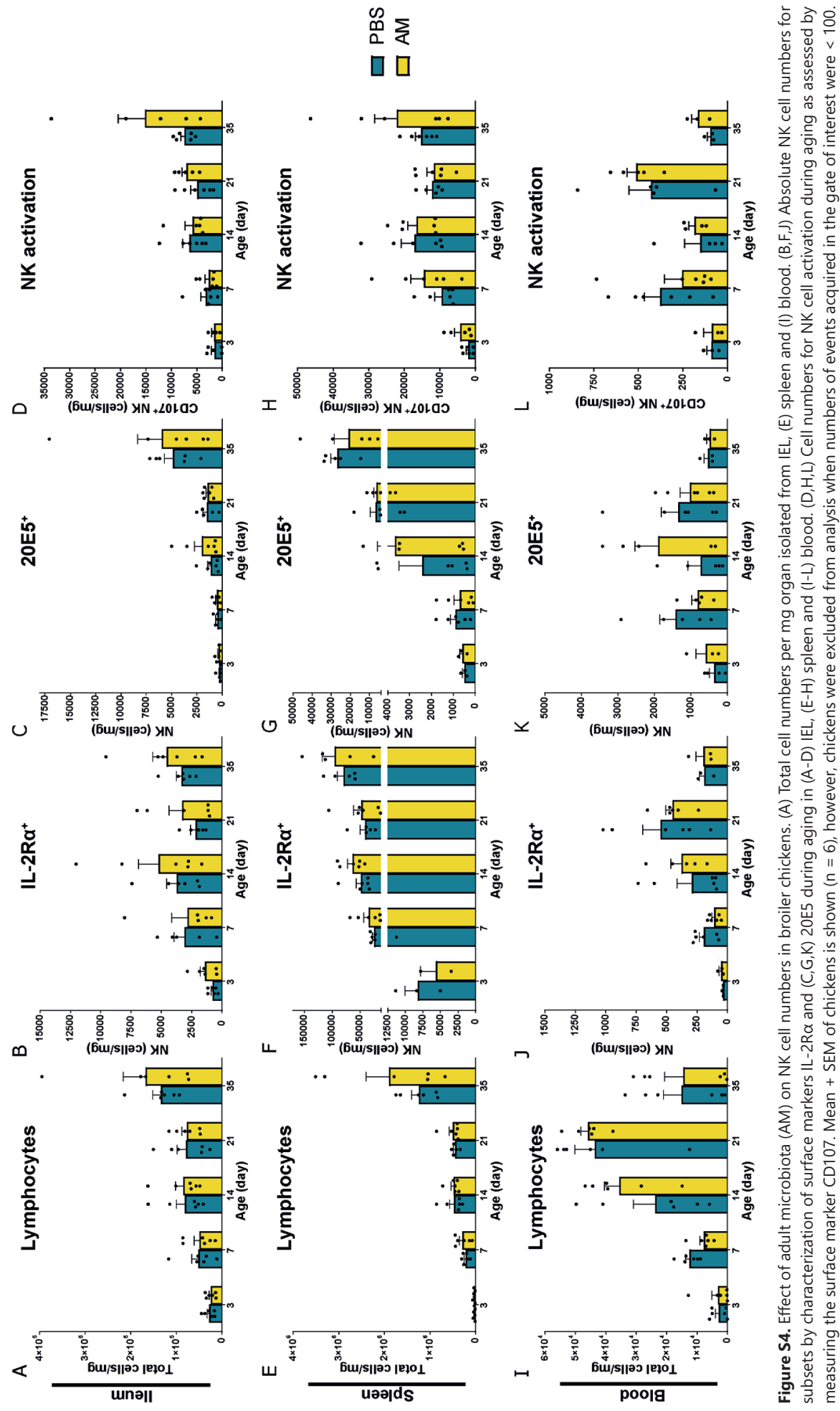




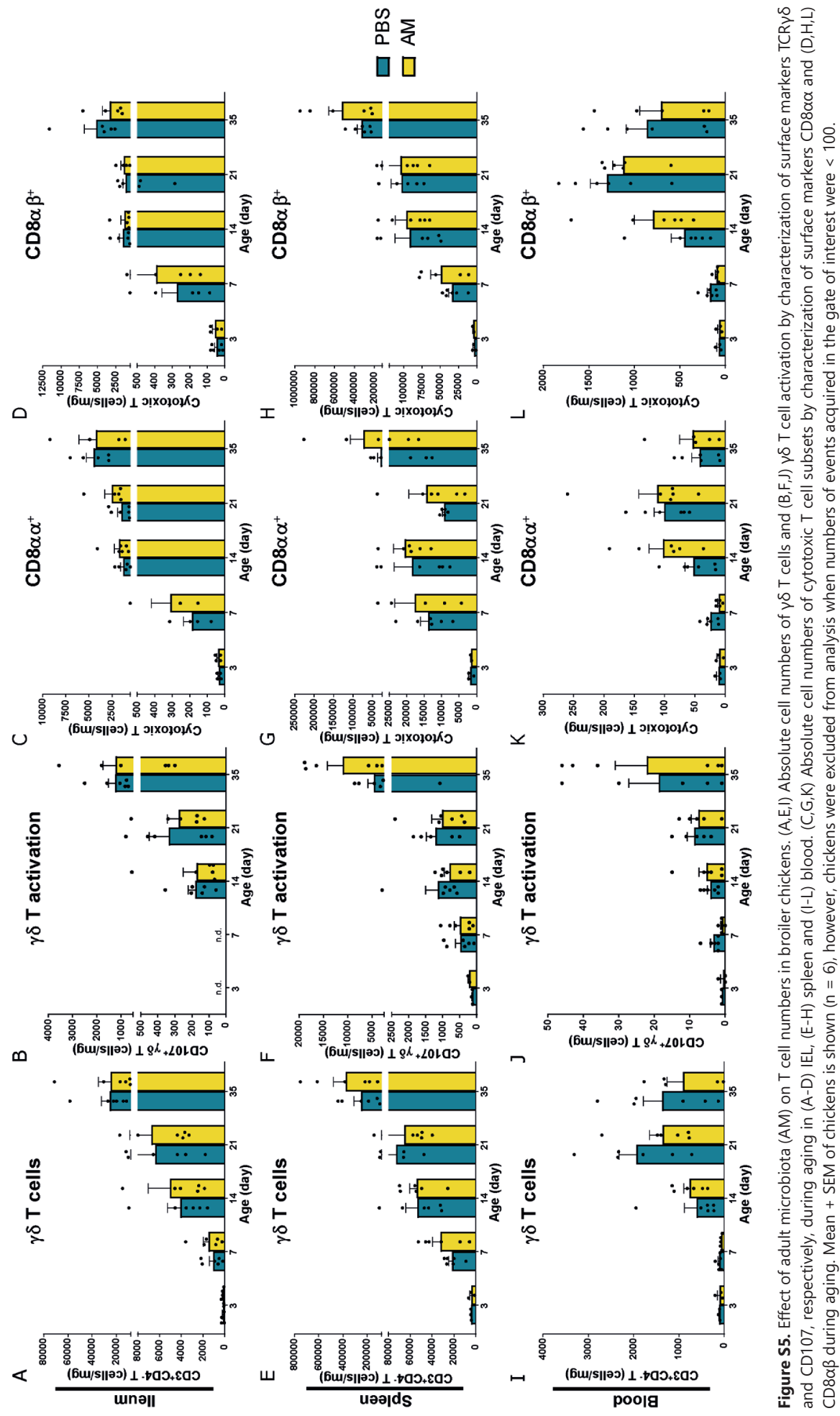




\section{References}

1. OECD/FAO. OECD-FAO Agricultural Outlook 2019-2028. 2019; Rome: OECD Publishing, Paris/Food and Agriculture Organization of the United Nations. doi: 10.1787/agr_outlook-2019-en.

2. Skunca D, Tomasevic I, Nastasijevic I, Tomovic V, Djekic I. Life cycle assessment of the chicken meat chain. J Clean Prod. 2018;184:440450. doi:10.1016/j.jclepro.2018.02.274.

3. Kogut MH. The effect of micr obiome modulation on the intestinal health of poultry. Anim Feed Sci Technol. 2019;250:32-40. doi:https://doi.org/10.1016/j.anifeedsci.2018.10.008.

4. Pedroso AA, Lee MD. The composition and role of the microbiota in chickens. 2015:21-50; In: Niewold T (ed). Intestinal Health. Wageningen: Wageningen Academic Publishers. doi: 10.3920/978-90-8686-792-9_2.

5. Cressman MD, Yu Z, Nelson MC, Moeller SJ, Lilburn MS, Zerby HN. Interrelations between the microbiotas in the litter and in the intestines of commercial broiler chickens. Appl Environ Microbiol. 2010;76(19):6572-6582.

6. Oakley BB, Kogut MH. Spatial and Temporal Changes in the Broiler Chicken Cecal and Fecal Microbiomes and Correlations of Bacterial Taxa with Cytokine Gene Expression. Front Vet Sci. 2016;3:11. doi:10.3389/fvets.2016.00011.

7. Jurburg SD, Brouwer MSM, Ceccarelli D, van der Goot J, Jansman AJM, Bossers A. Patterns of community assembly in the developing chicken microbiome reveal rapid primary succession. MicrobiologyOpen. 2019;8(9). doi:10.1002/mbo3.821.

8. Dominguez-Bello $\mathrm{M}$, Costello EK, Contreras $\mathrm{M}$, et al. Delivery mode shapes the acquisition and structure of the initial microbiota across multiple body habitats in newborns. Proc Natl Acad Sci U S A. 2010;107(26):11971-11975. doi:10.1073/pnas.1002601107.

9. Borewicz K, Suarez-Diez M, Hechler C, et al. The effect of prebiotic fortified infant formulas on microbiota composition and dynamics in early life. Scientific Reports. 2019;9(1). doi:10.1038/s41598-018-38268-x.

10. Borewicz K, Gu F, Saccenti E, et al. The association between breastmilk oligosaccharides and faecal microbiota in healthy breastfed infants at two, six, and twelve weeks of age. Scientific Reports. 2020;10(1). doi:10.1038/s41598-020-61024-z.

11. Coloe PJ, Bagust TJ, Ireland L. Development of the normal gastrointestinal microflora of specific pathogen-free chickens. J Hyg. 1984;92(1):79-87. doi:10.1017/s0022172400064056.

12. Inman CF, Haverson K, Konstantinov SR, et al. Rearing environment affects development of the immune system in neonates. Clin Exp Immunol. 2010;160(3):431-439. doi:10.1111/j.1365-2249.2010.04090.x.

13. Mulder IE, Schmidt B, Lewis M, et al. Restricting microbial exposure in early life negates the immune benefits associated with gut colonization in environments of high microbial diversity. PLoS ONE. 2011;6(12):e28279. doi:10.1371/journal.pone.0028279.

14. Schokker D, Jansman AJM, Veninga G, et al. Perturbation of microbiota in one-day old broiler chickens with antibiotic for 24 hours negatively affects intestinal immune development. BMC Genomics. 2017;18(1). doi:10.1186/s12864-017-3625-6.

15. Xi Y, Shuling N, Kunyuan T, et al. Characteristics of the intestinal flora of specific pathogen free chickens with age. Microb Pathog. 2019;132:325-334. doi:10.1016/j.micpath.2019.05.014.

16. Ballou AL, Ali RA, Mendoza MA, et al. Development of the Chick Microbiome: How Early Exposure Influences Future Microbial Diversity. Front Vet Sci. 2016;3:2. doi:10.3389/fvets.2016.00002.

17. Polansky O, Sekelova Z, Faldynova M, Sebkova A, Sisak F, Rychlik I. Important metabolic pathways and biological processes expressed by chicken cecal microbiota. Appl Environ Microbiol. 2016;82(5):1569-1576. doi:10.1128/AEM.03473-15.

18. Volf J, Polansky O, Varmuzova K, et al. Transient and prolonged response of chicken cecum mucosa to colonization with different gut microbiota. PLoS ONE. 2016;11(9). doi:10.1371/journal.pone.0163932.

19. Mead GC. Prospects for 'Competitive exclusion' treatment to control salmonellas and other foodborne pathogens in poultry. Veterinary Journal. 2000;159(2):111-123. doi:10.1053/tvjl.1999.0423.

20. Crhanova $M$, Hradecka $H$, Faldynova $M$, et al. Immune response of chicken gut to natural colonization by gut microflora and to Salmonella enterica serovar enteritidis infection. Infect Immun. 2011;79(7):2755-2763. doi:10.1128/IAI.01375-10.

21. Yin $Y$, Lei $F$, Zhu L, et al. Exposure of different bacterial inocula to newborn chicken affects gut microbiota development and ileum gene expression. ISME Journal. 2010;4(3):367-376. doi:10.1038/ismej.2009.128.

22. Pedroso $A A$, Batal $A B$, Lee MD. Effect of in ovo administration of an adult-derived microbiota on establishment of the intestinal microbiome in chickens. Am J Vet Res. 2016;77(5):514-526. doi:10.2460/ajvr.77.5.514.

23. Kerr AK, Farrar AM, Waddell LA, et al. A systematic review-meta-analysis and meta-regression on the effect of selected competitive exclusion products on Salmonella spp. prevalence and concentration in broiler chickens. Prev Vet Med. 2013;111(1-2):112-125. doi:10.1016/j.prevetmed.2013.04.005.

24. Ceccarelli $D$, van Essen-Zandbergen A, Smid B, et al. Competitive exclusion reduces transmission and excretion of extendedspectrum- $\beta$-lactamase-producing Escherichia coli in broilers. Appl Environ Microbiol. 2017;83(11). doi:10.1128/AEM.03439-16.

25. Uni Z, Smirnov A, Sklan D. Pre- and posthatch development of goblet cells in the broiler small intestine: Effect of delayed access to feed. Poult Sci. 2003;82(2):320-327. doi:10.1093/ps/82.2.320.

26. Göbel TWF, Kaspers B, Stangassinger M. NK and T cells constitute two major, functionally distinct intestinal epithelial lymphocyte subsets in the chicken. Int Immunol. 2001;13(6):757-762. doi:10.1093/intimm/13.6.757.

27. Klasing KC, Leshchinsky TV. Functions, costs and benefits of the immune system during development and growth. 1999; In: Adams NJ, and Slotow R (eds)(Proc. 22. Int. Ornithol. Congr. H. Birdlife South Africa, Johannesburg):2817-2835.

28. Sharma JM, Tizard I. Avian cellular immune effector mechanisms - a review. Avian Pathol. 1984;13(3):357-376. doi:10.1080/03079458408418541.

29. Jansen CA, van de Haar PM, van Haarlem D, et al. Identification of new populations of chicken natural killer (NK) cells. Dev Comp Immunol. 2010;34(7):759-767. doi:10.1016/j.dci.2010.02.009. 
30. Jansen CA, De Geus ED, Van Haarlem DA, et al. Differential lung NK cell responses in avian influenza virus infected chickens correlate with pathogenicity. Sci Rep. 2013;3. doi:10.1038/srep02478.

31. Meijerink N, van Haarlem DA, Velkers FC, Stegeman AJ, Rutten VPMG, Jansen CA. Analysis of chicken intestinal natural killer cells, a major IEL subset during embryonic and early life. Dev Comp Immunol. 2021;114. doi:10.1016/j.dci.2020.103857.

32. Leong JW, Chase JM, Romee R, et al. Preactivation with IL-12, IL-15, and IL-18 induces cd 25 and a functional high-affinity il-2 receptor on human cytokine-induced memory-like natural killer cells. Biol Blood Marrow Transplant. 2014;20(4):463-473. doi:10.1016/j.bbmt.2014.01.006.

33. Vervelde L, Matthijs MGR, van Haarlem DA, de Wit JJ, Jansen CA. Rapid NK-cell activation in chicken after infection with infectious bronchitis virus M41. Vet Immunol Immunopathol. 2013;151(3-4):337-341. doi:10.1016/j.vetimm.2012.11.012.

34. Bertzbach LD, van Haarlem DA, Härtle S, Kaufer BB, Jansen CA. Marek's disease virus infection of natural killer cells. Microorg. 2019;7(12). doi:10.3390/microorganisms7120588.

35. Round JL, Mazmanian SK. The gut microbiota shapes intestinal immune responses during health and disease. Nature Reviews Immunology. 2009;9(5):313-323. doi:10.1038/nri2515.

36. Hooper LV, Littman DR, Macpherson AJ. Interactions between the microbiota and the immune system. Science. 2012;336(6086):12681273. doi:10.1126/science.1223490.

37. Macpherson AJ, Harris NL. Interactions between commensal intestinal bacteria and the immune system. Nature Reviews Immunology. 2004;4(6):478-485. doi:10.1038/nri1373.

38. Rooks MG, Garrett WS. Gut microbiota, metabolites and host immunity. Nature Reviews Immunology. 2016;16(6):341-352. doi:10.1038/nri.2016.42.

39. Broom LJ, Kogut MH. The role of the gut microbiome in shaping the immune system of chickens. Vet Immunol Immunopathol. 2018;204:44-51. doi:10.1016/j.vetimm.2018.10.002.

40. van der Eijk JAJ, Rodenburg TB, de Vries $\mathrm{H}$, et al. Early-life microbiota transplantation affects behavioural responses, serotonin and immune characteristics in chicken lines divergently selected on feather pecking. Sci Rep. 2020;10(1). doi:10.1038/s41598-02059125-w.

41. Metzler-Zebeli BU, Siegerstetter S-, Magowan E, Lawlor PG, O'Connell NE, Zebeli Q. Fecal microbiota transplant from highly feed efficient donors affects cecal physiology and microbiota in low- And high-feed efficient chickens. Front Microbiol. 2019;10(JULY). doi:10.3389/fmicb.2019.01576.

42. Butler VL, Mowbray CA, Cadwell K, et al. Effects of rearing environment on the gut antimicrobial responses of two broiler chicken lines. Vet Immunol Immunopathol. 2016;178:29-36. doi:10.1016/j.vetimm.2016.06.004.

43. Aziz N, Bonavida B. Activation of natural killer cells by probiotics. Forum Immunopathol Dis Ther. 2016;7(1-2):41-55. doi:10.1615/ ForumlmmunDisTher.2016017095.

44. Carrillo-Bustamante P, Kesmir C, de Boer RJ. The evolution of natural killer cell receptors. Immunogenetics. 2016;68(1):3-18. doi:10.1007/s00251-015-0869-7.

45. Temperley ND, Berlin S, Paton IR, Griffin DK, Burt DW. Evolution of the chicken Toll-like receptor gene family: A story of gene gain and gene loss. BMC Genomics. 2008;9. doi:10.1186/1471-2164-9-62.

46. Sonnenberg G, Artis D. Innate Lymphoid Cell Interactions with Microbiota: Implications for Intestinal Health and Disease. Immunity. 2012;37(4):601-610. doi:10.1016/j.immuni.2012.10.003.

47. Yitbarek A, Astill J, Hodgins DC, Parkinson J, Nagy É, Sharif S. Commensal gut microbiota can modulate adaptive immune responses in chickens vaccinated with whole inactivated avian influenza virus subtype H9N2. Vaccine. 2019;37(44):6640-6647. doi:10.1016/j. vaccine.2019.09.046.

48. Siwek M, Slawinska A, Stadnicka K, Bogucka J, Dunislawska A, Bednarczyk M. Prebiotics and synbiotics - In ovo delivery for improved lifespan condition in chicken. BMC Vet Res. 2018;14(1). doi:10.1186/s12917-018-1738-Z.

49. Gao P, Ma C, Sun Z, et al. Feed-additive probiotics accelerate yet antibiotics delay intestinal microbiota maturation in broiler chicken. Microbiome. 2017;5(1):91.

50. Brisbin JT, Parvizi P, Sharif S. Differential cytokine expression in T-cell subsets of chicken caecal tonsils co-cultured with three species of Lactobacillus. Benefic Microbes. 2012;3(3):205-210. doi:10.3920/BM2012.0014.

51. Nielsen MM, Witherden DA, Havran WL. $\gamma \delta$ T cells in homeostasis and host defence of epithelial barrier tissues. Nat Rev Immunol. 2017;17(12):733-745. doi:10.1038/nri.2017.101.

52. Yang $Y, X u C, W u ~ D$, et al. $\gamma \delta T$ cells: Crosstalk between microbiota, chronic inflammation, and colorectal cancer. Front Immunol. 2018;9(JUN). doi:10.3389/fimmu.2018.01483.

53. Tanoue T, Morita S, Plichta DR, et al. A defined commensal consortium elicits CD8 T cells and anti-cancer immunity. Nature. 2019;565(7741):600-605. doi:10.1038/s41586-019-0878-z.

54. De Geus ED, Jansen CA, Vervelde L. Uptake of particulate antigens in a nonmammalian lung: Phenotypic and functional characterization of avian respiratory phagocytes using bacterial or viral antigens. J Immunol. 2012;188(9):4516-4526. doi:10.4049/ jimmunol.1200092.

55. Kubasova T, Kollarcikova M, Crhanova M, et al. Contact with adult hen affects development of caecal microbiota in newly hatched chicks. PLoS ONE. 2019;14(3). doi:10.1371/journal.pone.0212446.

56. Kers JG, Velkers FC, Fischer EAJ, et al. Take care of the environment: housing conditions affect the interplay of nutritional interventions and intestinal microbiota in broiler chickens. Animal Microbiome. 2019;1(1):10. doi:10.1186/s42523-019-0009-z.

57. Verlaet A, van der Bolt N, Meijer B, et al. Toll-like receptor-dependent immunomodulatory activity of pycnogenol ${ }^{\circledR}$. Nutrients. 2019;11(2). doi:10.3390/nu11020214. 
58. Ramiro-Garcia J, Hermes GDA, Giatsis C, et al. NG-Tax, a highly accurate and validated pipeline for analysis of $16 \mathrm{~S}$ rRNA amplicons from complex biomes. F1000Research. 2016;5:1791. doi:10.12688/f1000research.9227.1.

59. Poncheewin W, Hermes GDA, van Dam JCJ, Koehorst JJ, Smidt H, Schaap PJ. NG-Tax 2.0: A Semantic Framework for High-Throughput Amplicon Analysis. Frontiers in Genetics. 2020;10. doi:10.3389/fgene.2019.01366.

60. Quast C, Pruesse E, Yilmaz P, et al. The SILVA ribosomal RNA gene database project: improved data processing and web-based tools. Nucleic Acids Res. 2013;41; 2012/11/30:590. doi:10.1093/nar/gks1219.

61. McMurdie PJ, Holmes S. phyloseq: an R package for reproducible interactive analysis and graphics of microbiome census data. PLoS ONE. 2013;8; 2013/05/01(4):e61217. doi:10.1371/journal.pone.0061217.

62. Lahti L, Shetty S, Blake T, Salojarvi J. Tools for microbiome analysis in R. Version 1.5.28. 2017. Available online at http://microbiome. github.com/microbiome.

63. Oksanen J, Blanchet FG, Kindt R, Legendre P, O'Hara R, Simpson GL, Solymos P, Stevens MHH, Wagner HJ. Vegan: community ecology package. R package version 1.17-4. 2010. Available online at http://cran.r-project.org.

64. Morgan M. DirichletMultinomial: Dirichlet-Multinomial Mixture Model Machine Learning for Microbiome Data. R package version 1.28.0. 2019. Available online at https://bioconductor.org/packages/DirichletMultinomial/. doi:10.18129/B9.bioc.DirichletMultinomial.

65. Faith DP. The Role of the Phylogenetic Diversity Measure, PD, in Bio-informatics: Getting the Definition Right. Evolutionary Bioinformatics Online. 2006;2:277-283. doi:10.1177/117693430600200008.

66. Lozupone CA, Hamady M, Kelley ST, Knight R. Quantitative and qualitative beta diversity measures lead to different insights into factors that structure microbial communities. Appl Environ Microbiol. 2007;73; 2007/01/16(5):1576-1585. doi:10.1128/ aem.01996-06.

67. Anderson MJ. A new method for non-parametric multivariate analysis of variance. Austral Ecol. 2001;26(1):32-46. doi:10.1046/ j.1442-9993.2001.01070.x.

68. Holmes I, Harris K, Quince C. Dirichlet multinomial mixtures: Generative models for microbial metagenomics. PLoS ONE. 2012;7(2). doi:10.1371/journal.pone.0030126.

69. Richards-Rios P, Leeming G, Fothergill J, Bernardeau M, Wigley P. Topical Application of Adult Cecal Contents to Eggs Transplants Spore-Forming Microbiota but Not Other Members of the Microbiota to Chicks. Appl Environ Microbiol. 2020;86(5). doi:10.1128/ AEM.02387-19.

70. Donaldson EE, Stanley D, Hughes RJ, Moore RJ. The time-course of broiler intestinal microbiota development after administration of cecal contents to incubating eggs. PeerJ. 2017;5:e3587.

71. Jahromi MZ, Bello MB, Abdolmaleki M, Yeap SK, Hair-Bejo M, Omar AR. Differential activation of intraepithelial lymphocytenatural killer cells in chickens infected with very virulent and vaccine strains of infectious bursal disease virus. Dev Comp Immunol. 2018;87:116-123. doi:10.1016/j.dci.2018.06.004.

72. Abdolmaleki M, Yeap SK, Tan SW, et al. Effects of newcastle disease virus infection on chicken intestinal intraepithelial natural killer cells. Front Immunol. 2018;9(JUN). doi:10.3389/fimmu.2018.01386.

73. Ganal SC, Sanos SL, Kallfass C, et al. Priming of Natural Killer Cells by Nonmucosal Mononuclear Phagocytes Requires Instructive Signals from Commensal Microbiota. Immunity. 2012;37(1):171-186. doi:10.1016/j.immuni.2012.05.020.

74. Poggi A, Benelli R, Venè R, et al. Human gut-associated natural killer cells in health and disease. Front Immunol. 2019;10(MAY). doi:10.3389/fimmu.2019.00961.

75. Kannaki TR, Reddy MR, Shanmugam M, Verma PC, Sharma RP. Chicken toll-like receptors and their role in immunity. World's Poult Sci J. 2010;66(4):727-738. doi:10.1017/S0043933910000693.

76. Straub C, Neulen M-, Sperling B, et al. Chicken NK cell receptors. Dev Comp Immunol. 2013;41(3):324-333. doi:10.1016/j. dci.2013.03.013.

77. Jansen CA, Van Haarlem DA, Sperling B, et al. Identification of an activating chicken Ig-like receptor recognizing avian influenza viruses. J Immunol. 2016;197(12):4696-4703. doi:10.4049/jimmunol.1600401.

78. Fink LN, Zeuthen LH, Christensen HR, Morandi B, Frøkiær H, Ferlazzo G. Distinct gut-derived lactic acid bacteria elicit divergent dendritic cell-mediated NK cell responses. Int Immunol. 2007;19(12):1319-1327. doi:10.1093/intimm/dxm103.

79. Koizumi S-, Wakita D, Sato T, et al. Essential role of Toll-like receptors for dendritic cell and NK1.1+ cell-dependent activation of type 1 immunity by Lactobacillus pentosus strain S-PT84. Immunol Lett. 2008;120(1-2):14-19. doi:10.1016/j.imlet.2008.06.003.

80. Troy EB, Kasper DL. Beneficial effects of Bacteroides fragilis polysaccharides on the immune system. Front Biosci. 2010;15(1):25-34. doi:10.2741/3603.

81. Bhagat G, Naiyer AJ, Shah JG, et al. Small intestinal CD8+TCRy $\delta+N K G 2 A+$ intraepithelial lymphocytes have attributes of regulatory cells in patients with celiac disease. J Clin Invest. 2008;118(1):281-293. doi:10.1172/JCI30989.

82. Luu M, Weigand $K$, Wedi $F$, et al. Regulation of the effector function of CD8+ T cells by gut microbiota-derived metabolite butyrate. Sci Rep. 2018;8(1). doi:10.1038/s41598-018-32860-x.

83. Chai J-, Lillehoj HS. Isolation and functional characterization of chicken intestinal intra-epithelial lymphocytes showing natural killer cell activity against tumour target cells. Immunology. 1988;63(1):111-117.

84. Gómez Del Moral M, Fonfría J, Varas A, Jiménez E, Moreno J, Zapata AG. Appearance and development of lymphoid cells in the chicken (Gallus gallus) caecal tonsil. Anat Rec. 1998;250(2):182-189. doi:10.1002/(SICI)1097-0185(199802)250:23.0.CO;2-5. 

CHAPTER 4

\section{Glucose oligosaccharide and long-chain glucomannan feed additives induce enhanced activation of intraepithelial NK cells and relative abundance of commensal lactic acid bacteria in broiler chickens}

Nathalie Meijerink ${ }^{1}$, Jean E. de Oliveira², Daphne A. van Haarlem¹, Guilherme Hosotani², David M. Lamot $^{3}$, J. Arjan Stegeman', Victor P.M.G. Rutten ${ }^{1,5}$, Christine A. Jansen ${ }^{1}$

1 Department Biomolecular Health Sciences, Division Infectious Diseases and Immunology, Faculty of Veterinary Medicine, Utrecht University, Utrecht, the Netherlands

2 Cargill R\&D Center Europe, Vilvoorde, Belgium

3 Cargill Animal Nutrition and Health Innovation Center, Velddriel, the Netherlands

4 Department Population Health Sciences, Division Farm Animal Health, Faculty of Veterinary Medicine, Utrecht University, Utrecht, the Netherlands

${ }^{5}$ Department of Veterinary Tropical Diseases, Faculty of Veterinary Science, University of Pretoria, Pretoria, South Africa 


\section{Abstract}

Restrictions on the use of antibiotics in the poultry industry stimulate the development of alternative nutritional solutions to maintain or improve poultry health. This requires more insight in the modulatory effects of feed additives on the immune system and microbiota composition. Compounds known to influence the innate immune system and microbiota composition were selected and screened in vitro, in ovo, and in vivo. Among all compounds, 57 enhanced NK cell activation, 56 increased phagocytosis, and 22 increased NO production of the macrophage cell line HD11 in vitro. Based on these results, availability and regulatory status, six compounds were selected for further analysis. None of these compounds showed negative effects on growth, hatchability, and feed conversion in in ovo and in vivo studies. Based on the most interesting numerical results and highest future potential feasibility, two compounds were analyzed further. Administration of glucose oligosaccharide and long-chain glucomannan in vivo both enhanced activation of intraepithelial NK cells and led to increased relative abundance of lactic acid bacteria (LAB) amongst ileum and caeca microbiota after seven days of supplementation. Positive correlations between NK cell subsets and activation, and relative abundance of LAB suggest the involvement of microbiota in the modulation of the function of intraepithelial NK cells. This study identifies glucose oligosaccharide and long-chain glucomannan supplementation as effective nutritional strategies to modulate the intestinal microbiota composition and strengthen the intraepithelial innate immune system.

Keywords: broiler chickens, innate, adaptive, NK cells, T cells, IELs, microbiota, glucose oligo-saccharide, long-chain glucomannan, in vitro-, in ovo-, in vivo screening 


\section{Introduction}

Restrictions on the use of antibiotics in the poultry industry encourage the development of new strategies to maintain or improve poultry health such as nutritional solutions ${ }^{1,2}$. Nutrients are digested in the intestine, a site of interplay between feed constituents, microbiota, and the immune system, which may all affect the development of the immune system and its function in chickens ${ }^{3-6}$. Nutritional modulation of innate immune responses is likely to be most beneficial for the health of chickens during the first week of life, since a high susceptibility to disease due to an immature adaptive immune system ${ }^{7}$. In addition, the intestinal microbiota is highly dynamic at this age, resulting in a higher susceptibility to intestinal infections, which emphasizes the importance of the innate immune system in young chickens ${ }^{4}$. Identification and use of feed additives that either modulate the immune system directly or indirectly through changes in the microbiota may contribute to improved resistance against pathogens during the early life of chickens.

Immediately post-hatch, the immune competence in chickens depends on maternal antibodies in addition to the innate immune system, of which natural killer (NK) cells and macrophages are key players ${ }^{8,9}$. The adaptive immune system is not fully developed upon hatch and functional $\mathrm{T}$ and $\mathrm{B}$ cell responses are only observed after approximately two to three weeks of life ${ }^{8,9}$. In previous studies in chickens, CD3- IL-2R $\alpha^{+}$NK cells were identified as a major intraepithelial NK-subset in the intestine $\mathrm{e}^{6,10,11}$, and in addition, intraepithelial CD3 ${ }^{-} 205^{+}$NK cells were reported ${ }^{6,11}$. Furthermore, intraepithelial lymphocytes (IELs) also include high numbers of $\gamma \delta \mathrm{T}$ cells and cytotoxic CD8 ${ }^{+} T$ cells ${ }^{10}$. Macrophages, $\mathrm{B}$ cells, and $\mathrm{CD} 4^{+} \mathrm{T}$ cells are located directly underneath the epithelium, the latter two mainly in Peyer's patches ${ }^{12,13}$. Early feed modulation was shown before to increase resistance to pathogens by activation of macrophages and consequently subsequent adaptive reactivity ${ }^{1,14,15}$. Furthermore, both IL-2R $\alpha^{+}$and $20 \mathrm{E} 5^{+}$NK cells were shown to be important in the first response to viral ${ }^{16-18}$ and Salmonella Enteritidis ${ }^{19}$ infections in chickens.

Activation of chicken NK cells is characterized by increased surface expression of CD107 ${ }^{20}$, and activation of macrophages may be assessed by the analysis of phagocytosis s $^{21,22}$ and nitric oxide (NO) production ${ }^{23}$. Enhanced CD107 expression on NK cells is due to degranulation of vesicles containing perforin and granzyme, and results in apoptosis of the infected target cell ${ }^{24}$. Phagocytosis by macrophages leads to direct killing of internalized bacteria and antigen presentation to T cells ${ }^{25}$, whereas NO production induces apoptosis in target cells, resulting in the killing of intracellular pathogens ${ }^{26}$. Analyzing the direct effects of feed constituents on the activation of NK cells and macrophages in vitro will provide preliminary information on the innate immune responsiveness in vivo.

The development of the intestinal innate and adaptive immune system in chickens is influenced by exposure to microbiota, or their metabolites, and feed directly post-hatch ${ }^{27}$. Supplementation of probiotics like Lactobacilli and Bifidobacteria, and prebiotics like yeast and plant polysaccharides have been reported to lead to increased expression of genes implicated in innate and adaptive immune responses in chickens by binding to pathogen-associated molecular patterns (PAMPs) ${ }^{28-31}$. In addition, supplementation of plant polysaccharides affected numbers and cytotoxicity of NK cells, macrophages, $\mathrm{T}$ and $\mathrm{B}$ cells in chickens and humans $\mathrm{s}^{32-42}$. Natural ${ }^{43-46}$ and synthetic ${ }^{47}$ compounds, and immunomodulatory drug $\mathrm{s}^{48}$ have been shown to activate the innate and adaptive immune system, as demonstrated mainly by cytotoxicity assays. Despite many studies on immune modulation by feed compounds, their effects on NK cell subsets and activation have not been investigated in chickens before. Generation of more knowledge on modulatory properties of feed additives affecting innate immune cells will aid in finding feed strategies that increase immune responsiveness during the early life of chickens. 
Modulation of the intestinal microbiota through feed has been studied extensively in chickens $\mathbf{s}^{49,50}$, and positive effects on performance ${ }^{51}$ and health ${ }^{52}$ have been recognized. Pro- and prebiotics ${ }^{53}$, plant and mushroom polysaccharides ${ }^{32}$, and plant extracts ${ }^{54}$ were shown to affect microbial composition and functionality. Nevertheless, combined analyses of feed additives affecting the microbiota and the function of innate immune cells are lacking in chickens.

In the present study, we investigated whether feed supplementation stimulated NK cells either directly or indirectly by modulation of the intestinal microbiota composition in young broiler chickens. A total of 69 potential feed additives were selected based on their characteristic of affecting the innate immune system, the intestinal microbiota, or both. The selection was narrowed down by assessing stimulatory properties on NK cells and macrophages in vitro, followed by assessing possible negative effects on embryonic development and hatch upon in ovo administration, and on performance traits upon in vivo administration. Based on these experiments, availability and regulatory aspects on use, glucose oligosaccharide, and long-chain glucomannan were subsequently investigated for their effect on innate and adaptive immune cells, and the composition of the intestinal microbiota. Our data identified glucose oligosaccharide and long-chain glucomannan as feed additives that positively affect NK cells, an important cell type of the innate immune system, and modulate the intestinal microbiota in broiler chickens. In this way, these feed additives may contribute to improved resistance to infections and hence the health of broiler chickens.

\section{Materials and Methods}

\section{Overall experimental design}

A total of 69 compounds were selected based on their characteristic of affecting innate immunity, microbiota, or both, in chickens or other species either described in the literature or based on earlier studies performed by our industrial collaborator (Cargill Inc., USA). Compounds were screened in vitro for activation of NK cells and macrophages and based on the observed effect on these innate immune cells, their availability in larger quantities and legally approved use in feed, six compounds were subjected to further analyses. Their impact on embryonic development and hatch were assessed following in ovo administration. In addition, their effect on performance traits upon feed supplementation directly post-hatch were studied. Based on the most interesting numerical results of these studies and highest potential to be produced in large scale, two polysaccharides (glucose oligosaccharide and long-chain glucomannan) were selected and supplemented to the diets of broiler chickens directly post-hatch to study their effects on NK cells and microbiota composition until three weeks of age. As glucose oligosaccharide and long-chain glucomannan were selected for testing in the final in vivo experiment, these compounds are highlighted in the in vitro screening alongside compounds showing contrasting effects.

\section{Screening of the effect of compounds on activation of NK cells and macrophages in vitro}

Selected compounds included plant extracts, fermentation products, vitamins, drugs, lipids, fungus extracts, polysaccharides, acid/salts, blend of essential oils and organic acids, yeasts, modified sugars, simple sugars, and emulsifiers (Table S1). Powdered compounds were dissolved in DMSO (Sigma-Aldrich, the Netherlands) before pre-dilutions were made at $10^{3} \mathrm{ppm} / \mathrm{ml}$ in complete RPMI medium (RPMI 1640 GlutaMAX-I supplemented with $10 \%$ heat-inactivated FCS and $50 \mathrm{U} / \mathrm{ml}$ penicillin/streptomycin; Gibco, United Kingdom), which were stored at $4^{\circ} \mathrm{C}$. DMSO diluted 1:5 in complete RPMI medium was used 
as the solvent control in all assays, which is equal to the highest amount of DMSO in the compound solutions. Compounds were added at concentrations of 10,50, and $100 \mathrm{ppm}$ and screened for their effect on NK cell activation using the CD107 assay. Possible effects on the macrophage cell line HD11 were determined by assessing phagocytosis and NO production. Each of the CD107, phagocytosis, and $\mathrm{NO}$ assays were performed in three independent experiments.

\section{NK cell and T cell activation in vitro and in vivo as assessed in the CD107 assay}

To study the possible effects of compounds on the activation of NK cells and cytotoxic CD8 ${ }^{+} \mathrm{T}$ cells, enhanced surface expression of CD107a as a consequence of degranulation was determined by flow cytometry ${ }^{20}$. For the in vitro screening, splenocytes of 14 day-old chicken embryos were isolated as previously described. In these spleens, a population of cells that resemble mammalian NK cells and lack surface expression of T or B cell-specific antigens is abundantly present ${ }^{55}$, which are referred to as NK cells. ED14 NK cells were viably frozen and stored until use at $-140^{\circ} \mathrm{C}$ in complete IMDM medium (IMDM 2 mM Glutamax I supplemented with 8\% heat-inactivated FCS (Lonza, the Netherlands), 2\% heat-inactivated chicken serum, $100 \mathrm{U} / \mathrm{ml}$ penicillin, and $100 \mu \mathrm{g} / \mathrm{ml}$ streptomycin; Gibco) with $50 \%$ FCS and 10\% DMSO, as described before ${ }^{11}$. Frozen ED14 NK cells were thawed and resuspended in complete IMDM medium at $4 \times 10^{6}$ cells $/ \mathrm{ml}$.

Next, $1 \times 10^{6}$ cells in $0.5 \mathrm{ml}$ were incubated with $5 \mu \mathrm{l}(10 \mathrm{ppm}), 25 \mu \mathrm{l}(50 \mathrm{ppm})$, and $50 \mu \mathrm{l}(100 \mathrm{ppm})$ compound solutions in the presence of $1 \mu \mathrm{l} / \mathrm{ml}$ GolgiStop (Beckton Dickinson (BD) Biosciences, the Netherlands) and $0.5 \mu \mathrm{l} / \mathrm{ml}$ mouse-anti-chicken-CD107a-APC during $4 \mathrm{~h}$ at $37^{\circ} \mathrm{C}, 5 \% \mathrm{CO}_{2}$. Complete IMDM medium was used as the negative control, $50 \mu \mathrm{l}$ of 1:5 DMSO as the solvent control, and a combination of $100 \mathrm{ng} / \mathrm{ml}$ phorbol 12-myristate 13-acetate (PMA, Sigma-Aldrich) and $500 \mathrm{ng} / \mathrm{ml}$ lonomycin (Sigma-Aldrich) was used as the positive control. After incubation, cells were washed in PBA (PBS (Lonza) containing $0.5 \%$ bovine serum albumin and $0.1 \%$ sodium azide) and stained with the in vitro CD107 antibody panel (Table 1). Monoclonal antibodies anti-CD3 and -CD41/61 were used to exclude T cells and thrombocytes from the analyses of NK cell activation. Subsequently, cells were washed in PBS, stained with a viability dye, and fluorescence was analyzed by flow cytometry as described in section Phenotypic characterization of IELs and splenic leukocytes by flow cytometry. The fold change in CD107 expression upon incubation with the compounds was expressed relative to the negative control of each sample, which was set at $100 \%$.

In the in vivo experiment, cells were similarly incubated in the presence of Golgistop and CD107a-APC and stained with the in vivo CD107 antibody panel (Table 1). In addition to NK cell activation, T cell activation was analyzed in cells expressing CD3 and CD8 $\alpha$. The percentage of CD107 expression was determined within the total NK or CD8 $\alpha^{+} \mathrm{T}$ cell population. 
Table 1. Flow cytometry staining reagents.

\begin{tabular}{|c|c|c|c|}
\hline Cell Population & $\begin{array}{l}\text { Primary Antibody } \\
\text { (Mouse-Anti-Chicken) }\end{array}$ & Clone/Isotype & Secondary Antibody \\
\hline \multirow{5}{*}{$\begin{array}{l}\text { Peripheral } \\
\text { cells }\end{array}$} & CD45-PE 1 & LT40/lgM & - \\
\hline & Bu-1-FITC 1 & AV20/lgG1 & - \\
\hline & $\mathrm{CD}_{3}-\mathrm{PB}^{1}$ & $\mathrm{CT}-3 / \lg \mathrm{G} 1$ & - \\
\hline & CD4-APC ${ }^{1}$ & $\mathrm{CT}-4 / \mathrm{lgG} 1$ & - \\
\hline & $\mathrm{CD} 8 \alpha-\mathrm{PE} / \mathrm{Cy} 5^{1}$ & $\mathrm{CT}-8 / \mathrm{lgG} 1$ & - \\
\hline \multirow[t]{4}{*}{ NK cells } & CD45-FITC ${ }^{1}$ & LT40/IgM & - \\
\hline & CD3-APC ${ }^{1}$ & СT3/lgG1 & - \\
\hline & IL-2R $\alpha-U N L^{2}$ & $28-4 / \operatorname{lgG} 3$ & Goat-anti-mouse-IgG3-PE ${ }^{1}$ \\
\hline & 20E5-BIOT² & $\lg 1$ & Streptavidin (SA)-PercP5 \\
\hline \multirow[t]{5}{*}{ T cells } & CD3-PE ${ }^{1}$ & CT3/lgG1 & - \\
\hline & CD4-APC ${ }^{1}$ & $\mathrm{CT} 4 / \lg \mathrm{G} 1$ & - \\
\hline & 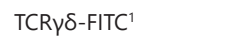 & TCR-1/lgG1 & - \\
\hline & $C D 8 \alpha-U N L^{1}$ & $\mathrm{EP} 72 / \lg \mathrm{g} 2 \mathrm{~b}$ & Goat-anti-mouse-lgG2b-APC/Cy $7^{1}$ \\
\hline & $\mathrm{CD} \beta \beta-\mathrm{BIOT}^{1}$ & $\mathrm{EP} 42 / \lg \mathrm{G} 2 \mathrm{a}$ & SA-PercP5 \\
\hline \multicolumn{4}{|c|}{ Activation of NK and T cells } \\
\hline \multirow{3}{*}{$\begin{array}{l}\text { in vitro } \\
\text { CD107 }\end{array}$} & CD107a-APC ${ }^{3}$ & LEP-100 I 5G10/lgG1 & - \\
\hline & CD41/61-FITC ${ }^{4}$ & $11 \mathrm{C} 3 / \operatorname{lgG} 1$ & - \\
\hline & CD3-PE ${ }^{1}$ & CT3/lgG1 & - \\
\hline \multirow{4}{*}{$\begin{array}{l}\text { in vivo } \\
\text { CD107 }\end{array}$} & CD107a-APC 3 & LEP-100 I 5G10/lgG1 & - \\
\hline & CD41/61-FITC ${ }^{4}$ & $11 \mathrm{C} 3 / \operatorname{lgG} 1$ & - \\
\hline & CD3-PE ${ }^{1}$ & $\mathrm{CT} 3 / \mathrm{lgG} 1$ & - \\
\hline & $C D 8 \alpha-U_{N L^{1}}$ & $\mathrm{EP} 72 / \mathrm{lg} \mathrm{G} 2 \mathrm{~b}$ & $\begin{array}{l}\text { Goat-anti-mouse-lgG2b-Alexa Fluor } \\
\text { (AF) } 790^{6}\end{array}$ \\
\hline
\end{tabular}

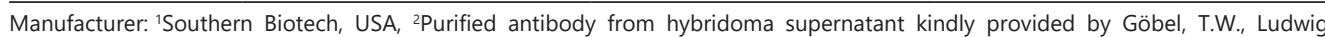
Maximilian University, Germany, ${ }^{3}$ Developmental Studies Hybridoma Bank (DSHB), University of lowa, USA, ${ }^{4}$ Serotec, United Kingdom, ${ }^{5} \mathrm{BD}$ Biosciences, the Netherlands, ${ }^{6}$ Biolegend, USA.

\section{Assessment of the effect of compounds on the phagocytic activity of the macrophage cell line HD11 in vitro}

To determine the effects of the compounds on the activation of macrophage-like HD11 cells, a phagocytosis assay was performed. The chicken HD11 cell line ${ }^{56}$, stored at $-140^{\circ} \mathrm{C}$ in complete RPMI medium with $50 \%$ FCS and $10 \%$ DMSO, was thawed and used after 3 to 20 passages. HD11 cells were cultured in complete RPMI medium in $75-\mathrm{cm}^{2}$ cell culture flasks (Corning B.V., the Netherlands) at $37^{\circ} \mathrm{C}, 5 \% \mathrm{CO}_{2^{\prime}}$ and passaged twice every week. HD11 cells were harvested from cell culture flasks when cells were at $\sim 90 \%$ confluency, using a 0.05\% trypsin/EDTA solution (Gibco). Subsequently, HD11 cells were counted and resuspended in complete RPMI medium at a concentration of $2 \times 10^{5} \mathrm{cell} / \mathrm{s} / \mathrm{ml}$. Cells were seeded at $1 \mathrm{ml} /$ well in 24-well cell culture plates (Corning Costar, the Netherlands) and cultured overnight at $37^{\circ} \mathrm{C}$ and $5 \% \mathrm{CO}_{2}$. After culture, HD11 cells were incubated with 10 (10 ppm), 50 (50 ppm), and $100(100 \mathrm{ppm}) \mu \mathrm{l} /$ well of compound solutions and incubated for 24 hours at $37^{\circ} \mathrm{C}$ and $5 \%$ $\mathrm{CO}_{2}$. As described previously, $1 \mu \mathrm{m}$ crimson red-fluorescent beads (carboxylate-modified FluoSpheres, Invitrogen, the Netherlands) were used as targets for phagocytosis ${ }^{21,22}$. After 24 hours of incubation, $1 \times 10^{7}$ LPS (Sigma-Aldrich)-coated beads ${ }^{21}$ were added to the wells with compound-incubated cells and wells were incubated for another 4 hours at $37^{\circ} \mathrm{C}, 5 \% \mathrm{CO}_{2}$ to allow the cells to engulf the beads. 
The controls included incubation with $100 \mu \mathrm{l} /$ well of complete RPMI medium followed by adding $1 \times$ $10^{7}$ uncoupled beads as the negative control to determine baseline phagocytosis and $100 \mu \mathrm{l} /$ well of 1:5 DMSO, followed by the addition of $1 \times 10^{7}$ LPS-coated beads as the solvent control. Furthermore, incubation with $100 \mu \mathrm{l} /$ well of complete RPMI medium followed by adding $1 \times 10^{7}$ LPS-coated beads was used as the reference control and $100 \mu \mathrm{l} /$ well of complete RPMI medium followed by the addition of $1 \times 10^{7} \lg Y$ (Agrisera $A B$, Sweden)-opsonized beads ${ }^{22}$ as the positive control to determine the highest level of phagocytosis. After the 4 hours of incubation, supernatants were harvested, and the adherent cells were washed twice in PBS at room temperature (RT), followed by harvesting the cells using warm $5 \mathrm{mM}, \mathrm{pH} 8$ UltraPure EDTA (Sigma-Aldrich). HD11 cells were transferred to 96-well U-bottom plates (Greiner Bio-One B.V., the Netherlands), and subsequently washed in PBS, stained for viability, and analyzed by flow cytometry as described in section Phenotypic characterization of IELs and splenic leukocytes by flow cytometry. The percentage of total bead uptake was determined in viable HD11 cells using the gating strategy described in De Geus et al. (2012), which included consecutive selection of the HD11 cell population, viable cells, and total amount of beads. The fold change of bead uptake upon incubation with the compounds was expressed relative to the bead uptake of LPS-coated beads in unstimulated cells. This reference control was set at $100 \%$.

\section{$\mathrm{NO}$ assay to assess activation of the macrophage cell line HD11 in vitro}

Possible effects of compounds on nitric oxide (NO) production by HD11 cells were measured by the Griess assay conducted on the culture supernatant ${ }^{23}$. HD11 cells were cultured, harvested, and resuspended in complete RPMI medium at $2 \times 10^{5} \mathrm{cells} / \mathrm{ml}$ as described for the phagocytosis assay. Cells were seeded at $1 \mathrm{ml} /$ well in 24-well cell culture plates (Corning Costar, Corning Life Sciences B.V., the Netherlands) and cultured overnight at $37^{\circ} \mathrm{C}$ and $5 \% \mathrm{CO}_{2}$. Next, the HD11 cells were incubated with $10(10 \mathrm{ppm}), 50(50 \mathrm{ppm})$, and $100(100 \mathrm{ppm}) \mu \mathrm{l} /$ well of compound solutions and $100 \mu \mathrm{l} /$ well of complete RPMI medium was added as the negative control, $100 \mu \mathrm{l} /$ well of 1:5 DMSO as the solvent control, and $100 \mathrm{ng} / \mathrm{ml}$ of lipopolysaccharides (LPS, Sigma-Aldrich) targeting E. coli O127:B8 as the positive control. After 48 hours incubation at $37^{\circ} \mathrm{C}$ and $5 \% \mathrm{CO}_{2^{\prime}} 50 \mu \mathrm{l}$ of supernatant was harvested and transferred to three individual wells of a 96-well flat-bottom plate (Corning Costar, the Netherlands) for measurement of the nitrite concentration. A 3.13-200 $\mu \mathrm{M}$ nitrite $\left(\mathrm{NaNO}_{2}\right)$ standard dilution series (Sigma-Aldrich, the Netherlands) was included to generate a standard curve. Griess assay reagents were made by dissolving $\mathrm{N}$-(1-naphtyl) ethylenediamine at $3 \mathrm{~g} / \mathrm{l}$ and sulfanilamide at $10 \mathrm{~g} / \mathrm{l}$ (both from Sigma-Aldrich) in 2.5\% phosphoric acid (Supelco, Merck, USA). These reagents were mixed $1: 1$ and $50 \mu \mathrm{l}$ was added to the wells with cell culture supernatants and standards. The plate was then gently shaken in the dark at RT for $10 \mathrm{~min}$ at $700 \mathrm{rpm}$ on a plate shaker (Schüttler MTS 4, IKA, Germany). The Griess reagents mixture turned purple upon reaction with nitrite ions in the cell culture supernatant. Finally, the optical density (OD) of each well was measured at $550 \mathrm{~nm}$ using a FLUOstar Omega microplate reader (BMG Labtech, Germany) and the nitrite concentration of each sample was determined according to the nitrite standard curve. 


\section{Screening of the effect of compounds following in ovo administration}

Possible negative effects of the compounds on embryonic growth, hatchability, and the number of peripheral blood cells were determined. A commercial hatchery (Morren B.V., the Netherlands) supplied 1200 Ross 308 14-day old embryonated eggs, they were weighed and incubated under optimal conditions in the experimental hatchery at the Cargill Animal Nutrition Innovation Center (the Netherlands). At embryonic day (ED) 15, eggs were randomly distributed in a complete randomized block design with six blocks including 20 treatment groups per block and 10 eggs per treatment in each block (replicates). At ED18, eggs were disinfected with alcohol spray, followed by injection of $1 \mathrm{ml}$ of the respective compound solutions into the amniotic fluid using a 23-gauge disposable needle according to Tako et al. (2004). $\mathrm{NaCl}$ saline solutions (0.4\%) contained $0.02 \%, 0.2 \%$, and $2 \%$ of feed additive for the polysaccharides (P1, P2, P8), simple sugar (SS2), modified sugar (MS3), and $0.0015 \mathrm{mg}, 0.0030 \mathrm{mg}$, and $0.0060 \mathrm{mg}$ for the lipid (L1). Eggs that were not injected acted as the negative control group and $0.4 \% \mathrm{NaCl}$ saline solution was injected as the solvent control. At ED19 and ED20, weights of eggs in the different groups were recorded and twelve eggs per group (two per replicate) were randomly selected and sacrificed to determine the weights of the embryo, yolk, and liver to assess embryonic growth by the use of yolk reserves. The relative embryo, yolk, and liver weights were expressed as a percentage of egg weight and used to determine the ratios between relative yolk and liver weight as a parameter of embryonic growth. At ED21 of the hatching period, twelve chicks per group (two per replicate) were randomly selected, weighed, and sacrificed to record weights of the chick, and remaining yolk and liver to assess embryonic growth. Numbers of internally or externally cracked eggs and hatched chicks were recorded and used to determine percentage hatchability. Numbers of unhatched eggs were counted, opened, and classified as dead embryos. In addition, four hatched chicks per group from different blocks were randomly chosen and sacrificed, and blood $(\sim 1 \mathrm{ml})$ was collected in EDTA tubes (VACUETTE ${ }^{\circledR}$ K3E EDTA, Greiner Bio-One, the Netherlands) to determine white blood cell counts. One in ovo screening was performed. The number of replicates per treatment within the in ovo experiment was based on power analysis, which was adjusted for the specific facility and aligned to the 4 Rs to reduce the use of animals in research by having a solid experimental design. For these calculations, we relied on historic data, obtained within the same experimental facility and with similar type of nutritional interventions.

The numbers of lymphocyte subsets were determined as described previously ${ }^{57}$ by staining peripheral blood using BD Trucount ${ }^{\mathrm{TM}}$ Tubes (BD Biosciences, the Netherlands) according to the manufacturer's instructions. First, $200 \mu \mathrm{l}$ of whole blood was fixed by mixing thoroughly with $40 \mu \mathrm{l}$ Transfix ${ }^{\oplus}$ (Thermo Fisher Scientific, the Netherlands), and subsequently diluted 1:50 in PBA. Next, $20 \mu$ l of the monoclonal antibody mix (Table 1) was added to BD Trucount Tubes followed by $50 \mu$ l of diluted blood, and this mixture was incubated for $15 \mathrm{~min}$ in the dark at RT. Subsequently, $450 \mu \mathrm{l} \mathrm{PBA}$ was added to the tubes, and cells and beads were measured by flow cytometry (FACSCANTO II Flowcytometer, BD Biosciences, the Netherlands). Per sample, 20,000 beads were collected and data were analyzed with FlowJo software (FlowJo LCC, BD Biosciences, USA). According to the manufacturer's instructions, cells positive for CD45 (lymphocytes) were gated and within this gate, CD3 negative and BU-1 negative cells (NK cells), BU-1 positive cells (B cells), and CD3 positive cells (T cells) were determined. The absolute number of immune cells per $\mu \mathrm{L}$ blood was calculated using the following formula: (number positive cell events/20,000 (number of beads)) * $(48,550$ (number of beads per test)/ 1 (test volume)).

\section{Screening of the effect of compounds on growth performance in vivo} For the in vivo screening of possible effects on performance traits, 720 one-day-old Ross 308 broiler chicks were obtained from a commercial hatchery (Welp Hatchery, USA), weighed, and randomly 
distributed in a complete randomized block design with 12 blocks including 12 dietary groups per block and five chickens per group in each block (replicates) at the Cargill Animal Nutrition Innovation Center (USA). Chicks were allocated into 144 pens, received water and standard or compound-supplemented commercial starter and grower feeds ad libitum. The 12 diets included standard diet (negative control) or feed supplemented with either of the selected compounds: $0.0625 \%$ Saccharomyces cerevisiae fermentation product (SCFP, positive control; XPC Ultra ${ }^{\mathrm{TM}}$, Diamond V, Cedar Rapids, IA, USA), polysaccharides P1 $(0.02 \%, 0.2 \%)$, P2 $(0.2 \%)$, P5 $(0.02 \%)$, P8 $(0.02 \%, 0.2 \%)$, simple sugars SS2 $(0.2 \%)$, SS2-S $(0.02 \%, 0.2 \%$, Sigma-Aldrich), and modified sugar MS3 $(0.02 \%)$, respectively. Per treatment group $(n=60)$, chickens were weighed at a regular interval and feed intake was measured. Body weights and feed intake were used to calculate average daily gain and feed conversion ratio (FCR), respectively, at days $0,7,13$, and 21 . FCR was calculated by dividing the amount of feed uptake by the growth of the chickens at 0 to 7,7 to 14 , and 14 to 21 days of age. Housing temperature, ventilation, and lighting were according to recommendations for breeder chickens (Aviagen, 2018) and industry standards. All procedures were approved by the Animal Care and Use Committee of Cargill (USA). The in vivo screening was performed once, and the number of replicates incorporated were based on power analysis. The power analysis was adjusted for the specific facility and aligned to the $4 R$ s to reduce the use of animals in research by having a solid experimental design. For these calculations, we relied on historic data, obtained within the same experimental facility and with similar type of nutritional interventions.

\section{In vivo supplementation of glucose oligosaccharide and long-chain glucomannan and their modulatory properties on immune cells and microbiota composition}

For the subsequent in vivo experiment studying the effect of glucose oligosaccharide and long-chain glucomannan on NK cells and microbiota composition, 15 sixteen-, seventeen-, and eighteen-day old embryonated eggs were obtained from a single Ross 308 broiler breeder flock at a commercial hatchery (Lagerwey, the Netherlands). Eggs were disinfected with 3\% hydrogen peroxide and placed in disinfected egg hatchers in one stable at the facilities of the Department of Population Health Sciences, Faculty of Veterinary Medicine, Utrecht University, the Netherlands. The treatment of eggs with a low concentration of hydrogen peroxide does not influence the intestinal microbiota composition, since the development of the intestinal microbiota was not affected by this treatment in a previous study ${ }^{6}$. Directly upon hatch, chickens were weighed, labelled, and housed in one of the three different floor pens according to their feed group, with a solid wall separating the pens. Pens were lined with wood shavings $\left(2 \mathrm{~kg} / \mathrm{m}^{2}\right)$, and water and standard or compound-supplemented commercial starter and grower feeds were provided ad libitum (Research Diet Services, the Netherlands). A standard lighting and temperature scheme for Ross broiler chickens was used. The animal experiments were approved by the Dutch Central Authority for Scientific Procedures on Animals and the Animal Experiments Committee (registration number AVD1080020174425) of Utrecht University (the Netherlands) and all procedures were done in full compliance with all relevant legislation. The in vivo experiment was performed once in which the number of chickens included per group was based on power analysis.

Before the start of the experiment, environmental swabs (FLOQSwabs ${ }^{\oplus}$, COPAN, Italy) were taken of the hatchers and floor pens before hatching as well as of the hatchers after hatching. Swabs were stored at RT in $0.5 \mathrm{ml}$ DNA/RNA Shield (Zymo Research, USA) to determine the microbiota composition of the environment. Directly post-hatch, chicks $(n=15)$ were provided with standard diet (control) or feed supplemented with $0.2 \%$ of either glucose oligosaccharide (P1) referred to as feed 1 (F1), or long-chain glucomannan (P2) referred to as feed 2 (F2). At days 7, 14, and 21, five chickens per 
feed group were randomly selected and sacrificed to collect ileum tissue $( \pm 10 \mathrm{~cm}$ distal from Meckel's diverticulum), spleen, and contents of ileum (distal from Meckel's diverticulum) and caeca for immunology and microbiota analyses. To calculate absolute cell numbers, ileum segments and spleens were weighed immediately after collection of the tissues, prior to isolation of cells. After isolation, cell numbers in the resulting suspension were calculated. This resulted in the total cell number, expressed as IELs per mg ileum or leukocytes per mg spleen. To calculate the absolute numbers of NK and T cells within the live IEL or leukocyte populations, the percentages of cells positive for the markers expressed on these cell types were used, which were determined in the flow cytometry analyses. Absolute cell numbers were calculated using the following formula: (absolute number IELs or leukocytes per mg tissue) $\times$ (percentage positive cells in the gate of interest of the live lymphocyte population). Intestinal contents were collected using sterile plastic cell culture loops, subsequently transferred into $2 \mathrm{ml}$ sterile tubes containing $0.5 \mathrm{ml}$ DNA/ RNA Shield (Zymo Research), and stored at RT for DNA extraction. All chickens were weighed prior to post-mortem analyses to calculate body weight gain over the previous feeding period.

\section{Isolation of immune cells from the intestine and the spleen}

The procedure to isolate IELs was performed as described previously and does not result in contamination with immune cells from the lamina propria ${ }^{11}$. lleum segments were washed with PBS to remove the contents, cut in sections of $1 \mathrm{~cm}^{2}$, and washed again. Subsequently, the IELs were collected by incubating the sections for $15 \mathrm{~min}$ in a shaking incubator at $200 \mathrm{rpm}$ at $37^{\circ} \mathrm{C}$ in EDTA-medium (HBSS 1x (Gibco) supplemented with 10\% heat-inactivated FCS (Lonza) and 1\% 0.5M EDTA (UltraPure ${ }^{\text {TM }}$, Invitrogen, the Netherlands)) after which the supernatants were harvested. This procedure was repeated three times using the remaining tissue sections. Subsequent supernatants containing the IELs were pooled and centrifuged for $5 \mathrm{~min}$ at $1200 \mathrm{rpm}$ at $20^{\circ} \mathrm{C}$ (Allegra ${ }^{\mathrm{TM}} \mathrm{X}-12 \mathrm{R}$ Centrifuge, Beckman Coulter, the Netherlands). Cells were then resuspended in PBS, IELs were isolated using Ficoll-Paque Plus (GE Healthcare, the Netherlands) density gradient centrifugation for $12 \mathrm{~min}$ at $1700 \mathrm{rpm}$ at $20^{\circ} \mathrm{C}$, washed in PBS by centrifugation for $5 \mathrm{~min}$ at $1300 \mathrm{rpm}$ at $4^{\circ} \mathrm{C}$, and resuspended at $4.0 \times 10^{6} \mathrm{cells} / \mathrm{ml}$ in complete IMDM medium. Spleens were homogenized using a $70 \mu \mathrm{m}$ cell strainer (BD Biosciences, the Netherlands) to obtain a single cell suspension. Next, leukocytes were isolated by Ficoll-Paque density gradient centrifugation ( $20 \mathrm{~min}, 2200 \mathrm{rpm}, 20^{\circ} \mathrm{C}$ ), washed in PBS, and resuspended at $4.0 \times 10^{6}$ cells/ $\mathrm{ml}$ in complete IMDM medium as described for IELs. Cell suspensions were analyzed for subsets and activation of NK and T cells as described in the next section and for the CD107 assay, respectively.

\section{Phenotypic characterization of IELs and splenic leukocytes by flow cytometry}

Presence of NK and T cell subsets were determined among IELs and splenic leukocytes at 7, 14, and 21 days of age as described previously ${ }^{11}$. Cell populations $\left(1 \times 10^{6}\right)$ were stained with a panel of antibodies specific for surface markers known to be expressed on NK cells as well as with anti-CD3 to exclude T cells from analysis. In addition, cells were stained with a panel of antibodies specific for surface markers that distinguish $\gamma \delta \mathrm{T}$ cell- and cytotoxic $\mathrm{CD} 8^{+} \mathrm{T}$ cell-subsets (Table 1 ). Staining with primary and secondary antibodies was performed in $50 \mu \mathrm{l}$ PBA. Cells were incubated for $20 \mathrm{~min}$ at $4^{\circ} \mathrm{C}$ in the dark, washed twice by centrifugation for $5 \mathrm{~min}$ at $1300 \mathrm{rpm}$ at $4^{\circ} \mathrm{C}$ in PBA, after primary staining, and in PBS after secondary staining. Subsequently, to be able to exclude dead cells from analysis, cells were stained in $100 \mu$ PBS with a viability dye (Zombie Aqua ${ }^{\mathrm{TM}}$ Fixable Viability Kit, Biolegend, the Netherlands) for 15 min at RT in the dark, washed twice in PBA, and resuspended in $200 \mu \mathrm{l} \mathrm{PBA}$. Of each sample, either $150 \mu \mathrm{l}$ or a maximum of $1 \times 10^{6}$ viable cells were analyzed using a CytoFLEX LX Flow Cytometer (Beckman Coulter), and data were analyzed with FlowJo software (FlowJo LCC, BD Biosciences, USA). 
The gating strategies used to analyze NK cells, $\gamma \delta T$ cells, and cytotoxic CD8 ${ }^{+} T$ cells were described previously, 19 . In short, gating included consecutive selection for lymphocytes (FSC-A vs. SSC-A), singlets (FSC-A vs. FSC-H), viable cells (Live/Dead marker-negative), followed by the selection of specific cellular subsets and upregulation of the activation marker CD107 according to the staining panels. NK cell subsets were gated on $\mathrm{CD}^{-}$cells expressing either IL-2R $\alpha$ or 20E5. To assess NK cell activation, $C D 3^{-} C D 41 / 61^{-}$cells were gated within the live cells and expression of CD107 within this subset was assessed. $T$ cell subsets were gated by selecting $C D 3^{+} C D 4^{-}$cells that were positive for $\mathrm{TCR} \gamma \delta(\gamma \delta)$ or negative $\left(\mathrm{CD} 8^{+} \alpha \beta\right)$ and subsequently, expression of $\mathrm{CD} 8 \alpha \alpha$ and $\mathrm{CD} 8 \alpha \beta$ within both $\gamma \delta$ and $\mathrm{CD} 8{ }^{+} \alpha \beta$ T cells was assessed. To assess T cell activation, $\mathrm{CD} 3{ }^{+} \mathrm{CD} 41 / 61^{-}$were gated within the live cells and subsequently CD8 $\alpha^{+}$T cells were selected in which expression of CD107 was assessed.

\section{Microbiota composition of ileum and caeca}

DNA was purified from ileal and caecal samples using the ZymoBIOMICS DNA Kit according to the manufacturer's instructions (Zymo Research, USA). Bacterial 16S ribosomal RNA genes were then amplified by running one PCR cycle while incorporating a cy-5 fluorescent labeled nucleotide, as described previously for labeling samples in microarray analysis ${ }^{58}$. Labeled PCR amplicons were then hybridized on a microarray chip containing probes for intestinal bacteria previously selected as biomarkers for broiler performance and intestinal health ${ }^{58}$. Microarray annotation for probes included sequential numbers added after bacteria genus or species in order to differentiate more than one probe with the same name. The fluorescence signal of each probe was read using a fluorescence array image reader (Sensovation AG, Germany). Intensity was used as a parameter to determine semi quantitative relative fluorescence values for each probe, which were used to compare relative abundance of each microbiota taxa between feed groups according to the experimental design.

In addition, Pearson's correlations were calculated between immune cells and intestinal microbial taxa that were significantly increased in each feed group. Positive and negative correlation values were reported between the microbial taxa mentioned in Table 2 and NK cell activation and subsets in the IELs and spleen for the respective intestinal segment, diet and age, and depicted in a heatmap. Correlation (r) values from 0 to 1 (positive) and 0 to -1 (negative) are shown, where $0-0.2(0--0.2)$ is interpreted as no/negligible correlation, $0.2-0.5(-0.2--0.5)$ as weak correlation, $0.5-0.8(-0.5--0.8)$ as moderate correlation, and $0.8-1(-0.8--1)$ as strong correlation.

\section{Statistical Analysis}

First, normal distribution of the data was confirmed using the Shapiro-Wilk test. For the in vitro screening, differences in each of the assays between compounds and solvent controls were analyzed using one-way ANOVA. For the in ovo screening, differences in embryonic growth, hatchability, and white blood cell counts between feed groups were analyzed using a mixed-model ANOVA, where all variables were classed as fixed or random effects; diet was used as the fixed effect and block as the random effect. For the in vivo screening on performance traits, differences in performance traits between feed groups were analyzed using a mixed-model ANOVA, where diet was classed as the fixed effect and block as the random effect. For the in vivo experiment with glucose oligosaccharide and long-chain glucomannan, differences in numbers of IELs, splenic leukocytes, NK cell and T cell subsets, and percentages of CD107 expression in the IEL population and spleen between the feed groups were analyzed using one-way ANOVA. Regarding the analysis of microbiota composition, raw fluorescence intensity data for each probe on each microarray chip were compiled and submitted to data quality control. The selected data treatment to reduce chip-to-chip variation was to standardize 
it to the shifting point of three, according to the option available in JMP Genomics, as described previously for microarray analysis ${ }^{59}$. The standardized data were then analyzed using mixed-model ANOVA, where intestinal segment, diet, age, and their three-way interaction were classed as fixed effects and chip was classed as the random effect. Results were used to produce clustering plots utilizing hierarchical clustering, with distances between clusters defined by the Ward's method ${ }^{60}$. Differences in standardized LS means were also used for principal component analysis and volcano plots for pair-wise comparisons. Correlations between immune cells and microbial taxa were analyzed using the Pearson product-moment correlation procedure. The statistical analyses for the in vitro screening and the immunological data of the in vivo experiment were performed using GraphPad Prism 9 software (GraphPad Software, USA). The statistical analyses for the in ovo and in vivo screening were performed using R software version 3.5.1 (The R Foundation for Statistical computing, Austria) and for the microbiota data of the in vivo experiment using JMP Genomics 9 software (SAS Institute 2017, USA). A $p$ value of $<0.05$ was considered statistically significant, a value of $0.05<p<0.1$ was referred to as a trend and in case the $p$ value did not belong to one of these categories; it was referred to as a numerical difference. Microarray standardized LS means of fluorescence intensities were compared using false discovery rate (FDR) adjusted $p$-values set at $<0.05$.

\section{Results}

\section{Compounds are able to induce activation of NK cells and macrophages}

Sixty-nine compounds were screened in vitro for their capacity to induce enhanced CD107 expression on NK cells as well as to stimulate bead uptake and NO production by macrophage-like HD11 cells. Stimulation with 57 compounds resulted in enhanced surface expression of CD107 on NK cells compared to the solvent control (Fig. S1). Viability of NK cells was not affected by stimulation with the compounds in the concentration range that was used (Fig. 1A right panel). Stimulation with compounds P1 and P2 resulted in enhanced CD107 expression at all concentrations (Fig. 1A left panel), with the highest levels of CD107 expression at $10 \mathrm{ppm}$ for both $\mathrm{P} 1(108.0 \pm 7.1 \%)$ and P2 (103.3 $\pm 13.7 \%)$ compared to the solvent control $(87.2 \pm 0.8 \%)$. In contrast, stimulation with compound PE1 resulted in a lower CD107 expression at 50 and $100 \mathrm{ppm}$ compared to the solvent control. Exposure of the macrophage cell line HD11 to 56 out of 69 compounds resulted in increased bead uptake compared to the solvent control (Fig. S2). Stimulation with compound P1 resulted in increased bead uptake at 10 and 50 ppm, whereas increased bead uptake was observed upon stimulation with $\mathrm{P} 2$ at all concentrations compared to the control (Fig. 1B left panel). Phagocytoses were most pronounced with stimulation at $10 \mathrm{ppm}$ for P1 $(115.9 \pm 4.3 \%)$ and $\mathrm{P} 2(116.0 \pm 2.2 \%)$ compared to the solvent control $(86.3 \pm 5.1 \%$, Fig. 1B left panel). In contrast, stimulation with compound PE2 resulted in diminished bead uptake at all concentrations (Fig. 1B left panel). The viability of HD11 cells was not affected by P2, whereas exposure to P1 at 50 and $100 \mathrm{ppm}$ and to PE2 (all concentrations) reduced viability of the cells (Fig. 1B right panel). Exposure to 22 out of 69 compounds increased NO production by macrophage-like HD11 cells compared to the solvent control (Fig. S3). Stimulation with compound P1 increased NO production in all concentrations and highest at $50 \mathrm{ppm}(96.8 \pm 6.8 \mu \mathrm{M})$ compared to no NO production in the presence of the solvent control (Fig. 1C). In contrast to P1, exposure to compound P2 did not result in NO production whereas stimulation with compound L1 led to low NO production at 10 ppm only (Fig. 1C). Out of the compounds that showed positive effects on the activation of NK cells and macrophages, six compounds including P1, P2, P8, SS2, MS3, and L1 were selected and investigated further in the in ovo screening. 
A

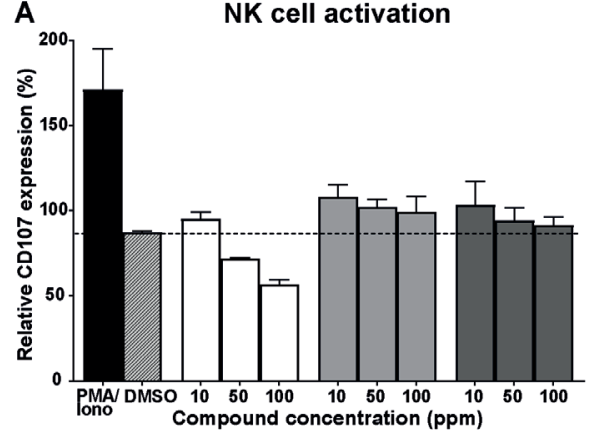

B

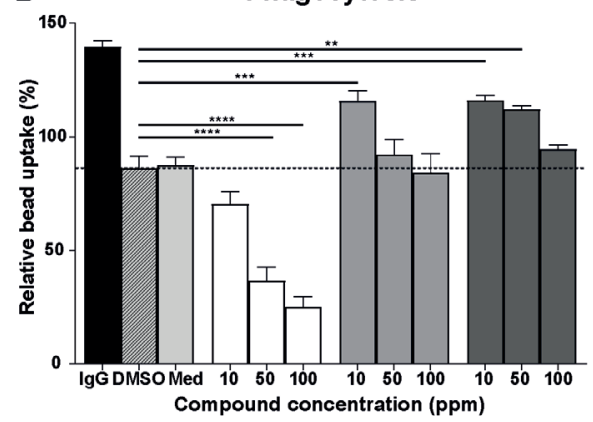

C

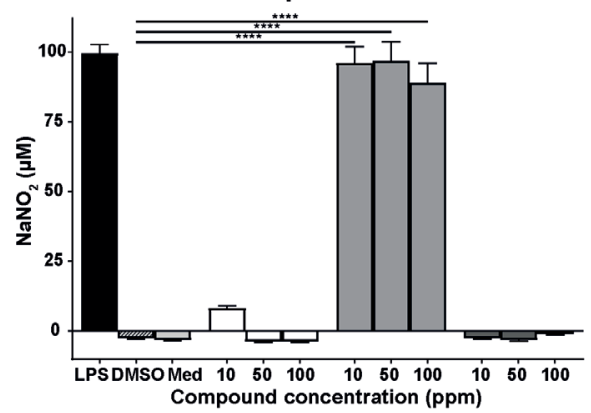

Viability NK cells

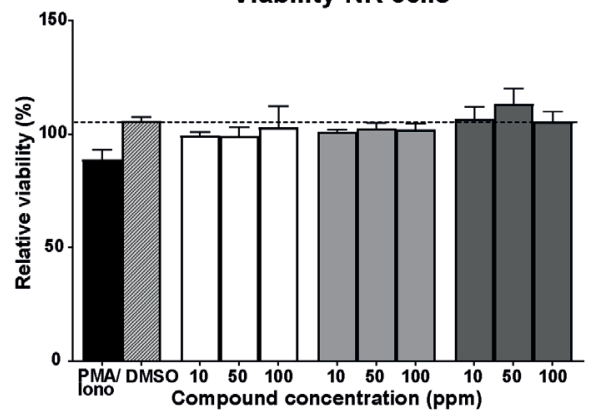

믈 $P$ 1
담

Viability HD11 cells

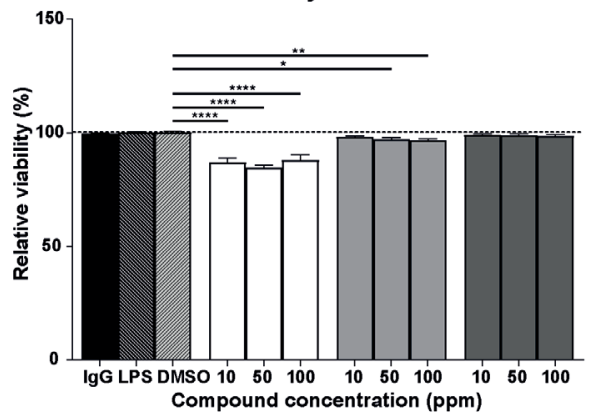

品

Figure 1. In vitro screening of feed compounds for their effect on the activation of NK cells and macrophages. (A) The effect of compounds on NK cell activation measured by the CD107 expression on NK cells (\%). Expression of CD107 is expressed relative to the negative control, which was set at 100\% (left panel). Viability of ED14 NK cells after exposure to compounds, which is expressed relative to the negative control set at 100\% (right panel). PMA/lonomycin was included as the positive control, 1:5 DMSO as the solvent control, and $n=3$ per compound concentration. (B) The effect of compounds on the phagocytosis of macrophage-like HD11 cells (\%). Phagocytosis is expressed relative to the reference control, which was set at $100 \%$ (left panel). Viability of HD11 cells after exposure to compounds, which is expressed relative to the negative control set at $100 \%$ (right panel). IgY-coated beads are included as positive control to determine the highest level of phagocytosis, LPS-coated beads in 1:5 DMSO as the solvent control, and uncoupled beads in complete RPMI medium as the negative control to determine baseline phagocytosis, $n=3$ per compound concentration. (C) The effect of compounds on the NO production of macrophage-like HD11 cells by measuring nitrite concentration ( $\mu$ M). LPS stimulation was included as the positive control, 1:5 DMSO as the solvent control, complete RPMI medium as the negative control, and all samples were in triplicates, $\mathrm{n}=9$ per compound concentration. Mean + SEM is shown of the compounds of polysaccharides (P1, P2), plant extracts (PE1, PE2), and lipids (L1); the dotted line represents the level of the solvent control and statistical significance is indicated as ${ }^{*} p<0.05,{ }^{* *} p<0.01,{ }^{* *} p<0.001$, and ${ }^{* * *} p<0.0001$. 


\section{In ovo administration of the selected compounds showed no adverse effects on embryonic development and hatching}

Next, polysaccharides P1, P2, P8, simple sugar SS2, modified sugar MS3, and lipid L1 were injected into the amniotic fluid of ED18 chicken eggs and possible negative impact on embryonic growth, hatchability of eggs, and peripheral immune cell numbers in the chickens immediately post-hatch were determined. In ovo injection of compounds did not give rise to differences in relative yolk utilization and liver growth compared to the controls during embryonic development (Fig. 2A). Injection of the compounds did not affect hatchability except for MS3 administered at a concentration of $2.0 \%$, which decreased hatchability to $67.3 \pm 13.4 \%$ compared to $100 \%$ of the controls (Fig. $2 \mathrm{~B}$ ). No differences in numbers of lymphocytes, NK, B and T cells in the blood were observed in chicks from eggs that were injected with the compounds compared to chicks that hatched from eggs that received the solvent control (Fig. 2C and Fig. S4). Next, the effect of five out of these six compounds on performance traits was investigated. In this study, compound L1 was excluded since a practical route of administration to broiler chickens in vivo was not available.

A

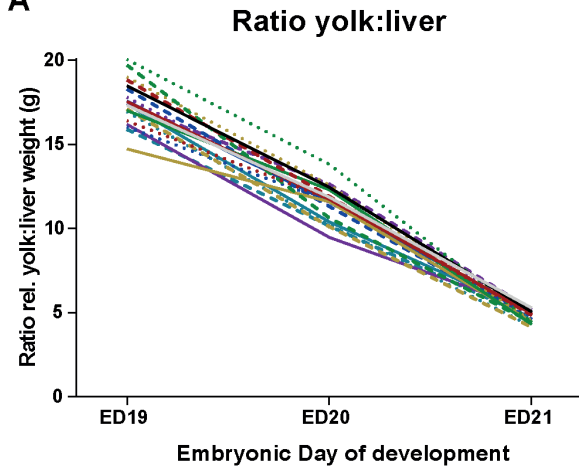

B

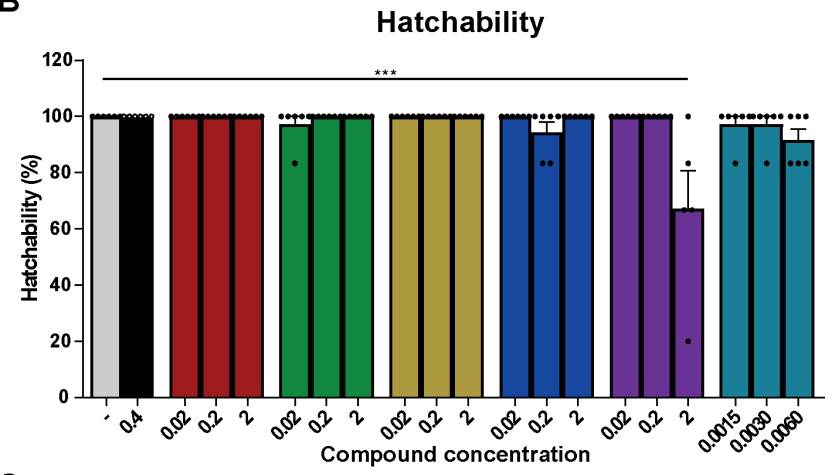

Lymphocytes

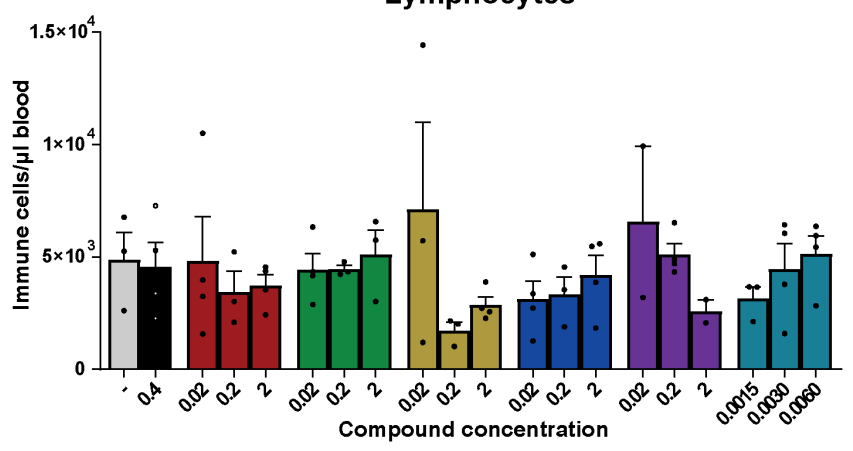

Figure 2. In ovo screening of feed compounds for their effect on embryonic growth, hatching, and peripheral lymphocyte numbers. (A) Effect of compound injections in embryos on the ratio of relative yolk and liver weight during late embryonic development, $\mathrm{n}=6$ per group per ED. (B) Effect of compound injections on hatchability (\%) at ED21, $n=6$ per group. (C) Effect of compound injections on the numbers of lymphocytes in whole blood in chicks immediately post-hatch. Non-injected eggs were included as the negative control and $0.4 \%$ saline solution as the solvent control, $n=4$ per group. Mean + SEM is shown of compounds of polysaccharides ( $P 1$, P2, P8), simple sugars (SS2), modified sugars (MS3), and lipids (L1) in different concentrations $(0.02,0.2,2 \%$, or $0.0015,0.0030,0.0060$ $\mathrm{mg}$ ) and statistical significance is indicated as ${ }^{* \star} p<0.001$. 


\section{No negative effects were observed on performance traits upon in vivo administration}

To determine possible negative effects on growth performance, diets supplemented with these five compounds were fed to broiler chickens from 0 days of age onwards. Two additional compounds, polysaccharide P5 and simple sugar SS2-S, were included in this study. These compounds are structurally related to P2 and SS2, respectively, and have been shown to enhance NK cell and macrophage activation in prior in vitro assays. No differences in body weight were observed among feed groups at $0,7,13$, and 21 days of age (Fig. S5A). Furthermore, no differences in FCR as a result of the supplemented feeds compared to the standard diet (negative control) were observed (Fig. S5B). Comparisons of the feed additives with the positive control, which was feed supplemented with $0.0625 \%$ SCFP, showed that the FCR from 7 to 13 and 13 to 21 days of age of chickens in the P8 $0.2 \%$ group was significantly higher compared to the positive control $(1.34 \pm 0.01$ vs. $1.22 \pm 0.01$ and $1.28 \pm 0.01$ vs. $1.16 \pm 0.01$, respectively). A similar observation was made when comparing the P8 $0.2 \%$ group with three other feed additives (SS2 0.2\%; P1 0.02\%; MS3 0.02\%) during these age periods, while the FCR of chickens in the other groups were in between the FCR of P8 $0.2 \%$ and the three other feed additives (Fig. S5B). None of the feed additives showed significant adverse effects on growth performance. P1 and P2 were the most favorable to select based on the numerical results of chicken body weight and FCR, and highest future potential feasibility. These two polysaccharides were studied in subsequent in vivo analyses for possible effects on immune cells and microbiota composition.

\section{Glucose oligosaccharide and long-chain glucomannan significantly increase activation of intraepithelial NK cells seven days after in vivo supplementation}

Growth performance of broiler chickens was not affected by the administration of the supplemented feeds compared to the control diet (Fig. S6A). Furthermore, no significant differences were observed in numbers of IELs (Fig. S6B) and splenic leukocytes (Fig. S6C) of chickens in the supplemented feed groups compared to the standard diet group. Nevertheless, at 7 days of age, IELs were numerically higher in chickens that received feed containing long-chain glucomannan (F2, $\left.8.3 \times 10^{4} \pm 2.6 \times 10^{4}\right)$ compared to the control group $\left(4.4 \times 10^{4} \pm 6.0 \times 10^{3}\right.$, Fig. S6B). Next, NK cell subsets and NK cell activation were assessed in the IEL population and spleen (Fig. 3A). At 7 days of age, intraepithelial IL-2R $\alpha^{+}$NK cells were numerically lower in chickens of the $\mathrm{F} 1$ group and $20 \mathrm{E5}{ }^{+} \mathrm{NK}$ cells were numerically higher in the F2 group compared to the control group, however, no significant differences were observed (Fig. 3B,D). Significantly enhanced CD107 expression on intraepithelial NK cells was observed at 7 days of age in both F1 and F2 groups, which was $21.3 \pm 2.2 \%$ for F1 and $25.9 \pm 1.7 \%$ for F2, compared to the control group $(10.89 \pm 1.1 \%$, Fig. $3 F)$. In the spleen at day 14 , IL-2R $\alpha^{+}$NK cells were numerically lower in the $F 1$ group and 20E5+ NK cells were numerically higher in the F2 group compared to the control group, but no significant differences were observed (Fig. 3C,E). Expression of CD107 on splenic NK cells was similar in chickens receiving one of the supplemented feeds compared to chickens that received a standard diet during aging (Fig. 3G).

Furthermore, possible effects of the feed additives on the numbers of $T$ cell subsets were analyzed (Fig. 4A). Numbers of $\gamma \delta T$ cells and CD8 ${ }^{+} T$ cells were not significantly different between the $F 1$ and F2 feed regimens compared to the standard diet in both the IEL population (Fig. 4B,D) and spleen (Fig. $4 C, E)$. Nevertheless, numerical differences were observed at all ages in $\gamma \delta T$ cells and $C D 8^{+} T$ cells in the F1 and F2 groups compared to the control group in IELs (Fig. 4B,D) and spleen (Fig. 4C,E). Likewise, no significant differences were observed in numbers of $\gamma \delta T$ cells and $C D 8^{+} T$ cells expressing either CD8 $\alpha \alpha$ or $C D 8 \alpha \beta$ (Fig. S7). Expression of CD107 on intraepithelial CD8 ${ }^{+}$T cells (including both $\gamma \delta$ and 
$\alpha \beta$ T cells) was numerically increased in the F2 group at day 14 and 21 compared to the control group (Fig. S8A). In the spleen, CD107 expression on CD8 ${ }^{+} \mathrm{T}$ cells was numerically increased in the $\mathrm{F} 2$ group at 7 days of age compared to the control group (Fig. S8B).

A

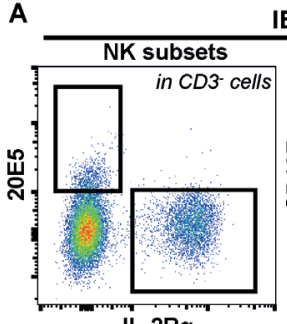

IL-2Ra
IELS

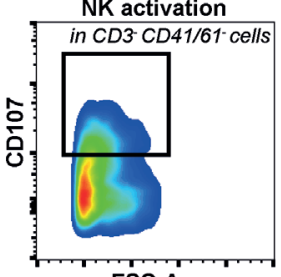

FSC-A

B

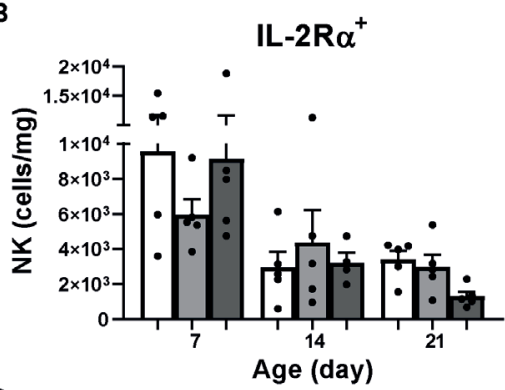

D

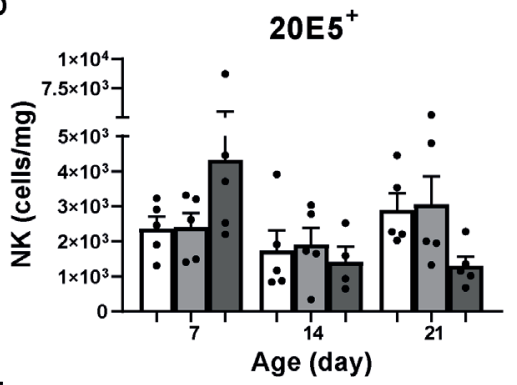

F

CD107 $^{+}$

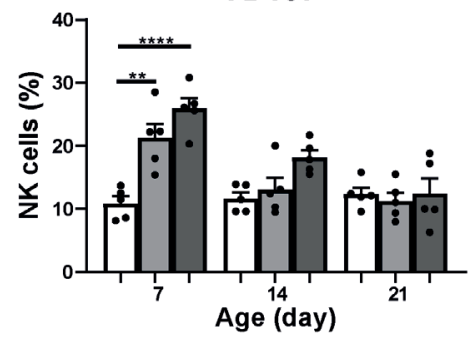

Spleen

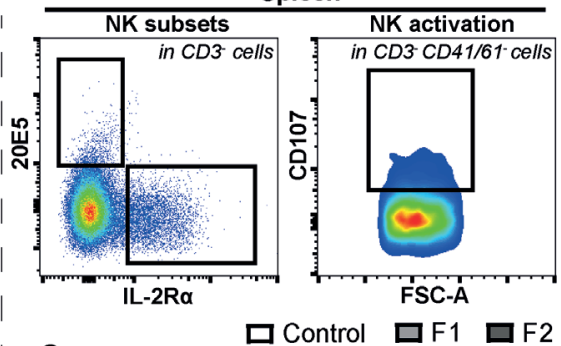

C

\section{IL-2R $\alpha^{+}$}

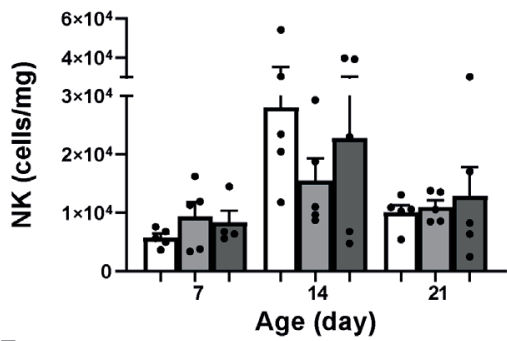

E
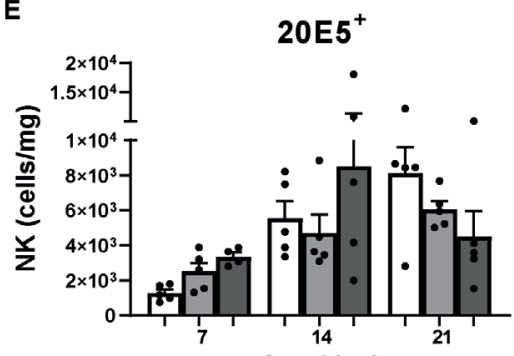

G

Age (day)

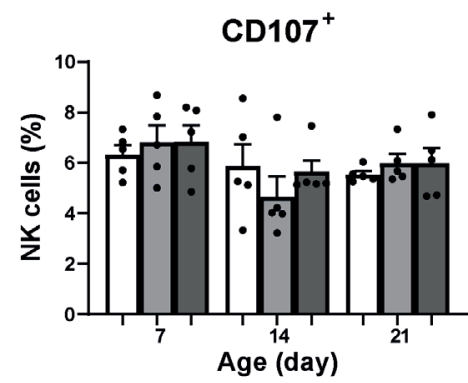

Figure 3. Effect of feeding glucose oligosaccharide and long-chain glucomannan to broiler chickens on NK cell subsets and NK cell activation. (A) CD3 negative cells expressing either IL-2R $\alpha$ or 20E5, and CD3 and CD41/61 negative cells expressing CD107 in the IEL population (left panel) and spleen (right panel). (B) Numbers (cells/mg) of intraepithelial IL-2R $\alpha^{+}$, (D) 20E5+ NK cells, and (F) percentages of NK cells expressing CD107 in broiler chickens provided different diets in the course of time. (C) Numbers (cells/mg) of splenic IL-2R $\alpha^{+}$, (E) 20E5 NK cells, and (G) percentages of NK cells expressing CD107 in broiler chickens provided different diets in the course of time. Mean + SEM is shown $(n=5)$ of chickens provided standard diet (control), feed supplemented with glucose oligosaccharide (F1) or long-chain glucomannan (F2) and statistical significance is indicated as ${ }^{* *} p<0.01$ and ${ }^{* * *} p<0.0001$. 
A

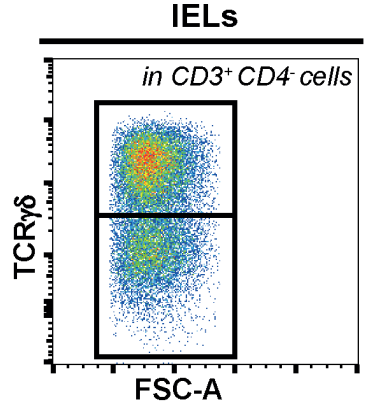

B

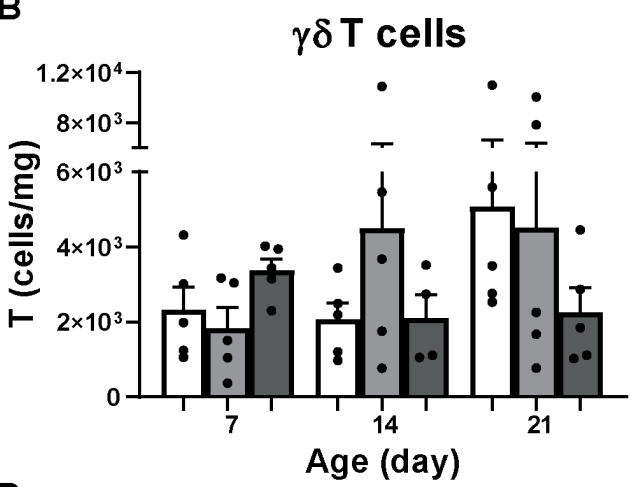

D
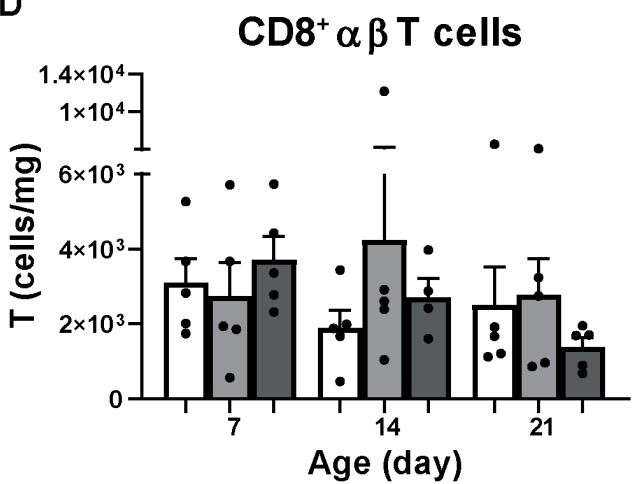

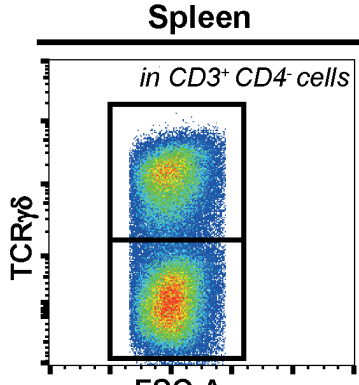

$\gamma \delta \mathrm{T}$ cells

CD8 $8^{+}$

$\alpha \beta$ T cells

C

E
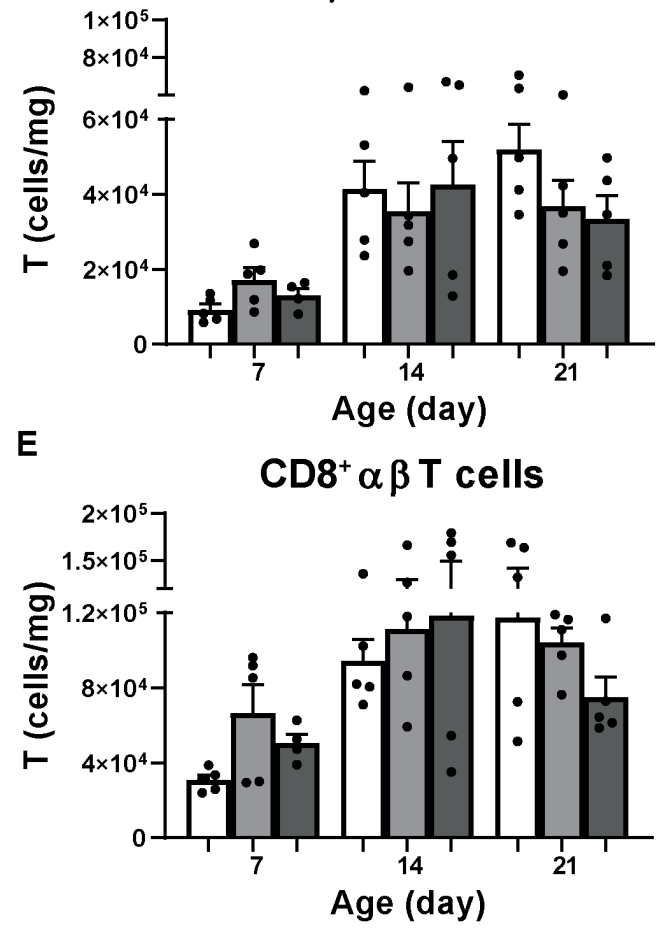

Figure 4. Effect of feeding glucose oligosaccharide and long-chain glucomannan to broiler chickens on T cell subsets. (A) CD3 positive and CD4 negative cells that are TCR $\gamma \delta$ positive ( $\gamma \delta$ T cells) or negative (CD8 ${ }^{+} \alpha \beta T$ cells) in the IEL population (left panel) and spleen (right panel). (B) Numbers (cells/mg) of intraepithelial $\gamma \delta T$ cells and (D) CD8+ $\alpha \beta$ T cells in broiler chickens fed with different diets in the course of time. (C) Numbers (cells/mg) of splenic $\gamma \delta$ T cells and (E) CD8 ${ }^{+} \alpha \beta$ T cells in broiler chickens fed with different diets in the course of time. Mean + SEM is shown $(n=5)$ of chickens provided standard diet (control), feed supplemented with glucose oligosaccharide (F1), or long-chain glucomannan (F2).

In vivo supplementation of glucose oligosaccharide and long-chain glucomannan led to increased relative abundance of lactobacillus species in the intestinal microbiota

Microbial analysis revealed that a total of 98 bacterial taxa identified by the probes were significantly different between the three feed groups among all tested factors (intestinal segment, age, diet, 
and their three-way interaction). By factorial analysis of the main effects, most of these significant differences were due to intestinal segment (78 bacterial taxa), followed by age (66 bacterial taxa), and diet (10 bacterial taxa). Principal component analysis (Fig. 5) confirmed that microbiota profiles grouped first by caeca (left) and ileum (right) and subsequently by age (7, 14, and 21 days of age), however, grouping by age was affected by feed treatment (Control, F1, or F2). By factorial analysis of the three-way interaction; effects between feed groups at different ages in the ileum or caeca, F1 supplementation significantly increased the relative abundance of Lactobacillus species ( $L$. reuteri, $L$. crispatus, and L. gasseri) in both ileum and caeca at 7 and 14 days of age, compared to the control group (2nd column Table 2, Fig. 6 and Fig. S9). The significantly increased intestinal bacteria of the F2 group compared to the control group were more variable in taxa in the intestinal segments and during aging, ranging from increased relative abundance of L. crispatus and Bifidobacterium in the ileum at 7 days of age and more proteolytic bacteria like E. coli and Serratia marcescens in the caeca at that same age, to increased relative abundance of Lachnospiraceae in the caeca at 14 days and L. panis in the ileum at 21 days of age ( 3 rd column Table 2, Fig. 6 and Fig. S9). The significant differences in microbiota composition between F1 and F2 were more evident in chickens at 7 days of age, whereas microbiota profiles of chickens in both feed groups were more similar at days 14 and 21 (4th column Table 2, Fig. 6 and Fig. S9). At 7 days of age, F1 was associated with significantly higher relative abundance of $L$. reuteri in the ileum and Ruminococcus and L. reuteri in the caeca (4th column Table 2, Fig. 6). In comparison, F2 was associated with significant higher relative abundance of L. crispatus, L. jenseni, and Enterococcus taxa in the ileum, while in the caeca to a variety of more strictly anaerobic bacteria such as Faecalibacterium, Lachnospiraceae, D. formicigenerans, S. marcescens, and B. gallinarum (4th column Table 2, Fig. 6). In addition, the significantly increased relative abundance of Lactobacillus species in the ileum and caeca were higher in the F1 compared to F2 group at 7 days of age (Fig. 6), 14, and 21 (Fig. S9). Pathogenic bacteria were found only in the caeca at 14 days of age; significant higher relative abundance of Salmonella was observed in the intestinal microbiota of chickens in the control group and similar relative abundance of $C$. jejuni compared to the F2 group, although F2 was associated with higher relative abundance of $C$. jejuni compared to F1 (4th column Table 2, Fig. S9).

\section{Positive correlations between NK cell activation and lactic acid bacteria upon in vivo supplementation by glucose oligosaccharide and long-chain glucomannan}

As supplementation of the polysaccharides affected NK cells and microbiota composition, we analyzed whether these effects were related by performing a Pearson's correlation analysis. At 7 days of age, strong positive correlations (0.8-1.0) were observed between CD107 expression on splenic NK cells and Lactobacillus crispatus 2 and 3 in the ileum microbiota of chickens in the control group (Fig. 7A). In addition, strong negative correlations (-0.8--1.0) were found between CD107 expression on splenic NK cells and Lactobacillus 3 and Lactobacillus reuteri 1, splenic IL-2R $\alpha^{+}$NK cell numbers, and Clostridium bartletii 1 and 2 as well as splenic 20E5+ NK cell numbers and Lactobacillus jenseni and Lactobacillus crispatus 3 in the ileum microbiota of chickens in the control group at day 7 (Fig. 7A). In comparison, in the F1 group, strong positive correlations were observed between CD107 expression on intraepithelial NK cells and Lactobacillus 3 and Clostridium bartletii 2, intraepithelial IL-2R $\alpha^{+}$NK cell numbers and Clostridium bartletii 1 as well as CD107 expression on splenic NK cells and Bifidobacterium 2 and Lactobacillus crispatus 3 (Fig. 7A). In the F2 group, a strong negative correlation was found between intraepithelial IL-2R $\alpha^{+}$NK cell numbers and Bifidobacterium 2 and a strong positive correlation between intraepithelial 20E5+ NK cell numbers and Clostridium bartletii 2 in the ileum microbiota of chickens at 7 days of age (Fig. 7A). In addition, strong positive correlations were observed between numbers of IL-2R $\alpha^{+}$NK cells in the spleen and Enterococcus, Lactobacillus 3, Lactobacillus reuteri 1, and Lactobacillus 


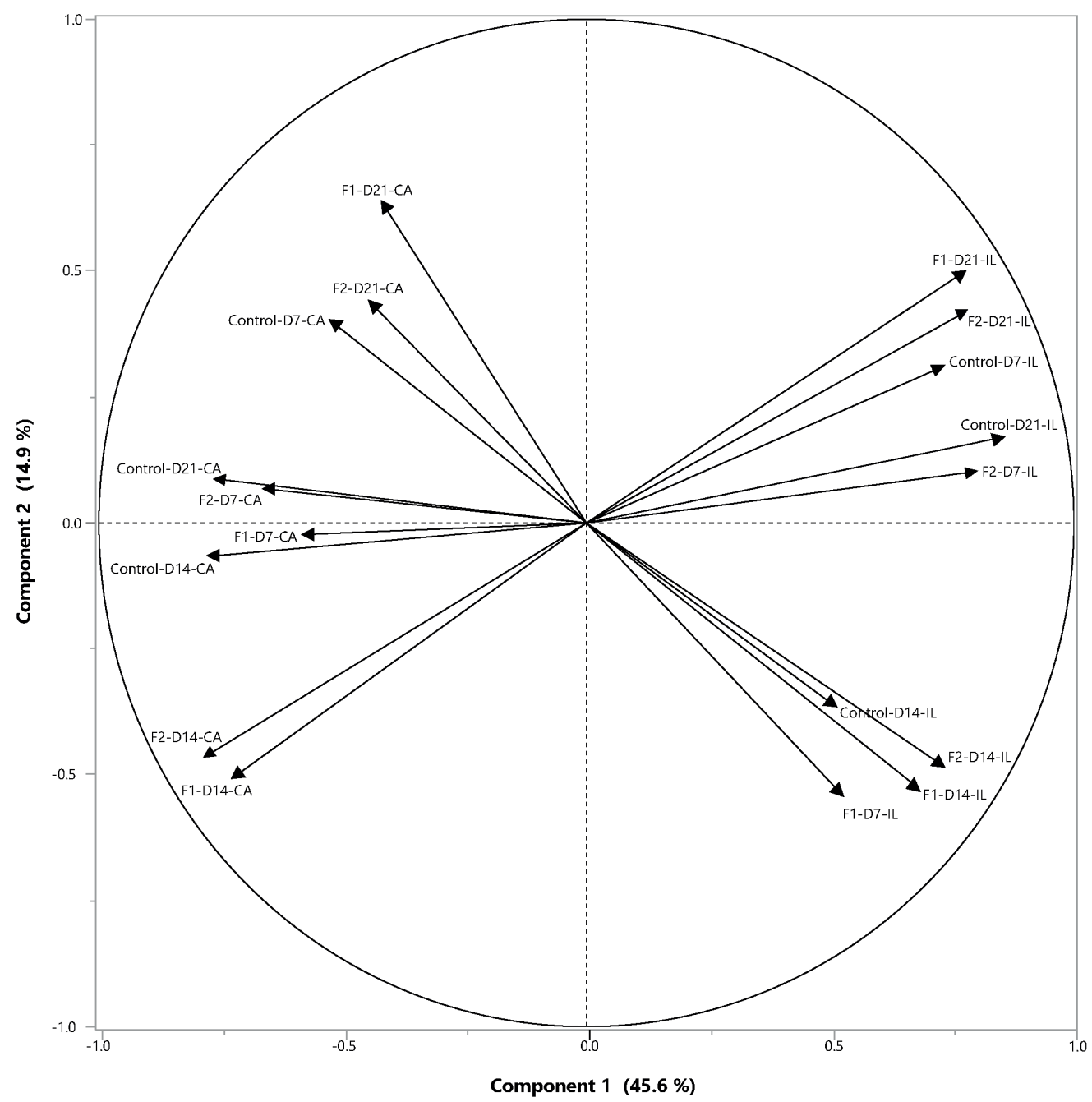

Figure 5. Effect of feeding glucose oligosaccharide and long-chain glucomannan to broiler chickens on microbiota composition in ileum and caeca. Principal component analysis of microbiota profiles from the ileum (IL) and caeca (CA) at day 7, 14, and 21 (D7, D14, and D21) of broiler chickens provided standard diet (control), feed supplemented with glucose oligosaccharide (F1), or long-chain glucomannan (F2). Per intestinal segment, age, and feed group $n=5$.

crispatus 3, whereas a strong negative correlation was seen with Clostridium bartletii 1 in the ileum microbiota of chickens in the F2 group at 7 days of age. Furthermore, strong positive correlations were observed between splenic 20E5 ${ }^{+}$NK cell numbers and Enterococcus and Lactobacillus crispatus 3 in the ileum microbiota of chickens in the F2 group at 7 days of age (Fig. 7A). No, weak, or moderate correlations were observed between CD107 expression on NK cells and numbers of NK cell subsets in the IEL population and spleen and the relative abundance of microbial taxa due to the respective feed in the caeca at 7 days of age (Fig. 7B) and in the ileum and caeca at 14 days of age (Fig. S10A) and 21 days of age (Fig. S10B). 
Table 2. Intestinal microbial taxa that are significantly increased with the respective feed at different ages in broiler chickens. The microbial taxa of which the standardized LS means were significantly increased due to the respective feed, as determined by factorial analysis of pairwise comparisons between feed groups at different ages in the ileum or the caeca. Feed groups included standard diet (control), and feed supplemented with glucose oligosaccharide (F1) or long-chain glucomannan (F2), with statistical significance of FDR adjusted p-values set at $<0.05$.

\begin{tabular}{|c|c|c|c|}
\hline Age/Intestinal Segment & Control vs. F1 & Control vs. F2 & F1 vs. F2 \\
\hline 7 days/ileum & $\begin{array}{c}\text { C: Clostridium bartletii } 1 \\
\text { and 2, Lactobacillus jenseni, } \\
\text { Bifidobacterium 2, Enterococcus } \\
\text { sp.i } \\
\text { F1: Lactobacillus reuteri 1, } \\
\text { Lactobacillus 3; }\end{array}$ & $\begin{array}{c}\text { C: Clostridium bartletii } 1 \text { and 2; } \\
\text { F2: Lactobacillus crispatus 2, } \\
\text { Bifidobacterium 2; }\end{array}$ & $\begin{array}{c}\text { F1: Lactobacillus 3, Lactobacillus } \\
\text { reuteri 1; } \\
\text { F2: Lactobacillus crispatus 2, } \\
\text { Lactobacillus crispatus 3, Lactobacillus } \\
\text { jenseni, Enterococcus sp.; }\end{array}$ \\
\hline 7 days/caeca & $\begin{array}{c}\text { C: Lachnospiraceae Incertae } \\
\text { Sedis 6, Dorea formicigen- } \\
\text { erans, Fusobacterium group 2, } \\
\text { Sanguibacter; } \\
\text { F1: Lactobacillus reuteri 1, } \\
\text { Lactobacillus 3, Lachnospiraceae } \\
\text { Incertae Sedis 4, Ruminococcus sp.; }\end{array}$ & $\begin{array}{c}\text { C: Ruminococcus sp.; } \\
\text { F2: E. coli 1, E. coli 2, Serratia } \\
\text { marcescens; }\end{array}$ & $\begin{array}{l}\text { F1: Ruminococcus Incertae Sedis } \\
\text { siraeum, Ruminococcaceae } \\
\text { unclassified, Lactobacillus } 3, \\
\text { Lactobacillus reuteri } 1 ; \\
\text { F2: Faecalibacterium 1, } \\
\text { Lachnospiraceae Incertae Sedis 6, } \\
\text { Lachnospiraceae unclassified, Dorea } \\
\text { formicigenerans, Serratia marcescens, } \\
\text { Bifidobacterium gallinarum; }\end{array}$ \\
\hline 14 days/ileum & $\begin{array}{c}\text { C: Lachnospiraceae Incertae Sedis } \\
\text { 2, Bacteroides uncult, Listeria; } \\
\text { F1: Lactobacillus reuteri 1, } \\
\text { Lactobacillus 3; }\end{array}$ & $\begin{array}{l}\text { C: Lachnospiraceae Incertae } \\
\text { Sedis 2, Bacteroides uncult, } \\
\text { Clostridium bartletii 2; } \\
\text { F2: none }\end{array}$ & None \\
\hline 14 days/caeca & $\begin{array}{l}\text { C: Ruminococcus unclassified, } \\
\text { Clostridiales unclassified, Incertae } \\
\text { Sedis XIII unclassified; } \\
\text { F1: Lactobacillus sp., Lactobacillus } \\
\text { gasseri 2, Lactobacillus crispatus 3; }\end{array}$ & $\begin{array}{l}\text { C: Salmonella 1, Parabacteroides } \\
\text { 2, Rikenellaceae Alistipes 1, } \\
\text { Salinococcus, Clostridiales } \\
\text { unclassified, Incertae Sedis XIII } \\
\text { unclassified; } \\
\text { F2: Lachnospiraceae Incertae } \\
\text { Sedis 3; }\end{array}$ & $\begin{array}{c}\text { F1: Lachnospiraceae Incertae Sedis 11; } \\
\text { F2: Campylobacter jejuni; }\end{array}$ \\
\hline 21 days/ileum & None & $\begin{array}{c}\text { C: Lactobacillus crispatus 2, } \\
\text { Bifidobacterium 2, Enterococcus } \\
\text { hirae; } \\
\text { F2: Lactobacillus panis; }\end{array}$ & None \\
\hline 21 days/caeca & $\begin{array}{c}\text { C: Lachnospiraceae unclassified, } \\
\text { Ruminococcus Incertae Sedis; } \\
\text { F1: none }\end{array}$ & $\begin{array}{c}\text { C: Lachnospiraceae unclassified, } \\
\text { Ruminococcus Incertae Sedis, } \\
\text { Citrobacter; } \\
\text { F2: Bacteria unclassified; }\end{array}$ & $\begin{array}{l}\text { F1: Agreia; } \\
\text { F2: none }\end{array}$ \\
\hline
\end{tabular}

\section{Discussion}

Currently, feed additives supplemented to the diet to improve growth performance of broiler chickens with the additional benefit of modulation of immune responsiveness or intestinal microbiota composition are of high interest.

The stimulatory effects of glucose oligosaccharide and long-chain glucomannan on the activation of NK cells and macrophages in vitro was in agreement with the reported immunomodulatory properties of plant-based polysaccharides observed in humans and chickens ${ }^{32,45}$. Furthermore, embryonic development, hatchability, and numbers of peripheral immune cells at day of hatch were not affected adversely by in ovo application of both polysaccharides, which indicates that they are safe for use as feed supplements. Moreover, this safety opens the option of in ovo application of both polysaccharides in addition to or instead of supplementation in the feed, to be investigated in future experiments. In the present study, in ovo application of these polysaccharides did neither affect the normal reduction of yolk sac reserves nor increase of liver weight ${ }^{61}$, whereas a higher reduction would have suggested an improved metabolic rate of the developing embryo ${ }^{60,62}$. This may be due to the low digestibility of the polysaccharides in 


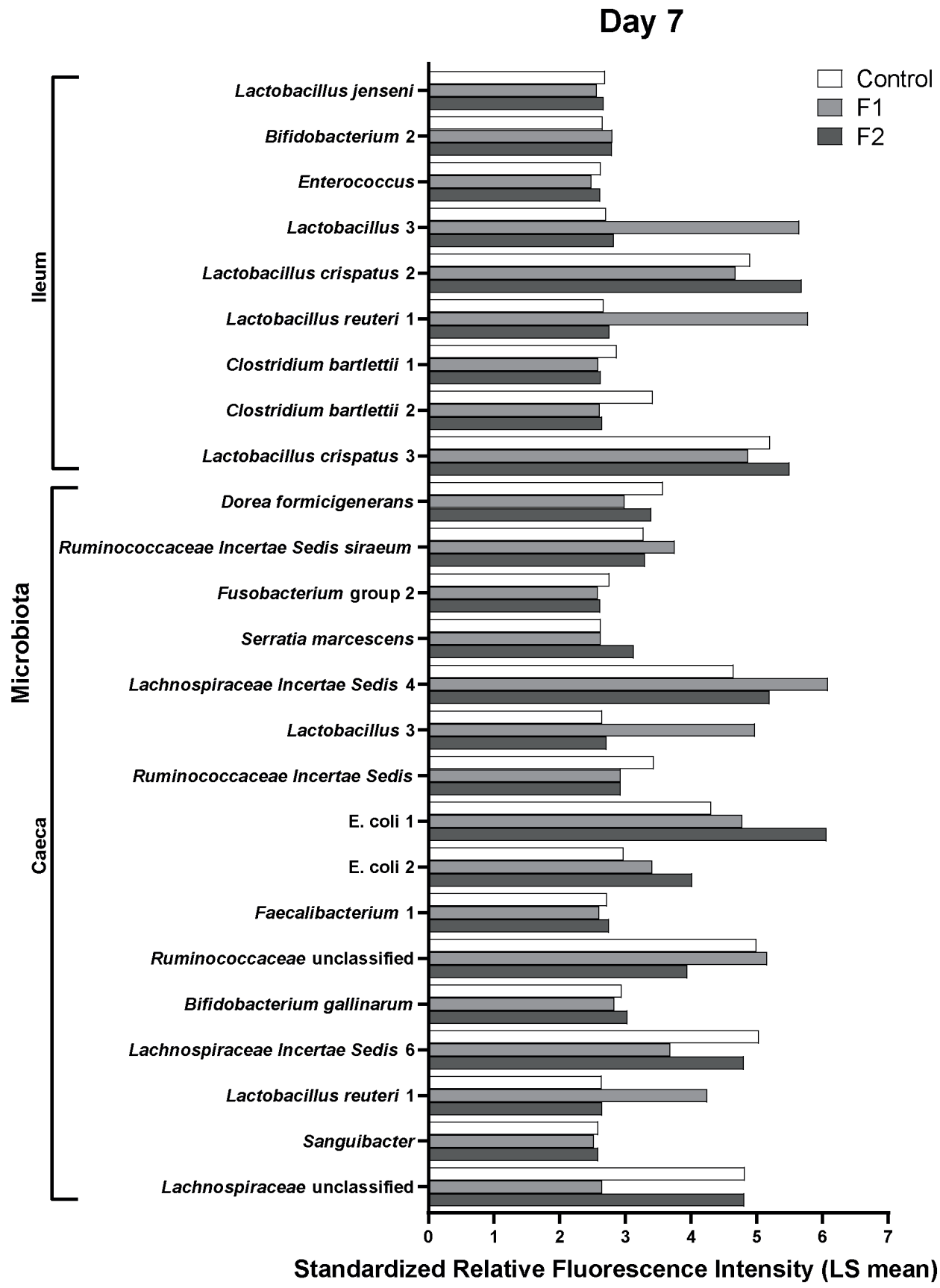

Figure 6. Relative abundance of intestinal microbial taxa significantly increased with the respective feed at day 7 in broiler chickens. Standardized relative fluorescence intensities (LS means) of the microbial taxa in the ileum and caeca as measured by the microarray (Table 2) that were significantly higher with standard diet (control), feed supplemented with glucose oligosaccharide (F1), or long-chain glucomannan (F2) at day 7 in broiler chickens. LS mean per microbial target and diet group are shown $(n=5)$ with statistical significance of FDR adjusted $p$-values set at $<0.05$. 
addition to the limited nutrient absorption capacity in chicken embryos due to lack of adequate nutrient transporters in the small intestine ${ }^{63}$. Both glucose oligosaccharide and long-chain glucomannan may be considered as prebiotics, which are undigestible materials consumed by gut microbiota ${ }^{64}$, thereby modifying and selectively favoring beneficial microbes toward a healthier microbiota.

Our finding that growth performance of broiler chickens was not affected by administration of both polysaccharides directly post-hatch was in agreement with other studies, although some prebiotics have shown beneficial effects on performance traits ${ }^{65-68}$. Supplementation with glucose oligosaccharide and long-chain glucomannan immediately post-hatch did increase intraepithelial NK cell activation early in life. This in vivo observation was in agreement with the enhanced NK cell activation observed in vitro and indicates direct immunomodulation by the polysaccharides as shown before with other polysaccharides ${ }^{69}$. In addition, the relation between the in vitro assays and in vivo situation suggests that these assays are useful tools to screen the immunomodulatory effects of feed constituents and may contribute to the reduction of animal experiments ${ }^{70}$. The enhanced activation of intraepithelial NK cells was observed after the first seven days of supplementation and subsequently decreased to levels similar to those observed in the control group. NK cells were shown to be involved in trained immunity in livestock ${ }^{71}$ and humans ${ }^{72}$. Initial exposure to $\beta$-glucans and BCG vaccination induced responses of monocytes and NK cells, respectively, thereby priming these innate cells, and a subsequent exposure to bacterial components led to increased innate responses, conferring innate memory. In humans, trained NK cells have been shown upon a secondary stimulus to undergo a secondary expansion and have the capacity to more rapid degranulation and production of cytokines, resulting in a higher protective immunity status ${ }^{72,73}$. It could be hypothesized that the signs of early NK cell activation in our study are a consequence of training by the polysaccharides and that a secondary stimulus such as an infection could increase NK cell responses. Future studies should investigate whether these polysaccharides enhance NK cell (re-)activation in chickens in response to infections. In this study, no significant changes in numbers and activation of T cells in the IEL population and spleen were observed after supplementation, whereas other polysaccharides did increase percentages of intraepithelial $T$ cells in chickens $^{74}$ and $\mathrm{T}$ cell activation in mammals ${ }^{75,76}$. This may be due to the different polysaccharides used in our study or that glucose oligosaccharide or long-chain glucomannan supplementation may have more pronounced effects on T cells during an infection, as was shown for another polysaccharide ${ }^{77}$.

The microbiota compositions of the ileum and caeca were modulated by feed supplemented with either glucose oligosaccharide or long-chain glucomannan resulting in increased relative abundance of LAB such as Lactobacillus and Bifidobacterium, in agreement with other studies in broiler chickens ${ }^{78,79}$. This confirms that both the polymerized carbohydrate and the mannose polymer are fermented by $L A B$. The differences in relative abundance of intestinal microbial taxa were most evident at 7 days of age and subsequently decreased until 21 days of age, when, as shown previously, a mature stable microbiota composition has established in the intestine. Relative abundance of LAB was found to be higher in the case of glucose oligosaccharide supplementation compared to long-chain glucomannan supplementation, but the latter also stimulated relative abundance of more strictly anaerobic bacteria such as Faecalibacterium and Lachnospiraceae. In addition to being fermented by LAB, Faecalibacterium and Lachnospiraceae, long-chain glucomannan may be fermented by a specific strain and, due to its microbial fermentation, result in metabolites to be utilized by other bacteria that increase in abundance 80,81 . Besides LAB, both Faecalibacterium ${ }^{82}$ and Lachnospiracea ${ }^{83}$ have been shown to be beneficial for human intestinal health since intestinal and metabolic disorders were associated with depletion of these species.

Then, we investigated whether the effects of supplemented feeds on NK cells and microbiota were related. Although similar analysis of correlation between immune cells and specific microbial taxa has 


\section{Correlation day 7}

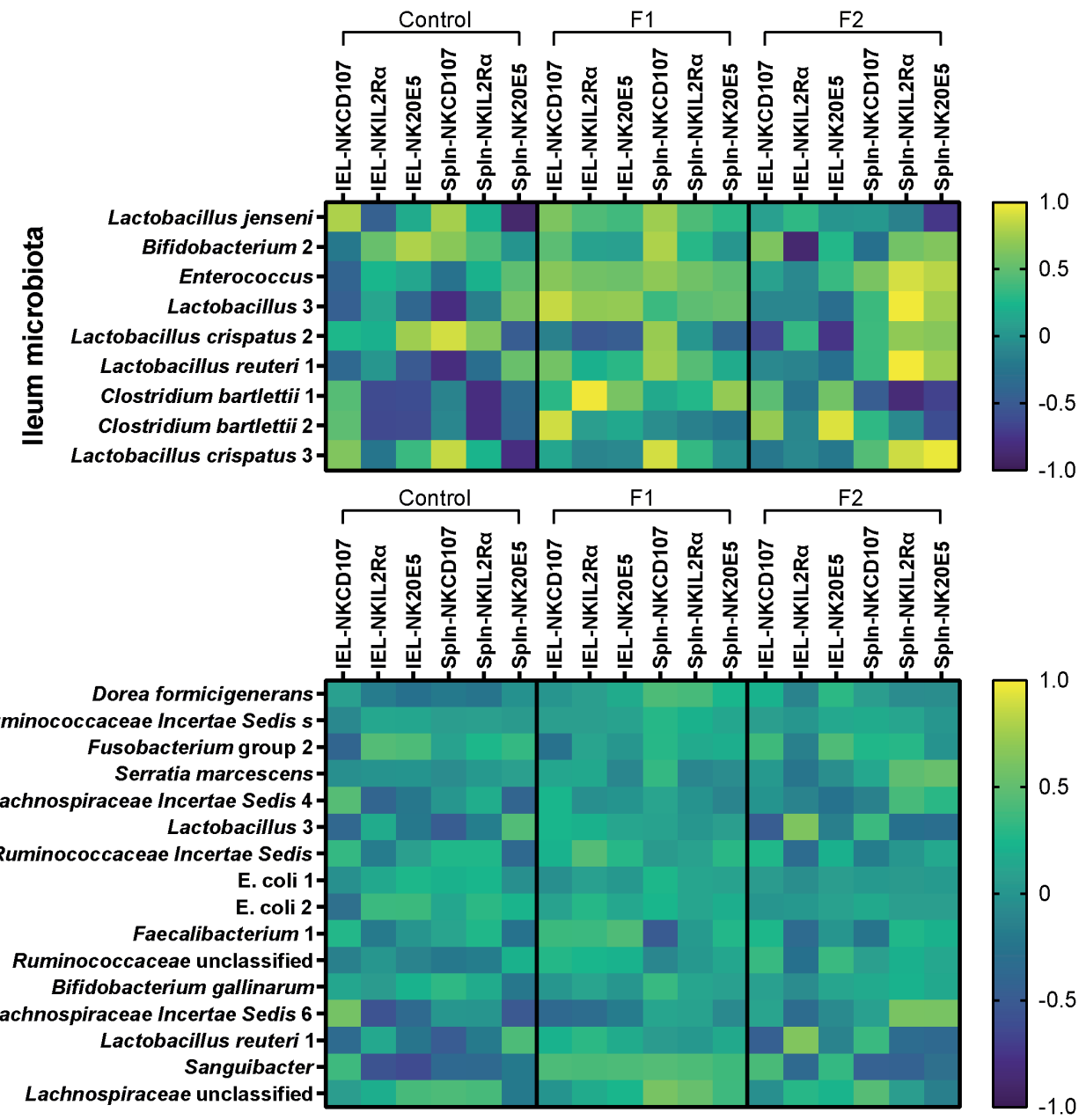

Figure 7. Correlations between intestinal microbial taxa and intraepithelial and splenic NK cells at day 7 in broiler chickens. (A) Correlation values between microbial taxa in the ileum or (B) caeca significantly increased with the respective feed and percentages of NK cell activation or numbers of NK cell subsets of the ileum (IELs) and spleen (Spln) per diet group (control, F1, F2) at day 7 in broiler chickens. Pearson's correlation ( $r$ ) values are depicted in a heatmap as positive (yellow) or negative (dark blue) correlations.

been performed in mice ${ }^{84}$, it has not been conducted in chickens. Both supplemented feeds enhanced CD107 expression on intraepithelial NK cells and increased relative abundance of commensal LAB at 7 days of age. Therefore, the positive correlations between NK cell activation and relative abundance of $L$. reuteri 1, Lactobacillus 3, L. crispatus 2, and Bifidobacterium 2 in the ileum at 7 days of age due to glucose oligosaccharide and long-chain glucomannan supplemented diets indicate involvement of $L A B$ in the modulation of the function of NK cells. While glucose oligosaccharide showed positive correlations between relative abundance of $L A B$ and NK cell subsets as well as activation of both intraepithelial and splenic NK cells, long-chain glucomannan mainly showed positive correlations with NK cells in the spleen. This suggests that glucose oligosaccharide affects NK cells both locally 
by interaction with $L A B$, and systemically by a yet hypothetical interaction with antigen presenting cells (APCs) that have interacted with intestinal $L A B$ or translocation of microbial products into the circulation ${ }^{85,86}$. The effects of long-chain glucomannan are suggested to be more systemic rather than local according to the correlation analysis, where splenic NK cells are affected through the hypothetical mechanisms mentioned earlier.

Enhancement of NK cell activation by microbiota has been shown previously in humans and mice ${ }^{87}$, induced either directly or indirectly via APCs ${ }^{88}$. Intestinal APCs recognize microbial species through Toll-like receptors (TLRs), resulting in the production of cytokines such as interleukin-12 (IL-12), which induces NK as well as T cell responses such as IFNY production ${ }^{89,90}$. It has been hypothesized that intraepithelial NK cells interact directly with microbiota by recognition of nonmethylated CpG motifs of bacterial DNA, mainly via TLR9, which enhances their cytotoxic activity ${ }^{88,91}$. TLR9 is absent in the chicken genome, however, TLR21 may act as a functional homologue to mammalian TLR9 in recognizing CPG motifs ${ }^{92}$. Furthermore, metabolites such as short-chain fatty acids are produced by microbiota and can be utilized as an energy substrate for microbial species as well as affect the function of intestinal epithelial cells, NK cells, APCs, and T cells ${ }^{93,94}$. These metabolites should be kept in mind when developing feed strategies to indirectly modulate the immune system via the microbiota ${ }^{95}$. Early feeding with glucose oligosaccharide and long-chain glucomannan may also improve health during adult life; either by preventing colonization of harmful bacteria or by the stimulation of the immune system and thereby increasing resistance against pathogens as previously shown for other dietary constituents in chickens ${ }^{1,7}$ and humans ${ }^{96}$.

In conclusion, this study showed that early feeding of glucose oligosaccharide and long-chain glucomannan stimulates intraepithelial NK cell activation as well as a relative abundance of commensal lactic acid bacteria in young broiler chickens. Although both feed additives had no effect on growth performance under non-challenging conditions, they may have an added value to performance by eliciting stronger immune responses during challenging conditions. Future studies should investigate the impact of feeding of these polysaccharides during experimental infection to validate their potency to improve resistance against bacteria and viruses in broiler chickens.

\section{Acknowledgements}

We acknowledge the animal caretakers of both the Cargill Animal Nutrition and Health Innovation Center and the Department Population Health Sciences, division Farm Animal Health, Faculty of Veterinary Medicine, Utrecht University, for their help during the animal experiments. We thank R.H.G.A. van den Biggelaar for his help during the isolation of immune cells and G.J.A. Arkesteijn for maintaining optimal working conditions of the Flow Cytometry and Cell Sorting Facility, Faculty of Veterinary Medicine, Utrecht University. This research was funded by the Dutch Research Council (NWO) in conjunction with Cargill Animal Nutrition and Health in the context of stimulating Public-Private research collaboration, grant number 868.15.020 and part of the research program of NWO Earth and Life Sciences (ALW). 


\section{Supplementary data}

Table S1. Clarification of compounds used in the in vitro screening.

\begin{tabular}{|c|c|c|}
\hline Group & Code & Compound \\
\hline \multirow[t]{23}{*}{ Plant extract } & PE1 & Andrographolide ${ }^{1}$ \\
\hline & PE2 & Apigenin ${ }^{1}$ \\
\hline & PE3 & Astragalus Membranaceus ${ }^{2}$ \\
\hline & PE4 & Garlic extract $1^{2}$ \\
\hline & PE5 & Garlic oil ${ }^{1}$ \\
\hline & PE6 & Luteolin ${ }^{1}$ \\
\hline & PE7 & Resveratrol $^{1}$ \\
\hline & PE8 & Andrographis paniculata ${ }^{1}$ \\
\hline & PE9 & Tocopherol (blend; delta-, beta-, gamma, alpha alpha-tocopherol) ${ }^{3}$ \\
\hline & PE10 & Curcuma longa ${ }^{3}$ \\
\hline & PE11 & Polyphenols/Antioxidants ${ }^{3}$ \\
\hline & PE12 & Onion extract ${ }^{3}$ \\
\hline & PE13 & Grape extract ${ }^{3}$ \\
\hline & PE14 & Rosmaniric acid ${ }^{3}$ \\
\hline & PE15 & Thymol $^{3}$ \\
\hline & PE16 & Citral $^{3}$ \\
\hline & PE17 & Geraniol $^{3}$ \\
\hline & PE18 & Garlic extract $2^{3}$ \\
\hline & PE19 & Eugenol$^{3}$ \\
\hline & PE20 & Para-cymene $^{3}$ \\
\hline & PE21 & Cinnamic aldehyde ${ }^{3}$ \\
\hline & PE22 & Carvacrol $^{3}$ \\
\hline & PE23 & Plant extract blend of zinc and selenium ${ }^{3}$ \\
\hline \multirow[t]{2}{*}{ Fermentation product } & FP1 & 2,3-Butanediol ${ }^{1}$ \\
\hline & FP2 & 2-Ketogulonic acid ${ }^{3}$ \\
\hline \multirow[t]{2}{*}{ Vitamin } & $\mathrm{V} 1$ & $\beta$-Carotene ${ }^{1}$ \\
\hline & V2 & Retinoic acid ${ }^{1}$ \\
\hline \multirow[t]{2}{*}{ Drug } & D1 & Lenalidomide $^{1}$ \\
\hline & D2 & Pomalidomide $^{1}$ \\
\hline \multirow[t]{3}{*}{ Lipid } & L1 & Linoleic acid, conjugated ${ }^{1}$ \\
\hline & L2 & Medium-chain fatty acids ${ }^{3}$ \\
\hline & L3 & Mannosylerythritol lipids ${ }^{3}$ \\
\hline \multirow[t]{2}{*}{ Fungus extract } & FE1 & Shiitake mushroom² \\
\hline & FE2 & Tremella fuciformis ${ }^{2}$ \\
\hline \multirow[t]{5}{*}{ Polysaccharide } & P1 & Glucose oligosaccharide ${ }^{3}$ \\
\hline & $\mathrm{P} 2$ & Glucomannan - long chain ${ }^{3}$ \\
\hline & P3 & Polydextrose ${ }^{3}$ \\
\hline & P4 & Resistant dextrin ${ }^{3}$ \\
\hline & P5 & Glucomannan - short chain ${ }^{3}$ \\
\hline
\end{tabular}




\begin{tabular}{|c|c|c|}
\hline & P6 & Glucomannan - medium chain ${ }^{3}$ \\
\hline & P7 & Scleroglucan $^{3}$ \\
\hline & P8 & Glucan $^{3}$ \\
\hline & P9 & Algae (whole product) - Euglena gracilis ${ }^{3}$ \\
\hline & P10 & Algae extract - Euglena gracilis ${ }^{3}$ \\
\hline & P11 & Algae (whole product) - Ulva lactuca ${ }^{3}$ \\
\hline & P12 & Algae extract - Ulva lactuca ${ }^{3}$ \\
\hline & P13 & Algae extract - Laminaria sp. and/or Ascophyllum nodosum ${ }^{3}$ \\
\hline & P14 & Yeast cell wall 1 - Saccharomyces cerevisiae ${ }^{3}$ \\
\hline & P15 & Yeast cell wall 2 - Saccharomyces cerevisiae ${ }^{3}$ \\
\hline & P16 & Yeast cell wall 3 - Saccharomyces cerevisiae ${ }^{3}$ \\
\hline & P17 & Algae insoluble polysaccharides ${ }^{3}$ \\
\hline & P18 & Chitosan $^{3}$ \\
\hline & P19 & Chitosan oligosaccharide $^{3}$ \\
\hline Acid/Salt & AS1 & Calcium butyrate $95 \%^{3}$ \\
\hline Blend & B1 & Blend of essential oils and organic acids ${ }^{3}$ \\
\hline Yeast & Y1 & Yeast culture - Saccharomyces cerevisiae ${ }^{3}$ \\
\hline \multirow[t]{6}{*}{ Modified sugar } & MS1 & Alkyl glycoside $-83.5 \%^{3}$ \\
\hline & MS2 & Alkyl glycoside - mixture ${ }^{3}$ \\
\hline & MS3 & Alkyl glycoside $-95 \%^{3}$ \\
\hline & MS4 & Oligodextran ${ }^{3}$ \\
\hline & MS5 & Glucuronolactone $^{3}$ \\
\hline & MS6 & Octyl- $\beta$-D-glucoside ${ }^{3}$ \\
\hline \multirow[t]{2}{*}{ Simple sugar } & SS1 & Erythrulose $^{3}$ \\
\hline & SS2 & Keto-sugar $^{3}$ \\
\hline \multirow[t]{5}{*}{ Emulsifier } & E1 & Sugar ester (100\% di-, tri-, polyester) ${ }^{3}$ \\
\hline & E2 & Sugar ester ( $50 \%$ mono-, $50 \%$ di-, tri-, polyester) ${ }^{3}$ \\
\hline & E3 & Citrilated mono-diglycerides ${ }^{3}$ \\
\hline & E4 & Sorbitan monostearate $1^{3}$ \\
\hline & E5 & Sorbitan monostearate $2^{3}$ \\
\hline
\end{tabular}

${ }^{1}$ Sigma-Aldrich, the Netherlands, ${ }^{2}$ Shaanxi Yuwangtang Biotechnology Development Co. Ltd., China, ${ }^{3}$ Cargill Inc., USA. 


\section{NK cell activation}

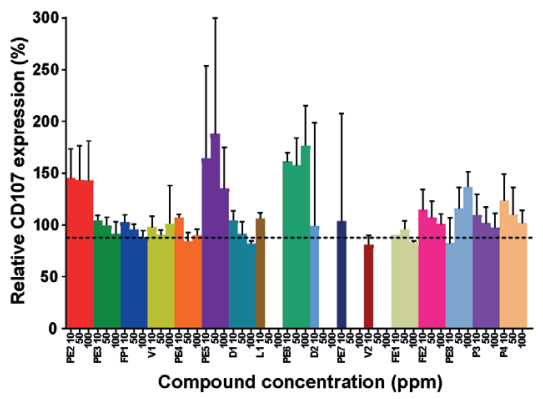

NK cell activation

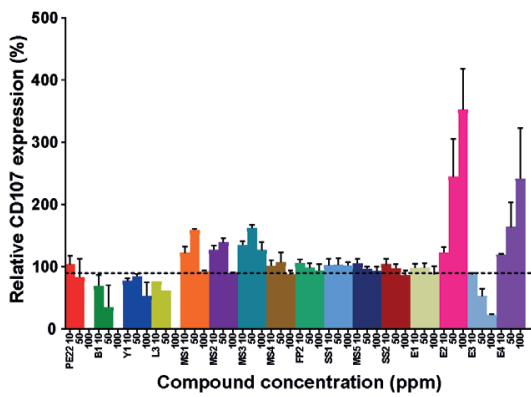

NK cell activation

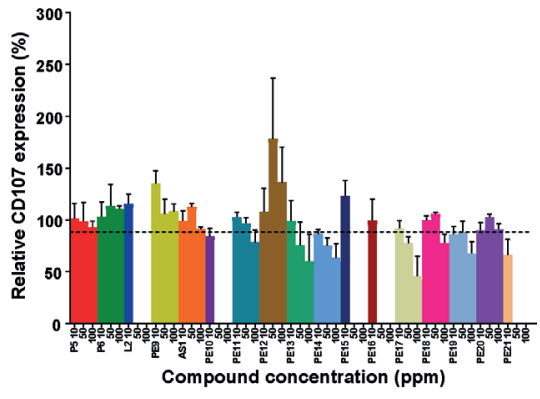

NK cell activation

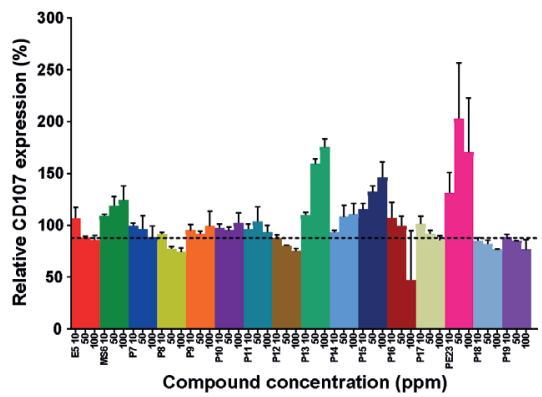

Figure S1. In vitro screening of feed compounds for their effect on NK cell activation. NK cell activation was measured by expression of CD107 on NK cells (\%) and CD107 expression is expressed relative to the negative control, which was set at $100 \%$. Mean + SEM is shown $(n=3)$ of the compounds plant extracts $(P E)$, fermentation products $(F P)$, vitamins $(V)$, drugs $(D)$, lipids $(L)$, fungus extracts $(F E)$, polysaccharides $(P)$, acid/salts (AS), blend of essential oils and organic acids (B), yeasts (Y), modified sugars (MS), simple sugars (SS) and emulsifiers $(\mathrm{E})$ and dotted line represents level of the solvent control.

\section{Phagocytosis}

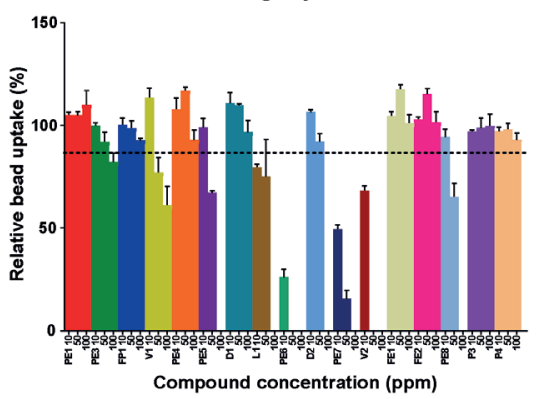

Phagocytosis

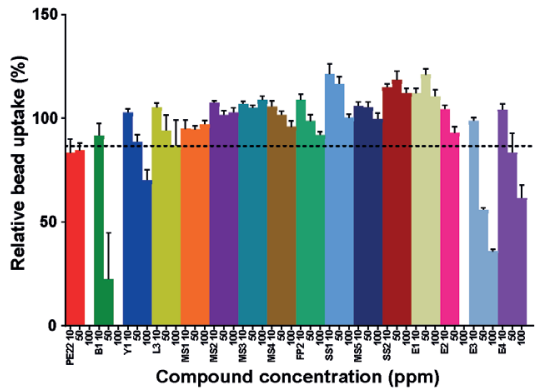

Phagocytosis

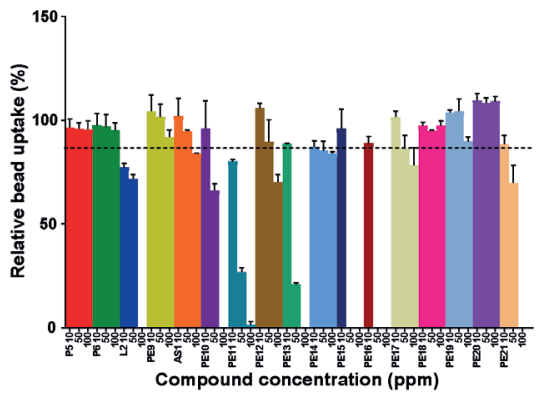

Phagocytosis

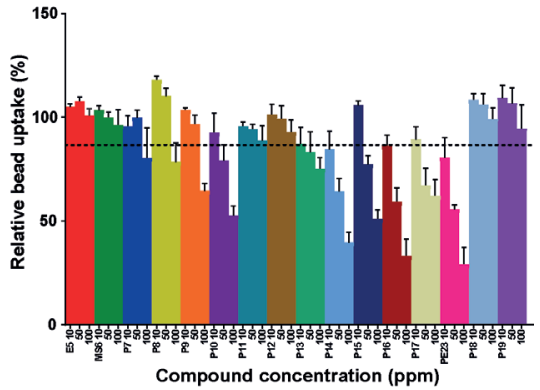

Figure S2. In vitro screening of feed compounds for their effect on phagocytosis of macrophages. Phagocytosis of macrophage-like HD11 cells (\%) is expressed relative to the reference control which was set at $100 \%$. Mean + SEM is shown $(n=3)$ of the compounds plant extracts $(P E)$, fermentation products $(F P)$, vitamins $(V)$, drugs $(D)$, lipids $(L)$, fungus extracts $(F E)$, polysaccharides $(P)$, acid/salts (AS), blend of essential oils and organic acids (B), yeasts (Y), modified sugars (MS), simple sugars (SS) and emulsifiers (E) and dotted line represents level of the solvent control. 


\section{NO production}

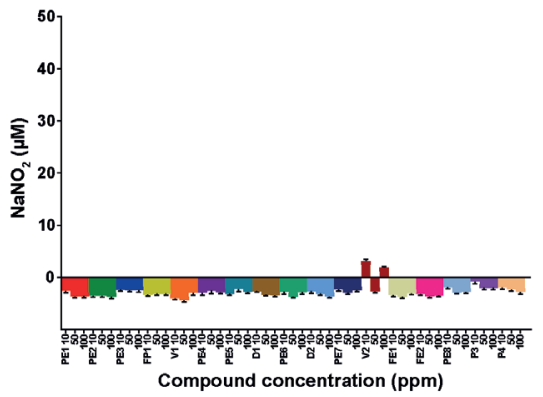

NO production

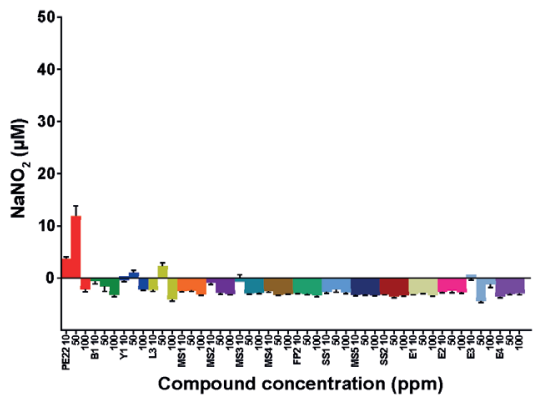

NO production

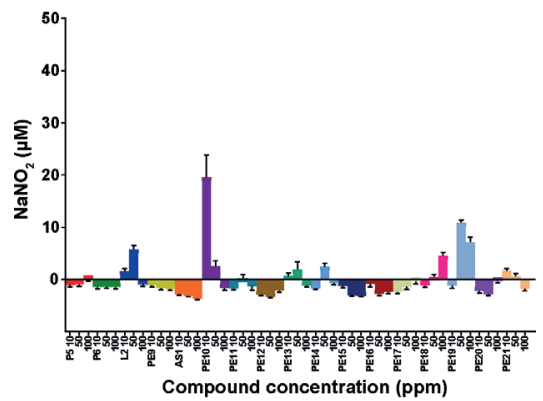

NO production

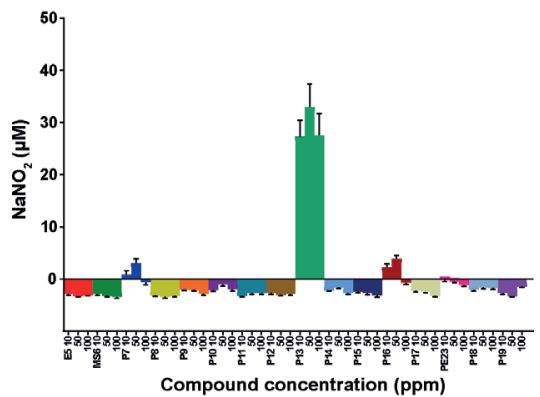

Figure S3. In vitro screening of feed compounds for their effect on NO production of macrophages. NO production of macrophage-like HD11 cells was assessed by measuring nitrite concentration $(\mu M)$. Mean + SEM is shown $(n=9)$ of the compounds plant extracts (PE), fermentation products $(F P)$, vitamins $(V)$, drugs $(D)$, lipids $(L)$, fungus extracts (FE), polysaccharides $(P)$, acid/salts (AS), blend of essential oils and organic acids (B), yeasts $(\mathrm{Y})$, modified sugars (MS), simple sugars (SS) and emulsifiers (E).

A

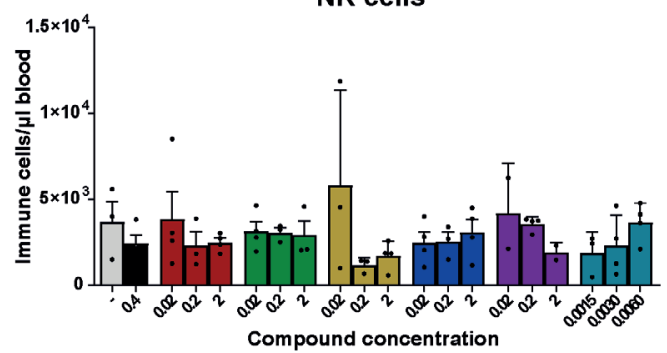

$$
\begin{array}{|lll}
\square \mathrm{Neg} & \square \mathrm{P} 1 & \text { 口 SS2 } \\
\text { NaCl } & \text { 口P2 } & \text { 口 MS3 } \\
& \square \mathrm{P} 8 & \square \mathrm{L1}
\end{array}
$$

B

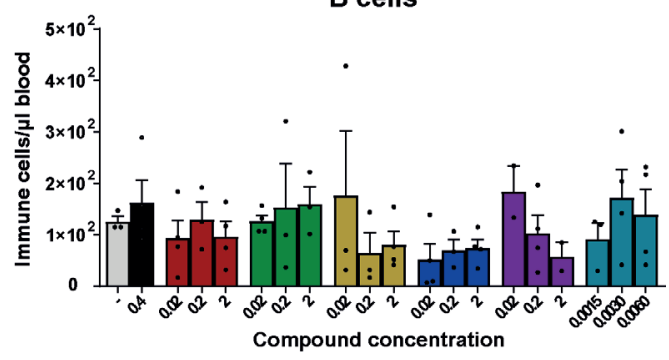

C

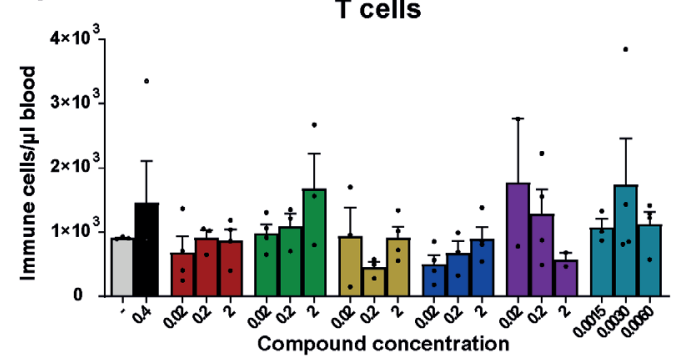

Figure S4. In ovo screening of feed compounds for their effect on peripheral immune cell numbers. (A) Effect of compound injections in ED18 eggs on numbers of NK cells, (B) B cells and (C) T cells in whole blood immediately post-hatch. Non-injected eggs were included as negative control and $0.4 \%$ saline solution as solvent control. Mean + SEM are shown $(n=4)$ of the compounds polysaccharides (P1, P2, P8), simple sugars (SS2), modified sugars (MS3) and lipids (L1) in different concentrations $(0.02,0.2,2 \%$ or $0.0015,0.0030$, $0.0060 \mathrm{mg}$ ). 
A
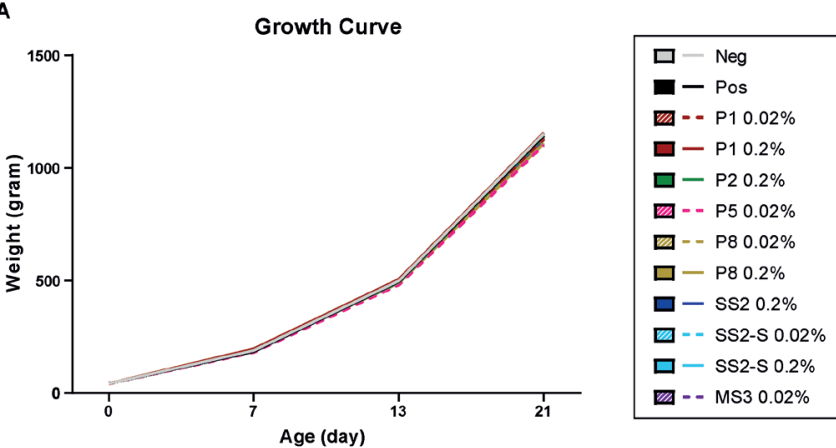

B

Feed Conversion Ratio

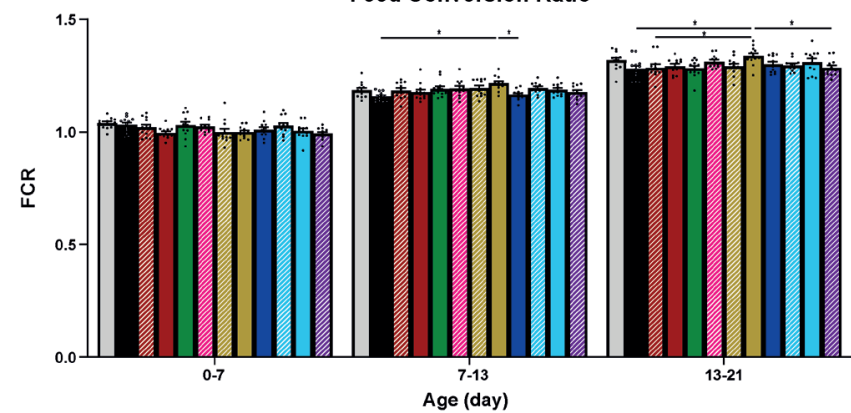

Figure S5. In vivo screening of diets for their effect on performance traits in broiler chickens. (A) Effect of diet on weights ( $\mathrm{g}$ ) of chickens during aging. (B) Effect of diet on feed conversion ratio (FCR) during the periods 0-7, 7-13 and 13-21 days of aging. Mean + SEM is shown $(n=12)$ of broiler chickens provided standard diet (negative control), feed supplemented with $0.0625 \%$ SCFP (positive control), or compounds in different concentrations; P1 $(0.02 \%, 0.2 \%)$, P2 $(0.2 \%)$, P5 $(0.02 \%)$, P8 $(0.02 \%, 0.2 \%)$, SS2 $(0.2 \%)$, SS2-S $(0.02 \%$, $0.2 \%$, Sigma-Aldrich) and MS3 (0.02\%), and statistical significance is indicated as * $p<0.05$.

A

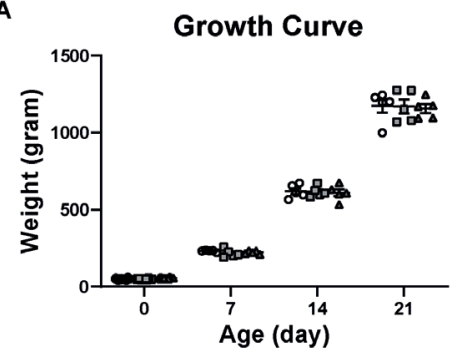

B

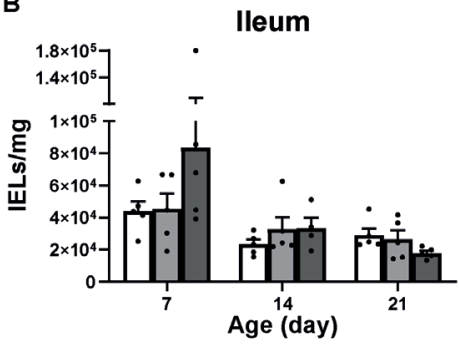

- $\square$ Control

- $\square \mathrm{F1}$

$\Delta \square$ F2

C

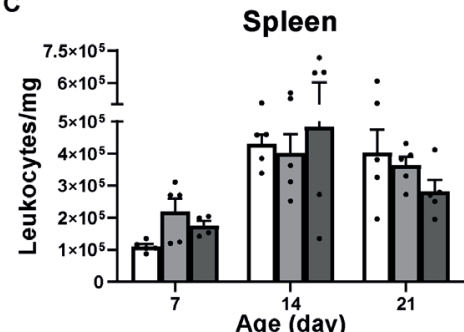

Figure S6. Effect of feeding glucose oligosaccharides and long-chain glucomannan to broiler chickens on growth and IELs and splenic leukocytes. (A) Weights (g) of chickens provided different diets in the course of time. (B) Numbers (cells/mg) of IELs isolated from the ileum and (C) splenic leukocytes in broiler chickens provided different diets in course of time. Mean + SEM is shown $(n=5)$ of chickens provided standard diet (control), feed supplemented with glucose oligosaccharide (F1) or long-chain glucomannan (F2). 
A

IELS

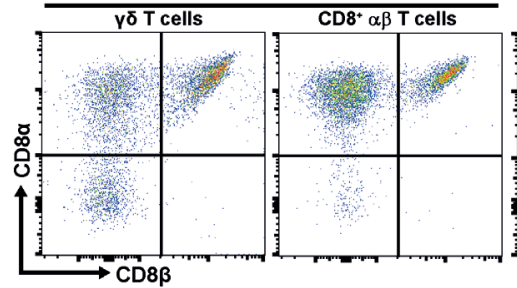

B

CD8 $\alpha \alpha^{+}$

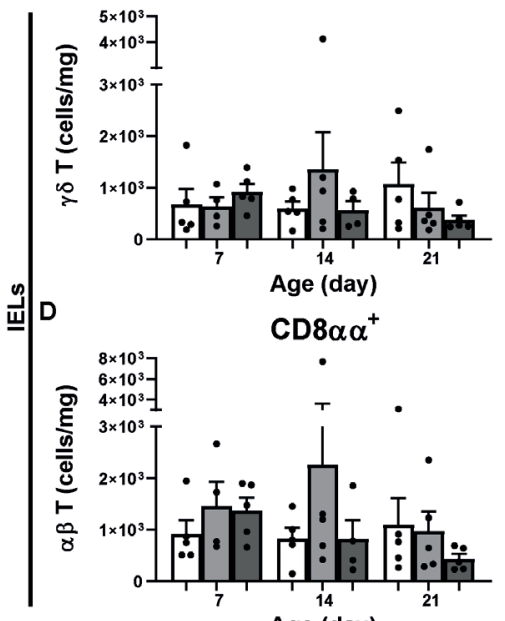

Age (day)
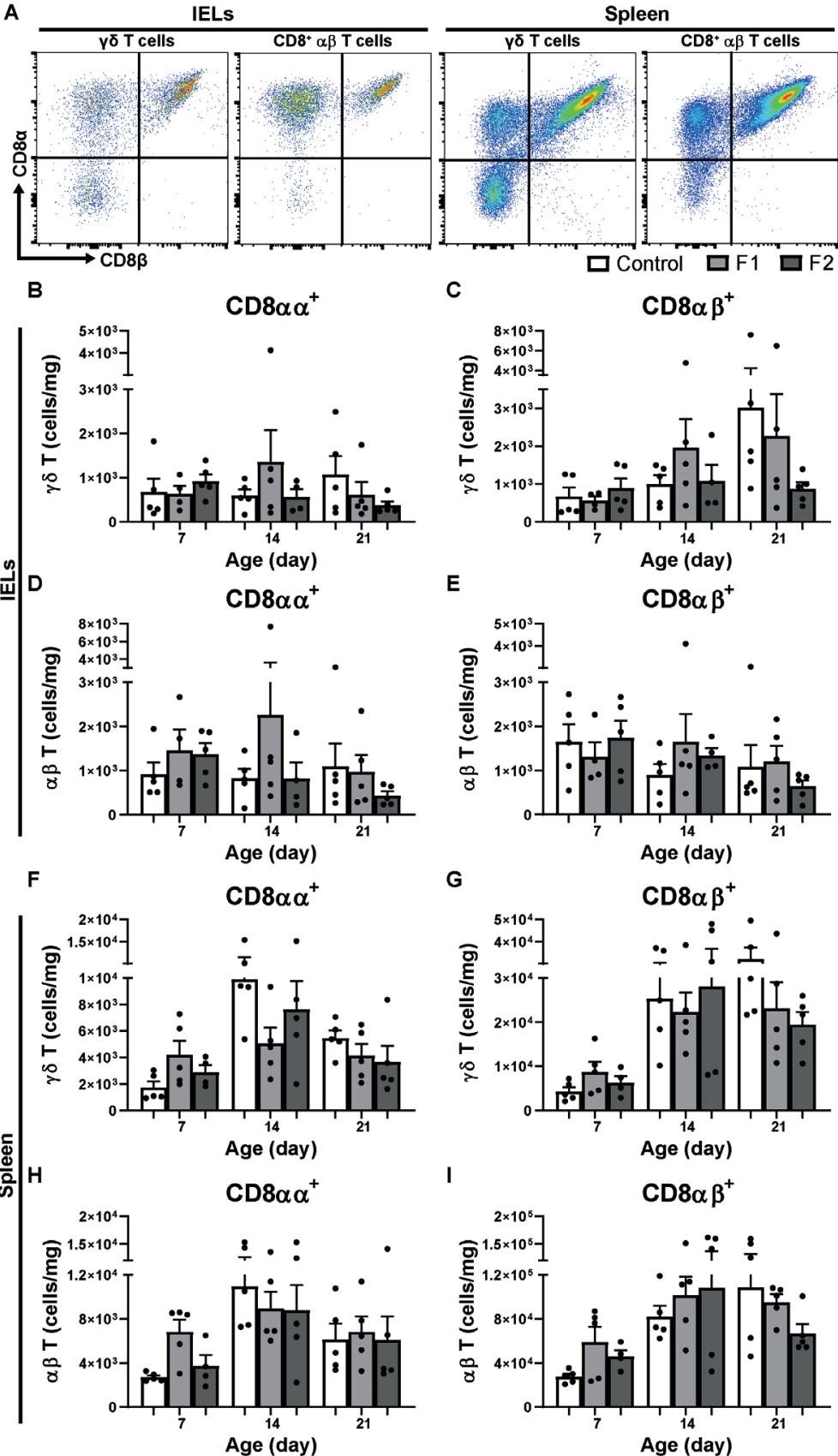

c

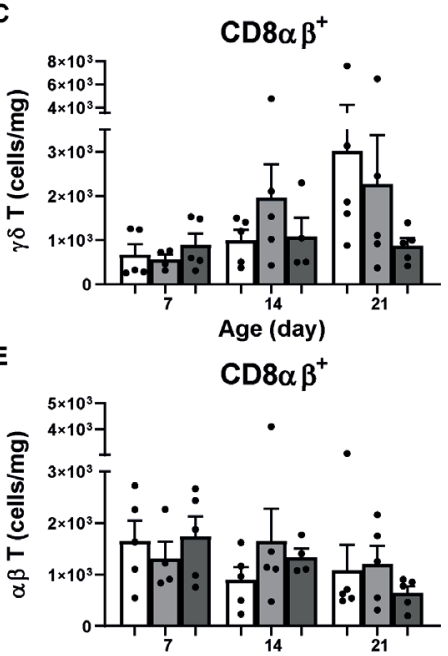

G

Age (day)

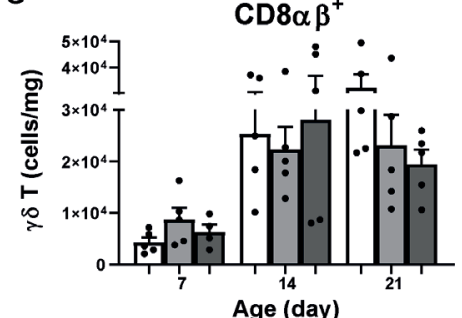

I

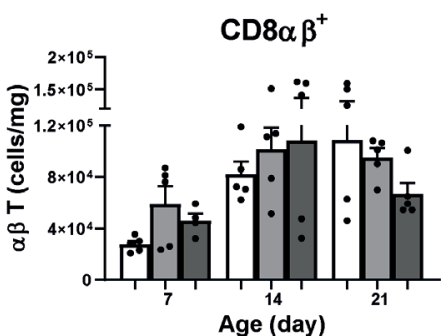

Figure S7. Effect of feeding glucose oligosaccharides and long-chain glucomannan to broiler chickens on intraepithelial and splenic $\gamma \delta$ $T$ cells and cytotoxic T cells expressing either CD8 $\alpha \alpha$ or $C D 8 \alpha \beta$. (A) $\gamma \delta$ T cells and CD8 ${ }^{+} \alpha \beta$ T cells expressing either CD $8 \alpha \alpha$ or $C D 8 \alpha \beta$ in the IEL population (first and second panels) and spleen (third and fourth panels). (B) Numbers (cells/mg) of intraepithelial CD $8 \alpha \alpha^{+} \gamma \delta$ T cells, (C) CD $8 \alpha \beta^{+} \gamma \delta$ T cells, (D) cytotoxic CD8 $\alpha \alpha^{+} \alpha \beta$ T cells and (E) CD8 $\alpha \beta^{+} \alpha \beta$ T cells in broiler chickens provided different diets in the course of time. (F) Numbers (cells/mg) of splenic CD8 $\alpha \alpha^{+} \gamma \delta$ T cells, (G) CD8 $\alpha \beta^{+} \gamma \delta$ T cells, (H) cytotoxic CD8 $\alpha \alpha^{+} \alpha \beta$ T cells and (I) $C D 8 \alpha \beta^{+} \alpha \beta$ T cells in broiler chickens provided different diets in the course of time. Mean + SEM is shown $(n=5)$ of chickens provided standard diet (control), feed supplemented with glucose oligosaccharide (F1) or long-chain glucomannan (F2). 
A

IELs

\section{CD107 ${ }^{+}$}

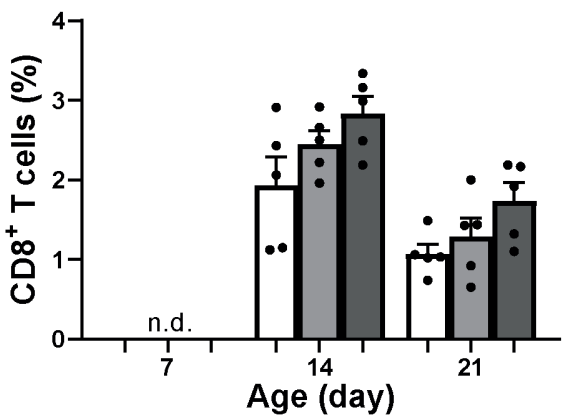

B
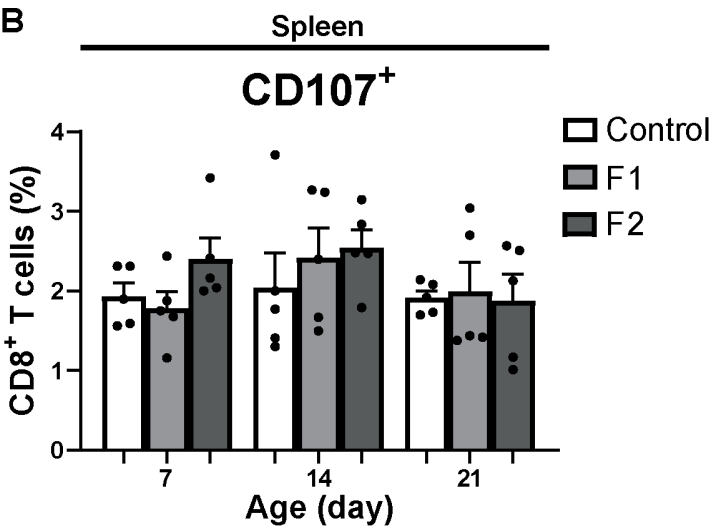

Figure S8. Effect of feeding glucose oligosaccharides and long-chain glucomannan to broiler chickens on intraepithelial and splenic $T$ cell activation. (A) Percentages of CD8 $8^{+}$cells expressing CD107 (including both $\gamma \delta$ and $\alpha \beta$ T cells) in the IEL population and (B) spleen in broiler chickens provided different diets in the course of time. Mean + SEM is shown $(n=5)$ of chickens provided standard diet (control), feed supplemented with glucose oligosaccharide (F1) or long-chain glucomannan (F2), for chickens at day 7 in the IEL population percentages were not determined (n.d.) due to numbers of events acquired in the gate of interest were $<100$. 


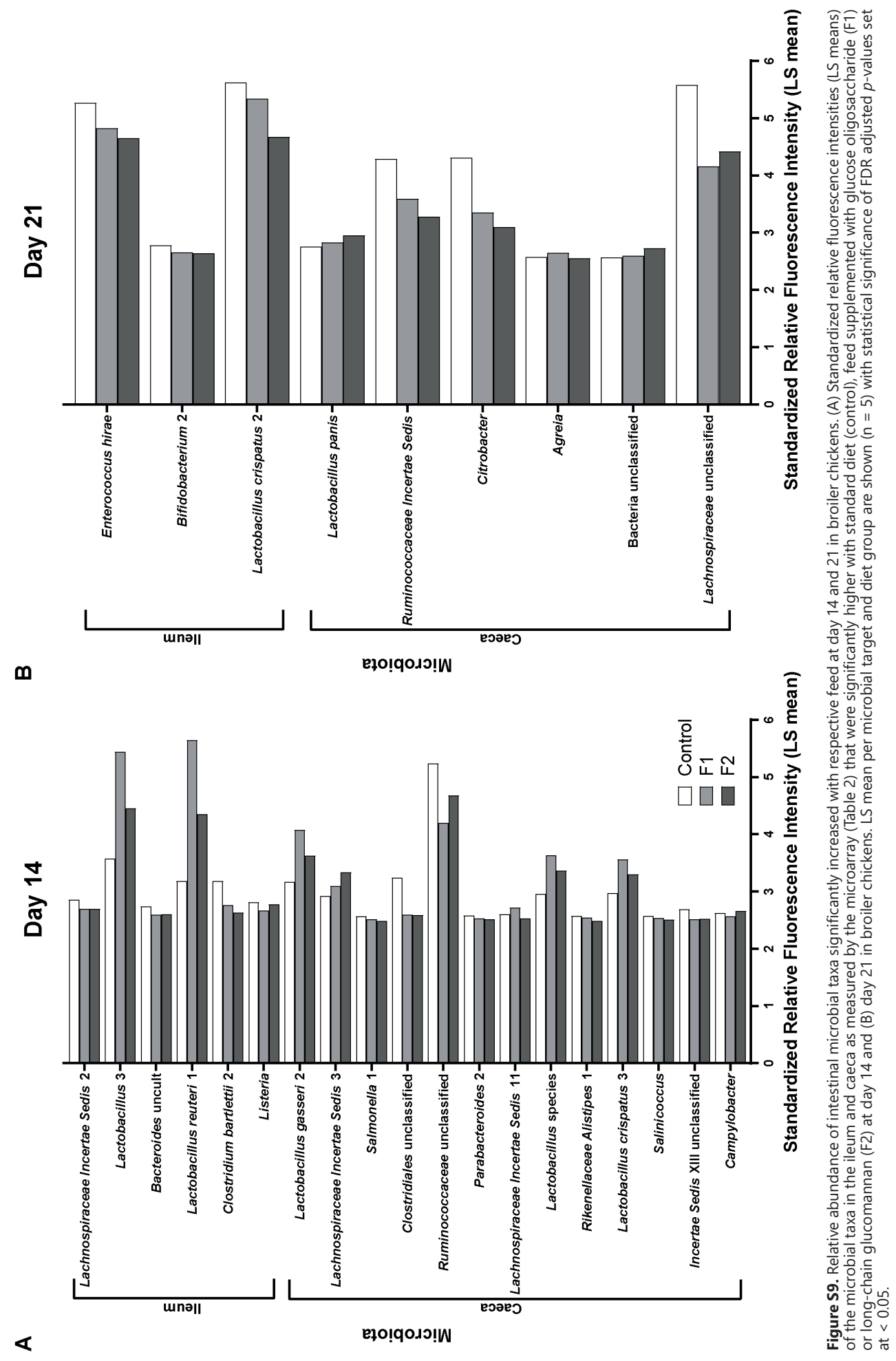




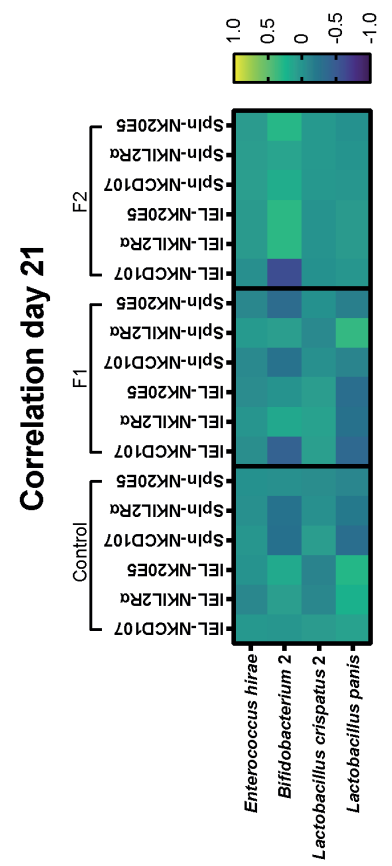

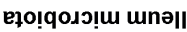

m

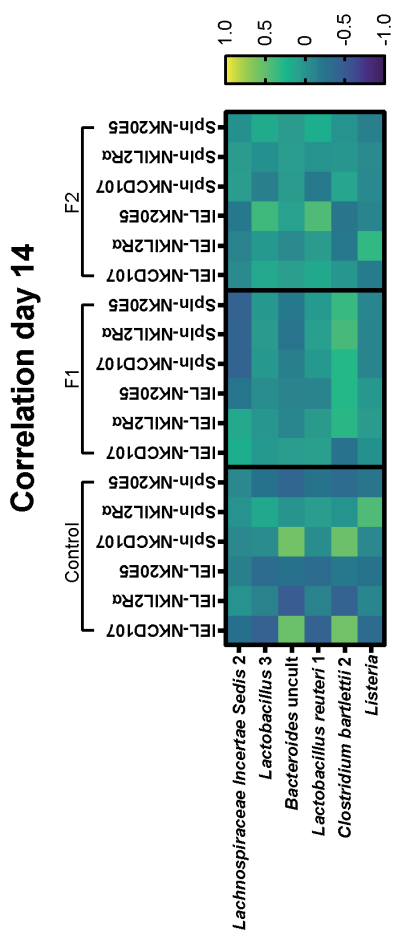

4

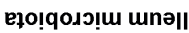

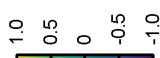

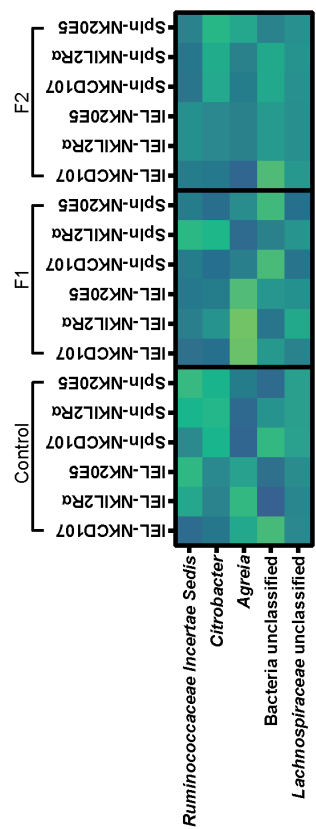

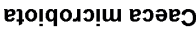

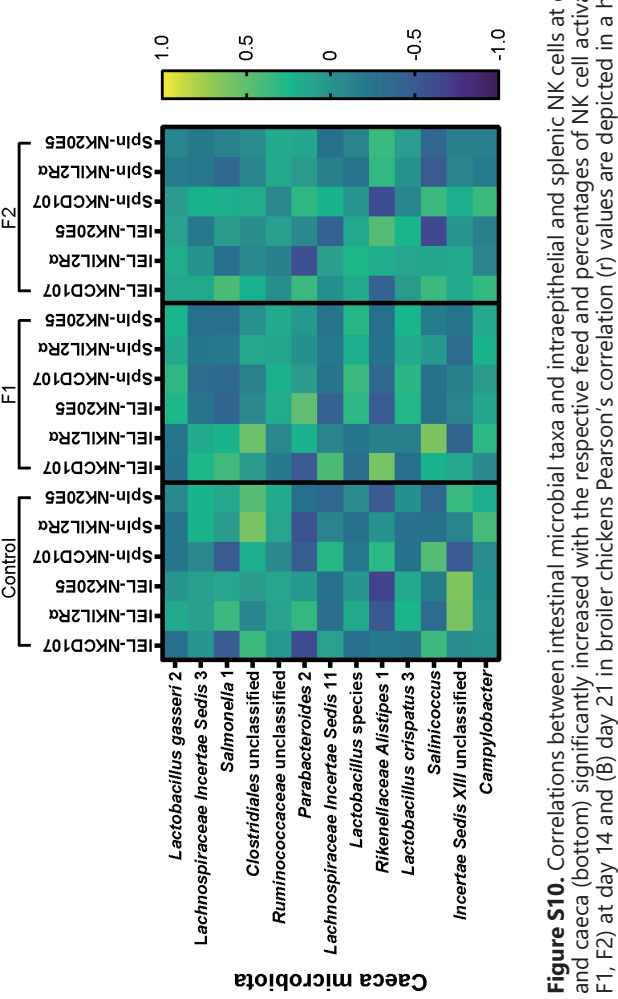

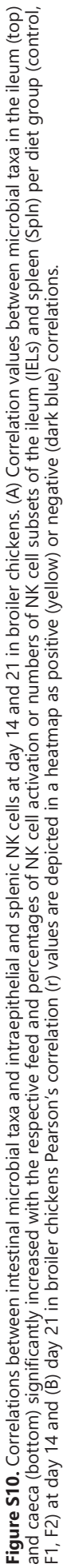




\section{References}

1. Taha-Abdelaziz K, Hodgins DC, Lammers A, Alkie TN, Sharif S. Effects of early feeding and dietary interventions on development of lymphoid organs and immune competence in neonatal chickens: A review. Vet Immunol Immunopathol. 2018;201:1-11. doi:10.1016/j.vetimm.2018.05.001.

2. Torok VA, Allison GE, Percy NJ, Ophel-Keller K, Hughes RJ. Influence of antimicrobial feed additives on broiler commensal posthatch gut microbiota development and performance. Appl Environ Microbiol. 2011;77(10):3380-3390. doi:10.1128/ AEM.02300-10.

3. Schokker D, Jansman AJM, Veninga G, et al. Perturbation of microbiota in one-day old broiler chickens with antibiotic for 24 hours negatively affects intestinal immune development. BMC Genomics. 2017;18(1). doi:10.1186/s12864-017-3625-6.

4. Broom LJ, Kogut MH. The role of the gut microbiome in shaping the immune system of chickens. Vet Immunol Immunopathol. 2018;204:44-51. doi:10.1016/j.vetimm.2018.10.002.

5. Yin Y, Lei F, Zhu L, et al. Exposure of different bacterial inocula to newborn chicken affects gut microbiota development and ileum gene expression. ISME J. 2010;4(3):367-376. doi:10.1038/ismej.2009.128.

6. Meijerink N, Kers JG, Velkers FC, et al. Early Life Inoculation With Adult-Derived Microbiota Accelerates Maturation of Intestinal Microbiota and Enhances NK Cell Activation in Broiler Chickens. Front Vet Sci. 2020;7. doi:10.3389/fvets.2020.584561.

7. Kogut MH. Impact of nutrition on the innate immune response to infection in poultry. J Appl Poult Res. 2009;18(1):111-124. doi:10.3382/japr.2008-00081.

8. Bar-Shira E, Sklan D, Friedman A. Establishment of immune competence in the avian GALT during the immediate post-hatch period. Dev Comp Immunol. 2003;27(2):147-157. doi:10.1016/S0145-305X(02)00076-9.

9. Klasing KC, Leshchinsky TV. Functions, costs and benefits of the immune system during development and growth. 1999; In: Adams NJ, and Slotow R (eds)(Proc. 22. Int. Ornithol. Congr. H. Birdlife South Africa, Johannesburg):2817-2835.

10. Göbel TWF, Kaspers B, Stangassinger M. NK and T cells constitute two major, functionally distinct intestinal epithelial lymphocyte subsets in the chicken. Int Immunol. 2001;13(6):757-762. doi:10.1093/intimm/13.6.757.

11. Meijerink N, van Haarlem DA, Velkers FC, Stegeman AJ, Rutten, V. P. M. G., Jansen CA. Analysis of chicken intestinal natural killer cells, a major IEL subset during embryonic and early life. Dev Comp Immunol. 2021;114. doi:10.1016/j.dci.2020.103857.

12. Lawn AM, Rose ME, Bradley JWA, Rennie MC. Lymphocytes of the intestinal mucosa of chickens. Cell Tissue Res. 1988;251(1):189195. doi:10.1007/BF00215464.

13. Lillehoj HS, Trout JM. Avian gut-associated lymphoid tissues and intestinal immune responses to Eimeria parasites. Clin Microbiol Rev. 1996;9(3):349-360. doi:10.1128/cmr.9.3.349.

14. Biddle A, Stewart L, Blanchard J, Leschine S. Untangling the genetic basis of fibrolytic specialization by lachnospiraceae and ruminococcaceae in diverse gut communities. Diversity. 2013;5(3):627-640. doi:10.3390/d5030627.

14. Swaggerty $\mathrm{CL}$, Callaway $\mathrm{TR}$, Kogut MH, Piva A, Grilli E. Modulation of the immune response to improve health and reduce foodborne pathogens in poultry. Microorg. 2019;7(3). doi:10.3390/microorganisms7030065.

15. Kidd MT. Nutritional modulation of immune function in broilers. Poult Sci. 2004;83(4):650-657. doi:10.1093/ps/83.4.650.

16. Abdolmaleki M, Yeap SK, Tan SW, et al. Effects of newcastle disease virus infection on chicken intestinal intraepithelial natural killer cells. Front Immunol. 2018;9(JUN). doi:10.3389/fimmu.2018.01386.

17. Jahromi MZ, Bello MB, Abdolmaleki M, Yeap SK, Hair-Bejo M, Omar AR. Differential activation of intraepithelial lymphocytenatural killer cells in chickens infected with very virulent and vaccine strains of infectious bursal disease virus. Dev Comp Immunol. 2018;87:116-123. doi:10.1016/j.dci.2018.06.004.

18. Jansen CA, De Geus ED, Van Haarlem DA, et al. Differential lung NK cell responses in avian influenza virus infected chickens correlate with pathogenicity. Sci Rep. 2013;3. doi:10.1038/srep02478.

19. Meijerink N, van den Biggelaar RHGA, van Haarlem DA, Stegeman JA, Rutten VPMG, Jansen CA. A detailed analysis of innate and adaptive immune responsiveness upon infection with Salmonella enterica serotype Enteritidis in young broiler chickens. Vet Res. 2021;52(1):109. doi:10.1186/s13567-021-00978-y.

20. Jansen $C A$, van de Haar PM, van Haarlem D, et al. Identification of new populations of chicken natural killer (NK) cells. Dev Comp Immunol. 2010;34(7):759-767. doi:10.1016/j.dci.2010.02.009.

21. De Geus ED, Jansen CA, Vervelde L. Uptake of particulate antigens in a nonmammalian lung: Phenotypic and functional characterization of avian respiratory phagocytes using bacterial or viral antigens. J Immunol. 2012;188(9):4516-4526. doi:10.4049/ jimmunol.1200092.

22. van den Biggelaar, R. H. G. A., van Eden W, Rutten, V. P. M. G., Jansen CA. Nitric oxide production and fc receptor-mediated phagocytosis as functional readouts of macrophage activity upon stimulation with inactivated poultry vaccines in vitro. Vaccines. 2020;8(2):1-16. doi:10.3390/vaccines8020332.

23. Ariaans MP, Matthijs MGR, van Haarlem D, et al. The role of phagocytic cells in enhanced susceptibility of broilers to colibacillosis after Infectious Bronchitis Virus infection. Vet Immunol Immunopathol. 2008;123(3-4):240-250. doi:10.1016/j.vetimm.2008.02.003.

24. Henkart PA. Lymphocyte-mediated cytotoxicity: Two pathways and multiple effector molecules. Immunity. 1994;1(5):343-346. doi:10.1016/1074-7613(94)90063-9.

25. Hirayama $D$, lida $T$, Nakase $H$. The phagocytic function of macrophage-enforcing innate immunity and tissue homeostasis. Int J Mol Sci. 2018;19(1). doi:10.3390/ijms19010092.

26. MacMicking J, Xie Q-, Nathan C. Nitric oxide and macrophage function. Annu Rev Immunol. 1997; 15. doi:10.1146/annurev. immunol.15.1.323. 
27. Bar-Shira E, Friedman A. Development and adaptations of innate immunity in the gastrointestinal tract of the newly hatched chick. Dev Comp Immunol. 2006;30(10):930-941. doi:10.1016/j.dci.2005.12.002.

28. Alizadeh M, Rogiewicz A, McMillan E, Rodriguez-Lecompte JC, Patterson R, Slominski BA. Effect of yeast-derived products and distillers dried grains with solubles (DDGS) on growth performance and local innate immune response of broiler chickens challenged with Clostridium perfringens. Avian Pathol. 2016;45(3):334-345. doi:10.1080/03079457.2016.1155693.

29. Shashidhara RG, Devegowda G. Effect of dietary mannan oligosaccharide on broiler breeder production traits and immunity. Poult Sci. 2003;82(8):1319-1325. doi:10.1093/ps/82.8.1319.

30. Baurhoo B, Ferket P, Ashwell CM, de Oliviera J, Zhao X. Cell walls of saccharomyces cerevisiae differentially modulated innate immunity and glucose metabolism during late systemic inflammation. PLoS ONE. 2012;7(1). doi:10.1371/journal.pone.0030323.

31. Roberts T, Wilson J, Guthrie A, et al. New issues and science in broiler chicken intestinal health: Emerging technology and alternative interventions. J Appl Poult Res. 2015;24(2):257-266. doi:10.3382/japr/pfv023.

32. Guo FC, Savelkoul HFJ, Kwakkel RP, Williams BA, Verstegen MWA. Immunoactive, medicinal properties of mushroom and herb polysaccharides and their potential use in chicken diets. World's Poult Sci J. 2003;59(4):427-440. doi:10.1079/WPS20030026.

33. Ritz BW, Nogusa S, Ackerman EA, Gardner EM. Supplementation with active hexose correlated compound increases the innate immune response of young mice to primary influenza infection. J Nutr. 2006;136(11):2868-2873.

34. Arreola R, Quintero-Fabián S, Lopez-Roa RI, et al. Immunomodulation and anti-inflammatory effects of garlic compounds. J Immunol Res. 2015;2015. doi:10.1155/2015/401630.

35. Falchetti R, Fuggetta MP, Lanzilli G, Tricarico M, Ravagnan G. Effects of resveratrol on human immune cell function. Life Sci. 2001;70(1):81-96. doi:10.1016/S0024-3205(01)01367-4.

36. Leischner C, Burkard M, Pfeiffer MM, Lauer UM, Busch C, Venturelli S. Nutritional immunology: Function of natural killer cells and their modulation by resveratrol for cancer prevention and treatment. Nutr J. 2016;15(1). doi:10.1186/s12937-016-0167-8.

37. Ren Y, Yuan C, Deng Y, et al. Cytotoxic and natural killer cell stimulatory constituents of Phyllanthus songboiensis. Phytochemistry. 2015;111:132-140. doi:10.1016/j.phytochem.2014.12.014.

38. Sheeja K, Kuttan G. Modulation of natural killer cell activity, antibody-dependent cellular cytotoxicity, and antibody-dependent complement-mediated cytotoxicity by andrographolide in normal and Ehrlich ascites carcinoma-bearing mice. Integr Cancer Ther. 2007;6(1):66-73. doi:10.1177/1534735406298975.

39. Kilani-Jaziri S, Mustapha N, Mokdad-Bzeouich I, El Gueder D, Ghedira K, Ghedira-Chekir L. Flavones induce immunomodu latory and anti-inflammatory effects by activating cellular anti-oxidant activity: a structure-activity relationship study. Tumor Biol. 2016;37(5):6571-6579. doi:10.1007/s13277-015-4541-5.

40. Murali VP, Kuttan G. Curculigoside augments cell-mediated immune responses in metastatic tumor-bearing animals. Immunophar macol Immunotoxicol. 2016;38(4):264-269. doi:10.1080/08923973.2016.1188401.

41. Zhang Y, Song TT, Cunnick JE, Murphy PA, Hendrich S. Daidzein and genistein glucuronides in vitro are weakly estrogenic and activate human natural killer cells at nutritionally relevant concentrations. J Nutr. 1999;129(2):399-405.

42. Hart AN, Zaske LA, Patterson KM, Drapeau C, Jensen GS. Natural killer cell activation and modulation of chemokine receptor profile in vitro by an extract from the cyanophyta Aphanizomenon flos-aquae. J Med Food. 2007;10(3):435-441. doi:10.1089/jmf.2007.401.

43. Lessard $M$, Dupuis $M$. Differential modulation of chicken lymphocyte blastogenesis and cytotoxic activity of natural killer cells in vitro by retinol, retinoic acid and beta-carotene. Nutr Res. 1994;14(8):1201-1217. doi:10.1016/S0271-5317(05)80247-7.

44. Lai $\mathrm{H}-$, Chang C-, Yang C-, et al. Activation of NK cell cytotoxicity by the natural compound 2,3-butanediol. J Leukocyte Biol. 2012;92(4):807-814. doi:10.1189/jlb.0112024.

45. Grudzien M, Rapak A. Effect of natural compounds on NK cell activation. J Immunol Res. 2018;2018. doi:10.1155/2018/4868417.

46. Nichenametla SN, South EH, Exon JH. Interaction of conjugated linoleic acid, sphingomyelin, and butyrate on formation of colonic aberrant crypt foci and immune functions in rats. J Toxicol Environ Health Part A. 2004;67(6):469-481.

47. Vannucci L, Fiserová A, Sadalapure K, et al. Effects of N-acetyl-glucosamine-coated glycodendrimers as biological modulators in the B16F10 melanoma model in vivo. Int J Oncol. 2003;23(2):285-296.

48. Reddy N, Hernandez-llizaliturri FJ, Deeb G, et al. Immunomodulatory drugs stimulate natural killer-cell function, alter cytokine production by dendritic cells, and inhibit angiogenesis enhancing the anti-tumour activity of rituximab in vivo. $\mathrm{Br} \mathrm{J}$ Haematol. 2008;140(1):36-45. doi:10.1111/j.1365-2141.2007.06841.x.

49. Yadav $S$, Jha R. Strategies to modulate the intestinal microbiota and their effects on nutrient utilization, performance, and health of poultry. J Animal Sci Biotechnol. 2019;10(1). doi:10.1186/s40104-018-0310-9.

50. Apajalahti JHA, Kettunen A, Bedford MR, Holben WE. Percent G+C Profiling Accurately Reveals Diet-Related Differences in the Gastrointestinal Microbial Community of Broiler Chickens. Appl Environ Microbiol. 2001;67(12):5656-5667. doi:10.1128/ AEM.67.12.5656-5667.2001.

51. Torok VA, Hughes RJ, Mikkelsen LL, et al. Identification and characterization of potential performance-related gut microbiotas in broiler chickens across various feeding trials. Appl Environ Microbiol. 2011;77(17):5868-5878. doi:10.1128/AEM.00165-11.

52. Kogut MH. The effect of microbiome modulation on the intestinal health of poultry. Anim Feed Sci Technol. 2019;250:32-40. doi:10.1016/j.anifeedsci.2018.10.008.

53. Roto SM, Rubinelli PM, Ricke SC. An introduction to the avian gut microbiota and the effects of yeast-based prebiotic-type compounds as potential feed additives. Front Vet Sci. 2015;2(SEP). doi:10.3389/fvets.2015.00028.

54. Zhu N, Wang J, Yu L, Zhang Q, Chen K, Liu B. Modulation of growth performance and intestinal microbiota in chickens fed plant extracts or virginiamycin. Front Microbiol. 2019;10(JUN). doi:10.3389/fmicb.2019.01333.

55. Göbel TWF, Chen CH, Shrimpf J, et al. Characterization of avian natural killer cells and their intracellular CD3 protein complex. Eur J Immunol. 1994;24(7):1685-1691. doi:10.1002/eji.1830240734. 
56. Beug H, von Kirchbach A, Döderlein G, Conscience J-, Graf T. Chicken hematopoietic cells transformed by seven strains of defective avian leukemia viruses display three distinct phenotypes of differentiation. Cell. 1979;18(2):375-390. doi:10.1016/00928674(79)90057-6.

57. Seliger C, Schaerer B, Kohn M, et al. A rapid high-precision flow cytometry based technique for total white blood cell counting in chickens. Vet Immunol Immunopathol. 2012;145(1-2):86-99. doi:10.1016/j.vetimm.2011.10.010.

58. van der Hoeven-Hangoor E, van der Vossen, J. M. B. M., Schuren FHJ, et al. lleal microbiota composition of broilers fed various commercial diet compositions. Poult Sci. 2013;92(10):2713-2723. doi:10.3382/ps.2013-03017.

59. Druyan S, De Oliveira JE, Ashwell CM. Focused microarrays as a method to evaluate subtle changes in gene expression. Poult Sci. 2008;87(11):2418-2429. doi:10.3382/ps.2007-00513.

60. De Oliveira JE, Druyan S, Uni Z, Ashwell CM, Ferket PR. Metabolic profiling of late-term turkey embryos by microarrays. Poult Sci. 2013;92(4):1011-1028.

61. Yadgary L, Uni Z. Yolk sac carbohydrate levels and gene expression of key gluconeogenic and glycogenic enzymes during chick embryonic development. Poult Sci. 2012;91(2):444-453. doi:10.3382/ps.2011-01669.

62. De Oliveira JE, Uni Z, Ferket PR. Important metabolic pathways in poultry embryos prior to hatch. Worlds Poult Sci J. 2008;64(4):488499.

63. Tako E, Ferket PR, Uni Z. Effects of in ovo feeding of carbohydrates and beta-hydroxy-beta-methylbutyrate on the development of chicken intestine. Poult Sci. 2004;83(12):2023-2028.

64. Davani-Davari D, Negahdaripour M, Karimzadeh I, et al. Prebiotics: definition, types, sources, mechanisms, and clinical applications. Foods. 2019;8(3):92.

65. Froebel LK, Jalukar S, Lavergne TA, Lee JT, Duong T. Administration of dietary prebiotics improves growth performance and reduces pathogen colonization in broiler chickens. Poult Sci. 2019;98(12):6668-6676. doi:10.3382/ps/pez537.

66. Abaza IM, Shehata MA, Shoieb MS, Hassan II. Evaluation of some natural feed additive in growing chicks diets. Int J Poult Sci. 2008;7(9):872-879. doi:10.3923/ijps.2008.872.879.

67. Al-Khalaifah H. Benefits of probiotics and/or prebiotics for antibiotic-reduced poultry. Poult Sci. 2018;97(11):3807-3815. doi:https://doi.org/10.3382/ps/pey160.

68. Arsenault RJ, Lee JT, Latham R, Carter B, Kogut MH. Changes in immune and metabolic gut response in broilers fed $\beta$-mannanase in $\beta$-mannan-containing diets. Poult Sci. 2017;96(12):4307-4316. doi:10.3382/ps/pex246.

69." wiaętkiewicz S, Arczewska-Włosek A, Józefiak D. Immunomodulatory efficacy of yeast cell products in poultry: A current review. World's Poult Sci J. 2014;70(1):57-68. doi:10.1017/S0043933914000051.

70. Doke SK, Dhawale SC. Alternatives to animal testing: A review. Saudi Pharm J. 2015;23(3):223-229. doi:10.1016/j.jsps.2013.11.002.

71. Byrne KA, Loving CL, McGill JL. Innate Immunomodulation in Food Animals: Evidence for Trained Immunity? Front Immunol. 2020;11. doi:10.3389/fimmu.2020.01099.

72. Netea MG, Domínguez-Andrés J, Barreiro LB, et al. Defining trained immunity and its role in health and disease. Nat Rev Immunol. 2020;20(6):375-388. doi:10.1038/s41577-020-0285-6.

73. Kleinnijenhuis J, Quintin J, Preijers F, et al. BCG-induced trained immunity in NK cells: Role for non-specific protection to infection. Clin Immunol. 2014;155(2):213-219. doi:10.1016/j.clim.2014.10.005.

74. Guo Y, Ali RA, Qureshi MA. The influence of $\beta$-glucan on immune responses in broiler chicks. Immunopharmacol Immunotoxicol. 2003;25(3):461-472. doi:10.1081/IPH-120024513.

75. Avci FY, Li X, Tsuji M, Kasper DL. Carbohydrates and T cells: A sweet twosome. Semin Immunol. 2013;25(2):146-151. doi:10.1016/j. smim.2013.05.005.

76. Chen X, Sheng Z, Qiu S, et al. Purification, characterization and in vitro and in vivo immune enhancement of polysaccharides from mulberry leaves. PLoS ONE. 2019;14(1). doi:10.1371/journal.pone.0208611.

77. Kallon S, Li X, Ji J, et al. Astragalus polysaccharide enhances immunity and inhibits H9N2 avian influenza virus in vitro and in vivo. J Animal Sci Biotechnol. 2013;4(1). doi:10.1186/2049-1891-4-22.

78. Teng P-, Kim WK. Review: Roles of prebiotics in intestinal ecosystem of broilers. Front Vet Sci. 2018;5(OCT). doi:10.3389/ fvets.2018.00245.

79. Saminathan M, Sieo CC, Kalavathy R, Abdullah N, Ho YW. Effect of prebiotic oligosaccharides on growth of Lactobacillus strains used as a probiotic for chickens. Afr.J.Microbiol.Res. 2011;5(1):57-64.

80. Flint HJ, Duncan SH, Scott KP, Louis P. Links between diet, gut microbiota composition and gut metabolism. Proc Nutr Soc. 2014;760. doi:10.1017/S0029665114001463.

81. Rinttilä T, Apajalahti J. Intestinal microbiota and metabolites-Implications for broiler chicken health and performance. J Appl Poult Res. 2013;22(3):647-658. doi:10.3382/japr.2013-00742.

82. Martín R, Miquel S, Benevides $L$, et al. Functional characterization of novel Faecalibacterium prausnitzii strains isolated from healthy volunteers: A step forward in the use of F. prausnitzii as a next-generation probiotic. Front Microbiol. 2017;8(JUN). doi:10.3389/fmicb.2017.01226.

83. Frank DN, St. Amand AL, Feldman RA, Boedeker EC, Harpaz N, Pace NR. Molecular-phylogenetic characterization of microbial community imbalances in human inflammatory bowel diseases. Proc Natl Acad Sci U S A. 2007;104(34):13780-13785. doi:10.1073/ pnas.0706625104.

84. Faas MM, Liu Y, Borghuis T, van Loo-Bouwman CA, Harmsen H, de Vos P. Microbiota Induced Changes in the Immune Response in Pregnant Mice. Front Immunol. 2020;10. doi:10.3389/fimmu.2019.02976.

85. Clarke TB, Davis KM, Lysenko ES, Zhou AY, Yu Y, Weiser JN. Recognition of peptidoglycan from the microbiota by Nod1 enhances systemic innate immunity. Nat Med. 2010;16(2):228-231. doi:10.1038/nm.2087. 
86. Macpherson AJ, Harris NL. Interactions between commensal intestinal bacteria and the immune system. Nature Reviews Immunology. 2004;4(6):478-485. doi:10.1038/nri1373.

87. Aziz N, Bonavida B. Activation of natural killer cells by probiotics. Forum Immunopathol Dis Ther. 2016;7(1-2):41-55. doi:10.1615/ ForumlmmunDisTher.2016017095.

88. Ferlazzo G, Rizzello V, Bonaccorsi I, Dongarr ML, Fink LN. Role of natural killer and dendritic cell crosstalk in immunomodulation by commensal bacteria probiotics. J Biomed Biotechnol. 2011;2011. doi:10.1155/2011/473097.

89. Hessle C, Andersson B, Wold AE. Gram-positive bacteria are potent inducers of monocytic interleukin-12 (IL-12) while gram-negative bacteria preferentially stimulate IL-10 production. Infect Immun. 2000;68(6):3581-3586. doi:10.1128/IAI.68.6.35813586.2000 .

90. Trinchieri G. Interleukin-12 and the regulation of innate resistance and adaptive immunity. NatRev Immunol. 2003;3(2):133-146. doi:10.1038/nri1001.

91. Ivanova D, Krempels R, Ryfe J, Weitzman K, Stephenson D, Gigley JP. NK cells in mucosal defense against infection. BioMed Res Int. 2014;2014. doi:10.1155/2014/413982.

92. Brownlie R, Zhu J, Allan B, et al. Chicken TLR21 acts as a functional homologue to mammalian TLR9 in the recognition of CpG oligodeoxynucleotides. Mol Immunol. 2009;46(15):3163-3170. doi:10.1016/j.molimm.2009.06.002.

93. Levy M, Blacher E, Elinav E. Microbiome, metabolites and host immunity. Curr Opin Microbiol. 2017;35:8-15. doi:10.1016/j. mib.2016.10.003.

94. Shapiro $\mathrm{H}$, Thaiss CA, Levy M, Elinav E. The cross talk between microbiota and the immune system: Metabolites take center stage. Curr Opin Immunol. 2014;30(1):54-62. doi:10.1016/j.coi.2014.07.003.

95. Skelly AN, Sato Y, Kearney S, Honda K. Mining the microbiota for microbial and metabolite-based immunotherapies. Nat Rev Immunol. 2019;19(5):305-323. doi:10.1038/s41577-019-0144-5.

96. Esch, B. C. A. M. V., Porbahaie M, Abbring S, et al. The Impact of Milk and Its Components on Epigenetic Programming of Immune Function in Early Life and Beyond: Implications for Allergy and Asthma. Front Immunol. 2020;11. doi:10.3389/fimmu.2020.02141. 


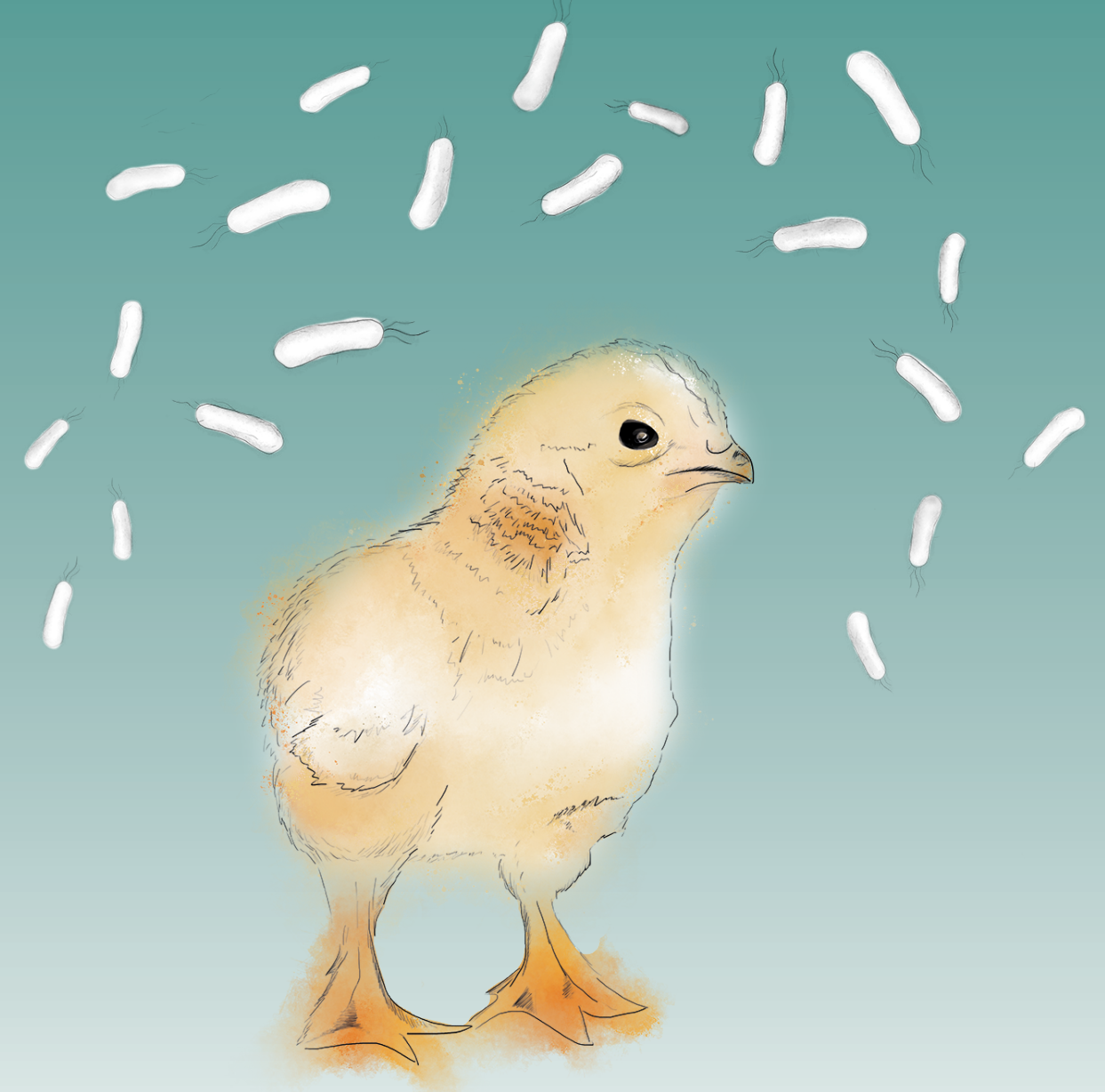


CHAPTER 5

\section{A detailed analysis of innate and adaptive immune responsiveness upon infection with Salmonella enterica serotype Enteritidis in young broiler chickens}

Nathalie Meijerink', Robin H.G.A. van den Biggelaar1, Daphne A. van Haarlem¹, J. Arjan Stegeman², Victor P.M.G. Rutten ${ }^{1,3}$, Christine A. Jansen ${ }^{1}$

1 Department Biomolecular Health Sciences, Division Infectious Diseases and Immunology, Faculty of Veterinary Medicine, Utrecht University, Utrecht, the Netherlands

2 Department Population Health Sciences, Division Farm Animal Health, Faculty of Veterinary Medicine, Utrecht University, Utrecht, the Netherlands

3 Department of Veterinary Tropical Diseases, Faculty of Veterinary Science, University of Pretoria, Pretoria, South Africa 


\section{Abstract}

Salmonella enterica serotype Enteritidis (SE) is a zoonotic pathogen which causes foodborne diseases in humans as well as severe disease symptoms in young chickens. More insight in innate and adaptive immune responses of chickens to SE infection is needed to understand elimination of SE. Seven-day-old broiler chickens were experimentally challenged with SE and numbers and responsiveness of innate and adaptive immune cells as well as antibody titers were assessed. SE was observed in the ileum and spleen of SE-infected chickens at 7 days post-infection (dpi). At $1 \mathrm{dpi} \mathrm{numbers} \mathrm{of} \mathrm{intraepithelial} \mathrm{cytotoxic}$ $\mathrm{CD}^{+} \mathrm{T}$ cells were significantly increased alongside numerically increased intraepithelial IL-2R $\alpha^{+}$and $20 \mathrm{E} 5^{+}$natural killer (NK) cells at 1 and $3 \mathrm{dpi}$. At both time points, activation of intraepithelial and splenic NK cells was significantly enhanced. At $7 \mathrm{dpi}$ in the spleen, presence of macrophages and expression of activation markers on dendritic cells were significantly increased. At $21 \mathrm{dpi}$, SE-induced proliferation of splenic $\mathrm{CD}^{+}$and $\mathrm{CD} 8^{+} \mathrm{T}$ cells was observed and SE-specific antibodies were detected in sera of all SE-infected chickens. In conclusion, SE results in enhanced numbers and activation of innate cells and we hypothesized that in concert with subsequent specific $T$ cell and antibody responses, reduction of $\mathrm{SE}$ is achieved. A better understanding of innate and adaptive immune responses important in the elimination of SE will aid in developing immune-modulation strategies, which may increase resistance to $S E$ in young broiler chickens.

Keywords: Broiler chickens, Salmonella enterica serotype Enteritidis, innate, adaptive, immunity, NK cells, antigen-presenting cells, T cells, IELs 


\section{Introduction}

Salmonella enterica serotype Enteritidis (SE) is one of the leading causes of foodborne diseases in humans, most often due to poultry products that are not well prepared. In chickens infected with faecal Salmonellae via oral or respiratory routes, SE colonizes the intestinal tract and disseminate systemically to tissues such as the liver and spleen ${ }^{1,2}$. In young chickens it can lead to severe disease and death, whereas adult chickens are often subclinically infected with $\mathrm{SE}$, carrying the bacteria in their intestines ${ }^{3}$. Prevention of $\mathrm{SE}$ infection in poultry is thus important for health and welfare of young chickens and to avoid substantial economic production losses in the poultry sector as a consequence. In addition, SE prevention in poultry is significant for the health and wellbeing of humans as well as to avoid loss of productivity and health care costs. Since therapeutic treatment of SE infection in chickens with antibiotics is not advised due to limited effectiveness and risk of antibiotic resistance, the use of immune-modulatory strategies to increase the resistance to SE is encouraged ${ }^{4}$. More insight in innate and adaptive immune responses and their interaction in response to SE infection in young broiler chickens will facilitate the design of these strategies.

In young chickens, immunity largely depends on maternal antibodies as well activity of the innate immune system, with natural killer (NK) cells and macrophages as key players, ${ }^{5,6}$. Due to the low numbers of NK cells that can be isolated from the caecum ${ }^{7,8}$ and since the ileum is generally considered as a site of immune activation with many lymphoid structures ${ }^{9,10}$, we set out to study immune responses induced by SE in the ileum. NK cells are particularly abundant amongst intestinal intraepithelial lymphocytes (IELs) $)^{11,12}$, which are also rich in $\gamma \delta T$ cells and $C D 8^{+} T$ cells expressing the $\alpha \beta T$ cell receptor $(T C R)^{11,13}$. Macrophages, dendritic cells (DCs) and $C D 4^{+} T$ cells are located directly underneath the intestinal epithelium ${ }^{14}$. The adaptive immune system is not fully developed yet upon hatch and functional $\mathrm{T}$ and $B$ cell responses are observed after approximately two to three weeks of life ${ }^{5,6}$.

The early response of the innate immune system in chickens within one week post SE infection is characterized by the upregulation of genes associated with "defense/pathogen response"15, inflammation ${ }^{16-18}$, NK cell-mediated cytotoxicity ${ }^{19}$ and production and secretion of the cytokine IFN $\gamma^{20}$. Other studies have shown the influx of heterophils and macrophages in the spleen and $\gamma \delta \mathrm{T}$ cells in the caecum, and expression of activation-related genes in the respective cell types during the first response to SE in chickens ${ }^{21,22}$. However, the effect of SE infection on the function of NK cells in chickens has not been studied so far. In chickens, intraepithelial NK cells comprise a major CD3- IL-2R $\alpha^{+}$subset ${ }^{11,12}$ and a minor CD3-20E5 ${ }^{+}$subset ${ }^{12}$, both having cytotoxic capacity but to different degrees. In mice and humans, high cytotoxic activity ${ }^{23,24}$ and IFN- $\gamma$ production ${ }^{24,25}$ by NK cells have been shown to result in resistance to Salmonella enterica serotype Typhimurium. This suggests an important role for NK cells as well in the first response to SE.

The development of $\mathrm{T}$ and $\mathrm{B}$ cell responses is initiated with the activation of professional antigen presenting cells (APCs) such as DCs and macrophages. The presence ${ }^{26}$ and activity of intestinal macrophages ${ }^{27-29}$, identified by the expression of mannose receptor C-type 1-like B (MRC1LB) ${ }^{30}$, and bone marrow-derived DCs ${ }^{31}$ increases during SE infection. Furthermore, oral infection of one-day-old specific-pathogen-free chickens with SE elicits increased mRNA expression of chemokines and macrophages are attracted to the ileum within 24 hours $^{32}$. In addition, it has been shown that decreased activity of peritoneal macrophage is associated with increased susceptibility for systemic dissemination of SE in chickens ${ }^{33}$. On the other hand, Salmonella species have been found to resist killing by macrophages in mammals ${ }^{34}$ and chickens $s^{35,36}$ and even use macrophages as a carrier for systemic dissemination ${ }^{37}$. Despite their involvement in SE infection, a detailed analysis of the effect of SE infection on the function of APCs in chickens has not been performed to date. 
Initial T cell responsiveness to SE in chickens has been observed within one week, including increased presence of $\gamma \delta T$ cells in the intestine, blood and spleen after vaccination with live-attenuated SE or SE infection as compared to SE negative chickens ${ }^{20,22}$. In addition, enhanced mRNA expression of cytotoxic activity-related genes was observed in the spleen of SE-infected compared to uninfected chickens $^{38}$. Two and three weeks post-vaccination or infection, a second increase in presence of $\gamma \delta T$ cells was observed in the spleen and intestine, respectively, compared to non-immunized chickens ${ }^{22}$. $\mathrm{CD} 4{ }^{+}$helper T cells and $\mathrm{CD}^{+}$cytotoxic $\mathrm{T}$ cells were also shown to increase in presence within one week post-SE infection and vaccination in the intestine, as compared to uninfected and non-vaccinated chickens ${ }^{20,26,27}$. CD8 ${ }^{+}$cytotoxic T cells kill infected host cells, while $\mathrm{CD}^{+}$helper $\mathrm{T}$ cells release cytokines like IL-2 and IFN $y$ to further stimulate NK cells, and macrophages and CD8 ${ }^{+}$cytotoxic T cells respectively, and promote the differentiation of B cells into antibody-producing plasma cells. Antibody responses involved in elimination of SE partly depend on maternal antibodies and on the production of IgA in the intestine ${ }^{39,40}$, as well as $\lg \mathrm{M}, \lg \mathrm{A}$ and $\lg \mathrm{Y}$ antibodies in blood ${ }^{40,41}$. Whereas previous studies have focussed on specific aspects of the immune responses, the present study combines cellular assays to analyze both the innate and adaptive immune responses in the IEL population and spleen upon SE infection in chickens.

In this study, we investigated how Salmonella enterica serotype Enteritidis infection in young broiler chickens affects presence and activation of innate and adaptive immune cells in the IEL population and spleen to obtain more insight in the contribution of the immune system to elimination of the infection. Numbers of SE in ileum and spleen were determined alongside differences in kinetics of presence and activation status of NK cell and APC subsets between uninfected and infected chickens. The subsequent adaptive responses were determined including presence and activation of $\gamma \delta$ T cell, CD4 ${ }^{+}$ and $C D 8^{+} \mathrm{T}$ cell subsets, and serum antibody levels. Hence the present study provides an extensive overview of intraepithelial and systemic immune responses that are evoked by SE infection in young broiler chickens. Based on the phenotypical and functional data obtained, we will hypothesize on how the various elements of the immune system interact and contribute to elimination of the SE infection, and on potential strengthening of immune responsiveness by immunomodulation strategies, which may prevent SE infection and colonization, and thus increase chicken health and welfare as well as safety of food of chicken origin.

\section{Materials and methods}

\section{Animals and husbandry}

A total of 30 respectively 35 Ross 308 seventeen- and eighteen-day old embryonated eggs were obtained from the same parent flock of a commercial hatchery (Lagerwey, the Netherlands). Eggs were disinfected with $3 \%$ hydrogen peroxide and placed in disinfected hatchers in two different stables (ED17 eggs: uninfected chickens and ED18 eggs: SE-infected chickens) at the facilities of the Department of Population Health Sciences, Faculty of Veterinary Medicine, Utrecht University, the Netherlands. Cleaning the eggs with a low concentration of hydrogen peroxide is a standard procedure. It is highly unlikely that it influences intestinal microbiota composition as described in a previous study ${ }^{42}$. Directly upon hatch, chickens were weighed, labelled and female and male chickens were equally distributed in floor pens of 2 $\times 2 \mathrm{~m}$ lined with wood shavings $\left(2 \mathrm{~kg} / \mathrm{m}^{2}\right)$, and received water and standard Salmonella-free commercial starter and grower feeds ad libitum (Research Diet Services, the Netherlands). A standard lighting and temperature scheme for Ross broiler chickens was used for both stables. 
The animal experiment was approved by the Dutch Central Authority for Scientific Procedures on Animals and the Animal Experiments Committee (registration number AVD1080020174425) of Utrecht University (the Netherlands) and all procedures were done in full compliance with all relevant legislation.

\section{Experimental design}

Before the start of the experiment at day 3, five chickens per group (uninfected $(n=30)$ and SE-infected $(n=35))$ were randomly selected and sacrificed for collection of ileum $( \pm 10 \mathrm{~cm}$ distal from Meckel's diverticulum) and spleen to confirm absence of SE before inoculation. At day 7 ( 0 days post-infection (dpi)), five chickens of the SE-infected group only, were randomly selected and sacrificed for collection of ileum and spleen, to determine baseline levels of the various immune parameters as well as absence of SE before infection. Subsequently, chickens of the SE-infected group were challenged at day 7 ( $0 \mathrm{dpi})$ by oral inoculation of $0.25 \mathrm{ml}$ brain heart infusion $(\mathrm{BHI})$ medium containing $1.12 \times 10^{6}$ colony-forming units (CFUs) SE, whereas chickens in the other stable (uninfected) were inoculated with $0.25 \mathrm{ml} \mathrm{BHI}$ medium. At days 8 (1 dpi), 10 (3 dpi), 14 (7 dpi), 21 (14 dpi) and 28 (21 dpi), five chickens per group were randomly selected and sacrificed for collection of ileum and spleen to determine bacterial CFUs as well as numbers and function of NK cells and T cells. At days 7, 8, 10 and 14 also spleen APCs were assessed. At days 7, 14,21 and 28 blood (at least $5 \mathrm{ml}$ ) was collected in EDTA tubes (VACUETTE ${ }^{\circledR}$ K3E EDTA, Greiner Bio-One, the Netherlands) for determination of SE-specific antibody levels. At day 28, splenic lymphocytes were also used to assess SE-induced T cell reactivity in a proliferation assay. The use of five chickens per group per time point was calculated using power analysis (Sample size \& power calculator, LASEC, China). All chickens were weighed prior to post-mortem analyses to determine the growth curve. To calculate absolute cell numbers, ileum segments and spleens were weighed immediately after collection of the tissues, prior to isolation of cells. After isolation, cell numbers in the resulting suspension were calculated. This resulted in the total cell number, expressed as IELs per mg ileum or leukocytes per mg spleen. To calculate the absolute numbers of NK cells, APCs and T cells within the live IEL or leukocyte populations, the percentages of cells positive for the markers expressed on these cell types were used which were determined in the flow cytometry analyses. Absolute cell numbers were calculated using the following formula: (absolute number IELs or leukocytes per mg tissue) $\times$ (percentage positive cells in the gate of interest of the live lymphocyte or leukocyte population).

\section{SE culture}

The Salmonella enterica serotype Enteritidis strain (K285/93 Nal res) was kindly provided by Dr. E. Broens, director of the Veterinary Microbiological Diagnostic Center (VMDC) of the Faculty of Veterinary Medicine, Utrecht University, and cultured as described previously ${ }^{43}$. In short, from an overnight culture of the SE strain on blood agar (Oxoid, the Netherlands) a single colony was used to inoculate $45 \mathrm{ml} \mathrm{BHI}$ medium (Oxoid), which was incubated aerobically overnight at $200 \mathrm{rpm}$ in a shaking incubator (Certomat BS-1, B. Braun Biotech international, Sweden) at $37^{\circ} \mathrm{C}$. The OD value of a sample of the SE culture diluted 1:10 in PBS was measured using a Ultrospec 2000 (Pharmacia Biotech, Sweden), the SE concentration was calculated from a previously determined growth curve, and SE were diluted in $\mathrm{BHI}$ medium to $4 \times 10^{6}$ $\mathrm{CFU} / \mathrm{ml}$, to constitute the inoculum. The exact SE concentration of the inoculum, determined by counting the number of CFUs of plated serial dilutions after overnight culture, was $4.49 \times 10^{6} \mathrm{CFU} / \mathrm{ml}$.

For the T cell proliferation assay, SE was fixed by resuspending $3.8 \times 10^{9}$ CFUs in $100 \mu$ PBS with $1 \%$ formaldehyde (Sigma-Aldrich, the Netherlands) and incubation for $5 \mathrm{~min}$ at RT, while the suspension was vortexed shortly every minute. After fixation, the bacteria were washed four times in $1 \mathrm{ml}$ PBS by centrifugation at $15000 \times g$ to remove the supernatants (Heraeus Pico 17 Centrifuge, Thermo Fisher 
Scientific). Finally, the bacteria were resuspended in $380 \mu \mathrm{I}$ X-VIVO 15 cell culture medium (Lonza, the Netherlands) with $50 \mu \mathrm{g} / \mathrm{ml}$ gentamycin (Gibco ${ }^{\mathrm{TM}}$, the Netherlands) to create a concentration of $10^{7} \mathrm{CFU} /$ $\mathrm{ml}$ and stored at $4^{\circ} \mathrm{C}$ until further use.

\section{Isolation of cells}

The procedures to isolate IELs from ileum and leukocytes from spleen were performed as described previously ${ }^{12,44}$. lleum segments were washed with PBS to remove the contents, cut into sections of $1 \mathrm{~cm}^{2}$ and washed again. Subsequently, the IELs were collected by incubating three times in a shaking incubator (Certomat BS-1) at $200 \mathrm{rpm}$ for $15 \mathrm{~min}$ at $37^{\circ} \mathrm{C}$ in EDTA-medium (HBSS 1x (Gibco ${ }^{\oplus}$ ) supplemented with $10 \%$ heat-inactivated FCS (Lonza) and 1\% 0.5M EDTA- $\mathrm{Na}_{2}$ (UltraPure ${ }^{\mathrm{TM}}$, Invitrogen, the Netherlands)). Supernatants were collected and centrifuged for $5 \mathrm{~min}$ at $335 \times \mathrm{g}$ at $20^{\circ} \mathrm{C}$ (Allegra ${ }^{\mathrm{TM}} \mathrm{X}-12 \mathrm{R}$ Centrifuge, Beckman Coulter, the Netherlands). Pellets were then resuspended in PBS at a concentration of $10 \mathrm{ml}$ per gram tissue and an aliquot of $100 \mu \mathrm{l}$ was used for bacteriological analysis. PBS was added to the remaining suspension up to $20 \mathrm{ml}$ and IELs were isolated using Ficoll-Paque Plus (GE Healthcare, the Netherlands) density gradient centrifugation for $12 \mathrm{~min}$ at $673 \times \mathrm{g}$ at $20^{\circ} \mathrm{C}$, washed in PBS by centrifugation for $5 \mathrm{~min}$ at $393 \times g$ at $4^{\circ} \mathrm{C}$ and resuspended at $4.0 \times 10^{6}$ cells $/ \mathrm{ml}$ in complete medium (IMDM $2 \mathrm{mM}$ glutamax I supplemented with $8 \%$ heat-inactivated FCS (Lonza), 2\% heat-inactivated chicken serum, $100 \mathrm{U} / \mathrm{ml}$ penicillin and $100 \mu \mathrm{g} / \mathrm{ml}$ streptomycin; Gibco ${ }^{\circledR}$ ). Spleens were homogenized using a $70 \mu \mathrm{m}$ cell strainer (Beckton Dickinson (BD) Biosciences, NJ, USA) and the single-cell suspension was diluted in PBS at a concentration of $10 \mathrm{ml}$ per gram tissue. An aliquot of $100 \mu \mathrm{l}$ was used again for bacteriological analysis. Next, leukocytes were isolated by Ficoll-Paque Plus density gradient centrifugation (20 min, $1126 \times g$, $20^{\circ} \mathrm{C}$ ), washed in PBS and resuspended at $4.0 \times 10^{6} \mathrm{cell} / \mathrm{s} / \mathrm{ml}$ in complete medium as described for ileum.

Whole blood was allowed to coagulate by leaving it undisturbed for 1 hour at room temperature (RT), centrifuged for $10 \mathrm{~min}$ at $2095 \times \mathrm{g}$ at $15^{\circ} \mathrm{C}$ and subsequently, serum was collected and stored at $-20^{\circ} \mathrm{C}$ until further use.

\section{Quantitative bacteriology of ileum and spleen}

At $-4,0,1,3,7,14$ and 21 dpi, the numbers of Salmonella colonies in ileum and spleen were determined by plating $100 \mu \mathrm{l}$ of the cell suspensions of either the ileum segments or homogenized spleens with a spatula on RAPID'Salmonella Medium plates (Bio-Rad, the Netherlands). Plates were incubated overnight at $37^{\circ} \mathrm{C}$ and subsequently, purple colonies were quantified and SE was expressed as CFU per gram tissue. The limit of detection (LOD) was 100 CFU per gram tissue.

\section{Phenotypic characterization of lymphocytes by flow cytometry}

Presence and activation of NK and T cell subsets were determined among IELs and splenocytes at 0, 1, 3, 7,14 and $21 \mathrm{dpi}$ as described previously ${ }^{12,42}$. Lymphocyte populations $\left(1 \times 10^{6}\right)$ were stained with a panel of antibodies specific for surface markers known to be expressed on NK cells, as well as with anti-CD3 to exclude $T$ cells from the analyses. In addition, cells were stained with a panel of antibodies specific for surface markers that distinguishes $\gamma \delta T$ cell, $C D 4^{+}$and $C D 8^{+} T$ cell subsets (Table 1). Staining with primary and secondary antibodies was performed in $50 \mu \mathrm{l}$ PBS (Lonza) containing $0.5 \%$ bovine serum albumin and $0.1 \%$ sodium azide (PBA). Cells were incubated for 20 min at $4^{\circ} \mathrm{C}$ in the dark, washed twice by centrifugation for 5 min at $393 \times g$ at $4^{\circ} \mathrm{C}$ in PBA, after primary staining, and in PBS after secondary staining. Subsequently, to be able to exclude dead cells from analysis, lymphocytes were stained in 100 $\mu \mathrm{l}$ PBS with a viability dye (Zombie Aqua ${ }^{\mathrm{TM}}$ Fixable Viability Kit, Biolegend, the Netherlands) for 15 min at 
RT in the dark, washed twice in PBA and resuspended in $200 \mu \mathrm{PBA}$. Of each sample, either $150 \mu \mathrm{l}$ or a maximum of $1 \times 10^{6}$ viable cells were analyzed using a CytoFLEX LX Flow Cytometer (Beckman Coulter), and data was analyzed with FlowJo software (FlowJo LCC, BD Biosciences). The gating strategies used to analyze NK cells, $\gamma \delta T$ cells and cytotoxic CD8 ${ }^{+} \mathrm{T}$ cells are depicted in Fig. S1.

Table 1. Flow cytometry staining reagents.

\begin{tabular}{|c|c|c|c|}
\hline $\begin{array}{l}\text { Cell } \\
\text { population }\end{array}$ & $\begin{array}{l}\text { Primary antibody } \\
\text { (mouse-anti-chicken) }\end{array}$ & Clone / Isotype & Secondary antibody \\
\hline \multirow[t]{4}{*}{ NK cells } & CD45-FITC $^{1}$ & $\mathrm{LT} 40$ / IgM & - \\
\hline & CD3-APC ${ }^{1}$ & $\mathrm{CT} 3 / \lg \mathrm{G} 1$ & - \\
\hline & IL-2R $\alpha-U N L^{2}$ & $28-4 / \lg G 3$ & Goat-anti-mouse-IgG3-PE ${ }^{1}$ \\
\hline & $20 \mathrm{E} 5-\mathrm{BIOT}^{2}$ & $\lg \mathrm{G} 1$ & Streptavidin (SA)-Perc $\mathrm{P}^{6}$ \\
\hline \multirow[t]{5}{*}{ T cells } & CD3-PE ${ }^{1}$ & $\mathrm{CT} 3 / \lg \mathrm{G} 1$ & - \\
\hline & CD4-APC ${ }^{1}$ & $\mathrm{CT} 4$ / IgG1 & - \\
\hline & TCR $\gamma \delta-$ FITC $^{1}$ & TCR-1 / lgG1 & - \\
\hline & $C D 8 \alpha-U N L^{1}$ & $\mathrm{EP72} / \lg \mathrm{g} 2 \mathrm{~b}$ & Goat-anti-mouse-IgG2b-APC/Cy $7^{1}$ \\
\hline & $\mathrm{CD} 8 \beta-\mathrm{BIOT}^{1}$ & $\mathrm{EP} 42 / \lg \mathrm{g} 2 \mathrm{a}$ & SA-PercP ${ }^{6}$ \\
\hline \multirow[t]{10}{*}{ APCs } & CD41/61-FITC ${ }^{4}$ & $11 \mathrm{C} 3 / \lg \mathrm{g} 1$ & - \\
\hline & $\mathrm{Bu}-1-\mathrm{AF} 647^{1}$ & $\mathrm{AV} 20$ / IgG1 & - \\
\hline & $\mathrm{CD}-\mathrm{FITC}^{1}$ & $\mathrm{CT} 3 / \lg \mathrm{G} 1$ & - \\
\hline & CD4-PE/Cy $7^{1}$ & $\mathrm{CT} 4$ / IgG1 & - \\
\hline & MRC1LB-PE ${ }^{1}$ & KUL01 / IgG1 & - \\
\hline & CD11-biotin ${ }^{2}$ & $5 \mathrm{C} 7 / \lg \mathrm{G} 1$ & SA-Brilliant Violet (BV) $605^{7}$ \\
\hline & MHCIIUNL $^{1}$ & $\mathrm{Cia} / \mathrm{Ig} \mathrm{M}$ & Rat-anti-mouse-IgM-BV421 (RMM-1) ${ }^{7}$ \\
\hline & ${ }^{*} \mathrm{CHIR}-\mathrm{AB} 1-\mathrm{UNL}^{2}$ & $8 D 12 / \lg G 2 a$ & Rat-anti-mouse-IgG2a-PerCP/Cy5.5 (RMG2a-62) \\
\hline & ${ }^{*} \mathrm{CD} 40-\mathrm{UNL}^{5}$ & AV79 / IgG2a & Rat-anti-mouse-IgG2a-PerCP/Cy5.5 (RMG2a-62) \\
\hline & ${ }^{*} \mathrm{CD} 80-\mathrm{UNL}^{5}$ & IAH:F864:DC7 / lgG2a & Rat-anti-mouse-IgG2a-PerCP/Cy5.5 (RMG2a-62) \\
\hline \multicolumn{4}{|l|}{ Assay } \\
\hline \multirow[t]{6}{*}{ CD107 } & CD107a-APC 3 & LEP-100 I 5G10 / lgG1 & - \\
\hline & CD41/61-FITC ${ }^{4}$ & $11 \mathrm{C} 3 / \lg \mathrm{g} 1$ & - \\
\hline & $\mathrm{CD} 3-\mathrm{PE}^{1}$ & $\mathrm{CT} 3 / \lg \mathrm{G} 1$ & - \\
\hline & $\mathrm{CD} 8 \alpha-\mathrm{UNL}_{1}$ & $\mathrm{EP72} / \lg \mathrm{G} 2 \mathrm{~b}$ & Goat-anti-mouse-IgG2b-Alexa Fluor (AF) $790^{8}$ \\
\hline & $28-4-U N L^{2}$ & $\lg G 3$ & Goat-anti-mouse-lgG3-APC/Cy $7^{1}$ \\
\hline & 20E5-BIOT² & $\lg G 1$ & SA-PercP ${ }^{6}$ \\
\hline \multirow[t]{6}{*}{ IFNY } & $\mathrm{CD} 3-\mathrm{PE}^{1}$ & $\mathrm{CT} 3 / \lg \mathrm{G} 1$ & - \\
\hline & TCRy $\delta$-FITC ${ }^{1}$ & TCR-1 / lgG1 & - \\
\hline & $\mathrm{CD} 8 \alpha-\mathrm{UNL}^{1}$ & $\mathrm{EP72} / \lg \mathrm{g} 2 \mathrm{~b}$ & Goat-anti-mouse-IgG2b-AF790 ${ }^{8}$ \\
\hline & $28-4-U N L^{2}$ & $\lg 33$ & Goat-anti-mouse-lgG3-APC/Cy $7^{1}$ \\
\hline & $20 \mathrm{E} 5-\mathrm{BIOT}^{2}$ & $\lg \mathrm{G} 1$ & SA-PercP ${ }^{6}$ \\
\hline & IFNY-APC ${ }^{3}$ & MAb80 / lgG1 & - \\
\hline \multirow{4}{*}{$\begin{array}{l}\text { T cell } \\
\text { proliferation }\end{array}$} & CD3-FITC $^{1}$ & $\mathrm{CT} 3 / \lg \mathrm{G} 1$ & - \\
\hline & CD4-PE/Cy $7^{1}$ & $\mathrm{CT} 4$ / IgG1 & - \\
\hline & $\mathrm{CD} 8 \alpha-\mathrm{APC}^{1}$ & СТ8 / lgG1 & - \\
\hline & IL-2R $\alpha-U N L^{2}$ & $28-4 / \lg G 3$ & Goat-anti-mouse-IgG3-PE ${ }^{1}$ \\
\hline
\end{tabular}

Three APC antibody panels were prepared each containing surface markers plus one out of three antibodies indicated $\left({ }^{*}\right)$. Manufacturer: 'Southern Biotech, AL, USA. ${ }^{2}$ Purified antibody from hybridoma supernatant ${ }^{44}$, kindly provided by Göbel, T.W., Ludwig Maximilian University, Germany. ${ }^{3}$ Developmental Studies Hybridoma Bank (DSHB), University of Iowa, IA, USA. ${ }^{4}$ Serotec, United Kingdom. ${ }^{5}$ Bio-Rad. ${ }^{6} \mathrm{BD}$ Biosciences. ${ }^{7}$ Biolegend. ${ }^{8}$ Jackson ImmunoResearch Laboratories, PA, USA. 


\section{CD107 assay}

Activation of NK cells and cytotoxic CD8 ${ }^{+}$T cells was determined using the CD107 assay, which measures the increased surface expression of CD107a that results from degranulation, the release of cytotoxic granules ${ }^{44}$. Briefly, lymphocytes isolated from the IEL population and spleen were resuspended in complete medium, and $1 \times 10^{6}$ lymphocytes in $0.5 \mathrm{ml}$ were incubated in the presence of $1 \mu \mathrm{l} / \mathrm{ml}$ GolgiStop (BD Biosciences) and $0.5 \mu \mathrm{l} / \mathrm{ml}$ mouse-anti-chicken-CD107a-APC for 4 hours at $37^{\circ} \mathrm{C}, 5 \% \mathrm{CO}_{2}$. After incubation, lymphocytes were washed in PBA and stained as described in section "Phenotypic characterization of lymphocytes by flow cytometry" with monoclonal antibodies for NK and T cells, and anti-CD41/61 to exclude thrombocytes from analyses, as mentioned in the CD107 panel (Table 1). Cells were washed in PBS, stained for viability and analyzed by flow cytometry.

\section{IFNץ assay}

Expression of intracellular IFN $\gamma$ was determined in (subsets of) NK cells, $\gamma \delta T$ cells, $C D 4^{+}$and $C D 8^{+} T$ cells, using the assay adapted from Ariaans et al. ${ }^{45}$. Lymphocytes isolated from the IEL population and spleen were resuspended in complete medium, and $1 \times 10^{6}$ lymphocytes in $0.5 \mathrm{ml}$ were incubated in the presence of $1 \mu \mathrm{l} / \mathrm{ml}$ Brefeldin A (Sigma Aldrich) for 4 hours at $41^{\circ} \mathrm{C}, 5 \% \mathrm{CO}_{2}$. After incubation, lymphocytes were washed in PBA and stained as described in section "Phenotypic characterization of lymphocytes by flow cytometry" with surface markers as mentioned in the IFNY panel (Table 1). Cells were washed in PBS, stained for viability and washed again in PBA. Then, lymphocytes were permeabilized differently as described by Ariaans et al. ${ }^{45}$. Lymphocytes were incubated in $200 \mu \mathrm{l}$ of a mixture of BD FACS ${ }^{\text {TM }}$ Permeabilizing Solution 2 and BD FACS ${ }^{\text {TM }}$ Lysing Solution prepared according to manufacturer's instructions (BD Biosciences) for 8 min at RT, immediately followed by centrifugation for 2 min at $393 \times g$ at $4^{\circ} \mathrm{C}$. Cells were washed twice in PBA, stained intracellularly with anti-IFN $\gamma$-APC in $50 \mu$ PBA for 20 min at $4^{\circ} \mathrm{C}$ in the dark, washed in PBA and finally analyzed by flow cytometry.

\section{Phenotypic characterization of APCs by flow cytometry}

Splenocytes isolated at 0, 1, 3 and 7 dpi from infected and uninfected chickens were transferred to a 96 wells V-bottom plate and stained with antibodies of the APC panel to distinguish APC subsets (Table 1). Staining with primary and secondary antibodies $\left(1 \times 10^{6}\right)$ was performed in $50 \mu \mathrm{PBA}$, incubated for 20 $\min$ at $4^{\circ} \mathrm{C}$ in the dark and washed twice by centrifugation for 3 min at $393 \times g$ at $4^{\circ} \mathrm{C}$ in PBA. Finally, the cells were stained in $50 \mu \mathrm{l}$ PBS with ViaKrome 808 viability dye (Beckman Coulter) for $20 \mathrm{~min}$ at $4^{\circ} \mathrm{C}$. Cells were washed in PBA and analyzed by flow cytometry as described in section "Phenotypic characterization of lymphocytes by flow cytometry", using $180 \mu$ l.

Based on the APC subset staining, a t-distributed Stochastic Neighbor Embedding (t-SNE) analysis was performed using FlowJo software to identify cell subsets using an unbiased approach. From each sample of splenocytes at 7 dpi of infected $(n=5)$ and uninfected $(n=5)$ chickens, 10000 cells were taken and concatenated into one FCS file that represented all individual chickens. The t-SNE was performed based on expression levels of CD3, CD41/61, MRC1LB, CD4, Bu-1, CD11, MHCIl and CHIR-AB1 using published automated optimized parameters ${ }^{46}$. Based on the t-SNE, three APC subsets were identified based on selection of MRC1LB and CD11 positive cells, which were negative for CD3, CD4 and CD41/61. Activation status of the subsets was subsequently evaluated using the expression percentages of immunoglobulin $Y$ receptor $C H I R-A B 1$, co-stimulatory molecules $C D 40$ and $C D 80$, and the geometric mean fluorescent intensity (gMFI) of MHCll. CHIR-AB1 was included as an activation marker since surface expression of this marker has been described to be induced on macrophages upon stimulation with LPS or IFN- $\gamma$, which was recently confirmed by van den Biggelaar et al. ${ }^{47,48}$. 


\section{Fluorescence-activated cell sorting of NK cell and APC subsets}

Based on marker expression, two NK cell subsets and three APC subsets were separated by fluorescence-activated cell sorting (FACS) to gain more insight into their functional identity. To distinguish NK cell subsets, splenocytes were stained with mouse-anti-chicken-CD3-APC, -Bu-1-FITC, -28-4 and -20E5-biotin. For secondary antibody staining goat-anti-mouse-lgG3-PE and SA-BV421 were used. To identify APC subsets, splenocytes were stained with mouse-anti-chicken-MRC1LB-PE and -CD11-biotin. Secondary staining with SA-BV605 was used to fluorescently label CD11-biotin. To assess viability, the cells were stained with the Zombie Aqua ${ }^{\mathrm{TM}}$ Fixable Viability Kit. Primary and secondary antibody staining of cells used the same conditions as described in section "Phenotypic characterization of lymphocytes by flow cytometry". Finally, the cells were resuspended in PBA (NK cells) or PBA with 2 mM EDTA-Na 2 (APC subsets), and isolated by FACS using the BD influx ${ }^{\mathrm{TM}}$ Cell Sorter and 405-, 488-, 638-, 561- and 640-nm lasers. The cells were gated for viability and subsequently sorted into CD3- Bu-1-28-4+ (IL-2R $\left.\alpha^{+} \mathrm{NK}\right), \mathrm{CD}^{-}$

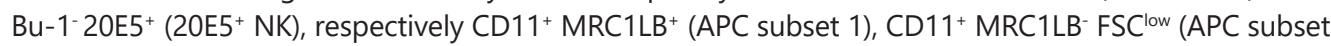
2a) and CD11 ${ }^{+}$MRC1LB- FSChigh (APC subset 2b). The sorted NK cell subsets were collected in $350 \mu$ l RLT buffer (Qiagen, the Netherlands) with 1\% 2-mercaptoethanol (Sigma Aldrich). The sorted APC subsets, and the original (unsorted) cell population as a control, were centrifuged at $393 \times g$ for 5 min and then lysed in $600 \mu \mathrm{l} \mathrm{RLT}$ buffer with 1\% 2-mercaptoethanol. Cell lysates of sorted cell subsets and the control cell population were then stored at $-20^{\circ} \mathrm{C}$ until RNA isolation and $\mathrm{qPCR}$ analysis.

\section{Gene expression of separated subsets of NK cells and APCs}

Target genes (Table 2) were selected based on literature to define functional differences between subsets of NK cells respectively APCs. RNA was isolated from lysates of sorted NK and APC subsets, and control cells, using the RNeasy Mini Kit (Qiagen) according to the manufacturer's instructions, including a DNase treatment using the RNase-Free DNase Set (Qiagen). Next, cDNA was prepared using the reverse transcriptase from the iScript cDNA Synthesis Kit (Bio-Rad) according to the manufacturer's instructions. RT-qPCRs were performed with primers and either FAM-TAMRA-labeled TaqMan probes combined with TaqMan Universal PCR Master Mix or SYBR Green Master Mix without probes (all from Thermo Fisher Scientific), as indicated in Table 2. Primers were used at $400 \mathrm{nM}$ (SYBR-Green) or $600 \mathrm{nM}$ (Taqman) and probes at $100 \mathrm{nM}$. RT-qPCRs were performed with a CFX Connect and analyzed with CFX Maestro software (both from Bio-Rad). All RT-qPCRs were evaluated for proper amplification efficiency (95-105\%) using serial dilutions of reference cDNA either from splenocytes that were stimulated with Concanavalin A for 24 hours or from HD11 cells that were stimulated with LPS for 3 hours. RT-qPCRs were performed in triplicate for every sample. For the NK cell subsets, mRNA levels are expressed as 40-Ct and the cycle threshold value $(\mathrm{Ct})$ was corrected for variations in RNA preparation and sampling using the GAPDH Ct values, as described elsewhere ${ }^{49}$. Higher gene expression of NFIL 3 and $I L-7 \alpha$ is indicative of the cytokine-producing NK cell subset in humans ${ }^{50-54}$, whereas higher expression of PRF1 is indicative of the cytotoxic NK cell subset in humans and chickens ${ }^{52,55}$. Gene expression levels of the APC subsets are shown relative to those of unsorted splenocytes. Furthermore, $\mathrm{Ct}$ gene expression values were normalized to housekeeping genes 285 and GAPDH. Changes in gene expression after sorting was expressed as $2^{-\triangle \triangle C t}$, according to the Livak method ${ }^{56}$. Enrichment of cells expressing CD 14, TLR4, MERTK and MAFB after sorting of cells was considered indicative for a monocyte/macrophage phenotype, whereas enrichment of cells expressing ZBTB46, XCR1 and FLT3 after sorting was considered indicative for a DC phenotype, in accordance with previous studies ${ }^{57,58}$. 
Table 2. Primers and TaqMan probe sequences used for RT-qPCR.

\begin{tabular}{|c|c|c|c|c|c|}
\hline Cell type & Genes & NCBI Reference & TaqMan / SYBR-Green & Type & Sequence $\left(5^{\prime}-3^{\prime}\right)$ \\
\hline \multirow[t]{8}{*}{ NK cells } & NFIL3 & XM_017014743.1 & SYBR-Green & Forward & TGAATGCCATCAGTTGAGC \\
\hline & & & & Reverse & GAGAGGCGGAGAATGTGAGT \\
\hline & $I L-7 R \alpha$ & NM_001080106.1 & SYBR-Green & Forward & ATTCTGGGAAAGCAGGATCAAG \\
\hline & & & & Reverse & CTTACACAGTCGCTCCAGAGTTATTT \\
\hline & PRF1 & XM_004945690.3 & SYBR-Green & Forward & ACCCGCACCAAAAGATGAAG \\
\hline & & & & Reverse & TAATTCGCACACCCCTAAACG \\
\hline & GAPDH & NM_204305.1 & SYBR-Green & Forward & GTGGTGCTAAGCGTGTTATC \\
\hline & & & & Reverse & GCATGGACAGTGGTCATAAG \\
\hline \multirow[t]{22}{*}{ APCs } & CD14 & NM_001139478.1 & TaqMan & Forward & GGACGACTCCACCATTGACAT \\
\hline & & & & Reverse & GGAGGACCTCAGGAACCAGAA \\
\hline & & & & Probe & AATGATCTTCCTGATTTGCAGACTGCCAA \\
\hline & TLR4 & NM_001030693.1 & SYBR-Green & Forward & GTCCCTGCTGGCAGGAT \\
\hline & & & & Reverse & TGTCCTGTGCATCTGAAAGCT \\
\hline & MERTK & NM_204988.1 & SYBR-Green & Forward & TGTGGAAGGATGGCAGGGAG \\
\hline & & & & Reverse & GCACGGATGCTGAATGTAGAGG \\
\hline & $M A F B$ & NM_001030852.1 & SYBR-Green & Forward & AGGACCGGTTCTCGGATGAC \\
\hline & & & & Reverse & CCTCGGAGGTGCCTGTTG \\
\hline & INOS & NM_204961.1 & SYBR-Green & Forward & TGGGTGGAAGCCGAAATA \\
\hline & & & & Reverse & GTACCAGCCGTTGAAAGGAC \\
\hline & ZBTB46 & XM_015296613.2 & SYBR-Green & Forward & CTGGACCTGTGGAAGAGGAAAC \\
\hline & & & & Reverse & CGGTAGTGGGAGGCAATCTC \\
\hline & $X C R 1$ & NM_001024644.2 & SYBR-Green & Forward & CCTTCGGGTGGATTTTTGGT \\
\hline & & & & Reverse & CGCTGTAGTAGCCAATGGAGAA \\
\hline & FLT3 & NM_004119.3 & SYBR-Green & Forward & CATTCGGACCCAGTACATGTTTAC \\
\hline & & & & Reverse & TGAGCCGTAGAAGAGCAGGTATAA \\
\hline & GAPDH & NM_204305.1 & SYBR-Green & Forward & GTGGTGCTAAGCGTGTTATC \\
\hline & & & & Reverse & GCATGGACAGTGGTCATAAG \\
\hline & 285 & XR_00378040.1 & TaqMan & Forward & GGCGAAGCCAGAGGAAACT \\
\hline & & & & Reverse & GACGACCGATTTGCACGTC \\
\hline & & & & Probe & AGGACCGCTACGGACCTCCACCA \\
\hline
\end{tabular}

All sequences have been described previously except for NFIL3 and PRF157,58,79.

\section{T cell proliferation assay}

Splenocytes isolated at $21 \mathrm{dpi}$ from uninfected and SE-infected chickens were labelled with CellTrace Violet (CTV, Invitrogen) to measure proliferation by flow cytometry. The cells were resuspended at $5 \times 10^{6} \mathrm{cells} / \mathrm{ml}$ in PBS with $5 \mu \mathrm{M}$ CTV and incubated for $20 \mathrm{~min}$ at RT, while the cell suspension was vortexed every $5 \mathrm{~min}$. Next, the labeling was quenched by the addition of $5 \mathrm{ml}$ complete medium for every $\mathrm{ml}$ of CTV staining solution and incubated for $5 \mathrm{~min}$ at RT. Cells were centrifuged for $5 \mathrm{~min}$ at $335 \times \mathrm{g}$ at $20^{\circ} \mathrm{C}$ and resuspended at $2.5 \times 10^{6} \mathrm{cells} / \mathrm{ml}$ in X-VIVO 15 cell culture medium (Lonza) with $50 \mathrm{U} / \mathrm{ml}$ penicillin-streptomycin, $50 \mu \mathrm{M}$ 2-mercaptoethanol (Sigma Aldrich) and $50 \mu \mathrm{g} / \mathrm{ml}$ gentamycin $\left(G_{i b c o}{ }^{\mathrm{TM}}\right)$. Aliquots of $200 \mu \mathrm{l}$ cell suspension containing 500000 splenocytes were added to the wells 
of a 96 wells round-bottom cell culture plate. Fixed SE was added to the splenocytes at $10^{4}, 10^{5}$ or $10^{6}$ CFU/well. As a positive control, splenocytes were stimulated with $1 \mu \mathrm{g} / \mathrm{ml}$ mouse-anti-chicken-CD3, 1 $\mu \mathrm{g} / \mathrm{ml}-\mathrm{CD} 28$ and 1:50 diluted conditioned supernatant from COS-7 cells transfected with a pcDNA1 vector (Invitrogen) encoding for recombinant chicken IL-2 (a kind gift from prof. Pete Kaiser and Lisa Rothwell), in accordance with a previous publication ${ }^{59}$. Cells were incubated for four days at $41^{\circ} \mathrm{C}$ and $5 \% \mathrm{CO}_{2}$. After incubation, cells were transferred to a 96 wells $\mathrm{V}$-bottom plate and stained in PBS with ViaKrome 808 viability dye (Beckman Coulter). Next, cells were stained with antibodies of the T cell proliferation panel (Table 1). Primary and secondary staining of cells were conducted in $30 \mu \mathrm{l}$ PBA and incubated for $20 \mathrm{~min}$ at $4^{\circ} \mathrm{C}$ in the dark. Stained cells were washed twice by centrifugation for 3 min at $393 \times g$ at $4^{\circ} \mathrm{C}$ in PBA and resuspended in $100 \mu \mathrm{l}$ followed by flow cytometry analysis as described in section "Phenotypic characterization of lymphocytes by flow cytometry", using $80 \mu \mathrm{l}$.

\section{SE-specific antibody titers in serum}

To detect titers of SE-specific antibodies in the sera collected at $0,7,14$ and $21 \mathrm{dpi}$, the commercially available Salmonella Enteritidis Antibody Test (IDEXX SE Ab X2 Test) was used according to manufacturer's instructions (IDEXX Europe, the Netherlands). Positive and negative controls were included in the kit, and serum samples were analyzed in duplicate. Endpoint titers were calculated with the following formula:

$10^{\wedge}\left(1.5 \times \log _{10}((\right.$ sample $\mu$ - negative control $\mu) /($ positive $\mu$ - negative control $\left.\mu))+3.47\right)$.

\section{Statistical analysis}

First, the data were tested for fitting a normal distribution using the Shapiro-Wilk test. Differences in numbers of IELs or leukocytes, NK cell and T cell subsets and percentages of CD107 and IFNY expression in the IELs and spleen between the uninfected and SE-infected groups as well as within each group in the course of time were analyzed using one-way ANOVA tests. Differences in SE-CFUs per gram ileum and spleen as well as SE-specific antibody titers in serum were analyzed using Kruskal-Wallis tests accompanied by Dunn's multiple comparisons tests. Differences in numbers and percentages of the splenic APC subsets 1 and 2a between the uninfected and SE-infected groups were analyzed using one-way ANOVA tests, while subset $2 \mathrm{~b}$ was analyzed using the Kruskal-Wallis test as the data was not normally distributed. All statistical analyses were performed using GraphPad Prism 9 software (GraphPad Software, CA, USA). A $p$-value of $<0.05$ was considered statistically significant and a value of $0.05<p<0.1$ is referred to as a trend, in case the $p$-value did not belong to one of these categories it is referred to as a numerical difference.

\section{Results}

\section{Highest presence of SE in ileum and spleen at $7 \mathrm{dpi}$ while intestinal infiltration of IELs was observed at $1 \mathrm{dpi}$}

SE was not observed at -4 and 0 dpi before SE inoculation, in both the IEL population of the ileum and the spleen of chickens of both groups (Fig. 1A, B). After inoculation SE was detected in the ileum of SE-infected chickens only at $7 \mathrm{dpi}$ (Fig. 1A). In the spleen, SE was observed at 7, 14 and $21 \mathrm{dpi}$ with the highest bacterial counts at $7 \mathrm{dpi}$, which subsequently decreased in course of time (Fig. 1B). SE was not detected in the ileum and spleen of uninfected chickens at any of the time points (Fig. 1A, B). One uninfected chicken showed counts of Proteus in the spleen at $7 \mathrm{dpi}$ and was therefore excluded from 
further analyses. Infection with SE did not affect the weight of the chickens, as growth curves were similar between uninfected and SE-infected chickens (Fig. 1C). A significant increase in numbers of IELs was found in SE-infected chickens at $1 \mathrm{dpi}$ compared to uninfected chickens (Fig. 1D). IELs were numerically higher at 3 dpi in SE-infected chickens and declined over time to numbers similar to those observed in uninfected chickens (Fig. 1D). The numbers of splenic leukocytes were similar between uninfected and SE-infected chickens at all time points (Fig. 1E).

A

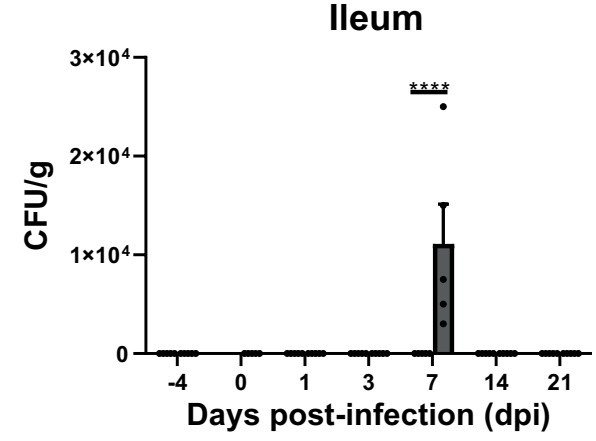

C

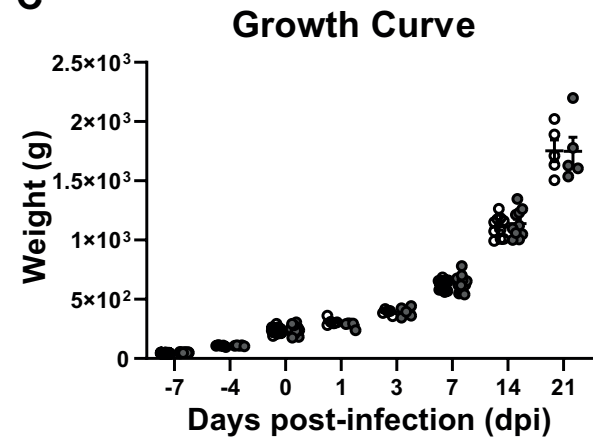

D

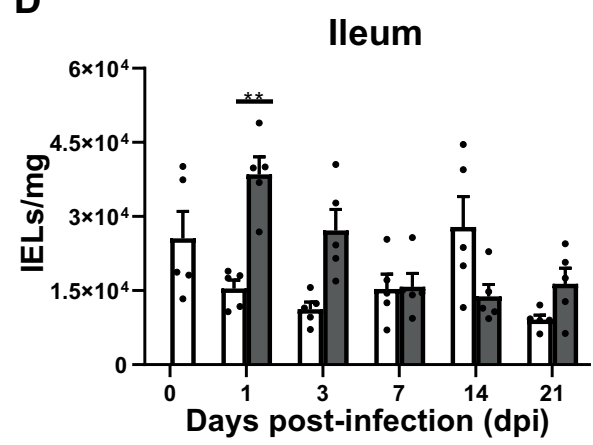

B

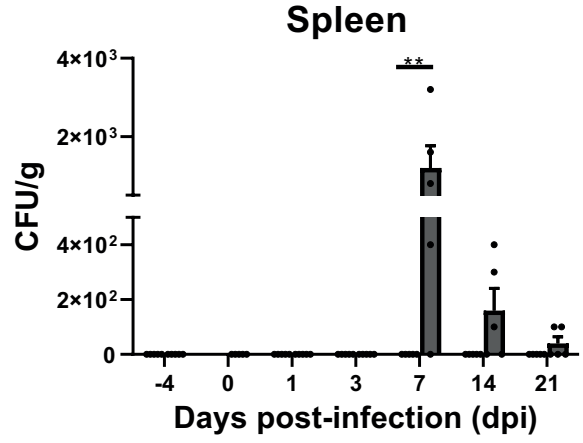

○ $\square$ uninf

- $\square$ SE-inf

E

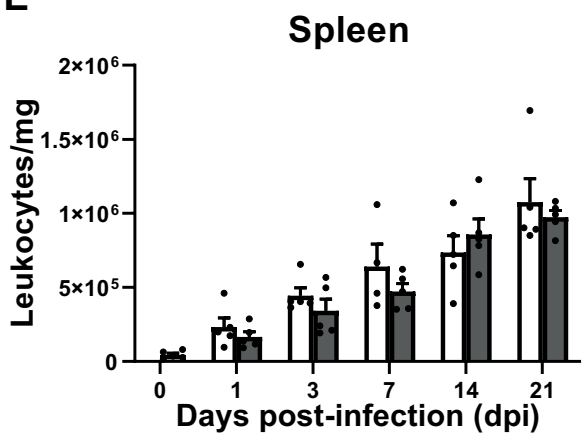

Figure 1. The bacterial load in course of time after SE infection and its effect on growth and numbers of IELs and splenic leukocytes. (A) Salmonella enterica serotype Enteritidis (CFU/g) in the ileum and (B) spleen of uninfected (uninf) and SE-infected chickens (SE-inf). The LOD was 100 CFU per gram tissue. (C) Bodyweights (g) of uninfected and SE-infected chickens in the course of time. (D) Numbers (cells/mg) of IELs per mg ileum and E leukocytes per mg spleen of uninfected and SE-infected chickens in the course of time. Mean + SEM per treatment and time point is shown $(\mathrm{n}=5)$ and statistical significance is indicated as ${ }^{* *} p<0.01$ and ${ }^{* * * *} p<0.0001$. 


\section{Enhanced activation of intraepithelial NK cells upon SE infection}

Numbers of intraepithelial and splenic NK cell subsets were determined to investigate differences between uninfected and SE-infected chickens at several time points post-infection. NK cell subsets were distinguished by membrane expression of IL-2R $\alpha$ or $20 \mathrm{E} 5$ (Fig. S1). Although no significant differences were observed in numbers of intraepithelial NK cell subsets, IL-2R $\alpha^{+}$and $20 \mathrm{E} 5^{+} \mathrm{NK}$ cells were numerically higher in SE-infected chickens at 1, 3 and 7 dpi compared to uninfected chickens (Fig. 2A, B). Furthermore, intraepithelial 20E5 ${ }^{+}$NK cells were numerically lower at $14 \mathrm{dpi}$ and higher at $21 \mathrm{dpi}$ in SE-infected compared to uninfected chickens (Fig. 2B). In the control group, no significant differences were observed in the numbers of intraepithelial NK cells in course of time. In the spleen, numbers of IL-2R $\alpha^{+}$and 20E5+ NK cells were similar between uninfected and SE-infected chickens and both increased in course of time (Fig. S2A, B). To obtain more insight in functional differences between IL-2R $\alpha^{+}$and $20 \mathrm{E} 5^{+} \mathrm{NK}$ cells, mRNA levels of genes deemed to be relevant were determined in the spleen. The IL-2R $\alpha^{+}$subset showed numerical higher mRNA levels of the NK cell lineage marker NFIL3 and IL-7R $\alpha^{+}$as compared to the $20 \mathrm{E5}^{+}$subset, whereas the $20 \mathrm{E5} 5^{+}$subset showed a numerical higher mRNA level of perforin as compared to the IL-2R $\alpha^{+}$subset (PRF1, Fig. S2C).

A

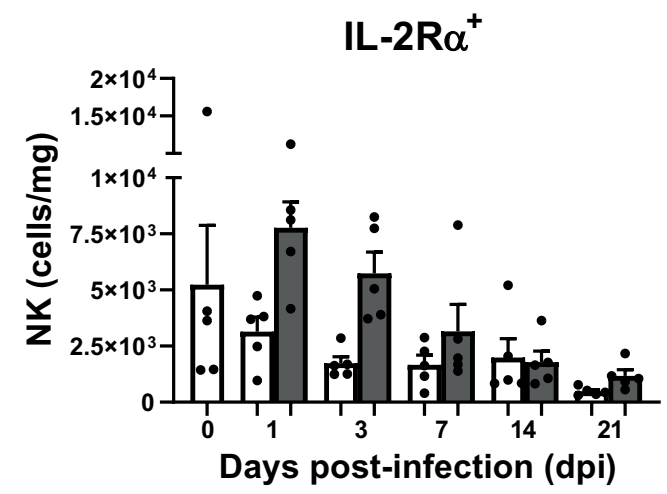

B

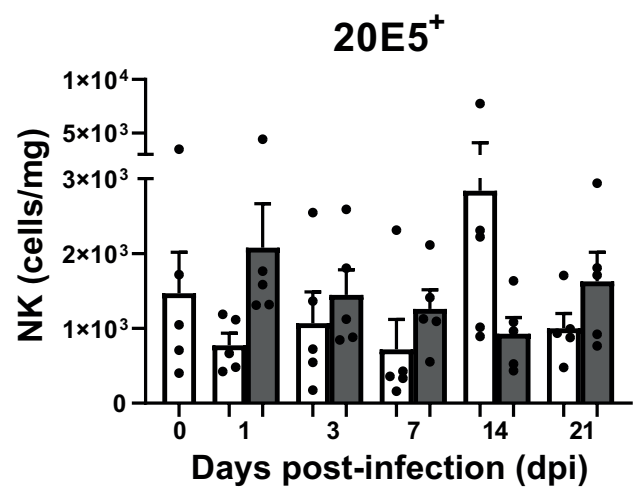

Figure 2. Effect of SE infection on numbers of intraepithelial NK cells in broiler chickens. (A) Numbers (cells/mg) of intraepithelial $\mathrm{IL}-2 \mathrm{R} \alpha^{+}$and (B) 20E5 $5^{+} \mathrm{NK}$ cells per mg ileum, in uninfected (uninf) and SE-infected (SE-inf) chickens. Mean + SEM per treatment and time point is shown $(n=5)$.

To determine possible changes in NK cell activation upon SE infection, CD107 surface expression and intracellular IFN $\gamma$ were analyzed in intraepithelial and splenic NK cells (Fig. 3A). Intraepithelial NK cells showed a significant increase in surface expression of CD107 and IFNY production in SE-infected chickens at $1 \mathrm{dpi}$ and $3 \mathrm{dpi}$ compared to uninfected chickens (Fig. 3B, C). In the spleen, a significant increase in surface expression of CD107 was observed at $1 \mathrm{dpi}$ and $3 \mathrm{dpi}$, and a significant increase in IFN $\gamma$ production was observed at $1 \mathrm{dpi}, 3 \mathrm{dpi}$ and $7 \mathrm{dpi}$ in SE-infected compared to uninfected chickens (Fig. 3D, E).

\section{Increased presence of APCS in the spleens of SE-infected chickens}

To investigate whether infection with SE affects the composition of the APC population, amongst splenocytes, these were stained for APC surface markers and analyzed by flow cytometry. A t-SNE 
A IELs

Spleen
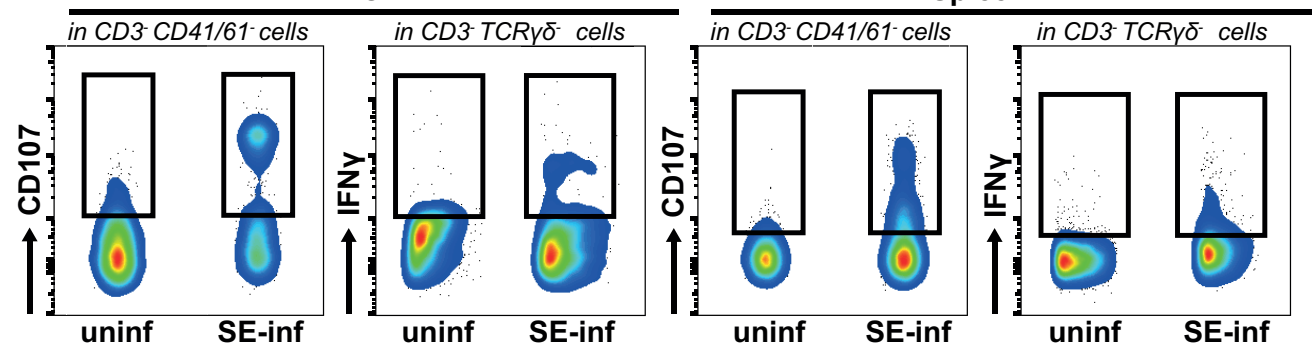

B
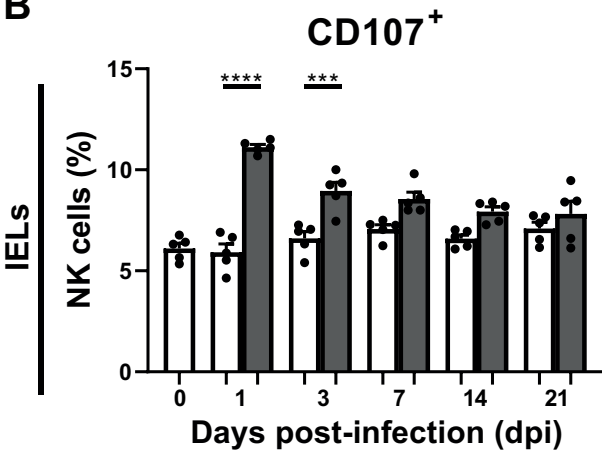

C
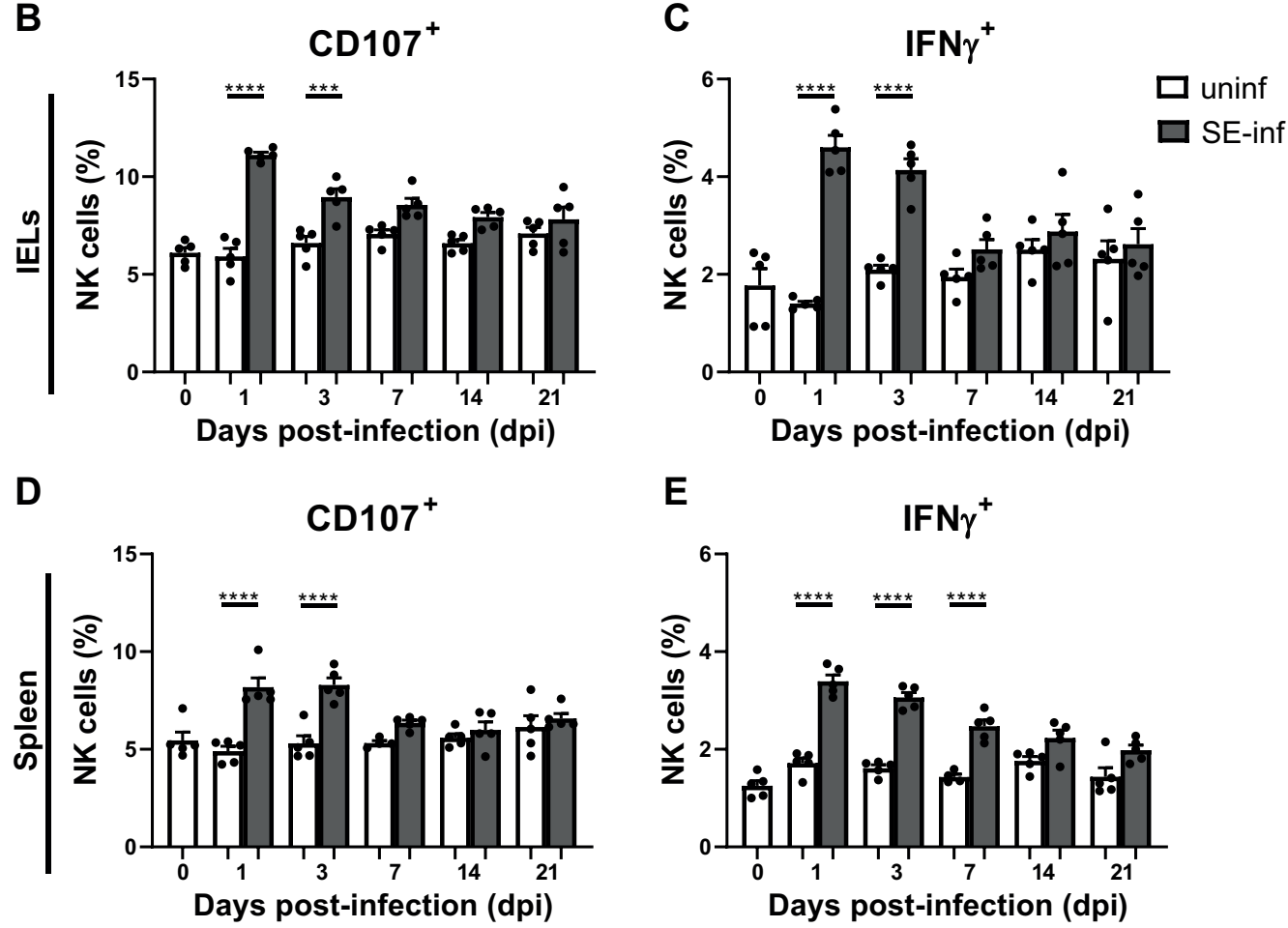

Figure 3. NK cell activation in the IEL population and spleen of broiler chickens upon SE infection. (A) Gating strategy for NK cells expressing surface CD107 and intracellular IFNY in the IEL population (first and second panels) and spleen (third and fourth panels), (B) Percentages of intraepithelial NK cells expressing CD107 and (C) IFNY in uninfected (uninf) and SE-infected (SE-inf) chickens in the course of time. (D) Percentages of splenic NK cells expressing CD107 and (E) IFNY in uninfected and SE-infected chickens. Mean + SEM per treatment and time point is shown $(n=5)$, for uninfected chickens at 7 dpi in spleen $n=4$. Statistical significance is indicated as *** $p<0.001$ and ${ }^{* * * *} p<0.0001$.

analysis was used to determine the differences in APCs between uninfected and SE-infected chickens (Fig. 4A-C, Fig. S3A). This analysis clustered cells that have high similarity and separated cells that are unrelated based on the APC surface markers that were used, leading to an unbiased visualization of all cell populations. Two populations were found overrepresented in the spleen of SE-infected chickens (Fig. 4B). By gating for subset 1 and 2 and assessing their expression of APC markers, subset 1 was identified as CD11 ${ }^{+}$MRC1LB+ and subset 2 as CD11+ MRC1LB- (Fig. 4C first panel). In addition, subset 2 could be further divided into two subsets, distinguished by FSC-A and SSC-A characteristics, that 
were further analyzed separately (Fig. 4C second panel). The APC subsets were sorted (Fig. S3B, C), and qPCR was performed to compare the expression levels of macrophage- and DC-specific genes between sorted cells and the unsorted total APC population. High expression levels of the monocyte/ macrophage genes CD14, TLR4, MERTK and MAFB (Fig. 4D) observed on the CD11 ${ }^{+} \mathrm{MRC1LB}^{+}$cells, indicates that this subset 1 includes macrophages (hereafter referred to as macrophages). The $C D 11^{+}$ MRC1LB- FSChigh subset $2 b$ includes DCs as reflected by high expression of the DC genes ZBTB46, XCR1 and FLT3 (hereafter referred to as FSChigh DCs) (Fig. 4D). The increase in expression of either macrophage- or DC-specific genes was less clear in the CD11+ MRC1LB- FSClow subset 2a, however, DC-specific genes were most abundantly expressed (hereafter referred to as FSClow DCs) (Fig. 4D). Next, the percentages (Fig. 4E, Fig. S4A, B) and numbers (Fig. 4F, Fig. S4C, D) of the three APC subsets were followed over time in the spleens of uninfected and SE-infected chickens. Due to limited cell numbers, the analysis of APCs could not be performed for two chickens at $0 \mathrm{dpi}$. At $7 \mathrm{dpi}$, the percentage of macrophages was significantly increased in SE-infected compared to uninfected chickens (Fig. 4E). The FSClow (Fig. S4A, C) and FSChigh (Fig. S4B, D) DCs were similar in SE-infected compared to uninfected chickens at all time points, although a slight increase in both the percentages and numbers of FSChigh DCs was observed at 7 dpi (Fig. S4B, D).

\section{APCs become activated in spleens of SE-infected chickens}

To assess the activation status of the three APC subsets in response to SE infection, expression levels of immunoglobulin Y receptor CHIR-AB1, co-stimulatory molecules CD40 and CD80, and MHCII were evaluated by flow cytometry (Fig. S5). The macrophages of SE-infected chickens showed significantly decreased expression of the activation markers CD40 (1 and $7 \mathrm{dpi}$ ) and CD80 (7 dpi), whereas expression of CHIR-AB1 and MHCIl was similar compared to uninfected chickens (Fig. 5A, D, G, J). Before infection, FSClow DCs of uninfected chickens showed a higher expression of $\mathrm{MHCll}$ (Fig. 5K, L) and more cells that were positive for the costimulatory molecules CD40 (Fig. 5E, F) and CD80 (Fig. 5H, I) compared to FSChigh DCs. At 7 dpi, FSClow DCs showed significantly increased expression of CHIR-AB1 (Fig. 5B), CD40 (Fig. 5E) and CD80 (Fig. 5H) in SE-infected chickens as compared to uninfected chickens. The FSChigh DCs showed at 7 dpi significantly increased expression of CHIR-AB1 (Fig. 5C) and MHCII (Fig. 5L) in SE-infected compared to uninfected chickens. In course of time, expression of CD40 by macrophages significantly increased at $1 \mathrm{dpi}, 3 \mathrm{dpi}$ and $7 \mathrm{dpi}$ as compared to $0 \mathrm{dpi}$ in the control group (Fig. 5D). In addition, CD80 expression by macrophages significantly increased at $3 \mathrm{dpi}$ as compared to $0 \mathrm{dpi}$ in the control group (Fig. 5G).

\section{Increased presence of intraepithelial cytotoxic T cells at $1 \mathrm{dpi}$ and proliferation of SE-induced splenic T cells ex vivo at $21 \mathrm{dpi}$}

Numbers of $\gamma \delta$ T cells and cytotoxic $\left(C D 8^{+}\right) \alpha \beta$ T cells were determined in course of time in the IEL population and spleen of uninfected and SE-infected chickens (Fig. S1). Although numbers of intraepithelial $\gamma \delta$ T cells did not significantly differ, they were numerically higher at 1 and $3 \mathrm{dpi}$, as well as at $21 \mathrm{dpi}$ in SE-infected compared to uninfected chickens (Fig. 6A). A significant increase in numbers of intraepithelial cytotoxic CD8 ${ }^{+} T$ cells was observed at $1 \mathrm{dpi}$, and at $3 \mathrm{dpi}$ and $21 \mathrm{dpi}$ intraepithelial cytotoxic $\mathrm{CD}^{+} \mathrm{T}$ cells were numerically higher in SE-infected compared to uninfected chickens (Fig. $6 \mathrm{~B})$. In the spleen, $\gamma \delta \mathrm{T}$ cells were numerically decreased at $1 \mathrm{dpi}$ but increased at $3 \mathrm{dpi}$ in SE-infected compared to uninfected chickens (Fig. 6C). Numbers of splenic cytotoxic CD8 ${ }^{+} \mathrm{T}$ cells were similar between uninfected and SE-infected chickens during the course of infection (Fig. 6D). Next, $\gamma \delta T$ cells and cytotoxic $\alpha \beta$ T cells were analyzed for their CD8 $\alpha \alpha$ and CD8 $\alpha \beta$ expression (Fig. S1). Numbers of intraepithelial CD8 $\alpha \alpha^{+} \gamma \delta$ T cells (Fig. S6A) were significantly increased at $1 \mathrm{dpi}$ and $21 \mathrm{dpi}$, and 
A

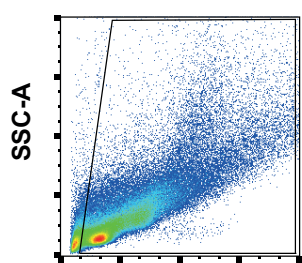

FSC-A

B

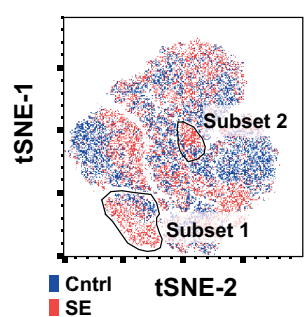

D

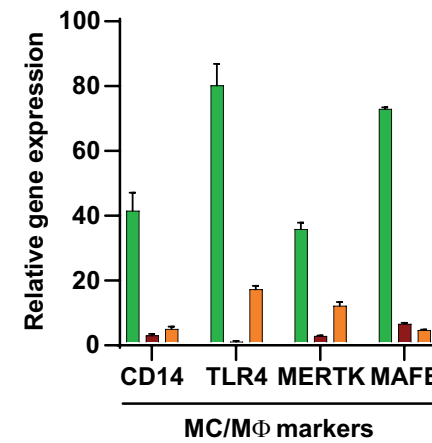

E

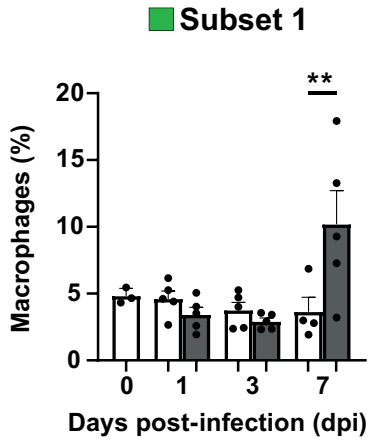

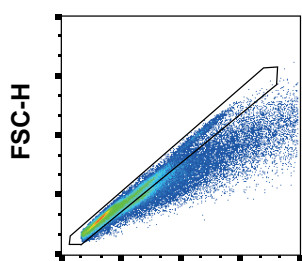

FSC-A

C

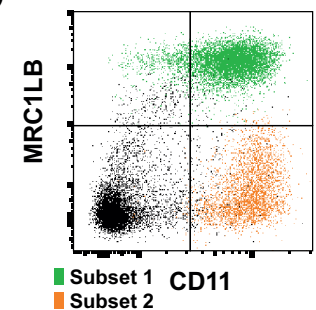

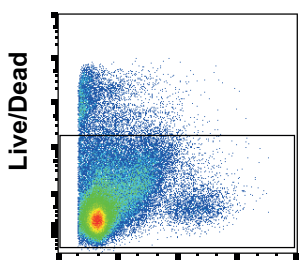

FSC-A

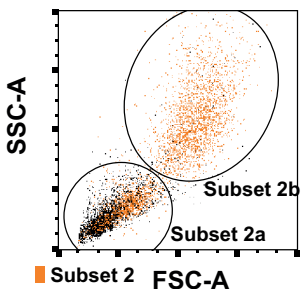

Subset 1 (CD11 ${ }^{+}$MRC1LB $\left.^{+}\right)$

Subset 2a (CD11+ MRC1LB- FSC ${ }^{\text {low }}$ )

Subset 2b (CD11+ MRC1LB- FSC ${ }^{\text {high }}$ )

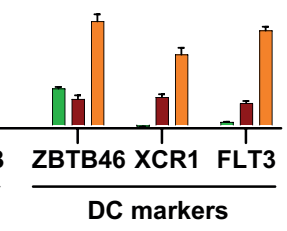

F
Subset 1 $\left(\times 10^{4}\right)$

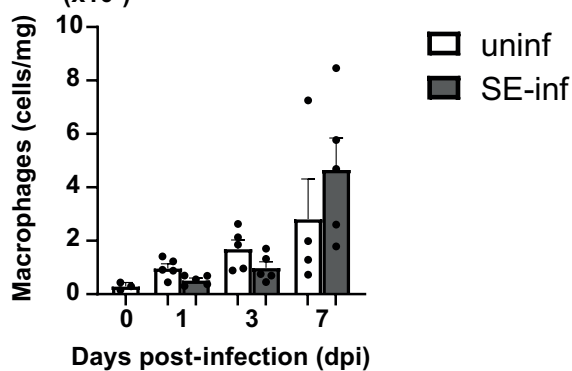

Figure 4. Phenotypic characterization of splenic APCs upon SE infection. (A) Splenocytes were gated for size, excluding debris (FSC-A vs SSC-A), singlets (FSC-A vs FSC-H) and viability (Live/Dead marker-negative) consecutively. (B) A t-SNE analysis was performed on spleen samples of $7 \mathrm{dpi}$ uninfected (uninf, blue) and SE-infected (SE-inf, red) chickens combined. Based on the t-SNE analysis, two population (subset 1 and subset 2) were found enriched among the splenocytes of SE-infected chickens. (C) The populations were evaluated for expression of MRC1LB versus CD11. Subset 2 was evaluated for its FSC-A vs SSC-A scatter pattern and further subdivided into subset $2 a$ and subset $2 b$. (D) Subset $1\left(C^{2} 11^{+}\right.$MRC1LB $\left.^{+}\right)$, subset $2 a\left(C D 11^{+}\right.$MRC1LB- FSClow) and subset $2 b\left(C D 11^{+}\right.$MRC1LB $^{-}$ FSChigh) were sorted by FACS to assess gene expression of immune markers by RT-qPCR relative to the total splenocyte population. (E) The presence (\%) and (F) numbers (cells/mg spleen) of macrophages in uninfected and SE-infected chickens were assessed over time. Mean + SEM per treatment and time point is shown $(n=5)$, for uninfected chickens at 0 dpi $n=3$ and at 7 dpi $n=4$. Statistical significance is indicated as ${ }^{*} p<0.01$. 
A

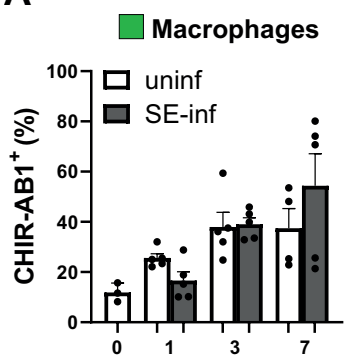

Days post-infection (dpi)

D

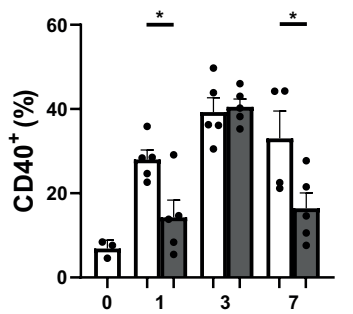

G

Days post-infection (dpi)

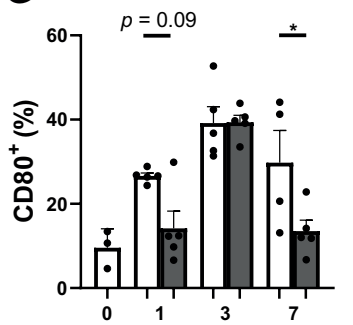

Days post-infection (dpi)

J

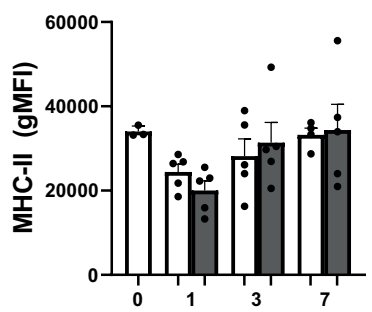

Days post-infection (dpi)
B

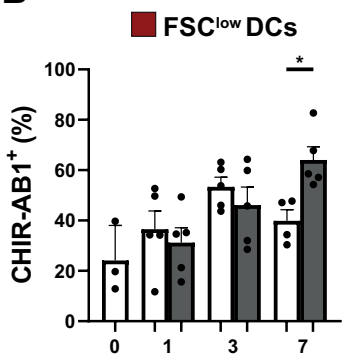

E

Days post-infection (dpi)

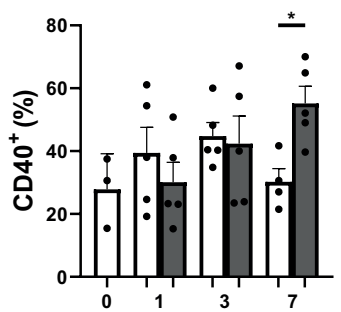
H

Days post-infection (dpi)

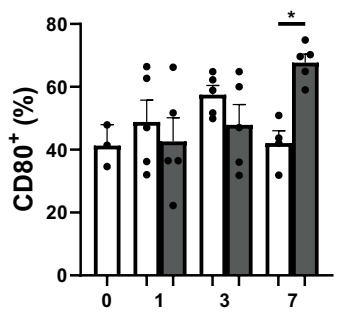

Days post-infection (dpi)

K

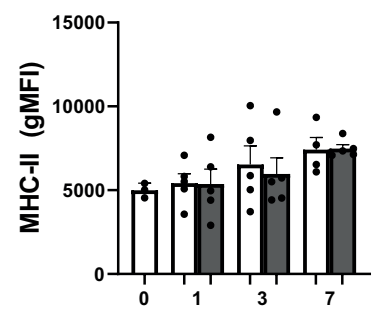

Days post-infection (dpi)
C

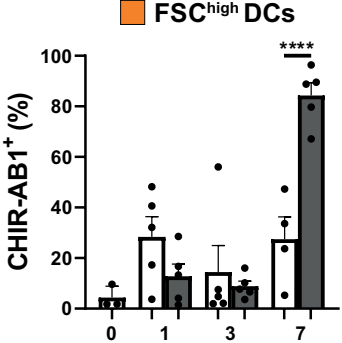

F

Days post-infection (dpi)

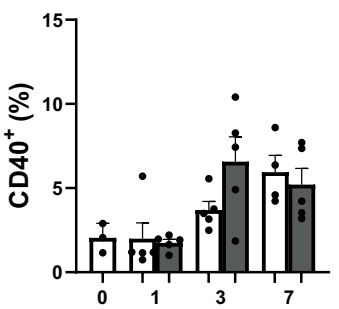

Days post-infection (dpi)

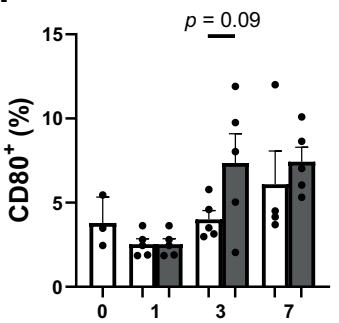

Days post-infection (dpi)

L

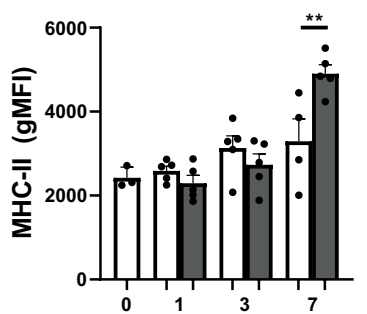

Days post-infection (dpi)

Figure 5. Activation marker expression by splenic APC subsets upon SE infection. (A) Macrophages, (B) FSC ${ }^{\text {low }}$ DCs and (C) FSC high DCs were assessed over time for CHIR-AB1, (D-F) CD40, (G-I) CD80 and (J-L) MHCIl expression in uninfected (uninf) and SE-infected (SE-inf) chickens. For CHIR-AB1, CD40 and CD80, the percentage of cells in each APC subset expressing the respective markers is shown, and for $\mathrm{MHCll}$ the geometric mean fluorescent intensity (gMFI) of each subset, in accordance with the gating strategy depicted in Fig. S5. Mean + SEM per treatment and time point is shown $(n=5)$, for uninfected chickens at 0 dpi $n=3$ and at 7 dpi $n=4$. Statistical significance is indicated as ${ }^{*} p<0.05,{ }^{* *} p<0.01$, ${ }^{* \star * *} p<0.0001$. 
CD8 $\alpha \beta^{+} \gamma \delta$ T cells (Fig. S6B) were numerically higher at those time points in SE-infected compared to uninfected chickens. Similarly, numbers of intraepithelial cytotoxic CD8 $\alpha \alpha^{+} T$ cells (Fig. S6C) were significantly increased at $1 \mathrm{dpi}$ and they were numerically higher at $21 \mathrm{dpi}$. The cytotoxic CD8 $\alpha \beta^{+} \mathrm{T}$ cells (Fig. S6D) were numerically higher at those time points in SE-infected compared to uninfected chickens. In the spleen, $C D 8 \alpha \alpha^{+} \gamma \delta \mathrm{T}$ cell numbers (Fig. S6E) were significantly increased at $14 \mathrm{dpi}$, whereas numbers of $C D 8 \alpha \beta^{+} \gamma \delta$ T cells (Fig. S6F) were similar in SE-infected versus uninfected chickens. Numbers of splenic cytotoxic CD8 $\alpha \alpha^{+}$(Fig. S6G) and CD8 $\alpha \beta^{+}$(Fig. S6H) T cells as well helper CD4 ${ }^{+} \mathrm{T}$ cells (Fig. S7) were similar between uninfected and SE-infected chickens during the course of infection. Finally, no significant differences were observed in T cell activation, determined by CD107 and IFNY expression, in the IEL population and spleen between uninfected and SE-infected chickens (Fig. S8). Although expression of CD107 was numerically higher at 3 dpi by intraepithelial and splenic CD8 ${ }^{+} T$ cells (comprising both $\gamma \delta$ and $\alpha \beta$ TCRs, Fig. S8A, B respectively), as well as expression of IFN $\gamma$ by splenic CD4 ${ }^{+} \mathrm{T}$ cells (Fig. S8D) in SE-infected compared to uninfected chickens. Expression of IFNY by $T$ cell subsets in the IEL population could not be determined due to too low cell numbers.

SE-induced proliferation of T cells, isolated from spleen at $21 \mathrm{dpi}$, was determined ex vivo (Fig. 7A). Increased proliferation of $\mathrm{SE}$-induced $\mathrm{CD}^{+}{ }^{+}$as well as $\mathrm{CD} 8^{+} \mathrm{T}$ cells isolated from SE-infected chickens was observed. This proliferation was antigen dose-dependent, whereas $T$ cells from uninfected chickens did not proliferate upon exposure to inactivated SE (Fig. 7B, C).

A

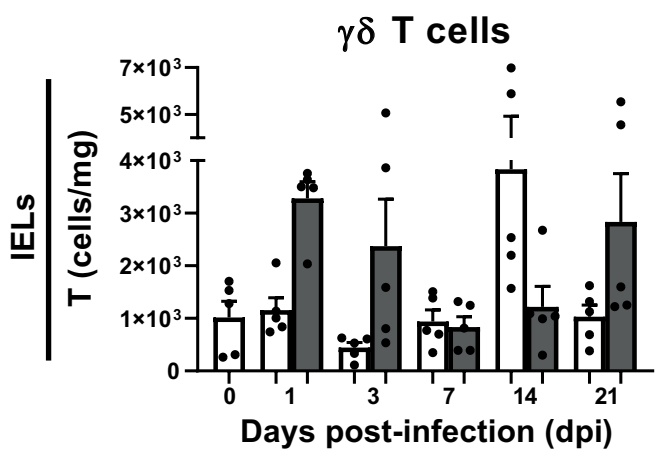

C

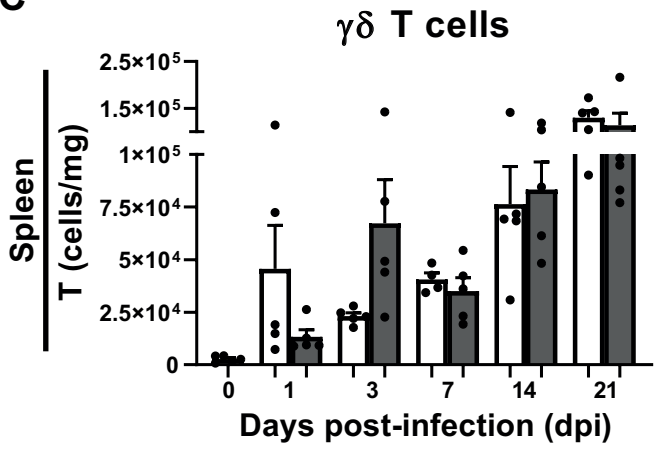

B

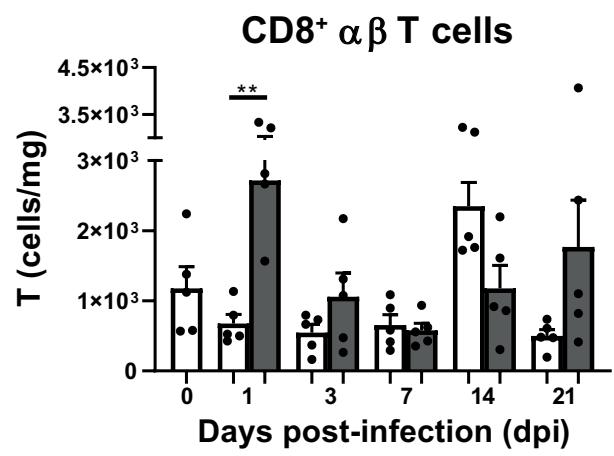

D

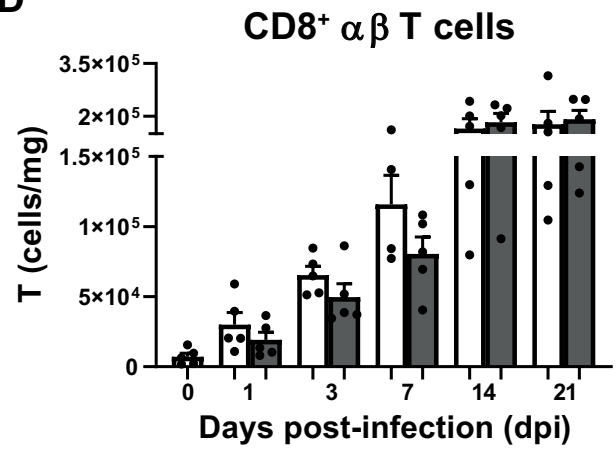

Figure 6. Numbers of intraepithelial and splenic T cells in broiler chickens upon SE infection. (A) Numbers (cells/mg) of intraepithelial $\gamma \delta T$ cells and (B) CD8 ${ }^{+} \alpha \beta$ T cells per mg ileum in uninfected (uninf) and SE-infected (SE-inf) chickens. (C) Numbers (cells/mg) of splenic $\gamma \delta T$ cells and (D) CD8 ${ }^{+} \alpha \beta$ T cells per mg spleen in uninfected and SE-infected chickens. Mean + SEM per treatment and time point is shown $(n=5)$, for uninfected chickens at 7 dpi in spleen $n=4$. Statistical significance is indicated as ** $p<0.01$. 


\section{High SE-specific antibody responses were found in all SE-infected chickens after three weeks of infection}

The presence of SE-specific antibodies was determined in serum before and after infection in uninfected and SE-infected chickens. In SE-infected chickens, SE-specific antibodies were first detected at $7 \mathrm{dpi}$, when two out of five chickens showed low antibody titers, that increased in course of time (Fig. 8). At 14 dpi SE-specific antibodies were observed in all SE-infected chickens although two chickens showed only low titers. At 21 dpi all SE-infected chickens showed high SE-specific antibody responses. SE-specific antibodies were not found in sera of the uninfected chickens (Fig. 8).

A

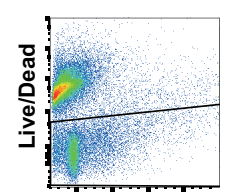

FSC-A

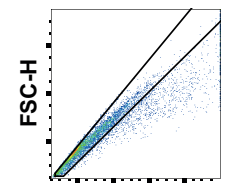

FSC-A

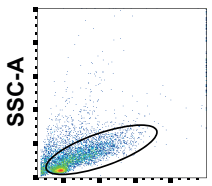

FSC-A

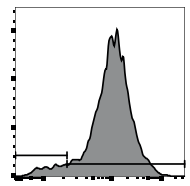

CD3

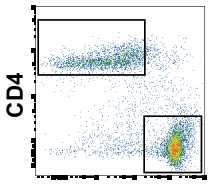

CD8

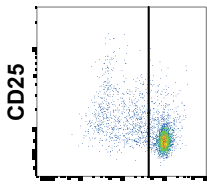

CTV
B

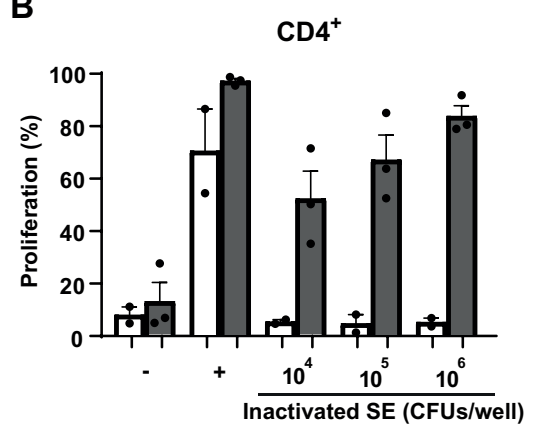

C
$\mathrm{CD}^{+}$

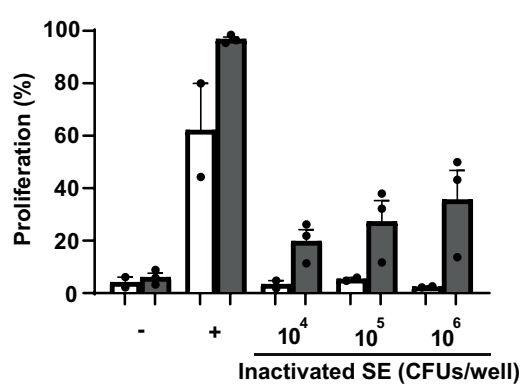

Figure 7. Ex vivo proliferation of SE-induced splenic CD4+ and $C D 8^{+} T$ cells. (A) The gating strategy shows the consecutive selection for viable cells (Live/Dead marker-negative), single cells (FSC-A vs FSC-H), lymphocytes (FSC-A vs SSC-A) and CD3 $3^{+}$cells. T cells were subdivided into $\mathrm{CD}^{+}$and $\mathrm{CD} 8^{+} \mathrm{T}$ cells. The final gating step selects on $\mathrm{T}$ cell subsets which have divided at least once based on dilution of the cell proliferation dye CellTrace Violet (CTV). (B) The percentage of cells that have proliferated is shown for splenic $\mathrm{CD}^{+}$and (C) CD8+ $\mathrm{T}$ cells after four days of stimulation with different doses of formaldehyde-inactivated SE expressed in CFU/well, none stimulated controls (-), or after stimulation with anti-CD3, anti-CD28 and recombinant chicken IL-2 (+). All splenocyte samples were stimulated and measured in triplicate for each of the conditions. Mean + SEM is shown; $n=2$ for uninfected (uninf) and $n=3$ for SE-infected (SE-inf) chickens.

\section{Discussion}

In the current study we aimed to provide a detailed analysis of SE-related innate and adaptive immune responses in young broiler chickens up to four weeks of age, to better understand how the immune response contributes to the elimination of infection in course of time. For this purpose, the presence and function of NK cells, various types of APCs and T cells in ileum, as the present infection site and in spleen, as indication of systemic dissemination of $\mathrm{SE}$, were investigated, as well as SE-specific antibody responses in serum, another systemic dissemination indicator. Seven-day-old broiler chickens were successfully infected with SE as was demonstrated by the detection of SE-induced T cell proliferation and SE-specific antibodies from 2-3 weeks after infection. Presence of SE was detected in ileum only at 
day 7 post-infection and in spleen from day 7 onwards and most likely, the number of bacteria in these tissues were below the detection limit during the first week post-infection as observed previously ${ }^{43}$. In the first week post-infection, significant increases in numbers of intraepithelial cytotoxic $T$ cells and splenic macrophages were observed in SE-infected compared to uninfected chickens. This was paralleled by a significant increase in NK cell activation in the IEL population and spleen as well as DC activation in the spleen in SE-infected compared to uninfected chickens. At $21 \mathrm{dpi}$, antibody titers in serum were significantly increased in SE-infected compared to uninfected chickens. These immune responses were paralleled by a reduction in SE counts.

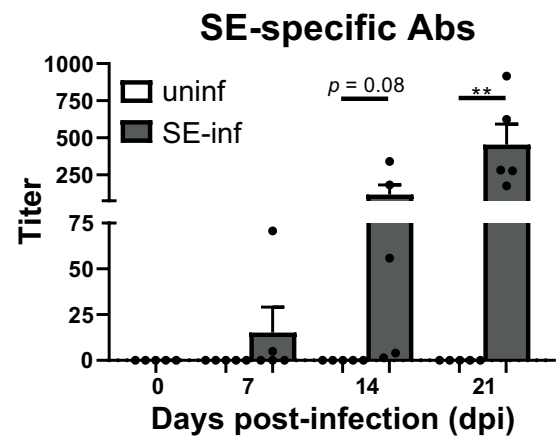

Figure 8. Serum antibody titers in broiler chickens as a response to SE infection. Titers of SE-specific antibodies in sera of uninfected (uninf) and SE-infected (SE-inf) chickens in course of time. Mean + SEM per treatment and time point is shown $(n=5)$ and statistical significance is indicated as ** $p<0.01$.

Although presence of SE was demonstrated in ileum and spleen of infected chickens, SE infection did not affect growth nor induced severe disease symptoms. This observation was similar to previous studies in young broiler chickens that were infected at seven or nine to eleven days of age ${ }^{40,60}$ and in layer chickens that were infected during adult life $\mathrm{e}^{20,43}$. The absence of severe disease symptoms is related to the SE-dose, which was chosen to avoid welfare issues in the chickens. Although SE has been detected in the small intestine ${ }^{61,62}$, other studies reported the presence of SE for a longer period in the caecum ${ }^{40,41}$. This suggests that SE may prefer colonization in the caecum rather than the ileum.

The enhanced activation of intraepithelial and splenic NK cells upon SE infection, represented by enhanced CD107 expression and IFNY production, is in agreement with other studies in chickens showing upregulated mRNA levels of intestinal IFN $\gamma^{20}$ and cytotoxicity-related NK cell genes ${ }^{19}$ in young chickens. Our observations are also supported by studies in humans and mice, which reported increased cytotoxicity ${ }^{23}$, IFNY production ${ }^{25}$ and CD107 expression ${ }^{24}$ of intestinal and systemic NK cells after Salmonella enterica serotype Typhimurium infection. The enhanced NK cell activation paralleled numerically increased intraepithelial IL-2R $\alpha^{+}$and $20 \mathrm{E} 5{ }^{+} \mathrm{NK}$ cells. Although we did not observe a distinct population of IL-2R $\alpha^{+} 20 \mathrm{E} 5^{+}$cells, we cannot exclude the possibility that these cells exist at a very low frequency. Due to incompatibility of available reagents, we were not able to determine CD107 and IFNY expression within the IL-2R $\alpha^{+}$and $20 \mathrm{E} 5^{+} \mathrm{NK}$ cell subsets. For that reason, we sorted IL-2R $\alpha^{+}$and $20 \mathrm{E} 5^{+}$ NK cells to perform RT-qPCR and both NK cell subsets showed mRNA levels of NFIL3, IL-7R and PRF1 genes albeit to different degrees, suggesting that both may be implicated in cytokine production ${ }^{50-52}$ as well as cytotoxic activity ${ }^{52,55}$ in response to SE infection. The observation that both NK cell subsets are involved in cytotoxicity is confirmed by previous studies in chickens, in which both NK cell subsets in the IEL population and spleen showed CD107 expression ${ }^{12}$ and intraepithelial IL-2R $\alpha^{+}$NK cells exerted cytotoxicity ${ }^{11}$. In humans, the peripheral IL-2R $\alpha^{+}$NK cell population expanded and increased their cytotoxic activity after Toll-like receptor (TLR) stimulation ${ }^{63}$. As SE might activate NK cells directly through TLRs ${ }^{63-65}$, more intraepithelial NK cells as well as enhanced activation, such as cytotoxicity and IFNY production, may 
increase the resistance of chickens against SE infection. IFN $\gamma$ has been reported to activate macrophages resulting in improved clearance of engulfed bacteria ${ }^{66}$ and enhanced antigen presentation by APCS inducing $T$ cell responses ${ }^{67}$. Although we were unable to demonstrate a direct relation between NK cell activation and SE counts in the first week post-infection, we hypothesize that NK cells play an important role in the resistance against SE infection. This can be either via direct killing of infected cells or indirectly by influencing other innate and adaptive immune cells via the production of IFN $\mathrm{F}$.

The systemic spread of SE infection as observed, coincided with a significantly increased presence of $\mathrm{CD} 11^{+} \mathrm{MRC} 1 \mathrm{LB}^{+}$macrophages in the spleen at $7 \mathrm{dpi}$ when bacterial counts were highest. Previous studies have shown that $\mathrm{MRC1LB}^{+}$macrophages are largely present in peri-ellipsoid lymphocyte sheaths of the spleen ${ }^{68}$, and have a role in clearing blood-borne bacteria in chickens ${ }^{69}$, equivalent to that of the marginal zone macrophages in mammals ${ }^{70}$. Therefore, these macrophages are suggested to be involved in clearing the SE from 7 dpi onwards. Expression levels of $\mathrm{MHCll}$ found on MRC1LB ${ }^{+}$ macrophages were higher than those found on the DC subpopulations, which was a surprising finding. In a recent publication, similarly high $\mathrm{MHCll}$ expression levels were observed for chicken splenic $\mathrm{MRC}_{1 \mathrm{LB}^{+}}$macrophages but this level of MHCll expression was also found on DCs ${ }^{71}$. The FSChigh DCs were numerically higher in presence and showed significant increased expression of CHIR-AB1 and $\mathrm{MHCll}$ at $7 \mathrm{dpi}$ in SE-infected chickens compared to uninfected chickens, indicating a role in antigen presentation. The FSC low DCs did not increase in numbers but showed a significant increased expression of the activation markers CHIR-AB1, CD40 and CD80 in SE-infected chickens. The two DC subsets were highly similar, and might comprise DCs at different stages of maturation with the FSClow subset being more mature based on the expression of CD40, CD80 and MHCII1,58,72. These results suggest that the increased presence of macrophages clear bacteria initially and the increased activation of DC subsets contribute to antigen presentation to stimulate the adaptive immune responses, all together resulting in further reduction of SE in infected chickens.

Whereas the APC subsets are likely to contribute to the clearance of the bacteria, it has also been suggested that they may worsen the impact of infection by acting as a carrier for Salmonellae $e^{32}$ and contribute to systemic dissemination ${ }^{37}$, since this bacterium is able to survive intracellularly in chicken macrophages $\mathrm{s}^{35,36}$ and $\mathrm{DCs^{72 }}$. It would be interesting for future studies to determine whether SE can be detected by qPCR in splenic APCs. The ability of Salmonellae to inhibit activation of APCs might explain why NK cells showed earlier activation than APCs and the high presence of SE found at 7 dpi in our study. Other studies have demonstrated that Salmonella-infected APCs secrete IL-12/IL-18 resulting in enhanced expression of the early activation marker IL-2R $\alpha^{+}$on NK cells, thereby inducing their activation by increased cytotoxicity and IFN $\gamma$ production ${ }^{24}$. This IFN $\gamma$ production can subsequently stimulate additional macrophages to clear phagocytosed bacteria ${ }^{73}$ or impair intracellular survival by direct killing of infected-macrophages ${ }^{24}$, which might be involved in the reduction of SE towards and after 7 dpi observed in our study.

T cell presence, SE-induced T cell proliferation and SE specific antibodies were addressed as well. All infected chickens in our study had circulating antibodies specific for SE after three weeks, which was in agreement with other studies ${ }^{40,41}$. The observed $T$ cell responses are similar to increased numbers of intestinal and splenic $\gamma \delta$ and cytotoxic $\alpha \beta$ T cells in response to SE early and three weeks after infection $20,26,27$, as well as to increased $C D 8 \alpha \alpha^{+} \gamma \delta$ T cell numbers ${ }^{22,74}$ in prior studies in chickens. The significant increase in cytotoxic $C D 8 \alpha \alpha^{+} T$ cell numbers, however, has not been shown before in chickens in response to $S E$ infection. The more innate-like nature of $\gamma \delta T$ cell responses early after infection have been recognized as well as the antigen-specific responses of cytotoxic CD8 ${ }^{+} T$ cells approximately two weeks after infection, whereas the functional difference between CD8 $\alpha \alpha$ and CD8 $\alpha \beta$ expression is less 
clear $^{75-78}$. Although expression of CD107 and IFN $y$ production by intraepithelial and splenic $\gamma \delta T$ cells, cytotoxic $C D 8^{+} \mathrm{T}$ cells, and splenic helper $\mathrm{CD} 4^{+} \mathrm{T}$ cells did not significantly differ between uninfected and SE-infected chickens, proliferation of SE-induced splenic T cells of SE-infected chickens ex vivo was observed three weeks after infection and not in uninfected chickens. Although the effect of the initial increased presence of T cells on the numbers of SE in the first week post-infection could not be determined, the SE-specific T cells and antibodies in course of infection are suggested to reduce the number of SE.

In conclusion, this study shows that Salmonella enterica serotype Enteritidis infection in young broiler chickens firstly induces local and systemic activation of NK cells (1, 3, $7 \mathrm{dpi})$ as well as presence of intraepithelial T cells (1 dpi), followed by increased presence of macrophages and activation of DCs (7 dpi). Subsequently, proliferation of T cells in the spleen and antibody responses in serum ( $21 \mathrm{dpi})$ are induced, all together paralleled by a reduction in SE counts. These insights in understanding the role of NK cell and APC subsets and responses of adaptive immune cells upon SE infection will aid in developing immune-modulation strategies to stimulate innate cells. The potential strengthening of immune responsiveness by vaccines or feed strategies during early life may increase resistance and may prevent SE infection and colonization in young broiler chickens.

\section{Acknowledgements}

We thank the animal caretakers and F.C. Velkers of the Department Population Health Sciences, division Farm Animal Health, Faculty of Veterinary Medicine, Utrecht University, for their help during the animal experiments and SE inoculation. We acknowledge E. Broens and A.J. Timmerman of the VMDC, Faculty of Veterinary Medicine, Utrecht University, for kindly providing the SE strain and help with the SE culture. We are thankful to I.S. Ludwig for her help during the isolation of immune cells and we acknowledge G.J.A. Arkesteijn for maintaining optimal working conditions of the Flow Cytometry and Cell Sorting Facility, Faculty of Veterinary Medicine, Utrecht University. This work is part of the research programme of NWO Earth and Life Sciences (ALW) with project number 868.15.020, which is financed by the Dutch Research Council (NWO) and by Cargill Animal Nutrition and Health. 


\section{Supplementary data}
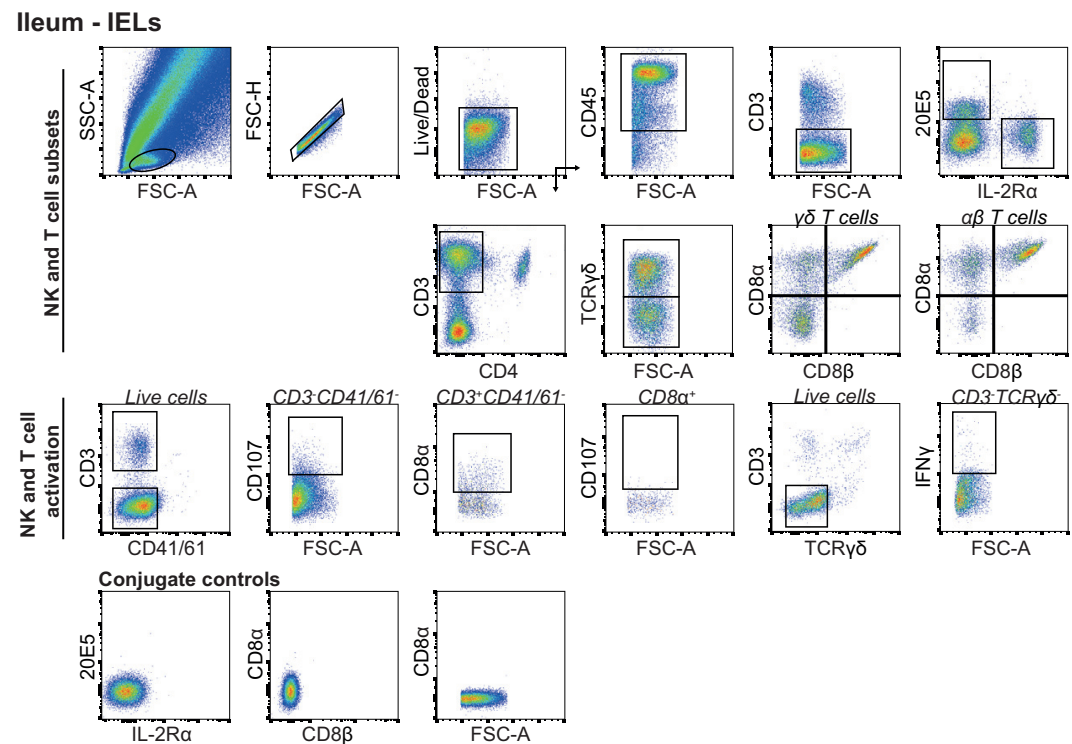

Spleen
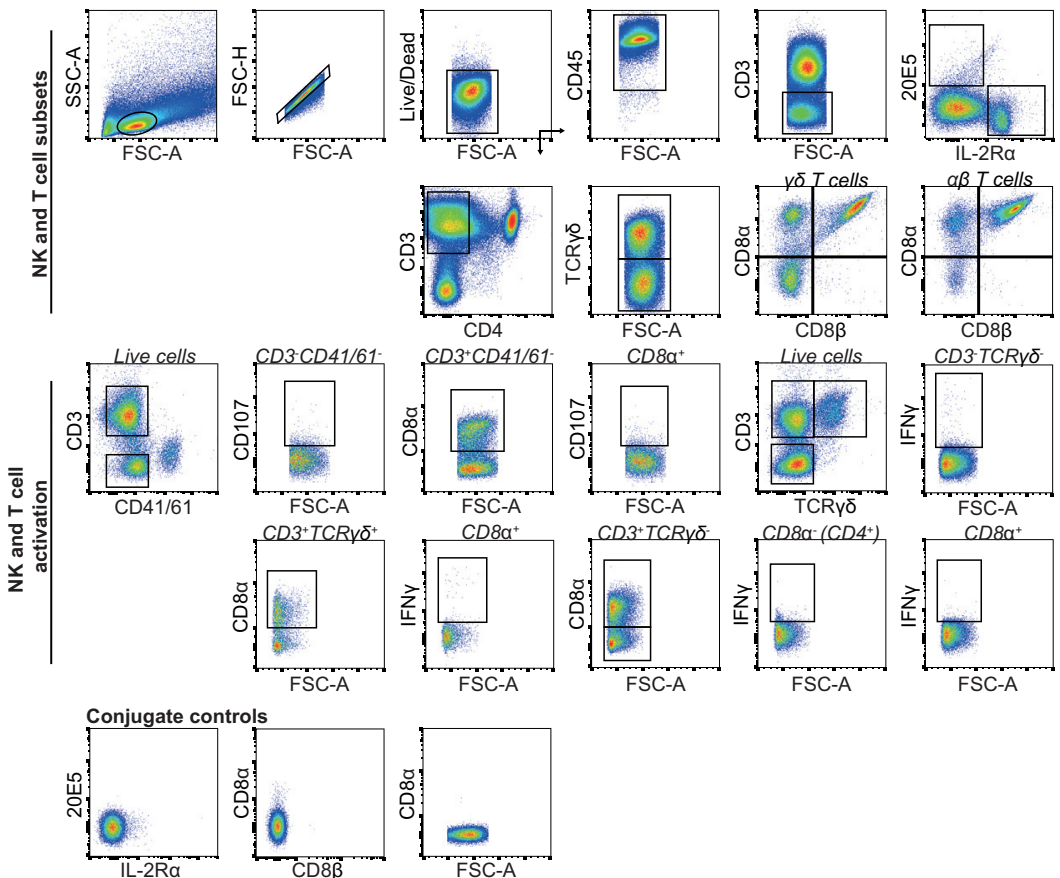

Figure S1. Gating strategy of IELs and splenic lymphocytes in broiler chickens. Gating strategy included consecutive selection for lymphocytes (FSC-A vs SSC-A), singlets (FSC-A vs FSC-H) and viable cells (Live/Dead marker-negative) followed by selection of NK and T cell subsets in ileum and spleen. Furthermore, activation of NK and T cells was analyzed by surface expression of CD107 and intracellular expression of IFNY. Conjugate controls are shown for IELs and splenic lymphocytes. 
A

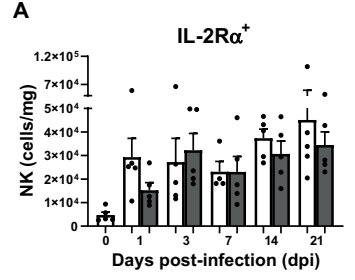

C

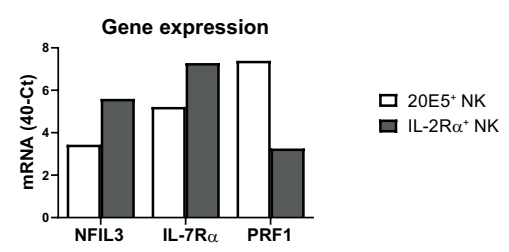

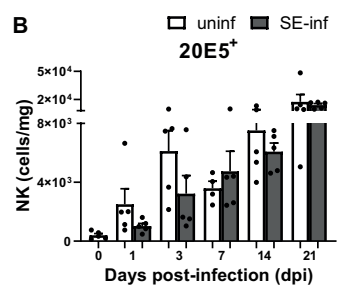

Figure S2. Effect of SE infection on numbers of splenic NK cells in broiler chickens. (A) Numbers (cells/mg) of splenic IL-2R $\alpha^{+}$and (B) 20E5 $5^{+}$NK cells per $m g$ spleen in uninfected (uninf) and SE-infected (SE-inf) chickens in the course of time. (C) Gene expression levels of NK cell lineage marker (NFIL3), IL-7R $\alpha$ and perforin 1 (PRF1) by RT-qPCR in sorted IL-2R $\alpha^{+}$and $20 \mathrm{E} 5^{+}$NK cell subsets. Mean + SEM per treatment and time point is shown ( $\mathrm{n}$ = 5), for uninfected chickens at $7 \mathrm{dpi} \mathrm{n}=4$ and for gene expression levels $n=1$.

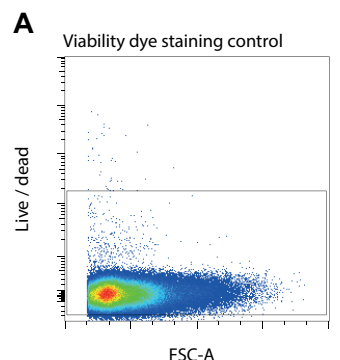

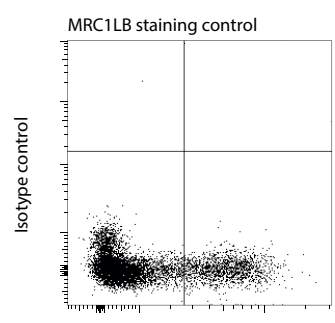

CD11

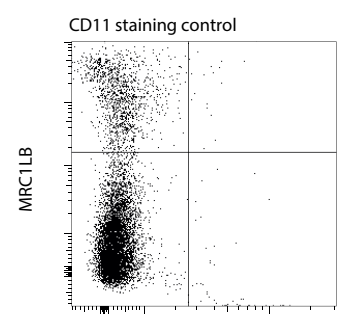

Conjugate control

B

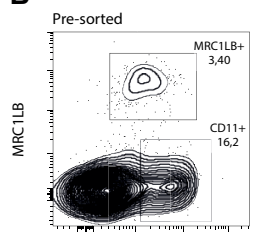

CD11

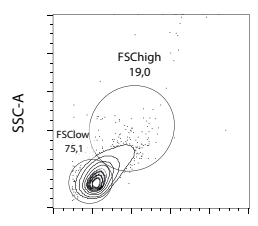

FSC-A

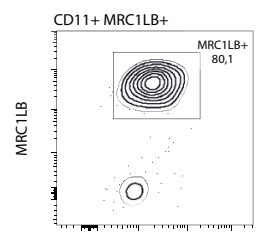

CD11

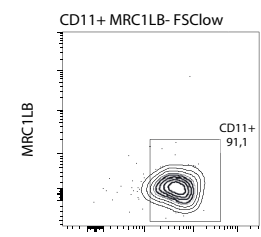

CD11

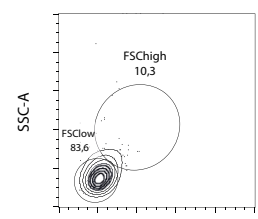

FSC-A

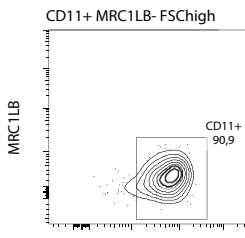

CD11

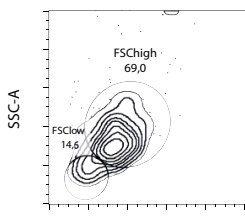

FSC-A

C

\begin{tabular}{|l|l|l|l|}
\hline $\begin{array}{l}\text { Absolute numbers of } \\
\text { sorted cells }\end{array}$ & CD11+ MRC1LB+ & $\begin{array}{l}\text { CD11+ MRC1LB- } \\
\text { FSClow }\end{array}$ & $\begin{array}{l}\text { CD11+ MRC1LB- } \\
\text { FSChigh }\end{array}$ \\
\hline Sample 1 & 3043 & 4441 & 1519 \\
Sample 2 & 4670 & 7062 & 2275 \\
Sample 3 & 6379 & 9868 & 3053 \\
\hline
\end{tabular}

Figure S3. Staining and sorting controls associated with Fig. 4. (A) The staining controls for the gating strategy are shown. The left panel depicts splenocytes without the viability dye. The middle and right panels show splenocytes that are gated according to Fig. $4 \mathrm{~A}$, but without the primary antibodies that bind MRC1LB and CD11, respectively. (B) The graphs show the gating strategy and purity of a representative sample of splenocytes that was sorted into CD11+ MRC1LB+, CD11+ MRC1LB- FSClow and CD11+ MRC1LB- FSChigh subpopulations. The splenocytes that are gated as CD11+ MRC1LB in the upper panels are shown in the lower panels to visualize their FSC-A vs SSC-A pattern. (C) The absolute numbers of sorted APC subpopulations are shown. 
A

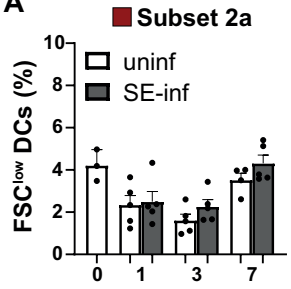

Days post-infection (dpi)

\section{C}

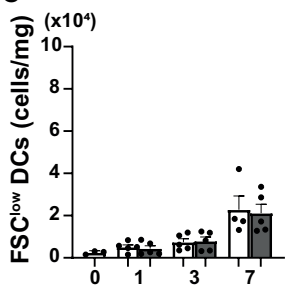

Days post-infection (dpi)
B

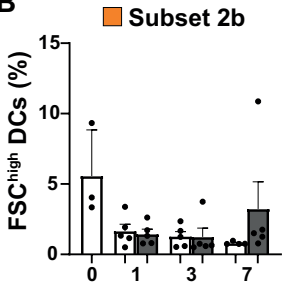

Days post-infection (dpi)

D

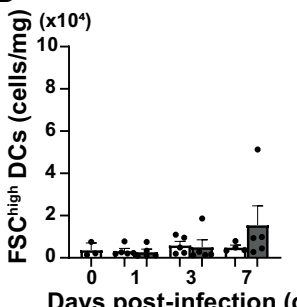

Figure S4. Phenotypic characterization of splenic APCs upon SE infection. (A-B) The presence (\%) and (C-D) numbers (cells/mg spleen) of FSClow DCs and and FSChigh DCS in uninfected (uninf) and SE-infected (SE-inf) chickens were assessed over time. Mean + SEM per treatment and time point is shown $(n=5)$, for uninfected chickens at 0 dpi $n=3$ and at 7 dpi $n=4$. Statistical significance is indicated as ${ }^{* \star} p<0.01$.

\section{A $\square$ Macrophages}

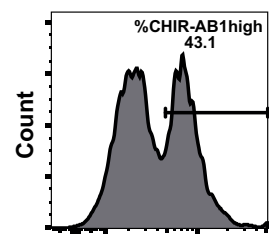

CHIR-AB1

B $\square$ FSC $^{\text {low DCs }}$

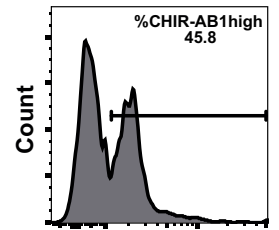

CHIR-AB1

\section{C $\square$ FSC $^{\text {high }}$ DCs}

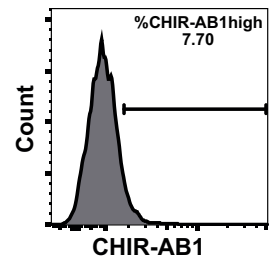

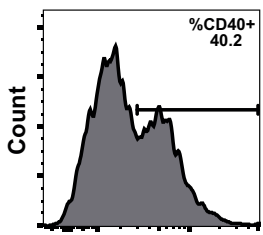

CD40
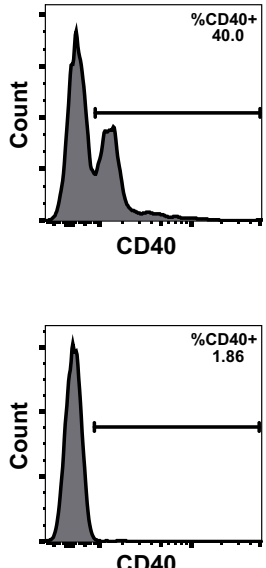

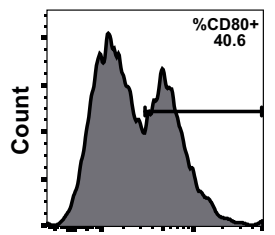

CD80
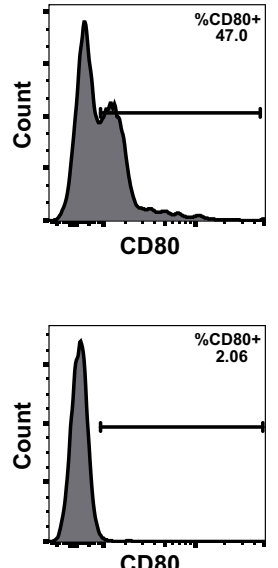

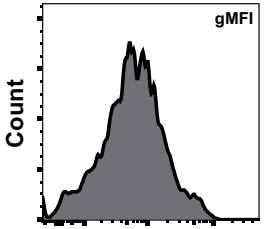

MHC-II

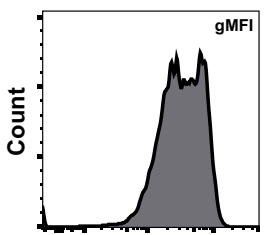

MHC-II

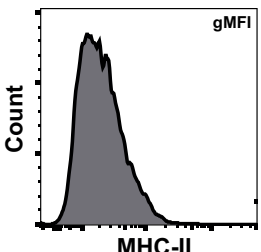

Figure S5. The gating strategy used to determine the activation status of the APC subsets as depicted in Fig. 5. The three identified splenic APC subsets (A) macrophages, (B) FSClow DCs and (C) FSChigh DCs were assessed for CHIR-AB1, CD40, CD80 and MHCIl. For CHIR-AB1, CD40 and CD80, the cells expressing the respective markers were selected and expressed as a percentage. The expression of $\mathrm{MHCll}$ by each subset was expressed as the geometric mean fluorescent intensity (gMFI). 
A

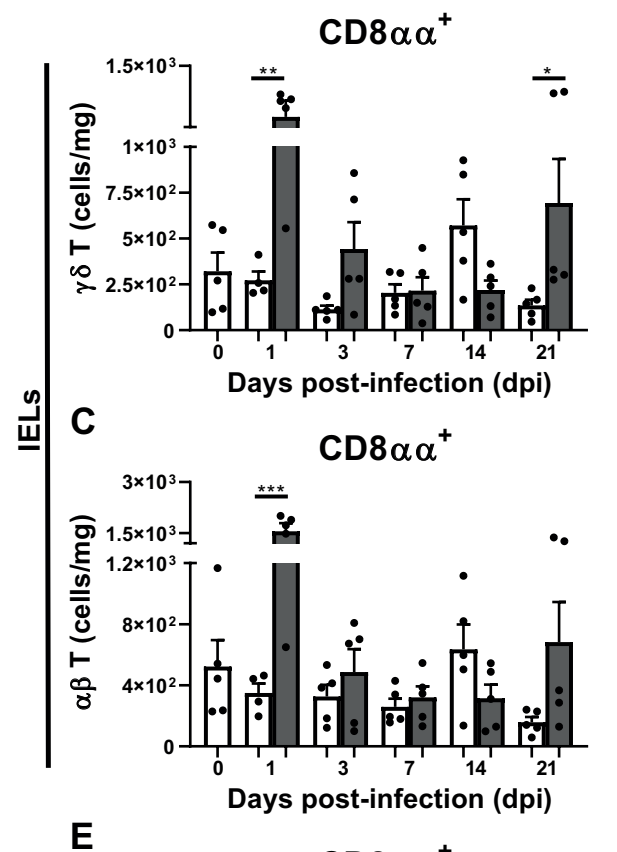

B

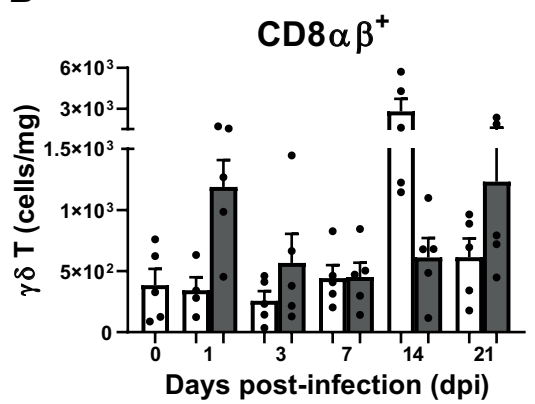

D

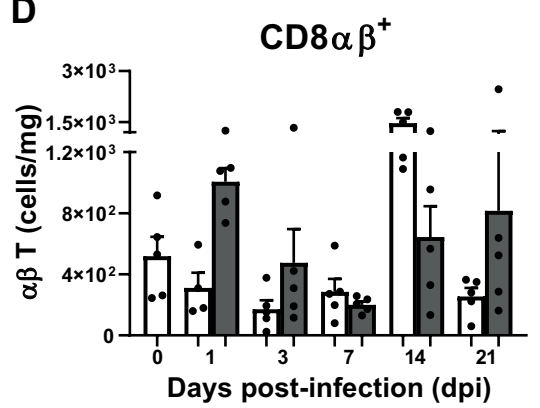

$\mathbf{F}$

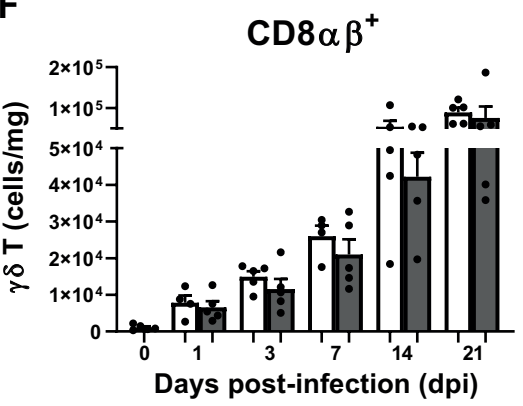

H

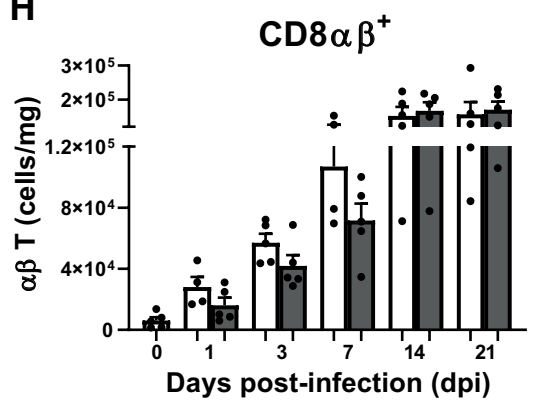

Figure S6. Numbers of intraepithelial and splenic $\gamma \delta$ T cells and cytotoxic T cells expressing either CD8 $\alpha \alpha$ or CD8 $\alpha \beta$ in broiler chickens upon SE infection. (A) Numbers (cells/mg) of intraepithelial CD $8 \alpha \alpha^{+} \gamma \delta$ T cells, (B) CD $8 \alpha \beta^{+} \gamma \delta$ T cells, (C) cytotoxic CD8 $\alpha \alpha^{+} T$ cells and (D) CD8 $\alpha \beta^{+} T$ cells per mg ileum in uninfected (uninf) and SE-infected (SE-inf) chickens in the course of time. (E) Numbers (cells/mg) of splenic CD8 $\alpha \alpha^{+} \gamma \delta$ T cells, (F) CD8 $\alpha \beta^{+} \gamma \delta T$ cells, (G) cytotoxic CD8 $\alpha \alpha^{+} T$ cells and (H) CD8 $\alpha \beta^{+} T$ cells per mg spleen in uninfected and SE-infected chickens. Mean + SEM per treatment and time point is shown $(n=5)$, for uninfected chickens at 1 dpi in the IELs and spleen $n=4$ due to numbers of events acquired in the gate of interest were $<100$, and at $7 \mathrm{dpi}$ in spleen $n=4$. Statistical significance is indicated as ${ }^{*} p<0.05,{ }^{* *} p<0.01$. ${ }^{* *} p<0.001$. 


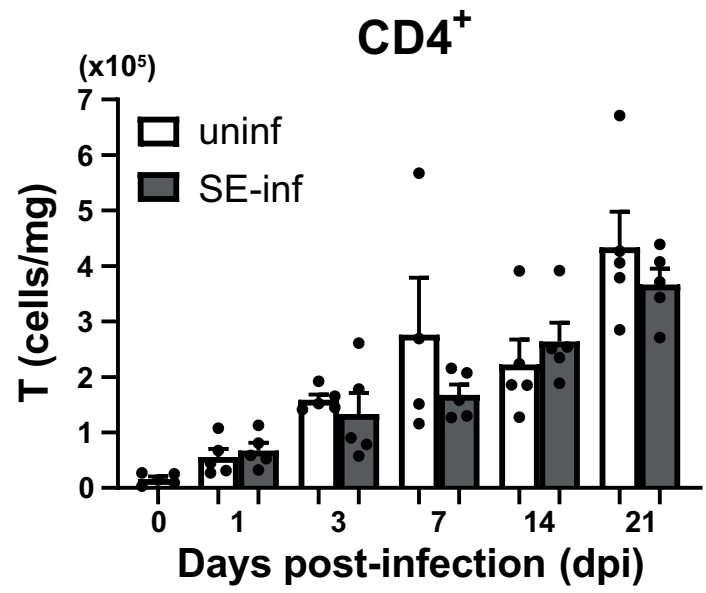

A

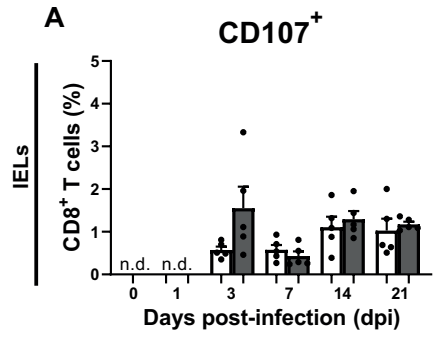

B

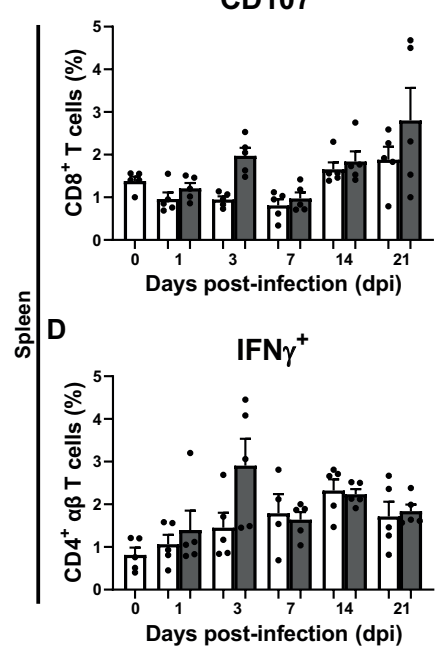

Figure S7. Numbers of $\mathrm{CD} 4^{+} \mathrm{T}$ cells in the spleen of broiler chickens upon SE infection. Numbers (cells/mg) of splenic $\mathrm{CD}^{+} \alpha \beta$ T cells per mg spleen in uninfected (uninf) and SE-infected (SE-inf) chickens in the course of time. Mean + SEM per treatment and time point is shown $(n=5)$, for uninfected chickens at $7 \mathrm{dpi} n=4$.

\section{$\square$ uninf \\ $\square$ SE-inf}

C
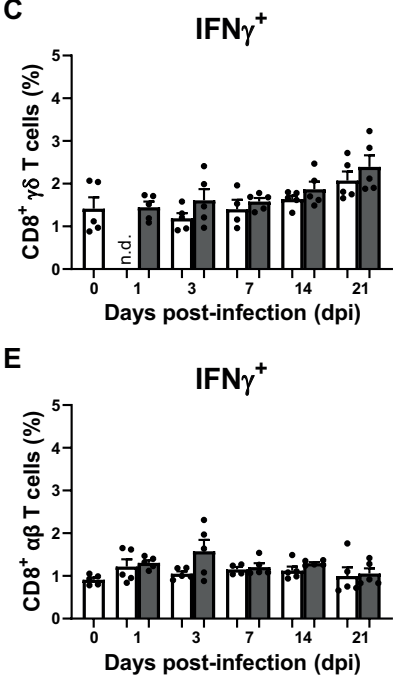

Figure S8. T cell activation in the IEL population and spleen of broiler chickens upon SE infection. (A) Percentages of intraepithelial CD8 ${ }^{+} T$ cells expressing CD107 (including both $\gamma \delta$ and $\alpha \beta T$ cells) in uninfected (uninf) and SE-infected (SE-inf) chickens in the course of time. (B) Percentages of splenic CD8 ${ }^{+}$T cells expressing CD107 (including both $\gamma \delta$ and $\alpha \beta T$ cells), (C) CD8 ${ }^{+} \gamma \delta T$ cells expressing IFN $\gamma_{\text {, }}$ (D) CD4 ${ }^{+} \alpha \beta$ T cells expressing IFN $\gamma$ and (E) CD8 ${ }^{+} \alpha \beta$ T cells expressing IFN $\gamma$ in uninfected (uninf) and SE-infected (SE-inf) chickens over time. Mean + SEM per treatment and time point is shown $(n=5)$, for uninfected chickens at $7 \mathrm{dpi}$ in spleen $n=4$ and at 1 and $3 \mathrm{dpi}$ in the IELs percentages were not determined (n.d.) due to numbers of events acquired in the gate of interest were $<100$. 


\section{References}

1. Kallapura G, Morgan MJ, Pumford NR, et al. Evaluation of the respiratory route as a viable portal of entry for Salmonella in poultry via intratracheal challenge of Salmonella Enteritidis and Salmonella Typhimurium. Poult Sci. 2014;93(2):340-346. doi:10.3382/ps.2013-03602.

2. Kallapura G, Kogut MH, Morgan MJ, et al. Fate of Salmonella Senftenberg in broiler chickens evaluated by challenge experiments. Avian Pathol. 2014;43(4):305-309. doi:10.1080/03079457.2014.923554.

3. Suzuki S. Pathogenicity of Salmonella enteritidis in poultry. Int J Food Microbiol. 1994;21(1-2):89-105. doi:10.1016/01681605(94)90203-8.

4. World Organisation for Animal Health, (OIE). Prevention, detection and control of Salmonella in poultry. OIE, ed. 28th ed. Office International des Epizooties; 2019.

5. Sharma JM, Tizard I. Avian cellular immune effector mechanisms - a review. Avian Pathol. 1984;13(3):357-376. doi:10.1080/03079458408418541.

6. Klasing KC LT. Functions, costs and benefits of the immune system during development and growth. 1999;In: Adams NJ, and Slotow R (eds)(Proc. 22. Int. Ornithol. Congr. H. Birdlife South Africa, Johannesburg):2817-2835.

7. Chai J-, Lillehoj HS. Isolation and functional characterization of chicken intestinal intra-epithelial lymphocytes showing natural killer cell activity against tumour target cells. Immunology. 1988;63(1):111-117.

8. Gómez Del Moral M, Fonfría J, Varas A, Jiménez E, Moreno J, Zapata AG. Appearance and development of lymphoid cells in the chicken (Gallus gallus) caecal tonsil. Anat Rec. 1998;250(2):182-189. doi:10.1002/(SICI)1097-0185(199802)250:23.0.CO;2-5.

9. Simon K, De Vries Reilingh G, Kemp B, Lammers A. Development of lleal cytokine and immunoglobulin expression levels in response to early feeding in broilers and layers. Poult Sci. 2014;93(12):3017-3027. doi:10.3382/ps.2014-04225.

10. Befus $A D$, Johnston N, Leslie GA, Bienenstock J. Gut-associated lymphoid tissue in the chicken. I. Morphology, ontogeny, and some functional characteristics of Peyer's patches. J Immunol. 1980;125(6):2626-2632.

11. Göbel TWF, Kaspers B, Stangassinger M. NK and T cells constitute two major, functionally distinct intestinal epithelial lymphocyte subsets in the chicken. Int Immunol. 2001;13(6):757-762. doi:10.1093/intimm/13.6.757.

12. Meijerink N, van Haarlem DA, Velkers FC, Stegeman AJ, Rutten, V. P. M. G., Jansen CA. Analysis of chicken intestinal natural killer cells, a major IEL subset during embryonic and early life. Dev Comp Immunol. 2021;114. doi:10.1016/j.dci.2020.103857.

13. Fenzl L, Göbel TW, Neulen M-. $\gamma \delta$ T cells represent a major spontaneously cytotoxic cell population in the chicken. Dev Comp Immunol. 2017;73:175-183. doi:10.1016/j.dci.2017.03.028.

14. Lillehoj HS, Trout JM. Avian gut-associated lymphoid tissues and intestinal immune responses to Eimeria parasites. Clin Microbiol Rev. 1996;9(3):349-360. doi:10.1128/cmr.9.3.349.

15. Schokker D, De Koning D-, Rebel JMJ, Smits MA. Shift in chicken intestinal gene association networks after infection with Salmonella. Comp Biochem Physiol Part D Genomics Proteomics. 2011;6(4):339-347. doi:10.1016/j.cbd.2011.07.004.

16. Li $X$, Zhang $P$, Jiang $X$, et al. Differences in expression of genes in the MyD88 and TRIF signalling pathways and methylation of TLR4 and TRIF in Tibetan chickens and DaHeng S03 chickens infected with Salmonella enterica serovar enteritidis. Vet Immunol Immunopathol. 2017;189:28-35. doi:10.1016/j.vetimm.2017.05.003.

17. Matulova M, Varmuzova K, Sisak F, et al. Chicken innate immune response to oral infection with Salmonella enterica serovar Enteritidis. Vet Res. 2013;44(1). doi:10.1186/1297-9716-44-37.

18. Swaggerty CL, Kogut MH, Ferro PJ, Rothwell L, Pevzner IY, Kaiser P. Differential cytokine mRNA expression in heterophils isolated from Salmonella-resistant and -susceptible chickens. Immunology. 2004;113(1):139-148. doi:10.1111/j.1365-2567.2004.01939.x.

19. Schokker D, Smits MA, Hoekman AJW, Parmentier HK, Rebel JMJ. Effects of Salmonella on spatial-temporal processes of jejunal development in chickens. Dev Comp Immunol. 2010;34(10):1090-1100. doi:10.1016/j.dci.2010.05.013.

20. Carvajal BG, Methner U, Pieper J, Berndt A. Effects of Salmonella enterica serovar Enteritidis on cellular recruitment and cytokine gene expression in caecum of vaccinated chickens. Vaccine. 2008;26(42):5423-5433. doi:10.1016/j.vaccine.2008.07.088.

21. Sekelova Z, Stepanova H, Polansky O, et al. Differential protein expression in chicken macrophages and heterophils in vivo following infection with Salmonella Enteritidis. Vet Res. 2017;48(1). doi:10.1186/s13567-017-0439-0.

22. Berndt A, Pieper J, Methner U. Circulating $\gamma \delta T$ cells in response to Salmonella enterica serovar enteritidis exposure in chickens. Infect Immun. 2006;74(7):3967-3978. doi:10.1128/IAI.01128-05.

23. Schafer R, Eisenstein TK. Natural killer cells mediate protection induced by a Salmonella aroA mutant. Infect Immun. 1992;60(3):791797.

24. Lapaque N, Walzer T, Méresse S, Vivier E, Trowsdale J. Interactions between human NK cells and macrophages in response to Salmonella infection. J Immunol. 2009;182(7):4339-4348. doi:10.4049/jimmunol.0803329.

25. Harrington L, Srikanth CV, Antony R, Shi HN, Cherayil BJ. A role for natural killer cells in intestinal inflammation caused by infection with Salmonella enterica serovar Typhimurium. FEMS Immunol Med Microbiol. 2007;51(2):372-380. doi:10.1111/j.1574695X.2007.00313.x.

26. Schokker D, Peters THF, Hoekman AJW, Rebel JMJ, Smits MA. Differences in the early response of hatchlings of different chicken breeding lines to Salmonella enterica serovar Enteritidis infection. Poult Sci. 2012;91(2):346-353. doi:10.3382/ps.2011-01758.

27. Van Hemert S, Hoekman AJW, Smits MA, Rebel JMJ. Immunological and gene expression responses to a Salmonella infection in the chicken intestine. Vet Res. 2007;38(1):51-63. doi:10.1051/vetres:2006048.

28. van Hemert S, Hoekman AJW, Smits MA, Rebel JMJ. Gene expression responses to a Salmonella infection in the chicken intestine differ between lines. Vet Immunol Immunopathol. 2006;114(3-4):247-258. doi:10.1016/j.vetimm.2006.08.007. 
29. van Hemert S, Hoekman AJW, Smits MA, Rebel JMJ. Early host gene expression responses to a Salmonella infection in the intestine of chickens with different genetic background examined with cDNA and oligonucleotide microarrays. Comp Biochem Physiol Part D Genomics Proteomics. 2006;1(3):292-299. doi:10.1016/j.cbd.2006.05.001.

30. Staines K, Hunt LG, Young JR, Butter C. Evolution of an expanded mannose receptor gene family. PLoS ONE. 2014;9(11). doi:10.1371/journal.pone.0110330.

31. Kamble NM, Jawale CV, Lee JH. Activation of chicken bone marrow-derived dendritic cells induced by a Salmonella Enteritidis ghost vaccine candidate. Poult Sci. 2016;95(10):2274-2280. doi:10.3382/ps/pew158.

32. Chappell L, Kaiser P, Barrow P, Jones MA, Johnston C, Wigley P. The immunobiology of avian systemic salmonellosis. Vet Immunol Immunopathol. 2009;128(1-3):53-59. doi:10.1016/j.vetimm.2008.10.295.

33. Gomes AVS, Quinteiro-Filho WM, Ribeiro A, et al. Overcrowding stress decreases macrophage activity and increases Salmonella Enteritidis invasion in broiler chickens. Avian Pathol. 2014;43(1):82-90. doi:10.1080/03079457.2013.874006.

34. Vazquez-Torres $\mathrm{A}, \mathrm{Xu} \mathrm{Y}$, Jones-Carson J, et al. Salmonella pathogenicity island 2-dependent evasion of the phagocyte NADPH oxidase. Science. 2000;287(5458):1655-1658. doi:10.1126/science.287.5458.1655.

35. Jones MA, Wigley P, Page KL, Hulme SD, Barrow PA. Salmonella enterica serovar Gallinarum requires the Salmonella pathogenicity island 2 type III secretion system but not the Salmonella pathogenicity island 1 type III secretion system for virulence in chickens. Infect Immun. 2001;69(9):5471-5476. doi:10.1128/IAl.69.9.5471-5476.2001.

36. Jones MA, Hulme SD, Barrow PA, Wigley P. The Salmonella pathogenicity island 1 and Salmonella pathogenicity island 2 type III secretion systems play a major role in pathogenesis of systemic disease and gastrointestinal tract colonization of Salmonella enterica serovar Typhimurium in the chicken. Avian Pathol. 2007;36(3):199-203. doi:10.1080/03079450701264118.

37. Vazquez-Terres A, Jones-Carson J, Bäumler AJ, et al. Extraintestinal dissemination of Salmonella by CD18-expressing phagocytes. Nature. 1999;401(6755):804-808. doi:10.1038/44593.

38. Sekelova Z, Polansky O, Stepanova $H$, et al. Different roles of CD4, CD8 and $\gamma \delta$ T-lymphocytes in naive and vaccinated chickens during Salmonella Enteritidis infection. Proteomics. 2017;17(13-14). doi:10.1002/pmic.201700073.

39. Desmidt M, Ducatelle R, Mast J, Goddeeris BM, Kaspers B, Haesebrouck F. Role of the humoral immune system in Salmonella enteritidis phage type four infection in chickens. Vet Immunol Immunopathol. 1998;63(4):355-367. doi:10.1016/ S0165-2427(98)00112-3.

40. Zhen W, Shao Y, Gong X, et al. Effect of dietary Bacillus coagulans supplementation on growth performance and immune responses of broiler chickens challenged by Salmonella enteritidis. Poult Sci. 2018;97(8):2654-2666. doi:10.3382/ps/pey119.

41. Berthelot-Hérault F, Mompart F, Zygmunt MS, Dubray GE, Duchet-Suchaux M. Antibody responses in the serum and gut of chicken lines differing in cecal carriage of Salmonella enteritidis. Vet Immunol Immunopathol. 2003;96(1-2):43-52. doi:10.1016/ S0165-2427(03)00155-7.

42. Meijerink N, Kers JG, Velkers FC, et al. Early Life Inoculation With Adult-Derived Microbiota Accelerates Maturation of Intestinal Microbiota and Enhances NK Cell Activation in Broiler Chickens. Front Vet Sci. 2020;7. doi:10.3389/fvets.2020.584561.

43. Van De Reep L, Nielen M, Verstappen, K. M. H. W., Broens EM, Van Den Broek J, Velkers FC. Response to a Salmonella Enteritidis challenge in old laying hens with different vaccination histories. Poult Sci. 2018;97(8):2733-2739. doi:10.3382/ps/pey134.

44. Jansen CA, van de Haar, P. M., van Haarlem D, et al. Identification of new populations of chicken natural killer (NK) cells. Dev Comp Immunol. 2010;34(7):759-767. doi:10.1016/j.dci.2010.02.009.

45. Ariaans MP, van de Haar, P. M., Lowenthal JW, van Eden W, Hensen EJ, Vervelde L. ELISPOT and intracellular cytokine staining: Novel assays for quantifying $T$ cell responses in the chicken. Dev Comp Immunol. 2008;32(11):1398-1404. doi:10.1016/j. dci.2008.05.007.

46. Belkina AC, Ciccolella CO, Anno R, Halpert R, Spidlen J, Snyder-Cappione JE. Automated optimized parameters for T-distributed stochastic neighbor embedding improve visualization and analysis of large datasets. Nat Commun. 2019;10(1). doi:10.1038/ s41467-019-13055-y.

47. van den Biggelaar, R. H. G. A., van Eden W, Rutten, V. P. M. G., Jansen CA. Nitric oxide production and fc receptor-mediated phagocytosis as functional readouts of macrophage activity upon stimulation with inactivated poultry vaccines in vitro. Vaccines. 2020;8(2):1-16. doi:10.3390/vaccines8020332.

48. Viertlboeck BC, Schweinsberg S, Hanczaruk MA, et al. The chicken leukocyte receptor complex encodes a primordial, activating, high-affinity IgY Fc receptor. Proc Natl Acad Sci U S A. 2007;104(28):11718-11723. doi:10.1073/pnas.0702011104.

49. Eldaghayes I, Rothwell L, Williams A, et al. Infectious bursal disease virus: Strains that differ in virulence differentially modulate the innate immune response to infection in the chicken bursa. Viral Immunol. 2006;19(1):83-91. doi:10.1089/vim.2006.19.83.

50. Michaud A, Dardari R, Charrier E, Cordeiro P, Herblot S, Duval M. IL-7 enhances survival of human CD56bright NK cells. J Immunother. 2010;33(4):382-390. doi:10.1097/CJl.0b013e3181cd872d.

51. Male V, Nisoli I, Kostrzewski T, et al. The transcription factor E4bp4/Nfil3 controls commitment to the NK lineage and directly regulates Eomes and Id2 expression. J Exp Med. 2014;211(4):635-642. doi:10.1084/jem.20132398.

52. Wendt K, Wilk E, Buyny S, Buer J, Schmidt RE, Jacobs R. Gene and protein characteristics reflect functional diversity of CD56 dim and CD56bright NK cells. J Leukocyte Biol. 2006;80(6):1529-1541. doi:10.1189/jlb.0306191.

53. Di Santo JP. A defining factor for natural killer cell development. Nat Immunol. 2009;10(10):1051-1052. doi:10.1038/ni1009-1051.

54. Luevano M, Madrigal A, Saudemont A. Transcription factors involved in the regulation of natural killer cell development and function: An update. Front Immunol. 2012;3(OCT). doi:10.3389/fimmu.2012.00319.

55. Sarson AJ, Abdul-Careem MF, Read LR, Brisbin JT, Sharif S. Expression of cytotoxicity-associated genes in Marek's disease virus-infected chickens. Viral Immunol. 2008;21(2):267-272. doi:10.1089/vim.2007.0094. 
56. Livak KJ, Schmittgen TD. Analysis of relative gene expression data using real-time quantitative PCR and the 2- $\triangle \triangle C T$ method. Methods. 2001;25(4):402-408. doi:10.1006/meth.2001.1262.

57. Manh $T-V$, Marty $H$, Sibille $P$, et al. Existence of conventional dendritic cells in gallus gallus revealed by comparative gene expression profiling. J Immunol. 2014;192(10):4510-4517. doi:10.4049/jimmunol.1303405.

58. van den Biggelaar, R. H. G. A., Arkesteijn GJA, Rutten, V. P. M. G., van Eden W, Jansen CA. In vitro Chicken Bone Marrow-Derived Dendritic Cells Comprise Subsets at Different States of Maturation. Front Immunol. 2020;11. doi:10.3389/fimmu.2020.00141.

59. Sundick RS, Gill-Dixon C. A Cloned Chicken Lymphokine Homologous to Both Mammalian IL-2 and IL-15. J Immunol. 1997;159(2):720-725.

60. Raehtz S, Hargis BM, Kuttappan VA, Pamukcu R, Bielke LR, McCabe LR. High molecular weight polymer promotes bone health and prevents bone loss under salmonella challenge in broiler chickens. Front Physiol. 2018;9(APR). doi:10.3389/fphys.2018.00384.

61. Khan MI, Fadl AA, Venkitanarayanan KS. Reducing colonization of Salmonella Enteritidis in chicken by targeting outer membrane proteins. J Appl Microbiol. 2003;95(1):142-145. doi:10.1046/j.1365-2672.2003.01953.x.

62. Gast RK, Regmi P, Guraya R, Jones DR, Anderson KE, Karcher DM. Colonization of internal organs by Salmonella Enteritidis in experimentally infected laying hens of four commercial genetic lines in conventional cages and enriched colony housing. Poult Sci. 2019;98(4):1785-1790. doi:10.3382/ps/pey541.

63. Rudnicka K, Matusiak A, Chmiela M. CD25 (IL-2R) expression correlates with the target cell induced cytotoxic activity and cytokine secretion in human natural killer cells. Acta Biochim Pol. 2015;62(4):885-894. doi:10.18388/abp.2015_1152.

64. Adib-Conquy M, Scott-Algara D, Cavaillon J-, Souza-Fonseca-Guimaraes F. TLR-mediated activation of NK cells and their role in bacterial/viral immune responses in mammals. Immunol Cell Biol. 2014;92(3):256-262. doi:10.1038/icb.2013.99.

65. Marcenaro E, Ferranti B, Falco M, Moretta L, Moretta A. Human NK cells directly recognize Mycobacterium bovis via TLR2 and acquire the ability to kill monocyte-derived DC. Int Immunol. 2008;20(9):1155-1167. doi:10.1093/intimm/dxn073.

66. Okamura M, Lillehoj HS, Raybourne RB, et al. Differential responses of macrophages to Salmonella enterica serovars Enteritidis and Typhimurium. Vet Immunol Immunopathol. 2005;107(3-4):327-335. doi:10.1016/j.vetimm.2005.05.009.

67. Pallmer K, Oxenius A. Recognition and regulation of T cells by NK cells. Front Immunol. 2016;7(JUN). doi:10.3389/fimmu.2016.00251.

68. Hu T, Wu Z, Bush SJ, et al. Characterization of subpopulations of chicken mononuclear phagocytes that express TIM4 and CSF1R. J Immunol. 2019;202(4):1186-1199. doi:10.4049/jimmunol.1800504.

69. Yu K, Gu MJ, Pyung YJ, et al. Characterization of splenic MRC1(hi)MHCII(lo) and MRC1(lo)MHCll(hi) cells from the monocyte/ macrophage lineage of White Leghorn chickens. Vet Res. 2020;51(1):73. doi:10.1186/s13567-020-00795-9.

70. A-Gonzalez N, Guillen JA, Gallardo G, et al. The nuclear receptor $L X R \propto$ controls the functional specialization of splenic macrophages. Nat Immunol. 2013;14(8):831-839. doi:10.1038/ni.2622.

71. Sutton KM, Morris KM, Borowska D, et al. Characterization of Conventional Dendritic Cells and Macrophages in the Spleen Using the CSF1R-Reporter Transgenic Chickens. Front Immunol. 2021;12. doi:10.3389/fimmu.2021.636436.

72. Kamble NM, Jawale CV, Lee JH. Interaction of a live attenuated Salmonella Gallinarum vaccine candidate with chicken bone marrow-derived dendritic cells. Avian Pathol. 2016;45(2):235-243. doi:10.1080/03079457.2016.1144919.

73. Rosenberger CM, Brett Finlay B. Macrophages inhibit Salmonella Typhimurium replication through MEK/ERK kinase and phagocyte NADPH oxidase activities. J Biol Chem. 2002;277(21):18753-18762. doi:10.1074/jbc.M110649200.

74. Pieper J, Methner U, Berndt A. Characterization of avian $\gamma \delta$ T-cell subsets after Salmonella enterica serovar typhimurium infection of chicks. Infect Immun. 2011;79(2):822-829. doi:10.1128/IAI.00788-10.

75. Gangadharan D, Cheroutre H. The CD8 isoform CD8 $\alpha$ is not a functional homologue of the TCR co-receptor CD8 $\alpha \beta$. Curr Opin Immunol. 2004;16(3):264-270. doi:10.1016/j.coi.2004.03.015.

76. Cheroutre H, Lambolez F, Mucida D. The light and dark sides of intestinal intraepithelial lymphocytes. Nat Rev Immunol. 2011;11(7):445-456. doi:10.1038/nri3007.

77. Pasman L, Kasper DL. Building conventions for unconventional lymphocytes. Immunol Rev. 2017;279(1):52-62. doi:10.1111/ imr.12576.

78. Leishman AJ, Naidenko OV, Attinger A, et al. T cell responses modulated through interaction between CD8 $\alpha$ and the nonclassical MHC class I molecule, TL. Science. 2001;294(5548):1936-1939. doi:10.1126/science.1063564.

79. van Haarlem DA, van Kooten, P. J. S., Rothwell L, Kaiser P, Vervelde L. Characterisation and expression analysis of the chicken interleukin-7 receptor alpha chain. Dev Comp Immunol. 2009;33(9):1018-1026. doi:10.1016/j.dci.2009.05.001. 
5 

CHAPTER 6

\section{Long-chain glucomannan supplementation modulates immune responsiveness and intestinal microbiota resulting in improved resistance to Salmonella enterica serotype Enteritidis in broiler chickens}

Nathalie Meijerink', Jean E. de Oliveira², Daphne A. van Haarlem¹, David M. Lamot ${ }^{3}$, Francisca C. Velkers ${ }^{4}$, Hauke Smidt ${ }^{5}$, J. Arjan Stegeman ${ }^{4}$, Victor P.M.G. Rutten ${ }^{1,6}$, Christine A. Jansen ${ }^{1}$

${ }^{1}$ Department Biomolecular Health Sciences, Division Infectious Diseases and Immunology, Faculty of Veterinary Medicine, Utrecht University, Utrecht, the Netherlands

${ }^{2}$ Cargill R\&D Center Europe, Vilvoorde, Belgium

${ }^{3}$ Cargill Animal Nutrition and Health Innovation Center, Velddriel, the Netherlands

${ }^{4}$ Department Population Health Sciences, Division Farm Animal Health, Faculty of Veterinary Medicine, Utrecht University, Utrecht, the Netherlands

${ }^{5}$ Laboratory of Microbiology, Wageningen University \& Research, Wageningen, the Netherlands

${ }^{6}$ Department of Veterinary Tropical Diseases, Faculty of Veterinary Science, University of Pretoria, Pretoria, South Africa 


\section{Abstract}

The zoonotic pathogen Salmonella enterica serotype Enteritidis (SE) causes severe disease in young chickens. Restriction on antibiotic use require alternative SE control strategies such as nutritional solutions to improve the resistance of chickens. In this study, chickens were fed long-chain glucomannan (GM) or standard diet and challenged with SE at seven days of age. During three weeks post-infection (dpi), we determined numbers and responsiveness of NK and T cells in ileum and spleen, and SE-specific antibody titers in serum. Microbiota compositions in ileum and caeca were determined, as well as correlations of these with numbers and function of immune cells. GM supplementation resulted in reduced SE numbers in intestine and spleen as compared to the standard diet. At 3 dpi, intraepithelial NK cell numbers were significantly higher, while intraepithelial NK cell activation tended to be higher at $7 \mathrm{dpi}$. At $14 \mathrm{dpi}$, numbers of intraepithelial cytotoxic $C D 8^{+} \mathrm{T}$ cell numbers tended be higher and SE-specific antibody titers were numerically higher. Furthermore, relative abundance of commensal lactic acid bacteria (LAB) significantly increased with GM supplementation post-infection. Higher relative abundance of streptococci was associated with reduced SE in ileal and caecal contents at 21 dpi. Relative abundance of streptococci was negatively correlated with SE in the spleen whereas positively correlated with splenic NK cell activation and a SE-specific antibody response. These correlations suggest involvement of commensal LAB in NK cell responsiveness coinciding with SE reduction. GM supplementation increases SE resistance in young chickens by immune system stimulation and intestinal microbiota modulation.

Keywords: Long-chain glucomannan, Salmonella Enteritidis, immunity, NK cells, T cells, IELs, intestinal microbiota, poultry, broiler chickens 


\section{Introduction}

Salmonella enterica serotype Enteritidis (SE) is a zoonotic pathogen that may cause severe disease and death in young chickens as well as subclinical infections in adult chickens ${ }^{1}$. Moreover, SE-contaminated poultry products are amongst the leading causes of foodborne diseases in humans ${ }^{2}$. Faecal salmonellae infect chickens via the oral or respiratory route, colonize the intestinal tract and disseminate to organs such as liver and spleen resulting in a systemic infection ${ }^{3,4}$. Prevention of SE infection in poultry is thus important for health and welfare of chickens and to avoid substantial economic losses in the poultry sector and food recalls. In addition, SE prevention in poultry is relevant for the health and wellbeing of humans in terms of food safety as well as to avoid loss of productivity and medical costs ${ }^{5,6}$. Therapeutic treatment of SE infection in chickens with antibiotics is restricted nowadays due to limited effectiveness against Salmonella strains, the risk of residues in poultry products, and potential induction of antibiotic resistance ${ }^{7}$. This encourages the search for immune-modulatory strategies to increase the resistance to SE.

Immune responsiveness in young chickens largely depends on maternal antibodies and the innate immune system, since the adaptive immune system is not fully developed yet ${ }^{8,9}$. Natural killer (NK) cells are key players of innate immunity and are abundantly present among the intraepithelial lymphocytes (IELs) in the intestine, in addition to $\gamma \delta T$ cells and cytotoxic CD8 $8^{+}$cells ${ }^{10-12}$. Directly underneath the intestinal epithelium, macrophages, $B$ cells and helper CD4+ $T$ cells predominate ${ }^{13,14}$. Apart from epithelial cells, IELs constitute the first cellular defense barrier in response to intestinal SE colonization. In a previous paper, we reported increased numbers of intraepithelial IL-2R $\alpha^{+}$and $20 \mathrm{E} 5^{+} \mathrm{NK}$ cells in the first days post SE infection. In parallel, NK cell activation was significantly enhanced in the IEL population and spleen of SE-infected compared to uninfected chickens, as reflected by CD107 and IFN $Y$ expression as parameters for cytotoxicity and cytokine production respectively ${ }^{15}$. The adaptive immune system gradually takes over clearance of SE by responses of $\gamma \delta T$ cells, cytotoxic T cells and antibody production ${ }^{15-18}$. Both gamma delta and cytotoxic T cells can express the CD8 $\alpha \alpha$ or CD8 $\alpha \beta$ co-receptor. The role of $C D 8 \alpha \alpha^{+} T$ cells has not been fully elucidated in contrast to the antigen-experienced $C D 8 \alpha \beta^{+}$cytotoxic responses. $C D 8 \alpha \alpha^{+}$T cells are involved in maintaining the integrity of the intestinal barrier as well as in NK-like cytotoxicity in response to pathogens ${ }^{19-21}$. In a previous study we observed increased numbers of both intraepithelial $C D 8 \alpha \alpha^{+}$and $C D 8 \alpha \beta^{+} \gamma \delta$ T- and cytotoxic T cells in response to SE infection, which were more pronounced for the $C D 8 \alpha \alpha^{+}$subset suggesting an important role for these T cells in defense against pathogens ${ }^{15}$. We hypothesize that stimulation of intraepithelial NK and T cells through feed supplementation during the early life of chickens may aid in increasing their resistance to SE infection.

Nutritional solutions have resulted in a reduction in colonization and shedding of salmonellae in chickens. Depending on the type of modulatory compounds, this was shown to occur directly through binding of feed additives to the bacteria, induction of competitive exclusion, modulation of the intestinal microbiota composition or by direct stimulation of numbers and function of immune cells ${ }^{22-27}$. In addition, changes in the intestinal microbiota by feed supplementation indirectly modulates the immune system, since interactions occur between commensal species and immune cells ${ }^{28-31}$. Feed additives, that have shown to reduce Salmonella enterica strains when provided directly after hatch, include probiotics ${ }^{32-37}$, prebiotics ${ }^{38-40}$ and polysaccharides ${ }^{41}$. Furthermore, administration of probiotics to embryos by in ovo inoculation has shown to reduce SE infection incidence after hatch ${ }^{42,43}$. In a previous study we showed that long-chain glucomannan (GM), a water-soluble polysaccharide that acts as a prebiotic, increased numbers and activation of NK cells in the IEL population as well as the relative abundance of lactic acid bacteria (LAB) in the intestine ${ }^{29}$. However, it is not known yet whether 
and how the increased numbers and function of NK cells and the effects on microbiota composition of GM supplementation increases the resistance to infections such as that with SE.

In this study, we investigated the protective potential and underlying mechanisms of GM supplementation, directly after hatch, against experimental SE challenge in seven-day-old broiler chickens. We analyzed effects of GM on the presence of SE in the intestine and spleen, numbers and functional aspects of NK and T cells in the IELs and spleen, serum antibody titers and intestinal microbiota composition until three weeks after challenge. The present study showed that GM supplementation leads to increased resistance to SE infection in young broiler chickens, by stimulation of the innate immune system, modulation of the intestinal microbiota composition as well as by their interaction. These findings contribute to understanding the effects of feed supplementation that results in higher immune-mediated resistance of young broiler chickens to infections and may contribute to reducing zoonotic infections, as well as use of antibiotics, hence increasing both animal health and welfare, and food safety for humans.

\section{Materials and methods}

\section{Animals and husbandry}

A total of 36 Ross 308 seventeen- and eighteen-day old embryonated eggs were obtained from the same parent flock of a commercial hatchery (Lagerwey, the Netherlands). Upon arrival at the facilities of the Department of Population Health Sciences, Faculty of Veterinary Medicine, Utrecht University, the Netherlands, eggs were disinfected with $3 \%$ hydrogen peroxide, a standard procedure in the facilities ${ }^{28}$ and placed in disinfected egg hatchers in one stable. Directly upon hatch, chickens were weighed, labelled and randomly housed according to their feed group in two floor pens separated by a solid wall. Both female and male chickens were included in the study. Each pen was divided in two equal subunits (A, B). Pens were lined with wood shavings $\left(2 \mathrm{~kg} / \mathrm{m}^{2}\right)$, and water and feed was provided ad libitum. Standard (control, $n=36$ ) or long-chain glucomannan supplemented $(G M ; 0.2 \%$ GM inclusion in complete standard diet $(100 \%), \mathrm{n}=36$ ) Salmonella-free commercial starter and grower feeds were provided (Research Diet Services, the Netherlands). A standard lighting and temperature scheme for Ross broiler chickens was used, equal for both pens.

The animal experiment was approved by the Dutch Central Authority for Scientific Procedures on Animals and the Animal Experiments Committee (registration number AVD1080020174425) of Utrecht University (the Netherlands) and all procedures were done in full compliance with all relevant legislation.

\section{Experimental design}

To determine microbiota composition of the environment, swabs (FLOQSwabs ${ }^{\oplus}$, COPAN, Italy) of the hatchers and floor pens were taken before hatching and once more of the hatchers after hatching. Swabs were stored at room temperature (RT) in $0.5 \mathrm{ml}$ DNA/RNA Shield (Zymo Research, CA, USA) until DNA purification. Additional swab samples of the hatchers after hatching were taken to confirm embryos were Salmonella-free by bacterial counts on MSRV plates (Veterinair Centrum Someren, the Netherlands). At three days post-hatch, six chickens per feed group (three per subunit A/B) were randomly selected and sacrificed, for collection of ileum $( \pm 10 \mathrm{~cm}$ distal from Meckel's diverticulum) and spleen to confirm absence of SE before experimental inoculation. Before inoculation, at seven days post-hatch ( 0 days "post-infection" (dpi)), six chickens per feed group (three per subunit A/B) 
were randomly selected and sacrificed to collect ileum, spleen and contents of ileum (distal from Meckel's diverticulum) and caeca to determine the baseline levels of the various parameters and once more absence of SE. Also, intestinal contents were collected using a sterile plastic cell culture loop, subsequently transferred into $2 \mathrm{ml}$ sterile tubes containing $0.5 \mathrm{ml} \mathrm{DNA} / R N A$ Shield (Zymo Research), and stored at RT for DNA extraction. Chickens of both groups were challenged at $0 \mathrm{dpi}$ by oral inoculation with $0.25 \mathrm{ml}$ brain heart infusion (BHI) medium containing $1.55 \times 10^{6} \mathrm{SE}$ colony-forming units (CFUs) in the control group and $1.78 \times 10^{6} \mathrm{SE}$-CFUs in the GM group. At 3, 7, 14 and $21 \mathrm{dpi}$, six chickens per group (three per subunit $A / B$ ) were randomly selected, weighed prior to post-mortem analyses to calculate body weight gain and sacrificed for collection of ileum, spleen and contents of ileum and caeca to determine bacterial CFUs, numbers and function of NK cells and T cells, and microbiota composition. In addition, at 0, 7, 14 and $21 \mathrm{dpi}$, blood (at least $5 \mathrm{ml}$ ) was collected in EDTA tubes (VACUETTE ${ }^{\circledR}$ K3E EDTA, Greiner Bio-One, the Netherlands) for determination of SE-specific antibody levels. To calculate absolute cell numbers, ileum segments and spleens were weighed immediately after collection of the tissues, prior to isolation of cells.

\section{SE culture}

Salmonella enterica serotype Enteritidis (strain K285/93 Nalres) was kindly provided by Dr. E. Broens, Veterinary Microbiological Diagnostic Center, the Faculty of Veterinary Medicine, Utrecht University, and cultured as described previously ${ }^{44}$. In short, from an overnight culture of the SE strain on blood agar (Oxoid, the Netherlands) two single colonies were used to inoculate two volumes of $45 \mathrm{ml} \mathrm{BHI}$ medium (Oxoid), and both cultures were incubated aerobically overnight and $37^{\circ} \mathrm{C}$ in a shaking (200 rpm) incubator (Certomat BS-1, B. Braun Biotech international, Sweden). Samples of the SE cultures were diluted 1:10 in PBS were and OD values measured using a Ultrospec 2000 (Pharmacia Biotech, Sweden). SE concentrations were calculated from a previously determined growth curve, and SE were diluted in $\mathrm{BHI}$ medium to $4 \times 10^{6} \mathrm{CFUs} / \mathrm{ml}$, to constitute the inocula. Serial dilutions of the two inocula were plated for overnight culture and number of CFUs were counted to determine the exact SE concentrations; $6.20 \times 10^{6} \mathrm{CFUs} / \mathrm{ml}$ (control) and $7.12 \times 10^{6} \mathrm{CFUs} / \mathrm{ml}(\mathrm{GM}$ ).

\section{Quantitation of SE in ileum and spleen}

At $-4,0,7,14$ and $21 \mathrm{dpi}$, the numbers of Salmonella colonies on the plates were counted and were the parameter for numbers of SE in ileum and spleen. From the cell suspensions of either the ileum segments or homogenized spleens, $100 \mu \mathrm{l}$ was plated with a spatula on RAPID' Salmonella Medium plates (Bio-Rad, the Netherlands). Plates were incubated overnight at $37^{\circ} \mathrm{C}$ and the purple colonies were counted. SE numbers were expressed as CFU per gram tissue to depict SE load as well as number of chickens positive for $\mathrm{SE}$, as has been described previously ${ }^{44}$. The limit of detection (LOD) was 100 CFU per gram tissue.

\section{Isolation of cells and serum}

Isolation of IELs from ileum and leukocytes from spleen was conducted according to the procedure described previously 12,45 . Briefly, ileum segments were washed with PBS to remove contents, cut in sections of $1 \mathrm{~cm}^{2}$ and washed again. Subsequently, the IELs were collected by incubating the sections three times for $15 \mathrm{~min}$ in a shaking incubator (Certomat BS-1) at $200 \mathrm{rpm}$ and $37^{\circ} \mathrm{C}$ in $20 \mathrm{ml}$ EDTA-medium: HBSS $1 \times\left(\right.$ Gibco $\left.^{\oplus}\right)$ supplemented with $10 \%$ heat-inactivated FCS (Lonza) and 1\% $0.5 \mathrm{M}$ EDTA (UltraPure ${ }^{\mathrm{TM}}$, Invitrogen, the Netherlands). Supernatants were collected and centrifuged for $5 \mathrm{~min}$ at $335 \times g$ and $20^{\circ} \mathrm{C}$ (Allegra ${ }^{\mathrm{TM}} \mathrm{X}-12 \mathrm{R}$ Centrifuge, Beckman Coulter, the Netherlands). Pellets were then 
resuspended in PBS at a concentration of $10 \mathrm{ml}$ per gram tissue and an aliquot of $100 \mu \mathrm{l}$ was used for quantitation of SE. PBS was added to the remaining suspension up to $20 \mathrm{ml}$ and IELs were isolated using Ficoll-Paque Plus (GE Healthcare, the Netherlands) density gradient centrifugation for 12 min at $673 \times g$ and $20^{\circ} \mathrm{C}$, washed in PBS by centrifugation for 5 min at $393 \times g$ and $4^{\circ} \mathrm{C}$ and resuspended at 4.0 $\times 10^{6}$ cells $/ \mathrm{ml}$ in complete medium (IMDM $2 \mathrm{mM}$ glutamax I supplemented with $8 \%$ heat-inactivated FCS (Lonza), 2\% heat-inactivated chicken serum, $100 \mathrm{U} / \mathrm{ml}$ penicillin and $100 \mu \mathrm{g} / \mathrm{ml}$ streptomycin; $\mathrm{Gibco}^{\circledast}$ ). Spleens were homogenized using a $70 \mu \mathrm{m}$ cell strainer (Beckton Dickinson (BD) Biosciences, $\mathrm{NJ}$, USA) and the single-cell suspension was diluted in PBS at a concentration of $10 \mathrm{ml}$ per gram tissue. An aliquot of $100 \mu \mathrm{l}$ was used again for SE quantitation. Next, leukocytes were isolated by Ficoll-Paque Plus density gradient centrifugation $\left(20 \mathrm{~min}, 1126 \times \mathrm{g}, 20^{\circ} \mathrm{C}\right)$, washed in PBS and resuspended at $4.0 \times$ $10^{6} \mathrm{cells} / \mathrm{ml}$ in complete medium as described for ileum.

After isolation, cell numbers in the resulting suspension were calculated. This resulted in the total cell number, expressed as IELs per mg ileum or leukocytes per mg spleen. To calculate the absolute numbers of NK and T cells within the live IEL or leukocyte populations, the percentages of cells positive for the markers expressed on these cell types were used which were determined in the flow cytometry analyses. Absolute cell numbers were calculated using the following formula: (absolute number IELs or leukocytes per mg tissue) $\times$ (percentage positive cells in the gate of interest of the live lymphocyte population).

Whole blood was allowed to coagulate for 1 hour at RT, centrifuged for $10 \mathrm{~min}$ at $2095 \times g$ and $15^{\circ} \mathrm{C}$, subsequently serum was collected and stored at $-20^{\circ} \mathrm{C}$ until further use.

\section{Phenotypic characterization of lymphocytes by flow cytometry}

At 0,3 and $7 \mathrm{dpi}$, numbers of NK and T cell subsets among IELs and splenocytes were measured, subsequently at 14 and 21 dpi only the numbers of $T$ cell subsets was determined. Lymphocytes (1 $\times 10^{6}$ ) were stained with a panel of antibodies specific for surface markers known to be expressed on NK cells, as well as with anti-CD3 to enable exclusion of T cells from analyses. Another aliquot of cells was stained with a panel of antibodies specific for surface markers that distinguish $\gamma \delta^{+}, C D 4^{+}$and $\mathrm{CD}^{+} \mathrm{T}$ cell subsets (Table 1). Staining with primary and secondary antibodies (Table 1 ) was performed in $50 \mu \mathrm{l} \mathrm{PBA} \mathrm{(PBS} \mathrm{(Lonza)} \mathrm{containing} 0.5 \%$ bovine serum albumin and $0.1 \%$ sodium azide). Cells were incubated for $20 \mathrm{~min}$ at $4^{\circ} \mathrm{C}$ in the dark with staining antibodies and washed twice by centrifugation for 5 min at $393 \times g$ and $4^{\circ} \mathrm{C}$ either in PBA after incubation with the primary antibodies, or in PBS after incubation with the secondary antibodies. Subsequently, to be able to exclude dead cells from analysis, lymphocytes were stained in $100 \mu \mathrm{l}$ PBS with a viability dye according to the manufacturer's instructions (Zombie Aqua ${ }^{\mathrm{TM}}$ Fixable Viability Kit, Biolegend, the Netherlands) for $15 \mathrm{~min}$ at RT in the dark, washed twice in PBA and resuspended in $200 \mu \mathrm{l} \mathrm{PBA}$. Of each sample, either $150 \mu \mathrm{l}$ or a maximum of $1 \times 10^{6}$ viable lymphocytes were measured using a CytoFLEX LX Flow Cytometer (Beckman Coulter), and data were analyzed with FlowJo software (FlowJo LCC, BD Biosciences). The gating strategies used to enable analyses of numbers and function of NK cells, $\gamma \delta T$ cells and cytotoxic CD8 ${ }^{+} T$ cells in the ileum are depicted in Fig. S1. The same gating strategy was used in the spleen and has been shown previously ${ }^{15}$. 
Table 1. Flow cytometry staining reagents.

\begin{tabular}{|c|c|c|c|}
\hline $\begin{array}{l}\text { Cell } \\
\text { population }\end{array}$ & $\begin{array}{l}\text { Primary antibody } \\
\text { (mouse-anti-chicken) }\end{array}$ & Clone / Isotype & Secondary antibody \\
\hline \multirow[t]{4}{*}{ NK cells } & CD45-FITC $^{1}$ & LT40 / lgM & - \\
\hline & CD3-APC ${ }^{1}$ & $\mathrm{CT} 3 / \lg \mathrm{G} 1$ & - \\
\hline & IL-2R $\alpha-U N L^{2}$ & $28-4 / \lg G 3$ & Goat-anti-mouse-IgG3-PE 1 \\
\hline & $20 \mathrm{E} 5-\mathrm{BIOT}^{2}$ & $\lg G 1$ & Streptavidin (SA)-PercP ${ }^{5}$ \\
\hline \multirow[t]{5}{*}{ T cells } & CD3-PE ${ }^{1}$ & $\mathrm{CT} 3 / \lg \mathrm{G} 1$ & - \\
\hline & $\mathrm{CD}_{4}-\mathrm{APC}^{1}$ & $\mathrm{CT} 4$ / IgG1 & - \\
\hline & 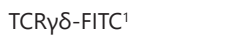 & TCR-1 / lgG1 & - \\
\hline & $\mathrm{CD} 8 \alpha-\mathrm{UNL}^{1}$ & $\mathrm{EP} 72 / \lg G 2 \mathrm{~b}$ & Goat-anti-mouse-lgG2b-APC/Cy $7^{1}$ \\
\hline & $\mathrm{CD} 8 \beta-\mathrm{BIOT}^{1}$ & EP42 / lgG2a & SA-PercP ${ }^{5}$ \\
\hline \multicolumn{4}{|l|}{ Assay } \\
\hline \multirow[t]{4}{*}{ CD107 } & CD107a-APC ${ }^{3}$ & LEP-100 I 5G10 / lgG1 & - \\
\hline & CD41/61-FITC 4 & $11 \mathrm{C} 3 / \lg \mathrm{G} 1$ & - \\
\hline & CD3-PE ${ }^{1}$ & $\mathrm{CT} 3 / \lg \mathrm{g} 1$ & - \\
\hline & $\mathrm{CD} 8 \alpha-U N L^{1}$ & $\mathrm{EP72} / \lg \mathrm{G} 2 \mathrm{~b}$ & Goat-anti-mouse-IgG2b-Alexa Fluor (AF) $790^{6}$ \\
\hline \multirow[t]{4}{*}{ IFNY } & CD3-PE ${ }^{1}$ & $\mathrm{CT} 3 / \lg \mathrm{G} 1$ & - \\
\hline & TCRy $\delta-$ FITC $^{1}$ & $\mathrm{TCR}-1 / \lg \mathrm{G} 1$ & - \\
\hline & $C D 8 \alpha-U N L^{1}$ & $\mathrm{EP72} / \lg \mathrm{G} 2 \mathrm{~b}$ & Goat-anti-mouse-IgG2b-AF790 6 \\
\hline & IFNY-APC ${ }^{3}$ & MAb80 / lgG1 & - \\
\hline
\end{tabular}

Manufacturers: ${ }^{1}$ Southern Biotech, AL, USA, ${ }^{2}$ Purified antibody from hybridoma supernatant kindly provided by Göbel, T.W., Ludwig Maximilian University, Germany, ${ }^{3}$ Developmental Studies Hybridoma Bank (DSHB), University of lowa, IA, USA, ${ }^{4}$ Serotec, United Kingdom, ${ }^{5} \mathrm{BD}$ Biosciences, ${ }^{6}$ Jackson ImmunoResearch Laboratories, PA, USA.

\section{CD107 assay}

At $0,3,7,14$ and $21 \mathrm{dpi}$, activation of NK cells and cytotoxic CD8 ${ }^{+} \mathrm{T}$ cells was determined in IELs and splenocytes. For this purpose the CD107 assay was used, which measures enhanced surface expression of CD107a that results from degranulation ${ }^{45,46}$. Briefly, lymphocytes isolated from the IEL population and spleen were suspended in complete medium, and $1 \times 10^{6}$ lymphocytes in $0.5 \mathrm{ml}$ were incubated in the presence of $1 \mu \mathrm{l} / \mathrm{ml}$ GolgiStop (BD Biosciences) and $0.5 \mu \mathrm{l} / \mathrm{ml}$ mouse-anti-chicken-CD107a-APC for 4 hours at $37^{\circ} \mathrm{C}, 5 \% \mathrm{CO}_{2}$. After incubation, lymphocytes were washed in PBA and stained as described above with monoclonal antibodies for NK and T cells, and in addition anti-CD41/61 to exclude thrombocytes from analyses, as mentioned in the CD107 panel (Table 1). Cells were washed in PBS, stained for viability and analyzed by flow cytometry.

\section{IFNץ assay}

At $0,3,7,14$ and $21 \mathrm{dpi}$, expression of intracellular IFN $\gamma$ was determined in NK cells, $\gamma \delta \mathrm{T}$ cells, CD8 ${ }^{+} \mathrm{T}$ cells and $\mathrm{CD} 4^{+} \mathrm{T}$ cells, using an assay adapted from Ariaans and colleagues ${ }^{47}$. Briefly, lymphocytes isolated from the IEL population and spleen were suspended in complete medium, and $1 \times 10^{6}$ lymphocytes in $0.5 \mathrm{ml}$ were incubated in the presence of $1 \mu \mathrm{l} / \mathrm{ml}$ Brefeldin A (Sigma Aldrich) for 4 hours at $41^{\circ} \mathrm{C}, 5 \%$ $\mathrm{CO}_{2}$. After incubation, lymphocytes were washed in PBA and stained as described above with surface markers summarized in the IFNy panel (Table 1). Cells were washed in PBS, stained for viability and washed again in PBA. Next, lymphocytes were permeabilized differently as described by Ariaans et al. (2008). Here, lymphocytes were incubated in $200 \mu \mathrm{l}$ of a mixture of BD FACS ${ }^{\mathrm{TM}}$ Permeabilizing Solution 
2 and BD FACS ${ }^{\mathrm{TM}}$ Lysing Solution prepared according to manufacturer's instructions (BD Biosciences) for $8 \mathrm{~min}$ at RT and immediately centrifuged for $2 \mathrm{~min}$ at $393 \times g$ and $4^{\circ} \mathrm{C}$. Cells were washed twice in PBA, stained intracellularly with anti-IFNY-APC in $50 \mu \mathrm{l}$ PBA for $20 \mathrm{~min}$ at $4^{\circ} \mathrm{C}$ in the dark, washed in PBA and finally analyzed by flow cytometry.

\section{SE-specific antibody titers in serum}

To detect titers of SE-specific antibodies in the sera collected at 0, 7, 14 and $21 \mathrm{dpi}$, the commercially available Salmonella Enteritidis Antibody Test (IDEXX SE Ab X2 Test) was used according to manufacturer's instructions (IDEXX Europe, the Netherlands). Positive and negative controls were included in the kit, and serum samples were analyzed in duplicate. Endpoint titers were calculated using the following formula:

$10 \wedge\left(1.5 * \log _{10}((\right.$ sample $\mu$ - negative control $\mu) /($ positive $\mu$ - negative control $\left.\mu))+3.47\right)$.

\section{Microbiota composition of ileum and caeca}

DNA was purified from ileal and caecal samples stored in DNA/RNA Shield using the ZymoBIOMICS DNA Kit according to manufacturer's instructions (Zymo Research). Bacterial 16S ribosomal RNA genes were then amplified by running one PCR cycle while incorporating a cy-5 fluorescent labeled nucleotide, as described previously for labeling samples in microarray analysis ${ }^{48}$. Labeled PCR amplicons were then hybridized to a microarray chip coated with probes for intestinal bacteria previously selected as biomarkers for broiler performance and intestinal health ${ }^{48}$. Microarray annotation for probes included sequential numbers added after bacteria genus or species in order to avoid more than one probe with the same name. The microarray contains two probes for Salmonella and the Salmonella probe 2 has been validated to specifically capture Salmonella enterica serotype Enteritidis (Cargill Inc., proprietary). The fluorescence signal of each probe was read using a fluorescence array image reader (Sensovation AG, Germany). Fluorescence intensity of each probe was used as a parameter to determine relative abundance of each of the microbial taxa in the feed groups according to the experimental design.

In addition, Pearson's correlations were calculated of immune cells and activation, SE-specific antibodies and SE-CFUs with intestinal microbial taxa that were significantly increased in each feed group before and during SE infection. Correlation ( $r$ ) values from 0 to 1 (positive) and 0 to -1 (negative) are depicted in a heatmap, where $0-0.2(0--0.2)$ is interpreted as no/negligible correlation, $0.2-0.5(-0.2--0.5)$ as weak correlation, $0.5-0.8(-0.5--0.8)$ as moderate correlation and $0.8-1(-0.8--1)$ as strong correlation.

\section{Statistical analyses}

First, the data were tested for normal distribution using the Shapiro-Wilk test. Differences between feed groups as well as within each group in the course of time in SE-CFUs per gram ileum and spleen, and SE-specific antibody titers in serum were analyzed using Kruskal-Wallis tests, followed by Dunn's multiple comparisons tests. Differences between control and GM groups as well as within each group in the course of time in body weight, numbers of IELs or leukocytes, NK cell and T cell subsets as well as percentages of cells expressing CD107 and IFNY in IELs and spleen were analyzed using one-way ANOVA. The correlation between SE-CFUs and SE-specific antibody titers was analyzed using the Spearman's rank correlation test. Regarding analysis of microbiota composition, raw fluorescence intensity data for each probe on each microarray chip was compiled and submitted to data quality control. The data treatment selected to reduce chip-to-chip variation was to standardize it to a shifting 
point of 3, according to the option available in JMP Genomics, as described previously for microarray analysis ${ }^{49}$. The standardized data were then analyzed using mixed-model ANOVA, where intestinal segment, diet, age and their three-way interaction were classified as fixed effects and chip was classified as random effect. Results were used to produce clustering plots utilizing hierarchical clustering, with distances between clusters defined by the Ward's method ${ }^{50}$. Differences in standardized LS means of relative abundance were also used for principal component analysis and volcano plots for pair-wise comparisons. Correlations between immune parameters and microbial taxa were analyzed using the Pearson product-moment correlation procedure. All statistical analyses on immunology data were performed using GraphPad Prism 9 software (GraphPad Software, CA, USA) and on microbiota data using JMP Genomics 9 software (SAS Institute 2017, NC, USA). A $p$ value of $<0.05$ was considered statistically significant and a value of $0.05<p<0.1$ is referred to as a trend, in case the $p$-value did not belong to one of these categories the difference observed is referred to as "numerical". Significant differences between groups are depicted by " * " and within a group in course of time by "\# ". Microarray standardized LS means of fluorescence intensities were compared using false discovery rate (FDR) adjusted $p$-values set at $<0.05$.

\section{Results}

\section{GM supplementation resulted in a lower SE dissemination to spleen at $7 \mathrm{dpi}$}

SE counts were not observed in the ileum, nor in the spleen of chickens fed either a standard or a GM supplemented diet before SE inoculation (Table 2). After inoculation, numbers of SE in the ileum did not differ between the GM and the control group. In the GM group, SE was detected in the ileum in one out of six chickens at $14 \mathrm{dpi}$, and also in one out of six chickens in the control group at $7 \mathrm{dpi}$ (Table 2). In the spleen at $7 \mathrm{dpi}$, SE was detected in three out of six chickens in the GM group compared to five out of six chickens in the control group (Table 2). Although no significant differences in SE-CFUs were observed, SE-CFUs were numerically lower at $7 \mathrm{dpi}$ in the spleens of chickens which received a GM supplemented diet compared to the control group (Fig. 1A). At 14 dpi, SE in the spleen was detected in five out of six chickens in the GM group compared to three out of six chickens in the control group (Table 2). At $21 \mathrm{dpi}$, SE in the spleen was detected in one out of six chickens in both groups (Table 2). In course of time, numbers of SE in the spleen decreased between $7 \mathrm{dpi}$ and $21 \mathrm{dpi}$ in both groups (Fig. 1A). GM supplementation during SE infection did not affect growth performance of the chickens, as body weights were similar in chickens of control and GM groups (Fig. 1B). After SE inoculation, numbers of IELs were numerically higher at $14 \mathrm{dpi}$ in the GM group as compared to the control group (Fig. 1C). In the spleen, numbers of leukocytes post-infection were not significantly different between the GM and the control group (Fig. 1D).

Table 2. Number of chickens positive for SE in ileum and spleen.

Chickens with positive Salmonella counts in the ileum and spleen, $n=6$ per group and time point.

\begin{tabular}{lllll}
\hline SE presence & lleum & Spleen & \\
Dpi & Control & GM & Control & GM \\
\hline-4 & $0 / 6$ & $0 / 6$ & $0 / 6$ & $0 / 6$ \\
0 & $0 / 6$ & $0 / 6$ & $0 / 6$ & $0 / 6$ \\
7 & $1 / 6$ & $0 / 6$ & $5 / 6$ & $3 / 6$ \\
14 & $0 / 6$ & $1 / 6$ & $3 / 6$ & $5 / 6$ \\
21 & $0 / 6$ & $0 / 6$ & $1 / 6$ & $1 / 6$ \\
\hline
\end{tabular}


A

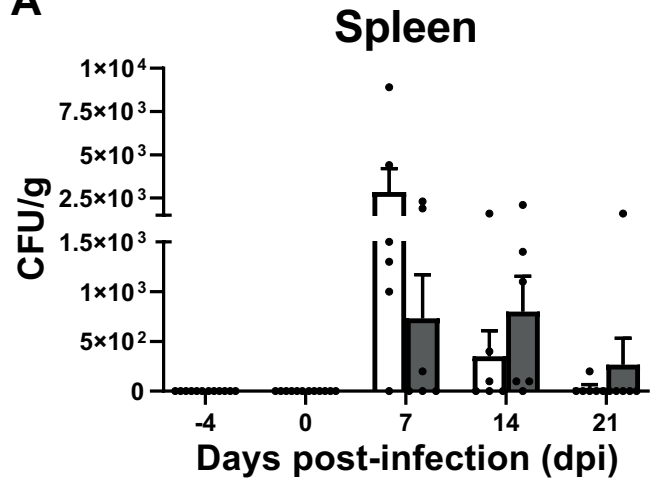

C

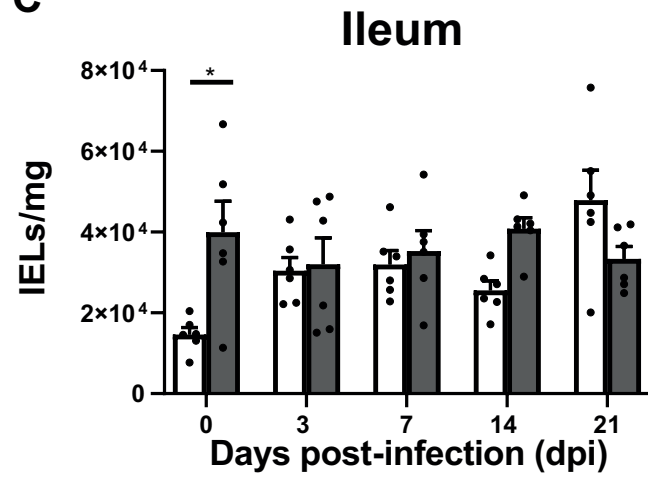

B

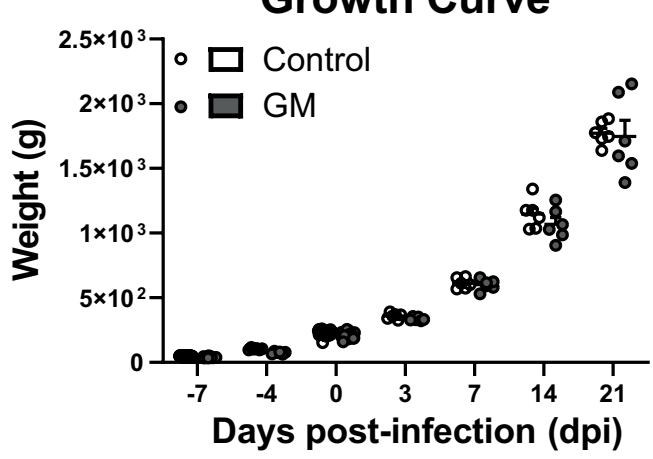

D

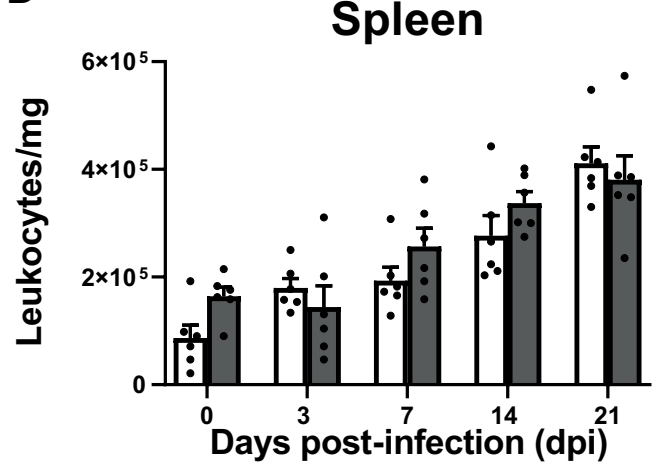

Figure 1. Effect of GM on SE load, chicken growth and numbers of IELs and splenic leukocytes before and during infection in broiler chickens. (A) Salmonella enterica serotype Enteritidis (SE, CFU/g) in the spleen of chickens either fed standard (control) or long-chain glucomannan supplemented (GM) diet before and during SE infection. (B) Body weights (g) of chickens either fed standard or GM diet in course of time before and during SE infection. (C) Numbers (cells/mg) of IELs in the ileum and (D) leukocytes in the spleen of chickens fed standard or GM diet in course of time before and during SE infection. Mean + SEM per diet group and time point are shown $(\mathrm{n}=6)$ and statistical significance between diet groups is indicated as * $(p<0.05)$.

\section{GM supplementation resulted in significantly higher numbers and an increase in activation of intraepithelial NK cells post SE infection}

The effect of GM supplementation on numbers of intraepithelial and splenic NK cell subsets was determined during SE infection. Post SE infection, intraepithelial IL-2R $\alpha^{+} N K$ cells were numerically higher and $20 \mathrm{E} 5^{+} \mathrm{NK}$ cell numbers significantly higher at $3 \mathrm{dpi}$ in the GM group compared to numbers in the control group (Fig. 2A, B). In course of time, numbers of intraepithelial IL-2R $\alpha^{+} \mathrm{NK}$ cells in the GM group remained similar before and during infection, whereas numbers in the control group significantly increased post-infection compared to $0 \mathrm{dpi}$ (Fig. 2A). Numbers of intraepithelial 20E5 ${ }^{+} \mathrm{NK}$ cells significantly increased at 3 dpi compared to 0 dpi in the GM group only (Fig. 2B). In the spleen, numbers of IL-2R $\alpha^{+}$and $20 \mathrm{E}^{+} \mathrm{NK}$ cells did not significantly differ between groups post-infection (Fig. 2C, D). In course of time, splenic IL-2R $\alpha^{+}$and $20 \mathrm{E} 5^{+} \mathrm{NK}$ cells numerically increased post-infection compared to $0 \mathrm{dpi}$ in the control group but not in the GM group (Fig. 2C, D).

To investigate possible changes in NK cell activation due to GM supplementation during SE infection, surface expression of CD107 and intracellular expression of IFN $\gamma$ were determined in NK cells isolated 
from the IEL population and spleen. Post SE infection, CD107 expression on intraepithelial NK cells tended to be higher at $7 \mathrm{dpi}$ in the GM group compared to the control group (Fig. 3A). No significant differences in IFNY expression of intraepithelial NK cells were observed between the GM and control group during SE infection (Fig. 3B). In the spleen, CD107 expression (Fig. 3C) and IFNY expression (Fig. 3D) of NK cells did not differ between the groups during SE infection. In course of time, CD107 expression on intraepithelial NK cells increased at $3 \mathrm{dpi}$ compared to $0 \mathrm{dpi}$ in both groups, although only significantly in the control group, and then decreased until $21 \mathrm{dpi}$ to the level of $0 \mathrm{dpi}$ in the control group (Fig. 3A). Furthermore, IFNy expression of intraepithelial and splenic NK cells and CD107 expression of splenic NK cells significantly increased at $3 \mathrm{dpi}$ compared to $0 \mathrm{dpi}$ in both groups and then decreased until $21 \mathrm{dpi}$ to levels before infection (Fig. 3B-D).

A

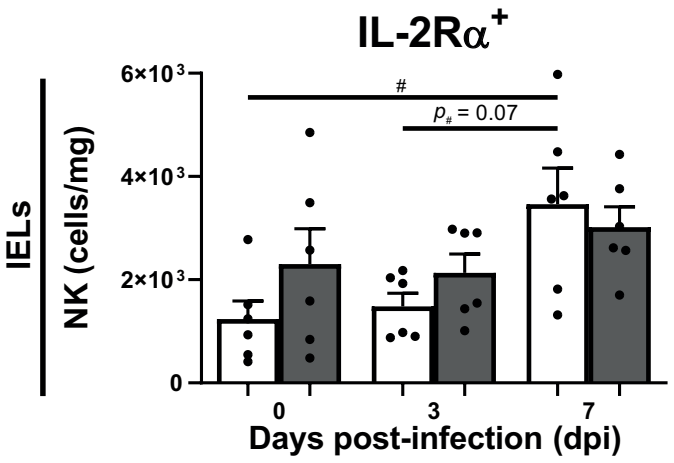

C

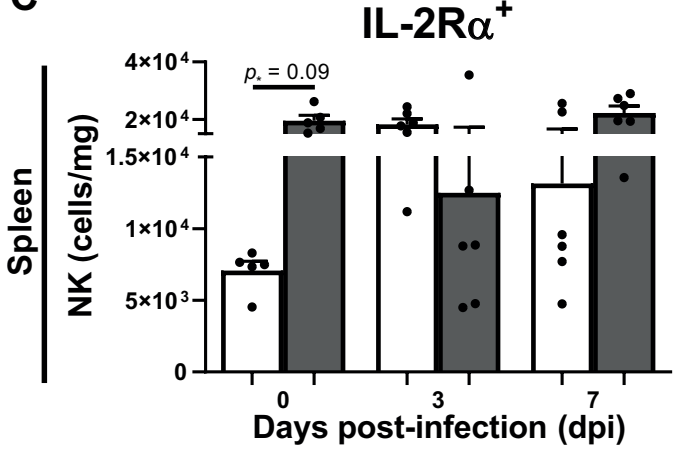

B

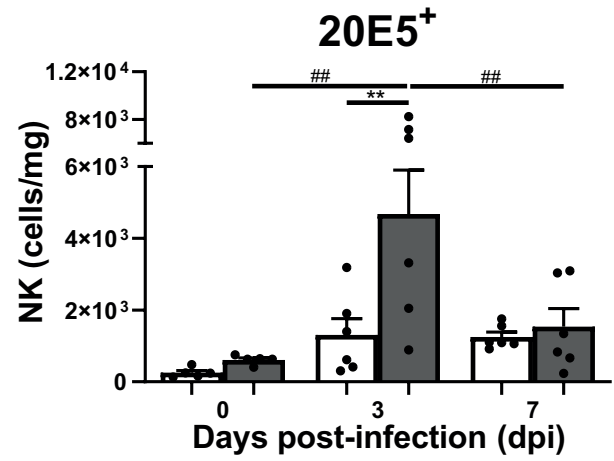

D

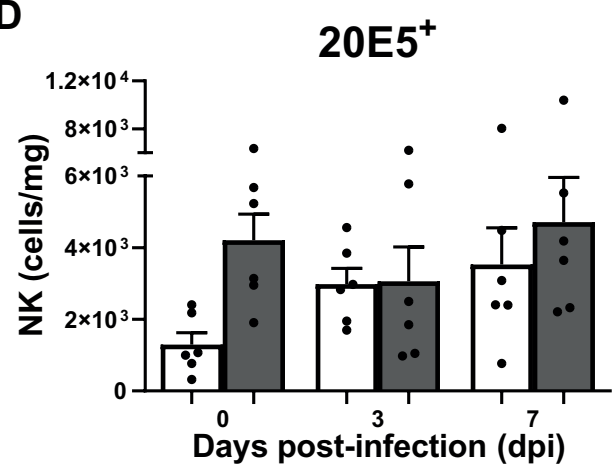

Figure 2. Effect of GM on numbers of intraepithelial and splenic NK cells before and during SE infection in broiler chickens. (A) Numbers (cells/mg) of intraepithelial IL-2R $\alpha^{+}$and (B) 20E5+ NK cells in chickens either fed standard (control) or long-chain glucomannan supplemented (GM) diet in course of time before and during SE infection. (C) Numbers (cells/mg) of splenic IL-2R $\alpha^{+}$and (D) 20E5+ NK cells in chickens either fed standard or GM diet before and during SE infection. Mean + SEM per diet group and time point are shown $(n=6)$, if $n=5$; one chicken was excluded due to numbers of events acquired in the gate of interest were $<100$. Statistical significance between diet groups is indicated as ** $(p<0.01)$ and in course of time within a group as \# $(p<0.05)$, \#\# (p<0.01).

\section{GM supplementation resulted in an increase of intraepithelial cytotoxic CD8 ${ }^{+} \mathrm{T}$ cell numbers post SE infection}

The effect of GM supplementation on numbers of intraepithelial and splenic $\gamma \delta$ T cells and cytotoxic $\left(C D 8^{+}\right) \alpha \beta$ T cells was investigated during SE infection. Post SE infection, intraepithelial $\gamma \delta \mathrm{T}$ cells were 
numerically higher and cytotoxic $C D 8^{+} \mathrm{T}$ cell numbers tended to be higher at $14 \mathrm{dpi}$ in the $\mathrm{GM}$ group compared to the control group (Fig. 4A, B). In course of time, intraepithelial $\gamma \delta T$ cells numerically increased post-infection compared to $0 \mathrm{dpi}$ in both groups. Intraepithelial cytotoxic $C D 8^{+} \mathrm{T}$ cell numbers remained similar post-infection compared to $0 \mathrm{dpi}$ in the GM group, whereas these cells numerically increased during this period in the control group (Fig. $4 A, B$ ). In the spleen, no differences in numbers of $\gamma \delta$ T cells (Fig. 4C) and cytotoxic CD8 ${ }^{+} T$ cells (Fig. 4D) were observed between the GM and control group during SE infection. In course of time, $\gamma \delta T$ cells and cytotoxic $C D 8^{+} T$ cells numerically increased post-infection compared to $0 \mathrm{dpi}$ in both groups (Fig. 4C, D).

A

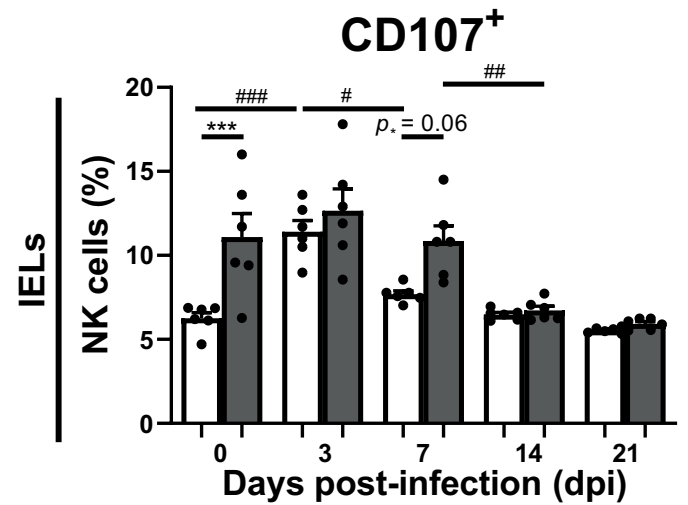

C

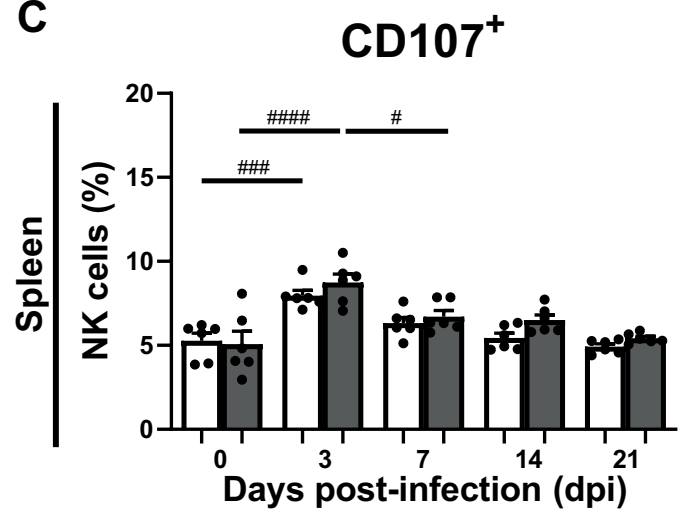

B

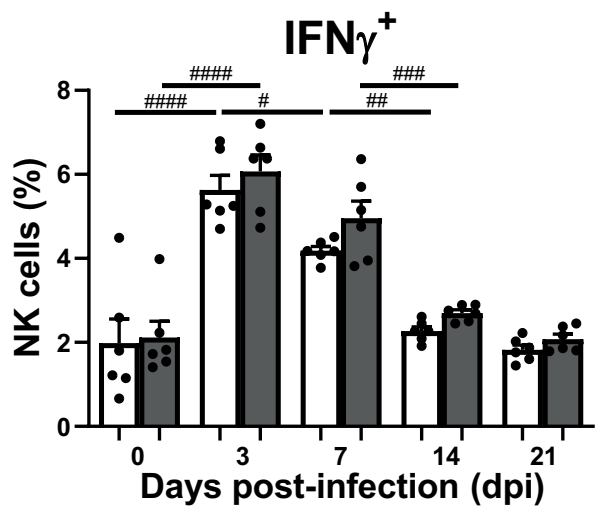

D

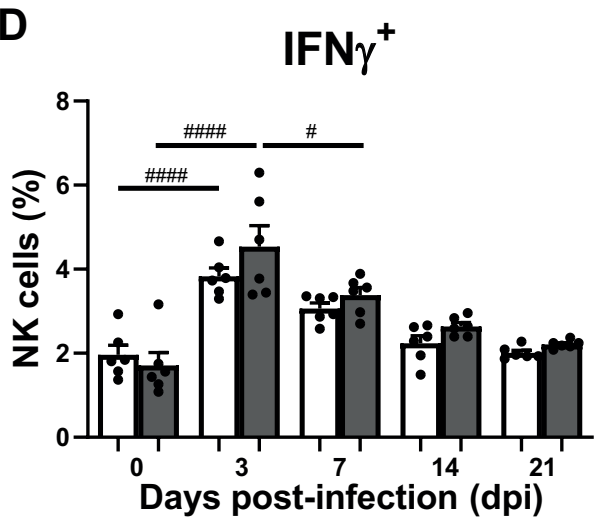

Figure 3. Effect of GM on NK cell activation in IELs and spleen before and during SE infection in broiler chickens. (A) Percentages of intraepithelial NK cells expressing CD107 and (B) IFNY in chickens either fed standard (control) or long-chain glucomannan supplemented (GM) diet in course of time before and during SE infection. (C) Percentages of splenic NK cells expressing CD107 and (D) IFNY in chickens either fed standard or GM diet before and during SE infection. Mean + SEM per diet group and time point are shown $(n=6)$ and statistical significance between diet groups is indicated as ${ }^{* *}(p<0.001)$ and in course of time within a group as \# $(p<0.05)$, \#\# ( $p<0.01)$, \#\#\# ( $p<0.001)$ and \#\#\#\# $(p<0.0001)$.

Next, $\gamma \delta$ T cells and cytotoxic $\alpha \beta$ T cells were analyzed for their CD8 $\alpha \alpha$ and CD8 $\alpha \beta$ expression. Both $\mathrm{CD} 8 \alpha \alpha^{+}$and $\mathrm{CD} 8 \alpha \beta^{+}$intraepithelial $\gamma \delta \mathrm{T}$ cells were numerically higher at $14 \mathrm{dpi}$ in the GM group compared to the control group (Fig. S2A, B). Intraepithelial cytotoxic CD8 $\alpha \alpha^{+}$T cells were numerically higher and $C D 8 \alpha \beta^{+} T$ cell numbers were significantly higher at $14 \mathrm{dpi}$ in the GM group compared to the 
control group (Fig. S2C, D). In course of time, CD8 $\alpha \alpha^{+}$and CD8 $\alpha \beta^{+}$intraepithelial $\gamma \delta$ T cells numerically increased post-infection compared to $0 \mathrm{dpi}$ in both groups. CD8 $\alpha \alpha^{+}$and $\mathrm{CD} 8 \alpha \beta^{+}$intraepithelial cytotoxic T cell numbers remained similar post-infection compared to 0 dpi in the GM group, whereas these cells numerically increased during this period in the control group (Fig. S2A-D). In the spleen, no differences in numbers of $C D 8 \alpha \alpha^{+}$and $C D 8 \alpha \beta^{+} \gamma \delta T$ cells nor cytotoxic CD8 $\alpha \alpha^{+}$and CD8 $\alpha \beta^{+} T$ cells were observed between the GM and control group during SE infection. In course of time, these splenic $T$ cells numerically increased post-infection compared to 0 dpi in both groups (Fig. S2E-H). Finally, possible changes in T cell activation due to GM supplementation during SE infection were determined. Post SE infection in the IELs and spleen, no differences in CD107 expression on CD8 ${ }^{+} \mathrm{T}$ cells (comprising both $\gamma \delta$ and $\alpha \beta$ TCRs) nor IFN $\gamma$ expression of CD8 $8^{+} \gamma \delta T$ cells, cytotoxic CD $8^{+} T$ cells and CD4 ${ }^{+} T$ cells (spleen only) between the GM and control group were observed (Fig. S3).

A

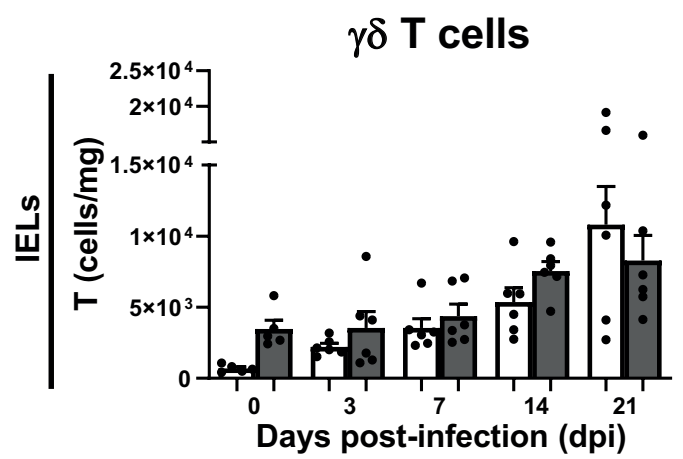

C

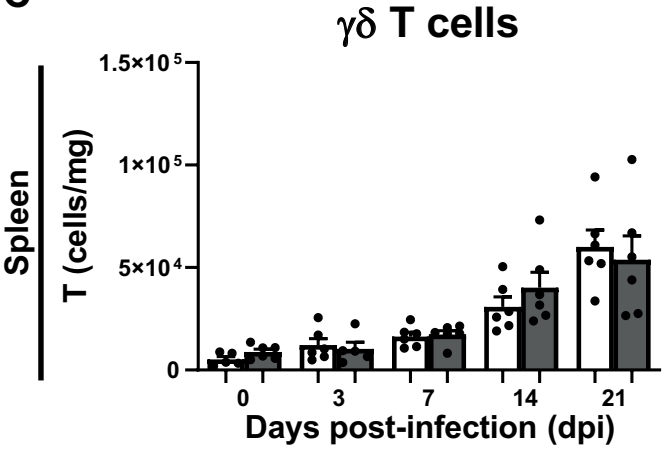

B

$\square$ Control $\square \mathrm{GM}$

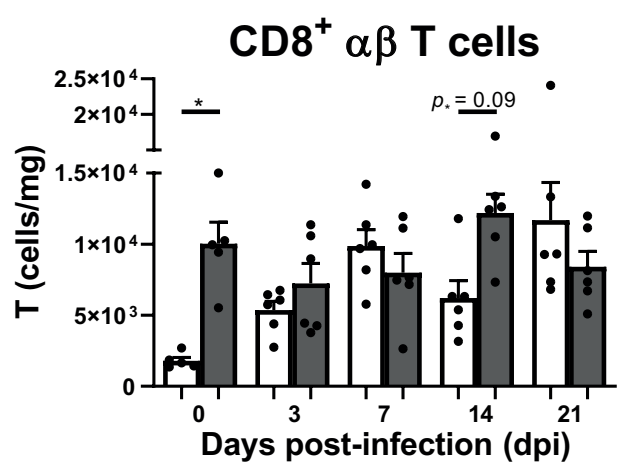

D

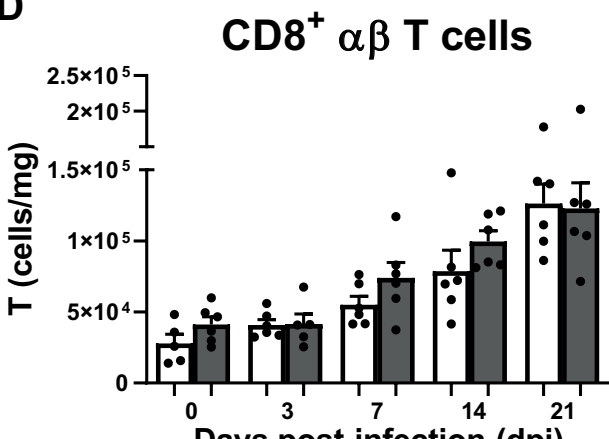

Figure 4. Effect of GM on numbers of intraepithelial and splenic T cells before and during SE infection in broiler chickens. (A) Numbers (cells/mg) of intraepithelial $\gamma \delta \mathrm{T}$ cells and (B) $\mathrm{CD}^{+} \alpha \beta \mathrm{T}$ cells in chickens either fed standard (control) or long-chain glucomannan supplemented (GM) diet in course of time before and during SE infection. (C) Numbers (cells/mg) of splenic $\gamma \delta T$ cells and (D) CD8+ $\alpha \beta$ T cells in chickens either fed standard or GM diet before and during SE infection. Mean + SEM per diet group and time point are shown ( $n=6)$, if $n=5$; one chicken was excluded due to numbers of events acquired in the gate of interest were < 100 . Statistical significance between diet groups is indicated as * $(p<0.05)$. 


\section{GM supplementation resulted in a higher SE-specific antibody response}

The effect of GM supplementation on titers of SE-specific antibodies in the serum was determined in course of time post SE infection. Although no significant differences were observed between the groups, all six chickens in the GM group showed SE-specific antibody titers at $14 \mathrm{dpi}$, which were numerically higher compared to antibody titers of the three out of six chickens in the control group (Fig. 5). At 21 dpi, all chickens showed SE-specific antibody responses and titers were numerically higher in the GM group compared to the control group (Fig. 5). In addition, a significant negative correlation between the antibody titers and SE-CFUs in the spleen was observed although it was weak $(r=-0.41$, Fig. S4).

\section{GM supplementation resulted in significantly higher relative abundance of lactic acid bacteria in microbiota of the ileum and caeca post SE infection}

Changes in the microbiota composition of the ileum and caeca due to GM supplementation during SE infection were determined using a microarray. Microbial analysis of standardized LS means as a parameter for relative abundance among the variables intestinal segment (caeca/ileum), age (dpi), diet (Control/ GM) and their three-way interaction, revealed a total of 86 bacterial taxa identified by the probes that were significantly different. By two-way hierarchical clustering between variables (clustered vertically) and significantly different bacterial taxa (clustered horizontally), microbiota profiles were divided first in two clusters (Fig. 6). In the left cluster, 7 out of the 11 profiles were samples from the ileum while in the right cluster 6 out of the 9 profiles were samples from the caeca. This indicates that with few exceptions, the variable intestinal segment was important to define clustering of microbiota profiles by similarity. Within these two clusters, further clustering seems to group microbiota profiles based on age and then diet.

Factorial interaction analysis of the relative abundance of microbial taxa showed differences in microbiota compositions post SE infection of which more Faecalibacterium 2 was detected in both ileum and caeca in the GM group, whereas more Lachnospiraceae 10 was observed in the caeca of chickens in the control group at 3 dpi (Table 3, Fig. S5). At 7 dpi, no differences were found in microbiota composition of the ileum between the two diet groups, however, in the caeca, higher levels of Salmonella probe 1 were detected in the GM group (Table 3, Fig. S5). The significant differences in microbial taxa between the control and GM group were more evident in chickens at 14 and $21 \mathrm{dpi}$, where GM supplementation induced higher relative abundance of LAB including Lactobacillus, Bifidobacterium, Streptococcus and Enterococcus species. At 14 dpi, more Bifidobacterium gallinarium and Lachnospiraceae 12 and 13 were observed in the GM group compared to more Lactobacillus jenseni and Lachnospiraceae 7 in the ileum of chickens in the control group (Table 3, Fig. 7). No differences in microbiota composition between groups were found in the caeca at $14 \mathrm{dpi}$ (Table 3). At $21 \mathrm{dpi}$, several streptococci, Lactobacillus gasseri 1 and Lactobacillus 4 showed a higher relative abundance in the GM group, whereas more Lactobacillus crispatus 3, Lachnospiraceae 1, 3 and 9, and Salmonella probe 2 were detected in the ileal microbiota in the control group (Table 3, Fig. 7). In the caecal microbiota of the GM group, more streptococci, Enterococcus hirae, Faecalibacterium 2 and Fusobacterium group 2 were found, compared to more Faecalibacterium 1, Lactobacillus crispatus 2, Lactobacillus reuteri 2 and Lachnospiraceae 5 in the control group (Table 3, Fig. 7). In course of time towards $21 \mathrm{dpi}$, the relative abundance of SE (Salmonella probe 2) significantly increased in the ileum (Fig. 8A) and caeca (Fig. 8B) of chickens fed the standard diet compared to GM supplemented diet. 


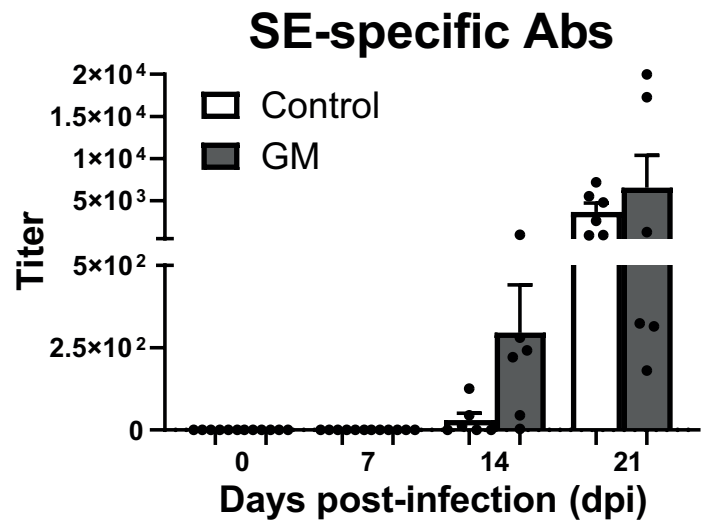

Figure 5. Effect of GM on serum antibody titers as a response to SE infection in broiler chickens. Titers of SE-specific antibodies in sera of chickens either fed standard (control) or long-chain glucomannan supplemented (GM) diet in course of time before and during SE infection. Mean + SEM per diet group and time point are shown $(n=6)$.

\begin{tabular}{|c|c|c|}
\hline $\begin{array}{l}\text { Dpi / } \\
\text { Intestinal segment }\end{array}$ & Control & GM \\
\hline 0 dpi / ileum & - & - \\
\hline $0 \mathrm{dpi} /$ caeca & - & Streptococcus alactolyticus \\
\hline $3 \mathrm{dpi} /$ ileum & - & Faecalibacterium 2 \\
\hline 3 dpi / caeca & Lachnospiraceae Incertae Sedis 10 & Agreia, Faecalibacterium 2 \\
\hline $7 \mathrm{dpi} /$ ileum & - & - \\
\hline $7 \mathrm{dpi} /$ caeca & - & Salmonella 1 \\
\hline 14 dpi / ileum & $\begin{array}{l}\text { Lactobacillus jenseni, Lachnospiraceae Incertae } \\
\text { Sedis } 7\end{array}$ & $\begin{array}{l}\text { Bifidobacterium gallinarium, } \\
\text { Sanguibacter, Lachnospiraceae } \\
\text { Incertae Sedis } 12 \text { and } 13, \\
\text { Salinococcus, Campylobacter }\end{array}$ \\
\hline $14 \mathrm{dpi} /$ caeca & - & - \\
\hline $21 \mathrm{dpi} /$ ileum & $\begin{array}{l}\text { Lactobacillus crispatus 3, } \\
\text { Lachnospiraceae Incertae Sedis 1, } 3 \text { and 9, } \\
\text { Rikenellaceae Alistipes } 1 \text { and 2, Salmonella } 2\end{array}$ & $\begin{array}{l}\text { Streptococcus group } 1 \text { and } 2 \text {, } \\
\text { Lactobacillus gasseri } 1 \\
\text { Lactobacillus 4, Lactobacillus sp. }\end{array}$ \\
\hline $21 \mathrm{dpi} /$ caeca & $\begin{array}{l}\text { Rikenellaceae Alistipes } 1 \text { and 2, } \\
\text { Faecalibacterium 1, Lactobacillus } \\
\text { crispatus 2, Lactobacillus reuteri 2, Lachnospiraceae } \\
\text { Incertae Sedis 5, Yersina enterocolitica, Bacillus } \\
\text { pumilus, Dorea formicigenerans, Salmonella } 2\end{array}$ & $\begin{array}{l}\text { Streptococcus group } 1 \text { and 2, } \\
\text { Enterococcus hirae, Faecalibacterium 2, } \\
\text { Fusobacterium group 2, } \\
\text { Ruminococcaceae unclassified, Clostridiales } \\
\text { unclassified, Bacteria unclassified }\end{array}$ \\
\hline
\end{tabular}

\section{Relative abundance of lactic acid bacteria correlate positively with NK cell activation and SE-specific antibodies, and negatively with SE-CFUs}

Since GM supplementation affected immune parameters and microbiota composition during SE infection, we analyzed whether these effects were related by performing a Pearson's correlation analysis. Positive and negative correlations were reported between significantly higher microbial taxa 

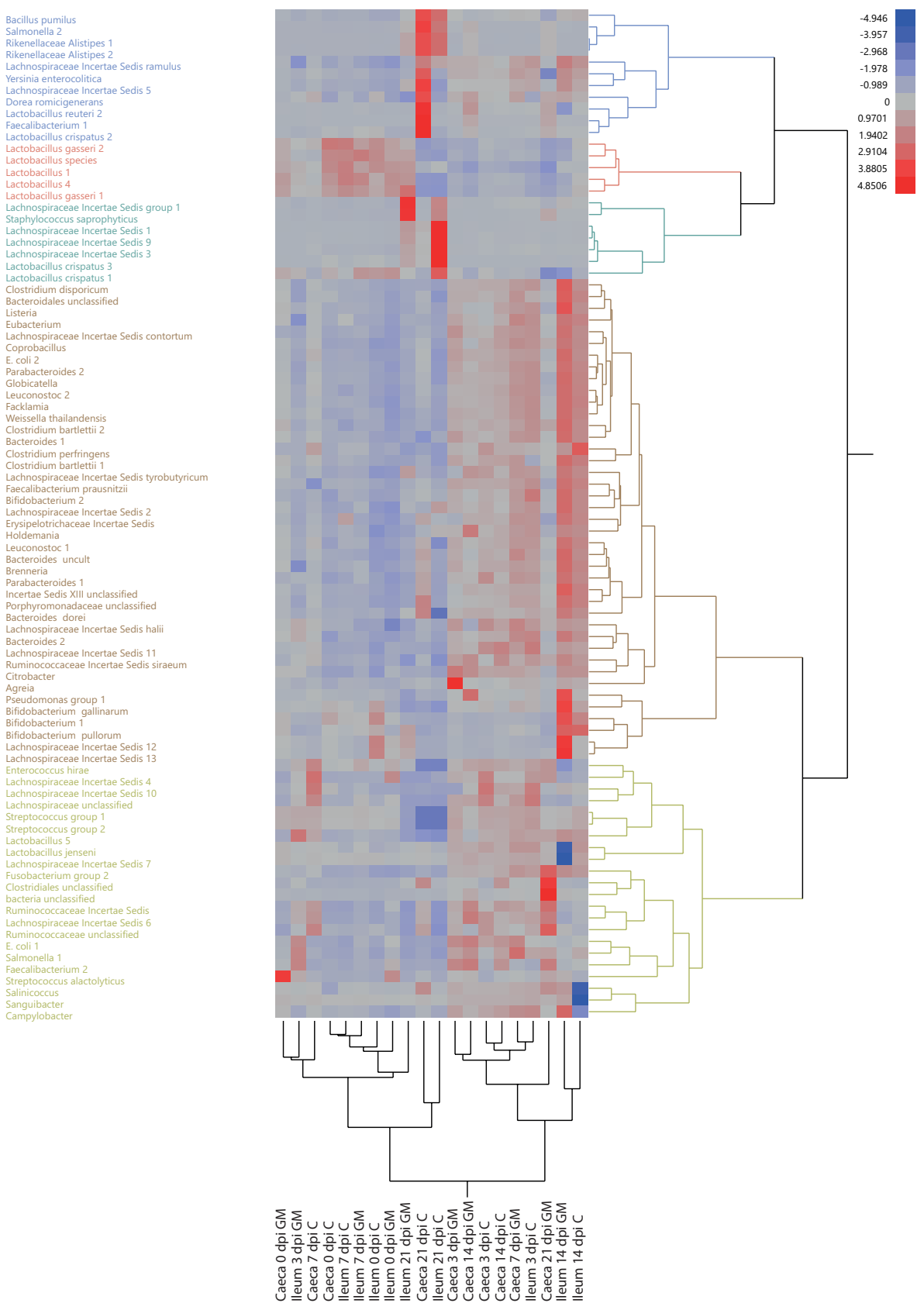

Figure 6. Effect of GM on microbiota composition in ileum and caeca before and during SE infection in broiler chickens. Hierarchical cluster analysis of relative abundance of microbial taxa targeted by the microarray in the ileum and caeca of broiler chickens either fed standard (C) or long-chain glucomannan supplemented (GM) diet at 0, 3, 7, 14 and 21 days post SE infection. The standardized relative fluorescence intensities of the microarray are depicted in a heatmap as low (blue) or high (red) relative abundance of microbial taxa. Microbiota clustered first by intestinal segment in ileum (left) or caeca (right) and subsequently by age in five clusters, but this clustering was affected by diet. Microbial taxa are colored by cluster (blue, red, green, brown, yellow). Per intestinal segment, age and diet $\mathrm{n}=6$ and with statistical significance of FDR adjusted $p$-values set at $<0.05$. 


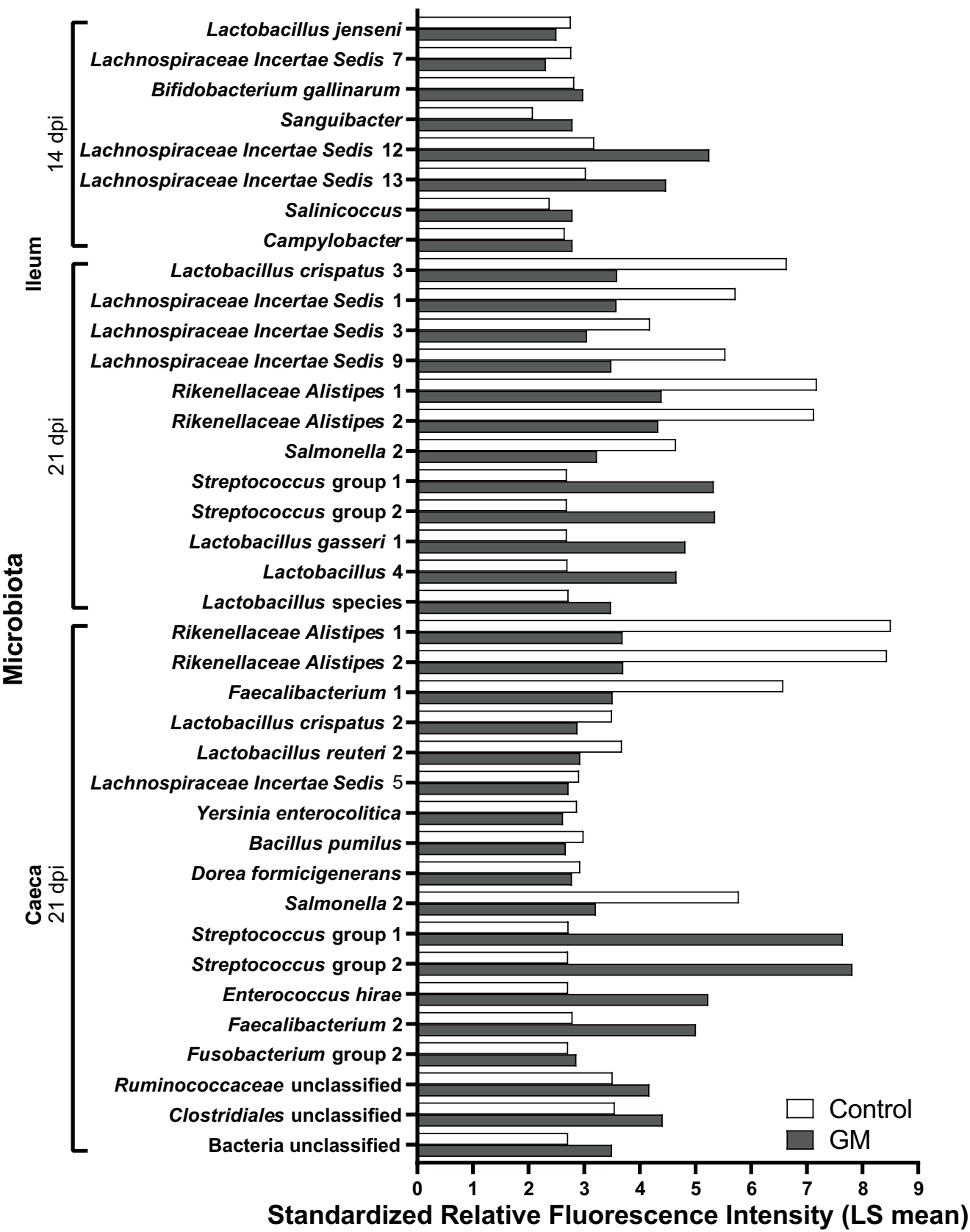

Figure 7. Intestinal microbial taxa significantly increased with diet at 14 and $21 \mathrm{dpi}$ of SE in broiler chickens. Standardized relative fluorescence intensities of the microbial taxa as measured by the microarray in the ileum and caeca (Table 3) that were significantly higher either with standard (control) or long-chain glucomannan supplemented (GM) diet at 14 and 21 dpi of SE in broiler chickens. LS mean per microbial taxa and diet group are shown $(n=6)$ with statistical significance of FDR adjusted $p$-values set at $<0.05$. 

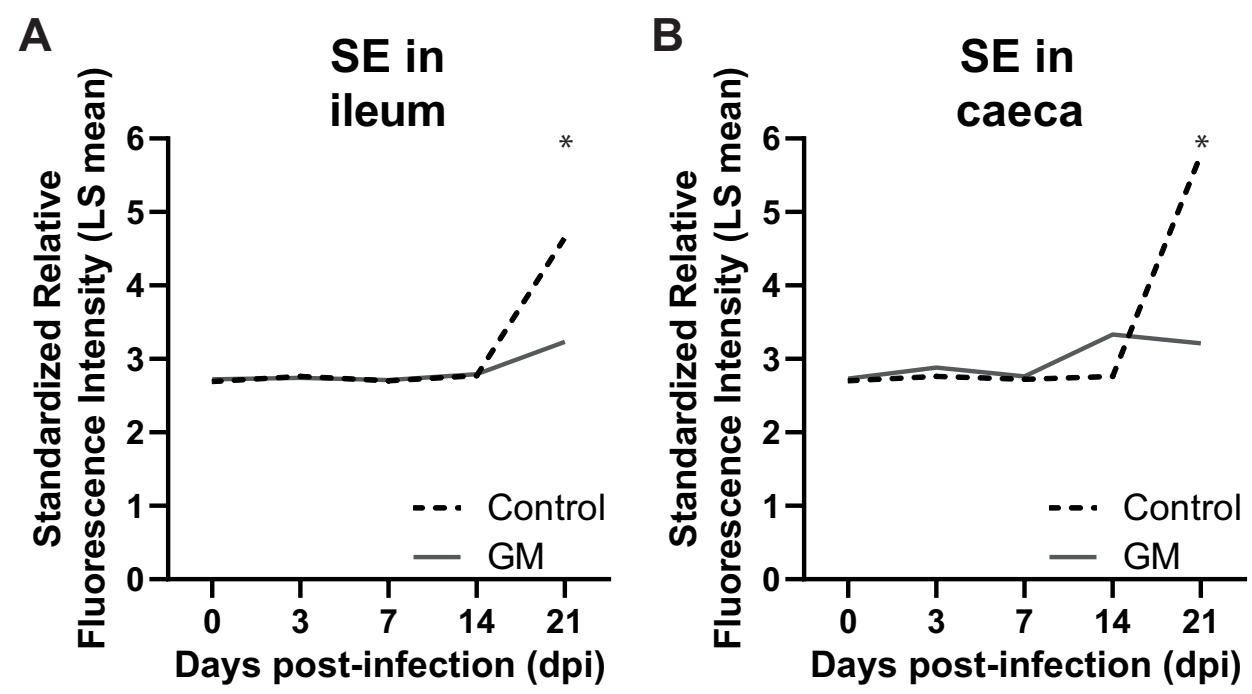

Figure 8. Effect of GM on relative abundance of SE in the microbiota of ileum and caeca before and during SE infection in broiler chickens. (A) Standardized relative fluorescence intensities of SE (Salmonella probe 2) as measured by the microarray in microbiota of the ileum and (B) caeca of chickens either fed standard (control) or long-chain glucomannan supplemented (GM) diet in course of time before and during SE infection. LS mean per diet group and time point are shown $(n=6)$ and statistical significance between diet groups is indicated as * (FDR adjusted $p$-values set at $<0.05$ ).

mentioned in Table 3, and SE-CFUs, SE-specific antibody titers, NK cell CD107 expression, NK cell IFNY expression, NK and T cell subsets in the IEL population and spleen for the respective intestinal segment, diet and age (Fig. 9 and Fig. S4). Based on our observations that GM supplementation significantly increased relative abundance of $L A B$, the correlations between $L A B$ and immune parameters are highlighted. At 3 dpi, no strong correlations were observed in ileal microbiota in both groups (Fig. S6A). In the caecal microbiota early post SE infection $(0-7 \mathrm{dpi})$, strong positive and negative correlations were observed of bacteria other than LAB (other bacteria) with intraepithelial NK cell subsets and splenic $\gamma \delta$ T cells in both groups (Fig. 6B). Most differences in the microbiota of ileum and caeca and strongest correlations were observed at 14 and $21 \mathrm{dpi}$ (Fig. 9). At $14 \mathrm{dpi}$ in the ileal microbiota, strong correlations between immune parameters and microbial taxa in the GM group were found in contrast to no strong correlations in the control group (Fig. 9A). In the GM group, strong positive correlations were observed between intraepithelial cytotoxic $\alpha \beta$ T cells and Bifidobacterium gallinarum, and of several other bacteria with SE-CFUs, intraepithelial IFN $\gamma^{+} \mathrm{NK}$ cells and splenic CD107 ${ }^{+} \mathrm{NK}$ cells and $\gamma \delta$ T cells (Fig. 9A).

At 21 dpi in the ileal microbiota in the GM group, a pattern in the correlations was observed with bacteria that had a lower (Lactobacillus crispatus 3 and other bacteria) or higher (bottom five LAB taxa) relative abundance compared to the control group (Fig. 9A). Lactobacillus crispatus 3 and other bacteria showed strong positive correlations with intraepithelial $\gamma \delta T$ cells and moderate negative correlations with splenic CD $107^{+} \mathrm{NK}$ cells. In contrast, LAB including streptococci in the ileal microbiota in the GM group at 21 dpi showed strong negative correlations with intraepithelial $\gamma \delta T$ cells and moderate positive correlations with SE-specific antibody titers and splenic CD107+ NK cells (Fig. 9A). In ileal microbiota in the control group at $21 \mathrm{dpi}$, Lactobacillus crispatus 3 and other bacteria showed strong negative and positive correlations with SE-specific antibody titers, intraepithelial and splenic

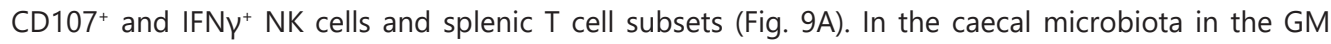


group at $21 \mathrm{dpi}$, a pattern in the correlations was observed again with bacteria that had a lower (two Lactobacillus species and other bacteria) or higher (bottom eight taxa) relative abundance compared to the control group (Fig. 9B). The two Lactobacillus species and several other bacteria showed strong positive correlations with SE-CFUs and splenic $\gamma \delta$ T cells and cytotoxic $\alpha \beta T$ cells. In contrast, LAB including streptococci in the caecal microbiota at $21 \mathrm{dpi}$ showed strong negative correlations with SE-CFUs and splenic $\gamma \delta$ T cells and cytotoxic $\alpha \beta$ T cells (Fig. 9B). In the caecal microbiota in the control group at $21 \mathrm{dpi}$, strong positive and negative correlations were observed of Lactobacillus crispatus 2 and other bacteria with SE-CFUs and intraepithelial and splenic T cell subsets (Fig. 9B).

\section{Discussion}

In the current study, we investigated the protective potential of long-chain glucomannan supplementation against experimental SE exposure in seven-day-old broiler chickens. The chickens were successfully infected with SE as was demonstrated by the presence of bacteria in ileum, caeca and spleen and the detection of SE-specific antibodies. At $7 \mathrm{dpi}$, chickens showed reduced numbers of SE in the spleen of chickens fed with the GM supplemented diet as compared to the standard diet. In addition, numbers of SE in the spleen decreased between 7 and $21 \mathrm{dpi}$ in both groups. Furthermore, a significantly lower relative abundance of SE was observed at $21 \mathrm{dpi}$ in the ileal and caecal microbiota of chickens that received the GM supplemented diet compared to the standard diet. These results are in agreement with a study showing lower presence of Salmonella enterica serotype Typhimurium (ST) in the intestine and liver of chickens due to galactoglucomannan supplementation ${ }^{38}$. The lower numbers of SE in the spleen and reduced SE colonization of the intestine indicates that GM supplementation may increase the resistance of chickens to SE infection. Several mechanisms could explain the reduction of SE due to GM supplementation.

First, GM supplementation may reduce SE due to stimulation of the immune system. GM supplemented chickens indeed showed increased numbers and activation of intraepithelial NK cells at respectively 3 and $7 \mathrm{dpi}$, as compared to chickens receiving the standard diet. A distinct population of IL-2R $\alpha^{+} 20 \mathrm{E} 5^{+}$ cells was not observed, although the possibility that these cells exist at a very low frequency cannot be excluded. The stimulatory impact of GM affecting numbers and function of intraepithelial NK and T cells before infection is similar to observations in uninfected chickens of the same age supplemented with $\mathrm{GM}^{29}$. GM supplementation enhanced expression of CD107 on intraepithelial NK cells but not IFNy expression before infection, and a similar trend was observed for intraepithelial CD8 ${ }^{+} \mathrm{T}$ cells. Early post-infection IFN $\gamma$ expression of NK cells was enhanced equally in both diet groups, which was similar to our previous study with SE-infected chickens fed a standard diet ${ }^{15}$. This suggests that GM results in increased responsiveness before and during the start of SE infection by stimulation of degranulation rather than IFNY production as effector pathways of these cells. Furthermore, GM supplementation was associated with stronger subsequent adaptive immune responsiveness that reduced $\mathrm{SE}$, since the numbers of intraepithelial cytotoxic $C D 8^{+} \mathrm{T}$ cells and $\mathrm{SE}$-specific serum antibody responses were higher at $14 \mathrm{dpi}$ compared to the standard diet. NK cells are likely to have contributed to these subsequent responses by secretion of cytokines ${ }^{51}$. Higher numbers of intraepithelial CD8 $\alpha \alpha^{+}$and CD8 $\alpha \beta^{+}$cytotoxic $T$ cells were observed in GM supplemented chickens compared to the standard diet post-infection, although only significant in the $C D 8 \alpha \beta^{+}$subset, suggesting a role in the defense against SE. Interestingly, the stimulatory effects of GM on the numbers of intraepithelial T cells is stronger under SE challenge as compared to non-challenged GM-supplemented chickens ${ }^{29}$. Our data are in line with studies that describe the effect of other polysaccharides such as yeast glucans and prebiotics, which were shown to reduce SE colonization and invasion by upregulated expression of intestinal innate-immunity-related 
A Correlation

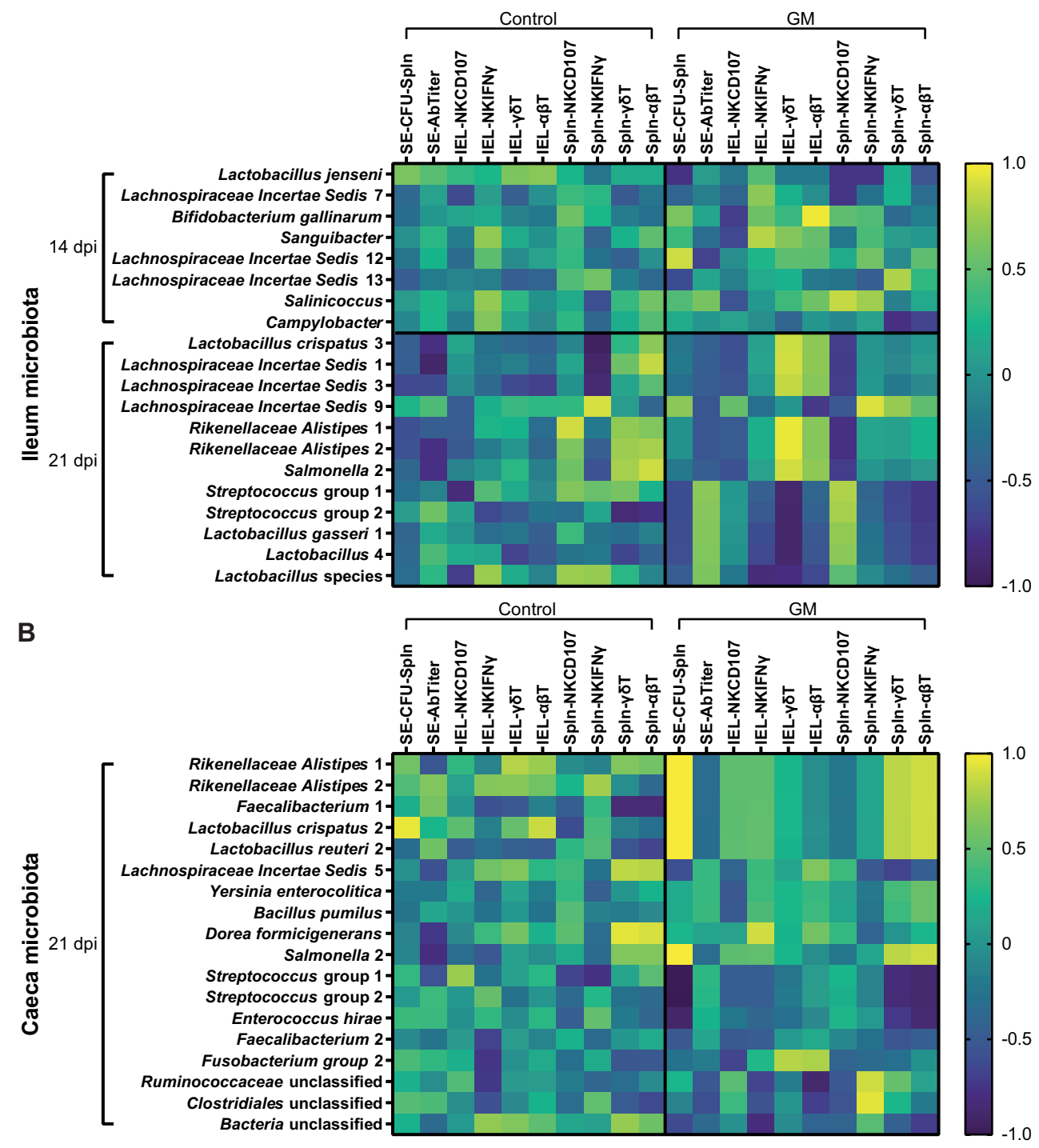

Figure 9. Correlation between microbial taxa and intraepithelial and splenic immune parameters at 14 and 21 dpi of SE in broiler chickens. (A) Correlation values between intestinal microbial taxa in the ileum or (B) caeca significantly increased with diet and splenic SE-CFUs, serum antibody titers, percentages of NK cell activation (CD107 or IFNY expression) or numbers of NK and T cell subsets of the ileum (IEL) and spleen (Spln) per diet (control, GM) at 14 and $21 \mathrm{dpi}$ of SE in broiler chickens. Pearson's correlation ( $r$ ) values are depicted in a heatmap as positive (yellow) or negative (dark blue) correlations.

genes ${ }^{39,41,52}$, enhanced killing of SE by macrophages ${ }^{53}$, and increased SE-specific intestinal IgA and serum IgG antibody responses ${ }^{41}$.

A second mechanism of GM supplementation potentially contributing to reduction of SE load is modulation of the intestinal microbiota composition, resulting in microbiota profiles different from 
the ones in chickens fed the standard diet during a SE infection. Differences in relative abundance of microbial taxa between the chickens in the two diet groups increased with time post-infection. GM supplemented chickens showed increased relative abundance of lactic acid bacteria (LAB) including Streptococcus species, Lactobacillus species and Bifidobacterium gallinarum in the ileum and caeca compared to the ones fed the standard diet. Before SE infection, the relative abundance of only streptococci was increased in the caeca of chickens receiving GM supplementation. This finding was different from previous observations where $G M$ increased relative abundance of multiple $L A B$ in the ileum and caeca of uninfected chickens at seven days of age ${ }^{29}$ and may result from variations in microbial composition among chickens ${ }^{54}$. The increased relative abundance of LAB during SE infection with GM supplementation is in agreement with other studies providing prebiotics during SE or ST infection ${ }^{39,40,52}$. The high relative abundance of streptococci was associated with the lower relative abundance of SE in both ileal and caecal microbiota at $21 \mathrm{dpi}$ in chickens receiving GM supplementation compared to the standard diet, suggesting competitive exclusion. This observation is in line with another study showing that commensal LAB strains including Enterococcus and Streptococcus contribute to a low-shedder phenotype of SE-infected chickens ${ }^{55}$. Furthermore, these LAB strains proved to have immunomodulatory properties on innate cells in humans ${ }^{56}$. For instance by production of metabolites like short-chain fatty acids (SCFAs), since they can function as energy substrate for the host and microbes ${ }^{57,58}$, but are also known to affect intestinal NK and T cell function in support of maintenance of intestinal homeostasis ${ }^{57,59-61}$. Production of SCFAs by LAB and other microorganisms able to use lactic acid as a substrate for SCFA production was shown previously to be increased during ST infection in chickens ${ }^{57}$. In addition, the production of organic acids such as lactic acid by Lactobacillus species may contribute to the reduction of SE, since these were shown to inhibit growth of SE and ST in vitro through acidification of the environment ${ }^{62}$. These findings indicate that GM supplementation results in a lower presence of SE most likely by competitive exclusion, microbial metabolites or indirect stimulation of innate immune cells via the microbiota.

Finally, we addressed the potential interference of the effects of GM supplemented diet between immune parameters and microbiota during SE infection. A negative correlation between relative abundance of commensal streptococci and numbers of SE in the spleen at $21 \mathrm{dpi}$ was revealed. Furthermore, positive correlations were found between relative abundance of Bifidobacterium gallinarum and numbers of intraepithelial cytotoxic T cells at $14 \mathrm{dpi}$, and between relative abundance of streptococci and splenic NK cell activation as well as SE-specific antibody responses at $21 \mathrm{dpi}$. In addition, GM-related positive correlations were observed between relative abundance of streptococci and intraepithelial NK cell activation as well as numbers of splenic IL-2R $\alpha^{+}$NK cells before SE infection, in agreement with previous findings of correlations between $L A B$ and immune cells ${ }^{29}$. These correlates indicate involvement of $L A B$ in the recruitment and functioning of immune cells that may contribute to the reduction of SE in GM supplemented chickens. GM is suggested to exert its effects indirectly on NK cells, mainly before, and on T cells, mainly post-infection, by local interactions with LAB or their metabolites in the intestine. Moreover, GM may have effects on those immune cells and antibody responses, systemically, due to translocation of microbial products into the circulation or a yet hypothetical interaction with antigen presenting cells that have interacted with intestinal $L A B^{63,64}$.

Another mechanism that can explain the observed reduction of SE in the intestine with GM supplementation involves the direct binding to SE of the mannose within the long-chain glucomannan, which thereby reduces attachment, hence, colonization of SE in the intestine of broiler chickens ${ }^{26}$.

In conclusion, supplementation of long-chain glucomannan stimulated recruitment and function of NK and T cells, as well as relative abundance of LAB in the intestinal microbiota, coinciding with the 
reduction of SE during infection in broiler chickens. Consequently, the resistance to SE colonization and systemic infection in young broiler chickens is improved, which may result in substantial health benefits for both chickens and humans. Although chickens fed the GM supplemented diet still became infected with SE, relative abundance of SE was lower in the intestine compared to chickens fed a standard diet. As a consequence, the lower SE colonization may also lead to reduced spread of SE within a flock and hence reduced food safety risks for humans. Future studies should investigate effects of providing the GM diet on spreading of SE from infected to uninfected chickens and flock prevalence of SE at time of slaughter to test this hypothesis. Furthermore, for practical application of GM future studies should perform larger scale trials including detailed monitoring of performance. The present study provides evidence for the potential to use long-chain glucomannan in practice to stimulate both the immune system and intestinal LAB in order to increase resistance of young chickens.

\section{Acknowledgements}

We thank the animal caretakers of the Department Population Health Sciences, division Farm Animal Health, Faculty of Veterinary Medicine, Utrecht University, for their help during the animal experiments. We acknowledge E. Broens, the Veterinary Microbiological Diagnostic Center, Faculty of Veterinary Medicine, Utrecht University, for kind provision of the SE strain. We are thankful to R.H.G.A. van den Biggelaar for his help during the isolation of immune cells and we acknowledge G.J.A. Arkesteijn for maintaining optimal working conditions of the Flow Cytometry and Cell Sorting Facility, Faculty of Veterinary Medicine, Utrecht University. This work is part of the research programme of NWO Earth and Life Sciences (ALW) with project number 868.15.020, which is financed by the Dutch Research Council (NWO) in conjunction with Cargill Animal Nutrition and Health in the context of stimulating Public-Private research collaboration. 


\section{Supplementary data}

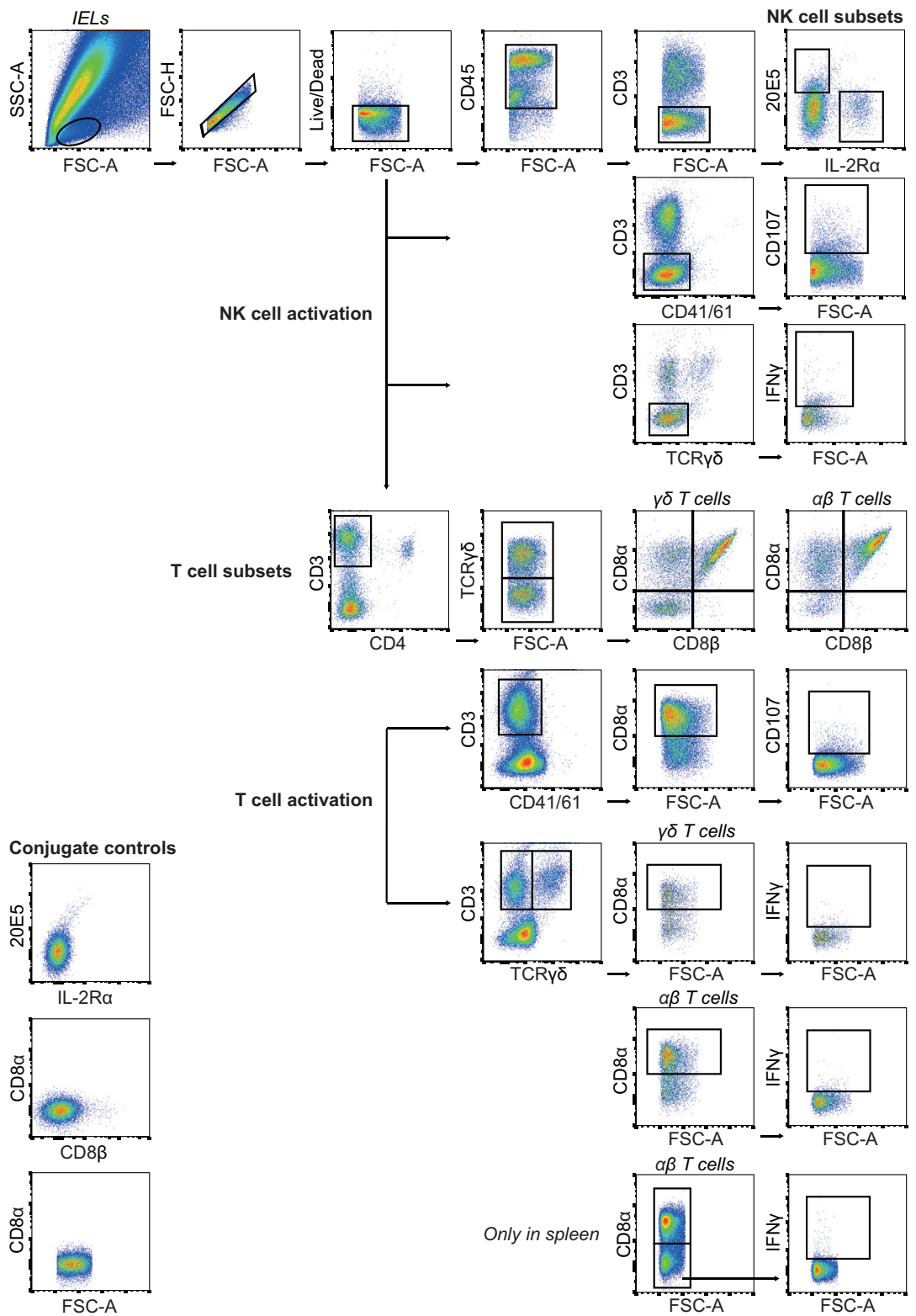

Figure S1. The gating strategies used to analyze numbers and function of NK cells, $\gamma \delta T$ cells and cytotoxic CD8 ${ }^{+} T$ cells in the ileum. Gating included consecutive selection for lymphocytes (FSC-A vs SSC-A), viable cells (Live/Dead marker-negative) followed by selection of the specific cellular subsets and the expression of activation markers by NK and T cells according to the staining panels (Table 1). NK cell subsets were gated on CD3- cells expressing either IL-2R $\alpha$ or 20 E5 and NK cell activation was gated on

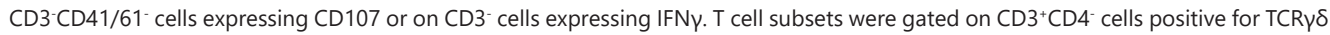
$(\gamma \delta)$ or negative $\left(C D 8^{+} \alpha \beta\right)$ with both $\gamma \delta$ and cytotoxic $\alpha \beta$ T cells expressing either CD8 $\alpha \alpha$ or CD8 $\alpha \beta$. T cell activation was gated on $\mathrm{CD}^{+}{ }^{+} \mathrm{CD} 41 / 61-\mathrm{CD} 8 \alpha^{+}$cells expressing $\mathrm{CD} 107$ or on $\mathrm{CD} 3^{+} \mathrm{TCR} \gamma \delta^{+} \mathrm{CD} 8 \alpha^{+}$and $\mathrm{CD} 3^{+} \mathrm{TCR} \gamma \delta{ }^{-} \mathrm{CD} 8 \alpha^{+}$cells expressing IFN $\gamma$, and only in spleen also $C D 3^{+} \mathrm{TCR} \gamma \delta \delta^{-C D 8} \alpha^{-}\left(\mathrm{CD} 4^{+}\right)$cells expressing IFN $\gamma$. 
A

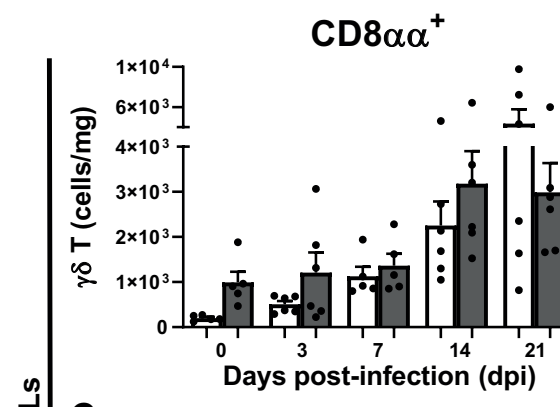

프

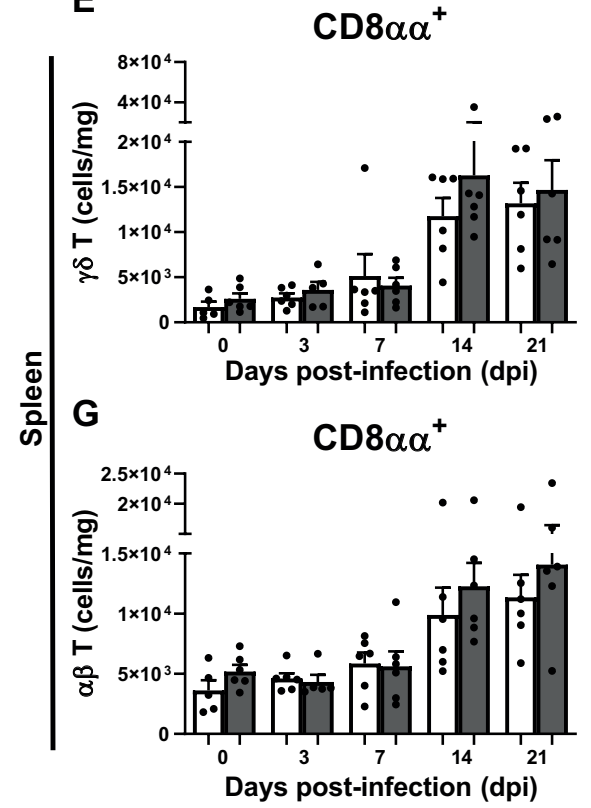

B

$\square$ Control $\square$ GM

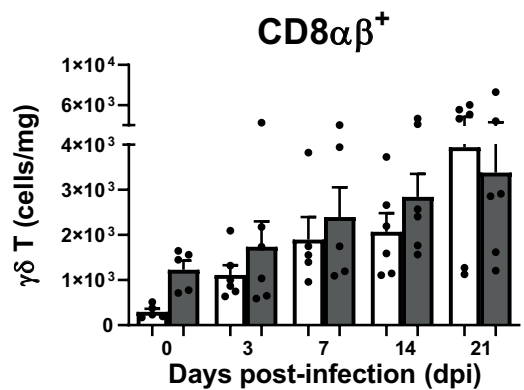

D

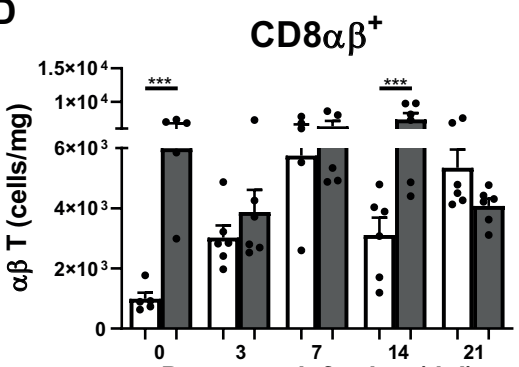

F

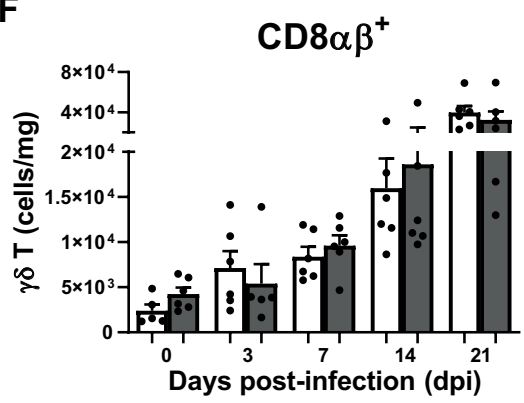

H

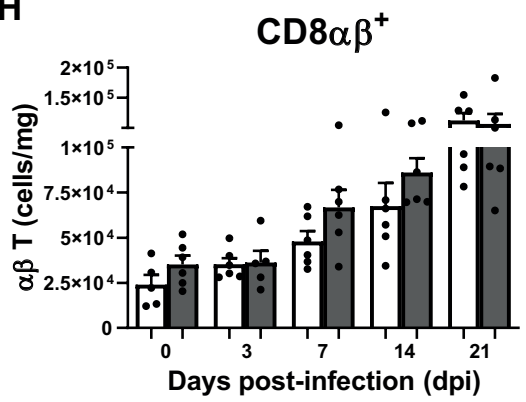

Figure S2. Effect of GM on numbers of intraepithelial and splenic $\gamma \delta$ T cells and cytotoxic T cells expressing either CD8 $\alpha \alpha^{+}$and $C D 8 \alpha \beta^{+}$ before and during SE infection in broiler chickens. (A) Numbers (cells/mg) of intraepithelial CD8 $\alpha \alpha^{+} \gamma \delta T$ cells, (B) CD8 $\alpha \beta^{+} \gamma \delta T$ cells, (C) cytotoxic CD8 $\alpha \alpha^{+} T$ cells and (D) CD8 $\alpha \beta^{+} T$ cells in chickens either fed standard (control) or long-chain glucomannan supplemented (GM) diet in course of time before and during SE infection. (E) Numbers (cells/mg) of splenic CD8 $\alpha \alpha^{+} \gamma \delta$ T cells, (F) CD8 $\alpha \beta^{+} \gamma \delta T$ cells, (G) cytotoxic CD8 $\alpha \alpha^{+} T$ cells and $(H) C D 8 \alpha \beta^{+} T$ cells in chickens either fed standard or GM diet before and during SE infection. Mean + SEM per diet group and time point are shown $(n=6)$, if $n=5$; one chicken was excluded due to numbers of events acquired in the gate of interest were $<100$. Statistical significance between diet groups is indicated as $* * *(p<0.001)$. 
A
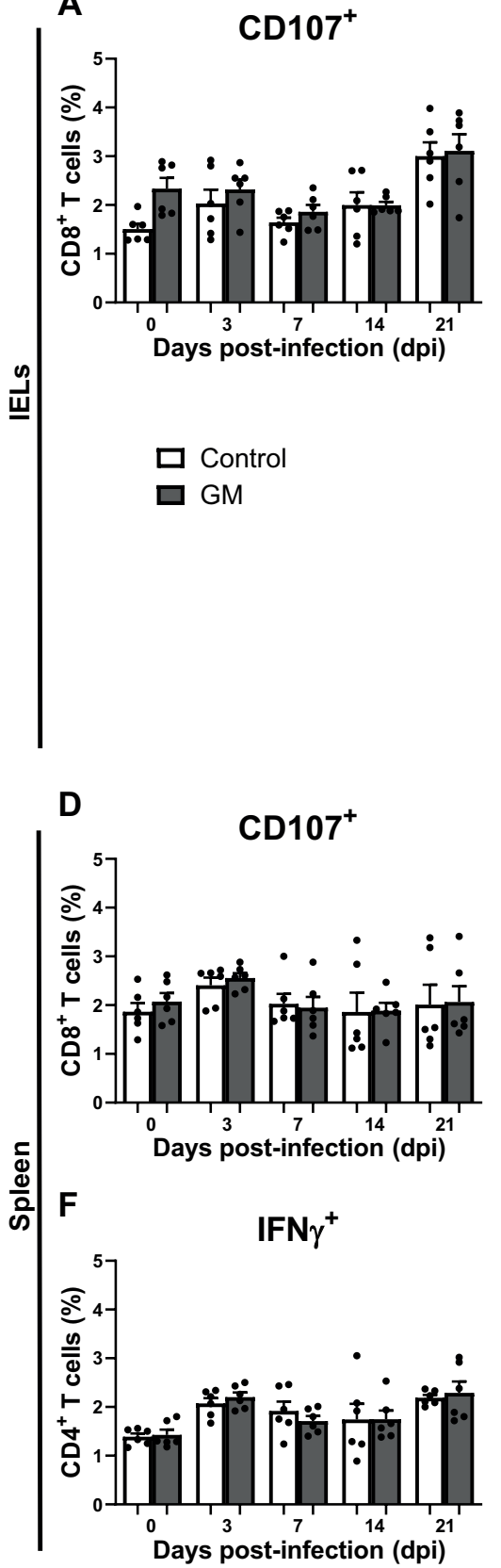

B

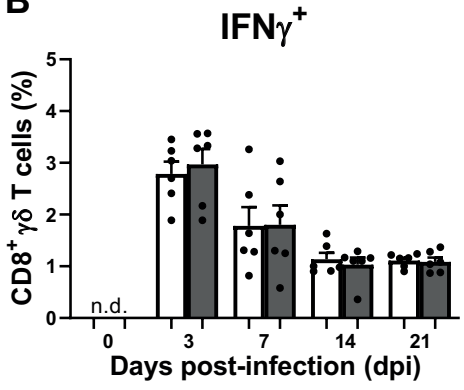

C

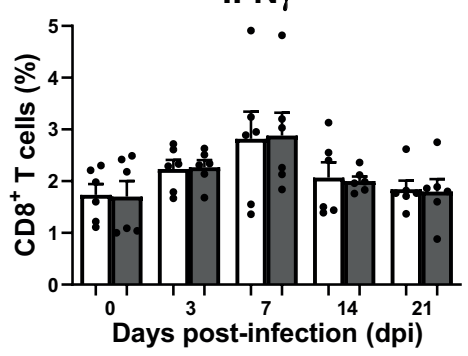

$E$
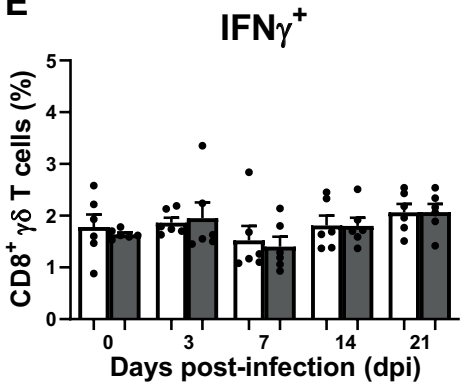

G

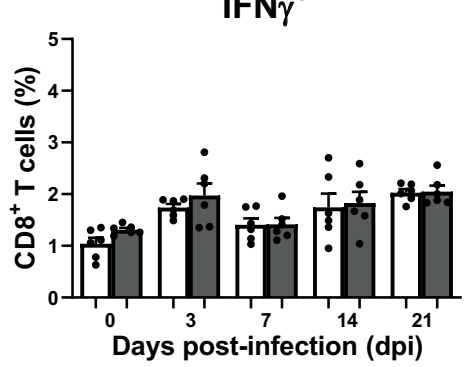

Figure S3. Effect of GM on T cell activation in IELs and spleen before and during SE infection in broiler chickens. (A) Percentages of intraepithelial CD8 ${ }^{+}$T cells expressing CD107 (including both $\gamma \delta$ and $\alpha \beta$ T cells), (B) CD8 ${ }^{+} \gamma \delta$ T cells expressing IFN $\gamma$ and (C) CD8 $8^{+} \alpha \beta$ T cells expressing IFN $\gamma$ in chickens either fed standard (control) or long-chain glucomannan supplemented (GM) diet in course of time before and during SE infection. (D) Percentages of splenic CD8 ${ }^{+}$T cells expressing CD107 (including both $\gamma \delta$ and $\alpha \beta T$ cells), (E) $C D 8^{+} \gamma \delta T$ cells

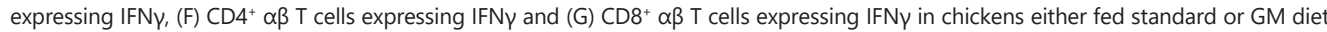
before and during SE infection. Mean + SEM per diet group and time point are shown $(n=6)$, for IFN $\gamma$ expression of $C D 8^{+} \gamma \delta T$ cells in the IEL population at 0 dpi percentages were not determined (n.d.) due to numbers of events acquired in the gate of interest were $<100$. 


\title{
Correlation
}

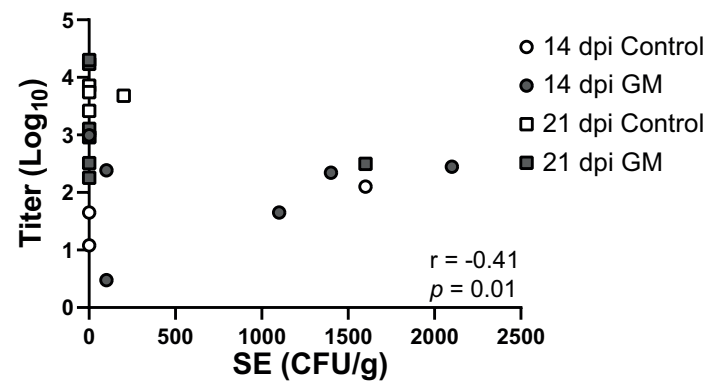

\begin{abstract}
Figure S4. Correlation between serum antibody titers and SE-CFUs in broiler chickens. Correlation between SE-specific antibody titers and splenic SE-CFUs of chickens either fed standard (control) or long-chain glucomannan (GM) diet using the Spearman rank correlation test. Statistical significance is indicated as $p=0.01$.
\end{abstract}

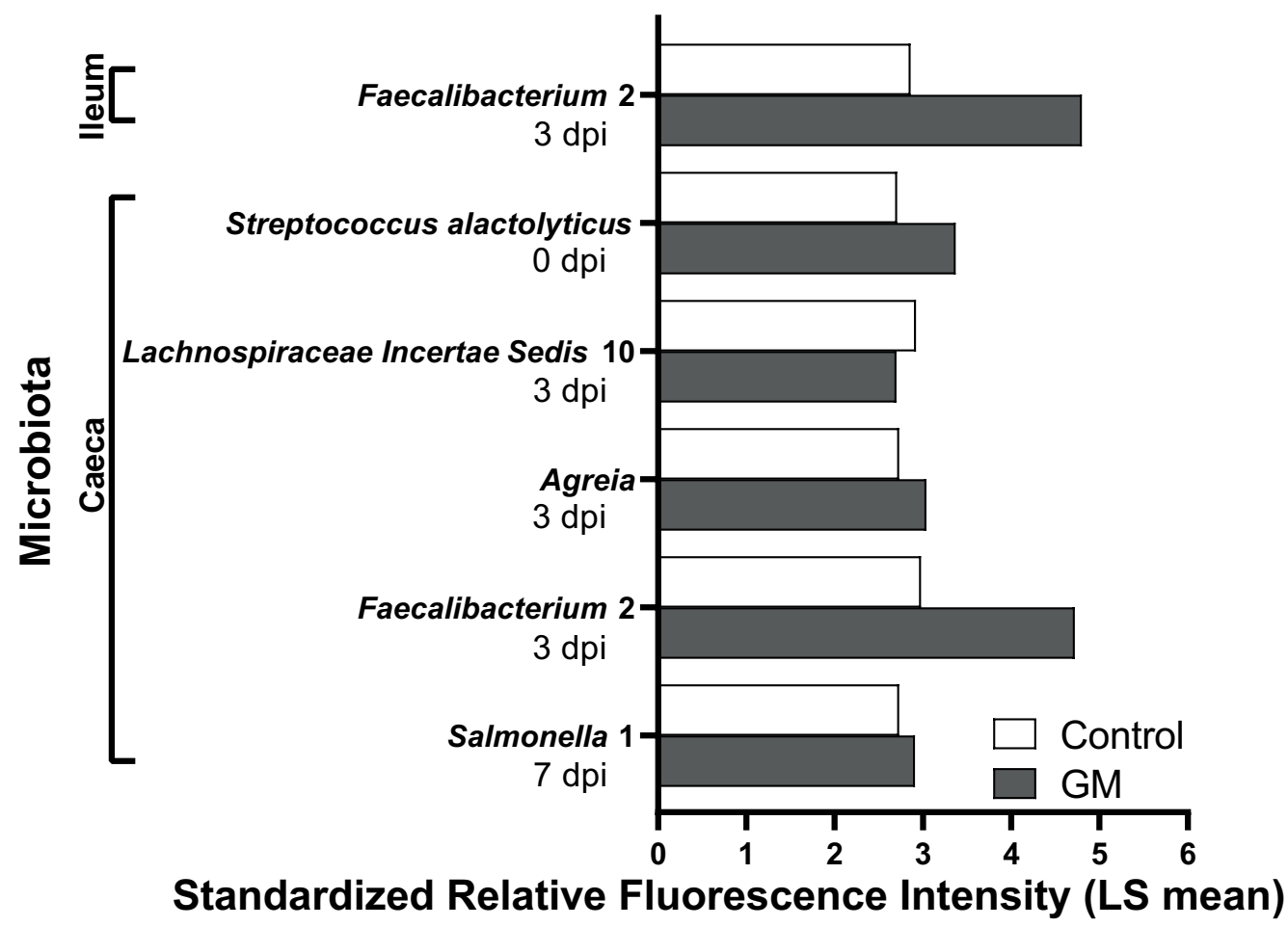

Figure S5. Intestinal microbial taxa significantly increased with diet at 0, 3 and $7 \mathrm{dpi}$ of SE in broiler chickens. Standardized relative fluorescence intensities of the microbial taxa as measured by the microarray in the ileum and caeca (Table 3) that were significantly increased either with standard (control) or long-chain glucomannan supplemented (GM) diet at 0, 3 and 7 dpi of SE in broiler chickens. LS mean per microbial taxa and diet group are shown $(n=6)$ with statistical significance of FDR adjusted $p$-values set at $<0.05$. 


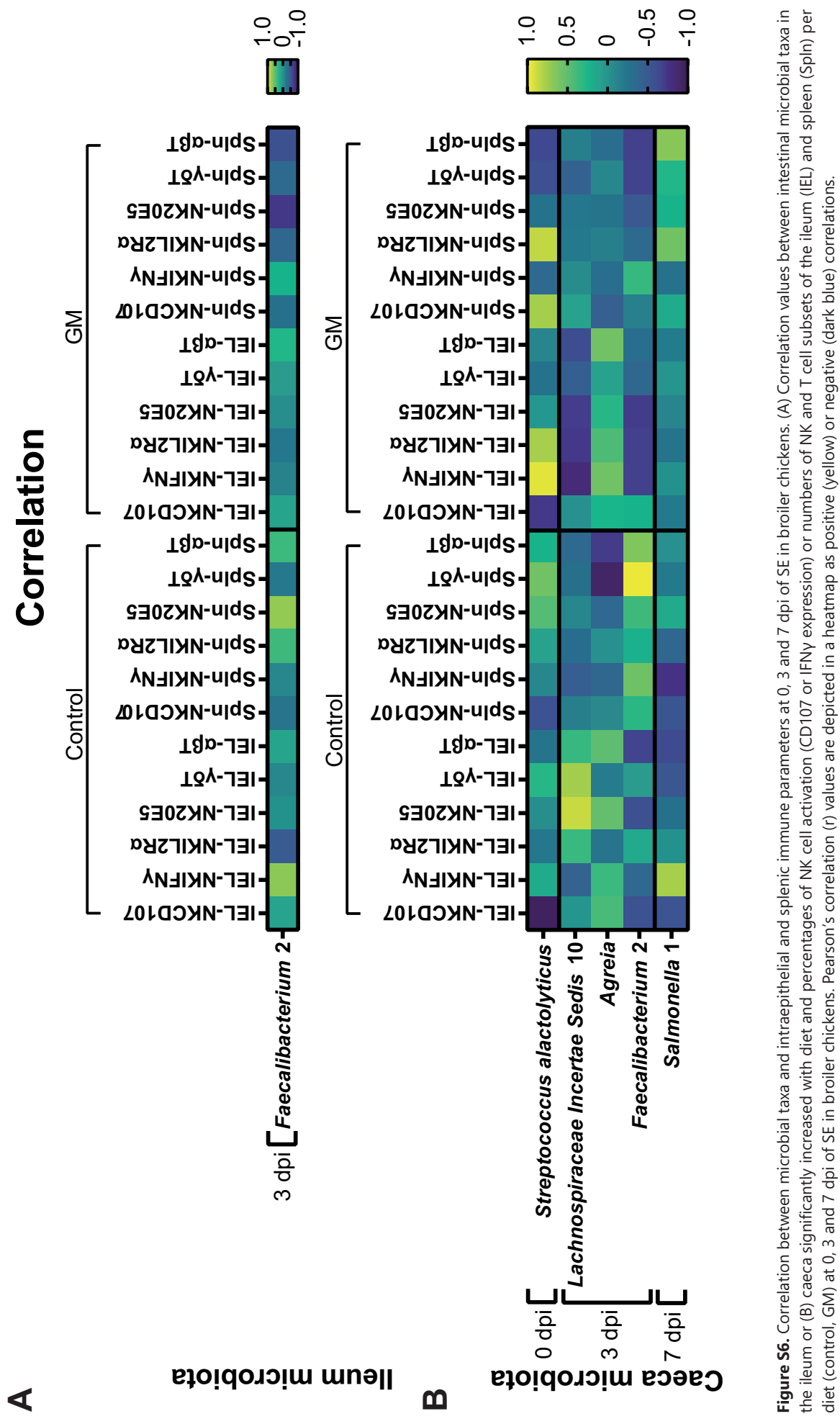




\section{References}

1. Suzuki S. Pathogenicity of Salmonella enteritidis in poultry. Int J Food Microbiol. 1994;21(1-2):89-105. doi:10.1016/01681605(94)90203-8.

2. Arlet G, Barrett TJ, Butaye P, Cloeckaert A, Mulvey MR, White DG. Salmonella resistant to extended-spectrum cephalosporins: prevalence and epidemiology. Microbes Infect. 2006;8(7):1945-1954. doi:10.1016/j.micinf.2005.12.029.

3. Kallapura G, Morgan MJ, Pumford NR, et al. Evaluation of the respiratory route as a viable portal of entry for Salmonella in poultry via intratracheal challenge of Salmonella Enteritidis and Salmonella Typhimurium. Poult Sci. 2014;93(2):340-346. doi:10.3382/ps.2013-03602.

4. Kallapura G, Kogut MH, Morgan MJ, et al. Fate of Salmonella Senftenberg in broiler chickens evaluated by challenge experiments. Avian Pathol. 2014;43(4):305-309. doi:10.1080/03079457.2014.923554.

5. Hoffmann S, Batz MB, Morris Jr. JG. Annual cost of illness and quality-adjusted life year losses in the united states due to 14 foodborne pathogens. J Food Protection. 2012;75(7):1292-1302. doi:10.4315/0362-028X.JFP-11-417.

6. Scharff RL. Food attribution and economic cost estimates for meat- And poultry-related illnesses. J Food Protection. 2020;83(6):959967. doi:10.4315/JFP-19-548.

7. World Organisation for Animal Health, (OIE). Prevention, detection and control of Salmonella in poultry. OIE, ed. 28th ed. Office International des Epizooties; 2019.

8. Sharma JM, Tizard I. Avian cellular immune effector mechanisms - a review. Avian Pathol. 1984;13(3):357-376. doi:10.1080/03079458408418541.

9. Klasing KC LT. Functions, costs and benefits of the immune system during development and growth. 1999;In: Adams NJ, and Slotow R (eds)(Proc. 22. Int. Ornithol. Congr. H. Birdlife South Africa, Johannesburg):2817-2835.

10. Fenzl L, Göbel TW, Neulen M-. $\gamma \delta$ T cells represent a major spontaneously cytotoxic cell population in the chicken. Dev Comp Immunol. 2017;73:175-183. doi:10.1016/j.dci.2017.03.028.

11. Göbel TWF, Kaspers B, Stangassinger M. NK and T cells constitute two major, functionally distinct intestinal epithelial lymphocyte subsets in the chicken. Int Immunol. 2001;13(6):757-762. doi:10.1093/intimm/13.6.757.

12. Meijerink N, van Haarlem DA, Velkers FC, Stegeman AJ, Rutten, V. P. M. G., Jansen CA. Analysis of chicken intestinal natural killer cells, a major IEL subset during embryonic and early life. Dev Comp Immunol. 2021;114. doi:10.1016/j.dci.2020.103857.

13. Lillehoj HS, Trout JM. Avian gut-associated lymphoid tissues and intestinal immune responses to Eimeria parasites. Clin Microbiol Rev. 1996;9(3):349-360. doi:10.1128/cmr.9.3.349.

14. Lawn AM, Rose ME, Bradley JWA, Rennie MC. Lymphocytes of the intestinal mucosa of chickens. Cell Tissue Res. 1988;251(1):189195. doi:10.1007/BF00215464.

15. Meijerink N, van den Biggelaar RHGA, van Haarlem DA, Stegeman JA, Rutten VPMG, Jansen CA. A detailed analysis of innate and adaptive immune responsiveness upon infection with Salmonella enterica serotype Enteritidis in young broiler chickens. Vet Res. 2021;52(1):109. doi:10.1186/s13567-021-00978-y.

16. Berndt A, Pieper J, Methner U. Circulating $\gamma \delta T$ cells in response to Salmonella enterica serovar enteritidis exposure in chickens. Infect Immun. 2006;74(7):3967-3978. doi:10.1128/IAI.01128-05.

17. Carvajal BG, Methner U, Pieper J, Berndt A. Effects of Salmonella enterica serovar Enteritidis on cellular recruitment and cytokine gene expression in caecum of vaccinated chickens. Vaccine. 2008;26(42):5423-5433. doi:10.1016/j.vaccine.2008.07.088.

18. Zhen W, Shao $Y$, Gong X, et al. Effect of dietary Bacillus coagulans supplementation on growth performance and immune responses of broiler chickens challenged by Salmonella enteritidis. Poult Sci. 2018;97(8):2654-2666. doi:10.3382/ps/pey119.

19. Gangadharan $D$, Cheroutre H. The CD8 isoform CD8 $\alpha$ is not a functional homologue of the TCR co-receptor CD8 $\alpha \beta$. Curr Opin Immunol. 2004;16(3):264-270. doi:10.1016/j.coi.2004.03.015.

20. Pasman L, Kasper DL. Building conventions for unconventional lymphocytes. Immunol Rev. 2017;279(1):52-62. doi:10.1111/ imr.12576.

21. Leishman AJ, Naidenko OV, Attinger A, et al. T cell responses modulated through interaction between CD8 $\alpha \alpha$ and the nonclassical MHC class I molecule, TL. Science. 2001;294(5548):1936-1939. doi:10.1126/science.1063564

22. Berge AC, Wierup M. Nutritional strategies to combat Salmonella in mono-gastric food animal production. Animal. 2012;6(4):557564. doi:10.1017/S1751731111002217.

23. Vandeplas $S$, Dubois Dauphin R, Beckers $Y$, Thonart $P$, Théwis A. Salmonella in chicken: Current and developing strategies to reduce contamination at farm level. J Food Protection. 2010;73(4):774-785. doi:10.4315/0362-028X-73.4.774.

24. Ashayerizadeh A, Dastar B, Shams Shargh M, Sadeghi Mahoonak A, Zerehdaran S. Fermented rapeseed meal is effective in controlling Salmonella enterica serovar Typhimurium infection and improving growth performance in broiler chicks. Vet Microbiol. 2017;201:93-102. doi:10.1016/j.vetmic.2017.01.007.

25. Rubinelli PM, Kim SA, Park SH, Roto SM, Ricke SC. Sodium bisulfate and a sodium bisulfate/tannin mixture decreases $\mathrm{pH}$ when added to an in vitro incubated poultry cecal or fecal contents while reducing Salmonella Typhimurium marker strain survival and altering the microbiome. J Environ Sci Health Part B Pestic Food Contamin Agric Wastes. 2017;52(8):607-615. doi:10.1080/036012 34.2017.1316159.

26. Spring P, Wenk C, Dawson KA, Newman KE. The Effects of Dietary Mannanoligosaccharides on Cecal Parameters and the Concentrations of Enteric Bacteria in the Ceca of Salmonella-Challenged Broiler Chicks. Poult Sci. 2000;79(2):205-211. doi:10.1093/ ps/79.2.205. 
27. Kogut MH. Impact of nutrition on the innate immune response to infection in poultry. J Appl Poult Res. 2009;18(1):111-124. doi:10.3382/japr.2008-00081.

28. Meijerink N, Kers JG, Velkers FC, et al. Early Life Inoculation With Adult-Derived Microbiota Accelerates Maturation of Intestinal Microbiota and Enhances NK Cell Activation in Broiler Chickens. Front Vet Sci. 2020;7. doi:10.3389/fvets.2020.584561.

29. Meijerink N, de Oliveira J,E., van Haarlem D,A., et al. Glucose Oligosaccharide and Long-Chain Glucomannan Feed Additives Induce Enhanced Activation of Intraepithelial NK Cells and Relative Abundance of Commensal Lactic Acid Bacteria in Broiler Chickens. Veterinary Sciences. 2021;8(6):110. doi:10.3390/vetsci8060110.

30. Round JL, Mazmanian SK. The gut microbiota shapes intestinal immune responses during health and disease. Nature Reviews Immunology. 2009;9(5):313-323. doi:10.1038/nri2515.

31. Hooper LV, Littman DR, Macpherson AJ. Interactions between the microbiota and the immune system. Science. 2012;336(6086):12681273. doi:10.1126/science.1223490.

32. Chen C-, Tsen H-, Lin C-, Yu B, Chen C-. Oral administration of a combination of select lactic acid bacteria strains to reduce the Salmonella invasion and inflammation of broiler chicks. Poult Sci. 2012;91(9):2139-2147. doi:10.3382/ps.2012-02237.

33. Thirabunyanon $\mathrm{M}$, Thongwittaya $\mathrm{N}$. Protection activity of a novel probiotic strain of Bacillus subtilis against Salmonella Enteritidis infection. Res Vet Sci. 2012;93(1):74-81. doi:10.1016/j.rvsc.2011.08.008.

34. Carter A, Adams M, La Ragione RM, Woodward MJ. Colonisation of poultry by Salmonella Enteritidis S1400 is reduced by combined administration of Lactobacillus salivarius 59 and Enterococcus faecium PXN-33. Vet Microbiol. 2017;199:100-107. doi:10.1016/j. vetmic.2016.12.029.

35. Krueger LA, Gaydos TA, Sims MD, Spangler DA. Avi-Lution supplemented at 250 or $500 \mathrm{mg}$ per $\mathrm{kg}$ in feed decreases the abundance of Salmonella Enteritidis in ceca of layer pullets. J Appl Poult Res. 2020. doi:10.1016/j.japr.2020.09.007.

36. Adhikari B, Hernandez-Patlan D, Solis-Cruz B, et al. Evaluation of the Antimicrobial and Anti-inflammatory Properties of Bacillus-DFM (Norum ${ }^{\mathrm{TM}}$ ) in Broiler Chickens Infected With Salmonella Enteritidis. Front Vet Sci. 2019;6. doi:10.3389/fvets.2019.00282.

37. Bae D, Kim D-, Chon J-, Song K-, Seo K-. Synergistic effects of the early administration of Lactobacillus kefiranofaciens DN1 and Kluyveromyces marxianus KU140723-05 on the inhibition of Salmonella Enteritidis colonization in young chickens. Poult Sci. 2020;99(11):5999-6006. doi:10.1016/j.psj.2020.07.032.

38. Rajani J, Dastar B, Samadi F, Karimi Torshizi MA, Abdulkhani A, Esfandyarpour S. Effect of extracted galactoglucomannan oligosaccharides from pine wood (Pinus brutia) on Salmonella typhimurium colonisation, growth performance and intestinal morphology in broiler chicks. Brit Poult Sci. 2016;57(5):682-692. doi:10.1080/00071668.2016.1200013.

39. Hughes R-, Ali RA, Mendoza MA, Hassan HM, Koci MD. Impact of dietary galacto-oligosaccharide (GOS) on chicken's gut microbiota, mucosal gene expression, and Salmonella colonization. Front Vet Sci. 2017;4(NOV). doi:10.3389/fvets.2017.00192.

40. Jazi V, Foroozandeh AD, Dastar B, Rezaie Koochaksaraie R, Toghyani M. Effects of Pediococcus acidilactici, mannan-oligosac charide, butyric acid and their combination on growth performance and intestinal health in young broiler chickens challenged with Salmonella Typhimurium. Poult Sci. 2018;97(6):2034-2043. doi:10.3382/ps/pey035.

41. Shao $Y$, Wang $Z$, Tian $X$, Guo $Y$, Zhang $H$. Yeast $\beta$-d-glucans induced antimicrobial peptide expressions against Salmonella infection in broiler chickens. Int J Biol Macromol. 2016;85:573-584. doi:10.1016/j.ijbiomac.2016.01.031.

42. De Oliveira JE, Van Der Hoeven-Hangoor E, Van De Linde, I. B., Montijn RC, Van Der Vossen, J. M. B. M. In ovo inoculation of chicken embryos with probiotic bacteria and its effect on posthatch Salmonella susceptibility. Poult Sci. 2014;93(4):818-829. doi:10.3382/ ps.2013-03409.

43. Teague KD, Graham LE, Dunn JR, et al. In ovo evaluation of FloraMax ${ }^{\circledR}-B 11$ on Marek's disease HVT vaccine protective efficacy, hatchability, microbiota composition, morphometric analysis, and Salmonella enteritidis infection in broiler chickens. Poult Sci. 2017;96(7):2074-2082. doi:10.3382/ps/pew494.

44. Van De Reep L, Nielen M, Verstappen, K. M. H. W., Broens EM, Van Den Broek J, Velkers FC. Response to a Salmonella Enteritidis challenge in old laying hens with different vaccination histories. Poult Sci. 2018;97(8):2733-2739. doi:10.3382/ps/pey134.

45. Jansen CA, van de Haar, P. M., van Haarlem D, et al. Identification of new populations of chicken natural killer (NK) cells. Dev Comp Immunol. 2010;34(7):759-767. doi:10.1016/j.dci.2010.02.009.

46. Wattrang E, Dalgaard TS, Norup LR, Kjærup RB, Lundén A, Juul-Madsen HR. CD107a as a marker of activation in chicken cytotoxic T cells. J Immunol Methods. 2015;419(1):35-47. doi:10.1016/j.jim.2015.02.011.

47. Ariaans MP, van de Haar, P. M., Lowenthal JW, van Eden W, Hensen EJ, Vervelde L. ELISPOT and intracellular cytokine staining: Novel assays for quantifying $T$ cell responses in the chicken. Dev Comp Immunol. 2008;32(11):1398-1404. doi:10.1016/j. dci.2008.05.007.

48. van der Hoeven-Hangoor E, van der Vossen, J. M. B. M., Schuren FHJ, et al. lleal microbiota composition of broilers fed various commercial diet compositions. Poult Sci. 2013;92(10):2713-2723. doi:10.3382/ps.2013-03017.

49. Druyan S, De Oliveira JE, Ashwell CM. Focused microarrays as a method to evaluate subtle changes in gene expression. Poult Sci. 2008;87(11):2418-2429. doi:10.3382/ps.2007-00513.

50. De Oliveira JE, Druyan S, Uni Z, Ashwell CM, Ferket PR. Metabolic profiling of late-term turkey embryos by microarrays. Poult Sci. 2013;92(4):1011-1028.

51. Cooper MA, Fehniger TA, Caligiuri MA. The biology of human natural killer-cell subsets. Trends in Immunology. 2001;22(11):633640. doi:https://doi.org/10.1016/S1471-4906(01)02060-9.

52. Pourabedin $M$, Chen $Q$, Yang $M$, Zhao X. Mannan- and xylooligosaccharides modulate caecal microbiota and expression of inflammatory-related cytokines and reduce caecal Salmonella Enteritidis colonisation in young chickens. FEMS Microbiol Ecol. 2017;93(1). doi:10.1093/femsec/fiw226. 
53. Babu US, Sommers K, Harrison LM, Balan KV. Effects of fructooligosaccharide-inulin on Salmonella-killing and inflammatory gene expression in chicken macrophages. Vet Immunol Immunopathol. 2012;149(1-2):92-96. doi:10.1016/j.vetimm.2012.05.003.

54. Choi JH, Kim GB, Cha CJ. Spatial heterogeneity and stability of bacterial community in the gastrointestinal tracts of broiler chickens. Poult Sci. 2014;93(8):1942-1950. doi:10.3382/ps.2014-03974.

55. Kempf F, Menanteau P, Rychlik I, et al. Gut microbiota composition before infection determines the Salmonella super- and low-shedder phenotypes in chicken. Microb Biotechnol. 2020;13(5):1611-1630. doi:10.1111/1751-7915.13621.

56. Van Den Bogert. B, Meijerink M, Zoetendal EG, Wells JM, Kleerebezem M. Immunomodulatory properties of streptococcus and veillonella isolates from the human small intestine microbiota. PLoS ONE. 2014;9(12). doi:10.1371/journal.pone.0114277.

57. Chang $\mathrm{CH}$, Teng PY, Lee TT, Yu B. The effects of the supplementation of multi-strain probiotics on intestinal microbiota, metabolites and inflammation of young SPF chickens challenged with Salmonella enterica subsp. enterica. Anim Sci J. 2019;90(6):737-746. doi:10.1111/asj.13205.

58. Rinttilä T, Apajalahti J. Intestinal microbiota and metabolites-Implications for broiler chicken health and performance. J Appl Poult Res. 2013;22(3):647-658. doi:10.3382/japr.2013-00742.

59. Poggi $A$, Benelli R, Venè R, et al. Human gut-associated natural killer cells in health and disease. Front Immunol. 2019;10(MAY). doi:10.3389/fimmu.2019.00961.

60. Shapiro $H$, Thaiss CA, Levy M, Elinav E. The cross talk between microbiota and the immune system: Metabolites take center stage. Curr Opin Immunol. 2014;30(1):54-62. doi:10.1016/j.coi.2014.07.003.

61. Levy M, Blacher E, Elinav E. Microbiome, metabolites and host immunity. Curr Opin Microbiol. 2017;35:8-15. doi:10.1016/j. mib.2016.10.003.

62. Yamazaki M, Ohtsu $H$, Yakabe $Y$, Kishima M, Abe H. In vitro screening of lactobacilli isolated from chicken excreta to control Salmonella Enteritidis and Typhimurium. Brit Poult Sci. 2012;53(2):183-189. doi:10.1080/00071668.2012.678814.

63. Clarke TB, Davis KM, Lysenko ES, Zhou AY, Yu Y, Weiser JN. Recognition of peptidoglycan from the microbiota by Nod1 enhances systemic innate immunity. Nat Med. 2010;16(2):228-231. doi:10.1038/nm.2087.

64. Macpherson AJ, Harris NL. Interactions between commensal intestinal bacteria and the immune system. Nature Reviews Immunology. 2004;4(6):478-485. doi:10.1038/nri1373. 
6 

CHAPTER 7

\section{Summarizing discussion}


The current ban on preventive treatment and restrictions on therapeutic application of antimicrobials in poultry production have led to an increase in intestinal health problems in chickens ${ }^{1,2}$. This urges for alternative strategies to maintain or improve health of broiler chickens. Young chickens are more susceptible to infectious diseases than adult ones since the adaptive immune system is not fully developed yet. Therefore, these strategies should aim to increase the resistance to pathogens early in the life of chickens. At that stage, the immune responsiveness of chickens to invading pathogens relies on the innate immune system and natural killer (NK) cells, abundantly present within the intestinal intraepithelial lymphocyte (IEL) population, are among the first to respond. Enhanced responsiveness of the innate immune system, with special emphasis on NK cells, is hypothesized to increase resistance of young chickens and consequently improve the health of young and adult broiler chickens.

The aim of this thesis was to investigate stimulation of immune responsiveness, modulation of intestinal microbiota and the interaction between these by nutritional supplementation applied in order to increase the resistance of young broiler chickens. For this purpose, NK and T cell subsets of IELs in the ileum as well as in other tissues were analyzed and quantified in course of time from late embryonic development up to three weeks of life. In parallel, functional parameters of NK cells were assessed and used as a baseline for subsequent studies pertaining modulation of NK cell number and function in broiler chickens (Chapter 2). Furthermore, the interaction between the immune system and modulated intestinal microbiota in young broiler chickens was investigated (Chapter $\mathbf{3}$ ). The potential of modulation of IELs and intestinal microbiota composition by nutritional strategies was explored and assessed (Chapter 4). Next, the role of NK cells in responsiveness to Salmonella enterica serotype Enteritidis (SE) infection, an important intestinal pathogen in chickens, was investigated (Chapter 5). Finally, to investigate the potency of feed supplementation to increase resistance to SE in young broiler chickens, stimulation of immune responsiveness and modulation of intestinal microbiota as well as the interaction between these, were assessed (Chapter 6).

\section{Analysis of NK cell subsets}

In chickens, NK cell research is in its infancy compared to mammals since no pan NK cell marker is present. Functional differences between NK cell subsets have not been described yet, unlike in mammalian species. In this thesis, insights in different chicken NK cell subsets and their function are provided which will aid in the development of strategies to stimulate immune responsiveness of NK cells at early life that may increase the resistance of chickens to infections. First, presence of different NK cell subsets was analyzed in the IELs and spleens of embryos and adult broiler chickens to identify the largest NK cell populations (unpublished experiments). For this, expression of previously identified markers, reported to be expressed on chicken CD3 negative cells showing NK cell function, including IL-2Ra $\alpha^{3}, 20 E 5^{4}, 7 C 1^{4}, 5 C 7^{4}, 21 E 3^{4}$ and NKp465, was determined. Chicken CD3- cells predominantly expressed IL-2R $\alpha, 20 \mathrm{E} 5$ and $5 \mathrm{C7}$ and these NK cell populations were subsequently determined in several tissues of broiler chickens in course of time from late embryonic development up to three weeks of life (Chapter 2). Throughout aging presence of $5 \mathrm{C7}^{+} \mathrm{NK}$ cells remained low in all tissues compared to $\mathrm{IL}-2 \mathrm{R} \alpha^{+}$and $20 \mathrm{E} 5^{+} \mathrm{NK}$ cells. A different tissue distribution of the latter two NK cell subsets was found, since IL-2R $\alpha^{+}$NK cells were most predominant in IELs and spleen whereas 20E5 ${ }^{+}$NK cells dominated in the blood and bone marrow (Chapter 2). A similar distribution between secondary lymphoid tissue and blood circulation was found for CD56 bright and CD56 dim NK cells in humans ${ }^{6}$ respectively, CD27 high and $\mathrm{CD} 27^{\text {low/ }}$ NK cells in mice ${ }^{7}$ and $\mathrm{CD}^{-}$and $\mathrm{CD}^{+}{ }^{+} \mathrm{NK}$ cells in cattle ${ }^{8}$. In addition, the human circulating CD56 bright NK cell subset constitutively express IL-2R $\alpha$, which is considered as an early activation marker ${ }^{9-11}$. These observations suggest that the chicken IL-2R $\alpha^{+}$and $20 \mathrm{E} 5^{+} \mathrm{NK}$ cells may resemble the NK cell subsets found in these mammalian species. The different tissue distribution may be related 
to homing properties, in situ maturation and/or a functional differences between the NK cell subsets, as was shown in humans and mice ${ }^{6,12-14}$. The high number of intraepithelial and splenic CD3- IL-2R $\alpha^{+}$ NK cells was in agreement with earlier studies in layer chickens $s^{3,4}$ and rats ${ }^{15}$. Interestingly, the majority of human intraepithelial NK cells do not constitutively express IL-2R $\alpha^{+16,17}$ but CD69 ${ }^{16}$, another early activation marker ${ }^{18}$. Other studies in chickens demonstrated mRNA expression of CD69 in intraepithelial IL-2R $\alpha^{+} N K$ cells ${ }^{19,20}$. These observations suggest that both of these chicken and human intraepithelial NK cell subsets show an activated phenotype but differences in the phenotypic in situ maturation of NK cell subsets exist between chickens and humans. In humans, a high phenotypical diversity within the tissue-resident CD56 bright NK cells is observed ${ }^{6}$. This heterogeneity and plasticity illustrates the complexity of NK cells and there is still no definitive understanding of their developmental pathways leading to the peripheral and tissue-resident NK cell subsets ${ }^{21-24}$. The current model of human NK cell development proposes a continuous development in which CD56 bright differentiate into CD56 ${ }^{\text {dim }} \mathrm{NK}$ cells, although some studies report that different precursor populations may independently develop into distinct NK cell subsets ${ }^{21,25}$. A similar model has been proposed for differentiation of murine CD27 high into CD27low/ NK cells ${ }^{26}$. The developmental pathways of chicken NK cells are currently unknown and future studies should investigate whether differentiation of chicken IL-2R $\alpha^{+}$into $20 \mathrm{E} 5^{+} \mathrm{NK}$ cells occurs. The majority of chicken NK cells expressed either IL-2R $\alpha$ or 20E5, which indicates that these NK cell subsets are important in the first line of defense against infectious agents. Hence, better understanding of their functional capacities is required and was subsequently investigated.

\section{Functional characteristics of NK cell subsets}

Activation of NK cells results, amongst others, in cell cytotoxicity by degranulation of cytotoxic granules and cytokine production such as IFN $\gamma^{27,28}$. These functional characteristics were investigated for both $\mathrm{IL}-2 \mathrm{R} \alpha^{+}$and $20 \mathrm{E} 5^{+} \mathrm{NK}$ cell subsets to determine whether they differed functionally as was shown for various subsets in mammalian species. Our data showed that both IL-2R $\alpha^{+}$and $20 \mathrm{E} 5^{+} \mathrm{NK}$ cells are implicated in cytotoxic activity based on the capacity to degranulate, as assessed by expression of the biomarker CD107, although the levels varied between the subsets in different tissues. In the IELs, expression of CD107 occurred mostly on IL-2R $\alpha^{+}$NK cells while in the spleen and blood CD107 was mainly expressed on 20E5 ${ }^{+}$NK cells (Chapter 2). Expression of CD107 on intestinal IL-2R $\alpha^{+}$NK cells is consistent with previous studies in chickens, that showed killing of target cells by this subset ${ }^{3,29}$. Furthermore, the CD107 expression on splenic 20E5+ NK cells was in agreement with preliminary data that suggest higher mRNA expression of perforin, a cytolytic protein found in the granules, in this NK cell subset compared to IL-2R $\alpha^{+}$NK cells (Chapter 5). In addition to cytotoxicity, both splenic IL-2R $\alpha^{+}$and 20E5+ NK cell subsets are suggested to be implicated in cytokine production by their expression of nuclear factor-interleukin 3 regulated (NFIL3) and IL-7R $\alpha$, genes indicative for the cytokine-producing human NK cell subset ${ }^{30,31}$, although IL-2R $\alpha^{+}$NK cells showed higher mRNA levels of these genes than 20E5+ NK cells (Chapter 5). The 20E5+ and IL-2R $\alpha^{+}$NK cell subsets in chickens show both degranulation-related cytotoxicity and cytokine production characteristics similar to the functional capacities of NK cell subsets found in cattle ${ }^{14}$ and pigs $^{32}$. However, this observation differs from the functional distinction observed in mice based on higher cytotoxicity and cytokine production of one NK cell subset (CD27 high or CD94 ${ }^{\text {high }}$ ) compared to the other $\left(\mathrm{CD} 27^{\text {low/- }} \text { or } \mathrm{CD} 94^{\text {low/ } /}\right)^{13,33}$ and the functionally distinct cytotoxic $\left(\mathrm{CD} 56^{\mathrm{dim}}\right)$ and cytokine-producing (CD56 bright) circulating NK cell subsets in humans ${ }^{12}$. As noted previously, the phenotypical divers tissue-resident CD56 bright NK cell population may parallel a high functional diversity and the tissue microenvironment is suggested to play an important role in shaping tissue-specific NK cell functions ${ }^{6,34}$. In this context, the degranulation-related cytotoxicity of splenic and intraepithelial IL-2R $\alpha^{+}$NK cells in chickens is in agreement with that of tissue-resident CD56 bright NK cells in spleen ${ }^{34}$ and IELs ${ }^{16}$ in humans based on CD107 expression and high levels of intracellular perforin. The higher 
level of degranulation-related cytotoxicity of IL-2R $\alpha^{+} \mathrm{NK}$ cells in IELs as opposed to spleen may also be explained by the tissue microenvironment as a result from exposure to the content of the gut lumen ${ }^{16,35}$. The ability of NK cells to be activated rapidly upon recognition of invading pathogens indicates that intraepithelial NK cells are the first innate cells to respond during intestinal infection ${ }^{3,36}$. Since Salmonella enterica serotype Enteritidis (SE) is one of the major intestinal pathogens $\mathrm{s}^{37,38}$, an experimental SE infection model was used to study immunity, as well as the possibility to manipulate this, during SE infection in chickens. The role of NK cells during Salmonella infection in chickens was not described yet and therefore, numbers and functions of NK cell subsets were investigated in broiler chickens that were challenged with SE at seven days of age. On the first days post SE infection, an increase in the numbers of both IL-2R $\alpha^{+}$and $20 \mathrm{E} 5^{+}$intraepithelial NK cells was observed paralleled by significantly enhanced degranulation-related cytotoxicity and IFNY production of NK cells in the IELs and spleen as compared to uninfected chickens (Chapter 5). Although few double positive 20E5 ${ }^{+} \mathrm{IL}-2 \mathrm{R} \alpha^{+} \mathrm{NK}$ cells appeared to be represented in response to SE infection, a distinct population was not observed whereas a double positive population has been observed in humans where the CD56 dim NK cell subset expressed IL-2R $\alpha$ upon pre-stimulation of $F_{c}$ receptors or combinatorial cytokine stimulation ${ }^{10,11,39}$. The mainly observed individual IL-2R $\alpha^{+}$and $20 \mathrm{EF}^{+} \mathrm{NK}$ cells may therefore imply that activation of these subsets in response to SE is mediated by an NKR ${ }^{40}$ or TLRs ${ }^{41-45}$ as was shown for human and murine NK cells during bacterial infection. Unfortunately, the functional characteristics of the individual IL-2R $\alpha^{+}$ and $20 \mathrm{E} 5^{+} \mathrm{NK}$ cell subsets with regard to degranulation-related cytotoxicity and IFNY production during SE infection could not be analyzed due to incompatibility of available reagents. This needs to be explored in future studies to provide more insight in the activity of the chicken NK cell subsets during infection. Nevertheless, similar to our study, an increase in the numbers of both intestinal NK cell subsets (CD27 ${ }^{\text {high }}$ and $C D 27^{\text {low/- }}$ ) and enhanced degranulation-related cytotoxicity and IFNY production of the total intestinal NK cell population was observed in Salmonella Typhimurium (ST)-infected mice ${ }^{46}$. Furthermore, both NK cell subsets in the spleen of mice were implicated in IFN $\gamma$ production post ST infection although mostly by CD27 high $N K$ cells ${ }^{47}$. Based on increased numbers of both NK cell subsets paralleled by enhanced NK cell activation in our study and those in mice, it is suggested that both IL-2R $\alpha^{+}$and $20 \mathrm{E} 5^{+} \mathrm{NK}$ cells are involved in the response against SE although one of these subsets might be more implicated in degranulation-related cytotoxicity or IFN $\gamma$ production compared to the other, which may depend on tissue localization of the subsets.

\section{Tissue distribution and functions of $\mathrm{CD}^{+} \gamma \delta \mathrm{T}$ cell and $\alpha \beta$ T cell subsets}

In addition to NK cells, intraepithelial $\gamma \delta \mathrm{T}$ cells and $\mathrm{CD}^{+} \alpha \beta \mathrm{T}$ cells may play a role in the innate respectively adaptive immune responses to intestinal infections. Both intraepithelial $\gamma \delta$ and $\alpha \beta$ T cells were shown to express $C D 8 \alpha \alpha$ or $C D 8 \alpha \beta^{48,49}$. Both CD8 isoforms are structurally similar, however, $C D 8 \alpha \beta^{+} \alpha \beta$ T cells are implicated in specific antigen-driven cytotoxicity whereas CD8 $\alpha \alpha^{+} \alpha \beta$ T cells show rapid NK-like non-specific cytotoxicity as well as anti-inflammatory cytokine production to maintain the intestinal barrier ${ }^{48,50}$. The rapid activation as well as the functions of $C D 8 \alpha \alpha^{+} \alpha \beta$ T cells are similar to $\gamma \delta T_{\text {cells }}^{48,50}$. In chickens, only few studies have investigated CD8 $\alpha \alpha$ and CD $8 \alpha \beta$ expression on T cells but in these studies no distinction was made between $\gamma \delta$ and $\alpha \beta$ T cells and therefore, expression of CD8 isoforms on both $\gamma \delta$ and $\alpha \beta$ T cells was determined. During early life of chickens, expression of CD $8 \alpha \alpha$ and $C D 8 \alpha \beta$ on both $\gamma \delta$ and $\alpha \beta$ T cells is similar in the IELs, spleen and blood, whereas in adult chickens expression of $C D 8 \alpha \beta$ on both $\gamma \delta$ and $\alpha \beta$ T cells is higher. Furthermore, the populations of $C D 8 \alpha \alpha^{+} \gamma \delta$ and $\alpha \beta$ T cells were most abundant in the IELs compared to other tissues throughout aging (Chapter 2). These findings are in agreement with studies in humans and mice in which most of the $C D 8^{+} \gamma \delta$ and $\alpha \beta$ T cells expressed $C D 8 \alpha \beta$ compared to $C D 8 \alpha \alpha$ and the highest numbers of $C D 8 \alpha \alpha^{+} \gamma \delta$ 
and $\alpha \beta$ T cells were found in the intestine ${ }^{51}$. Next, the involvement of CD8 $\alpha \alpha^{+}$and CD8 $\alpha \beta^{+} \gamma \delta$ T cells as well as $\alpha \beta$ T cells was investigated during SE infection in young chickens. Numbers of intraepithelial CD $8 \alpha \alpha^{+}$and $C D 8 \alpha \beta^{+} \gamma \delta$ T cells as well as $\alpha \beta$ T cells were increased early and three weeks post-infection as compared to uninfected chickens, although only significantly of the CD8 $\alpha \alpha^{+} \gamma \delta$ and $\alpha \beta T$ cells. In addition, although low levels of degranulation-related cytotoxicity and IFN $\gamma$ production were determined, only an increase in degranulation-related cytotoxicity of the total $\mathrm{CD} 8^{+} \mathrm{T}$ cell population was observed early post-infection in the IELs and spleen (Chapter 5). The increase in numbers of $C D 8 \alpha \alpha^{+}$and $C D 8 \alpha \beta^{+} \gamma \delta$ T as well as $\alpha \beta$ T cells in the early and late response to SE are consistent with increased percentages of $C D 8 \alpha^{+}$and $C D 8 \beta^{+}$cells in the caeca of chickens post SE immunization ${ }^{52}$ and $\mathrm{CD}^{+} \gamma \delta \mathrm{T}$ cells in the IELs of mice post ST infection ${ }^{53}$. Furthermore, the increase in degranulation-related cytotoxicity of intraepithelial $\mathrm{CD} 8^{+} \mathrm{T}$ cells was in agreement with enhanced lysis of target cells by intraepithelial T cells observed in mice post ST infection ${ }^{53}$. Based on data obtained and similar results in other studies, it is hypothesized that both $C D 8 \alpha \alpha^{+}$and $C D 8 \alpha \beta^{+} T$ cells, including $\gamma \delta$ and $\alpha \beta T$ cells, are involved in cytotoxicity by degranulation of granules in response to SE infection of young chickens. In addition, the CD8 $\alpha \alpha^{+} \gamma \delta$ and $\alpha \beta$ T cells are suggested to be implicated in maintaining the intestinal barrier as was shown in humans and mice ${ }^{48,50}$, since no clinical symptoms of chickens were observed during SE infection. Future studies should explore the cytotoxicity and anti-inflammatory cytokine production of the individual $C D 8 \alpha \alpha^{+}$and $C D 8 \alpha \beta^{+} \gamma \delta$ T cells as well as $\alpha \beta$ T cells, in order to elucidate the role of $C D 8 \alpha \alpha^{+}$and $C D 8 \alpha \beta^{+}$T cells during infection. Our results and other studies in chickens and mice show that the total IEL population; including NK cells ${ }^{54}, C D 8 \alpha \alpha / \alpha \beta^{+} \gamma \delta$ and $\alpha \beta T$ cells $s^{52,53}$, is important in the response to Salmonella since numbers of bacteria decreased during infection. In addition, our study emphasizes the importance of intraepithelial NK cells due to their significantly enhanced degranulation-related cytotoxicity and IFN $y$ production early in response to SE. Therefore, stimulation of immune responsiveness of these IELs and NK cells in particular by feed additives may increase the resistance of young chickens to pathogens like Salmonella.

\section{Modulation of the activity of IELs, with emphasis on NK cells}

Following the identification of important NK and T cell subsets, the ability to stimulate immune responsiveness of these cells by feed additives, directly and/or indirectly through modulation of intestinal microbiota, in order to increase resistance of young chickens was investigated. Firstly, the interaction between microbiota and NK and T cells in chickens was explored by exposure to adult-derived microbiota (AM) immediately post-hatch. AM inoculation accelerated the maturation of intestinal microbiota paralleled by an increase in the number of intraepithelial IL-2R $\alpha^{+} \mathrm{NK}$ cells and enhanced degranulation-related cytotoxicity of NK cells in the IELs and spleen of young broiler chickens. In addition, AM resulted in enhanced NK cell activation in three week old chickens and an increase in the number of CD8 $\alpha \alpha^{+} \alpha \beta$ T cells in the IEL population of two and three week old chickens (Chapter 3). The observed association between early life development of the immune system and intestinal microbiota that result from AM exposure are in agreement with continuous interaction between the intestinal immune system and microbiota observed in chickens ${ }^{55}$ and mammals ${ }^{56}$. Furthermore, the interaction indicates that modulation of microbiota composition can stimulate NK cell responsiveness in young and adult chickens as was shown previously in humans and mice ${ }^{57,58}$. Subsequently, the capacity of feed additives to stimulate immunity directly as well as indirectly by modulation of the intestinal microbiota composition was investigated. Nutritional compounds were consecutively screened for enhanced activity of NK cells and macrophages in vitro, and evaluated for safety during embryonic development in ovo and impact on performance traits in vivo. Based on these results, two plant polysaccharides; glucose oligosaccharide and long-chain glucomannan, were selected for further investigation in vivo. Both feed additives enhanced degranulation-related cytotoxicity of intraepithelial 
NK cells, which was positively correlated with increased relative abundance of lactic acid bacteria (LAB) in ileum and caeca of young chickens (Chapter 4). The enhanced degranulation-related cytotoxicity of NK cells in vitro and in vivo show that both feed additives have the ability to directly stimulate NK cell responsiveness, as was reported for other plant polysaccharides in chickens and humans ${ }^{59}$. The increased relative abundance of commensal $L A B$ suggests that both feed additives act as prebiotic for these microbes ${ }^{60}$ and is consistent with other studies that observed increased levels of LAB following feed supplementation, which consequently promoted chicken health ${ }^{61,62}$. Furthermore, the positive correlation between commensal LAB and NK cell responsiveness suggests that both feed additives are capable of indirect stimulation of NK cells by interaction with specific microbial species ${ }^{63}$. Possible mechanisms underlying the interaction of immune cells with microbiota and feed are given based on studies in humans and mice. In these studies, both diet and microbiota were shown to be involved in metabolic regulation of immune cell responses; controlling the uptake and catabolism of nutrients in quiescent and activated immune cells. Ligands derived from commensal microbes or diet may diffuse across the plasma membrane of IELs and bind to the cytosolic aryl hydrocarbon receptor (AHR), which was shown to be a crucial factor in maintaining numbers and regulating functions of IELs ${ }^{64-66}$. In addition, these ligands may bind to AHR and pattern recognition receptors (PRRs) of epithelial cells, and macrophages and dendritic cells located in the lamina propria resulting in production of cytokines that regulate functions of IELs ${ }^{35,67-71}$. Commensal microbes and feed additives may also regulate functions of IELs by recognition through NCRs and PRRs expressed on these cells $s^{35,70,72-75}$. Furthermore, the observed increased relative abundance of commensal LAB by feed supplementation may consequently result in increased levels of short-chain fatty acids in the gut ${ }^{76,77}$. These microbiota-derived metabolites may interact with IELs through G-protein coupled receptors (GPCRs) inducing anti- or proinflammatory functions ${ }^{78,79}$. The AHR and several PRRs, NCRs and GPCRs have been identified in chickens ${ }^{80-83}$ and future studies should explore whether these receptors are involved in the interaction of IELs with microbiota and diet similar to the pathways observed in humans and mice. Elucidating involvement of these interaction pathways in chickens will aid in developing strategies to boost the host immune defense.

Based on the findings reported in Chapter 4, we hypothesized that supplementation of long-chain glucomannan (GM) may increase the resistance of young chickens to pathogens and investigated this in our final study in which GM supplemented chickens were exposed to SE. GM supplementation indeed led to increased resistance of young chickens since numbers of SE in the spleen were decreased and significantly lower colonization of SE in the ileum and caeca was observed. These findings coincided with increased intraepithelial NK cell responsiveness and relative abundance of LAB post-infection. In addition, the relative abundance of commensal LAB was negatively correlated with SE while positively correlated with splenic NK cell cytotoxicity and serum SE-specific antibody responses three weeks post-infection (Chapter 6). The observed levels of commensal LAB and correlations may increase the resistance of chickens through competitive exclusion ${ }^{84}$ and by interaction with NK cells ${ }^{63}$. The enhanced degranulation-related cytotoxicity of intraepithelial NK cells observed in the GM group post SE infection might imply trained immunity or innate memory based on human and murine studies. These studies showed that microbiota- or diet-derived ligands are recognized by PRRs on NK cells upon primary exposure and results in an enhanced rapid memory response upon pathogenic secondary exposure ${ }^{74,85-87}$. Furthermore, the stimulation of $20 \mathrm{E} 5^{+}$intraepithelial NK cell numbers by GM post SE infection may result from proliferation or differentiation of IL-2R $\alpha^{+} N K$ cells into this subset ${ }^{21,25,26}$, which remains to be elucidated in future studies. Interestingly, GM stimulated numbers of intraepithelial CD8 ${ }^{+}$ $\alpha \beta T$ cells in two week old chickens more pronouncedly during infection compared to no infection as described in Chapter 4. These findings show that GM supplementation stimulates responsiveness of NK cells rather than T cells, which is suggested to be the key factor in the increased resistance of young 
chickens since NK cells showed higher responsiveness to SE compared to T cells (Chapter 5). Moreover, the enhanced NK cell responsiveness in young chickens by GM supplementation may increase the resistance to a variety of pathogens due to the non-specific nature of NK cells ${ }^{36}$. Therefore, interest is growing in innate and NK cell therapies in addition to specific adaptive immunotherapies such as vaccination. Stimulation of innate and NK cell responsiveness were reported as effective therapies to combat pathogens and cancer in humans ${ }^{88-90}$. Hence, stimulation of NK cell responsiveness in young chickens by nutritional strategies can be considered as an effective approach to increase resistance to pathogens and consequently improve health of chickens.

\section{Implications and future perspectives}

The aim of this thesis was to investigate whether immune responsiveness of young broiler chickens could be stimulated by nutritional strategies in order to increase the resistance and consequently health of chickens. Results of our studies showed that feed supplementation enhanced NK cell responsiveness in young broiler chickens, which resulted in increased resistance to Salmonella infection. Feed additives were shown to enhance NK cell responsiveness directly and/or indirectly by modulation of the intestinal microbiota and the interactions between microbes and immune cells. The knowledge obtained will aid in the development of nutritional strategies specifically targeting NK cells to strengthen the first line of defense of young chickens. Regarding long-chain glucomannan supplementation, practical application requires further investigation such as larger scale trials including detailed monitoring of performance and analysis of the transmission of SE from infected to uninfected chickens within a flock. In addition, the potential of GM diet to increase resistance of young chickens to a variety of pathogens should be validated by challenge experiments with different pathogens. In the research described in Chapter $\mathbf{2}$ and 5, two NK cell subsets are shown to be important in the response to SE and both subsets are suggested to be involved in degranulation-related cytotoxicity and cytokine production. Future studies should identify the ligands recognized by the 20E5 biomarker and develop a better pan NK-marker(s) in chickens to enhance our understanding of chicken NK cell biology. In addition, an extensive analysis of genes related to cytotoxic and regulatory function that are expressed in the NK cell subsets in different tissues will aid in the development of strategies targeting NK cell responsiveness. Furthermore, in the studies presented in Chapter 3, 4 and $\mathbf{6}$ interactions of immune cells with intestinal microbiota and diet components are observed. Assessment of the expression of AHR, PRRs, NCRs and GPCRs in NK cell subsets in future studies may elucidate the interaction pathways of NK cells with microbiota and diet, and contribute to the development of strategies to strengthen the first line of defense in young chickens. Eventually, this may lead to practical application of nutritional strategies that stimulate immune responsiveness, directly and/or indirectly by modulation of the intestinal microbiota, in order to increase the resistance of young chickens and consequently improve chicken health. 


\section{References}

1. McEwen SA, Angulo FJ, Collignon PJ, Conly JM. Unintended consequences associated with national-level restrictions on antimicrobial use in food-producing animals. Lancet Planet Health. 2018;2(7):e279-e282. doi:10.1016/S2542-5196(18)30138-4.

2. Mehdi $\mathrm{Y}$, Létourneau-Montminy $\mathrm{M}$-, Gaucher $\mathrm{M}$-, et al. Use of antibiotics in broiler production: Global impacts and alternatives. Anim Nutr. 2018;4(2):170-178. doi:10.1016/j.aninu.2018.03.002.

3. Göbel TWF, Kaspers B, Stangassinger M. NK and T cells constitute two major, functionally distinct intestinal epithelial lymphocyte subsets in the chicken. Int Immunol. 2001;13(6):757-762.

4. Jansen CA, van de Haar, P. M., van Haarlem D, et al. Identification of new populations of chicken natural killer (NK) cells. Dev Comp Immunol. 2010;34(7):759-767. doi:10.1016/j.dci.2010.02.009.

5. Walzer T, Bléry M, Chaix J, et al. Identification, activation, and selective in vivo ablation of mouse NK cells via NKp46. Proc Natl Acad Sci U S A. 2007;104(9):3384-3389. doi:10.1073/pnas.0609692104.

6. Melsen JE, Lugthart G, Lankester AC, Schilham MW. Human Circulating and Tissue-Resident CD56bright Natural Killer Cell Populations. Front Immunol. 2016;7(JUN). doi:10.3389/fimmu.2016.00262.

7. Hayakawa Y, Huntington ND, Nutt SL, Smyth MJ. Functional subsets of mouse natural killer cells. Immunol Rev. 2006;214(1):47-55. doi:10.1111/j.1600-065X.2006.00454.x.

8. Hamilton CA, Mahan $\mathrm{S}$, Bell $\mathrm{CR}$, et al. Frequency and phenotype of natural killer cells and natural killer cell subsets in bovine lymphoid compartments and blood. Immunology. 2017;151(1):89-97. doi:10.1111/imm.12708.

9. Rudnicka K, Matusiak A, Chmiela M. CD25 (IL-2R) expression correlates with the target cell induced cytotoxic activity and cytokine secretion in human natural killer cells. Acta Biochim Pol. 2015;62(4):885-894. doi:10.18388/abp.2015_1152.

10. Duggan MC, Campbell AR, McMichael EL, et al. Co-stimulation of the fc receptor and interleukin-12 receptor on human natural killer cells leads to increased expression of cd25. Oncolmmunology. 2018;7(2). doi:10.1080/2162402X.2017.1381813.

11. Leong JW, Chase JM, Romee R, et al. Preactivation with IL-12, IL-15, and IL-18 induces Cd25 and a functional high-affinity il-2 receptor on human cytokine-induced memory-like natural killer cells. Biol Blood Marrow Transplant. 2014;20(4):463-473. doi:10.1016/j.bbmt.2014.01.006.

12. Cooper MA, Fehniger TA, Caligiuri MA. The biology of human natural killer-cell subsets. Trends in Immunology. 2001;22(11):633640. doi:10.1016/S1471-4906(01)02060-9.

13. Hayakawa Y, Smyth MJ. CD27 dissects mature NK cells into two subsets with distinct responsiveness and migratory capacity. J Immunol. 2006;176(3):1517-1524. doi:10.4049/jimmunol.176.3.1517.

14. Boysen P, Olsen I, Berg I, Kulberg S, Johansen GM, Storset AK. Bovine CD2-/NKp46+ cells are fully functional natural killer cells with a high activation status. BMC Immunol. 2006;7. doi:10.1186/1471-2172-7-10.

15. Todd DJ, Greiner DL, Rossini AA, Mordes JP, Bortell R. An atypical population of NK cells that spontaneously secrete IFN- $\gamma$ and IL-4 is present in the intraepithelial lymphoid compartment of the rat. J Immunol. 2001;167(7):3600-3609. doi:10.4049/ jimmunol.167.7.3600.

16. Eiras $\mathrm{P}$, Leon $\mathrm{F}$, Camarero $\mathrm{C}$, et al. Intestinal intraepithelial lymphocytes contain a CD3- CD7+ subset expressing natural killer markers and a singular pattern of adhesion molecules. Scand J Immunol. 2000;52(1):1-6. doi:10.1046/j.1365-3083.2000.00761.x.

17. Leon F, Roldan E, Sanchez L, Camarero C, Bootello A, Roy G. Human small-iintestinal epithelium contains functional natural killer lymphocytes. Gastroenterology. 2003;125(2):345-356. doi:10.1016/S0016-5085(03)00886-2.

18. Borrego F, Peña J, Solana R. Regulation of CD69 expression on human natural killer cells: differential involvement of protein kinase C and protein tyrosine kinases. Eur J Immunol. 1993;23(5):1039-1043. doi:10.1002/eji.1830230509.

19. Abdolmaleki M, Yeap SK, Tan SW, et al. Effects of newcastle disease virus infection on chicken intestinal intraepithelial natural killer cells. Front Immunol. 2018;9(JUN). doi:10.3389/fimmu.2018.01386.

20. Jahromi MZ, Bello MB, Abdolmaleki M, Yeap SK, Hair-Bejo M, Omar AR. Differential activation of intraepithelial lymphocytenatural killer cells in chickens infected with very virulent and vaccine strains of infectious bursal disease virus. Dev Comp Immunol. 2018;87:116-123. doi:10.1016/j.dci.2018.06.004.

21. Cichocki F, Grzywacz B, Miller JS. Human NK cell development: One road or many? Front Immunol. 2019;10(AUG). doi:10.3389/ fimmu.2019.02078.

22. Bonanni V, Sciumè G, Santoni A, Bernardini G. Bone marrow NK cells: Origin, distinctive features, and requirements for tissue localization. Front Immunol. 2019;10(JULY). doi:10.3389/fimmu.2019.01569.

23. Sun JC, Lanier LL. NK cell development, homeostasis and function: Parallels with CD8 + T cells. Nat Rev Immunol. 2011;11(10):645657. doi:10.1038/nri3044.

24. Montaldo E, Del Zotto G, Della Chiesa M, et al. Human NK cell receptors/markers: A tool to analyze NK cell development, subsets and function. Cytometry Part A. 2013;83(8):702-713. doi:10.1002/cyto.a.22302.

25. Crinier A, Dumas P-, Escalière B, et al. Single-cell profiling reveals the trajectories of natural killer cell differentiation in bone marrow and a stress signature induced by acute myeloid leukemia. Cell Mol Immunol. 2021;18(5):1290-1304. doi:10.1038/ s41423-020-00574-8.

26. Chiossone L, Chaix J, Fuseri N, Roth C, Vivier E, Walzer T. Maturation of mouse NK cells is a 4-stage developmental program. Blood. 2009;113(22):5488-5496. doi:10.1182/blood-2008-10-187179.

27. Smyth MJ, Cretney E, Kelly JM, et al. Activation of NK cell cytotoxicity. Mol Immunol. 2005;42(4 SPEC. ISS.):501-510. doi:10.1016/j. molimm.2004.07.034. 
28. Horowitz A, Stegmann KA, Riley EM. Activation of natural killer cells during microbial infections. Front Immunol. 2012;2(JAN). doi:10.3389/fimmu.2011.00088.

29. Fenzl L, Göbel TW, Neulen M-. $\gamma \delta$ T cells represent a major spontaneously cytotoxic cell population in the chicken. Dev Comp Immunol. 2017;73:175-183. doi:10.1016/j.dci.2017.03.028.

30. Wendt K, Wilk E, Buyny S, Buer J, Schmidt RE, Jacobs R. Gene and protein characteristics reflect functional diversity of CD56 dim and CD56bright NK cells. J Leukocyte Biol. 2006;80(6):1529-1541. doi:10.1189/jlb.0306191.

31. Bi J, Wang X. Molecular Regulation of NK Cell Maturation. Front Immunol. 2020;11. doi:10.3389/fimmu.2020.01945.

32. Mair KH, Essler SE, Patzl M, Storset AK, Saalmüller A, Gerner W. NKp46 expression discriminates porcine NK cells with different functional properties. Eur J Immunol. 2012;42(5):1261-1271. doi:10.1002/eji.201141989.

33. Yu J, Wei M, Mao H, et al. CD94 defines phenotypically and functionally distinct mouse NK cell subsets. J Immunol. 2009;183(8):49684974. doi:10.4049/jimmunol.0900907.

34. Lugthart G, Melsen JE, Vervat C, et al. Human lymphoid tissues harbor a distinct CD69+CXCR6+ NK cell population. J Immunol. 2016;197(1):78-84. doi:10.4049/jimmunol.1502603.

35. Poggi A, Benelli R, Venè R, et al. Human gut-associated natural killer cells in health and disease. Front Immunol. 2019;10(MAY). doi:10.3389/fimmu.2019.00961.

36. Juul-Madsen HR, Viertlböeck B, Härtle S, Smith AL, Göbel TW. Innate Immune Responses. Innate Immune Responses. ; 2013:121-147.

37. Suzuki S. Pathogenicity of Salmonella enteritidis in poultry. Int J Food Microbiol. 1994;21(1-2):89-105. doi:10.1016/01681605(94)90203-8.

38. Arlet G, Barrett TJ, Butaye P, Cloeckaert A, Mulvey MR, White DG. Salmonella resistant to extended-spectrum cephalosporins: prevalence and epidemiology. Microbes Infect. 2006;8(7):1945-1954. doi:10.1016/j.micinf.2005.12.029.

39. Márquez M-, Millet C, Stekman H, et al. CD16 cross-linking induces increased expression of CD56 and production of IL-12 in peripheral NK cells. Cell Immunol. 2010;264(1):86-92. doi:10.1016/j.cellimm.2010.05.002.

40. Sivori S, Falco M, Carlomagno S, et al. A novel KIR-associated function: Evidence that CpG DNA uptake and shuttling to early endosomes is mediated by KIR3DL2. Blood. 2010;116(10):1637-1647. doi:10.1182/blood-2009-12-256586.

41. Marcenaro E, Ferranti B, Falco M, Moretta L, Moretta A. Human NK cells directly recognize Mycobacterium bovis via TLR2 and acquire the ability to kill monocyte-derived DC. Int Immunol. 2008;20(9):1155-1167. doi:10.1093/intimm/dxn073.

42. Chalifour A, Jeannin P, Gauchat J-, et al. Direct bacterial protein PAMP recognition by human NK cells involves TLRs and triggers $\alpha$-defensin production. Blood. 2004;104(6):1778-1783. doi:10.1182/blood-2003-08-2820.

43. Sivori $\mathrm{S}$, Falco M, Della Chiesa M, et al. CpG and double-stranded RNA trigger human NK cells by toll-like receptors: Induction of cytokine release and cytotoxicity against tumors dendritic cells. Proc Natl Acad Sci U S A. 2004;101(27):10116-10121. doi:10.1073/pnas.0403744101.

44. Sawaki J, Tsutsui $\mathrm{H}$, Hayashi N, et al. Type 1 cytokine/chemokine production by mouse NK cells following activation of their TLR/ MyD88-mediated pathways. Int Immunol. 2007;19(3):311-320. doi:10.1093/intimm/dxl148.

45. Souza-Fonseca-Guimaraes F, Adib-Conquy M, Cavaillon JM. Natural killer (NK) cells in antibacterial innate immunity: angels or devils? Mol Med. 2012;18(1):270-285. doi:10.2119/molmed.2011.00201.

46. Müller AA, Dolowschiak T, Sellin ME, et al. An NK Cell Perforin Response Elicited via IL-18 Controls Mucosal Inflammation Kinetics during Salmonella Gut Infection. PLoS Pathog. 2016;12(6). doi:10.1371/journal.ppat.1005723.

47. Kupz A, Scott TA, Belz GT, et al. Contribution of Thy $1+$ NK cells to protective IFN- $\gamma$ production during Salmonella Typhimurium infections. Proc Natl Acad Sci U S A. 2013;110(6):2252-2257. doi:10.1073/pnas.1222047110.

48. Van Kaer L, Olivares-Villagomez D. Development, homeostasis, and functions of intestinal intraepithelial lymphocytes. J Immunol. 2018;200(7):2235-2244. doi:10.4049/jimmunol.1701704.

49. Kadivar M, Petersson J, Svensson L, Marsal J. CD8 $\alpha \beta+\gamma \delta$ T cells: A novel T cell subset with a potential role in inflammatory bowel disease. J Immunol. 2016;197(12):4584-4592. doi:10.4049/jimmunol.1601146.

50. Cheroutre H, Lambolez F, Mucida D. The light and dark sides of intestinal intraepithelial lymphocytes. Nat Rev Immunol. 2011;11(7):445-456. doi:10.1038/nri3007.

51. Cheroutre H. Starting at the beginning: New perspectives on the biology of mucosal T cells. Vol 22. ; 2004.

52. Berndt A, Pieper J, Methner U. Circulating $\gamma \delta T$ cells in response to Salmonella enterica serovar enteritidis exposure in chickens. Infect Immun. 2006;74(7):3967-3978. doi:10.1128/IAl.01128-05.

53. Li Z, Zhang C, Zhou Z, Zhang J, Zhang J, Tian Z. Small intestinal intraepithelial lymphocytes expressing CD8 and T cell receptor $\gamma \delta$ are involved in bacterial clearance during salmonella enterica serovar typhimurium infection. Infect Immun. 2012;80(2):565574. doi:10.1128/IAI.05078-11.

54. Ashkar AA, Reid S, Verdu EF, Zhang K, Coombes BK. Interleukin-15 and NK1.1+ cells provide innate protection against acute Salmonella enterica serovar typhimurium infection in the gut and in systemic tissues. Infect Immun. 2009;77(1):214-222. doi:10.1128/IAI.01066-08.

55. Broom LJ, Kogut MH. The role of the gut microbiome in shaping the immune system of chickens. Vet Immunol Immunopathol. 2018;204:44-51. doi:10.1016/j.vetimm.2018.10.002.

56. Macpherson AJ, Harris NL. Interactions between commensal intestinal bacteria and the immune system. Nature Reviews Immunology. 2004;4(6):478-485. doi:10.1038/nri1373.

57. Ferlazzo G, Rizzello V, Bonaccorsi I, Dongarr ML, Fink LN. Role of natural killer and dendritic cell crosstalk in immunomodulation by commensal bacteria probiotics. J Biomed Biotechnol. 2011;2011. doi:10.1155/2011/473097. 
58. Makino S, Sato A, Goto A, et al. Enhanced natural killer cell activation by exopolysaccharides derived from yogurt fermented with Lactobacillus delbrueckii ssp. bulgaricus OLL1073R-1. J Dairy Sci. 2016;99(2):915-923. doi:10.3168/jds.2015-10376.

59. Guo FC, Savelkoul HFJ, Kwakkel RP, Williams BA, Verstegen MWA. Immunoactive, medicinal properties of mushroom and herb polysaccharides and their potential use in chicken diets. World's Poult Sci J. 2003;59(4):427-440. doi:10.1079/WPS20030026.

60. Davani-Davari D, Negahdaripour M, Karimzadeh I, et al. Prebiotics: definition, types, sources, mechanisms, and clinical applications. Foods. 2019;8(3):92.

61. Yadav S, Jha R. Strategies to modulate the intestinal microbiota and their effects on nutrient utilization, performance, and health of poultry. J Animal Sci Biotechnol. 2019;10(1). doi:10.1186/s40104-018-0310-9.

62. Kogut MH. The effect of microbiome modulation on the intestinal health of poultry. Anim Feed Sci Technol. 2019;250:32-40. doi:10.1016/j.anifeedsci.2018.10.008.

63. Faas MM, Liu Y, Borghuis T, van Loo-Bouwman CA, Harmsen H, de Vos P. Microbiota Induced Changes in the Immune Response in Pregnant Mice. Front Immunol. 2020;10. doi:10.3389/fimmu.2019.02976.

64. Li Y, Innocentin S, Withers DR, et al. Exogenous stimuli maintain intraepithelial lymphocytes via aryl hydrocarbon receptor activation. Cell. 2011;147(3):629-640. doi:10.1016/j.cell.2011.09.025.

65. Shin JH, Zhang L, Murillo-Sauca O, et al. Modulation of natural killer cell antitumor activity by the aryl hydrocarbon receptor. Proc Natl Acad Sci U S A. 2013;110(30):12391-12396. doi:10.1073/pnas.1302856110.

66. Li S, Bostick JW, Zhou L. Regulation of innate lymphoid cells by aryl hydrocarbon receptor. Front Immunol. 2018;8(JAN). doi:10.3389/fimmu.2017.01909.

67. Hoytema van Konijnenburg, D. P., Reis BS, Pedicord VA, Farache J, Victora GD, Mucida D. Intestinal Epithelial and Intraepithelial T Cell Crosstalk Mediates a Dynamic Response to Infection. Cell. 2017;171(4):783-794.e13. doi:10.1016/j.cell.2017.08.046.

68. Konjar Š, Ferreira C, Blankenhaus B, Veldhoen M. Intestinal barrier interactions with specialized CD8 T cells. Front Immunol. 2017;8(OCT). doi:10.3389/fimmu.2017.01281.

69. Jiang W, Wang X, Zeng B, et al. Recognition of gut microbiota by NOD2 is essential for the homeostasis of intestinal intraepithelial lymphocytes. J Exp Med. 2013;210(11):2465-2476. doi:10.1084/jem.20122490.

70. Sonnenberg G, Artis D. Innate Lymphoid Cell Interactions with Microbiota: Implications for Intestinal Health and Disease. Immunity. 2012;37(4):601-610. doi:10.1016/j.immuni.2012.10.003.

71. Gutiérrez-Vázquez C, Quintana FJ. Regulation of the Immune Response by the Aryl Hydrocarbon Receptor. Immunity. 2018;48(1):19-33. doi:10.1016/j.immuni.2017.12.012.

72. Martin B, Hirota K, Cua DJ, Stockinger B, Veldhoen M. Interleukin-17-Producing $\gamma \delta T$ Cells Selectively Expand in Response to Pathogen Products and Environmental Signals. Immunity. 2009;31(2):321-330. doi:10.1016/j.immuni.2009.06.020.

73. Rahman AH, Taylor DK, Turka LA. The contribution of direct TLR signaling to T cell responses. Immunol Res. 2009;45(1):25-36. doi:10.1007/s12026-009-8113-X.

74. Huang Y-, He T-, Cuan X-, Wang XJ, Hu J-, Sheng J. 1,4- $\beta$-D-Glucomannan from dendrobium officinale activates nf-kb via tlr4 to regulate the immune response. Molecules. 2018;23(10). doi:10.3390/molecules23102658.

75. Vandereyken M, James OJ, Swamy M. Mechanisms of activation of innate-like intraepithelial T lymphocytes. Mucosal Immunol. 2020;13(5):721-731. doi:10.1038/s41385-020-0294-6.

76. Novik G, Savich V. Beneficial microbiota. Probiotics and pharmaceutical products in functional nutrition and medicine. Microbes Infect. 2020;22(1):8-18. doi:10.1016/j.micinf.2019.06.004.

77. Stanley D, Hughes RJ, Moore RJ. Microbiota of the chicken gastrointestinal tract: Influence on health, productivity and disease. Appl Microbiol Biotechnol. 2014;98(10):4301-4310. doi:10.1007/s00253-014-5646-2.

78. Alvarez-Curto E, Milligan G. Metabolism meets immunity: The role of free fatty acid receptors in the immune system. Biochem Pharmacol. 2016;114:3-13. doi:10.1016/j.bcp.2016.03.017.

79. Husted AS, Trauelsen M, Rudenko O, Hjorth SA, Schwartz TW. GPCR-Mediated Signaling of Metabolites. Cell Metab. 2017;25(4):777796. doi:10.1016/j.cmet.2017.03.008.

80. Walker MK, Heid SE, Smith SM, Swanson HI. Molecular characterization and developmental expression of the aryl hydrocarbon receptor from the chick embryo. Comp Biochem Physiol C Pharmacol Toxicol Endocrinol. 2000;126(3):305-319. doi:10.1016/ S0742-8413(00)00119-5.

81. Kannaki TR, Reddy MR, Shanmugam M, Verma PC, Sharma RP. Chicken toll-like receptors and their role in immunity. World's Poult Sci J. 2010;66(4):727-738. doi:10.1017/S0043933910000693.

82. Straub C, Neulen M-, Sperling B, et al. Chicken NK cell receptors. Dev Comp Immunol. 2013;41(3):324-333. doi:10.1016/j. dci.2013.03.013.

83. Lagerström MC, Hellström AR, Gloriam DE, Larsson TP, Schiöth HB, Fredriksson R. The G protein-coupled receptor subset of the chicken genome. PLoS Comput Biol. 2006;2(6):493. doi:10.1371/journal.pcbi.0020054.

84. Ferreira AJP, Ferreira CSA, Knobl T, et al. Comparison of three commercial competitive-exclusion products for controlling Salmonella colonization of broilers in Brazil. J Food Protection. 2003;66(3):490-492. doi:10.4315/0362-028X-66.3.490.

85. Kleinnijenhuis J, Quintin J, Preijers F, et al. BCG-induced trained immunity in NK cells: Role for non-specific protection to infection. Clin Immunol. 2014;155(2):213-219. doi:10.1016/j.clim.2014.10.005.

86. Negi S, Das DK, Pahari S, Nadeem S, Agrewala JN. Potential role of gut microbiota in induction and regulation of innate immune memory. Front Immunol. 2019;10(OCT). doi:10.3389/fimmu.2019.02441.

87. Netea MG, Domínguez-Andrés J, Barreiro LB, et al. Defining trained immunity and its role in health and disease. Nat Rev Immunol. 2020;20(6):375-388. doi:10.1038/s41577-020-0285-6. 
88. Demaria O, Cornen S, Daëron M, Morel Y, Medzhitov R, Vivier E. Harnessing innate immunity in cancer therapy. Nature. 2019;574(7776):45-56. doi:10.1038/s41586-019-1593-5.

89. Bergman P, Raqib R, Rekha RS, Agerberth B, Gudmundsson GH. Host Directed Therapy Against Infection by Boosting Innate Immunity. Front Immunol. 2020;11. doi:10.3389/fimmu.2020.01209.

90. Liu S, Galat V, Galat4 Y, Lee YKA, Wainwright D, Wu J. NK cell-based cancer immunotherapy: from basic biology to clinical development. J Hematol Oncol. 2021;14(1). doi:10.1186/s13045-020-01014-w. 



\title{
APPENDICES
}

Nederlandse samenvatting

Dankwoord / Acknowledgements

\author{
Curriculum Vitae
}

List of Publications 


\section{Nederlandse samenvatting}

De pluimveehouderij levert een grote bijdrage aan de wereldvoedselvoorziening door de productie van eieren en vlees, die in de komende tien jaar sterk zal stijgen. Gezondheid en welzijn van vleeskuikens zijn van groot belang, echter, het verbod op preventief gebruik van antibiotica heeft geleid tot een toename van darmgezondheidsproblemen in pluimvee. Hierdoor is er behoefte aan alternatieve manieren om de weerstand van kippen tegen infecties te verhogen. Stimulatie van het immuunsysteem en modulatie van de darmmicrobiota samenstelling in kuikens door middel van voer is een mogelijkheid. Een verhoogde immuunresponsiviteit na uitkomst wordt verondersteld de weerstand van kuikens te verhogen tegen infecties zoals met Salmonella, een belangrijk darmpathogeen, en zal vervolgens leiden tot een verbeterde gezondheid van kippen.

\section{Het immuunsysteem}

Het afweersysteem is ruwweg in te delen in een aangeboren en een aangeleerd immuunsysteem, en beschermt een organisme tegen ziekteverwekkers zoals bacteriën en virussen. Nadat een pathogeen de lichaamsbarrières (zoals epitheelcellen en slijmvliezen) is gepasseerd zullen de aangeboren immuuncellen als eerste reageren. Het aangeboren immuunsysteem bestaat uit (witte bloed) cellen en moleculen die al vanaf de geboorte aanwezig zijn, algemene structuren van pathogenen herkennen en onmiddellijk bescherming bieden door vermeerdering van pathogenen te verhinderen. Natural killer (NK) cellen zijn belangrijke witte bloedcellen van het aangeboren immuunsysteem die snel bacteriën, virus-geïnfecteerde cellen en tumor cellen kunnen doden. Dit kan door middel van verschillende mechanismen, de bekendste is degranulatie; het afgeven van granulen waarin perforinen en granzymen zitten die leiden tot celdood van ziekteverwekkers. Daarnaast kunnen NK cellen andere witte bloedcellen, van zowel het aangeboren als het aangeleerde immuunsysteem, rekruteren en activeren door signaalmoleculen (chemokinen respectievelijk cytokinen) te produceren zoals het cytokine IFN $\gamma$. In een aantal zoogdieren wordt er onderscheid gemaakt tussen NK cel subgroepen die meer cytotoxisch zijn of meer cytokinen produceren.

Het aangeleerde immuunsysteem wordt geactiveerd door het pathogeen en aangeboren immuuncellen. Zogenaamde antigeen-presenterende cellen (dendritische cellen en macrofagen) presenteren pathogene fragmenten samen met instructies voor het opwekken van een efficiënte afweerreactie van aangeleerde immuuncellen. Dit kost echter tijd waardoor er op jonge leeftijd geen effectieve respons aanwezig is van het aangeleerde immuunsysteem. Dit afweersysteem omvat twee typen witte bloedcellen; B en T cellen die specifieke antigenen van pathogenen herkennen, vervolgens delen en uitrijpen tot effector cellen die de ziekteverwekkers opruimen. B cellen produceren antistoffen die pathogenen neutraliseren en herkenbaar maken voor andere immuuncellen, die ze vernietigen. $T$ cellen ruimen de geïnfecteerde cellen op (cytotoxische CD8 + T cellen) of helpen andere immuuncellen (helper CD4 + T cellen) om het pathogeen te vernietigen. Daarnaast wordt er onderscheid gemaakt tussen $C D 8 \alpha \beta+T$ cellen die antigeen-gestuurde cytotoxiciteit vertonen en CD8 $\alpha \alpha+T$ cellen die snelle NK-achtige cytotoxische responsen hebben maar ook betrokken zijn in het behouden van de darmbarrière. De functies en het snelle reactievermogen van $C D 8 \alpha \alpha+T$ cellen komen overeen met gamma delta $(\gamma \delta)$ T cellen en deze T cellen vormen een overbrugging tussen het aangeboren en aangeleerde immuunsysteem.

Kuikens zijn vatbaarder voor infecties dan volwassen kippen omdat het aangeleerde immuunsysteem nog niet volledig ontwikkeld is, waardoor ze voornamelijk afhankelijk zijn van aangeboren immuuncellen. Het stimuleren van de responsiviteit van het aangeboren immuunsysteem, met name van NK cellen 
onder andere door middel van voersupplementatie, zou de weerstand van kuikens kunnen verhogen tegen infecties.

\section{De darm; interactie immuunsysteem en darmmicrobiota}

De darm bevat veel immuuncellen welke niet mogen reageren op voercomponenten en commensale darmmicrobiota. De eerste fysieke barrière in de darm bestaat uit een slijmlaag met daaronder een laag van epitheelcellen. Tussen de epitheelcellen bevinden zich een groot aantal NK cellen, $\gamma \delta T$ cellen en CD8 + T cellen, genaamd de intra-epitheliale lymfocyten (IELs). Deze IELs reageren als eerste op binnengedrongen pathogenen en communiceren met de immuuncellen die onder het epitheel aanwezig zijn. De aantallen en functies van IELs zijn beïnvloedbaar door voer en darmmicrobiota. Darmmicrobiota omvatten de verzameling van levende micro-organismen aanwezig in de darm en zijn ook beïnvloedbaar door voer. Bepaalde darmmicrobiota, zoals melkzuurbacteriën, staan bekend om hun gunstig effect op de gezondheid, omdat ze het nestelen van pathogene bacteriën in de darm voorkomen en aanleiding zijn tot het rekruteren en stimuleren van de responsiviteit van immuuncellen. Modulatie van de darmmicrobiota samenstelling, gericht op gunstige darmmicrobiota, zou bij kunnen dragen aan een verhoogde weerstand van kuikens tegen infecties.

\section{Doel van dit proefschrift}

Dit proefschrift beschrijft studies naar mogelijke stimulatie van de immuunresponsiviteit, met name van NK cellen, en modulatie van de darmmicrobiota samenstelling, door middel van een voerstrategie, met als beoogt gevolg directe en indirecte stimulatie van immuunresponsiviteit, en een verhoogde weerstand van kuikens tegen met name de Salmonella infecties.

\section{In dit proefschrift}

In de studie van hoofdstuk 2 worden verschillende subgroepen en activiteiten van NK cellen in de IELs, milt, bloed en beenmerg beschreven tijdens de late embryonale fase tot en met drie weken oude vleeskuikens. In deze periode zijn ook de $\gamma \delta T$ en CD8+ T cel subgroepen onderzocht. Deze studie beschrijft twee grote NK cel subgroepen, namelijk IL-2R $\alpha+$ en 20E5+ NK cellen, waarvan IL-2R $\alpha+N K$ cellen voornamelijk aanwezig zijn in de IELs en milt, en 20E5 + NK cellen merendeels in het bloed en beenmerg. In gezonde kippen vertonen NK cellen weinig degranulatie-gerelateerde cytotoxiciteit. Dit mechanisme wordt in beide NK cel subgroepen aangetoond, voornamelijk door IL-2R $\alpha+$ NK cellen in de IELs en merendeels door 20E5 + NK cellen in de milt en bloed. Ook zijn beide subgroepen in de milt, merendeels IL-2R $\alpha+$ NK cellen, betrokken in cytokineproductie zoals weergegeven door de karakterisatie van NK cellen beschreven in hoofdstuk 5. Daarnaast beschrijft de studie van hoofdstuk 2 dat in kuikens evenveel CD8 $\alpha \alpha+$ en $C D 8 \alpha \beta+(\gamma \delta)$ T cellen aanwezig zijn in de IELs, milt en bloed, terwijl in oudere vleeskuikens de $(\gamma \delta)$ T cellen voornamelijk CD8 $\alpha \beta+$ zijn. De CD8 $\alpha \alpha+(\gamma \delta) T$ cellen zijn het meest aanwezig in de IELs in vergelijking met milt en bloed. Het inzicht in de distributie en activiteit van NK cellen, als ook van $\gamma \delta T$ en CD8 + T cellen, opgedaan in hoofdstuk 2, vormt de basis om vervolgens mogelijke stimulatie door darmmicrobiota en voer te onderzoeken. In de studie van hoofdstuk 3 wordt de relatie tussen het immuunsysteem en de darmmicrobiota onderzocht, door darmmicroben verkregen uit gezonde volwassen kippen (i.e. AM) toe te dienen aan pasgeboren vleeskuikens. Dit experiment toont aan dat vroege blootstelling aan AM de kolonisatie van caeca door microbiota versnelt, parallel aan een toename van het aantal intra-epitheliale IL-2R $\alpha+N K$ cellen en degranulatie-gerelateerde cytotoxiciteit van NK cellen in de IELs en milt van jonge vleeskuikens. Daarnaast leidt vroege AM blootstelling tot een toename van het aantal intra-epitheliale CD8 $\alpha \alpha+$ 
T cellen in oudere vleeskuikens. Deze resultaten suggereren dat stimulatie van NK cel responsiviteit mogelijk is door modulatie van de darmmicrobiota samenstelling op jonge leeftijd.

In de studie van hoofdstuk 4 wordt de potentie van voerstrategieën om de immuunresponsiviteit direct te stimuleren als ook indirect door modulatie van de darmmicrobiota samenstelling, onderzocht. Voeradditieven zijn achtereenvolgens getest op het stimuleren van de activiteit van aangeboren immuuncellen in vitro, en geëvalueerd op gebruiksveiligheid tijdens de embryonale ontwikkeling in ovo en invloed op productiekenmerken van vleeskuikens. Op basis van deze resultaten zijn twee plantaardige polysachariden, glucose oligosacharide en lange-keten glucomannaan, geselecteerd voor verder onderzoek in vleeskuikens. Beide voeradditieven verhoogden degranulatie-gerelateerde cytotoxiciteit van intra-epitheliale NK cellen, een fenomeen positief gecorreleerd met een toename van de relatieve aanwezigheid van commensale melkzuurbacteriën in het ileum en de caeca van jonge vleeskuikens. Vervolgens is onderzocht of de stimulatie van NK cel activiteit en modulatie van de darmmicrobiota samenstelling door het voeradditief lange-keten glucomannaan ook resulteert in een verhoogde weerstand tegen een Salmonella infectie in jonge vleeskuikens. Hiertoe was allereerst meer inzicht nodig in de rol van NK cellen in de afweerreactie tegen Salmonella. De studie beschreven in hoofdstuk 5 geeft weer dat NK cellen snel op Salmonella reageren door een toename in het aantal intra-epitheliale IL-2R $\alpha+$ en 20E5 + NK cellen, en een verhoogde activiteit, i.e. degranulatie-gerelateerde cytotoxiciteit en IFN $\gamma$ productie, in de IELs en milt. Daarnaast nam het aantal CD8 $\alpha \alpha+$ en $\mathrm{CD} 8 \alpha \beta+(\gamma \delta)$ T cellen toe, zowel vroeg als drie weken na infectie. In de studie van hoofdstuk 6 is uiteindelijk de potentie van het lange-keten glucomannaan als voeradditief, om de weerstand van jonge vleeskuikens tegen een Salmonella infectie te verhogen, beoordeeld. Het voeradditief toont inderdaad potentie om de weerstand van jonge vleeskuikens te verhogen, zoals af te leiden uit de gereduceerde hoeveelheden bacteriën in de milt en een verminderde kolonisatie van Salmonella in het ileum en de caeca. Deze observaties gaan gepaard met een toename in het aantal intra-epitheliale 20E5 + NK cellen, een verhoogde degranulatie-gerelateerde cytotoxiciteit van intra-epitheliale NK cellen en een toename in de relatieve aanwezigheid van commensale melkzuurbacteriën na infectie in vleeskuikens met een lange-keten glucomannaan voersupplementatie. Daarnaast is de relatieve aanwezigheid van commensale melkzuurbacteriën negatief gecorreleerd met het Salmonella aantal, terwijl positief gecorreleerd met degranulatie-gerelateerde cytotoxiciteit van NK cellen in de milt en Salmonella-specifieke antistoffen in het serum na infectie. De studies laten zien dat zowel directe als indirecte stimulatie van NK cel responsiviteit mogelijk is in jonge vleeskuikens wat zou kunnen leiden tot een verhoogde weerstand van kippen tegen verschillende infecties.

\section{Conclusie}

De studies beschreven in dit proefschrift tonen aan dat stimulatie van NK cel responsiviteit en modulatie van darmmicrobiota samenstelling, met als mogelijk gevolg indirecte stimulatie van immuunresponsiviteit, door middel van een voerstrategie resulteert in een verhoogde weerstand van kuikens tegen een Salmonella infectie. De praktische toepassing van het voeradditief vereist nader onderzoek op grotere schaal inclusief gedetailleerde monitoring van productiekenmerken. Dit proefschrift draagt bij aan de ontwikkeling van alternatieve manieren om de weerstand van jonge kuikens tegen infecties te verhogen en als gevolg daarvan de gezondheid van kippen te verbeteren. 


\section{Dankwoord / Acknowledgements}

Dan is het eindelijk tijd om dit laatste stuk te schrijven! Promoveren doe je natuurlijk niet alleen en ik wil dan ook iedereen bedanken die een bijdrage geleverd heeft aan dit proefschrift.

Allereerst wil ik graag Christine bedanken. Bedankt dat jij me de kans hebt gegeven om een PhD te doen binnen de kippengroep en voor je uitgebreide begeleiding tijdens het project. Ik heb je grote betrokkenheid en toegankelijkheid erg gewaardeerd; als er iets was kon ik altijd bij je terecht. Daarnaast hoefde het niet altijd over werk te gaan en kon er bijvoorbeeld tijdens het doorploegen van de FACS analyses, ook even tijd vrij worden gemaakt om die olympische schaatswedstrijd te kijken.

Victor, bedankt voor je betrokkenheid tijdens mijn PhD, ook na je pensioen. Jouw oog voor detail heeft geresulteerd in een beter proefschrift en ondanks dat je kritische blik nogal overweldigend was, heb ik er veel van geleerd. Arjan, bedankt voor je betrokkenheid en je neutrale en concrete houding tijdens het project.

Ik wil iedereen bedanken die betrokken is geweest binnen het FIRM-Broilers consortium. Bedankt/ Thanks Annelies, David, Egil, Francisca, Guilherme, Hauke en Jean, voor jullie kennis, expertise en hulp met de experimenten en dierproeven. Ook de dierverzorgers Carmen, Freek, Hanke en Marc bedankt voor de hulp en gezelligheid tijdens de dierproeven.

Ik wil de leden van mijn beoordelingscommissie bedanken voor hun tijd om dit proefschrift te lezen en beoordelen.

Naast het werk is een fijne werkomgeving ook erg belangrijk en ik wil de Immuno afdeling bedanken voor alle hulp en vooral de gezelligheid tijdens de lunch, koffie/thee pauzes, borrels en labuitjes. Allereerst, Daphne en Robin, mijn paranimfen en mede-chickies. Daphne, wat had ik zonder jou gemoeten met die dierproeven. Ontzettend bedankt voor al je kennis en hulp, en natuurlijk je gezelligheid. We hebben altijd veel gelachen tijdens de prakdagen (en ook op andere momenten), waardoor die lange dagen wel vol te houden waren. Robin, we zijn tegelijk gestart met onze PhD en konden de ups en downs in de afgelopen jaren delen. Je was altijd behulpzaam en vond het ook niet erg om met dierproeven wat eerder je bed uit te moeten om mee te helpen met prakken ;). Naast dat je een fijne collega was, ben je ook een fijn, gezellig persoon en een goede vriend geworden. Het was fijn om bij jou lekker te kunnen klagen en frustraties te delen, maar we hebben niet te vergeten ook veel lol gehad en genoten van de 'werktripjes' in Sardinië, Oxford en Seattle. Proost op het leven na een PhD!

Ook de latere mede-chickies. Adil, although we did not work together for that long it was fun to have you around during my last year. Miriam, we kenden elkaar al voordat je in de kippengroep kwam door een gezamenlijk congres in Rome en wist meteen dat je een leuke collega zou worden. Ondanks dat we kort hebben samengewerkt, hebben we wel gezellige koffie/borrel momentjes kunnen vinden zowel op als buiten het werk. Op naar de volgende!

Dankjewel aan de collega's in mijn beginperiode: Anouk, Charlotte, Elise, Manon, Marit, Michael, New, Peter, Stefanie and Susan, thanks for all the advice and fun at work. In het bijzonder Manon, bedankt voor je support en gezelligheid ook na je PhD, altijd fijn om met jou bij te kletsen! Andreja, we started together as roomies and shared frustrations but also fun moments. Thank you for your nice company at work and during trips in Sardinia and Seattle. Qingkang, it was fun working with you and I wish you the best in your future career. Coen en Daan, ondanks dat jullie bij het CBG en RIVM werkten 
bedankt voor jullie hulp en gezelligheid. Dat ik per ongeluk een glas rode wijn omstootte op je witte vest, Coen, mocht de pret niet drukken in Sardinië ;). Ook de nieuwe lichting collega's Arie-jan, Bart, Daniëlle, Emanuele, Kitty, Lena, Lobna, Naomi en Tabitha, thank you all for your help but most of all for the many laughs we had. Daniëlle, jij bent toch wel de grootste aanstichter van alle gezelligheid op de afdeling met al je spellen, pubquizen en muziekbingo's. Hopelijk kunnen we nog eens een voetbalwedstrijd meepakken!

Natuurlijk ook Aad, Alice, Femke, Ger, Irene, Karin, Peter, Tara en Willem bedankt voor het draaiende houden van de afdeling, hulp en advies maar ook de gezelligheid tijdens de borrels en labuitjes. Ger, ook bedankt voor al je hulp met de FACS experimenten! Els en Femke, bedankt dat jullie in mijn PhD begeleidingscommissie zaten.

A big thanks to Alberta, Guus, Malte, Nienke and Roel! Organizing the I\&I drinks was always fun with you, both during the meetings and at the actual event. Of course also all the people of the Infection \& Immunity programme that I met during these drinks or other events, thank you for all the nice interactions. Lisa, we leerden elkaar kennen tijdens de roadtrip rondom Seattle en konden het meteen goed met elkaar vinden. De daaropvolgende borrels en koffiemomentjes waren altijd fijn en gezellig om weer even bij te kletsen.

En niet te vergeten Alberta en Susanne, je bent nu al een tijdje Nederlands aan het leren Alberta dus dit moet lukken;). Toen de borrels niet meer konden door corona moesten we maar wat anders verzinnen. Dankjulliewel voor de gezellige etentjes waarbij ik m'n frustraties eruit kon gooien en op advies kon rekenen van onze zeergeleerde postdoc Suus. Maar bovenal de lol tijdens de fanatieke spelletjesavonden, kano-avonturen, skeelersessies, midgetgolfen en natuurlijk het weekendje Texel. Op naar de volgende activiteit!

Dan Dorothé, Marloes, Martijn, Rose en William van Team PANN 25+, bedankt voor alle gezelligheid tijdens de vergaderingen en de feesten zelf natuurlijk (al zijn die alweer een tijdje geleden). Hopelijk mogen we binnenkort weer! Ook Christiaan en Jasmijn bedankt voor de leuke en gezellige bijkletsmomentjes tijdens het wandelen, zwemmen of met een borrel. Jasmijn, ook super fijn dat je het design en de layout van mijn proefschrift wilde doen!

Ook wil ik mijn vriendinnen van de middelbare school bedanken: Ariëlla, Esther, Rike en Sandra. We zien elkaar niet zo vaak maar als we afspreken voelt het meteen weer vertrouwd. Bedankt voor jullie interesse, steun, en natuurlijk de gezelligheid! Rike, super leuk dat je de illustraties voor mijn proefschrift wilde maken!

Tenslotte wil ik graag mijn familie bedanken voor jullie betrokkenheid, interesse, steun en gezelligheid in de afgelopen jaren. In het bijzonder, lieve pap, Regina en Teunis dankjulliewel voor jullie interesse en voor de fijne afwisseling als ik bij jullie ben. Lieve mam, Chanel en Willem-jan, bedankt dat ik altijd bij jullie terecht kan. Om even frustratie te lozen maar vooral ook om mijn gedachten op wat anders te krijgen, de gezelligheid en onderlinge broer-zus plagerijen waardeer ik zeer. Ik ben blij met jullie als zus en broers! Mam, het was niet altijd makkelijk en wanneer het tegenzat kon ik altijd op jou rekenen, dankzij jouw steun kon ik toch telkens weer de motivatie vinden om door te gaan. Bedankt voor alles! 


\section{Curriculum Vitae}

Nathalie Meijerink was born on the $26^{\text {th }}$ of May 1991 in IJsselmuiden, the Netherlands. In 2009, she graduated from pre-university education (Gymnasium) at the Ichthus College in Kampen. Subsequently, she started her studies in Animal Sciences at Wageningen University in Wageningen. She conducted her bachelor thesis on typing of natural antibodies in serum and milk of dairy cows under supervision of dr. H.K. Parmentier at the Adaptation and Physiology Department of Wageningen University. She obtained her bachelor's degree in 2012, after which she continued with her master Animal Sciences at Wageningen University. During her master, Nathalie was an active member of the editorial committee of "De Veeteler", a monthly publication magazine for fellow students. She was admitted to the Research Master track of Animal Sciences in which Nathalie fulfilled an internship under supervision of Prof.dr. H.F.J. Savelkoul at the Cell Biology and Immunology Department of Wageningen University. Here, she worked on the different tasks of neutrophils and how these cells are triggered to form extracellular traps. Furthermore, Nathalie conducted a minor thesis on the relation between natural antibodies and osteochondrosis in pigs with different feeding strategies under supervision of dr. H.K. Parmentier. For her major thesis, Nathalie went to the University of Aberdeen in Scotland to conduct an internship under supervision of dr. H. Dooley at the Shark Immunology Group. Here, she worked on the therapeutic possibilities of shark-derived binding domains that target TNF- $\alpha$. Her supervisor in the Netherlands for this internship was dr. M. Forlenza of the Cell Biology and Immunology Department of Wageningen University. Nathalie obtained her master's degree with the specialization Applied Zoology, Cell Biology and Immunology in 2014. After her studies, Nathalie was a microbiology and serology analyst at a diagnostic laboratory "Veterinair Laboratorium Gelderland" in Epe, the Netherlands until 2016.

In 2016, Nathalie started her PhD project at the Infectious diseases and Immunology division of the Faculty of Veterinary Medicine at Utrecht University under supervision of Prof.dr. V.P.M.G. Rutten. Prof.dr. J.A. Stegeman and dr. C.A. Jansen. This project was part of the FIRM-Broilers consortium titled "Optimizing Flock health and performance by influencing Immune Responsiveness and the gut Microbiome by nutritional interventions in Broilers" and resulted in this thesis. During her PhD, Nathalie was chair of the scientific journal club at the Immunology division and she received a young investigator award by the American association of immunologists at the international veterinary immunology symposium in 2019.

Furthermore, Nathalie started in 2019 as volunteer regarding the organization of LGBTQI+ events at Foundation PANN. 


\section{List of Publications}

Meijerink N, van Haarlem DA, Velkers FC, Stegeman AJ, Rutten VPMG, Jansen CA. Analysis of chicken intestinal natural killer cells, a major IEL subset during embryonic and early life. Dev Comp Immunol. 2021;114. doi:10.1016/j.dci.2020.103857.

Meijerink N, Kers JG, Velkers FC, et al. Early life inoculation with adult-derived microbiota accelerates maturation of intestinal microbiota and enhances NK cell activation in broiler chickens. Front Vet Sci. 2020;7. doi:10.3389/fvets.2020.584561.

Meijerink N, de Oliveira JE, van Haarlem DA, et al. Glucose oligosaccharide and long-chain glucomannan feed additives induce enhanced activation of intraepithelial NK cells and relative abundance of commensal lactic acid bacteria in broiler chickens. Veterinary Sciences. 2021;8(6):110. doi:10.3390/vetsci8060110.

Meijerink N, van den Biggelaar RHGA, van Haarlem DA, Stegeman JA, Rutten VPMG, Jansen CA. A detailed analysis of innate and adaptive immune responsiveness upon infection with Salmonella enterica serotype Enteritidis in young broiler chickens. Vet Res. 2021;52(1):109. doi:10.1186/s13567-02100978-y.

Meijerink N, de Oliveira JE, van Haarlem DA, et al. Long-chain glucomannan supplementation modulates immune responsiveness and intestinal microbiota resulting in improved resistance to Salmonella enterica serotype Enteritidis in broiler chickens. Submitted

Kers JG, Meijerink N, Lamot DM, et al. Effects of a competitive exclusion or dietary medium-chain fatty acids intervention to alter intestinal microbiota on broiler health and performance after subclinical intestinal challenge. In preparation 


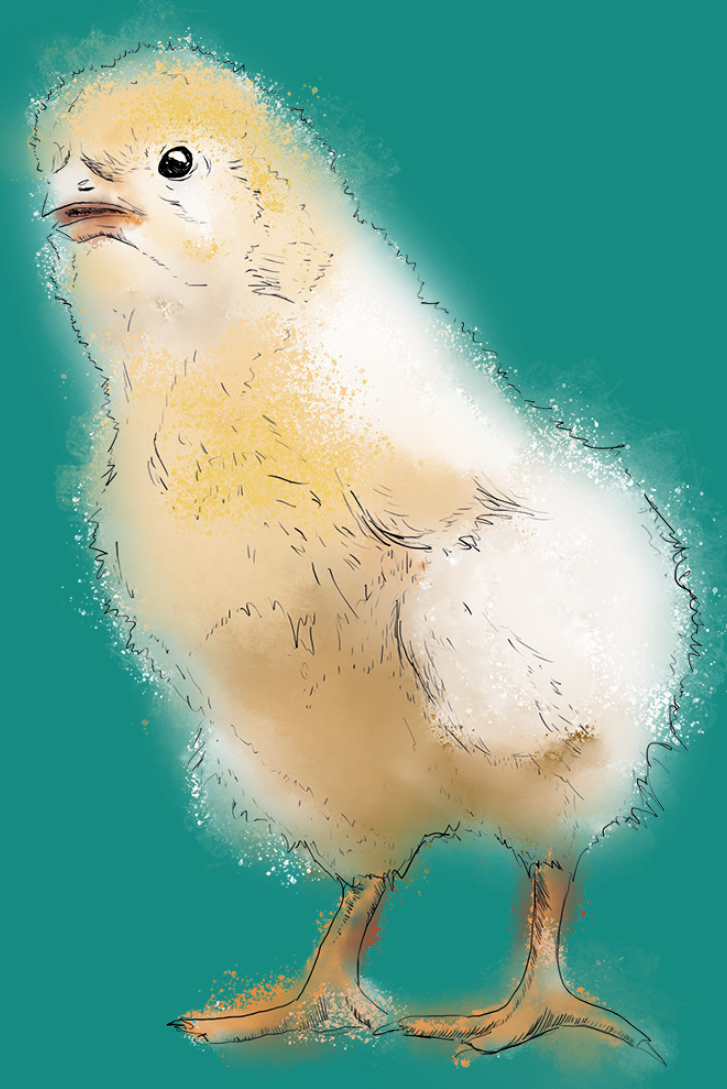

